Florida International University FIU Digital Commons

\title{
Determination of Human Scent Biomarkers for Race, Ethnicity and Gender
}

Lauren J. Colón Crespo

Florida International University, lauren.j.colon@gmail.com

DOI: 10.25148 /etd.FIDC000281

Follow this and additional works at: https:// digitalcommons.fiu.edu/etd

Part of the Analytical Chemistry Commons, and the Other Chemistry Commons

\section{Recommended Citation}

Colón Crespo, Lauren J., "Determination of Human Scent Biomarkers for Race, Ethnicity and Gender" (2016). FIU Electronic Theses and Dissertations. 2442.

https://digitalcommons.fiu.edu/etd/2442

This work is brought to you for free and open access by the University Graduate School at FIU Digital Commons. It has been accepted for inclusion in FIU Electronic Theses and Dissertations by an authorized administrator of FIU Digital Commons. For more information, please contact dcc@fiu.edu. 


\section{FLORIDA INTERNATIONAL UNIVERSITY}

Miami, Florida

\section{DETERMINATION OF HUMAN SCENT BIOMARKERS FOR RACE, ETHNICITY AND GENDER}

A dissertation submitted in partial fulfillment of the requirements for the degree of

DOCTOR OF PHILOSOPHY

in

CHEMISTRY

by

Lauren Janice Colón Crespo 
To: Dean Michael R. Heithaus

College of Arts, Sciences and Education

This dissertation, written by Lauren Janice Colón Crespo, and entitled Determination of Human Scent Biomarkers for Race, Ethnicity and Gender, having been approved in respect to style and intellectual content, is referred to you for judgment.

We have read this dissertation and recommend that it be approved.

Yong Cai

David Chatfield

Watson Lees

DeEtta Mills

Kenneth G. Furton, Major Professor

Date of Defense: March 10, 2016

The dissertation of Lauren Janice Colón Crespo is approved.

Dean Michael R. Heithaus

College of Arts, Sciences and Education

Andrés G. Gil

Vice President for Research and Academic Development and Dean of the University Graduate School

Florida International University, 2016 
(C) Copyright 2016 by Lauren Janice Colón Crespo

All rights reserved. 


\section{DEDICATION}

All the effort, determination, courage and passion that became essential to the pursuit of my doctoral degree are dedicated to those who have always made sure I don't forget the value of such qualities: my parents. To my mom, Agnes Crespo, who has not only been my motor in life, but has also been my friend, role model, cheerleader, and \#1 fan; and my dad, Luis Barreiro, who's faith in me has always been my north and has always managed to find ways to make me see the importance of not faltering my dreams. Thank you for believing in me so much and encouraging me to do my best, even at times in which things got tough. It is because of you that I am the person I am today, and for this I will be eternally grateful. I love you. 


\section{ACKNOWLEDGMENTS}

I would like to acknowledge my advisor, Dr. Kenneth G. Furton, for granting me the opportunity to be part of his research team, and helping me learn and gain experience on things that are beyond the reach of books. I would also like to thank the ColgatePalmolive Company for having provided financial support for my research over the last 4 years, and allowing me to be part of an extremely enriching and gratifying collaboration. In addition, I would like to thank Dr. Howard Holness, who has been key to the development and success of my research. Thank you for your endless patience, impeccable example, thoughtful guidance, and infallible support. To Danay Herrera, Alejandra Patino, and Carol Garcia, thank you for being such amazing students and research assistants. There is no doubt that this project would have never been the same without your dedication and hard work.

I would also like to acknowledge my committee members, Dr. Yong Cai, Dr. Watson Lees, Dr. David Chatfield and Dr. DeEtta Mills. Thank you for all your teachings and wise advice. To my lab mates, thank you for helping me grow as both a scientist and individual. In addition, I would like to thank Lorraine Sweeney, Atilda Alvarido, Magali (Maggie) Autie, and Pupi Tomassini, for having gone above and beyond to assist me since my beginnings as a graduate student; and to all those people who, at some point, made themselves available to help me surpass the challenges of my research project, thank you.

To my friends, Joana Antunes, Joao Marcelo Ribeiro, Deborah Soares and Claudia Sanchez, your unconditional love and support have always been a blessing. Thank you for always being there for me. To my roommate and friend, Adhly Huertas, 
thank you for your company on this journey and for being my study buddy for the past 9 years.

In addition to all my previous acknowledgments, I would like to say that I cannot imagine myself having accomplished my doctoral degree without the love and support of my family. For this reason, I want to thank my parents, Agnes Crespo and Luis Barreiro, for being my neverending source of strength and encouragement; my brother, Bryan Barreiro, for all the moral support and outstanding help in moments of crisis; and my boyfriend, Gabriel Rivera, for his incredible patience and disposition to ease my worries and make me happy. I love you all to the moon and back. Thank you for all of this and so much more. 


\section{ABSTRACT OF THE DISSERTATION}

\section{DETERMINATION OF HUMAN SCENT BIOMARKERS FOR RACE, ETHNICITY AND GENDER}

by

\section{Lauren Janice Colón Crespo}

Florida International University, 2016

Miami, Florida

\section{Professor Kenneth G. Furton, Major Professor}

Human scent has been the focal point of diverse scientific interests and research initiatives for the past several years. The knowledge gained about its composition has favored the advancement of multiple disciplines, and promoted the development of a wide variety of applications. Among these applications is the use of human scent as a resource for Forensic investigations, where scent profiles are often used as evidence to associate individuals to the scene of a crime. The characteristic nature of individual human scent has enabled this type of evidence to be used as a biometric tool for the differentiation of subjects. Nevertheless, the present study discusses a new perspective towards human scent's role and application in Forensic investigations. The foundation of this new perspective consists of employing human scent's biometric quality to classify individuals using common traits.

In this research study, underarm and hand odor samples were collected from Caucasian, Hispanic and East Asian individuals, of both genders. Subjects were also organized into 3 different age groups: 18-30, 35-50 and 55+ years. Headspace Solid Phase Micro-extraction Gas Chromatography Mass Spectrometry (HS-SPME-GC-MS) was used 
to create individual scent profiles for the evaluation of subject classification by age, gender and race/ethnicity. Individual classification was assessed through the identification of qualitative and quantitative patterns in the volatile organic compound (VOC) constituents that characterize human scent. Principal Component and Linear Discriminant analyses of the collected scent profiles, led to the identification and validation of characteristic VOC marker combinations for age, gender and race/ethnicity. Statistical analysis facilitated group classification and differentiation on the basis of these traits. Moreover, this study also evaluated the use of solvent extraction as a complementary technique to HS-SPME for human scent analysis. Findings from this assessment revealed that the simultaneous consideration of data from both extraction techniques favors an enhancement of the classification of subjects by means of human scent.

The discoveries achieved in this study represent a significant step for human scent as a forensic tool. The outcome of this research has cleared a new path for further human scent investigation, and highlighted its further relevance to forensic applications. 


\section{TABLE OF CONTENTS}

CHAPTER

PAGE

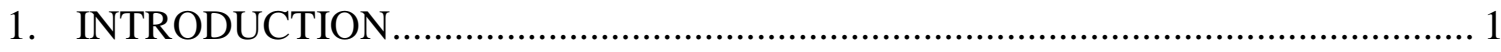

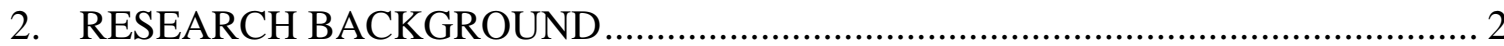

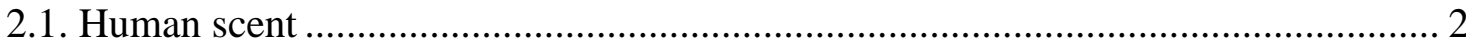

2.2. The human skin and its role in the production of scent.............................................. 3

2.3. Exudation and dispersion of human scent .............................................................. 8

2.4. Origin and composition of human scent in different body regions .......................... 9

2.5. Overview of past research work in the human scent field........................................ 11

2.5.1. Human scent and the expression of intrinsic features ....................................... 12

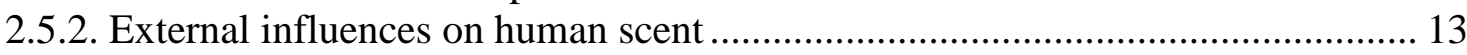

2.5.3. Human scent and its relationship with the phenotypical expression of traits .. 16

2.5.3.1. Race/Ethnicity ......................................................................................... 16

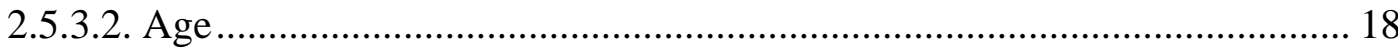

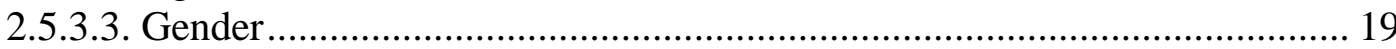

2.6. Human scent as a tool for the identification and association of individuals .......... 20

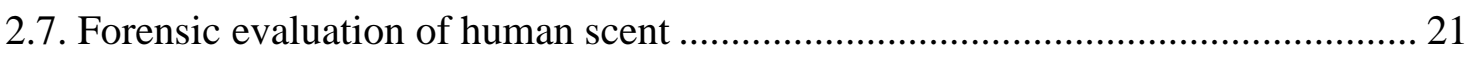

2.7.1. Human scent collection methods used in forensic investigations ..................... 23

2.7.2. Instrumental techniques for the analysis of human scent................................. 24

2.7.2.1. Solvent Extraction.................................................................................... 25

2.7.2.2. Solid Phase Micro-Extraction (SPME).................................................... 28

2.7.2.3. Gas Chromatography-Mass Spectrometry (GC-MS).................................. 31

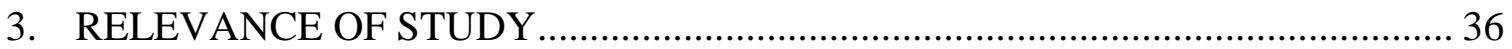

4. RESEARCH OBJECTIVES ………………………..................................... 38

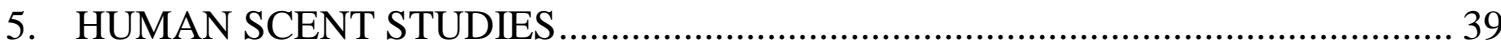

5.1. POPULATIONS UNDER STUDY AND THEIR RECRUITMENT ...................... 39

5.2. MATERIALS, OPTIMIZATION METHODS AND PROCEDURES................... 42

5.2.1. Materials for sample collection and handling .................................................. 42

5.2.2. Chemical standards and reagents .................................................................... 43

5.2.3. Optimization of research methods, conditions and procedures......................... 44

5.2.3.1. Optimization of GC-MS column and instrumental method for VOC

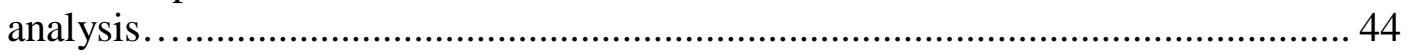

5.2.3.2. Optimization of material and conditions for HS-SPME .............................. 46

5.2.3.3. Optimization of extraction temperature for HS-SPME .............................. 47

5.2.3.4. Optimization of the underarm odor sample collection method ................... 48

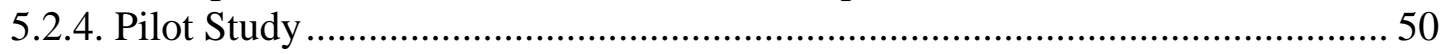

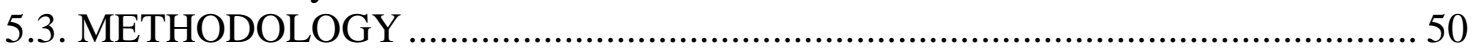

5.3.1. Preparation and analysis of soap and deodorant blanks.................................... 50

5.3.2. Pretreatment procedure of sample collection materials ...................................... 51 
5.3.3. Collection of underarm odor samples ……………....................................... 51

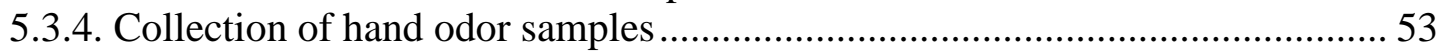

5.3.5. HS-SPME-GC-MS method for the analysis of VOCs in odor samples........... 54

5.3.6. Methods of statistical analysis ....................................................................... 55

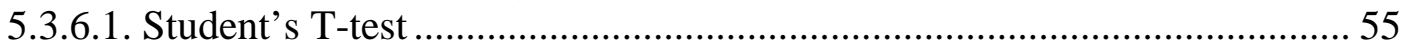

5.3.6.2. One Way Analysis of Variance (ANOVA) ................................................ 57

5.3.6.3. Tukey Kramer HSD test......................................................................... 59

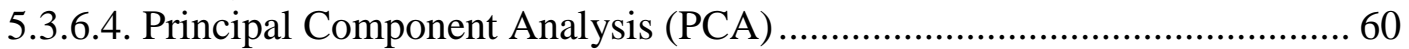

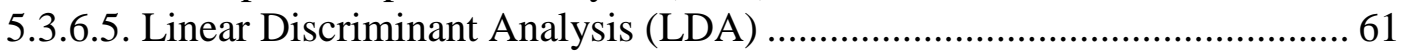

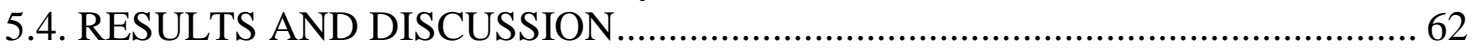

5.4.1. Results on the optimization of research methods, conditions and

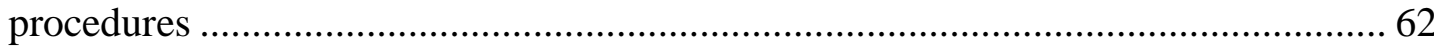

5.4.1.1. Determination of the best suited GC-MS column and method for VOC

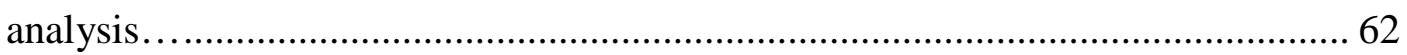

5.4.1.2. Determination of the best suited material and conditions for HS-SPME .. 68

5.4.1.3. Determination of best suited extraction temperature for HS-SPME ......... 76

5.4.1.4. Determination of best underarm odor sample collection method............... 79

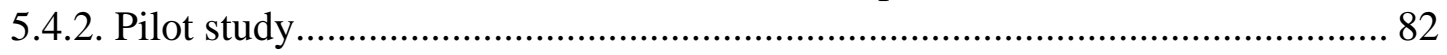

5.4.3. Determination of scent markers in underarm odor samples.............................. 85

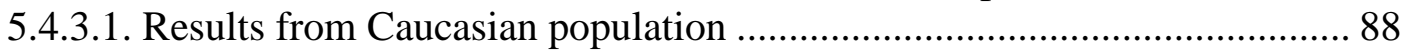

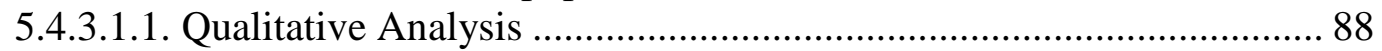

5.4.3.1.2. Gender comparison: a quantitative analysis of VOC profiles by age

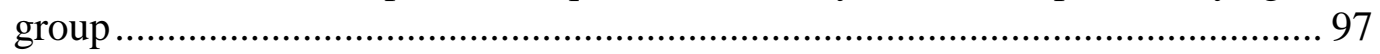

5.4.3.1.3. Age group comparison: a quantitative analysis of VOC profiles by

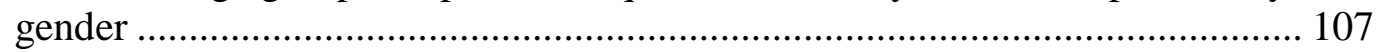

5.4.3.2. Results from Hispanic population............................................................ 126

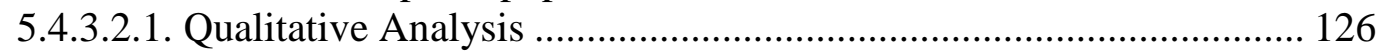

5.4.3.2.2. Gender comparison: a quantitative analysis of VOC profiles by

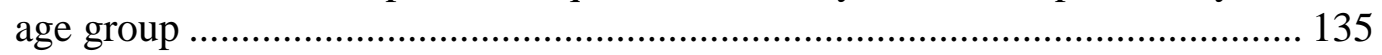

5.4.3.2.3. Age group comparison: a quantitative analysis of VOC profiles by

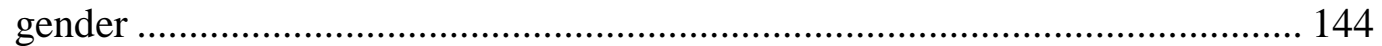

5.4.3.3. Results from East Asian population.......................................................... 163

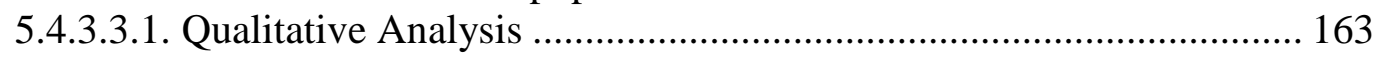

5.4.3.3.2. Gender comparison: a quantitative analysis of VOC profiles by

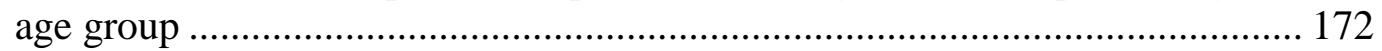

5.4.3.3.3. Age group comparison: a quantitative analysis of VOC profiles by

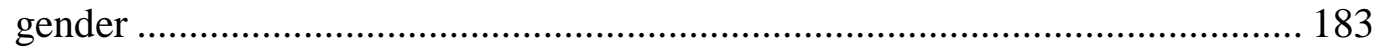

5.4.3.4. Overall comparison across all populations under study ............................ 201

5.4.3.4.1. Determination of VOC markers for individual classification by age.......................................................................................................... 203

5.4.3.4.2. Determination of VOC markers for individual classification by

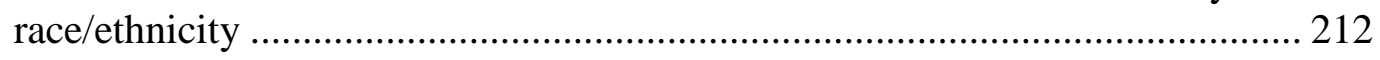

5.4.3.4.3. Determination of VOC markers for individual classification by

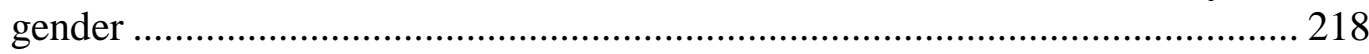
5.4.3.4.4. Determination of VOC markers for individual classification by 
race/ethnicity and gender... 225

5.4.3.4.5. Conclusion on the determination of scent markers in underarm odor samples

5.4.4. Determination of scent marker consistency in hand odor samples ................. 241

5.4.4.1. Determination of VOC markers for individual classification by age ...... 246

5.4.4.2. Determination of VOC markers for individual classification by race/ethnicity. 250

5.4.4.3. Determination of VOC markers for individual classification by gender..... 257

5.4.4.4. Determination of VOC markers for individual classification by race/ethnicity and gender 260 5.4.4.5. Confirmation of the forensic value of previously determined VOC marker combinations.

5.4.4.6. Conclusion on the determination of scent marker consistency in hand odor samples

6. EVALUATION OF HS-SPME AND SOLVENT EXTRACTION METHODS AS COMPLEMENTARY TECHNIQUES FOR THE EXTRACTION OF HUMAN SCENT SAMPLES 288

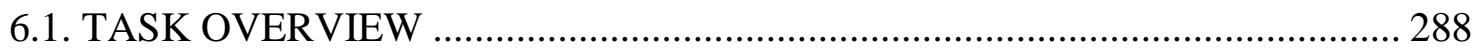

6.2. OPTIMIZATION OF SOLVENT EXTRACTION TECHNIQUE....................... 289

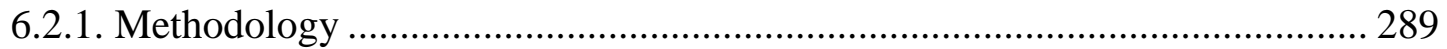

6.2.2. Results on the determination of best suited solvent extraction method .......... 290

6.3. COMPARISON OF SOLVENT EXTRACTION AND HS-SPME

TECHNIQUES IN THE EXTRACTION OF SCENT SAMPLES................................ 293

6.3.1. Methodology .......................................................................................... 293

6.3.2. Results on the evaluation of a solvent extraction method as a complementary technique for the HS-SPME of scent samples.................................. 294

6.4. CONCLUSION ON THE COMPLEMENTARITY OF SOLVENT

EXTRACTION AND HS-SPME METHODS........................................................... 301

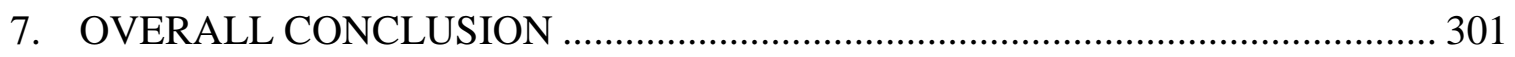

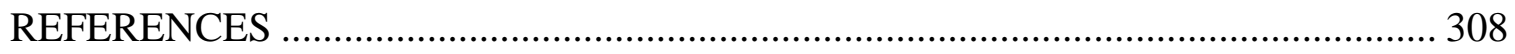

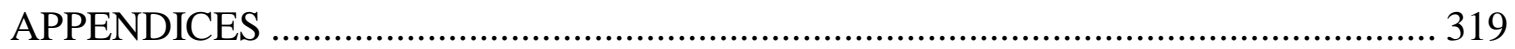

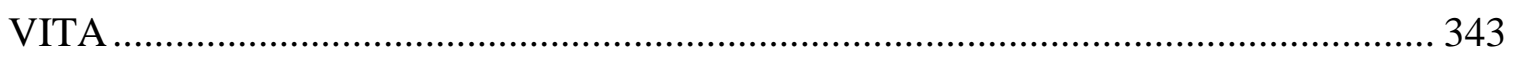




\section{LIST OF TABLES}

TABLE

PAGE

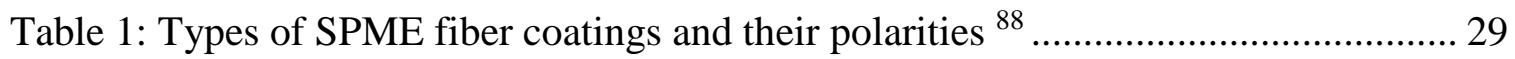

Table 2: Commercially available stationary phases and their polarity .......................... 33

Table 3: Demographic information of research participants ..................................... 41

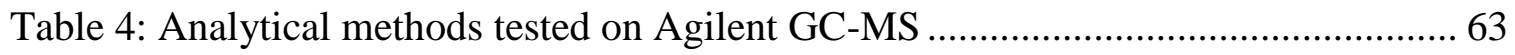

Table 5: Summary of results obtained for the comparison of analytical methods in the

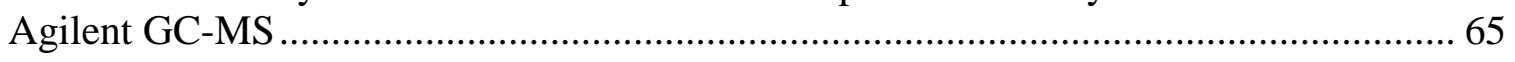

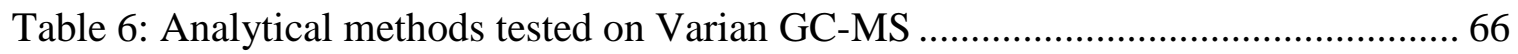

Table 7: Summary of results obtained for the comparison of analytical methods in the

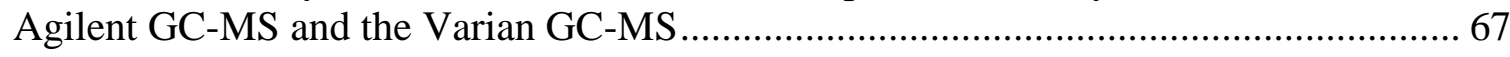

Table 8: Boiling point ranges defined for the selection of the best suited extraction

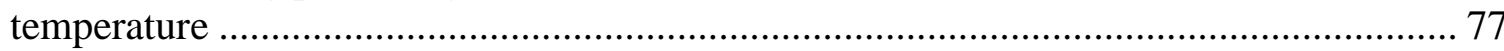

Table 9: Results of VOC extraction with different sampling methods.......................... 81

Table 10: Values for average amount extracted and standard deviation of VOCs

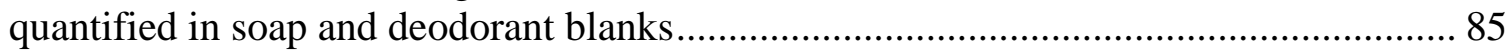

Table 11: VOCs emanating from Caucasian subjects' underarm odor samples detected and quantified by solid-phase microextraction GC/MS............................................... 95

Table 12: Average VOC amount for secondary list compounds in 18-30 year old

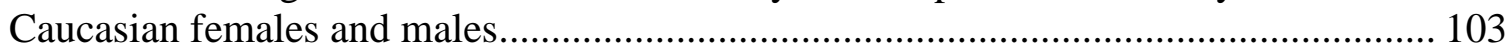

Table 13: Average VOC amount for secondary list compounds in 35-50 year old Caucasian females and males. 104

Table 14: Average VOC amount for secondary list compounds in 55+ year old Caucasian females and males. 104

Table 15: Compounds considered in secondary lists made for comparing Caucasian females of different age groups.

Table 16: Compounds considered in secondary lists made for comparing Caucasian males of different age groups. 
Table 17: VOCs emanating from Hispanic subjects’ underarm odor samples detected and quantified by solid-phase microextraction GC/MS

Table 18: Average VOC amount for secondary list compounds in 18-30 year old Hispanic females and males.

Table 19: Average VOC amount for secondary list compounds in 35-50 year old Hispanic females and males.....

Table 20: Average VOC amount for secondary list compounds in 55+ year old Hispanic females and males. 142

Table 21: Compounds considered in secondary lists made for comparing Hispanic females of different age groups

Table 22: Compounds considered in secondary lists made for comparing Hispanic males of different age groups 158

Table 23: VOCs emanating from East Asian subjects’ underarm odor samples detected and quantified by solid-phase microextraction GC/MS 170

Table 24: Average VOC amount for secondary list compounds in 18-30 year old East Asian females and males. 179

Table 25: Average VOC amount for secondary list compounds in 35-50 year old East Asian females and males 180

Table 26: Average VOC amount for secondary list compounds in 55+ year old East Asian females and males. 180

Table 27: Compounds considered in secondary lists made for comparing East Asian females of different age groups. 187

Table 28: Compounds considered in secondary lists made for comparing East Asian males of different age groups.

Table 29: VOC lists employed as criteria for subject differentiation by age group in Linear Discriminant Analysis

Table 30: VOC lists employed as criteria for subject differentiation by racial/ethnic group in Linear Discriminant Analysis.

Table 31: VOC lists employed as criteria for subject differentiation by gender group in Linear Discriminant Analysis 
Table 32: VOC lists employed as criteria for female differentiation by racial/ethnic group in Linear Discriminant Analysis

Table 33: VOC lists employed as criteria for male differentiation by racial/ethnic group in Linear Discriminant Analysis.

Table 34: Demographic information of the population surveyed for hand odor VOCs . 244

Table 35: VOC lists employed as criteria for subject differentiation by age group in

Linear Discriminant Analysis 250

Table 36: VOC lists employed as criteria for subject differentiation by race/ethnic group in Linear Discriminant Analysis. 256

Table 37: VOC lists employed as criteria for subject differentiation by gender group in Linear Discriminant Analysis 260

Table 38: VOC lists employed as criteria for racial/ethnic group differentiation in Linear Discriminant Analysis 266

Table 39: VOC list employed as criteria for female differentiation by racial/ethnic group in Linear Discriminant Analysis.

Table 40: VOC lists employed as criteria for male differentiation by racial/ethnic group in Linear Discriminant Analysis.

Table 41: Results obtained for the 5-fold cross validation of hand odor VOC marker combinations 276

Table 42: Demographic information of individuals included in external validation...... 277

Table 43: Classification accuracy results obtained from external validation 279

Table 44: Subject classification accuracy percentages obtained in hand and underarm odor assessments.

Table 45: VOCs found to be consistent in marker combinations identified for hand and underarm odor

Table 46: VOCs of most influence in subject differentiation by trait comparison using hand odor. 287 


\section{LIST OF FIGURES}

FIGURE

PAGE

Figure 1: Cross section diagram of the human skin and its components........................ 6

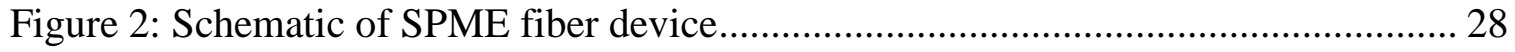

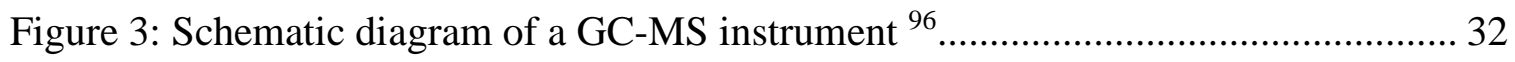

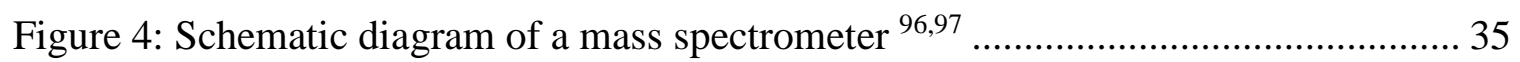

Figure 5: Description of pretreatment and sampling protocols .................................. 52

Figure 6: Results for RSD percentages achieved by methods tested in the Agilent

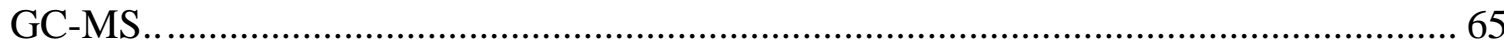

Figure 7: Results obtained for average total VOC abundance by each method tested

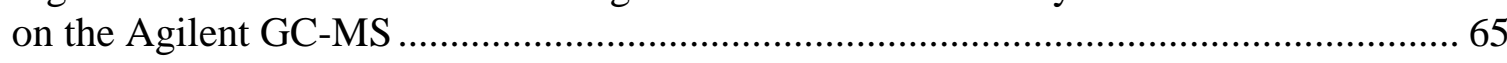

Figure 8: RSD percentages achieved by the Agilent GC-MS and the Varian GC-MS .... 68

Figure 9: Total VOC average amount extracted vs. extraction time per fiber................. 70

Figure 10: Individual VOC extraction patterns across different extraction times for the

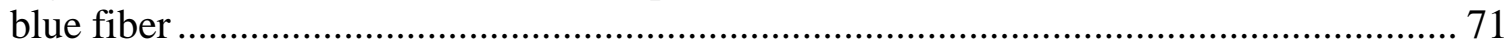

Figure 11: Individual VOC extraction patterns across different extraction times for the

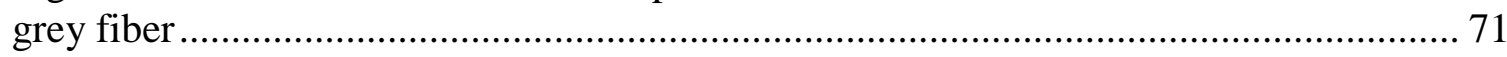

Figure 12: Individual VOC extraction patterns across different extraction times for the

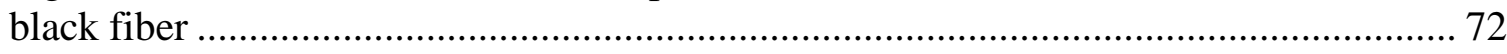

Figure 13: Average total VOC amount per extraction time........................................ 74

Figure 14: RSD percentages for total VOC amounts per extraction time ...................... 75

Figure 15: VOC amount for low boiling point compounds at different extraction

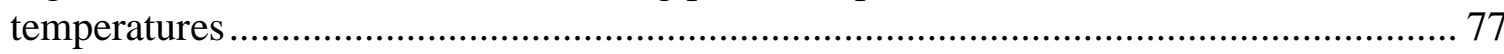

Figure 16: VOC amount for medium boiling point compounds at different extraction

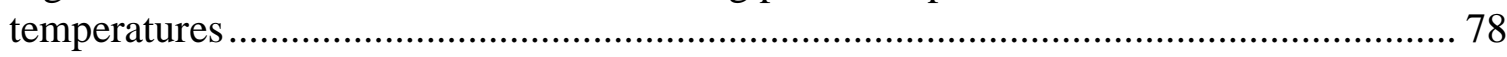

Figure 17: VOC amount for high boiling point compounds at different extraction temperatures 
Figure 18: Comparison of the average compound amount extracted from underarm odor samples of 3 females using different sampling methods

Figure 19: Scent profiles obtained for all 18-30 year old Caucasian females .................. 89

Figure 20: Scent profiles obtained for all 35-50 year old Caucasian females .................. 89

Figure 21: Scent profiles obtained for 55+ year old Caucasian females ........................ 90

Figure 22: Scent profiles obtained for all 18-30 year old Caucasian males .................... 90

Figure 23: Scent profiles obtained for all 35-50 year old Caucasian males .................... 91

Figure 24: Scent profiles obtained for all 55+ year old Caucasian males ...................... 91

Figure 25: Comparison of the average extracted amounts obtained for all 28 VOCs in

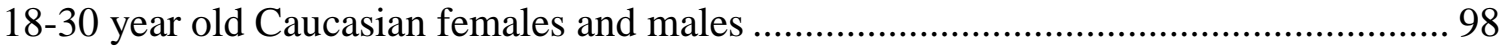

Figure 26: Comparison of the average extracted amounts obtained for all 28 VOCs in 35-50 year old Caucasian females and males ............................................................... 98

Figure 27: Comparison of the average extracted amounts obtained for all 28 VOCs in 55+ year old Caucasian females and males

Figure 28: PCA score plot of 18-30 year old Caucasian females and males including all VOCs.

Figure 29: PCA score plot (left) and loading plot (right) of 18-30 year old Caucasian females and males including VOCs from secondary list

Figure 30: PCA score plot of 35-50 year old Caucasian females and males including all VOCs.

Figure 31: PCA score plot (left) and loading plot (right) of 35-50 year old Caucasian females and males including VOCs from secondary list 106

Figure 32: PCA score plot of 55+ year old Caucasian females and males including all VOCs.

Figure 33: PCA score plot (left) and loading plot (right) of 55+ year old Caucasian females and males including VOCs from secondary list

Figure 34: Comparison of the average extracted amounts obtained for all 28 VOCs in Caucasian females of all age groups.... 108 
Figure 35: Comparison of the average extracted amounts obtained for all 28 VOCs in Caucasian males of all age groups. 108

Figure 36: Comparison of average extracted amounts obtained for the VOCs considered in the secondary lists of Caucasian females from different age groups

Figure 37: PCA score plot of 18-30 vs. 55+ year old Caucasian females including all VOCs.

Figure 38: PCA score plot (left) and loading plot (right) of 18-30 vs. 55+ year old Caucasian females including VOCs from secondary list.

Figure 39: PCA score plot of 18-30 vs. 35-50 year old Caucasian females including all VOCs.

Figure 40: PCA score plot (left) and loading plot (right) of 18-30 vs. 35-50 year old Caucasian females including VOCs from secondary list.

Figure 41: PCA score plot of 35-50 vs. 55+ year old Caucasian females including all VOCs

Figure 42: PCA score plot (left) and loading plot (right) of 35-50 vs. 55+ year old Caucasian females including VOCs from secondary list.....

Figure 43: Comparison of average extracted amounts obtained for the VOCs considered in the secondary lists of Caucasian males from different age groups

Figure 44: PCA score plot of 18-30 vs. 55+ year old Caucasian males including all VOCs

Figure 45: PCA score plot (left) and loading plot (right) of 18-30 vs. 55+ year old Caucasian males including VOCs from secondary list.

Figure 46: PCA score plot of 18-30 vs. 35-50 year old Caucasian males including all VOCs

Figure 47: PCA score plot (left) and loading plot (right) of 18-30 vs. 35-50 year old Caucasian males including VOCs from secondary list.

Figure 48: PCA score plot of 35-50 vs. 55+ year old Caucasian males including all VOCs.

Figure 49: PCA score plot (left) and loading plot (right) of 35-50 vs. 55+ year old Caucasian males including VOCs from secondary list. 
Figure 50: Scent profiles obtained for all 18-30 year old Hispanic females 127

Figure 51: Scent profiles obtained for all 35-50 year old Hispanic females 127

Figure 52: Scent profiles obtained for all 55+ year old Hispanic females. 128

Figure 53: Scent profiles obtained for all 18-30 year old Hispanic males. 128

Figure 54: Scent profiles obtained for all 35-50 year old Hispanic males. 129

Figure 55: Scent profiles obtained for all 55+ year old Hispanic males 129

Figure 56: Comparison of the average extracted amounts obtained for all 30 VOCs in 18-30 year old Hispanic females and males 136

Figure 57: Comparison of the average extracted amounts obtained for all 30 VOCs in 35-50 year old Hispanic females and males

Figure 58: Comparison of the average extracted amounts obtained for all 30 VOCs in 55+ year old Hispanic females and males.

Figure 59: PCA score plot of 18-30 year old Hispanic females and males including all VOCs.

Figure 60: PCA score plot (left) and loading plot (right) of 18-30 year old Hispanic females and males including VOCs from secondary list

Figure 61: PCA score plot of 35-50 year old Hispanic females and males including all VOCs.

Figure 62: PCA score plot (left) and loading plot (right) of 35-50 year old Hispanic females and males including VOCs from secondary list.....

Figure 63: PCA score plot of 55+ year old Hispanic females and males including all VOCs..

Figure 64: PCA score plot (left) and loading plot (right) of 55+ year old Hispanic females and males including VOCs from secondary list.

Figure 65: Comparison of the average extracted amounts obtained for all 30 VOCs in Hispanic females of all age groups 145

Figure 66: Comparison of the average extracted amounts obtained for all 30 VOCs in Hispanic males of all age groups 146 
Figure 67: Comparison of average extracted amounts obtained for the VOCs considered in the secondary lists of Hispanic females from different age groups

Figure 68: PCA score plot of 18-30 vs. 55+ year old Hispanic females including all VOCs.

Figure 69: PCA score plot (left) and loading plot (right) of 18-30 vs. 55+ year old Hispanic females including VOCs from secondary list.

Figure 70: PCA score plot of 18-30 vs. 35-50 year old Hispanic females including all VOCs

Figure 71: PCA score plot (left) and loading plot (right) of 18-30 vs. 35-50 year old Hispanic females including VOCs from secondary list

Figure 72: PCA score plot of 35-50 vs. 55+ year old Hispanic females including all VOCs.

Figure 73: PCA score plot (left) and loading plot (right) of 35-50 vs. 55+ year old Hispanic females including VOCs from secondary list....

Figure 74: Comparison of average extracted amounts obtained for the VOCs considered in the secondary lists of Hispanic males from different age groups

Figure 75: PCA score plot of 18-30 vs. 55+ year old Hispanic males including all VOCs.

Figure 76: PCA score plot (left) and loading plot (right) of 18-30 vs. 55+ year old Hispanic males including VOCs from secondary list....

Figure 77: PCA score plot of 18-30 vs. 35-50 year old Hispanic males including all VOCs.

Figure 78: PCA score plot (left) and loading plot (right) of 18-30 vs. 35-50 year old Hispanic males including VOCs from secondary list

Figure 79: PCA score plot of 35-50 vs. 55+ year old Hispanic males including all VOCs.

Figure 80: PCA score plot (left) and loading plot (right) of 35-50 vs. 55+ year old Hispanic males including VOCs from secondary list. 162

Figure 81: Scent profiles obtained for all 18-30 year old East Asian females 163

Figure 82: Scent profiles obtained for all 35-50 year old East Asian females 164 
Figure 83: Scent profiles obtained for all 55+ year old East Asian females. 164

Figure 84: Scent profiles obtained for all 18-30 year old East Asian males. 165

Figure 85: Scent profiles obtained for all 35-50 year old East Asian males. 165

Figure 86: Scent profiles obtained for all 55+ year old East Asian males. 166

Figure 87: Comparison of the average extracted amounts obtained for all 27 VOCs in 18-30 year old East Asian females and males

Figure 88: Comparison of the average extracted amounts obtained for all 27 VOCs in 35-50 year old East Asian females and males

Figure 89: Comparison of the average extracted amounts obtained for all 27 VOCs in 55+ year old East Asian females and males.

Figure 90: PCA score plot of 18-30 year old East Asian females and males including all VOCs.

Figure 91: PCA score plot (left) and loading plot (right) of 18-30 year old East Asian females and males including VOCs from secondary list

Figure 92: PCA score plot of 35-50 year old East Asian females and males including all VOCs.

Figure 93: PCA score plot (left) and loading plot (right) of 35-50 year old East Asian females and males including VOCs from secondary list.

Figure 94: PCA score plot of 55+ year old East Asian females and males including all VOCs.

Figure 95: PCA score plot (left) and loading plot (right) of 55+ year old East Asian females and males including VOCs from secondary list

Figure 96: Comparison of the average extracted amounts obtained for all 27 VOCs in East Asian females of all age groups

Figure 97: Comparison of the average extracted amounts obtained for all 27 VOCs in East Asian males of all age groups

Figure 98: Comparison of average extracted amounts obtained for the compounds in the VOC secondary lists of East Asian females from different age groups. 
Figure 99: PCA score plot of 18-30 vs. 55+ year old East Asian females including all VOCs.

Figure 100: PCA score plot (left) and loading plot (right) of $18-30$ vs. $55+$ year old East Asian females including VOCs from secondary list.

Figure 101: PCA score plot of 18-30 vs. 35-50 year old East Asian females including all VOCs.

Figure 102: PCA score plot (left) and loading plot (right) of 18-30 vs. 35-50 year old East Asian females including VOCs from secondary list

Figure 103: PCA score plot of 35-50 vs. 55+ year old East Asian females including all VOCs.

Figure 104: PCA score plot (left) and loading plot (right) of 35-50 vs. 55+ year old East Asian females including VOCs from secondary list.....

Figure 105: Comparison of average extracted amounts obtained for the compounds in the VOC secondary lists of East Asian males from different age groups.

Figure 106: PCA score plot of 18-30 vs. 55+ year old East Asian males including all VOCs.

Figure 107: PCA score plot (left) and loading plot (right) of 18-30 vs. 55+ year old

East Asian males including VOCs from secondary list

Figure 108: PCA score plot of 18-30 vs. 35-50 year old East Asian males including all VOCs.

Figure 109: PCA score plot (left) and loading plot (right) of 18-30 vs. 35-50 year old East Asian males including VOCs from secondary list..... 200

Figure 110: PCA score plot of 35-50 vs. 55+ year old East Asian males including all VOCs 200

Figure 111: PCA score plot (left) and loading plot (right) of 35-50 vs. 55+ year old East Asian males including VOCs from secondary list

Figure 112: Average VOC amounts per age group for all 26 VOCs in the initial compound list.

Figure 113: LDA canonical plot using all 26 VOCs as criteria for subject differentiation by age group. 205 
Figure 114: LDA canonical plot using compounds from secondary list as criteria for subject differentiation by age group

Figure 115: LDA canonical plot using compounds from stepwise regression method as criteria for subject differentiation by age group

Figure 116: Average VOC amounts per racial/ethnic group for all 26 VOCs in the initial compound list

Figure 117: LDA canonical plot using all 26 VOCs as criteria for subject differentiation by racial/ethnic group.

Figure 118: LDA canonical plot using compounds from secondary list as criteria for subject differentiation by racial/ethnic group

Figure 119: LDA canonical plot using compounds from stepwise regression method as criteria for subject differentiation by racial/ethnic group.

Figure 120: Average VOC amounts per gender group for all 26 VOCs in the initial compound list

Figure 121: LDA canonical plot using all 26 VOCs as criteria for subject differentiation by gender.

Figure 122: LDA canonical plot using compounds from secondary list as criteria for subject differentiation by gender

Figure 123: LDA canonical plot using compounds from stepwise regression method as criteria for subject differentiation by gender

Figure 124: Average VOC amounts per race/ethnicity and gender for all 26 VOCs in the initial compound list.

Figure 125: LDA canonical plot obtained through both the initial list and the stepwise regression methods for subject differentiation by race/ethnicity and gender

Figure 126: LDA canonical plot obtained using the initial VOC list for female subject differentiation by race/ethnicity.

Figure 127: LDA canonical plot obtained using the initial VOC list for male subject differentiation by race/ethnicity.

Figure 128: LDA canonical plot using compounds from stepwise regression method as criteria for female subject differentiation by race/ethnicity 
Figure 129: LDA canonical plot using compounds from stepwise regression method as criteria for male subject differentiation by race/ethnicity ..................................... 233

Figure 130: Hand odor profiles from Caucasian females of all age groups .................. 242

Figure 131: Hand odor profiles from Caucasian males of all age groups ..................... 242

Figure 132: Hand odor profiles from Hispanic females of all age groups..................... 243

Figure 133: Hand odor profiles from Hispanic males of all age groups....................... 243

Figure 134: Hand odor profiles from East Asian females of all age groups................... 244

Figure 135: Hand odor profiles from East Asian males of all age groups.................... 244

Figure 136: Average amounts of hand VOCs per age group.................................... 247

Figure 137: LDA canonical plot using all 26 VOCs as criteria for subject differentiation by age group using hand odor

Figure 138: Average amounts of hand VOCs per race/ethnic group............................ 251

Figure 139: LDA canonical plot using all 26 VOCs as criteria for subject differentiation by race/ethnic group using hand odor ............................................... 253

Figure 140: LDA canonical plot of stepwise regression VOCs as criteria for subject differentiation by race/ethnic group using hand odor .............................................. 255

Figure 141: Average amounts of hand VOCs per gender......................................... 257

Figure 142: LDA canonical plot using all 26 VOCs as criteria for subject

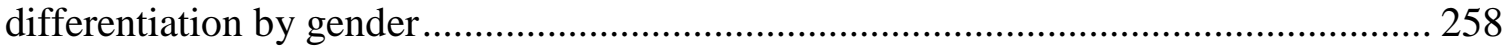

Figure 143: LDA canonical plot using compounds from stepwise regression method

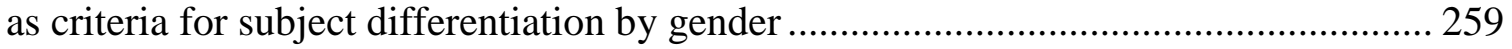

Figure 144: Average VOC amounts per race/ethnicity and gender .............................. 262

Figure 145: LDA canonical plot obtained using all 26 VOCs for subject differentiation by race/ethnicity and gender 264

Figure 146: LDA canonical plot obtained using the stepwise regression method for subject differentiation by race/ethnicity and gender.... 265 
Figure 147: LDA canonical plot obtained through both the initial list and the stepwise regression methods for female differentiation by race/ethnicity. 268

Figure 148: LDA canonical plot obtained using the initial VOC list for male subject differentiation by race/ethnicity.

Figure 149: LDA canonical plot obtained using VOCs from the stepwise regression method for male subject differentiation by race/ethnicity 272

Figure 150: Hand odor profiles of individuals considered for the external validation method.

Figure 151: VOC Amount extracted per compound for $1 \mathrm{~mL}$ and $2 \mathrm{~mL}$ Methanol extracts.

Figure 152: VOC Amount extracted per compound for $1 \mathrm{~mL}$ and $2 \mathrm{~mL}$ Methylene Chloride extracts

Figure 153: VOC Amount extracted per compound for $1 \mathrm{~mL}$ and $2 \mathrm{~mL}$ Hexane extracts.

Figure 154: Comparison of total VOC amounts obtained by different extract volumes and solvents tested.

Figure 155: Overlaid chromatograms depicting VOCs extracted by solvent extraction and HS-SPME from a human scent sample. 296

Figure 156: Differentiation of Caucasian females by age group using HS-SPME data. 297

Figure 157: Differentiation of Caucasian females by age group using compiled HS-SPME and solvent extraction data. 297

Figure 158: Differentiation of males by age group using HS-SPME data 298

Figure 159: Differentiation of males by age group using compiled HS-SPME and solvent extraction data 298

Figure 160: Differentiation of 18-30 year old females and males using HS-SPME data. 299

Figure 161: Differentiation of 18-30 year old females and males using compiled HS-SPME and solvent extraction data. 299

Figure 162: Differentiation of 55+ year old females and males using HS-SPME data.. 300 
Figure 163: Differentiation of 55+ year old females and males using compiled

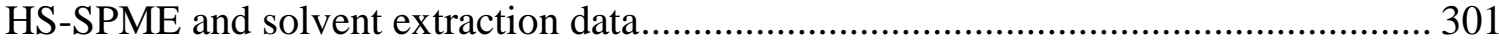




\section{ABBREVIATIONS \& ACRONYMS}

ATM

CAR/PDMS

CL

DVB/CAR/PDMS

GC-MS

HS - SPME

LDA

MS

PA

PC1

PC2

PCA

PDMS

PDMS/DVB

PEG

RSD

SPME

VOC
Automated Teller Machine

Carboxen Polydimethylsiloxane

Confidence Level

Divinylbenzene-carboxen-

polydimethylsiloxane

Gas Chromatography

Gass Chromatography - Mass Spectrometry

Headspace Solid Phase Microextraction

Linear Discriminant Analysis

Mass Spectrometry

Polyacrylate

First Principal Component

Second Principal Component

Principal Component Analysis

Polydimethylsiloxane

Polydimethylsiloxane-divinylbenzene

Carbowax-polyethylene glycol

Relative Standard Deviation

Solid Phase Micro-Extraction

Volatile Organic Compound 


\section{INTRODUCTION}

In 2007, 52 year old Mindy Schloss was reported missing in Anchorage, Alaska. ${ }^{1}$ After her disappearance, her car was found in a parking lot close to the airport. A few weeks later Schloss was found dead in the woods of Knik-Goose Bay Road in Wasilla, and her neighbor, Joshua Wade, was arrested. He was charged with bank fraud for having used Schloss' ATM card soon after her disappearance. After her death, the police found Wade's DNA in Schloss' car. Human scent trailing canines were also able to follow Wade's scent from Schloss' car, and several ATMs he had visited, to his house. The identification of Wade's human scent led investigators to obtain a search warrant, which resulted in the collection of additional incriminating evidence. As a result of this evidence, in 2010, Joshua Wade pleaded guilty to the murder of Mindy Schloss. In addition, he also pleaded guilty of having murdered Della Brown, another crime for which he had been previously acquitted in the year 2003. ${ }^{2}$

The resolution of criminal cases in a court of law depends on the presentation and admissibility of forensic evidence that can assist in the incrimination or exoneration of accused suspects. To obtain this kind of evidence, law enforcement officials conduct forensic investigations that focus on evaluating every aspect of a crime scene. As it was demonstrated in the State $v$ Wade case, human scent evidence obtained in these investigations can play a crucial role in the resolution of forensic cases. However, despite its usefulness, the admissibility of human scent evidence is often challenged in US courts of law. The reliability and evidentiary value of human scent has been questioned in multiple court cases over the years. ${ }^{3-6}$ These challenges have created a need to investigate human scent as a valuable type of forensic evidence, and continue to seek new 
information that can help overcome the current resistance to its use. The present dissertation has been written to enhance current human scent knowledge and promote the generation of scientific findings that support its use as a reliable tool in forensic investigations. In order to accomplish this goal, this dissertation presents a new perspective on the evaluation of human scent, and shows that there is more to its relevance in forensic cases than its role as an individualizing feature. There is an abundance of additional information that can be provided by human scent that is yet to be explored. Therefore, this dissertation focuses on the identification of human scent features that can be associated to traits that are shared between individuals from the same population. This innovative approach can not only increase the level of detail of the information obtained from an individual's human scent profile, but also enhance and support the potential of human scent's evidentiary power in the resolution of criminal cases.

\section{RESEARCH BACKGROUND}

\subsection{Human scent}

The term scent is defined as the effluvia from a substance that affects the sense of smell, as an odor left by an animal on a surface passed over, or as a characteristic or particular odor. ${ }^{7}$ The definition of this term provides a fitting description of what the general concept of scent involves. However, in order to make reference to the concept of human scent, it is necessary to enhance this definition by taking into consideration additional elements. An article published by Curran et al. in 2005, defines human scent as "the most abundant volatile organic compounds determined to be in the headspace above 
scent samples". ${ }^{8}$ This article states that human odor can also be influenced by other substances, and describes it as being composed of three different types of odor: primary, secondary and tertiary odors. The primary odor of an individual is said to be consistent over time and only impacted by genetic factors of an individual. On the other hand, the secondary odor is caused by the influence of diet and environmental factors on an individual, and the tertiary odor portrays the influence of outside sources that add exogenous components to an individual's odor profile (e.g., scented cosmetic products). ${ }^{8}$ The reason why human scent is considered a source of information for an individual's personal traits is because, regardless of the possible changes that exogenous sources or conditions can impose in the odor profile, primary odor constituents always remain. Hence, primary odor constituents play a key role in using human scent for individual characterization.

\subsection{The human skin and its role in the production of scent}

The skin, which is the largest organ of the human body, serves as a mechanical barrier to protect individuals from the external environment. ${ }^{9}$ As part of its role as a barrier, it is involved in the body's mechanisms for thermoregulation and homeostatic control, as well as with providing the human body innate immune defense. The skin consists of two major tissue layers, the epidermis and the dermis, followed by a layer of subcutaneous fat that serves as boundary for these layers and the rest of the body's interior. ${ }^{10}$ The subcutaneous fat layer plays an important role in cushioning corporal trauma, providing insulation and calorie reserve, as well as in endocrine functions like the regulation of hunger and energy metabolism, among others. ${ }^{10}$ In the same token, the 
dermis is considered to be a supporting matrix of polysaccharides and proteins that link for the production of macromolecules. The thickness of this particular layer varies from less than $0.5 \mathrm{~mm}$ to more than $5 \mathrm{~mm}$, and the two main protein fibers contained in its extracellular matrix are collagen and elastic tissue, which is known to be composed of elastin and elastin-associated microfibrils (Figure 1). ${ }^{10}$ Out of these two, collagen constitutes $80-85 \%$ of the dermis' dry weight and is what provides skin its tensile strength, while elastic fibers only constitute between $2-4 \%$ of the matrix and are what provide skin its elasticity and resilience. ${ }^{10}$ In addition to these components, the extracellular matrix also contains different non-collagenous glycoproteins that facilitate cell adhesion and motility, as well as glycosaminoglycan/proteoglycan macromolecules, which serve as ground substance between collagen and the elastic tissue and are in charge of maintaining dermal hydration. Among other important characteristics of the dermis are that it is also known to have a rich blood supply by means of a superficial and a deep vascular network, and that although hair follicles and skin glands are derived from the epidermis, it is in the dermis that they are deeply embedded. ${ }^{11}$ The dermis is attached to the epidermis through a network of proteins and glycoproteins that extends from inside basal keratynocites into the dermal surface. Besides facilitating the adhesion of both skin layers, components of this dermal-epidermal junction contribute to cell migration and epithelial-mesenchymal signalling events. ${ }^{10}$

The epidermis, the outermost of skin layers, is a stratified epithelium of typically 0.05-0.1 mm in thickness that is composed of four main cell types: keratinocytes, melanocytes, Merkel cells and Langerghans cells. The epidermal structure is subdivided into several different cell layers, all of which play a role in the formation of the epidermis 
as whole. The first of these layers, the basal layer (stratum basale or stratum germinativum), is where epidermal stem cells are located. Once these stem cells undergo cell division, the spinous cell layer (stratum spinosum) arises. It is from this second layer that cells move outwards and progressively differentiate to form the granular cell layer (stratum granulosum), and the cornified cell layer (stratum corneum). ${ }^{10}$ The complete process of cellular progression from the basal layer to the stratum corneum takes approximately 30 days. ${ }^{10}$ During the cell differentiation process, keratinocytes change their cuboidal basal layer form to become flat hexagonal corneocytes capable of covering a higher surface area in the stratum corneum, outermost layer of the epidermis. Cells in the stratum corneum lack nucleus and cytoplasmic organelles, but instead contain a highly insoluble cornified envelope within the plasma membrane that contains protein precursors and different lipids (e.g., fatty acids, sterols and ceramides). On the other hand, the rest of the cell types present in the epidermis posses different roles within the epidermal structure and function. For instance, melanocytes are in charge of distributing melanin pigment packages to keratinocytes, which provides color to the skin. The Langerhans' cells, which originate from bone marrow, are antigen-presenting cells and, as such, play an important role in adaptive immune responses in the skin. ${ }^{10}$ Moreover, Merkel cells serve as mechanosensory receptors in response to touch. ${ }^{10}$ 


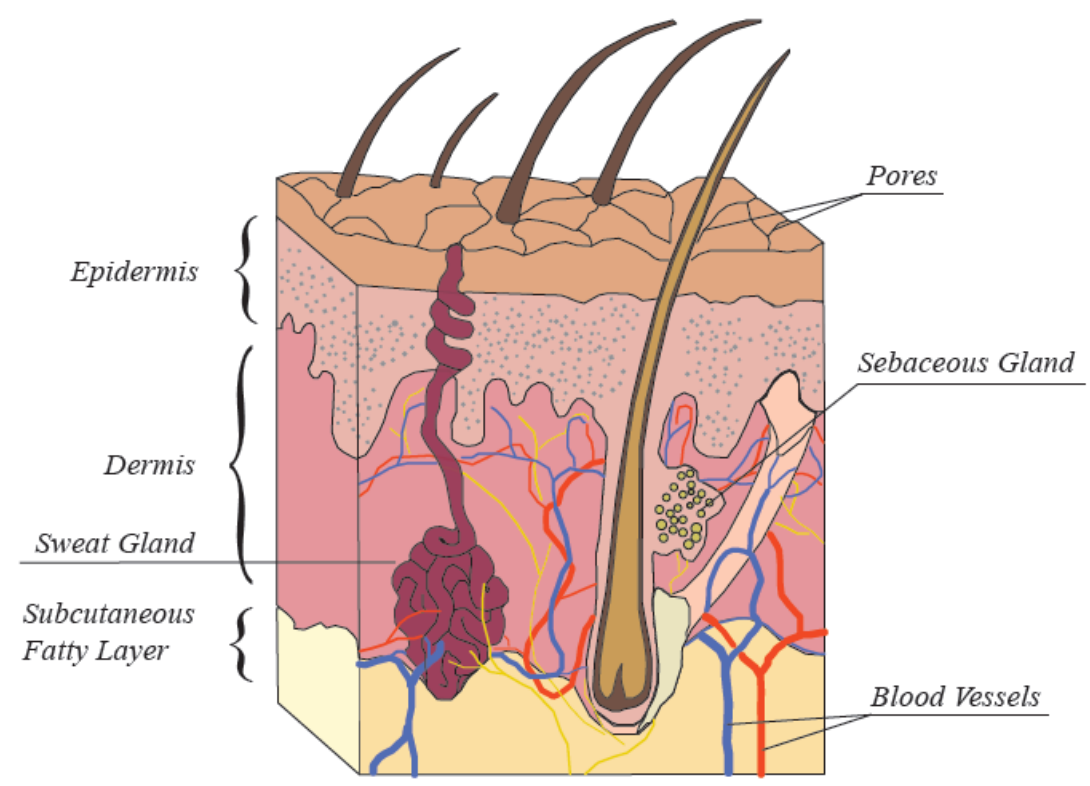

Figure 1: Cross section diagram of the human skin and its components

Besides the already mentioned epidermal and dermal components, there are other components that are of great importance to the human skin. The presence of hair and glands throughout the skin also play an important role on the human body. Distribution and abundance of these skin components vary according to the region of the body and the type of skin that predominates in each region. There are two main kinds of human skin: glabrous (non-hairy) and hair-bearing skin. Glabrous skin is located on the palms and soles, is known to be up to ten times thicker than skin in other body sites, has encapsulated sense organs within the dermis, and lacks hair follicles and sebaceous glands. On the contrary, hair-bearing skin has both hair follicles and sebaceous glands but lacks encapsulated sense organs. Sebaceous glands are usually attached to hair follicles, although they can also be found throughout the body on their own, and are responsible for sebum secretion. ${ }^{11}$ Sebum is a complex mixture of lipids that has been previously 
associated with providing the body photoprotection, antimicrobial activity, delivery of fat-soluble anti-oxidants to the skin's surface and pro- and anti-inflammatory activity by means and influence of certain lipids. ${ }^{12,13}$ Although sebum's definite function in the human body remains unknown, studies on its composition have revealed that it consists of squalene, esters of glycerol, wax and cholesterol, free cholesterol and fatty acids. ${ }^{13}$ According to these studies, triglycerides and fatty acids are the predominating components comprising $57.5 \%$ of sebum, followed by wax esters (26\%), squalene (12\%) and ultimately cholesterol, which along with its esters accounts for the $4.5 \%$ of total lipids. ${ }^{13,14}$

Sweat gland distribution and abundance are also factors that vary with body regions. The human body contains two to three million eccrine sweat glands distributed over almost its entire surface, while they are most abundant on the soles, palms and the forehead. ${ }^{15}$ Although the size of eccrine glands varies among individuals, and even within an individual, they have a tubular shape, an approximate length of four to eight $\mathrm{mm}$, and an outside diameter of 30 to $60 \mathrm{~mm} .{ }^{16}$ Eccrine secretions form at the gland's coil inside the dermis and flow through a duct that delivers them to the skin's surface. Eccrine sweat glands are known to be primarily responsible for the human body's thermoregulatory sweating, which is why it secretes large amounts of fluid in a relatively short time. ${ }^{10}$ Eccrine secretions are mostly water, but also contain amino acids, electrolytes and minerals. ${ }^{16}$ Since it originates in extracellular fluid, it is also known to contain very small concentrations of the same solutes as plasma. ${ }^{16}$ On the other hand, Apoeccrine glands are also known to continuously secrete an "eccrine like-watery fluid". ${ }^{17}$ However, difficulties with discriminating the secretions from eccrine and apoeccrine glands have 
not yet allowed to elucidate the exact composition of apoeccrine secretions. Apoeccrine glands are considered a mixed type gland, since they share qualities with both eccrine and apocrine glands. They are presumed to develop from eccrine glands, and are characterized by being present in hair-bearing skin, particularly in the axillae. ${ }^{17}$ The close relationship of apoeccrine and eccrine glands explains the morphological and secretory similarities, among other, that are shared by both types of glands. ${ }^{17-19}$ Nevertheless, as apocrine glands, apoeccrine glands are considered to play an important role on hairbearing skin, especially in the axillary region.

Contrary to eccrine and apoeccrine glands, apocrine sweat glands have a low secretory output (e.g., less than $1 \mathrm{~mL}$ per day in axillary organ). This difference makes of the role of apocrine glands be of less importance to the human body's thermal regulation. However, apocrine secretions are produced on a continuous basis in the gland's secretory coil and excreted through a duct that connects to the hair follicle canal near the skin surface. These secretions are also odorless, sterile, rich in lipids and proteins, and, for the most part, found in the genital, axillary and mammary areas of the body where there is a higher density of apocrine glands. ${ }^{16}$ Apocrine secretions have been recognized by different studies as having an influence on human chemical signaling, as well as being the main source for axillary odor. ${ }^{20,21}$

\subsection{Exudation and dispersion of human scent}

The human body serves as the main source and carrier of human scent. Humans shed their entire outermost skin layer every two to four weeks. This shedding process occurs at a rate of about a thousand cells/ $\mathrm{cm}^{2} / \mathrm{h}$ or approximately $5 / 10^{8}$ cells/day, and 
causes a constant deposition of dead skin cells into the environment. ${ }^{22}$ These dead skin cells, also known as rafts, are approximately 14 microns in size, 0.07 micrograms in weight, and can carry up to four viable microbial units. These microbial units are responsible for the generation of odorous vapors, or volatile organic compounds, as they engage in the breakdown of skin gland secretions.

Raft dispersion occurs by means of warm air currents that surround the human body. ${ }^{23}$ These currents originate at the feet, and travel up the rest of the body at an approximate speed of 125 feet per minute. ${ }^{23,24}$ Differences in temperature between the human body and the environment induce changes in the speed of these currents, and aid in transferring the rafts into the environment. The fact that rafts possess an aerodynamic shape facilitates their transmission away from the body and allows human scent to be dispersed. ${ }^{24}$ As dispersion occurs, human scent gets deposited in the environment, and remains as a distinctive mark of an individual's presence. The inevitable transmission and wide availability of human scent highlight its relevance in forensic investigations.

2.4. Origin and composition of human scent in different body regions

Variations in the characteristics of skin and its secretions, across different body regions, exert an important role in generating human scent. Body secretions depend on the type of skin gland producing them, and have been seen to serve as primal matter for the formation of body odor. Therefore, the innate differences across human body regions cause the exudation and deposition of different types of odors from individuals into the environment. 
In general, the human skin hosts a series of different bacteria populations in its surface, which exert metabolic activity on odorless skin gland secretions and transform them into odorous compounds. ${ }^{25}$ As part of its role as a barrier, the skin favors the growth of commensal bacteria that protect the host from pathogenic bacteria. Among these bacterial residents are Staphylococcus sp., Micrococcus sp., coryneforms and some gramnegative organisms. Each one of these microorganisms plays an important part in maintaining the delicate balance that exists between them and their host, and contributes to the host's healthy living. ${ }^{26}$ Nevertheless, the type and density of bacteria varies with anatomic location, local humidity, and certain qualities that are specific to each host. Among these qualities, the amount of sebum and sweat produced, the age, and hormonal status of an individual have been seen to influence bacterial population densities. ${ }^{27}$

The axillary region is among the main human body sites known for the production of scent. The axilla differs from other body sites in that it possesses multiple skin gland types (e.g., eccrine, sebaceous, apoeccrine and apocrine glands) that favor the creation of an occluded, moist, and nutrient rich, environment for dense bacterial colonization $\left(10^{6}\right.$ organisms $\left./ \mathrm{cm}^{2}\right)$. According to previous research, the predominant bacterial flora in the axilla are Corynebacterium sp. and Staphylococcus sp. ${ }^{25,28,29}$ These bacterial species biotransform apocrine secretions into odorous molecules that end up constituting characteristic and individual odor profiles. ${ }^{29-31}$ Past studies have reported having found steroid derivatives, sulfanylalkanols, and volatile short-chain fatty acids among the constituents of these profiles. ${ }^{31,32}$ In addition, they have also managed to associate different intensity levels of axillary odor with variations in the combinations and ratios of such compounds. 
Besides the axilla, human hands are body parts with great potential for the study of human scent. Despite the fact that hands have more exposure to the environment than the axillary vault, previous studies have shown that they can still portray odor profiles. ${ }^{33,34}$ The existence of this type of odor profiles makes of hands a valuable asset for the assessment of human scent, especially as a result of their ease to transfer odors. Human hands are characterized by a high density of eccrine sweat glands (700 glands $\left./ \mathrm{cm}^{2}\right) .{ }^{17}$ Hence the dominant secretions in this region consist of $99 \%$ water, and can arise as a result of both thermoregulatory and emotional body responses. ${ }^{17}$ The bacterial populations that reside in human hands are mostly staphylococci, corynebacteria, propionibacteria, gram negative bacilli, and yeasts, and past studies have identified a variety of chemical functionalities as hand odor constituents. ${ }^{35}$ Alcohols, acids, aldehydes, hydrocarbons, esters, ketones and nitrogen-containing compounds have been found to be the main components of hand odor. ${ }^{33}$

Despite of the differences in scent expression manifested by human hands and axillae, both body regions have proven to provide good insight on the nature of human scent. Both hands and axillae have been often surveyed for the evaluation of human scent constituents and the determination of their impact on characteristic odor profiles. ${ }^{33,36-38}$ Hence, a great number of the advancements accomplished in the human scent field could be considered direct results of these assessments.

\subsection{Overview of past research work in the human scent field}

Throughout the years, a wide series of research studies have focused on studying the origin, roles and composition of scent from different body regions. Scent samples 
have been collected from the axillae $e^{30,38,39}$, forearms ${ }^{21}$, hands ${ }^{4,40}$, feet ${ }^{41}$, back ${ }^{21}$, and the whole body of individuals ${ }^{42}$, among other sites, in an attempt to pursue this assessment. The mechanism through which human scent is generated, and the effect of bacterial flora populations on its expression, have been among the most common topics in human scent research..$^{25,43-45}$ Nonetheless, a great diversity of scientific investigations have been conducted with the intention of obtaining a better understanding of human scent's complex nature.

\subsubsection{Human scent and the expression of intrinsic features}

Different studies have evaluated scent and its ability to portray features that are intrinsic to the human body. In order to obtain a better understanding of this ability, multiple studies have turned to investigating the impact of genetics on scent expression. As a result, confounding evidence has been found to relate the highly polymorphic genes in the major histocompatibility complex (MHC) with human scent.

The genes in the MHC (human leukocyte antigen (HLA) for humans), are in charge of the human body's immune recognition system. These genes, are characterized by a high degree of genetic variation, that is thought to be maintained as a result of mating preferences ${ }^{46}$ Past studies on both mice and humans, have revealed that these preferences can be driven by olfactory cues. ${ }^{46-48}$ Findings of this nature, among many others, highlight the influence of genetics on mechanisms for chemical communication between individuals.

In the case of humans, considerable uncertainty and controversy have often characterized the topic of chemical communication by means of scent. Nevertheless, past 
studies have reported findings that demonstrate the impact of human scent on human responses and behaviors. Wedekind and Füri, in 1995 and 1997, revealed that men and women prefer the body odor of individuals whose MHC is dissimilar to their own. ${ }^{49,50}$ The discoveries made by Wedekind and Füri suggested a clear relationship between the MHC genes and the expression, and preference, of scent. However, the possible role of scent in human mating dynamics remained implicit until a study, by Herz and Cahill, was published in 1997. The Herz and Cahill study was able to confirm that female mating preferences rely more on odor cues than other sensory cues. ${ }^{51}$ Therefore, the research helped establish human scent as a factor of influence in mating preferences amongst humans. Despite of the need to further investigate human scent as a method of chemical communication, studies like these have already managed to expose its potential to exhibit intrinsic individual features.

\subsubsection{External influences on human scent}

As stated in the definition of human scent, an individual's odor can be affected by both internal and external conditions. For this reason, research studies have also evaluated the way in which external factors, that are independent from the body's natural processes and genetics, can exert an impact on odor profiles. There are different types of external influences (e.g., temperature and humidity, the types of cosmetic products used, etc.). However, the impact exerted by dietary habits and diseases are amongst the most studied factors of external impact on human scent.

The volatile organic compounds that constitute scent are thought to reflect the human body's metabolic conditions. Hence, under the influence of a disease, an 
individual's scent starts to display different changes. ${ }^{52}$ These changes have been assessed by multiple research studies in an attempt to investigate human scent as a resource for medical diagnosis. As a result, different human scent constituents have been identified as biomarkers for diseases, including cancer, metabolic disorders and infections. ${ }^{52-56}$ According to the National Institutes of Health Biomarkers Definitions Working Group, a biomarker is "a characteristic that is objectively measured and evaluated as an indicator of normal biological processes, pathogenic processes, or pharmacologic responses to a therapeutic intervention.” ${ }^{52,57}$ Therefore, the changes in scent caused by the impact of diseases, represent an important expression of the human body's condition. In this case, scent variations facilitate an objective alternative to diagnose health conditions and accurately pursue optimum medical treatment. However, the fact that human scent characteristics have already been associated to the impact of diseases, suggests there is potential to expand on this type of associations for many other factors. There is no doubt that this type of application provides hopeful insight on the possibility of using human scent to identify other types of biomarkers. This sort of accomplishment, would not only help expand the knowledge on this sort of scent attributes, but would also lead to significant advances in science and, in particular, the forensic field.

On the other hand, the influence of diet on human scent expression has also been widely investigated. ${ }^{58-61}$ For instance, a study by Havlicek et al. evaluated the effect of red meat consumption on the attractiveness of body odor. ${ }^{58}$ In this study, the axillary odor of 17 males was evaluated after their consumption of controlled "meat" and "nonmeat" diets. Subsequently, all samples were presented to a group of 30 females, which rated the samples on the basis of their intensity, pleasantness, sexual attractiveness and 
masculinity. The results obtained from repeated measures analysis of variance revealed that, when on the nonmeat diet, the scent of donors was judged as significantly more attractive, pleasant, and less intense. Therefore, red meat consumption was concluded to have a negative impact on the way females perceive body odor hedonicity. ${ }^{58}$ Other studies published by Wallace et al. and Hepper et al., also addressed the impact of diet in scent expression. ${ }^{59,60}$ The study by Wallace et al. evaluated the hand odor of identical twins and siblings, and revealed that it is significantly easier for women to match the odor to a specific twin when both twins follow different diets. ${ }^{59}$ Similarly to these findings, Hepper et al. also reported that diet does play a role on body odor, by showing that dogs are capable of discriminating between adult monozygotic twins on different diets, but not between infant twins that share the same diet. ${ }^{60}$ Both of these studies evaluate the impact of diet in scent profiles as a factor that could hinder the consideration of human scent as a characteristic trait in individuals. Nevertheless, work published by Hudson subsequently to these studies, stated that the highest similarity seen between the VOCs present in the hand odor profiles of monozygotic twins who share the same diet was an $86 \% .{ }^{61}$ This percentage demonstrates that the odor profiles from monozygotic twins can still be distinguished on the basis of slight differences, despite the impact from external factors (i.e., diet). Hence, Hudson's work supports the fact that human scent's characteristic features have their foundation on endogenous/genetic factors. These findings were further confirmed in 2011, when Pinc et al. published a study that showed trained dogs, used routinely and exclusively for scent identification lineups, were completely capable of discriminating individual scents of monozygotic twins under the same diet. ${ }^{62}$ Therefore, these two studies serve as example of how past research has found ways to test human 
scent as a resource for individual differentiation, and prove the importance of genetic influence on its expression. The characteristic odor of every human, also known as "body odor signature”, serves as an essential source of information about the individual who produces it. ${ }^{63}$ Human scent's characteristic nature is precisely the fundamental principle behind its use as a forensic tool.

2.5.3. Human scent and its relationship with the phenotypical expression of traits

A wide variety of research studies have sought to obtain information on the chemical constituents that make human scent a characteristic feature in individuals. ${ }^{30,36,38}$ The study of human scent's composition and characteristic nature has also led other research efforts to evaluate the extent to which scent differentiation is feasible in humans. ${ }^{33,34,40,62,64}$ Amongst the previously mentioned efforts, different studies have assessed the relationship between certain individual traits and human scent expression. In most cases, the evaluation of this type of relationship has resulted beneficial to the better understanding of scent as a human feature.

\subsubsection{Race/Ethnicity}

In recent years, researchers became interested in investigating the relationship between two specific traits: earwax type and race/ethnicity. ${ }^{65,66}$ A study by Yoshiura et al. reported that the single nucleotide polymorphism (SNP) in the ABCC11 gene was responsible for dictating the earwax type of an individual. ${ }^{65}$ As stated in other previous reports, the recessive dry cerumen phenotype was found to be abundant amongst East Asians, while uncommon in populations of European and African origins. ${ }^{65,66}$ These 
results, not only provided evidence to show that earwax expression is directly influenced by genetics, but also set the foundation to associate this trait with an individual's race/ethnicity. Moreover, other studies have also suggested a relationship between earwax type and axillary odor. ${ }^{65,66}$ The relationship between these two human features has been explained to be founded on the histological similarities between the ceruminous and apocrine glands. ${ }^{65,66}$ The histological similarities between these glands provided insight on the potential relevance of comparing earwax, or even body odor, as a characteristic between race/ethnic groups. Consequently, a study by Prokop-Prigge et al. used SPME-GC-MS to evaluate the VOCs emanating from earwax samples of Caucasian and East Asian individuals. ${ }^{67}$ The results revealed that the odor profiles obtained, for individuals of both races, did not portray significant qualitative differences. On the other hand, significant differences were noted in the amounts of earwax VOCs expressed by each group. ${ }^{67}$ In response to the findings reported by Prokop-Prigge et al. and all the other studies mentioned, the potential to assess human scent as a differentiating feature across race/ethnic populations has been emphasized. Therefore, new research efforts have been directed towards the next steps in human scent research: the assessment of individual differentiation and classification by race/ethnicity.

Besides earwax, there are other physiological traits that have been associated with variations in body odor amongst individuals of different race/ethnic backgrounds. Examples of these traits are the number and size of sweat glands in individuals across different populations. These traits have a direct impact on the volume of secretions made available for bacterial breakdown on the body, causing consequential differences in body odors across races/ethnicities. ${ }^{68}$ According to the literature, individuals from the Negroid 
race tend to have larger sized apocrine glands and a greater number of total sweat glands; followed by Caucasians and Asians. ${ }^{24,69}$ These physiological tendencies were supported by the work of Hurley and Shelley in 1960. In their study, it was revealed that Asians have little or no body odor, and that this is indicative of reduced or absent apocrine gland activity. ${ }^{69}$ Also, Negro subjects were found to consistently produce greater amounts of apocrine sweat, in comparison to individuals from other races. The high abundance of apocrine sweat revealed a direct correlation between the amount of sweat, and the size and abundance of sweat glands, in individuals from the Negroid race. ${ }^{69}$ On the other hand, another study has also demonstrated the feasibility of using human scent to associate an individual to its race/ethnicity. According to Bates et al., elephants can use olfactory cues to distinguish between Kenyan ethnic groups that represent different levels

of threat to their survival. ${ }^{70}$ Therefore, the findings reported by Bates et al. show that it is possible for some animals to detect variations in human scent across different ethnic groups, and use them as a resource for human identification. This sort of ability supports the need to further investigate the impact of race/ethnicity on human scent expression.

\subsubsection{Age}

In the same way research efforts have aimed to define the relationship between race/ethnicity and human scent, other studies have focused on obtaining a better understanding of the relationship between age and human scent. A study by Mitro et al. assessed body odor differences between age groups by evaluating the perceptual ratings and discrimination performance of 41 individuals. In this particular study, it was found that individuals were able to discriminate among age groups, and that such ability was 
mainly mediated by their discrimination of the body odor from "old-age" donors. ${ }^{71}$ On the other hand, other studies have focused on associating body odor constituents with specific age groups on the basis of their qualitative and quantitative nature in odor profiles. ${ }^{21,72}$ For instance, Gallagher et. al evaluated the odor profiles of 25 subjects that were classified in two different age groups: 19-40 years (young) and 41-79 years (old). ${ }^{21}$ As a result, quantitative differences between the chemical constituents of such profiles were attributed to aging, and several compounds were identified as biomarkers for increased age. ${ }^{21}$ Nevertheless, although there is no doubt that this kind of study provides a starting point to understanding body odor differences between individuals whose age is different, additional research is still required in this area of study.

\subsubsection{Gender}

Gender has also been evaluated as a trait that exerts influence on human scent. Multiple studies have assessed the analysis of human scent profiles with the objective of identifying features in body odor that can be independently associated to each of the sexes. ${ }^{30,31,36,37,73}$ One of these studies was published in 1996 by Zeng et al., and compared the components of both female and male axillary sweat. ${ }^{36}$ As a result, the study reported that there are both qualitative and quantitative differences between the components of sweat of the two genders. ${ }^{36}$ Moreover, a study by Penn et al. stated that, although it was not possible to identify marker compounds that were unique to any of the sexes, it was still possible to statistically discriminate individuals by gender using their odor profiles. ${ }^{37}$ According to Penn et al.'s results, the difference between genders relies on an assortment of marker compounds. Hence the study evaluated differences in the frequency of 
compounds, between female and male odor profiles, to accomplish gender differentiation. ${ }^{37}$ Regardless of the extent or focus of the investigation made by these different studies, most of them agree that there are differences in body odor that can be associated to gender. Obtaining a better understanding of the human scent differences between females and males would facilitate using this type of information in a wide range of applications, especially within the forensic field. Therefore, a need to expand on the current knowledge about these differences and find new ways to accurately apply new findings continues to persist.

\subsection{Human scent as a tool for the identification and association of individuals}

The fact that characteristic information of an individual can be obtained by assessing a human scent profile, results in a powerful resource for the identification of persons of interest. The value of human scent as a resource relies on the ability to use those characteristics to establish links between individuals and their features. These links are precisely what allows the identification, association or differentiation of individuals on the basis of their odor.

A study published by Curran et al. in 2007, surveyed hand odor profiles from a total of 60 subjects, and identified a series of low, medium and high frequency compounds that portrayed a high level of variability amongst them. ${ }^{33}$ On the basis of such findings, statistical analysis was performed to differentiate individuals by means of the chemical composition of their profiles. The results of the statistical analysis revealed that a high level of differentiation was possible between the subjects under study. ${ }^{33}$ The feasibility of using human scent to identify individuals was tested, once again, in another 
study published in 2010. The study, also published by Curran et al., revealed that using the "primary odor constituents" of the hand odor profiles from ten subjects, allowed the correct discrimination and identification of $99.54 \%$ of the individuals tested. ${ }^{40}$ Findings from these studies provide scientific evidence that supports the use of human scent as a forensic resource. Nevertheless, there are still a wide variety of topics in human scent research that need to be further explored for this resource to achieve its full potential. The incorporation of scientific findings from other fields (i.e., medicine, cosmetology, etc.) into forensic applications could help enhance the versatility and practicality of human scent. Hence, there is a lot more to be achieved in the journey of making human scent become an unchallenged forensic tool for individual differentiation.

\subsection{Forensic evaluation of human scent}

Human scent has been often used as a type of evidence in forensic investigations. Qualities like its ease of transfer and biometric value have made of human scent a useful resource to uncover details about different cases. The most common way of making use of human scent evidence is by using canines to identify suspects on the basis of their odor. Canines have been used as a resource for scent identification since the beginning of the $20^{\text {th }}$ century, as a result of their ability to make this sort of association. These animals have been found capable of identifying individuals by both tracking and trailing methods, which means they are capable of following an individual's tracks from both up close and from a distance. ${ }^{47}$ On account of the diversity of canine capabilities, there are different methods that can be used to have canines identify scent. As part of tracking methods, canines are capable of following fresh scent tracks to identify individuals, despite the 
presence of obstacles, other people, or even, changes in direction. ${ }^{74}$ On the contrary, trailing methods involve presenting a canine with a scent sample from a person of interest and having it differentiate that specific scent from other scent trails present in a search location. ${ }^{74}$ In this method, the canine can associate the odor and follow a specific path to the person of interest or to a new relevant location. ${ }^{75}$

On the other hand, some forensic investigations may also involve a protocol known as Scent Identification Line-ups. As part of this protocol, canines are presented with a scent sample that is collected at a crime scene, and is presumed to contain the odor of the perpetrator. ${ }^{47}$ As a result, the canine must identify the odor from the crime scene amongst an array of samples that includes the scent sample of the suspect. The purpose of the lineup approach is to identify the perpetrator by means of the scent traces collected. Therefore, in the event that the canine matches the odor from the crime scene with a sample from the array, he would indicate his match with a learned response. ${ }^{47}$ Despite the general concepts that give human scent's forensic investigation its relevance, there is ample variation in the ways different countries assess this type of evidence. In the case of the United States, human scent can be admissible to court upon complying with the standards set by the jurisdiction considering the evidence. This condition has, in many cases, caused setbacks in the acceptance of human scent as a forensic tool. Nevertheless, these setbacks have also stimulated the search for new methods to strengthen human scent evidence and improve its capability to overcome forensic challenges. 


\subsubsection{Human scent collection methods used in forensic investigations}

In all cases in which human scent is considered to be forensic evidence, the credibility and value of the evidence relies in the use of efficient sample collection methods and materials. There are several different methods of human scent collection that are normally employed when the samples are to be used in canine-assisted investigations. The first method is known as "direct scenting” and consists of having the canine smell the scent source (i.e., evidence containing scent traces) directly. The second method consists of using a sorbent material (usually a gauze pad) to swipe the object's surface and transfer the odor from the surface onto the gauze. Similarly, the third method consists of placing the sorbent material on the surface of the object for a period of time. Nevertheless, in the third method, scent absorption occurs from the sole contact of the collection material with the object's surface. All of these methods involve a high risk of contaminating and tampering other potential pieces of trace evidence that may be present in the object being used (e.g., DNA, fingerprints, etc.) Therefore, less invasive methods of human scent collection have also been developed, as alternatives to protect the integrity of forensic evidence. $^{74}$

The Scent Transfer Unit, or STU-100, is an airflow device that acts like a type of vacuum, and has been used before by different police agencies and the Federal Bureau of Investigation (FBI) to collect human scent samples. The vacuum is used to transfer scent, from the surface of pieces of evidence, onto a $5 \times 9$-inch sterile gauze pad that is contained in the device. ${ }^{74}$ This method allows the collection of scent samples without having to make contact with the evidence, which minimizes the evidence's deterioration. Similar to the STU-100, the Human Scent Collection System (HSCS) is also another 
device that can be used as a forensic vacuum to collect human scent samples. The HSCS is a cylindrical device that draws human scent onto a 4" x 4" gauze pad by means of a dynamic airflow. Therefore, it also has the advantage of not requiring physical contact with other pieces of forensic evidence in order to collect scent samples. ${ }^{75}$ Both the STU100 and the HSCS are portable, battery powered devices, and possess different settings for airflow speed. These sorts of features ease their use as sampling devices and facilitate the acquisition of human scent evidence during forensic investigations.

2.7.2. Instrumental techniques for the analysis of human scent

As previously mentioned, human scent is characterized by containing a variety of VOCs in very low concentrations. Therefore, the assessment of its qualitative and quantitative properties normally involves the use of sampling and/or preconcentration procedures. ${ }^{76}$ These procedures aim to maximize the detection of VOC constituents that may, otherwise, not reach the detection limits of analytical instrumentation. On the other hand, another important element in human scent analysis is analyte desorption. The fact that most sample collection methods rely on the use of sorbent materials (i.e., gauze pads), makes the use of solvent or thermal desorption steps a regular practice in human scent analysis. The incorporation of these steps into the analytical procedures ensures optimum analyte detachment from the collection material, and enhances the accuracy and efficiency of the analysis. Solvent Extraction (SE) is considered to be amongst the most common techniques that use solvents to enhance analyte desorption from sorbent materials. ${ }^{77}$ On the other hand, Solid Phase Micro-Extraction (SPME) is amongst the most common sampling techniques employed for human scent analysis. The SPME 
technique facilitates analyte preconcentration and, when used concurrently with Gas Chromatography-Mass Spectrometry, it can make use of direct thermal desorption to release the analytes. ${ }^{78}$

\subsubsection{Solvent Extraction}

Solvent extraction is a simple desorption technique that is normally pursued as a sample preparation step prior to instrumental analysis. The technique consists in using a liquid organic solvent to dissolve an analyte of interest (VOCs in the case of human scent.). ${ }^{79}$ The dissolution of analytes occurs as a result of their chemical affinity with the added solvent. The chemical affinity between a solvent and the analyte is determined by the similarity of their intermolecular forces. These forces dictate the solubility properties of organic compounds, which explains why polar analytes dissolve in polar solvents, and non polar solvents dissolve non polar analytes. ${ }^{80}$

The mass transfer process, through which an analyte is dissolved in an organic solvent, during solvent extraction, is called partition. ${ }^{81}$ During partition, the sample and the solvent are placed in contact with each other until the concentration of the analyte reaches equilibrium in both phases. ${ }^{81}$ The distribution constant for the system analyte/solvent/matrix during extraction is defined by Equation $1 .^{81}$

$$
K=\frac{C_{e x t}}{C_{r e s}}
$$

\section{Equation 1: Distribution constant for solvent extraction system}


The previous equation describes the distribution constant $(\mathrm{K})$ as the ratio between the concentration of the analyte in the solvent $\left(\mathrm{C}_{\text {ext }}\right)$ and the remaining concentration of the analyte in the sample $\left(\mathrm{C}_{\mathrm{res}}\right) \cdot{ }^{81}$ According to the equation, the magnitude of the distribution constant depends on the affinity between the solvent and the analyte. For this reason, there is a direct correlation between the distribution constant and the system's efficiency for extraction. The extraction efficiency $(\eta)$ for this type of system is described by Equation 2 in terms of the distribution constant $(\mathrm{K})$ and the phase ratio ( $\beta) .{ }^{81}$ Hence, the manipulation of those two values in every solvent extraction system is the key to maximizing extraction efficiency.

$$
\eta=\frac{K}{K+\beta}
$$

\section{Equation 2: Extraction efficiency for a solvent extraction system}

The fact that the solvent extraction method yields a relatively large volume of extract (normally 1 - $5 \mathrm{~mL}$ ) represents a drawback for the technique. ${ }^{82}$ The concentration of VOCs is usually low in scent samples, which leads to low preconcentration factors (1:50 - 1:250) when doing solvent extraction. ${ }^{82}$ These large volumes increase the amount of sample needed for further analysis, which may not always be favorable for subsequent instrumental techniques; especially those involving capillary columns (e.g., GC-MS). Moreover, the dilution factor can also increase the discrepancy between the concentration of VOCs in a scent sample and the sensitivity of the detector in the instrumental technique. Therefore, in an attempt to mitigate these challenges, analyte preconcentration is often assessed prior to instrumental analysis. ${ }^{82}$ One of the most basic preconcentration methods is done by using nitrogen or helium gas to evaporate the extract and reduce its 
volume. As a result, the concentration of the extracted analytes increases. The increase in concentration improves instrumental detection and sensitivity, which leads to achieving optimum analytical recoveries.

The use of solvent extraction has various advantages over the use of other extraction methods. This technique is considered to be a simple, fast, and compatible with high molecular mass compounds. ${ }^{83}$ Also, it does not require expensive equipment and allows the repeated analysis of samples. ${ }^{77}$ Nevertheless, like all techniques, it also has a few disadvantages. Since solvent extraction involves a dilution of the sample with an organic solvent, there is a chance for sample contamination by the solvent. In addition, solvent extraction may be more prone than other methods to lose any highly volatile compounds that may be present in the sample at the moment of extraction. ${ }^{77}$

Despite of the challenges that could arise from using solvent extraction, different research studies have successfully used this technique in the past to assess human skin VOCs. ${ }^{21,36,84}$ Hexane, methylene chloride and ether, have been some of the most commonly used solvents in this type of assessment. Besides their variety in solvent alternatives, these studies have also taken different approaches to pursue solvent extraction. Some studies have attempted direct solvent extraction from sweat samples ${ }^{21,85}$, while others have extracted different sample collection materials (e.g., cotton gauze pads). ${ }^{32,36,41,84}$ Nevertheless, in all cases solvent extraction has been a useful technique to obtain new information about human scent's composition and source. 


\subsubsection{Solid Phase Micro-Extraction (SPME)}

Solid Phase Micro-Extraction is a solvent-free sample preparation technique that consists of two separate processes. The first process involves the partition of analytes between the sample and a fiber coating, while the second involves analyte desorption from the fiber coating into an analytical instrument. ${ }^{78}$ The technique employs a simple and portable device to extract analytes from solid, liquid and gaseous samples. The device contains a thin fused-silica fiber, coated with an absorbent polymer that ranges between 7-100 $\mu \mathrm{m}$ in thinckness. ${ }^{78}$ In addition, the fiber is covered by a metal rod (fiber holder) for protection, which makes the device resemble a modified syringe. ${ }^{78}$

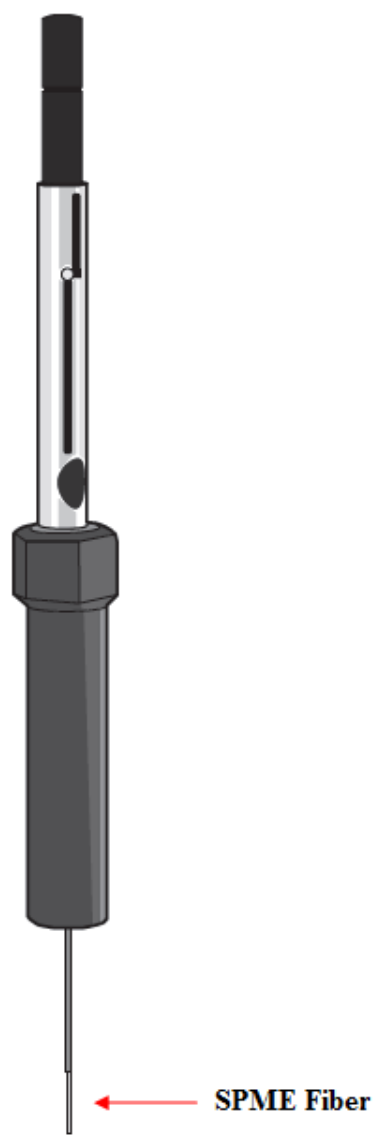

Figure 2: Schematic of SPME fiber device 
During extraction, the fiber is exposed to the sample, and analytes are concentrated in the polymer coating by means of absorption/adsorption processes. ${ }^{86} \mathrm{~A}$ fiber's selectivity for a compound is determined by the compound's volatility and affinity to the chemical properties (e.g., polarity) of its polymer coating. ${ }^{78}$ On the other hand, the amount of analyte extracted by a fiber is directly proportional to the volume of the fiber coating. ${ }^{78}$ The fact that the volume of fiber coating can impact the extraction proves that the sensitivity of the SPME technique is significantly dependent on the characteristics of the SPME fiber. Therefore, a variety of fiber coating chemistries have been developed to expand the list of compounds that can be sampled using SPME, and to enhance the efficiency of the sampling technique (Table 1).

\section{Table 1: Types of SPME fiber coatings and their polarities ${ }^{87}$}

\begin{tabular}{|l|c|}
\hline \multicolumn{1}{|c|}{ Fiber Coatings } & Polarity \\
\hline Polydimethylsiloxane (PDMS) & Non Polar \\
\hline Polyacrylate (PA) & Polar \\
\hline Carbowax-polyethylene glycol (PEG) & Polar \\
\hline Polydimethylsiloxane-divinylbenzene (PDMS/DVB) & Mid-Polar \\
\hline Carboxen Polydimethylsiloxane (CAR/PDMS) & Mid-Polar \\
\hline Divinylbenzene-carboxen- polydimethylsiloxane (DVB/CAR/PDMS) & Mid-Polar \\
\hline
\end{tabular}

There are two main methods of sampling using SPME: direct liquid sampling and headspace sampling. ${ }^{78}$ In the direct liquid sampling, the SPME fiber is exposed to be in direct contact with a liquid sample. As a result, analyte partitioning occurs between the fiber coating and the sample's matrix. On the other hand, in headspace sampling the sample is placed in a sealed container and the fiber is only exposed to the environment above the sample. For this reason, in this case, the SPME system consists of three phases: the sample, its headspace and the fiber coating. ${ }^{78}$ Nevertheless, in both modes of 
sampling, analyte recovery is dependent on the overall equilibrium between the phases involved. The sampling of volatile organic compounds, like those found in human scent, is the perfect example of an application in which headspace SPME is used. In this type of sampling, analyte partitioning occurs between the fiber coating and the sample's headspace, between the sample's headspace and the sample's collection material (e.g., cotton gauze pad), and between the sample's collection material and the fiber coating. Consequently, the amount of analyte absorbed/adsorbed by the fiber coating $\left(\mathrm{n}_{\mathrm{f}}\right)$, when extracting a finite sample volume, can be expressed by the following equation ${ }^{78}$ :

$$
\mathrm{n}_{\mathrm{f}}=\frac{K_{f s} V_{f} V_{s} C_{o}}{K_{f s} V_{f}+K_{h s} V_{h}+V_{s}}
$$

\section{Equation 3: Amount of analyte extracted in headspace SPME in cases of finite sample volume}

Equation 3 relates the analyte amount extracted by the SPME fiber with the sample volume and the analyte capacity for each of the phases involved in the SPME system. The term $V_{s}$ represents the volume of the sample, while $K_{f s} V_{f}$ is the analyte capacity for the fiber, $K_{h s} V_{h}$ the analyte capacity for the headspace, and $C_{o}$ the analyte's initial concentration in the sample. ${ }^{78}$ The associations established in Equation 3 explain the decrease observed in the amount of analyte extracted when the sample's volume is finite (small). Under this type of sample volume conditions, the decrease in the amount extracted occurs as a result of sample depletion. ${ }^{78}$ On the other hand, in cases in which the sample volume is relatively infinite to the fiber volume, the amount of analyte extracted $\left(\mathrm{n}_{\mathrm{f}}\right)$ can be described by Equation $4 .{ }^{78}$ Nonetheless, regardless of the sample 
volume, the analyte amount extracted by the fiber coating is always directly proportional to the analyte's initial concentration in the sample.

$$
\mathrm{n}_{\mathrm{f}}=K_{f s} V_{f} C_{o}
$$

\section{Equation 4: Amount of analyte extracted by SPME fiber in cases of infinite sample volume}

Solid Phase Micro-Extraction has been extensively used in past studies for a wide variety of applications (i.e., environmental studies, food studies, drug studies, etc.), including the analysis of human scent. ${ }^{21,38-40,88-94}$ The convenient qualities that characterize this technique have been the motor behind its success in so many fields. Besides serving as a feasible sample preparation method for samples in any state of matter, its ease of use, portability, low cost and easy automation have made of SPME an incredibly accessible technique. In addition, its sensitivity and ability to be used with different analytical instruments, like Gas Chromatography-Mass Spectrometry (GC-MS) and High Performance Liquid-Chromatography (HPLC), have made of SPME an incredibly versatile technique.

\subsubsection{Gas Chromatography-Mass Spectrometry (GC-MS)}

Gas Chromatography-Mass Spectrometry is an analytical technique that couples two separate analytical instruments: a gas chromatograph and a mass spectrometer. The gas chromatograph separates the constituents of a mixture in time, while the mass spectrometer provides details on their structural information. ${ }^{95}$ The concerted use of both

instruments facilitates the identification and quantitation of the chemical constituents in 
samples of interest. Samples in all states of matter (e.g., solid, liquid and gas) can be assessed using GC-MS; yet liquid samples are the most common. In order to be analyzed in the gas chromatograph, analytes need to be volatile or semi-volatile, and thermally stable. Hence, there are cases in which samples must undergo preparation procedures (e.g., derivatization) prior to analysis, in order to meet the GC-MS requirements.

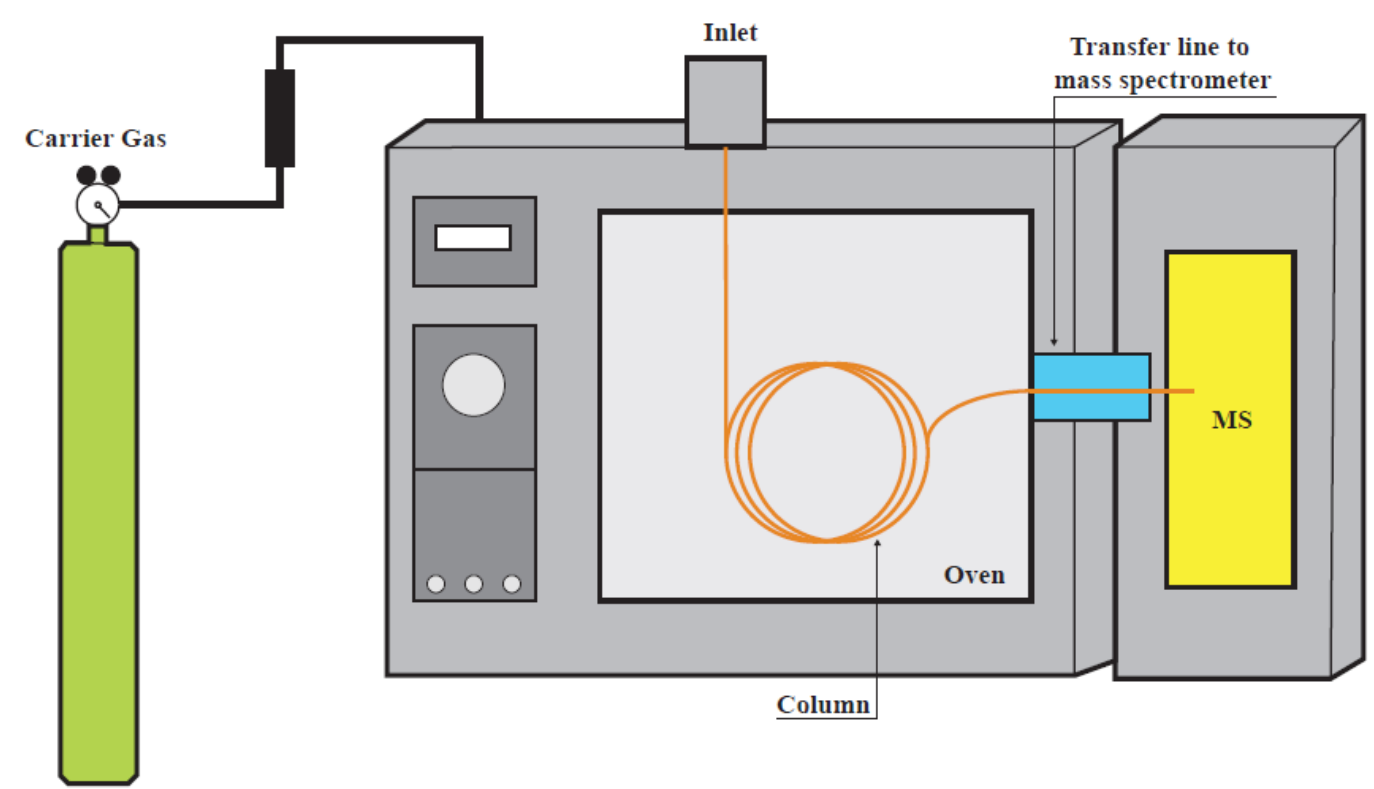

Figure 3: Schematic diagram of a GC-MS instrument ${ }^{95}$

When performing GC-MS analysis, a sample is introduced into the gas chromatograph through a heated injection port. This injection port can operate in two different modes, split or splitless. The modes are used to control the amount of sample that enters the column, depending on the concentration of analytes it contains. ${ }^{95}$ As it passes through the port, both the solvent and the analytes get vaporized. Then an inert carrier gas (in this case helium) transports the analytes to continue the instrumental assessment. Initially the carrier gas, also known as mobile phase, transports the gaseous 
sample through a capillary column. The column is an open capillary tube that is coated with a thin layer of stationary phase. It can vary in length $(10-60 \mathrm{~m})$, inner diameter $(0.10-0.53 \mathrm{~mm})$, thickness $(0.1-5 \mu \mathrm{m})$ and polarity, depending on the application it is used for. ${ }^{95,96}$ The most widely used stationary phase is made out of fused silica, yet there is a wide variety of stationary phases commercially available. Some examples of common stationary phases are listed in Table 2. ${ }^{96,97}$

Table 2: Commercially available stationary phases and their polarity

\begin{tabular}{|c|c|}
\hline Stationary Phase & Polarity \\
\hline Polydimethyl siloxane & Non Polar \\
\hline 5\% Phenyl-polydimethyl siloxane & Non Polar \\
\hline 5\% Phenyl- dimethyl arylene siloxane & Non Polar \\
\hline 50\% Phenyl-polydimethyl siloxane & Mid- Polar \\
\hline Polyethylene glycol & Polar \\
\hline Polyethylene glycol in Sol Gel Matrix & Polar \\
\hline 50\% Cyanopropyl- polydimethyl siloxane & Polar \\
\hline
\end{tabular}

Once in the column, the analytes separate on the basis of their partitioning between the mobile phase and the stationary phase. ${ }^{95}$ The compounds that have a higher affinity for the stationary phase will take longer to elute than those that show less affinity. After being separated, analytes are transferred into the mass spectrometer, where they become ionized and separated by their mass-to-charge ratio. ${ }^{95}$

There are different types of mass spectrometers which mainly vary in the type of ionization technique, and mass analyzer each employs. ${ }^{96}$ In GC-MS, Electron Impact ionization (EI) is the most widely used ionization method. ${ }^{95}$ As part of this technique, an 
electron beam is produced to interact with the eluted molecules until these get excited and become molecular ions. Also, it is precisely the further fragmentation of these molecular ions, at the ion source, that results in a multi peak mass spectra. ${ }^{95}$ On the other hand, after ionization occurs, the ions are transferred to a mass analyzer. Amongst the most commonly used GC-MS mass analyzers is the quadrupole. Quadrupole mass spectrometers are less expensive, more compact and more rugged than most other types of mass spectrometers, which has made them more accessible for analysis in different applications. As a mass analyzer, the quadrupole consists of four parallel electrode rods. These electrodes are electrically connected and create direct currents and alternating currents, in the form of a radio frequency electric field. ${ }^{95}$ As a result, the beam of ions being transferred from the ion source gets separated, and ions get filtered by their mass to charge ratio $(\mathrm{m} / \mathrm{z})$ to later reach a transducer. The transducer, usually an electron multiplier in GC-MS analysis, converts the beam of ions into an electrical signal that can be processed and displayed in a computer. ${ }^{96}$ The intensity of this signal is directly proportional to the abundance of ions with specific $\mathrm{m} / \mathrm{z}$ values ${ }^{95}$ Hence, the signal is used to create mass spectra that provide structural information of the analytes on interest. 


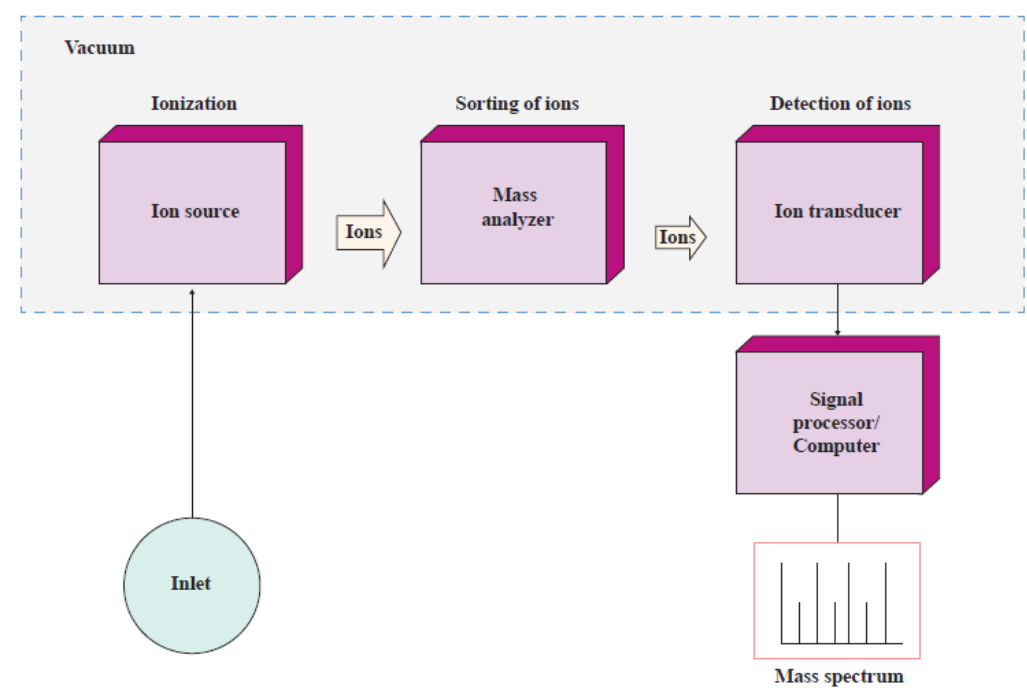

Figure 4: Schematic diagram of a mass spectrometer ${ }^{95,96}$

Gas Chromatography - Mass Spectrometry is considered to be the primary technique for the identification and quantitation of organic analytes in complex matrices. ${ }^{95}$ As such, the GC-MS technique has been essential to the advancement of countless fields (e.g., environmental, medical, food chemistry, etc.), including forensics. ${ }^{38,83,90,91,98}$ Among its applications in the forensic field, GC-MS is considered to be the primary method of analysis for human scent. Consequently, a great number of the discoveries achieved in the human scent field can be attributed to the analytical strenghts of the GC-MS technique. Therefore, the use of GC-MS in the assessment of new human scent investigations represents a promising approach for the achievement of compelling findings. 


\section{RELEVANCE OF STUDY}

Throughout the years, human scent has been presented as forensic evidence for the resolution of different criminal cases. ${ }^{99-102}$ The admissibility of human scent, in the form of canine scent evidence, into the United States court of law is dependent upon its compliance with court jurisdiction standards. ${ }^{103}$ For this reason, different aspects of this type of evidence have often been challenged in court. Despite the advances accomplished through different court cases that have involved canine scent evidence in trial proceedings, there continues to be a need to limit the challenges faced by this type of forensic evidence. ${ }^{101,104-106}$ The reliability of the canine and handler team, as well as the value of scent identification as incriminating or exonerating evidence, have been found among the main concerns regarding the use of canine scent evidence. ${ }^{61}$ These concerns have hampered different attempts of using canine evidence in court proceedings in the past, causing delays on the broad acceptance of human scent as reliable evidence in court. Nevertheless, a surge of different initiatives has emerged to address the current challenges. Among these initiatives, some have appealed to the use of scientific technologies and principles to strengthen human scent's evidentiary power. After being challenged, in 2005 the People v. Salcido case became the first to admit human scent evidence collected using the Scent Transfer Unit (STU-100) technology into trial. ${ }^{107}$ The successful admissibility of human scent evidence in this case demonstrates that there is great potential in continuing to explore scientific approaches that may aid in reducing the current challenges faced by this type of evidence in court.

The capability of using human scent information to associate an individual to a crime scene, and being able to confirm such information by means of well-established 
analytical instrumentation, represents a significant step for forensic science and the law enforcement community. Therefore, previous studies have assessed the identification and differentiation of individuals, by means of the scientific analysis of the VOC constituents of scent profiles..$^{34,38}$ These studies have been conducted with the intention of disclosing information that can enhance the credibility of human scent evidence in the court of law. In the same token, this dissertation intends to continue to expand on human scent knowledge and contribute to its evidentiary power.

This dissertation presents the first attempt to evaluate human scent as a unifying trait in populations, rather than an element for the identification of individualizing features. The use of analytical instruments to determine VOCs that are characteristic to different age groups, races/ethnicities, and genders, represents a big advancement to human scent research. Findings from this new approach can facilitate looking at the influence of specific traits on scent expression and using this to identify specific features in an individual. This capability enhances the accessibility to new pieces of information that can aid in forensic investigation and be of great impact to the law enforcement community.

In recent years, there have been attempts to use DNA for race determination as a way to identify suspects of committed crimes. In 2004, Derrick Todd Lee was convicted for a series of rape and murder cases in Louisiana after having his DNA analyzed and his racial origins broken down to percentages. ${ }^{108,109}$ The Derrick Todd Lee case shows that there is an interest in the law enforcement community to use information about traits, like race, to associate suspects to committed crimes. The determination of human scent features, that are indicative of specific traits in individuals, can be a new and useful tool 
for solving crimes. As this tool, human scent characteristics can be of extreme value in cases in which no DNA evidence is available, and may be useful in complementing other pieces of evidence that are crucial to the resolution of a case. Also, law enforcement officials may be able to simplify criminal investigations by using scent features to narrow the number of individuals who are considered suspects of a crime. Therefore, using human scent to identify specific traits in individuals has the potential to induce significant improvements in forensic investigations.

The identification of human scent characteristics, that can be associated to age, race/ethnicity and gender in individual scent profiles, provides new elements to corroborate canine investigative work. The ability to further confirm canine scent evidence will continue to reduce the challenges and controversies revolving this type of forensic evidence in courts. The findings presented in this dissertation enhance the current knowledge from human scent profiles, establish a path for further investigations in the human scent research field, and display great potential for a positive impact in the forensic and law enforcement communities. Hence, the following research exemplifies an innovative attempt to bolster forensic science and contribute to the pursuit of justice.

\section{RESEARCH OBJECTIVES}

This dissertation aims to obtain information on the nature and chemical composition of the human scent profiles of individuals from different populations. The evaluation of these profiles was meant to determine the relationship between specific traits and human scent expression for individuals from different groups. Human scent 
profiles were used to identify similarities and differences in the composition of scent, for individuals who shared, or did not share, traits across different populations.

The objective of this research was to identify possible features in individual scent profiles that could be considered biomarkers for three specific traits: gender, age and race/ethnicity. In addition to this assessment, a thorough evaluation and development of analytical methods and conditions was performed. This particular assessment intended to establish the best alternatives for the successful analysis of potential scent biomarkers. The tasks performed to satisfy this dissertation's goals are listed below.

a. Optimization of research methods, conditions and procedures for the collection and analysis of human scent samples

b. Identification of scent biomarkers in the underarm odor profiles of individuals from different gender, age and race/ethnic groups

c. Comparison of underarm odor profiles and hand odor profiles to determine biomarker consistency between body regions

d. Evaluation of solvent extraction as a complementary technique for Solid Phase Microextraction (SPME) in the analysis of human scent VOCs

e. Conduct statistical analysis for data interpretation and presentation of results

\section{HUMAN SCENT STUDIES}

\subsection{POPULATIONS UNDER STUDY AND THEIR RECRUITMENT}

Authorization (062012-00) was obtained, from the FIU Institutional Review Board (IRB), to recruit human subjects to participate in the research study. Subsequently, different approaches and methods were put into practice in efforts to recruit participants 
for the study. Face-to-face recruiting, as well as the diffusion of electronic communications (e.g., email) and word of mouth, served as the main methods employed for subject recruitment. Nevertheless, identifying and visiting residential, community and commercial areas (e.g., neighborhoods, community centers, churches, restaurants, etc.) with higher number of possible willing participants were also among the measures taken to accomplish a successful recruitment process. Moreover, flyers (Appendix 1 Appendix 4) were also created and distributed to provide adetailed explanation of the study to individuals during the recruitment process. The explanation was provided to facilitate their understanding about what the research study entailed and have potential participants become more comfortable with being committed to the study.

After all recruitment efforts, a total of 190 subjects were successfully sampled to obtain their underarm odor profiles. From these subjects, 105 were also sampled for their hand odor profiles. As part of the study, all participants were provided with a questionnaire prior to sampling. In this questionnaire they were asked to identify themselves under any of three different racial/ethnic group options: Caucasian, Hispanic and East Asian. In addition, they were asked to specify their age and gender. All subjects were then organized into three different age groups: 18 -30 years, 35-50 years and 55+ years. Table 3 presents the demographic information of all the individuals sampled for both underarm and hand odor profiles. 
Table 3: Demographic information of research participants

\begin{tabular}{|c|c|c|c|c|c|c|c|}
\hline \multirow[t]{2}{*}{ Assessment } & \multirow{2}{*}{ Age Group } & \multicolumn{6}{|c|}{ Caucasians } \\
\hline & & \multicolumn{3}{|c|}{ Females } & \multicolumn{3}{|c|}{ Males } \\
\hline \multirow{3}{*}{ 司 } & 18-30 years & \multicolumn{3}{|c|}{9} & \multicolumn{3}{|c|}{8} \\
\hline & $55+$ years & \multicolumn{3}{|c|}{3} & \multicolumn{3}{|c|}{1} \\
\hline & $\begin{array}{c}\text { Total } \\
\text { Subjects }\end{array}$ & \multicolumn{6}{|c|}{21} \\
\hline \multirow{6}{*}{ 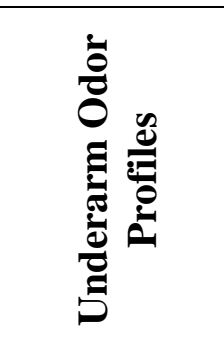 } & \multirow{2}{*}{ Age Group } & \multicolumn{2}{|c|}{ Caucasians } & \multicolumn{2}{|c|}{ Hispanics } & \multicolumn{2}{|c|}{ East Asians } \\
\hline & & Females & Males & Females & Males & Females & Males \\
\hline & 18-30 years & 10 & 10 & 10 & 10 & 10 & 10 \\
\hline & $35-50$ years & 10 & 10 & 10 & 10 & 10 & 8 \\
\hline & $55+$ years & 10 & 10 & 10 & 10 & 8 & 3 \\
\hline & $\begin{array}{c}\text { Total } \\
\text { Subjects }\end{array}$ & \multicolumn{6}{|c|}{169} \\
\hline \multirow{6}{*}{ 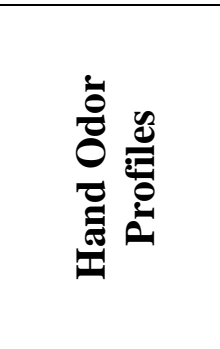 } & \multirow{2}{*}{ Age Group } & \multicolumn{2}{|c|}{ Caucasians } & \multicolumn{2}{|c|}{ Hispanics } & \multicolumn{2}{|c|}{ East Asians } \\
\hline & & Females & Males & Females & Males & Females & Males \\
\hline & 18-30 years & 6 & 6 & 6 & 6 & 6 & 6 \\
\hline & $35-50$ years & 6 & 6 & 6 & 6 & 6 & 6 \\
\hline & $55+$ years & 6 & 6 & 6 & 6 & 6 & 3 \\
\hline & $\begin{array}{c}\text { Total } \\
\text { Subjects }\end{array}$ & \multicolumn{6}{|c|}{105} \\
\hline
\end{tabular}

Out of the total number of participants sampled for underarm odor profiles, 81 were of Caucasian descent, and were divided between the Pilot Study and the underarm odor assessment. Since the Pilot Study was meant to only provide preliminary data, it involved fewer subjects and it solely considered individuals from the 18-30 year and 55+ year age categories. Nevertheless, all the other assessments involving human scent profiles targeted individuals in all of the pre-established age groups, and included larger numbers of subjects. A total of 60 Hispanic and 49 East Asian subjects were also sampled. All East Asian participants were specifically of Japanese, Chinese or Korean descent. Moreover, limitations on subject availability for this population caused the 18-30 year old category to be the only age group to include ten East Asian individuals per gender. On the other hand, the East Asian 35-50 year group included ten females and 
eight males, while the $55+$ year group included eight females and three males. This type of limitation was not experienced during the recruitment of Hispanic and Caucasian individuals. Therefore, both of these populations considered ten individuals per gender for each age group. Overall, the youngest of all individuals to participate in the study was 18 years old, and the eldest was 77 years old.

\subsection{MATERIALS, OPTIMIZATION METHODS AND PROCEDURES}

\subsubsection{Materials for sample collection and handling}

As part of this study, odor samples were collected using sterile cotton gauze pads (100\% cotton) as sorbent material. The gauze pads used were 2 inch. $\mathrm{x} 2$ inch., eight ply surgical sponges (Dukal Corporation, Syosset, NY), and served to collect the VOCs emanating from the skin of each subject. Clear, $10 \mathrm{~mL}$ glass vials containing a screw top with PTFE/Silicone septa (SUPELCO, Bellefonte, PA), were used to store clean gauzes prior to sampling and to seal samples in preparation for analysis once they had been collected. Gauze pads were handled at all times with the assistance of disinfected stainless steel tweezers (Fisher Scientific, Pittsburgh, PA) and using powder free nitrile exam gloves (Fisher Scientific, Pittsburgh, PA) to avoid any VOC cross contamination caused by direct contact with the analyst. Pads of $70 \% \mathrm{v} / \mathrm{v}$ antiseptic Isopropyl alcohol, (Professional Disposables International Inc., Orangeburg, NY) were used to clean and disinfect the stainless steel tweezers in between handling different gauzes, or even in between periods of handling a same gauze, to ensure their cleanliness at all times. Prior to GC-MS analysis, a dry bath incubator (Fisher Scientific, Pittsburgh, PA) was used to increase the temperature of the gauzes and facilitate VOC desorption from the sorbent 
material. Extraction of VOCs was performed using $50 / 30 \mu \mathrm{m}$ Divinylbenzene/Carboxen/Polydimethylsiloxane （DVB/CAR/PDMS; SUPELCO, Bellefonte, PA) Solid Phase Microextraction fibers.

\subsubsection{Chemical standards and reagents}

In addition to all materials employed for sample collection and handling, chemical standards were obtained from (Fisher Scientific, Pittsburgh, PA) and Sigma Aldrich (St. Louis, MO) for a series of volatile organic compounds. Initially, a VOC menu was created on the basis of the compounds that would be expected to be present in the types of samples being assessed in the study. Findings from previous human scent studies were used as a reference to create a general and comprehensive VOC menu that would include a variety of compound types and possibly mimic the chemical constitution of real odor samples. ${ }^{36,38,94,110,111}$ Nevertheless, as all sampling and analysis methods were optimized for the study, the initial compound menu was evaluated and compared with the constituents of real odor samples. As a result, a modified version of the VOC menu was created and used for further analysis. The revised set of VOCs was intended to provide a more accurate representation of the chemical constitution of real odor samples.

External calibration curves were prepared by creating a mix of all the VOC standard reference materials and making a series of dilutions to cover a range of six different analyte concentrations: 1 ppm, 5 ppm, 20 ppm, 40 ppm, 60 ppm, and 80 ppm. Mixed standards were placed in $2 \mathrm{~mL}$ glass vials with PTFE/Silicone lined caps (SUPELCO, Bellefonte, PA) and analyzed sequentially, on a weekly basis, in the GCMS. The weekly frequency with which calibration curves were analyzed served as a 
consistent measure to ensure accuracy in all SPME-GC-MS results. Analyte amounts were calculated by comparison with the calibration curves, using the Enhanced MSD ChemStation $^{\odot}$ (Agilent Technologies 1989-2006) analysis software. In addition, calibration curves were also used as a tool for compound identification during GC-MS analysis. The identity of all compounds was confirmed by comparing their retention times with those of the known chemical standards being included in each week's calibration curve mix.

\subsubsection{Optimization of research methods, conditions and procedures}

Prior to initiating the assessment of human scent samples for the determination of potential VOC biomarkers, a series of optimization studies were performed. Studies were conducted to determine which analytical methods and conditions were best suited for the HS-SPME and GC-MS analysis to be performed. Moreover, the procedure employed to collect underarm odor samples was also evaluated. The goal of optimizing the sampling procedure was to establish a method that would maximize and facilitate an efficient collection of underarm odor VOCs.

\subsubsection{Optimization of GC-MS column and instrumental method for VOC analysis}

A literature review of studies, that had considered similar types of human scent samples to those considered in this dissertation, was performed. ${ }^{30,36,38,110-112}$ As a result, a total of 27 VOCs (Appendix 5) were initially selected to take part in a chemical standard mix solution that would be used to mimic the chemical composition of authentic scent samples during optimization. The number of compounds included in the standard mix 
would vary in subsequent studies as a result of modifications made during the optimization process. The VOC selection for the mixed solution was made on the basis of the commercial availability of the chemical standards identified, and their potential capabilities to act as biomarkers for age, race/ethnicity and gender. Also, this selection included compounds of different functional groups and boiling point levels. The variety was intended to make the standard list a comprehensive representation of the expected composition of real human scent samples.

The determination of the most suited GC column was fulfilled by testing two different GC column chemistries, in two separate GC-MS instruments. The first instrument was a Varian GC-3800 gas chromatograph coupled to an ion trap Saturn 2000 Mass Spectrometer (Agilent Technologies, Inc., Santa Clara, CA). The second instrument was a quadrupole Agilent 6890 gas chromatograph coupled to a 5973 mass spectrometer (Agilent Technologies, Inc., Santa Clara, CA). The Varian GC contained a polar Sol-gel Wax column as stationary phase (SGE Analytical Science, Australia), while the Agilent GC contained a non polar HP-5MS UI column as stationary phase (Agilent Technologies, Inc., Santa Clara, CA). Both instruments used helium as a carrier gas.

Dilutions of the stock chemical standard mix were prepared, and mix solutions of 5 ppm, 20 ppm, 40 ppm, 60 ppm and 100 ppm were analyzed on both instruments during the optimization study. In addition, different analytical methods were tested on both instruments to identify the necessary conditions to achieve the best chromatographic separation of the VOCs being detected. The temperature ramp of the GC oven, time of solvent delay and GC injector mode (split vs. splitless) were manipulated, in both instruments, to evaluate the efficiency of each chromatographic method. The quality of 
analysis accomplished with each column, and analytical method, tested was determined under several conditions. The reproducibility, selectivity, and sensitivity of, each column and method, were evaluated to select the analytical method and column chemistry that would be used for analysis in subsequent studies.

\subsubsection{Optimization of material and conditions for HS-SPME}

The SPME fiber chemistry and headspace extraction time were two experimental conditions that were also optimized. Spiked samples were created by adding $5 \mu \mathrm{L}$ of a 100 ppm standard mix solution (Appendix 5) to clean cotton gauzes inside $10 \mathrm{~mL}$ glass vials. In preparation to HS-SPME, the samples were left to equilibrate for a period of one hour to ensure the presence of the VOCs in the vials' headspace.

Three different fiber chemistries were tested as part of the optimization: Polydimethylsiloxane/Divinylbenzene (PDMS/DVB - blue - mid-polar; SUPELCO, Bellefonte, PA), Divinylbenzene/Carboxen/Polydimethylsiloxane (DVB/CAR/PDMS grey - mid-polar; SUPELCO, Bellefonte, PA) and Carboxen/PDMS (CAR/PDMS - black

- bipolar; SUPELCO, Bellefonte, PA). The fiber chemistry selection was made on the basis of previous studies that had tested these SPME fibers for the extraction of similar VOCs. ${ }^{113}$ In addition, the grey fiber was also included for its consistent use in previous human scent research studies. ${ }^{61,75,114}$

The three fiber chemistries were tested by using them to perform HS-SPME on the spiked samples. Duplicate extractions were performed for each fiber at three different extraction times: 1 hour, 8 hours, and 21 hours. The evaluation of these three different extraction times was meant to promote an unbiased selection of the best suited SPME 
fiber. On the other hand, the extraction performance of each fiber, at each of the extraction times, was compared. The number of compounds detected, the average amount extracted for each VOC under consideration, and the consistency of VOC extraction were all contemplated as part of the comparison. As a result of this study, the best suited SPME fiber chemistry was selected, and tested once again under a wider range of extraction times. In this case, the extraction times tested were chosen to assess a comprehensive evaluation of the relationship between extraction time and fiber efficiency. In addition, this evaluation aimed to reduce the standard extraction time used in previous human scent studies, while ensuring optimum extraction efficiency. ${ }^{75}$

The time periods tested in this assessment were 30 minutes, 1 hour, 4 hours, 8 hours, 15 hours, 21 hours and 24 hours. In the same manner it was done for the

optimization of the SPME fiber chemistry, three spiked samples were prepared and equilibrated for each extraction time under study. All samples were extracted by exposing the previously selected fiber chemistry to the samples' headspace, for each of the extraction times being considered. Following GC-MS analysis, the reproducibility, number of compounds detected, and average amount extracted for each VOC, were compared across the different extraction time periods. As a result, a decision was made on the extraction time that would be officially used for all subsequent HS-SPME studies.

\subsubsection{Optimization of extraction temperature for HS-SPME}

To enhance VOC extraction from human scent samples, heating was considered in the HS-SPME procedure. A total of seven different extraction temperatures were tested to determine the temperature necessary to achieve an optimum VOC extraction: $\sim 22.0^{\circ} \mathrm{C}$ 
(room temperature), $25.0^{\circ} \mathrm{C}, 37.0^{\circ} \mathrm{C}, 50.0^{\circ} \mathrm{C}, 74.0^{\circ} \mathrm{C}, 90.0^{\circ} \mathrm{C}$ and $120.0^{\circ} \mathrm{C}$. Spiked samples were prepared for extraction at each temperature by adding $5 \mu \mathrm{L}$ of a $100 \mathrm{ppm}$ standard mix solution (Appendix 5) to clean gauze pads, and allowing them to equilibrate for 24 hours. Then, the spiked samples were placed in a dry bath incubator and extracted for four hours using DVB/CAR/PDMS (grey) SPME fibers. The selection of a four hour extraction time was made in an attempt to expedite the temperature optimization procedure, while continuing to maintain the conditions established from previous optimization studies. Heated extractions were performed in triplicate for all temperatures being tested, and the average VOC amount extracted for each compound was evaluated to determine the optimum extraction temperature.

\subsubsection{Optimization of the underarm odor sample collection method}

Three Hispanic 18-30 year old females (20, 22 and 24 years old), were used as sample subjects for the optimization of the underarm odor sampling procedure. All three subjects were provided unscented soap (Natural/Unscented beauty bar - Sensitive) and deodorant (Original Care - Unscented) products (Tom’s of Maine, Kennebunk, ME), and instructed to use them for two days prior to the sampling. During this period, participants were also instructed to avoid using any scented hygiene or cosmetic products, as well as to forego alcohol consumption. These measures were established to minimize the influence of any exogenous VOCs that such common habits might induce in the scent profiles to be studied. On the other hand, the length of the pretreatment period was determined in an effort to maintain a balance between the study's interests and the comfort of participants. On the day of the sampling, participants were asked to not 
shower prior to sample collection. This request was made in an effort to maximize the amount of VOCs that would be collected as samples.

Human scent samples were collected, in duplicate, from all three females by placing cotton gauze pads in both of their underarms. Gauze pads were set in place using disinfected stainless steel tweezers (Fisher Scientific, Pittsburgh, PA), and participants were instructed to hold them with their arms down while following four different sampling methods. Participants were asked to provide the samples while sitting for 15 minutes, sitting for 30 minutes, sitting for one hour, and walking outside for 15 minutes. All methods involving seated participants were conducted inside the laboratory, at an average temperature and humidity of $23.8{ }^{\circ} \mathrm{C}$ and $43.8 \%$, respectively. For the "walking method", all participants were asked to walk the same path outdoors, while the sample was collected. The length and manner of all sampling methods were initially selected considering the comfort and abilities of the individuals to be tested, as well as the practicality of their assessment.

After collection, all underarm odor samples were sealed in glass vials and left to equilibrate for 24 hours. Subsequently, samples were analyzed using the final methods and conditions established for HS-SPME and GC-MS, following the optimization studies. Upon completion of the analysis, the results obtained for each participant in each method were evaluated, and the best sampling procedure was selected. The reproducibility of the results provided by each method, and the average amount of VOCs extracted from the samples collected, were the main factors considered in the selection of the final underarm odor sampling procedure. 


\subsubsection{Pilot Study}

The results obtained from all optimization assessments were incorporated simultaneously, for the first time, during the development of a Pilot Study. The pilot study aimed to test the feasibility of using all the optimized parameters and procedures to accomplish a comprehensive analysis of scent samples. For this reason, authentic human scent samples were collected from the underarms of 21 individuals in this assessment. These samples were also analyzed using HS-SPME and GC-MS. The pilot study was meant to assist in modifying and finalizing the list of VOCs that would be considered in subsequent studies. In addition, the study was also intended to assist in obtaining information on the potential impact that unscented hygiene products may exert on the scent profiles of the individuals under study.

\subsection{METHODOLOGY}

5.3.1. Preparation and analysis of soap and deodorant blanks

The unscented soap and deodorant products, provided to all participants to be used as pre-treatment to the study, were sampled and used as blanks for further reference in the study. Both types of blanks were used to establish a profile of the potential VOC impact exerted by the pre-treatment products in individual scent profiles. Deodorant blanks were prepared by cutting three separate pieces, of approximately $1.2708 \mathrm{~g}$, from the top surface of the deodorant bar. Each individual piece was placed in a glass vial and analyzed by SPME-GC-MS to acquire a detailed profile of the VOCs present in the

product. In the same manner, three individual pieces of a soap bar of approximately $1.4296 \mathrm{~g}$ were also cut, stored in vials and analyzed using SPME-GC-MS. 


\subsubsection{Pretreatment procedure of sample collection materials}

On the basis of data from previously published research studies, VOCs that are commonly found in human scent have also been found in some sorbent materials prior to these being used for sample collection. ${ }^{38,115}$ Hence, in this study, cleaning procedures were performed to ensure that all sampling materials were "analytically clean". The analytical cleanliness ensured materials had no or very little human scent VOCs prior to their use, which was verified by SPME-GC-MS analysis prior to sample collection.

There were two main cleaning procedures in this study: the cleaning of glass vials and the cleaning of cotton gauze pads. Glass vials were used to contain cleaned gauze pads for SPME-GC-MS analysis. Therefore, prior to them being used, all vials were rinsed with acetone (HPLC grade, Sigma Aldrich, St. Louis, MO) and heated at $105^{\circ} \mathrm{C}$, for a period of 24 hours. The cleaning of cotton gauze pads served as a fundamental procedure to ensure no sorbent material contained any external VOC contaminants prior to sample collection. A pre-treatment protocol, published by Prada et al. in 2011, was used to clean gauze pads. The protocol consists of spiking each gauze pad with $1 \mathrm{~mL}$ of methanol and heating in an oven for one hour, at $105^{\circ} \mathrm{C} . \cdot^{34,116}$

\subsubsection{Collection of underarm odor samples}

To prepare for their participation in the study, all subjects were provided with unscented soap and deodorant products. Participants were asked to use these products, at least once per day, for a period of two days before the day of sampling. This period was intended to serve as a pretreatment to each individual's sample collection and help minimize the presence and impact of exogenous VOCs in their characteristic odor 
profiles. For this same reason, during the pretreatment stage, all participants were also asked to forgo the use of any scented hygiene or cosmetic products. Odor samples were collected twice from both of each subject's underarms (one sample per week on consecutive weeks), and all participants repeated the same pretreatment procedure prior to both sampling appointments. Between sampling appointments, participants were allowed to go back to using their regular hygiene products, and continue with their usual hygiene patterns, to minimize their discomfort with the sampling protocol. On the other hand, on the day of the sampling, participants were instructed to not shower prior to sample collection, so that VOCs could be maximized in the underarms. All participants were also asked to avoid alcohol consumption during the total period of their participation in the study, as a way to minimize any impact that this type of consumption may have on odor profiles.

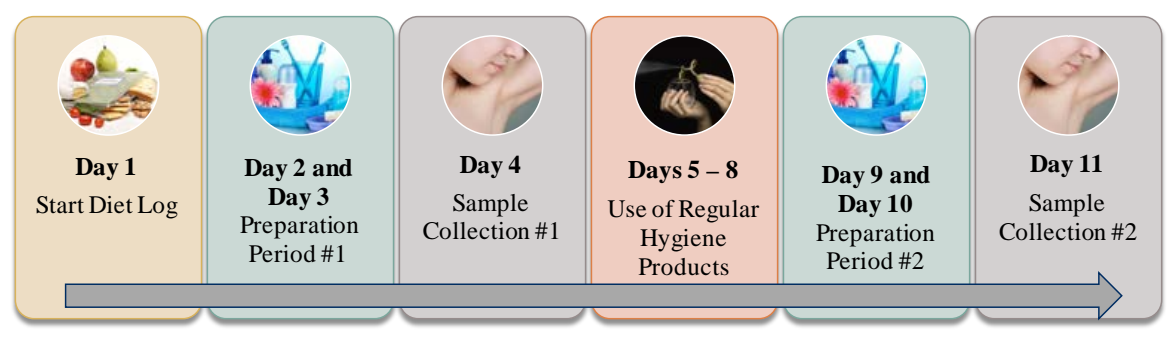

Figure 5: Description of pretreatment and sampling protocols

Underarm odor samples were collected by having individuals sit for one hour and hold a sterile cotton gauze pad in their underarm. Gauze pads were set flat against the skin with the assistance of disinfected stainless steel tweezers. Once sampling time had elapsed, the gauze pads were removed from the underarm and placed in sterile vials using stainless steel tweezers. Samples were then sealed inside the vials and left to equilibrate 
for a period of 24 hours. The equilibration period was established to let the VOCs be released from the collection material and facilitate SPME from the vials' headspace.

\subsubsection{Collection of hand odor samples}

A hand sampling protocol, published by Brown et al. in 2013, was followed to collect all hand samples studied in this research. ${ }^{33,34}$ The protocol was followed indoors prior to sample collection. As part of the protocol, subjects were asked to wash their hands and forearms for a period of 30 seconds, and rinse them with running tap water for a period of two minutes. Following the rinsing period, participants were asked to air dry their hands for a period of two minutes, and rub their hands and forearms for five minutes. The last step was an effort to reconstitute the depleted VOCs in the participant's skin. Once the five minute period had culminated, hand odor samples were collected by using disinfected stainless steel tweezers to place a cotton gauze pad flat in between the palms of the subjects. Then, subjects were instructed to keep their hands closed and hold the gauze pad for a period of ten minutes. Once the ten minutes had elapsed, the sample was placed and sealed inside a $10 \mathrm{~mL}$ glass vial. Triplicate samples were collected from each individual in a sequential manner, and were left to equilibrate for a period of 24 hours prior to HS-SPME.

In the case of the present study, a small modification was made to the cited protocol. The soap used for hand washing was the Tom's of Maine unscented soap that was provided to all participants as pre-treatment product. The cited protocol involved the use of olive oil soap during the hand washing process. The reason for this slight modification was that, in this study, it was crucial to have participants use the same soap 
in both their underarms and hands. The consistency would minimize external variables of potential impact to odor profiles and allow establish a fair comparison between the profiles obtained from both body regions.

\subsubsection{HS-SPME-GC-MS method for the analysis of VOCs in odor samples}

Following the equilibration period, all samples were placed in a digital dry bath incubator at $50^{\circ} \mathrm{C}$ and VOC extraction from the headspace of the samples was assessed. The HS-SPME was performed for a period of 15 hours using 50/30 $\mu \mathrm{m}$ DVB/CAR/PDMS (grey) SPME fibers. At the end of the 15 hour extraction period, SPME fibers were removed from the headspace vial, and inserted in the injection port of the gas chromatographer. Fibers were kept exposed for a total of ten minutes inside the GC injection port to ensure complete thermal desorption of all the compounds extracted. Chromatographic analysis and identification of all compounds was performed using an Agilent 6890 GC/5973 MS (Agilent Technologies, Inc., Santa Clara, CA). The instrument was equipped with a $0.25 \mathrm{~mm}$ x $30 \mathrm{~m}$ HP-5MS UI column, containing a phase film thickness of $0.25 \mu \mathrm{m}$. helium was employed, at a flow rate of $1.0 \mathrm{~mL} / \mathrm{min}$, as a carrier gas for the chromatographic system, while the instrument's injection port was kept at a temperature of $270^{\circ} \mathrm{C}$.

The temperature program employed as part of the chromatographic method, started VOC separation at a temperature of $40^{\circ} \mathrm{C}$. This temperature was held for a total of five minutes, after which temperature was increased at a rate of $10^{\circ} \mathrm{C} / \mathrm{min}$ to a temperature of $220^{\circ} \mathrm{C}$. Once having reached this temperature, the temperature was increased again at a rate of $30^{\circ} \mathrm{C} / \mathrm{min}$ until reaching $300^{\circ} \mathrm{C}$. The temperature was held at 
$300^{\circ} \mathrm{C}$ for a total of two minutes, providing a total run time of 32.67 minutes for analysis. The mass spectrometer transfer line and ion source, were maintained at $300^{\circ} \mathrm{C}$ and $230^{\circ} \mathrm{C}$, respectively. Mass spectra were scanned for a mass range of $45-410 \mathrm{amu}$ and the NIST 98 mass spectral reference library was used to identify the compounds detected.

In addition to the extraction and analysis of odor samples, a gauze and fiber blank were assessed for each sample collected. Prior to being used for sample collection, all gauze pads were extracted and analyzed to obtain a "baseline" VOC depiction of the collection material. The SPME fibers were cleaned and checked for cleanliness by exposing them to the hot injection port of the gas chromatographer for a period of 15 minutes. These blanks were meant to provide information on the presence of any background or contaminant VOCs in the materials, prior to these being used for sample collection and analysis. Therefore, to eliminate the impact of any background VOCs in the results obtained for actual odor samples, all sample results were corrected by both their corresponding gauze and fiber blank. Blank correction consisted of subtracting the amounts detected for any background VOCs in the blanks, from the amounts detected for those same compounds in the human scent sample.

\subsubsection{Methods of statistical analysis}

\subsubsection{Student's T-test}

The Student's T-test is a parametric statistical technique that is used to compare the means of two independent sample populations, and determine whether there is any significant difference between the two means. In this dissertation, this test was used to determine significant differences between the average VOC amounts evaluated in the 
overall comparisons of gender. Therefore, the Student's T-test was used for both the underarm and hand odor assessments. The assessment of this statistical technique aimed to provide additional details that would help explain the causes for gender differentiation.

The independent T-test method tests the null hypothesis that the two mean values being compared are equal. This is accomplished by comparing the subtraction of both mean values to determine their significant difference in comparison to zero. The following formula is used for such purposes ${ }^{117}$ :

$$
t=\frac{\bar{x}_{1}-\bar{x}_{2}}{s \sqrt{\left(\frac{1}{n_{1}}+\frac{1}{n_{2}}\right)}}
$$

\section{Equation 5: Formula for the calculation of the Student's T-test statistic}

In the previously stated formula, $\bar{x}_{1}$ and $\bar{x}_{2}$ represent the two sample means being compared, $n_{1}$ and $n_{2}$ are the sample sizes, and $\mathrm{s}$ is a pooled estimate of the standard deviation. The value of $s$ is obtained through the following formula ${ }^{117}$ :

$$
s=\sqrt{\left(\frac{\left(n_{1}-1\right) s_{1}^{2}+\left(n_{2}-1\right) s_{2}^{2}}{n_{1}+n_{2}-2}\right)}
$$

\section{Equation 6: Formula for the calculation of the pooled estimate of the standard deviation (s)}

The previous formula includes the two individual standard deviation values for each mean $\left(s_{1}\right.$ and $\left.s_{2}\right)$, and establishes that $n_{1}+n_{2}-2$ are the degrees of freedom for the statistic t. In this dissertation, the Student's T-test was performed using $J M P^{\circledR}$ 12.0.0 statistical software. 


\subsubsection{One Way Analysis of Variance (ANOVA)}

The One Way Analysis of Variance (ANOVA) is a parametric statistical technique that allows comparing the means of three or more independent sample groups to determine if there are any significant differences between them. ${ }^{117}$ Significant differences are determined after identifying whether the differences between the mean values are too great to be explained by random error. ${ }^{117}$ In this dissertation, ANOVA was initially used for the statistical analysis of data obtained during the method optimization process. In these cases, the statistical technique was assessed to ease the selection process of the best suited methods, conditions and procedures for sample collection and analysis. Furthermore, other instances in which ANOVA was used in this dissertation were the overall comparisons performed for age and race/ethnic groups, in both the hand and underarm odor assessments. In these cases, ANOVA was used to evaluate the average VOC amounts obtained for each group, and determine whether there were any differences between them that were greater than those caused by random error.

The One Way ANOVA method determines an estimate of the variance on the basis of two types of variation: within sample variation and between sample variation. This is made possible when testing for the null hypothesis, which states all samples belong to a population of mean $\mu$ and variance $\sigma_{0}^{2}$. The "within sample" estimate of the variance is obtained using the following equation ${ }^{117}$ :

$$
\sigma_{0}^{2}=\sum_{i} \sum_{j}\left(x_{i j}-\bar{x}_{i}\right)^{2} / h(n-1)
$$

\section{Equation 7: Formula for the "within sample" estimate of variance}


In this equation, $h$ represents the number of samples that contain $n$ members each. The term $x_{i j}$ is the $j$ th measurement of the $i$ th sample, while $\bar{x}_{i}$ represents the individual sample mean. Moreover, both the summation over $j$, and the division by $(n-1)$, provide the variance of each sample. The summation over $i$ and the division by $h$ average such variances ${ }^{117}$.

On the other hand, the "between sample" estimate of the variance is provided by the following equation ${ }^{117}$ :

$$
\sigma_{0}^{2}=n \sum_{i}\left(\bar{x}_{i}-\bar{x}\right)^{2} /(h-1)
$$

\section{Equation 8: Formula for the "between sample" estimate of variance}

The calculation of the "between sample" estimate of variance also involves a sum of squared terms divided by the number of degrees of freedom. In this case, the overall mean is represented by $\bar{x}$.

The calculation of these two estimates of the variance, serve to test the null hypothesis. If the two estimates are not found to be significantly different, the null hypothesis can be assumed to be correct. On the contrary, if it is incorrect, the between sample estimate will be greater than the "within sample" estimate. The possibility of significant difference between the two estimates is tested using a one-sided F-test. The Ftest considers the ratio of the two sample variances, or the squares of the standard deviations. This test is performed by using the following equation ${ }^{117}$ :

$$
F=s_{1}^{2} / s_{2}^{2}
$$

\section{Equation 9: F-test Formula}


The sample means are said to be significantly different when the calculated $F$ value is greater than the critical value for $\mathrm{F}$. The ANOVA data analysis in this dissertation was performed using $J M P^{\circledR} 12.0 .0$ statistical software.

\subsubsection{Tukey Kramer HSD test}

The Tukey Kramer's Honestly Significant Difference (HSD) test was used as a post hoc test for all cases in which ANOVA was performed in this dissertation. The test was used to perform pairwise comparisons of the mean values being compared, and determine between which mean values laid the significant difference stated by the ANOVA results. The Tukey Kramer HSD test relies on the studentized range statistic $(Q)$ to compare each pair of values. In order for the mean values to be considered statistically different, the calculated value of $Q$, which represents the difference between means, must be equal or greater to the critical value of $Q$ at the significance level being considered. ${ }^{118}$ In this dissertation, significant differences between the means being compared were calculated using $J M P^{\circledR}$ 12.0.0 statistical software. This software uses the following formula to obtain the experimental statistic $\left(Q^{*}\right)$, which considers the percentile of the studentized range distribution $(Q)$ that is required by the method's significance level ${ }^{119}$ :

$$
Q^{*}=\left(\frac{1}{\sqrt{2}}\right) Q
$$

\section{Equation 10: Formula for the calculation of the experimental studentized range statistic}




\subsubsection{Principal Component Analysis (PCA)}

Principal Component Analysis is a multivariate and nonparametric statistical technique used to reduce the number of variables in a data set. This reduction is done by using linear combinations of the observed variables to create a new set of uncorrelated variables, also known as principal components. The mathematical description of these principal components consists on defining them as the eigenvectors of the covariance matrix. Each eigenvector, or principal component, possesses an eigenvalue that discloses the amount of variance for which it is responsible in the data set considered. ${ }^{117}$

The first principal component accounts for the maximal amount of total variance in the data set. The second principal component accounts for the maximal amount of variance in the data that was not accounted in the first component ${ }^{120}$. Subsequently, all principal components would continue to state in decreasing order the highest possible level of variation achieved. Scoreplots and loading plots can be created to depict the effect of each principal component on the data set. The scoreplots graph the scores obtained for a pair of principal components (first and second components in the case of this dissertation). These plots often serve to identify clusters of data. On the other hand, the loading plots display the factor loadings of the principal component pair. In these specific plots, the closer the loading number is to one, the greater is the effect of a component on a specific variable.

Principal Component Analysis was used in this research to evaluate the potential differentiation of the populations under study, on the basis of the VOC amounts extracted from human scent samples. The PCA technique and all the graphical representations of its results were performed using $J M P^{\circledR} 12.0 .0$ statistical software. 


\subsubsection{Linear Discriminant Analysis (LDA)}

Linear discriminant analysis is a supervised pattern recognition method that is often used as a classification technique. In this method, objects are classified into groups on the basis of their values on continuous variables. In order to classify objects, a linear discriminant function (LDF) is employed. This function represents the linear combination of all continuous variables that is capable of achieving optimum object separation into groups, and is described as follows ${ }^{117}$ :

$$
Y=a_{1} X_{1}+a_{2} X_{2}+a_{n} X_{n}
$$

\section{Equation 11: Linear Discriminant Function}

In this dissertation, LDA was used to test the potential classification of individuals, who shared specific traits, into groups. The classification was assessed by gender, race/ethnicity, and age group, on the basis of the VOCs expressed in the scent profiles of all individuals under study. In these cases, the VOC amounts were considered the continuous variables used for subject classification. All LDA results in this research were obtained using $J M P^{\circledR}$ 12.0.0 statistical software, which considered the Mahalanobis Distance to assess group classification. In other words, this software classified individuals into groups whenever the squared distance between the subject and a group's multivariate mean (centroid) was at its minimum. Moreover, canonical plots were created using the same statistical software. In these plots, the axes define the two dimensions in which the groups are most separated, also known as the first two canonical variables. ${ }^{121}$

On the other hand, the points represent the different individuals under study and the multivariate mean of each group, in terms of these two variables. 


\subsection{RESULTS AND DISCUSSION}

5.4.1. Results on the optimization of research methods, conditions and procedures

5.4.1.1. Determination of the best suited GC-MS column and method for VOC analysis

Chemical standard reference solutions (Appendix 5) were prepared (5 ppm, 20 ppm, 40 ppm, 60 ppm and $100 \mathrm{ppm}$ ) and analyzed, using both the Varian GC-MS and the Agilent GC-MS, to determine the conditions for optimum chromatographic separation of the human scent VOCs being tested. Different analytical methods were created to test which GC provided the best selectivity, sensitivity and reproducibility for analysis. Ultimately, the methods were compared to determine the best suited GC column and analytical method for VOC analysis.

Three different analytical methods were developed and tested in the Agilent GCMS, each one with a different injector mode: splitless, split 10:1 and split 5:1. These modes were tested in order to choose the method that would provide the best sensitivity and reproducibility during VOC analysis. In addition, different oven temperature ramps were tested to maximize the chromatographic separation of VOC peaks and favor a high selectivity. The methods tested in the Agilent GC-MS appear in Table 4. 
Table 4: Analytical methods tested on Agilent GC-MS

\begin{tabular}{|c|c|c|c|c|c|c|c|}
\hline \multirow[b]{2}{*}{ Method } & \multirow[b]{2}{*}{$\begin{array}{l}\text { Injector } \\
\text { Mode }\end{array}$} & \multirow[b]{2}{*}{$\begin{array}{l}\text { Solvent } \\
\text { Delay } \\
\text { (min) }\end{array}$} & \multicolumn{5}{|c|}{ Sequence of Oven Temperature Cycles } \\
\hline & & & $\begin{array}{c}\text { Initial Oven } \\
\text { Conditions }\end{array}$ & $\begin{array}{l}\text { Rate of } \\
\text { Change } \\
\left({ }^{\circ} \mathrm{C} / \mathrm{min}\right)\end{array}$ & $\begin{array}{c}\text { Final } \\
\text { Temperature } \\
\left({ }^{\circ} \mathrm{C}\right)\end{array}$ & $\begin{array}{l}\text { Final } \\
\text { Time } \\
\text { (min) }\end{array}$ & $\begin{array}{l}\text { Run } \\
\text { Time } \\
\text { (min) } \\
\end{array}$ \\
\hline \multirow{5}{*}{ Method I } & \multirow{5}{*}{ Splitless } & \multirow{5}{*}{7.00} & \multirow{5}{*}{$\begin{array}{c}40^{\circ} \mathrm{C} \text { for } 5 \\
\min \end{array}$} & 10.00 & 110 & 1.50 & \multirow{5}{*}{32.67} \\
\hline & & & & 10.00 & 150 & 2.50 & \\
\hline & & & & 10.00 & 170 & 1.00 & \\
\hline & & & & 10.00 & 220 & 0.00 & \\
\hline & & & & 30.00 & 300 & 2.00 & \\
\hline \multirow{3}{*}{ Method II } & \multirow{3}{*}{$\begin{array}{l}\text { Split } \\
10: 1\end{array}$} & \multirow{3}{*}{2.00} & \multirow{3}{*}{$\begin{array}{c}40^{\circ} \mathrm{C} \text { for } 5 \\
\min \end{array}$} & 10.00 & 150 & 2.00 & \multirow{3}{*}{29.67} \\
\hline & & & & 10.00 & 220 & 0.00 & \\
\hline & & & & 30.00 & 300 & 2.00 & \\
\hline \multirow{3}{*}{ Method III } & \multirow{3}{*}{ Split 5:1 } & \multirow{3}{*}{2.00} & \multirow{3}{*}{$\begin{array}{c}40^{\circ} \mathrm{C} \text { for } 5 \\
\min \end{array}$} & 10.00 & 150 & 2.00 & \multirow{3}{*}{29.67} \\
\hline & & & & 10.00 & 220 & 0.00 & \\
\hline & & & & 30.00 & 300 & 2.00 & \\
\hline
\end{tabular}

The three methods tested in the Agilent GC-MS were compared. The comparison aimed to facilitate selecting the optimum analytical method to be used with the non polar HP-5MS UI column in this instrument. The relative standard deviation (RSD) percentage of the three methods was among the criteria evaluated. It was noted that, out of the three methods, Method I (Splitless) showed the lowest RSD percentages for the majority of the concentrations tested (Figure 6). Therefore, the splitless method was found to be the most reproducible. Method III (Split 5:1) was found to be the least effective method out of the three in this aspect. This particular method was not capable of detecting compounds at the 5 ppm concentration, contrary to the other two methods which, despite their differences in reproducibility, were still effective in detecting compounds in all concentrations. The limit of detection (LOD) accomplished with each method was also evaluated to test differences in method sensitivity. Once again, Method I (Splitless) provided the lowest/best LODs, achieved by any of the methods, for the majority of the compounds being tested. Therefore, this method showed the smallest LOD range out of 
the three methods (Table 5). In the case of both split methods, Method III (Split 5:1) displayed lower LODs for a higher number of compounds. However, contrary to Method II (Split 10:1), this method failed to deliver an efficient detection of compounds at low concentrations. This observation continued to demonstrate that Method III (Split 5:1) lacked the necessary qualities to deliver optimum analytical results.

The total peak area achieved for each of the concentrations tested was used as another means to evaluate method sensitivity and reproducibility. The evaluation of the results, from the three replicates analyzed for each concentration, revealed that Method I (Splitless) was both the most reproducible and provided the highest compound abundance out of all methods. The One way-ANOVA (significance level $=0.05$ ) and Tukey Kramer HSD tests were also performed to determine whether there was a significant difference between the average total peak area detected with each method. This finding was meant to confirm the superiority of Method I (Splitless) over the others (Figure 7). The results from these tests revealed that, for all concentrations being tested, Method I (Splitless) displayed a significantly higher abundance than those shown by the other two methods (all p-values < 0.0001). Therefore, Method I (Splitless) was proven to be the most efficient method to achieve maximum analytical sensitivity and reproducibility. In addition, the splitless method allowed a consistent detection of compounds at the lowest concentrations, which normally characterize human scent samples. After having considered all of the previously described criteria, Method I (Splitless) was selected as the most efficient analytical method for the HP-5MS UI column in the Agilent GC-MS. 
Table 5: Summary of results obtained for the comparison of analytical methods in the Agilent GC-MS

\begin{tabular}{|c|c|c|c|}
\hline Method Criteria Evaluated & $\begin{array}{c}\text { Method II } \\
\text { (Split 10:1) }\end{array}$ & $\begin{array}{c}\text { Method III } \\
\text { (Split 5:1) }\end{array}$ & $\begin{array}{c}\text { Method I } \\
\text { (Splitless) }\end{array}$ \\
\hline LOD (ng) Ranges & $9.64-32.73$ & $5.35-42.73$ & $5.06-23.42$ \\
\hline RSD (\%) range throughout all concentrations tested & $11.6-21.3$ & $2.2-22.7$ & $2.0-8.5$ \\
\cline { 2 - 3 } & \multicolumn{2}{|l}{}
\end{tabular}

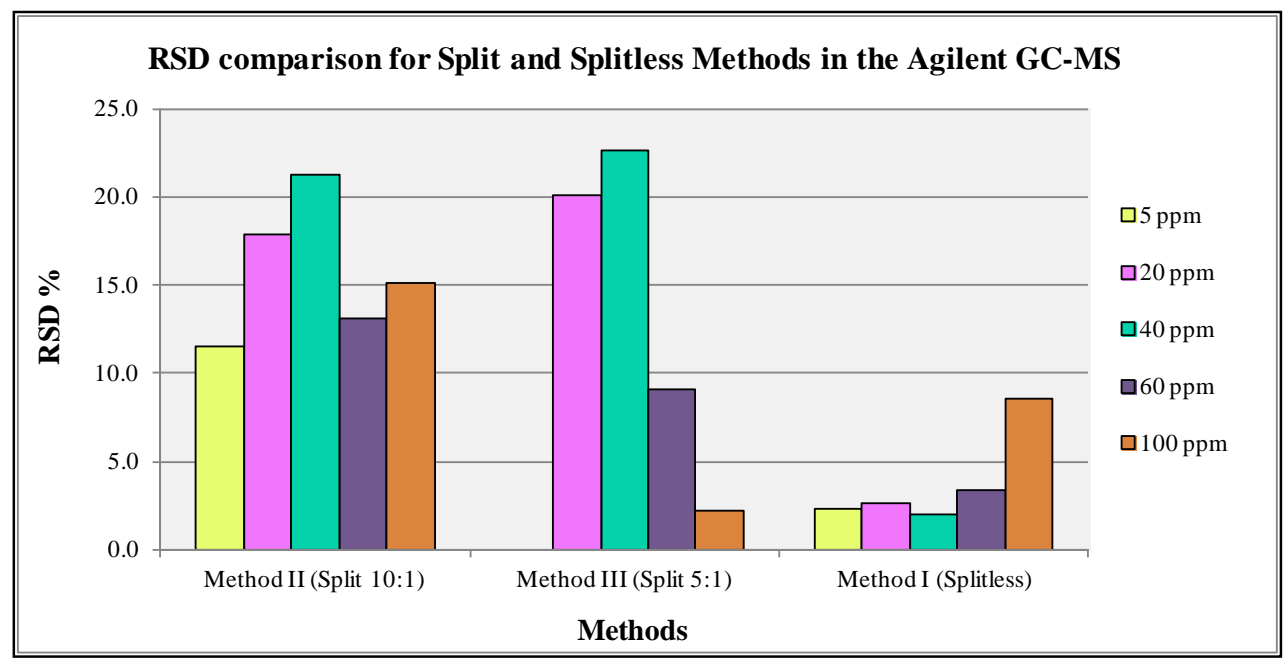

Figure 6: Results for RSD percentages achieved by methods tested in the Agilent GC-MS

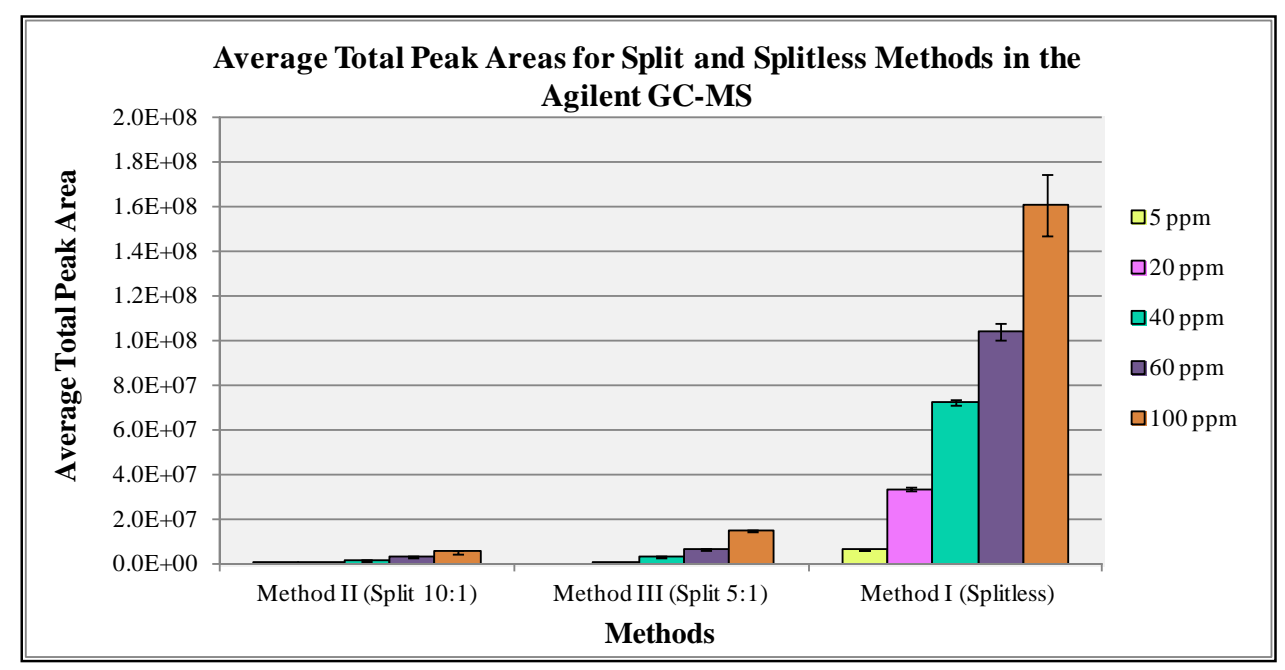

Figure 7: Results obtained for average total VOC abundance by each method tested on the Agilent GC-MS 
On the other hand, a method was also developed in the Varian GC-MS to be able to compare the optimum capabilities for analysis of both instruments. The GC injector modes and the oven's temperature ramp were, once again, among the most important parameters considered in the creation of the new analytical method. Details on the parameters of the Varian GC-MS method are described in Table 6.

Table 6: Analytical methods tested on Varian GC-MS

\begin{tabular}{|c|c|c|c|c|c|c|c|}
\hline \multirow[b]{2}{*}{ Method } & \multirow[b]{2}{*}{$\begin{array}{c}\text { Injector } \\
\text { Mode }\end{array}$} & \multirow[b]{2}{*}{$\begin{array}{l}\text { Solvent } \\
\text { Delay } \\
\text { (min) }\end{array}$} & \multicolumn{5}{|c|}{ Sequence of Oven Temperature Cycles } \\
\hline & & & $\begin{array}{c}\text { Initial Oven } \\
\text { Conditions }\end{array}$ & $\begin{array}{l}\text { Rate of } \\
\text { Change } \\
\left({ }^{\circ} \mathrm{C} / \mathrm{min}\right)\end{array}$ & $\begin{array}{c}\text { Final } \\
\text { Temperature } \\
\left({ }^{\circ} \mathrm{C}\right)\end{array}$ & $\begin{array}{l}\text { Final } \\
\text { Time } \\
\text { (min) }\end{array}$ & $\begin{array}{l}\text { Run } \\
\text { Time } \\
\text { (min) }\end{array}$ \\
\hline \multirow{3}{*}{$\begin{array}{l}\text { Method } \\
\text { IV }\end{array}$} & \multirow{3}{*}{ Split 8:1 } & \multirow{3}{*}{1.20} & \multirow{3}{*}{$\begin{array}{l}40^{\circ} \mathrm{C} \text { for } \\
1.25 \mathrm{~min}\end{array}$} & 4.5 & 50.00 & 0.00 & \multirow{3}{*}{32.00} \\
\hline & & & & 10 & 185.00 & 5.42 & \\
\hline & & & & 10 & 200.00 & 2.11 & \\
\hline
\end{tabular}

In the case of the Varian GC-MS, the best chromatographic separation and sensitivity was achieved using an 8:1 split ratio for the injector mode. Throughout the method development process, this injector mode was seen to provide the best selectivity and reproducibility for VOC analysis in this instrument. For this reason, a comparison was established between Method I (Splitless), from the Agilent GC-MS, and the newly created Method IV (split 8:1). This comparison was meant to facilitate the final selection of the optimum GC column and analytical method for VOC analysis in further studies. The fact that the two methods being compared represented the optimum capabilities of analysis of each instrument, made such an assessment possible.

The chemical standard reference solutions were analyzed using both the Agilent GC-MS and Varian GC-MS methods. The LODs obtained for each method were compared, and the Varian GC-MS method was seen to provide a higher sensitivity for the 
majority of the compounds tested (Table 7). The Varian GC-MS method was also seen to detect and quantify the highest number of compounds out of the two methods (Table 7). The RSD percentage for each method was calculated to determine which one displayed a higher level of reproducibility. Both methods provided similar reproducibility of analysis in some cases, but the Agilent GC-MS method was seen to display better reproducibility at the lowest concentration (5 ppm) (Figure 8). This observation suggested that the Agilent GC-MS method would be more consistent than the Varian GC-MS method in the analysis of low VOC concentrations in real scent samples. Nevertheless, the Varian GCMS method displayed lower RSD percentages in the two highest concentrations (60 ppm and $100 \mathrm{ppm})$.

Table 7: Summary of results obtained for the comparison of analytical methods in the Agilent GC-MS and the Varian GC-MS

\begin{tabular}{|c|c|c|}
\hline $\begin{array}{c}\text { Method Criteria Evaluated } \\
\text { concentrations tested }\end{array}$ & $\begin{array}{c}\text { Varian } \\
\text { GC-MS }\end{array}$ & $\begin{array}{c}\text { Agilent } \\
\text { GC-MS }\end{array}$ \\
\hline LOD (ng) Ranges & 24 & 20 \\
\hline Compounds detected and quantified throughout all & $4.21-24.38$ & $5.06-23.42$ \\
\hline RSD (\%) range throughout all concentrations tested & $2.0-8.5$ & $0.6-3.4$ \\
\hline
\end{tabular}




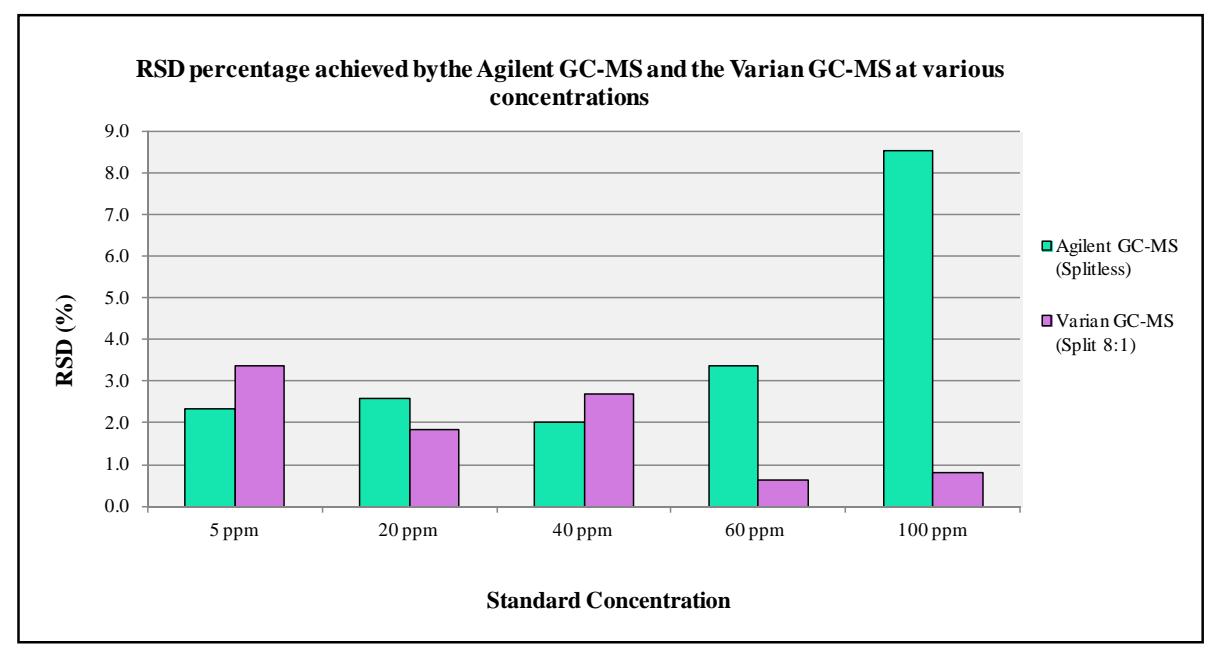

Figure 8: RSD percentages achieved by the Agilent GC-MS and the Varian GC-MS

After the overall comparison of the two GC columns and the corresponding analytical methods tested, it was concluded that the Varian GC-MS method and the Solgel Wax column provided the most efficient, sensitive and reproducible conditions for VOC analysis. However, as a result of limitations on instrument availability caused by frequent instrument malfunctions, VOC analysis was ultimately continued in further studies using the Agilent GC-MS instrument. Hence, the non polar HP-5MS UI column and the GC-MS Method I (splitless) were also used in further studies.

\subsubsection{Determination of the best suited material and conditions for HS-SPME}

The determination of the best suited material for HS-SPME was assessed by evaluating the extraction efficiency of three different fiber chemistries in the analysis of human scent VOCs. The three fiber chemistries tested were the PDMS/DVB or blue fiber, the DVB/CAR/PDMS or grey fiber, and the CAR/PDMS or black fiber. All three fiber chemistries were used to perform HS-SPME on mock human scent samples, created 
by spiking $5 \mu \mathrm{L}$ of a $100 \mathrm{ppm}$ mixture of chemical standards (Appendix 5) onto clean gauzes. In this assessment, each fiber was tested at three different extraction times: 1 hour, 8 hours, and 21 hours.

Different SPME fiber chemistries were seen to reach their highest extraction capabilities at different extraction times (Figure 9). The highest overall amount extracted by the blue fiber was obtained after having exposed the fiber for one hour to the samples’ headspace. On the other hand, both the grey and the black fibers showed a better extraction after an eight hour exposure. Out of the three fibers, the blue fiber showed the lowest total VOC amount (average) after the eight and 21 hour extractions. Nonetheless, the blue fiber proved to have reproducible extraction capabilities after being able to provide consistent VOC extraction across different replicates. Conversely, the black fiber extracted the highest total VOC amount (average) in the three extraction times being tested, but it was also observed to display the most inconsistent extraction pattern across replicates for all the extraction times. After the eight hour extraction, the total amounts extracted by the black fiber and the grey fiber were seen to be very close to each other (black fiber $=480.00 \mathrm{ng}$ and grey fiber $=478.85 \mathrm{ng}$ ). This observation led to a more strict evaluation of the extraction capabilities of the grey fiber. As a result, it was noted that, like the blue fiber, the grey fiber provided a consistent extraction of the different VOCs across different replicates. Therefore, in comparison to the black fiber, the grey fiber was found to have better qualities for HS-SPME analysis. 


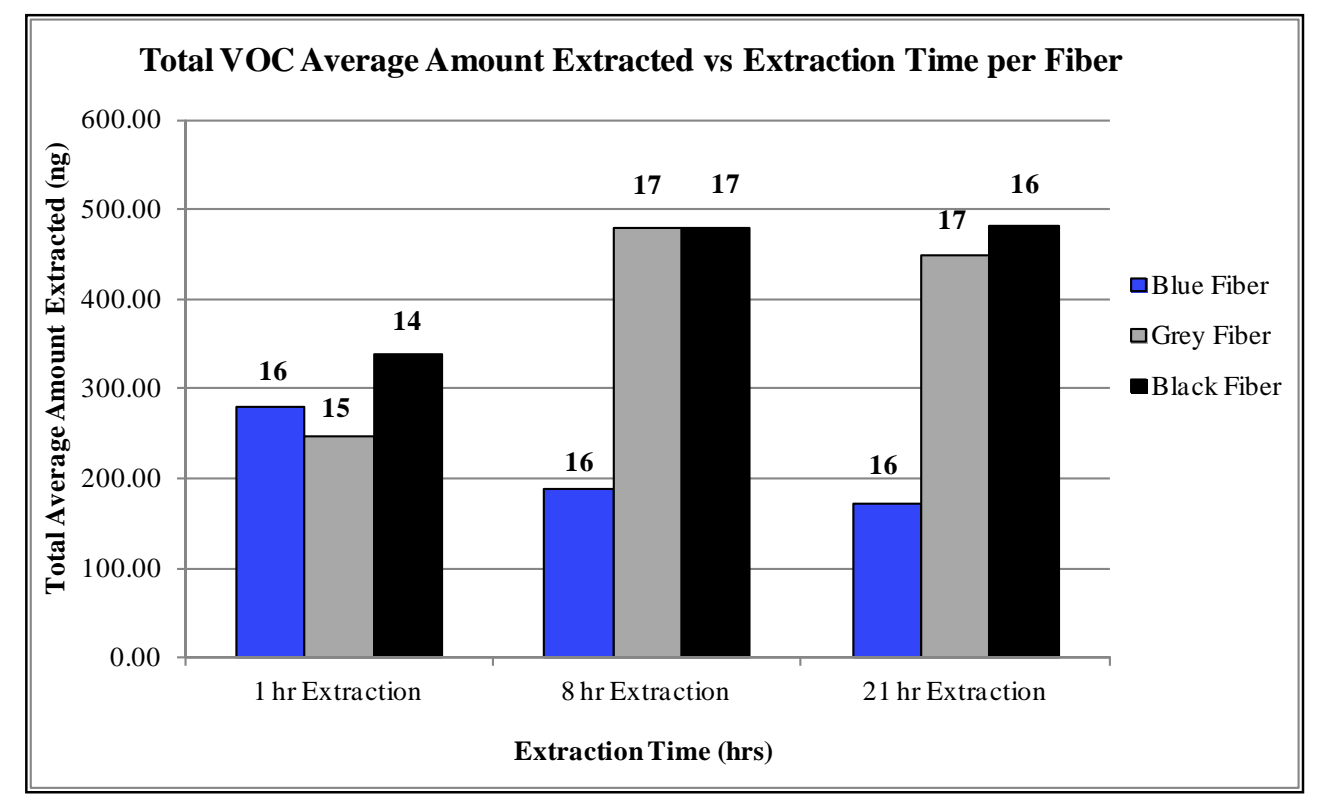

Figure 9: Total VOC average amount extracted vs. extraction time per fiber

The comparison of the blue, grey and black fibers also revealed differences in the way an increase in extraction time can impact each fiber's VOC extraction. As mentioned previously, the blue fiber displayed a consistent decrease in the total average VOC amount observed as extraction time increased (Figure 9). The extracted amount of nine compounds was seen to reduce between one hour and eight hours, while the amount of 11 VOCs was reduced between eight hours and 21 hours of extraction. On the other hand, both the grey and black fibers were characterized by an increase in the extraction of a large number of compounds from one hour to eight hours. Nevertheless, a decreasing trend was also seen in the extraction capability of both fibers for a large number of VOCs past eight hours of extraction. Therefore, in all cases of the HS-SPME fibers evaluated, the total average VOC amount was seen to depend on the ease with which each fiber was capable of extracting the different compounds under study as time passed. Being able to witness this type of fluctuations in HS-SPME analysis favored an unbiased selection of 
the best suited fiber chemistry, and enhanced the awareness for further fiber chemistry evaluations.

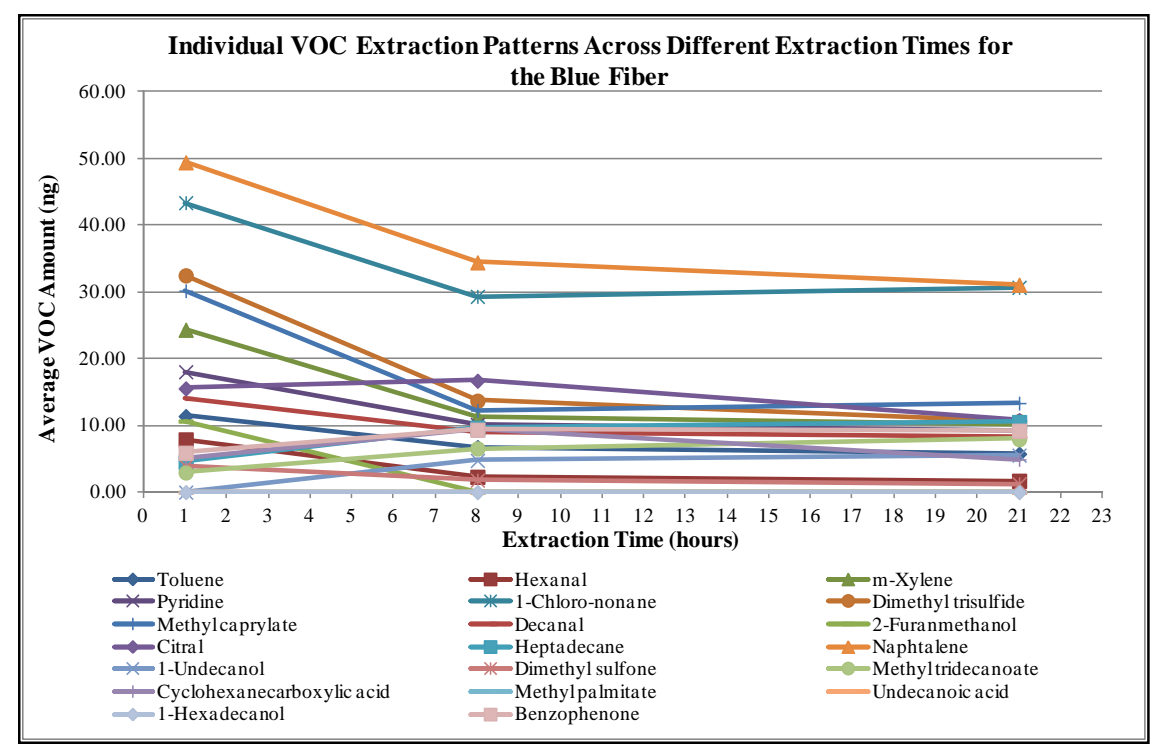

Figure 10: Individual VOC extraction patterns across different extraction times for the blue fiber

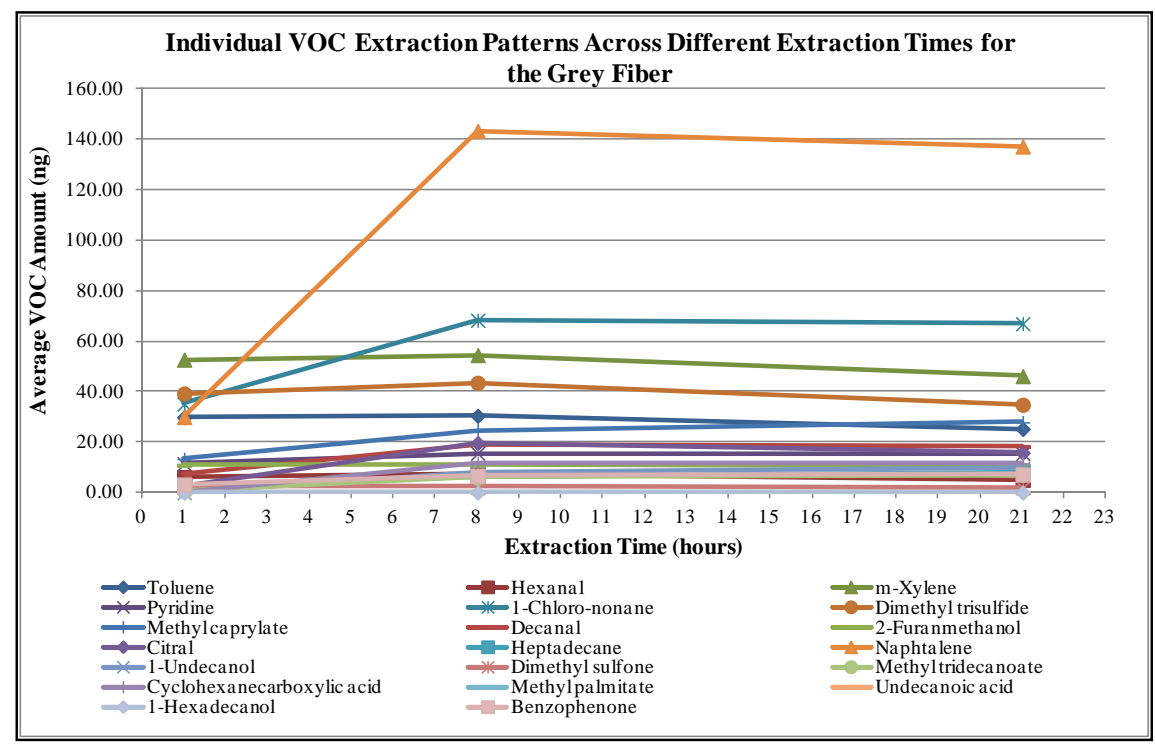

Figure 11: Individual VOC extraction patterns across different extraction times for the grey fiber 


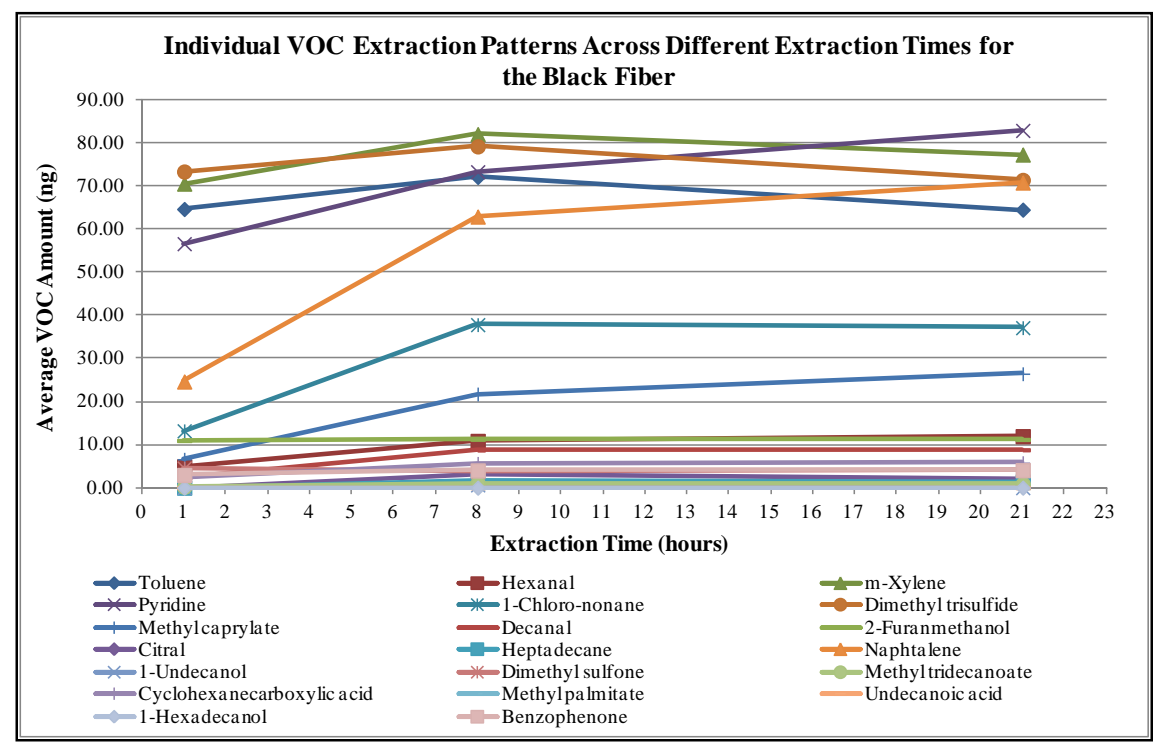

Figure 12: Individual VOC extraction patterns across different extraction times for the black fiber

Another aspect evaluated among the three different fiber chemistries under study, was their number of extracted compounds at each of the extraction times considered. Figure 9 shows the number of compounds extracted by each fiber, at each of the extraction times tested, at the top of the corresponding bars. Despite of the blue fiber being able to accomplish optimum VOC extraction in the least amount of time (one hour), it was noted that the number of compounds detected from its extraction was always below those detected for the black fiber. Also, only after the one hour extraction, the number of compounds detected for the blue fiber was seen to surpass those detected for the grey fiber. Therefore, this observation suggested that, out of the three non- polar fiber chemistries, the blue fiber showed the least overall affinity to the compounds being tested. Moreover, in the case of the black fiber, the number of compounds detected as being extracted was found to be comparable with those of the grey fiber for both the eight hour and 21 hour extractions. However, after the one hour extraction, the black fiber 
extracted fewer compounds than any other fiber chemistry. In addition to its already proven inconsistency, the low number of extracted compounds also implied the black fiber required longer extraction times to accomplish a better extraction of the VOCs being tested. On the contrary, the grey fiber showed to always be among the two fibers with the most amount of compounds extracted in all times tested. The fact that the grey fiber was able to maintain a consistent extraction across different extraction times, as well as across different replicates, suggested its capability to enhance the reproducibility of VOC analysis.

The grey fiber was not only found to be capable of ensuring the highest reproducibility possible for HS-SPME of VOCs, but was also able to extract a high number of compounds and high VOC amounts. The sum of all these qualities made the grey fiber outperform both the black and the blue fiber chemistries. Therefore, after thorough consideration of all aspects evaluated, the grey fiber was selected as the best suited fiber chemistry for HS-SPME. Consequently, an expanded evaluation of the extraction capabilities of this fiber, under a wider range of extraction times, was assessed. The expanded evaluation was done to determine and confirm a more specific time condition capable of maximizing VOC extraction with the grey fiber. This time, the fiber was exposed to the headspace of spiked samples, for periods of 30 minutes, 1 hour, 4 hours, 8 hours, 15 hours, 21 hours and 24 hours.

Initially, the average total VOC amount extracted by the grey fiber, at all times under study, was compared. The order of extraction times for increasing total VOC abundance was identified to be the following: 30 minutes $<1$ hour $<24$ hours $<4$ hours $<$ 15 hours $<21$ hours $<8$ hours. Nevertheless, a comparison was made using ANOVA and 
Tukey Kramer's HSD test to determine whether there was a statistically significant difference between the average VOC amounts extracted in each extraction time. The results to these tests did not reveal any significant difference between the VOC amounts of any groups. For this reason, additional criteria were evaluated to support the selection of the optimum extraction time for further studies.

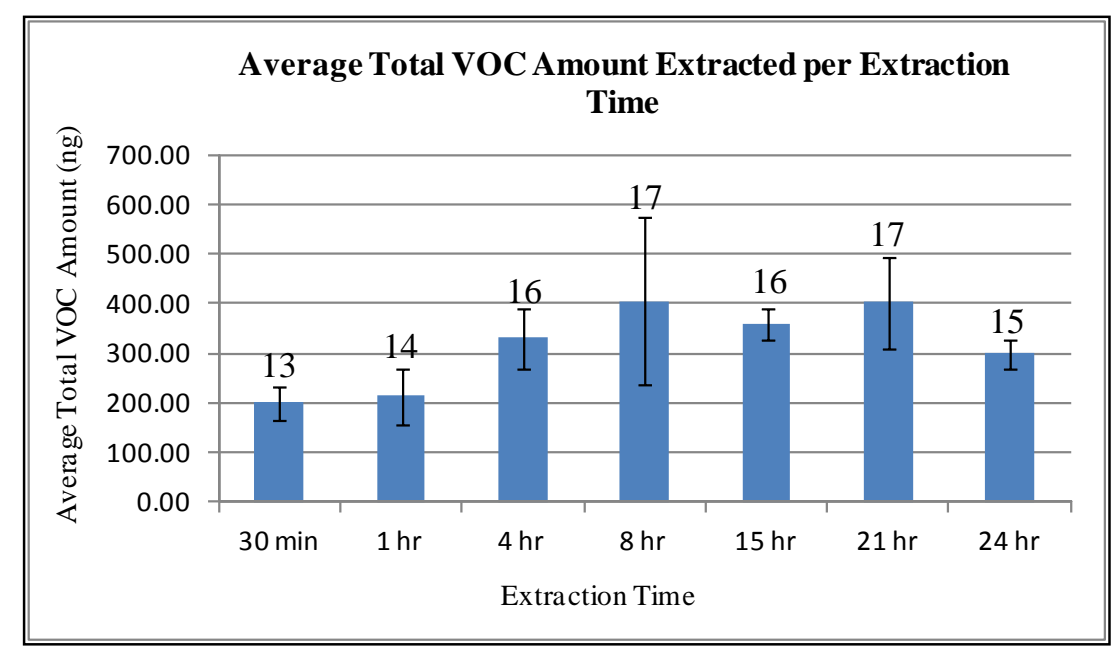

Figure 13: Average total VOC amount per extraction time

The difference between the average total amount extracted for each individual compound, at each extraction time, was among the other criteria considered in the present study. As part of such evaluation, it was determined that both the 15 hour and eight hour extractions provided the highest VOC amount for a total of five compounds. All other extraction times showed fewer VOCs for which the highest average amount was extracted. This finding explained the fact that both the 15 hour and the eight hour extraction were found to be among the top three methods to extract high total VOC amounts. The number of compounds extracted for each method was also compared (top of each bar on Figure 13). As a result of this comparison it was established that the 15 
hour and eight hour methods were among the most efficient. However, in the case of this particular criterion, both the eight hour extraction and the 21 hour extraction were seen to equally extract 17 compounds. Meanwhile, the 15 hour extraction and the four hour extraction were seen to equally extract 16 compounds. In that sense, the eight hour extraction and 21 hour extraction appeared to slightly outperform the latter methods. Moreover, the reproducibility of extraction during each of the time periods tested was also assessed to determine the best suited extraction time. The eight hour method and the 15 hour method had, so far, seemed comparable in their potential for VOC analysis. However, a comparison of the RSD percentages of all methods revealed that the 15 hour extraction method was more reproducible than, not only the eight hour extraction, but all the of the other extraction methods as well (Figure 14). The fact that the 15 hour extraction resulted so reproducible was interpreted as an asset that could enhance the reliability of further HS-SPME analysis.

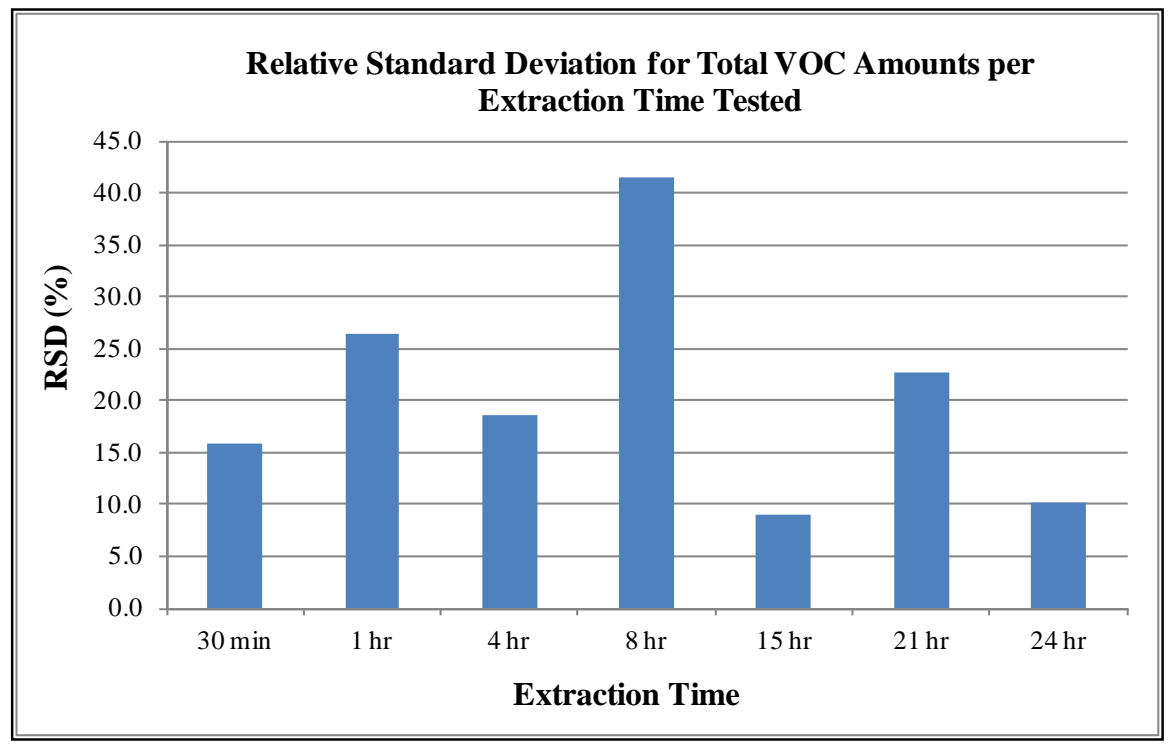

Figure 14: RSD percentages for total VOC amounts per extraction time 
After completion of the evaluation of all extraction times tested with the grey fiber, it was decided that the 15 hour extraction method was the best suited for use in subsequent HS-SPME studies. The reproducibility of extraction provided by this method, along with its capability to extract high abundances of a large number of compounds, classified this method as the most efficient for the analysis of the VOCs under study.

\subsubsection{Determination of best suited extraction temperature for HS-SPME}

The incorporation of heat during the HS-SPME procedure was also evaluated as a resource to enhance VOC extraction. Seven extraction temperatures were tested: $\sim 22.0^{\circ} \mathrm{C}$ (room temperature), $25.0{ }^{\circ} \mathrm{C}, 37.0^{\circ} \mathrm{C}, 50.0^{\circ} \mathrm{C}, 74.0^{\circ} \mathrm{C}, 90.0^{\circ} \mathrm{C}$ and $120.0{ }^{\circ} \mathrm{C}$. Spiked samples were prepared and placed in a dry bath incubator, where they were extracted for a period of four hours using the grey (DVB/CAR/PDMS) fibers.

The average amount extracted for each of the VOCs contained in the spiked samples was evaluated on the basis of their corresponding standard boiling points. Low, medium and high boiling point ranges were defined in an attempt to facilitate the analysis of each VOC's behavior in response to the changes in extraction temperature (Table 8). From this evaluation, it was observed that the amount extracted for those compounds with low and medium boiling points tended to decrease as extraction temperatures increased. Nevertheless, despite both types of compounds having displayed a decrease in their extracted amounts, the decrease for low boiling point compounds (Figure 15) was noted to be more drastic than for medium boiling point compounds (Figure 16). On the contrary, the relationship between the amount extracted for high boiling point compounds

and the extraction temperatures, was seen to be directly proportional. As temperatures 
increased, the amounts of high boiling point VOCs were also seen to increase (Figure 17). These observations suggested that as the extraction temperature increased, high boiling point compounds started displacing low and medium boiling point compounds from the SPME fiber's surface. This displacement led to a reduction in the extraction of low and medium boiling point compounds.

Table 8: Boiling point ranges defined for the selection of the best suited extraction temperature

\begin{tabular}{|c|c|c|}
\hline \multicolumn{3}{|c|}{ VOC Boiling Point Ranges } \\
\hline Low $\left({ }^{\circ} \mathrm{C}\right)$ & Medium $\left({ }^{\circ} \mathrm{C}\right)$ & $\operatorname{High}\left({ }^{\circ} \mathrm{C}\right)$ \\
\hline $110.6-168.0$ & $204.0-238.0$ & $246.0-421.3$ \\
\hline
\end{tabular}

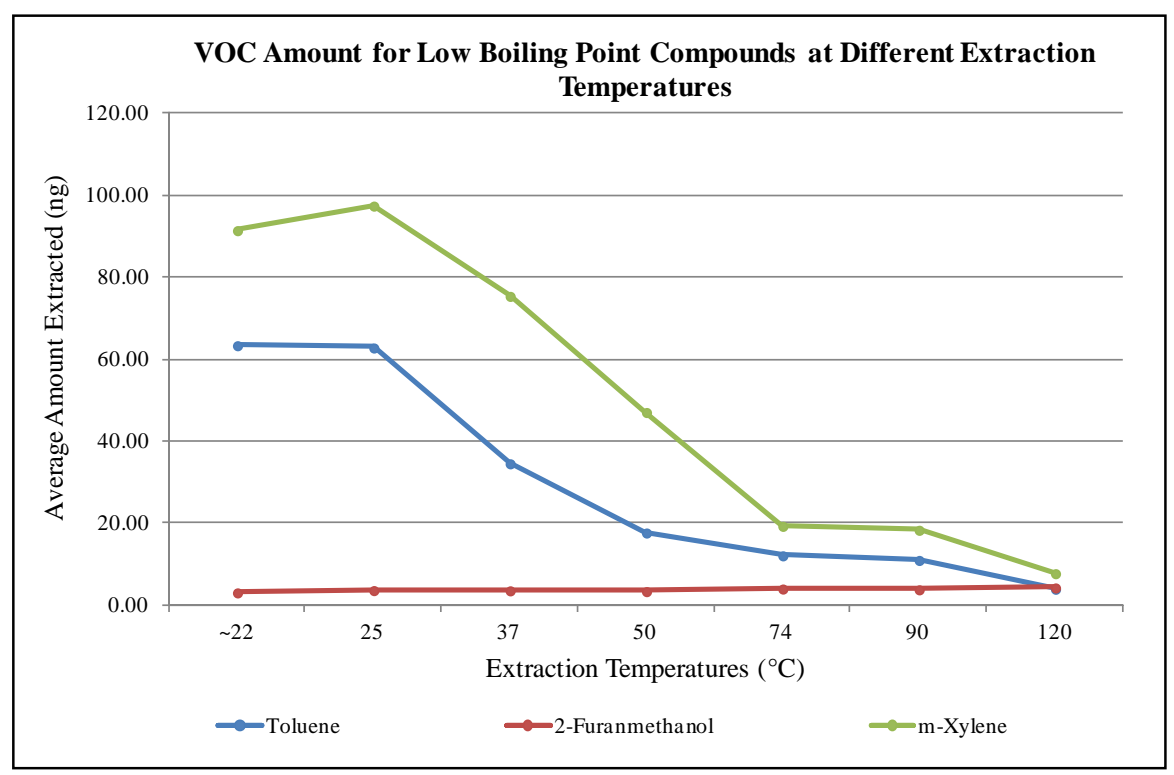

Figure 15: VOC amount for low boiling point compounds at different extraction temperatures 


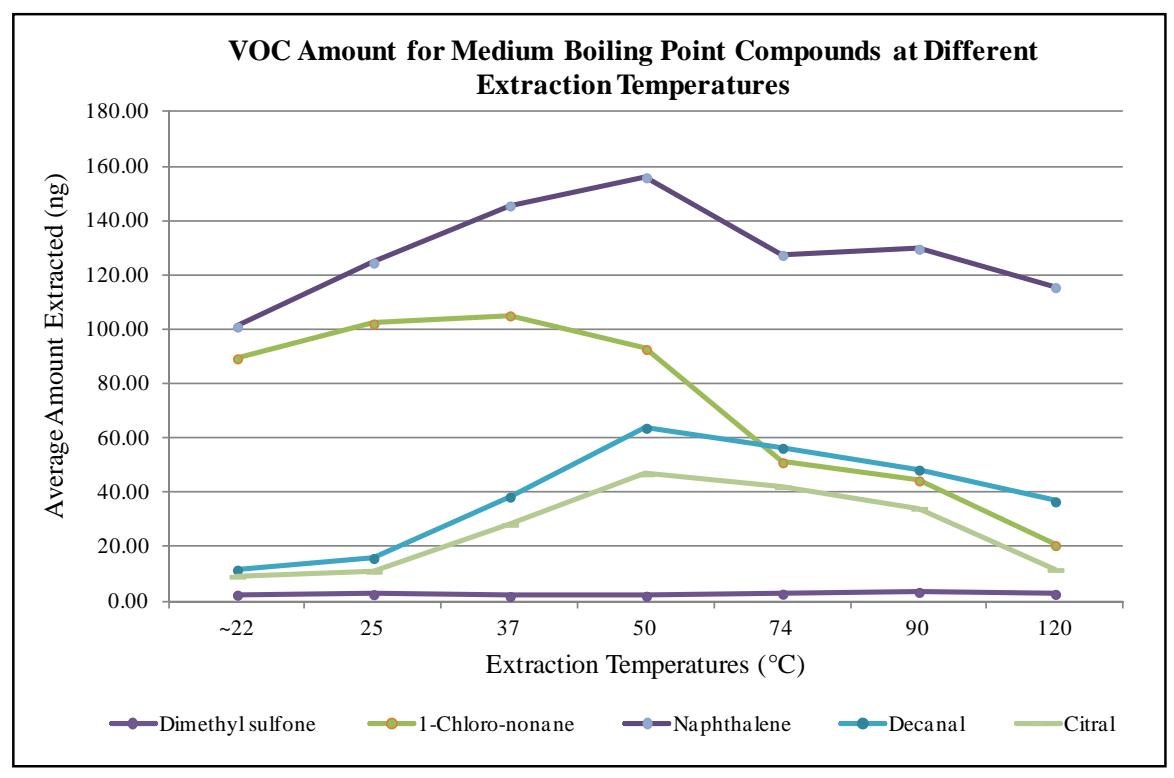

Figure 16: VOC amount for medium boiling point compounds at different extraction temperatures

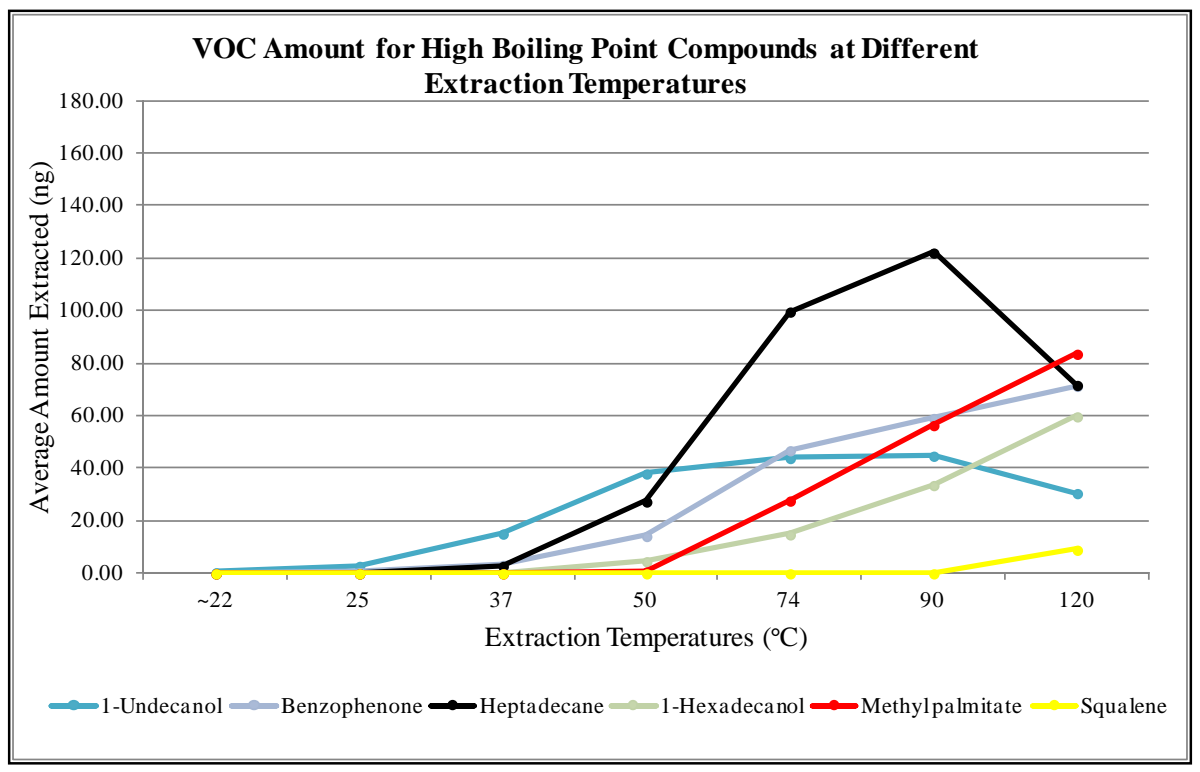

Figure 17: VOC amount for high boiling point compounds at different extraction temperatures

Heating the samples enhanced VOC extraction, by facilitating VOC desorption from the collection material (cotton gauze). However, the results from this assessment 
also revealed that different temperatures enhanced the extraction of certain compounds over others, such as medium and high boiling compounds (Figure 16 and Figure 17). Therefore, in an attempt to maximize overall extraction efficiency and maintain an unbiased acquisition of information from the scent samples to be analyzed in further studies, $50^{\circ} \mathrm{C}$ was selected as the best suited temperature for HS-SPME extraction.

\subsubsection{Determination of best underarm odor sample collection method}

The scent samples collected from the two underarms of three Hispanic females, allowed the construction of each of their scent profiles under the different conditions of the sampling methods tested. In order to be included in a scent profile, a VOC needed to be quantitated in two out of the four samples collected from each individual. Subsequently, an average of the total VOC amount extracted from all samples of an individual was calculated for each method. A One-Way Analysis of Variance (ANOVA) was performed for each subject to compare the average total amounts obtained with each sampling method (significance level $=0.05$ ). This comparison was intended to allow the identification of any significant differences between the amounts, and establish a correlation between that and a method's efficiency for VOC collection. The results from this analysis revealed that the average total amounts obtained by each method were not significantly different in any of the subjects ( $\mathrm{p}$ values $\geq 0.1$ ).

After not finding a statistically significant difference that could influence the

determination of the best suited method, compound abundance was considered as a criterion for the method's selection. Nevertheless, the total amounts extracted for each individual did not reveal any trend that would particularly highlight the efficiency of one 
sampling method over the others (Figure 18). For this reason, reproducibility was evaluated as a criterion in the method selection process. The scent profile of subject A displayed the highest total VOC abundance after engaging in the 15 minutes walking method, but a high relative standard deviation (RSD) was obtained for this average VOC amount (Table 9). The magnitude of the RSD value implied a lack of reproducibility across the different samples collected from Subject A. Therefore, in the case of this subject, this could not be considered the most efficient sampling method. After evaluating the VOC amounts extracted for Subject A in the other methods, it was established that Subject A portrayed the best results in the 30 minutes sitting method.

In the same manner, Subject B was seen to provide the best VOC results with the one hour sitting method (Table 9). Although, in both this method and the 30 minutes sitting method Subject B displayed very similar amounts, the standard deviation and RSD value obtained for the one hour sitting method reflected a higher reproducibility. On the other hand for Subject C, the highest total VOC amount was extracted as a result of the one hour sitting method. In this case, the results for standard deviation and RSD achieved in the one hour method were also better than those accomplished by the other methods tested (Table 9). 
Table 9: Results of VOC extraction with different sampling methods

\begin{tabular}{|c|c|c|c|c|}
\hline $\begin{array}{c}\text { Sampling } \\
\text { Method }\end{array}$ & $\begin{array}{c}\text { Subject } \\
\text { Sampled }\end{array}$ & $\begin{array}{c}\text { Average total amount } \\
\text { extracted (ng) }\end{array}$ & $\begin{array}{c}\text { Standard Deviation of } \\
\text { total amount extracted } \\
\text { (ng) }\end{array}$ & $\begin{array}{c}\text { RSD } \\
\text { (\%) }\end{array}$ \\
\hline 15 min walking & & & 86.98 & \\
\hline & Sub. A & 70.28 & 18.32 & 30 \\
\hline & Sub. B & 60.47 & 24.58 & 50 \\
\hline & Sub. C & 49.61 & & 65 \\
\hline 15 min sitting & & & 19.91 & 45 \\
\hline & Sub. A & 30.61 & 13.76 & 49 \\
\hline & Sub. B & 30.66 & 17.58 & 51 \\
\hline & Sub. C & 35.92 & 34.49 & 79 \\
\hline 30 min sitting & & & 59.09 & 28 \\
\hline & Sub. A & 67.98 & 8.85 & 99 \\
\hline & Sub. B & 75.15 & 24.19 & 26 \\
\hline & Sub. C & 31.90 & 19.17 & 13 \\
\hline
\end{tabular}

*Quantitated compounds: 2-Furanmethanol, Decanal, Citral, Methyl tridecanoate, Benzophenone, Heptadecane, 1-Hexadecanol, and Methyl palmitate

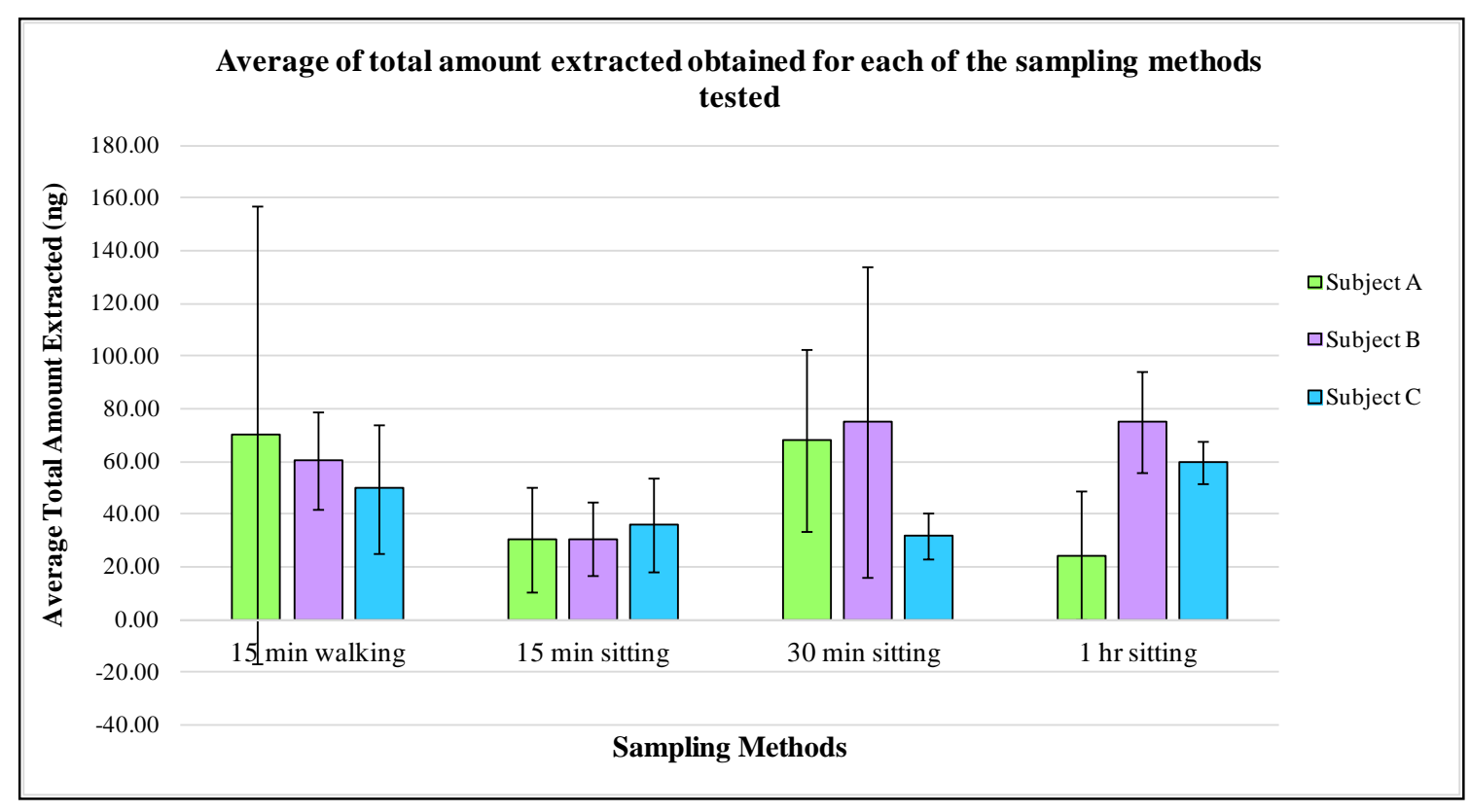

Figure 18: Comparison of the average compound amount extracted from underarm odor samples of three females using different sampling methods 
The magnitude of the standard deviations and RSD values calculated for this study suggested a great level of variability between the different samples collected from a same subject. A possible factor that could have contributed to this observation could be the presence of within-subject variations in scent profiles. Different samples may have contained different amounts of VOCs, sometimes even below the instrument's quantitation limits (Appendix 6). Therefore, variations in VOC detection and quantitation, caused by individual variability, may have contributed to high RSDs and standard deviations in this study. However, these two types of measurements can still highlight how efficient a sampling method can be against the others. If within-subject variability is considered a constant that is characteristic of all subjects, it will equally impact all methods and become negligible. In such a case, the reproducibility of a method would be indicating exactly how efficient the method is for the collection of underarm VOCs. On the basis of this statement, one hour sitting was selected as the optimum method to be used for sample collection. Two out of three females showed that this method was capable of providing a high compound abundance while still providing the best reproducibility possible in the collection of underarm odor samples. Therefore, the one hour sitting method was established as the most efficient way to assess sampling in further studies.

\subsubsection{Pilot study}

A total of 21 individuals between the ages of 18-30 years and 55+ years were sampled following the optimized procedure for the collection of underarm odor samples. The scent profiles of 12 females and nine males were evaluated, and their composition 
was used as a model to modify the VOC list that had originally been created by referencing previous literature. Using real samples as a reference to establish the expected constituents of human scent, in the samples to be collected, allowed tailoring the VOC list so that it could resemble more accurately the composition of authentic scent samples.

Compounds found in three out of four sample profiles from each subject, and showing a mass spectral library match of 80 or above, were identified first. Subsequently, compounds were selected and included in the VOC list on the basis of their frequency of occurrence in odor profiles, their potential impact for group differentiation, and the commercial availability of chemical reference standards. A total of 28 new compounds were selected from both female and male scent profiles, and incorporated into the original VOC list. On the other hand, 15 compounds that were initially present in the original VOC list were ultimately removed. The compounds were removed after noticing, throughout the different optimization studies, that they limited the analysis to be performed on authentic scent samples. Therefore, a resulting VOC list of 40 compounds (Appendix 6) would be considered in further studies to determine which VOCs could constitute an initial VOC list for the analysis of each group. A VOC's ability to pertain in each group's initial list would depend on its occurrence, and consistency over time, in the scent profiles of the individuals constituting the group.

In addition to the modification of the VOC list, the soap and deodorant provided to participants in this study were evaluated. The analysis of both types of blanks revealed that there was a potential for VOC contribution from the pretreatment products to individual scent profiles. From the 40 compounds included in the tailored VOC list, a 
total of 11 compounds were quantified in soap and deodorant blanks (Table 10). However, it was not always possible to quantify VOC contribution, as compound amounts varied and were sometimes below the limit of quantitation of the instrument. This was observed for 1-Hexadecanol in soap blanks, and 1-Octadecene, Dimethyl sulfone, Decanal, Methyl palmitate and Naphthalene in deodorant blanks. In these cases it was still possible to detect the compounds and, therefore, identify them as potential sources of influence. Nevertheless, these small VOC quantities were considered negligible on individual scent profiles. In an attempt to increase the accuracy of the VOC information obtained from soap and deodorant blanks, a spectral quality match of 80 or higher was required for compounds to be reported present in the blank samples. The consideration of a high spectral quality match value served to increase the level of certainty with which it can be established that the already mentioned compounds have an influence in VOC profiles.

The pilot study provided useful information that served as foundation to the development of all subsequent studies performed. 
Table 10: Values for average amount extracted and standard deviation of VOCs quantified in soap and deodorant blanks

\begin{tabular}{|c|c|c|c|c|}
\hline \multicolumn{5}{|c|}{$\begin{array}{c}\text { Average amount extracted and Standard Deviation for VOCs quantified in Soap and Deodorant } \\
\text { blanks }\end{array}$} \\
\cline { 2 - 5 } Compound Name & \multicolumn{2}{|c|}{ Soap Blank } & \multicolumn{2}{c|}{ Deodorant Blank } \\
\cline { 2 - 5 } & $\begin{array}{c}\text { Average Amount } \\
\text { extracted (ng) }\end{array}$ & $\begin{array}{c}\text { Standard } \\
\text { Deviation } \\
\text { (ng) }\end{array}$ & $\begin{array}{c}\text { Average } \\
\text { Amount } \\
\text { extracted (ng) }\end{array}$ & $\begin{array}{c}\text { Standard } \\
\text { Deviation } \\
\text { (ng) }\end{array}$ \\
\hline Naphthalene & 1.04 & 0.01 & N/D & N/D \\
\hline Trans-Beta-Ionone & 4.80 & 0.33 & N/D & N/D \\
\hline 2-Tridecanone & 4.02 & 0.06 & N/D & N/D \\
\hline Pentadecane & 2.83 & 0.56 & N/D & N/D \\
\hline Dodecanoic acid & 10.55 & 0.85 & N/D & N/D \\
\hline Isoamyl salicylate & 1.61 & 0.09 & N/D & N/D \\
\hline Benzophenone & 1.36 & 0.05 & N/D & N/D \\
\hline Alpha-Amylcinnamaldehyde & 81.85 & 11.12 & N/D & N/D \\
\hline Alpha-Hexylcinnamaldehyde & 1.74 & 0.36 & N/D & N/D \\
\hline Galaxolide & 2.25 & 0.03 & N/D & N/D \\
\hline Heptadecane & N/D & N/D & 130.23 & 18.52 \\
\hline
\end{tabular}

5.4.3. Determination of scent markers in underarm odor samples

Data analysis performed to determine scent markers in underarm odor samples consisted of two main approaches. The first approach evaluated traits (e.g., age and gender), within each racial/ethnic population under study, to identify human scent trends and peculiarities. This type of analysis enhances the availability of specialized human scent information, that can be directly associated to traits in individuals who belong to specific groups or populations. The first approach provides tools to attain details about an individual's specific features, and facilitates the interpretation of such features under the context of the specific group to which the individual belongs. On the contrary, the second approach used in data analysis, was developed to explore whether human scent can still be employed to point out specific traits that may be common amongst individuals, even when they do not belong to the same race/ethnic group. This approach served to test the possibility of identifying human scent biomarkers that are specific to age, gender, and 
race/ethnicity, as traits. The combination of these two approaches enhances the ability of odor profiles to provide details on individual traits. For this reason, the analysis and results presented have been structured around both approaches.

As part of the first approach for the evaluation of underarm odor samples, a series of VOCs was identified for each racial/ethnic population group under study. These VOCs were selected on the basis of their occurrence in the odor profiles of the individuals forming the different groups. In all cases, the occurrence of these VOCs in individual odor profiles was determined by looking at the consistency of their presence in the odor samples. Samples from the left underarm were not included in the assessment as a result of their inconsistency as replicates. Compounds were only considered to have occurred in an odor profile after having seen it present in the two samples collected from a subject's right underarm, during the period of consecutive weeks of study. This criterion was employed throughout all data analysis as a way to filter out compounds that could be present in odor profiles by influence of exogenous factors. Hence, the criterion was used as a measure to minimize the temporary impact of exogenous factors on the search for potential biomarkers that could facilitate group discrimination.

Following the analysis of compound occurrence in individual odor profiles, an "averaged profile” was created for each racial/ethnic group under study. This was done by calculating the average amount of each VOC, for each of the gender and age groups being considered per race/ethnic group (JMP ${ }^{\circledR}$ 12.0.0 statistical software). In this case, the averages were meant to provide a general depiction of what an odor profile should be like for an individual of a specific racial/ethnic population, on the basis of his/her corresponding age and gender. Averaged profiles provided insight on the overall 
influence that the different traits may have on an individual's scent. Therefore, the VOC constituents of the averaged profiles were used for both the qualitative and quantitative analysis of the different racial/ethnic populations under study. The standard error of the mean was also obtained for all the VOC average amounts calculated. These values were later on used to indicate the error of the average amounts in some of the graphical representations of the results. A careful visual evaluation of the average compound amounts, per gender and age groups, was performed for all racial/ethnic groups under study. From that evaluation, a series of compounds were selected to take part of "secondary lists". These secondary lists, along with the original compound list, served to establish a comparison between the different groups being considered; and to determine the efficiency of both VOC lists as discrimination criteria for each of the groups. In order to be part of a secondary list, a compound had to either show a difference of $100 \%$ or more on its average amount, between the groups being compared, or show a difference in terms of its occurrence. Nevertheless, some compounds were still considered part of secondary lists despite of having a difference lower than $100 \%$ in their average. These exceptions were made after finding the compounds' to be valuable for group discrimination (detailed in the results' section).

The same line of qualitative and quantitative evaluation was followed for the more generalized analysis. However, in this case, averaged profiles represented the independent influence of each trait (e.g., age and gender) on a pool of different individuals. Moreover, the data collected in these studies were analyzed using Principal Component Analysis (PCA). This statistical technique was used to evaluate the role of different VOCs in the potential discrimination of gender and age groups, within specific 
racial/ethnic groups. Principal Component Analysis served as a tool to graphically represent how each VOC can be associated to the clustering of individuals into different groups. In addition, it also served as a source of information on the level of discrimination power that can be attained by specific VOC variables when attempting to differentiate individuals from such groups. All score plots created were marked arbitrarily in an attempt to highlight the differentiation achieved between the groups and ease the understanding of the analysis. Linear Discriminant Analysis (LDA) was utilized as a resource to evaluate group differentiation, and the potential to classify different individuals on the basis of human scent characteristics displayed by odor profiles. This statistical technique was meant to facilitate the generalized analysis of all the collected data and allow the evaluation of scent profiles on a broader scale.

Overall, the data analysis performed in this study intended to provide an overview of the information that can be found in the odor profiles of different individuals, as a result of the traits that characterize them. The analysis aimed to obtain information that could be employed to classify members of a same group, and discriminate members of different groups. This objective was meant to be fulfilled while also identifying portions of information that could be directly associated with gender, age, and race or ethnicity, as common or shared traits.

\subsubsection{Results from Caucasian population}

\subsection{Qualitative Analysis}

An original VOC list consisting of 28 compounds was considered in the data analysis of the Caucasian population. These compounds were considered for both the 
qualitative and quantitative analysis of the different gender and age groups (Table 11) under study. The occurrence of all 28 VOCs was evaluated in the samples collected from the right underarm of all Caucasian individuals. Using this information, individual odor profiles were created and an overall depiction of the scent trends, displayed by females and males in all age groups, was obtained (Figure 19 - Figure 24).

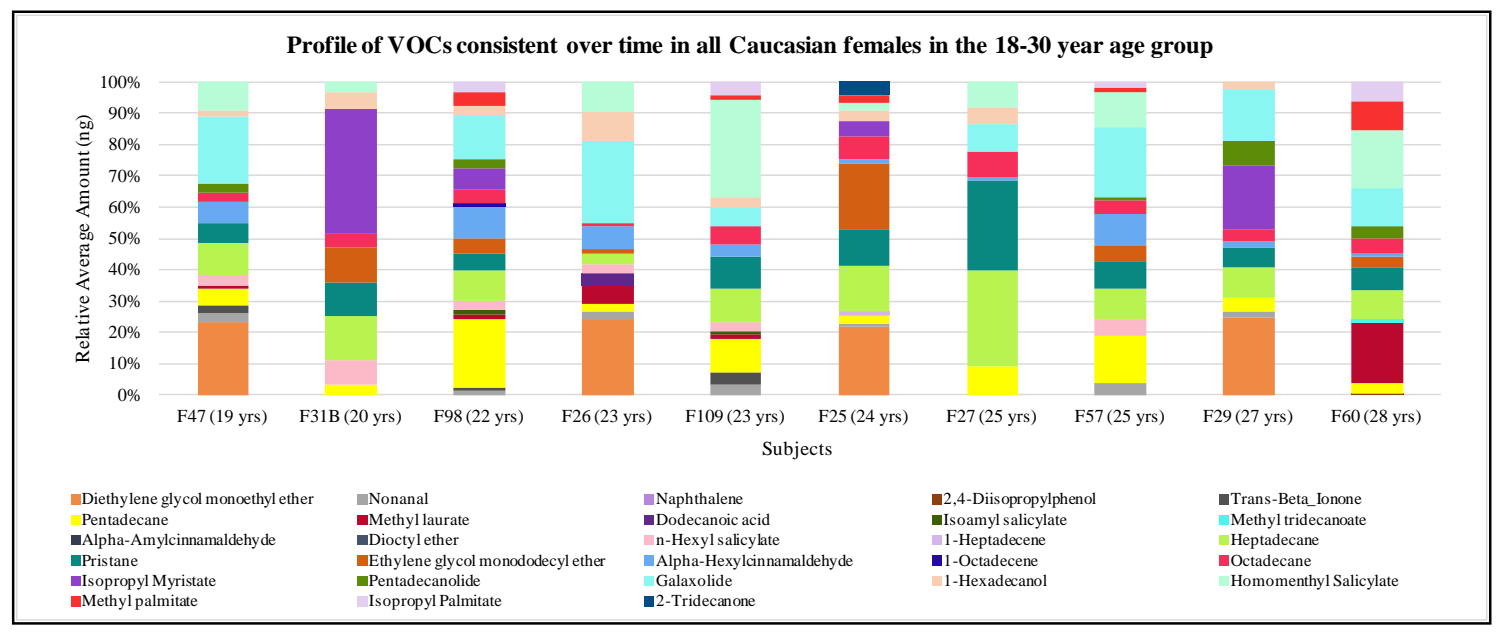

Figure 19: Scent profiles obtained for all 18-30 year old Caucasian females

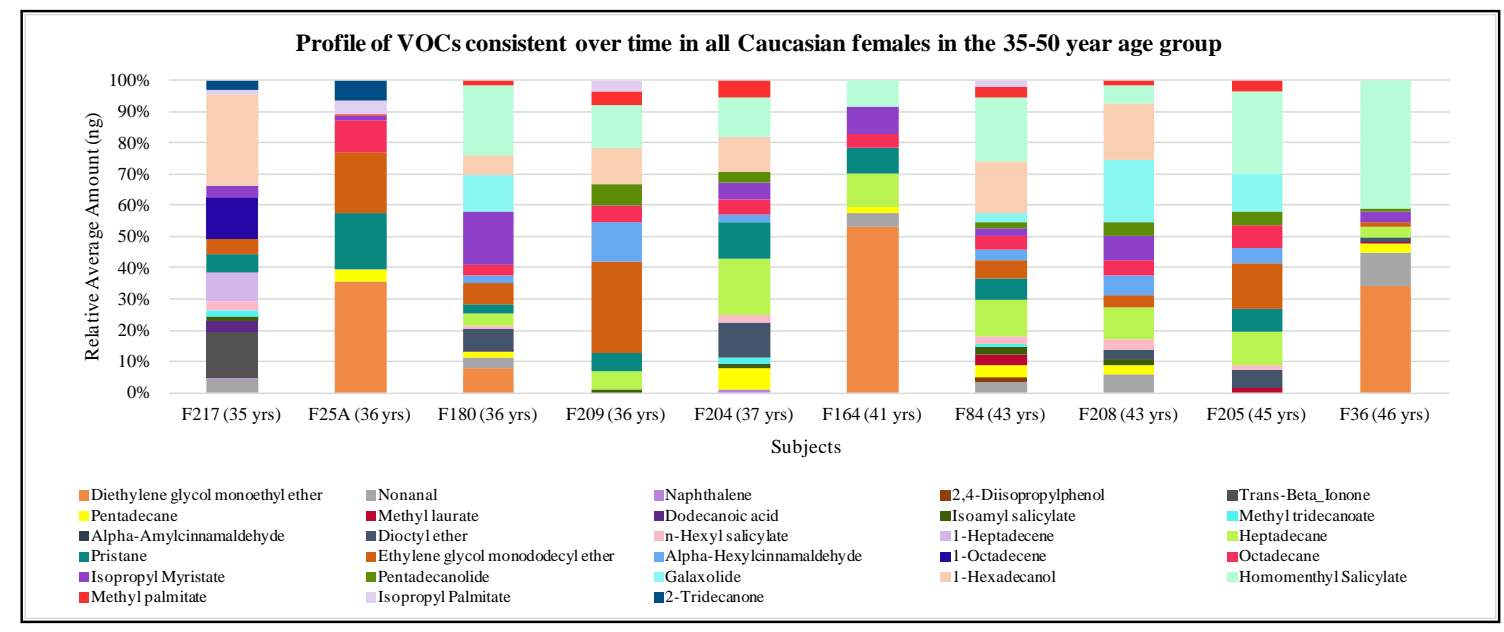

Figure 20: Scent profiles obtained for all 35-50 year old Caucasian females 


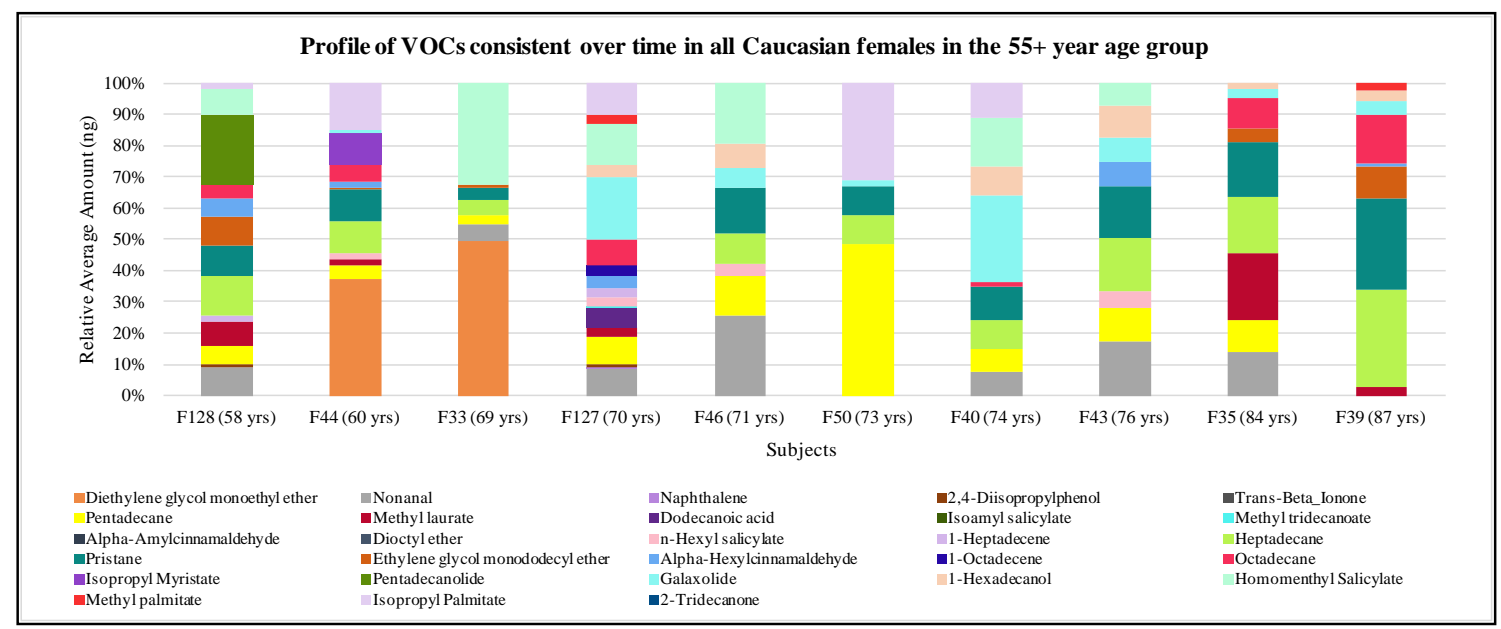

Figure 21: Scent profiles obtained for 55+ year old Caucasian females

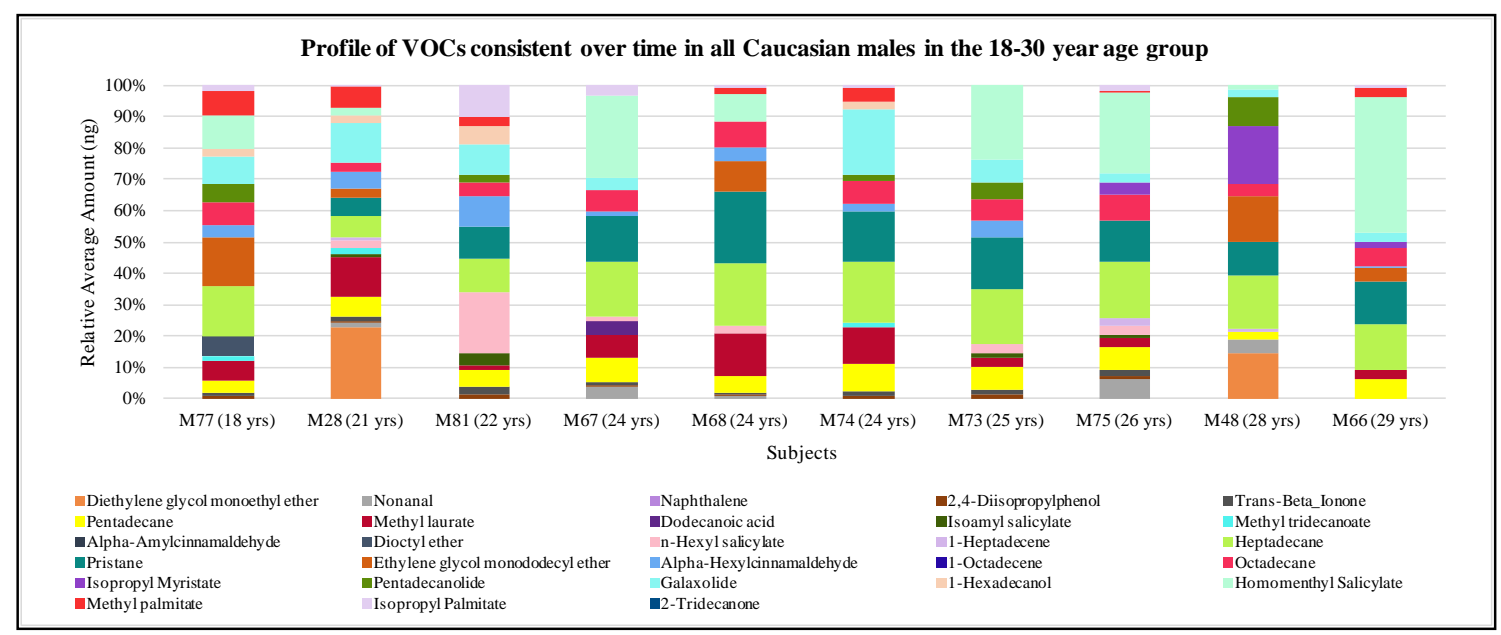

Figure 22: Scent profiles obtained for all 18-30 year old Caucasian males 


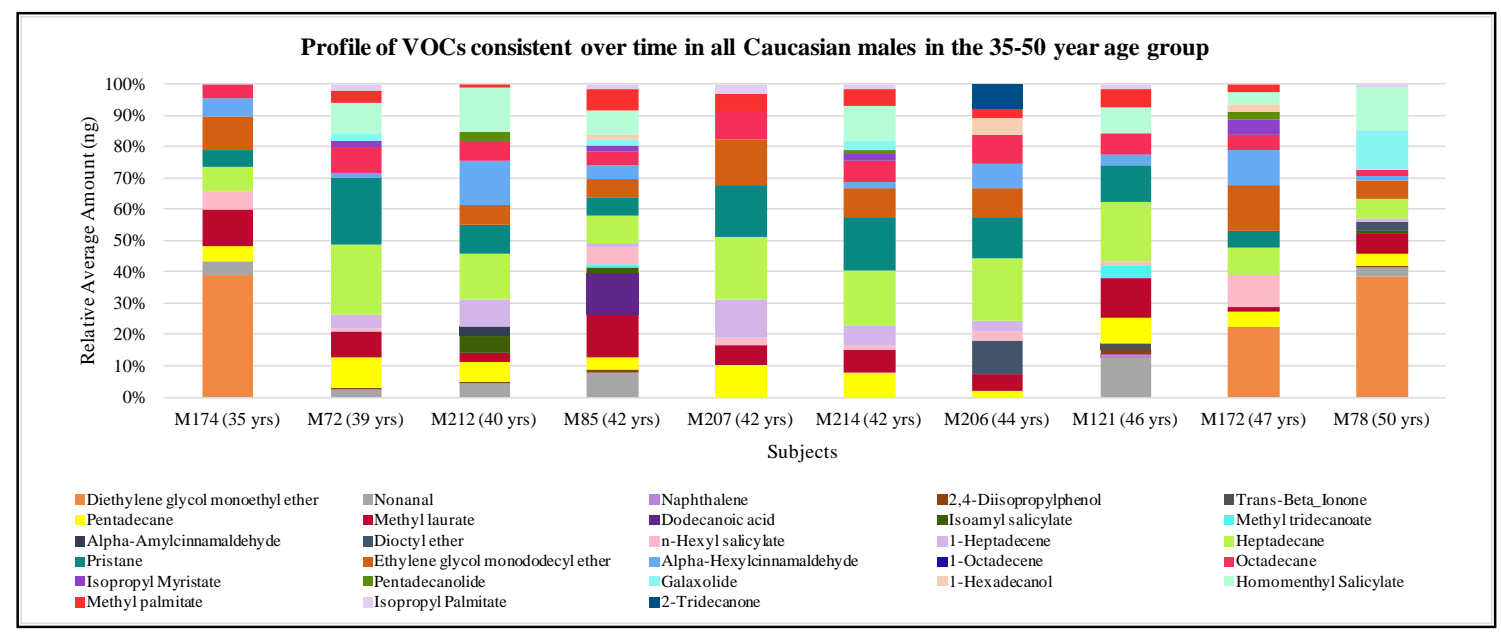

Figure 23: Scent profiles obtained for all 35-50 year old Caucasian males

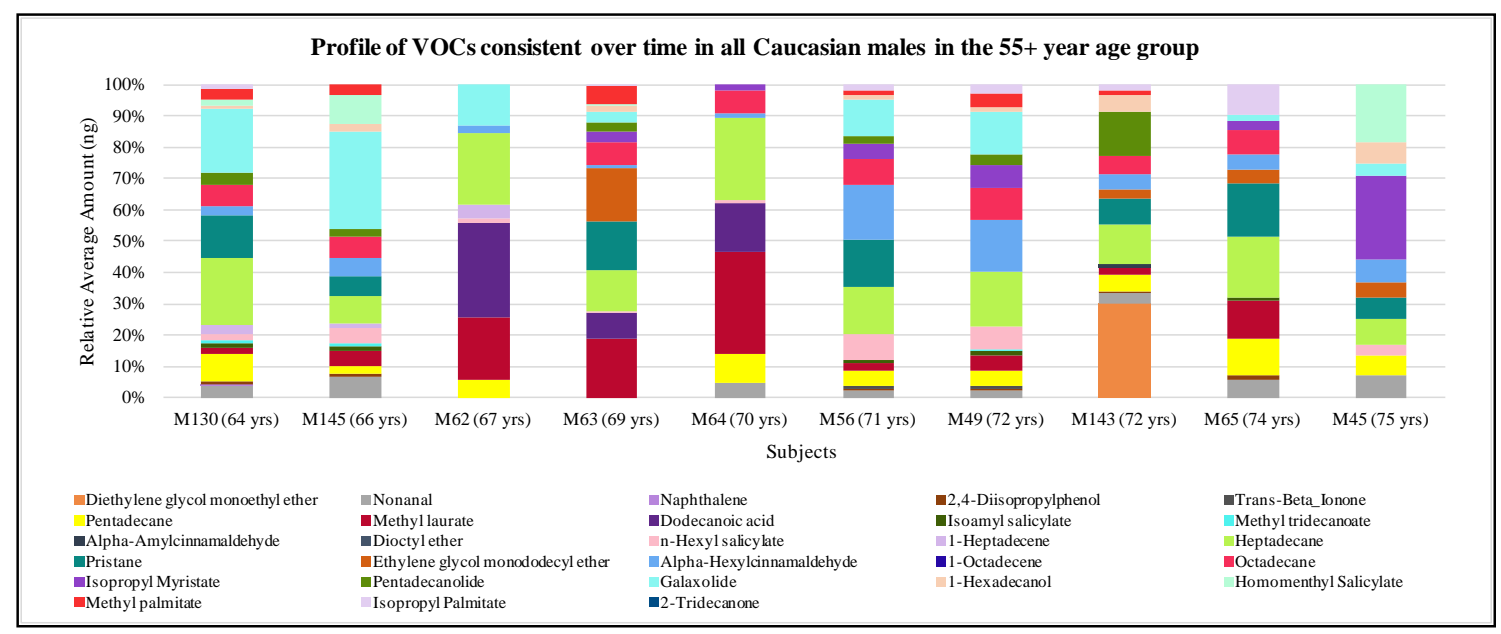

Figure 24: Scent profiles obtained for all 55+ year old Caucasian males

According to the information obtained from the odor profiles of individuals in the 18-30 year age group, 1-Hexadecanol occurred in Caucasian females twice as much as it occurred in Caucasian males (Table 11). On the other hand, the opposite seemed to occur in the case of 2,4-Diisopropylphenol, Trans-Beta-Ionone, Isoamyl salicylate, Methyl tridecanoate, and Isopropyl Palmitate. In the case of these particular compounds the occurrence in males was $100 \%$ or more than the occurrence of the same compounds in 
female odor profiles. Both 2-Tridecanone and 1-Octadecene were only found in female odor profiles for this age group, while Dioctyl ether was only found in males. Nevertheless, Naphthalene and Alpha-Amylcinnamaldehyde were not found in any gender for the 18-30 year group. On the other hand, Isopropyl palmitate was observed to have a higher occurrence in Caucasian males than in females, yet females displayed a higher average VOC amount than males for this compound $($ Females $=4.57 \mathrm{ng}$; males $=$ 2.84 ng.) The fact that Isopropyl palmitate was seen to be more abundant in females, agrees with previous reports in which this compound was established as a gender marker for females. ${ }^{37}$ Nevertheless, the fact that the compound revealed a higher occurrence in males suggests that there is a possibility that the occurrence and abundance of Isopropyl palmitate are not always necessarily directly proportional for Caucasian females and males. The qualitative analysis of odor profiles from Caucasian individuals in the 35-50 year age group showed that the occurrence of Naphthalene, 2-Tridecanone, Dioctyl ether, Isopropyl myristate , Pentadecanolide and 1-Hexadecanol in females was $100 \%$ or more than that of males in the same population (Table 11). On the other hand, it also showed that the occurrence of 2,4-Diisopropylphenol, Methyl laurate, and 1-Heptadecene in males surpassed that of females by $100 \%$ or more. Alpha-Amylcinnamaldehyde and 1Octadecene were both only found in one of the genders and both compounds were noted to occur in a low number of subjects (10\%). In regards to Isopropyl palmitate, a higher occurrence was observed in Caucasian males (60\%) than in Caucasian females (40\%) for this age group. Also, the average amount extracted for this compound in males was found to be slightly higher than that of females. Hence, in the case of this age group, the information obtained for Isopropyl palmitate is not in accordance with previous reports. ${ }^{37}$ 
In the case of Caucasian individuals in the 55+ year category, Trans-Beta-Ionone, Isoamyl salicylate, and Alpha-Amylcinnamaldehyde were only observed to occur in male odor profiles (Table 11). Conversely, 1-Octadecene was only noted in female profiles; specifically in the odor profiles of only $10 \%$ of the females in this age group. A total of ten different compounds showed to have an occurrence in males that surpassed that of females by $100 \%$ or more: 2,4-Diisopropylphenol, Trans-Beta-Ionone, Dodecanoic acid, Isoamyl salicylate, Methyl tridecanoate, $\mathrm{n}$-Hexyl salicylate, AlphaHexylcinnamaldehyde, Isopropyl Myristate, Pentadecanolide, and Methyl palmitate. On the other hand, the occurrence of Diethylene glycol monoethyl ether and 1-Heptadecene in female profiles dominated that of males by $100 \%$ or more.

Isopropyl palmitate was found in the odor profile of $50 \%$ of all $55+$ year old females and $60 \%$ of all males in the same age group. Once again, this shows that this compound was slightly more occurrent in male odor profiles than in females. Overall, throughout all Caucasian gender comparisons by age group, it was possible to note how the occurrence of this specific compound predominated mainly in male odor profiles. However, it was noticed that both in the $55+$ year age group and in the 18-30 year age group, the average amount for this compound resulted higher in females than in males. In these cases, the observations of this study continue to support the previous findings that describe Isopropyl palmitate as being a gender marker for females. ${ }^{21,37}$ In the 35-50 year age group, the only case in which males showed a higher average amount of Isopropyl palmitate than females, there was not much of a difference between the amounts (females: $1.70 \mathrm{ng}$ and males: $1.78 \mathrm{ng}$ ). The small difference between the amounts of both genders suggests surveying a larger number of subjects could be necessary. The 
evaluation of a greater number of subjects could lead to confirm whether the difference in average amounts is a result of mere gender differences, or if it's being influenced by chance. Nevertheless, it seems that there may be a possibility for compound occurrence to be more representative of odor profile variation across different individuals; while average amounts serve as a more accurate depiction of what a compounds' presence represents to each of the genders and their profiles.

A qualitative analysis of the odor profiles was also assessed by gender. This was done in an attempt to identify specific VOC trends as a result of changes in age (Table 11Table 11). As a result of this analysis, three different compounds were identified as having a decrease in occurrence as age increased for females: Trans-Beta-Ionone, nHexyl salicylate and Octadecane. However, no compounds appeared to consistently increase their occurrence with age in female odor profiles. In addition, it was noticed that Trans-Beta-Ionone, 2-Tridecanone, and Isoamyl salicylate were not present in the odor profiles of any 55+ year old female, and Naphthalene was not found in the odor profile of any 18-30 year old female. Dioctyl ether was only found present in the odor profiles of 35-50 year old females, while Alpha-Amylcinnamaldehyde was not found in any Caucasian female profiles at all. On the other hand, from the qualitative evaluation of male odor profiles across age groups, it was observed that Dioctyl ether and Homomenthyl Salicylate had a tendency to decrease their occurrence with age. On the contrary Nonanal, Alpha-Hexylcinnamaldehyde, and Isopropyl Myristate appeared to consistently increase their occurrence as males aged. 
Table 11: VOCs emanating from Caucasian subjects' underarm odor samples detected and quantified by solid-phase microextraction GC/MS

\begin{tabular}{|c|c|c|c|c|c|c|c|c|}
\hline \multirow{3}{*}{ CAS no. } & \multirow{3}{*}{$\begin{array}{c}\mathrm{RT} \\
(\mathrm{min})\end{array}$} & \multirow{3}{*}{ Compound Name } & \multicolumn{6}{|c|}{ Frequency of Occurrence in Subjects } \\
\hline & & & \multicolumn{3}{|c|}{ Females $(\mathrm{n}=30)$} & \multicolumn{3}{|c|}{ Males $(n=30)$} \\
\hline & & & $\begin{array}{l}18-30 \\
\text { years }\end{array}$ & $\begin{array}{l}35-50 \\
\text { years }\end{array}$ & $\begin{array}{c}55+ \\
\text { years }\end{array}$ & $\begin{array}{l}\text { 18-30 } \\
\text { years }\end{array}$ & $\begin{array}{l}35-50 \\
\text { years }\end{array}$ & $\begin{array}{c}55^{+} \\
\text {years }\end{array}$ \\
\hline $111-90-0$ & 11.168 & Diethylene glycol monoethyl ether ${ }^{\mathrm{c}}$ & $40 \%$ & $40 \%$ & $20 \%$ & $20 \%$ & $30 \%$ & $10 \%$ \\
\hline $124-19-6$ & 13.066 & Nonanal ${ }^{\mathrm{c}, \mathrm{d}}$ & $70 \%$ & $60 \%$ & $70 \%$ & $50 \%$ & $60 \%$ & $80 \%$ \\
\hline $91-20-3$ & 14.883 & Naphthalene $^{\text {c, d, e }}$ & $0 \%$ & $20 \%$ & $10 \%$ & $0 \%$ & $10 \%$ & $10 \%$ \\
\hline $2934-05-6$ & 18.800 & 2,4-Diisopropylphenol & $10 \%$ & $10 \%$ & $20 \%$ & $80 \%$ & $50 \%$ & $60 \%$ \\
\hline $79-77-6$ & 20.638 & Trans-Beta-Ionone $^{\text {c, e }}$ & $30 \%$ & $10 \%$ & $0 \%$ & $80 \%$ & $10 \%$ & $20 \%$ \\
\hline $593-08-8$ & 20.741 & 2-Tridecanone ${ }^{\text {c, e }}$ & $10 \%$ & $20 \%$ & $0 \%$ & $0 \%$ & $10 \%$ & $0 \%$ \\
\hline $629-62-9$ & 20.796 & Pentadecane ${ }^{c, \mathrm{e}}$ & $100 \%$ & $70 \%$ & $90 \%$ & $100 \%$ & $100 \%$ & $90 \%$ \\
\hline $111-82-0$ & 21.296 & Methyl laurate ${ }^{\text {c, d }}$ & $50 \%$ & $40 \%$ & $50 \%$ & $90 \%$ & $100 \%$ & $90 \%$ \\
\hline $143-07-7$ & 22.156 & Dodecanoic acid ${ }^{\mathrm{c}, \mathrm{d}, \mathrm{e}}$ & $10 \%$ & $10 \%$ & $10 \%$ & $10 \%$ & $10 \%$ & $30 \%$ \\
\hline $87-20-7$ & 22.331 & Isoamyl salicylate ${ }^{\mathrm{c}, \mathrm{e}}$ & $20 \%$ & $50 \%$ & $0 \%$ & $40 \%$ & $30 \%$ & $40 \%$ \\
\hline $1731-88-0$ & 23.263 & Methyl tridecanoate $^{\mathrm{d}}$ & $10 \%$ & $30 \%$ & $10 \%$ & $30 \%$ & $20 \%$ & $30 \%$ \\
\hline $122-40-7$ & 23.819 & Alpha-Amylcinnamaldehyde c, e & $0 \%$ & $0 \%$ & $0 \%$ & $0 \%$ & $10 \%$ & $10 \%$ \\
\hline $629-82-3$ & 24.037 & Dioctyl ether & $0 \%$ & $50 \%$ & $0 \%$ & $10 \%$ & $20 \%$ & $0 \%$ \\
\hline $6259-76-3$ & 24.383 & Hexyl salicylate ${ }^{c, d}$ & $70 \%$ & $60 \%$ & $40 \%$ & $60 \%$ & $80 \%$ & $80 \%$ \\
\hline $6765-39-5$ & 24.537 & 1-Heptadecene ${ }^{\mathrm{d}}$ & $10 \%$ & $10 \%$ & $90 \%$ & $30 \%$ & $70 \%$ & $30 \%$ \\
\hline $629-78-7$ & 24.657 & Heptadecane ${ }^{\text {c, e }}$ & $100 \%$ & $\mathbf{8 0 \%}$ & $90 \%$ & $100 \%$ & $100 \%$ & $100 \%$ \\
\hline 1921-70-6 & 24.764 & Pristane & $90 \%$ & $80 \%$ & $90 \%$ & $90 \%$ & $90 \%$ & $70 \%$ \\
\hline $4536-30-5$ & 24.965 & Ethylene glycol monododecyl ether & $60 \%$ & $80 \%$ & $50 \%$ & $50 \%$ & $80 \%$ & $40 \%$ \\
\hline $101-86-0$ & 25.521 & Alpha-Hexylcinnamaldehyde c, d, e & $60 \%$ & $60 \%$ & $50 \%$ & $\mathbf{8 0} \%$ & $90 \%$ & $100 \%$ \\
\hline $112-88-9$ & 26.102 & 1-Octadecene & $10 \%$ & $10 \%$ & $10 \%$ & $0 \%$ & $0 \%$ & $0 \%$ \\
\hline $593-45-3$ & 26.209 & Octadecane $^{c}$ & $100 \%$ & $\mathbf{8 0 \%}$ & $60 \%$ & $100 \%$ & $100 \%$ & $80 \%$ \\
\hline
\end{tabular}




\begin{tabular}{|c|c|c|c|c|c|c|c|c|}
\hline \multirow{3}{*}{ CAS no. } & \multirow{3}{*}{$\begin{array}{c}\mathrm{RT} \\
(\mathrm{min})\end{array}$} & \multirow{3}{*}{ Compound Name } & \multicolumn{6}{|c|}{ Frequency of Occurrence in Subjects } \\
\hline & & & \multicolumn{3}{|c|}{ Females $(n=30)$} & \multicolumn{3}{|c|}{ Males $(n=30)$} \\
\hline & & & $\begin{array}{l}18-30 \\
\text { years }\end{array}$ & $\begin{array}{l}35-50 \\
\text { years }\end{array}$ & $\begin{array}{c}55+ \\
\text { years }\end{array}$ & $\begin{array}{l}18-30 \\
\text { years }\end{array}$ & $\begin{array}{l}35-50 \\
\text { years }\end{array}$ & $\begin{array}{c}55+ \\
\text { years }\end{array}$ \\
\hline $110-27-0$ & 26.564 & Isopropyl Myristate $^{\mathrm{c}}$ & $40 \%$ & $80 \%$ & $10 \%$ & $30 \%$ & $40 \%$ & $60 \%$ \\
\hline $106-02-5$ & 26.808 & Pentadecanolide $^{\mathrm{c}}$ & $50 \%$ & $60 \%$ & $10 \%$ & $50 \%$ & $30 \%$ & $60 \%$ \\
\hline $1222-05-5$ & 27.068 & Galaxolide $^{\mathrm{c}, \mathrm{e}}$ & $80 \%$ & $40 \%$ & $80 \%$ & $90 \%$ & $40 \%$ & $\mathbf{8 0 \%}$ \\
\hline $36653-82-4$ & 27.274 & 1-Hexadecanol c, d & $80 \%$ & $60 \%$ & $60 \%$ & $40 \%$ & $30 \%$ & $70 \%$ \\
\hline $52253-93-7$ & 27.475 & Homomenthyl salicylate & $80 \%$ & $80 \%$ & $60 \%$ & $80 \%$ & $70 \%$ & $40 \%$ \\
\hline $112-39-0$ & 27.838 & Methyl palmitate ${ }^{c, d}$ & $50 \%$ & $70 \%$ & $20 \%$ & $70 \%$ & $80 \%$ & $60 \%$ \\
\hline $142-91-6$ & 28.821 & Isopropyl Palmitate ${ }^{\mathrm{c}, \mathrm{d}}$ & $40 \%$ & $40 \%$ & $50 \%$ & $80 \%$ & $60 \%$ & $60 \%$ \\
\hline
\end{tabular}

a. $\mathrm{RT}=$ Retention Time

b. Compounds with a frequency of occurrence equal or higher to $50 \%$ of subjects are denoted in bold

c. Compounds of possible exogenous origin - reported as being typically used as fragrance ingredients in consumer goods ${ }^{122}$

d. Reported by previous studies as present in human skin emanations, human VOC profiles, characteristic axillary odors or as individual axillary marker compounds ${ }^{21,33,36-38,123}$

e. Compounds were also quantified in unscented deodorant or soap blanks 


\subsection{Gender comparison: a quantitative analysis of VOC profiles by age group}

In order to perform a gender comparison, a quantitative analysis was performed for females and males within each age group. For this analysis, all individuals were organized and evaluated in accordance to their age group. In this manner, age was kept as a constant trait during each gender comparison. Differentiation of females and males within each age group was initially evaluated using all 28 compounds as discrimination criteria (Figure 25 - Figure 27). A visual evaluation of the average amount extracted per VOC, for each gender, was performed for all age categories. Consequently, tailored versions of the initial compound list (secondary lists) were created for gender discrimination in each of the age categories. The power of the compounds included in the secondary lists was compared to that of the initial compound list for gender discrimination within each age group. This comparison aimed to evaluate the potential of those compounds included in the secondary lists as gender biomarkers within the different age categories. In this case, keeping age as a constant variable during group comparisons made average compound amounts provide a general representation of each compound's contribution to an individual's VOC profile according to their gender. Moreover, it facilitated the assessment of a possible link between such contribution and gender, as a trait, in individuals. This approach allowed the creation of a generalized profile for each gender and eased the identification of those compounds that could serve as biomarkers to classify Caucasian individuals based on such trait. 


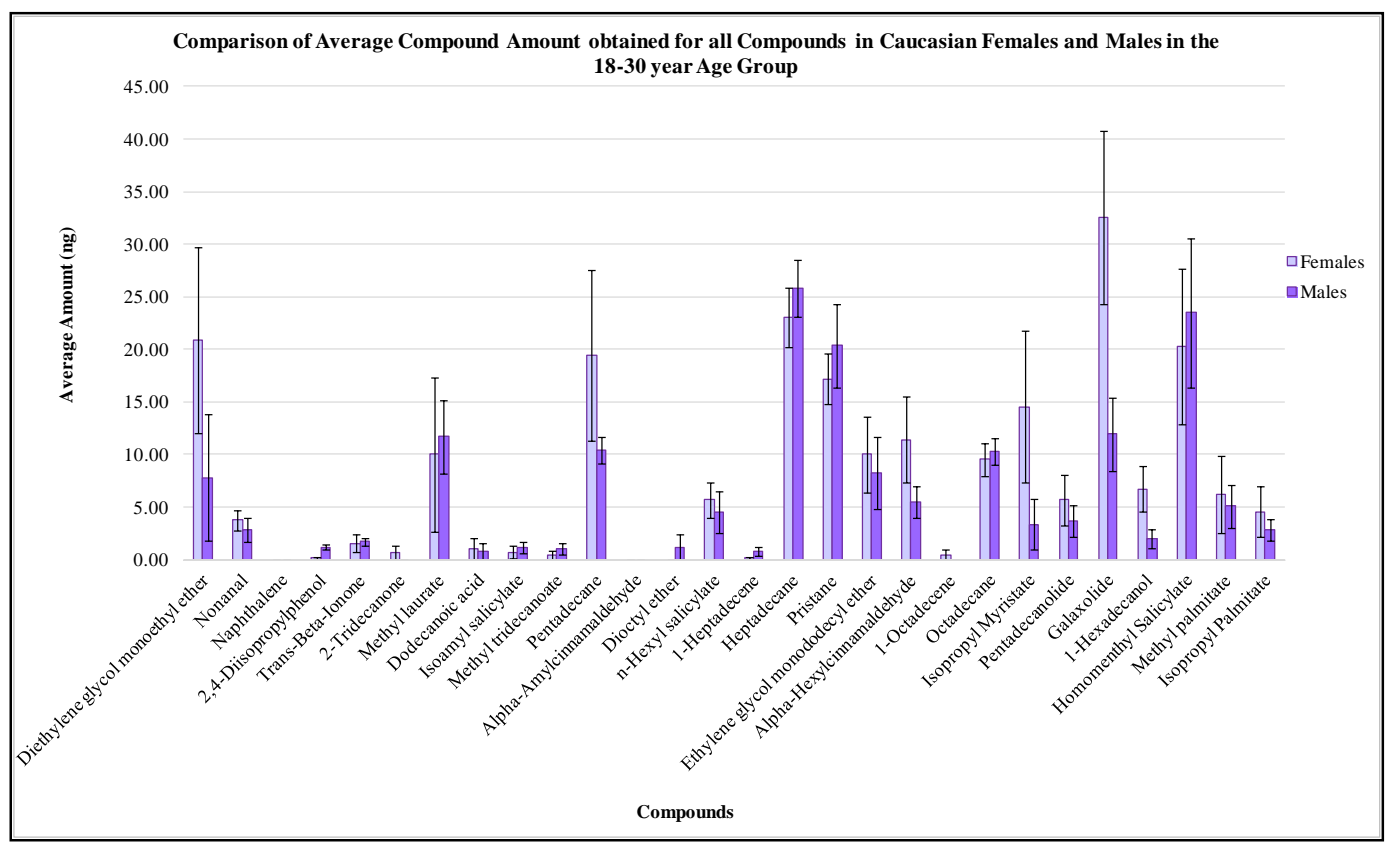

Figure 25: Comparison of the average extracted amounts obtained for all 28 VOCs in 18-30 year old Caucasian females and males

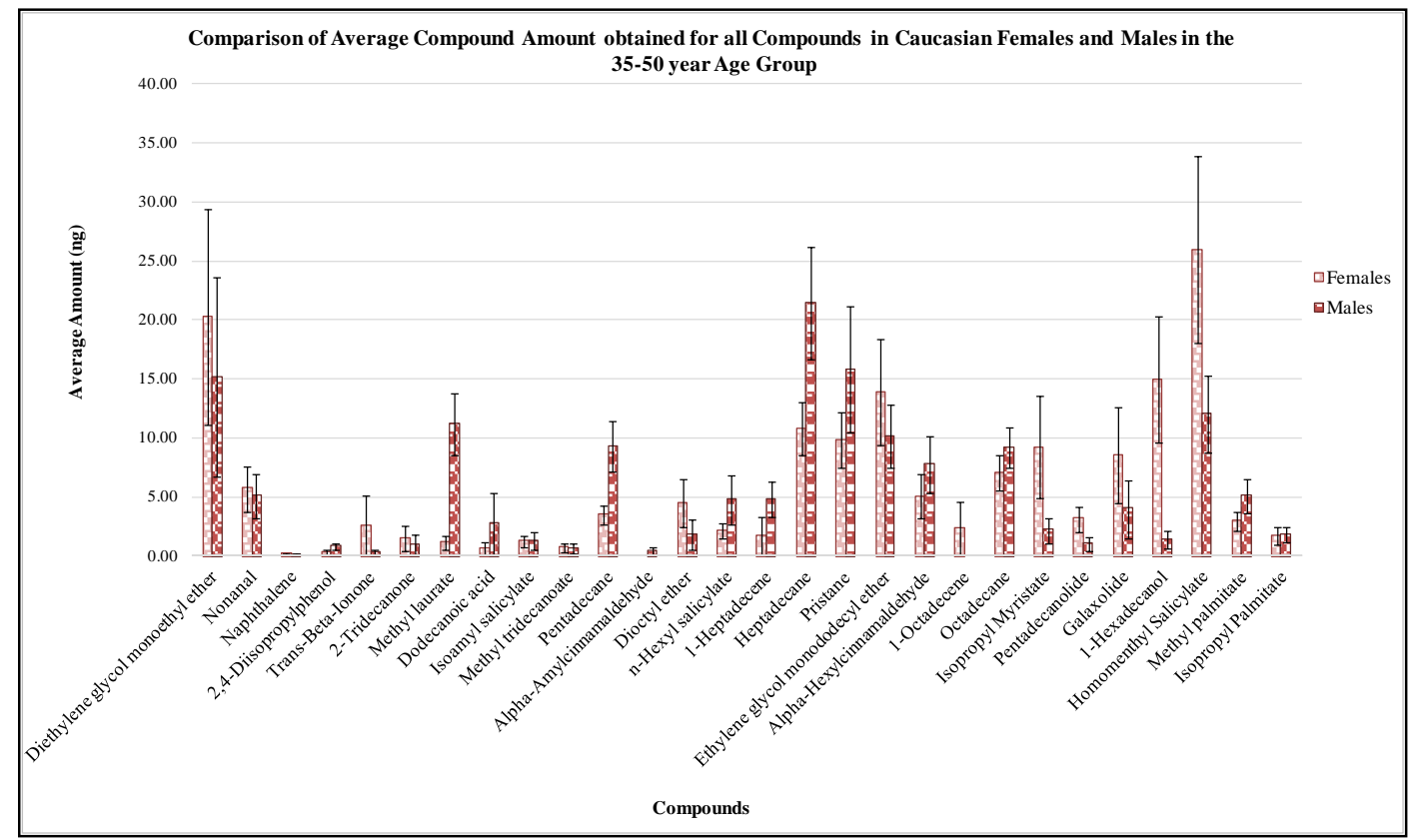

Figure 26: Comparison of the average extracted amounts obtained for all 28 VOCs in 35-50 year old Caucasian females and males 


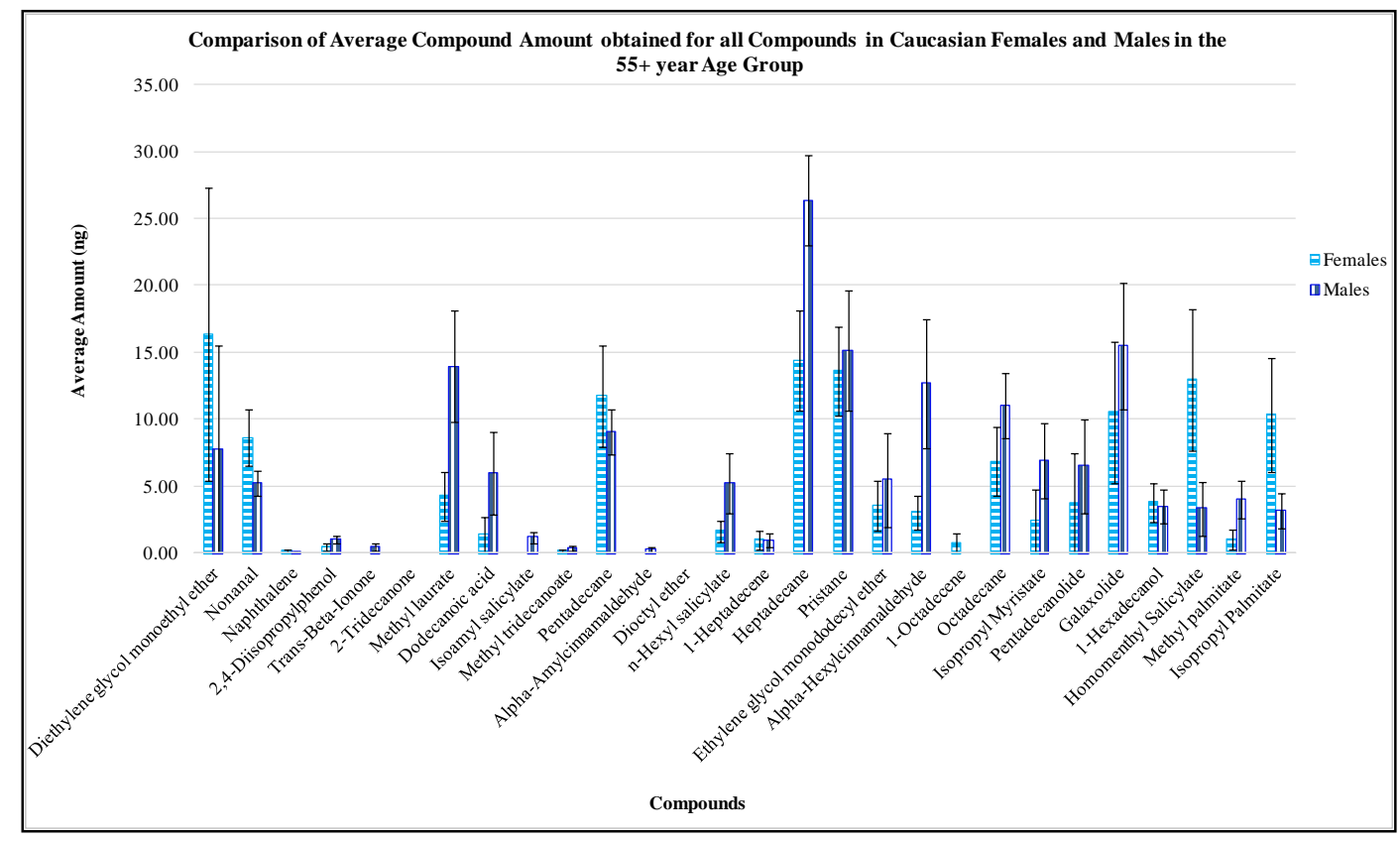

Figure 27: Comparison of the average extracted amounts obtained for all 28 VOCs in 55+ year old Caucasian females and males

\section{8-30 year Age Group}

From the initial list of 28 compounds, 11 compounds were selected for the secondary list of the 18-30 year age group (Table 12). These 11 compounds showed differences of $100 \%$ or more in the average amount between Caucasian females and males. In the case of both, the initial and the secondary lists, female odor profiles showed a tendency to have higher average VOC amounts than males. In specific, out of the 11 compounds that comprised the secondary list for this group, seven VOCs had a higher average amount in females and only four had a higher amount in males.

A PCA score plot was created using all 28 compounds to evaluate their potential as criteria for gender discrimination (Figure 28). This initial score plot revealed that using the 28 VOCs altogether allowed the formation of slight clusters for both genders. However, the level of spread between the subjects did not allow a clear distinction of the 
clusters being formed; hence it did not facilitate group differentiation. When considering the compounds from the secondary list as discrimination variables, a reduction in the spread between the subjects of each group was observed (Figure 29 - left). This enhanced the differentiation of the gender groups and made it possible to have a clearer depiction of the clusters being formed. Nevertheless, a total separation between the groups was not achieved because of a slight overlap that is observed between the groups.

In comparison to the initial PCA score plot, the score plot obtained for the compounds in the secondary list showed an increase in the variation of the first two principal components. The first PCA score plot showed 20.6\% of variation in PC1 and $17.5 \%$ in PC2, while the secondary list score plot showed a variation percent of $27.4 \%$ in PC1 and 18\% in PC2. These results show that using the compounds from the secondary list as discrimination criteria improved gender discrimination in the 18-30 year age group. According to the loading plot obtained for the secondary list score plot (Figure 29 - right). Alpha-Hexylcinnamaldehyde, Galaxolide, 1-Hexadecanol, 1-Octadecene, and 2,4-Diisopropylphenol were the VOCs with most impact on the variation of the first principal component (in decreasing order of influence). On the other hand, Methyl tridecanoate, 2,4-Diisopropylphenol, Isopropyl Myristate, Galaxolide, and AlphaHexylcinnamaldehyde were found to be the compounds with most impact on PC2 (also in decreasing order).

\section{5-50 year Age Group}

In the comparison made for the 35-50 year age group, it was noted that females had a higher average amount for 15 compounds, while males showed a higher amount for 
13 VOCs. A total of 15 compounds were selected for the secondary list of this age group, after being observed to have a $100 \%$ or higher difference in average amount between males and females (Table 13). From this selection, females showed a higher average amount than males for eight compounds. Therefore, as seen in the 18-30 year category, there was a tendency for 35-50 year old females to display amounts that surpassed those of males of the same age group, in the case of most VOCs.

The PCA score plot created with the initial 28 compounds showed that most subjects from both genders grouped together towards the center of the plot (Figure 30). However, once the compound list was reduced to take into consideration only those compounds in the secondary list, a clearer separation of the groups was observed (Figure 31 - left). The rearrangement of all subjects under the influence of the secondary list VOCs resulted in an enhancement in gender discrimination within this age group; even though a slight overlap was still observed between the groups towards the center of the scoreplot. A variation of $20.4 \%$ in PC1 and $16.5 \%$ in PC2 was observed in the initial PCA score plot, while the secondary list score plot displayed a $22.5 \%$ and $22 \%$ of variation in PC1 and PC2, respectively. The increase in variation in both principal components explains the enhancement in differentiation between the gender groups. Among the primary VOCs found to be driving the variation in PC1 are 1-Heptadecene, Dioctyl ether, Trans-Beta-Ionone, 1-Octadecene, and Galaxolide (in decreasing order). On the other hand, the loading plot also revealed 1-Hexadecanol, Methyl laurate, 1Octadecene, Pentadecane, and Trans-Beta-Ionone as the most influential compounds in the variation of PC2 (also in decreasing order) (Figure 31 - right). 
55+ year Age Group

Out of the original 28 compounds, 55+ year old females had a higher average VOC amount for nine compounds; while males in the same age group showed a higher average amount for 17 compounds. In the case of this age group, 16 compounds were identified as being noticeably different between females and males (Table 14). These compounds were then selected to comprise the secondary list for this age group. From these 16 compounds, males showed to have a higher average amount in 11 VOCs. Therefore, when comparing 55+ year old Caucasian females and males, most VOCs tend to be more abundant in male odor profiles than in females.

A PCA score plot was created to evaluate the potential for gender discrimination with the initial list of 28 compounds (Figure 32). As part of that score plot it was possible to observe a fairly defined cluster of female subjects. However, the wide spread of male subjects did not allow the identification of a clear cluster for the male gender and caused the majority of subjects to overlap with the female group. After the sole consideration of the secondary list compounds as differentiation variables, the score plot showed a tighter cluster formed by female subjects (Figure 33 - left). However, this was still not enough to be able to identify a clear cluster for males in this age group. Although it was observed that a larger number of male subjects separated from the female group, certain males continued to overlap with the female cluster. This overlap did not favor a total separation between both genders in this age group, and suggests that there might still be a need to further explore the potential of other compounds as gender discrimination criteria. When comparing 55+ year old females and males using the secondary list of VOCs, the first principal component showed a variation percent of 30.3\%, and the 2nd principal 
component of $18.5 \%$. The variation percent obtained for PC1 in this case, revealed an increase from the variation displayed by the first principal component in the initial score plot (23.9\%). However, the opposite was observed in the case of PC2. The use of the secondary list VOCs as gender discrimination criteria reduced its variation percentage from $19.1 \%$ to $18.5 \%$. The fact that the increase was only observed in one of the principal components explains why there was not an effective separation between genders in the age group. On the other hand, the loading plot (Figure 33 - right) obtained from using the secondary list VOCs showed that the compounds causing the most variation in PC1 were n-Hexyl salicylate, Alpha-Hexylcinnamaldehyde, Isoamyl salicylate, Trans-Beta-Ionone, and 2,4-Diisopropylphenol (in decreasing order). Moreover, the most influential compounds in PC2's variation were found to be Naphthalene, 1-Octadecene, Methyl tridecanoate, Homomenthyl Salicylate, and Dodecanoic acid (also in decreasing order).

Table 12: Average VOC amount for secondary list compounds in 18-30 year old Caucasian females and males

\begin{tabular}{|c|c|c|}
\hline $\begin{array}{c}\text { Secondary List of Compounds for } \\
\text { Males and Females of 18-30 years }\end{array}$ & $\begin{array}{c}\text { Female Average } \\
\text { Compound } \\
\text { Amount (ng) }\end{array}$ & $\begin{array}{c}\text { Male Average } \\
\text { Compound } \\
\text { Amount (ng) }\end{array}$ \\
\hline Diethylene glycol monoethyl ether & 20.88 & 7.81 \\
\hline 2,4-Diisopropylphenol & 0.14 & 1.21 \\
\hline 2-Tridecanone & 0.66 & 0 \\
\hline Methyl tridecanoate & 0.40 & 1.03 \\
\hline Dioctyl ether & 0 & 1.21 \\
\hline 1-Heptadecene & 0.14 & 0.77 \\
\hline Alpha-Hexylcinnamaldehyde & 11.40 & 5.51 \\
\hline 1-Octadecene & 0.48 & 0 \\
\hline Isopropyl Myristate & 14.50 & 3.36 \\
\hline Galaxolide & 32.56 & 11.92 \\
\hline 1-Hexadecanol & 6.73 & 1.99 \\
\hline
\end{tabular}


Table 13: Average VOC amount for secondary list compounds in 35-50 year old Caucasian females and males

\begin{tabular}{|c|c|c|}
\hline $\begin{array}{c}\text { Secondary List of Compounds for } \\
\text { Males and Females of 35-50 years }\end{array}$ & $\begin{array}{c}\text { Female Average } \\
\text { Compound } \\
\text { Amount (ng) }\end{array}$ & $\begin{array}{c}\text { Male Average } \\
\text { Compound } \\
\text { Amount (ng) }\end{array}$ \\
\hline 2,4-Diisopropylphenol & 0.25 & 0.80 \\
\hline Trans-Beta_Ionone & 2.56 & 0.24 \\
\hline Methyl laurate & 1.08 & 11.13 \\
\hline Dodecanoic acid & 0.57 & 2.69 \\
\hline Pentadecane & 3.46 & 9.26 \\
\hline Alpha-Amylcinnamaldehyde & 0 & 0.37 \\
\hline Dioctyl ether & 4.47 & 1.78 \\
\hline n-Hexyl salicylate & 2.12 & 4.78 \\
\hline 1-Heptadecene & 1.63 & 4.80 \\
\hline 1-Octadecene & 2.30 & 0 \\
\hline Isopropyl Myristate & 9.20 & 2.18 \\
\hline Pentadecanolide & 3.11 & 1.02 \\
\hline Galaxolide & 8.53 & 3.98 \\
\hline 1-Hexadecanol & 14.94 & 1.35 \\
\hline Homomenthyl Salicylate & 25.94 & 12.00 \\
\hline
\end{tabular}

Table 14: Average VOC amount for secondary list compounds in 55+ year old Caucasian females and males

\begin{tabular}{|c|c|c|}
\hline $\begin{array}{c}\text { Secondary List of Compounds for } \\
\text { Males and Females of 55+ years }\end{array}$ & $\begin{array}{c}\text { Female Average } \\
\text { Compound } \\
\text { Amount (ng) }\end{array}$ & $\begin{array}{c}\text { Male Average } \\
\text { Compound } \\
\text { Amount (ng) }\end{array}$ \\
\hline Diethylene glycol monoethyl ether & 16.35 & 7.77 \\
\hline Naphthalene & 0.14 & 0.04 \\
\hline 2,4-Diisopropylphenol & 0.41 & 0.96 \\
\hline Trans-Beta_Ionone & 0 & 0.43 \\
\hline Methyl laurate & 4.25 & 13.92 \\
\hline Dodecanoic acid & 1.35 & 5.93 \\
\hline Isoamyl salicylate & 0 & 1.18 \\
\hline Methyl tridecanoate & 0.13 & 0.36 \\
\hline Alpha-Amylcinnamaldehyde & 0 & 0.20 \\
\hline n-Hexyl salicylate & 1.63 & 5.19 \\
\hline Alpha-Hexylcinnamaldehyde & 3.01 & 12.65 \\
\hline 1-Octadecene & 0.72 & 0 \\
\hline Isopropyl Myristate & 2.36 & 6.87 \\
\hline Homomenthyl Salicylate & 12.92 & 3.28 \\
\hline Methyl palmitate & 1.00 & 4.00 \\
\hline Isopropyl Palmitate & 10.33 & 3.16 \\
\hline
\end{tabular}




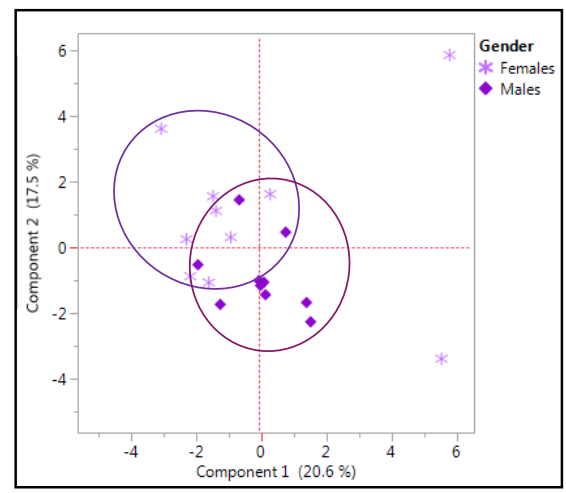

Figure 28: PCA score plot of 18-30 year old Caucasian females and males including all VOCs
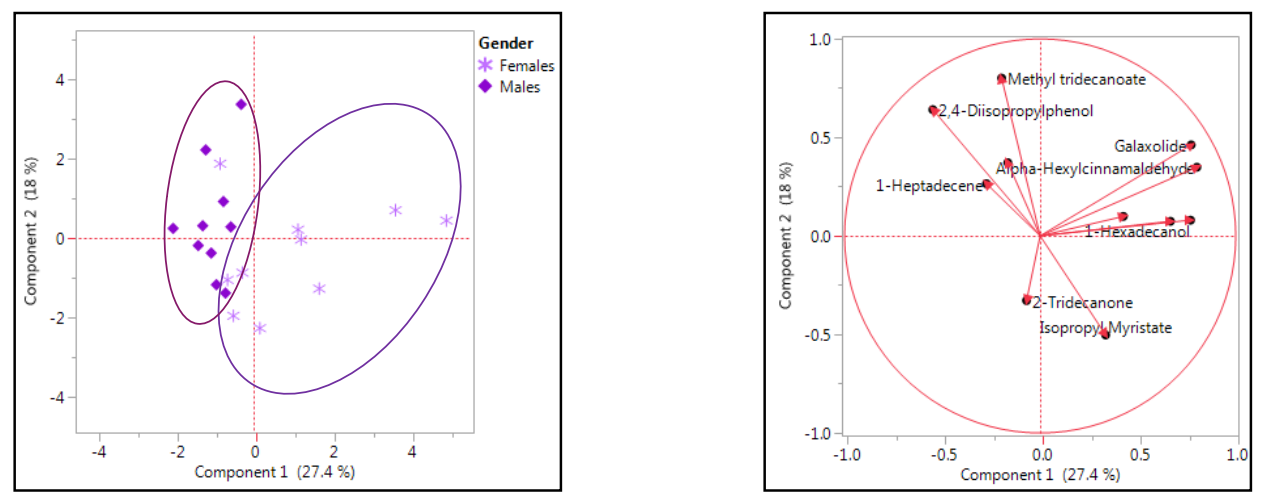

Figure 29: PCA score plot (left) and loading plot (right) of 18-30 year old Caucasian females and males including VOCs from secondary list

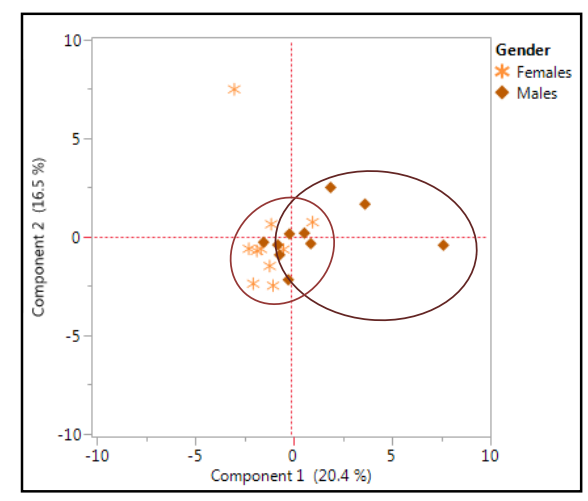

Figure 30: PCA score plot of 35-50 year old Caucasian females and males including all VOCs 

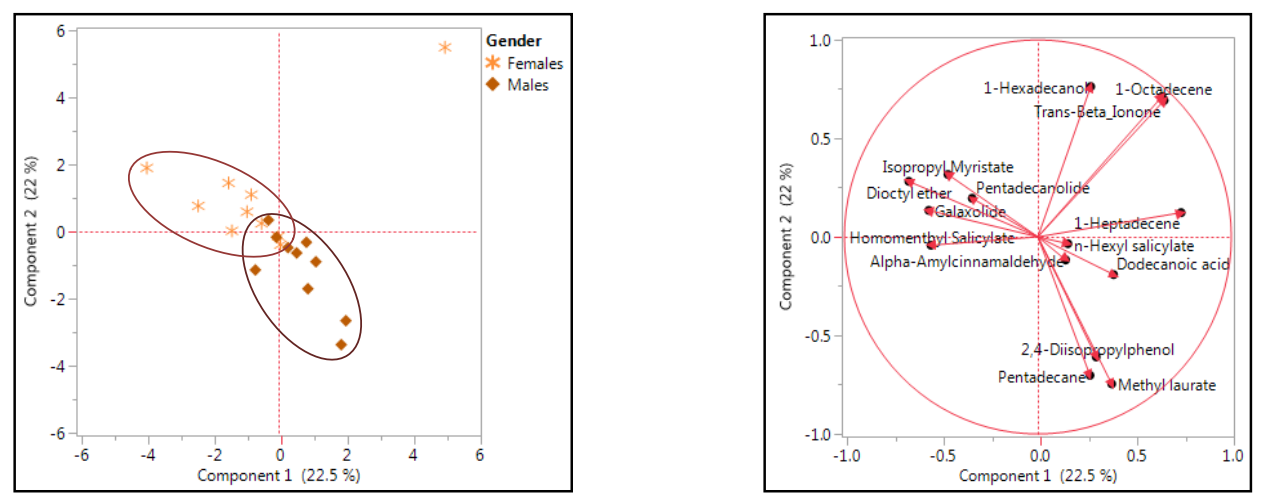

Figure 31: PCA score plot (left) and loading plot (right) of 35-50 year old Caucasian females and males including VOCs from secondary list

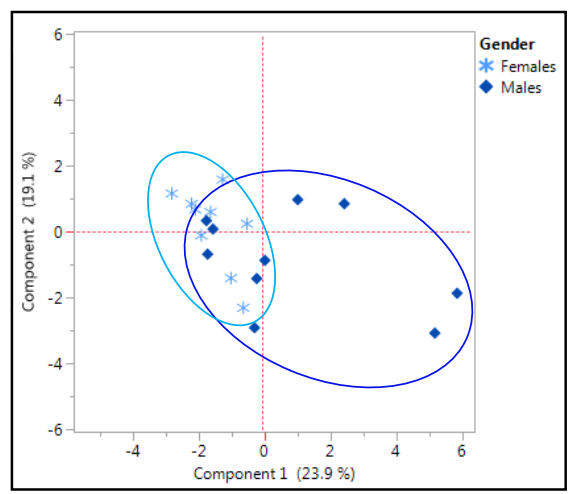

Figure 32: PCA score plot of 55+ year old Caucasian females and males including all VOCs
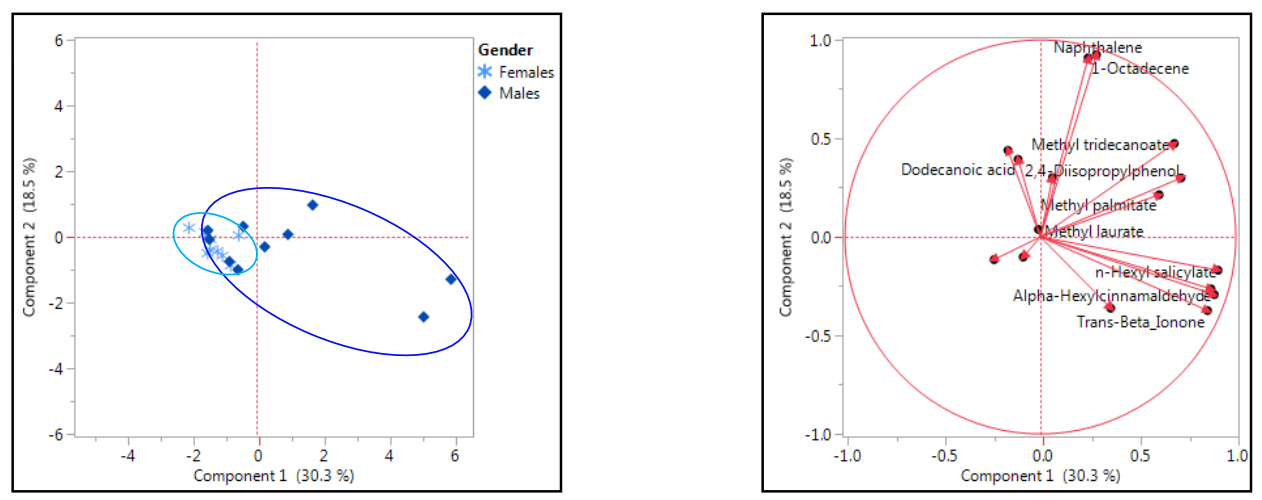

Figure 33: PCA score plot (left) and loading plot (right) of 55+ year old Caucasian females and males including VOCs from secondary list 
5.4.3.1.3. Age group comparison: a quantitative analysis of VOC profiles by gender

Odor profiles were grouped on the basis of gender to perform an age group comparison. By maintaining the gender of all subjects as a consistent variable, it was possible to compare the average amount extracted for each compound, in each of the different age groups (Figure 34 - Figure 35). A visual evaluation, of sets of two age groups at a time, was performed for females and males. This evaluation allowed surveying the average amount extracted per VOC for each age group, in each gender, and resulted in the creation of secondary lists for age group discrimination. In the case of this comparison, the averages provided a general representation of each compound's contribution to an individual's VOC profile according to their age group. Hence, the evaluation of the average VOC amounts facilitated the identification of those compounds that could serve as age markers, in each age group's generalized VOC profile, to classify Caucasian individuals of a same gender by their age. 


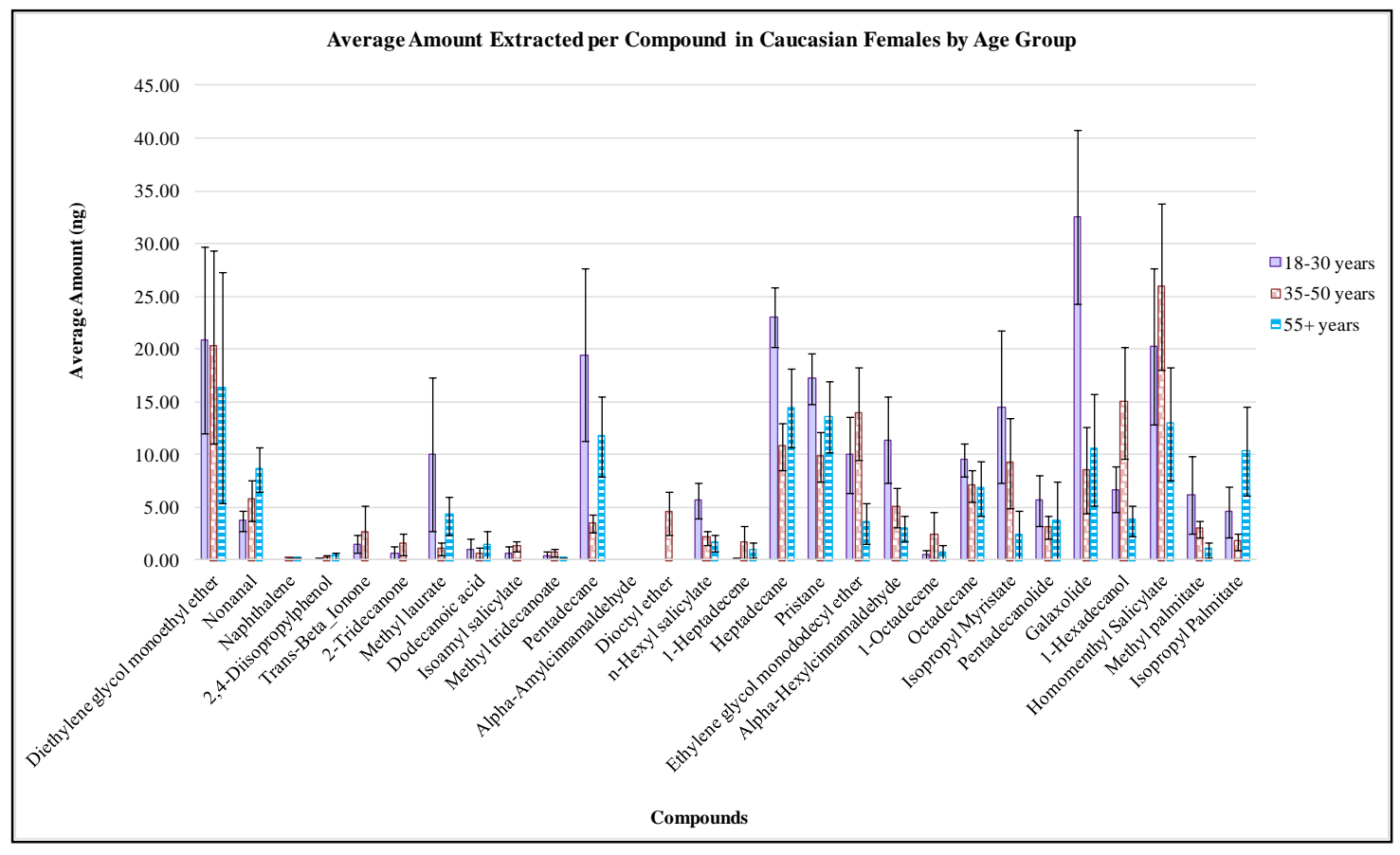

Figure 34: Comparison of the average extracted amounts obtained for all 28 VOCs in Caucasian females of all age groups

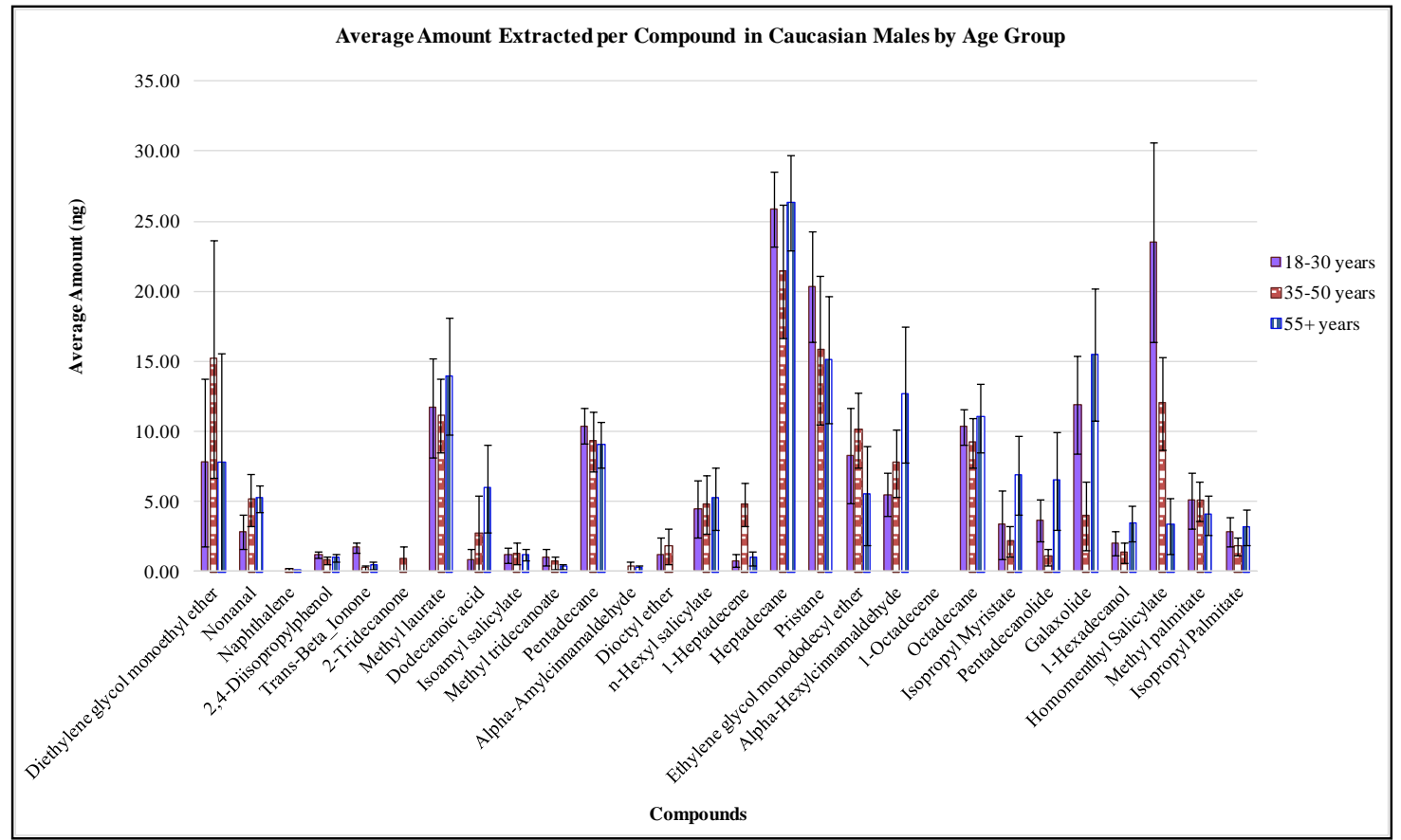

Figure 35: Comparison of the average extracted amounts obtained for all 28 VOCs in Caucasian males of all age groups 


\section{Comparison of Females of Different Age Groups}

An average compound amount comparison was assessed for Caucasian females in each age group. Through this comparison it was noted that, out of 28 compounds being considered, 18-30 year old females showed the highest average amount out of all groups for most compounds. Moreover, the 35-50 year old females had the highest average amount in the case of ten compounds, while 55+ year old females only did for 5 compounds: Nonanal, Naphthalene, 2,4-Diisopropylphenol, Dodecanoic acid, and Isopropyl Palmitate. According to Gallagher et al., who evaluated skin VOCs from the back and forearm of different individuals, Hexyl salicylate and AlphaHexylcinnamaldehyde are significantly more abundant in younger subjects (19-40 years) than in older subjects. ${ }^{21}$ The age group comparison in my study agreed with those results showing that females in the 18-30 year age group exhibit the greatest average compound amount for those two compounds. Moreover, it was noted that the average amount for these two compounds reduced with age. On the other hand, it had also been previously stated that Nonanal can be considered a biomarker for older subjects (41-79 year olds). ${ }^{21}$ In my study, the average amount for Nonanal increased with age. Although both the 55+ year and the 18-30 year categories showed equally high percentages of occurrence (70\%), the average amount obtained for Nonanal in females of the 55+ year category was more than twice of that observed for females in the youngest age group. Therefore, once again, these findings serve as supporting evidence for the statements made in Gallagher's study.

A total of 22 compounds, which showed differences of $100 \%$ or more in their amounts between any two of the age groups, were selected (Figure 36). Compounds that were found to be absent in one group while being present in others, were also considered 
to be noticeably different and incorporated into the selection. These compounds would help identify any possible differences being enforced between the groups by compound occurrence in itself. Out of the 22 compounds, 15 compounds showed a difference in average amount between individuals in the $18-30$ year category and the 55+ year category; while the 18-30 year and 35-50 year age groups only showed 12 noticeable differences (Table 15). When comparing the 35-50 year and 55+ year age groups a total of 15 compounds showed differences, indicating that females in the 55+ year group differ the most from those in the other two age categories (Table 15). When all three age group sets were compared, only three compounds out of 22 were seen to display differences in all comparisons (Methyl laurate, Methyl palmitate, and Isopropyl palmitate). The fact that such an observation was made could suggest that, rather than being discriminated by specific compound differences, age group discrimination for females may be relying in compound combinations. 


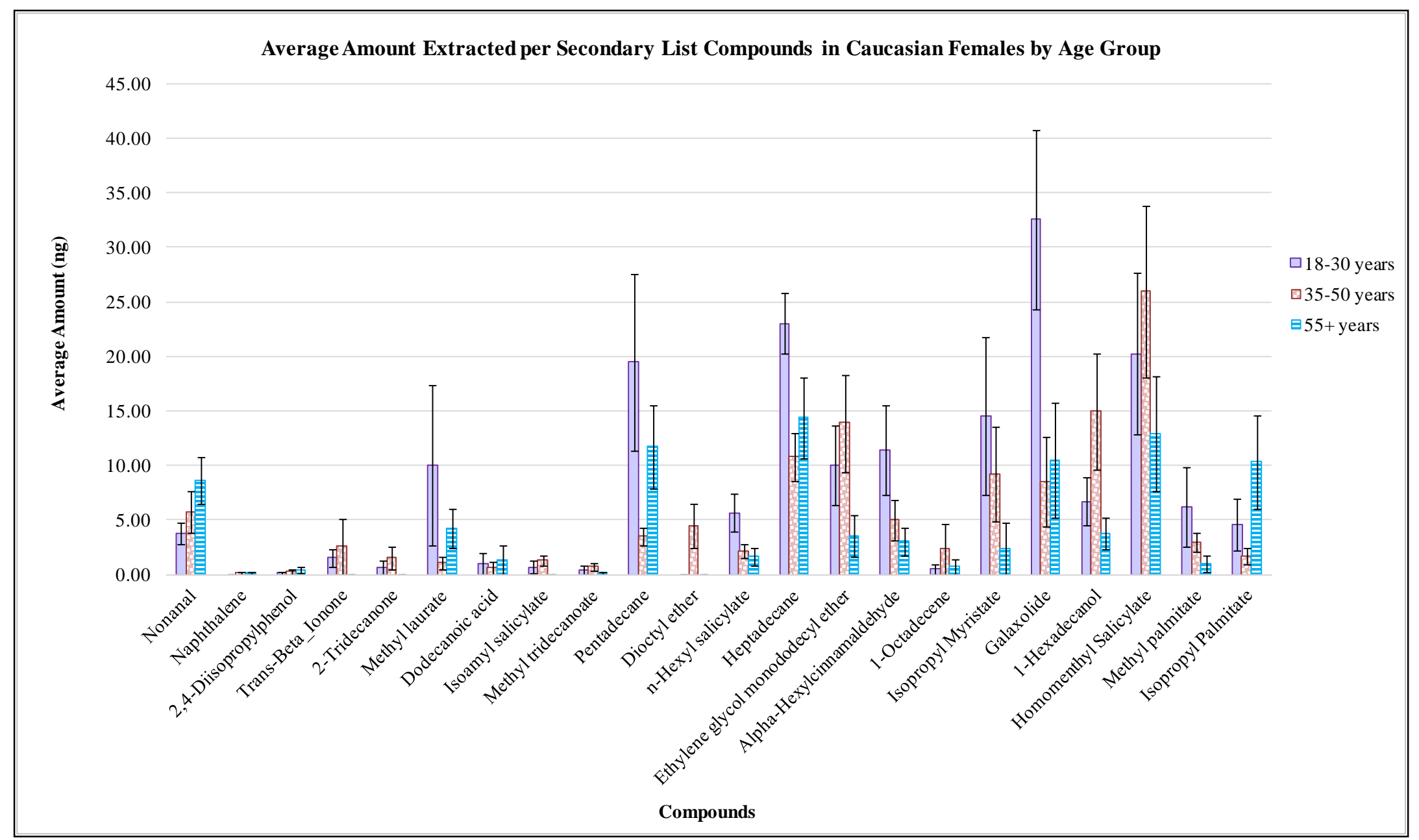

Figure 36: Comparison of average extracted amounts obtained for the VOCs considered in the secondary lists of Caucasian females from different age groups 
Table 15: Compounds considered in secondary lists made for comparing Caucasian females of different age groups

\begin{tabular}{|l|c|c|c|}
\hline $\begin{array}{c}\text { Compounds included in } \\
\text { Secondary Lists for Females }\end{array}$ & $\begin{array}{c}\mathbf{1 8 - 3 0} \text { vs. } \\
\mathbf{3 5 - 5 0} \text { years }\end{array}$ & $\begin{array}{c}\mathbf{1 8 - 3 0} \text { vs. } \\
\mathbf{5 5 +} \text { years }\end{array}$ & $\begin{array}{c}\mathbf{3 5 - 5 0} \text { vs. } \\
\mathbf{5 5 +} \text { years }\end{array}$ \\
\hline Nonanal & $\mathrm{X}$ & $\mathrm{X}$ & \\
\hline Naphthalene & & $\mathrm{X}$ & \\
\hline 2,4-Diisopropylphenol & & $\mathrm{X}$ & $\mathrm{X}$ \\
\hline Trans-Beta_Ionone & $\mathrm{X}$ & $\mathrm{X}$ & $\mathrm{X}$ \\
\hline 2-Tridecanone & & & $\mathrm{X}$ \\
\hline Methyl laurate & & $\mathrm{X}$ & $\mathrm{X}$ \\
\hline Dodecanoic acid & & $\mathrm{X}$ & $\mathrm{X}$ \\
\hline Isoamyl salicylate & $\mathrm{X}$ & & $\mathrm{X}$ \\
\hline Methyl tridecanoate & $\mathrm{X}$ & & $\mathrm{X}$ \\
\hline Pentadecane & $\mathrm{X}$ & $\mathrm{X}$ & \\
\hline Dioctyl ether & $\mathrm{X}$ & & \\
\hline n-Hexyl salicylate & & $\mathrm{X}$ & $\mathrm{X}$ \\
\hline Heptadecane & $\mathrm{X}$ & $\mathrm{X}$ & \\
\hline Ethylene glycol monododecyl ether & $\mathrm{X}$ & & $\mathrm{X}$ \\
\hline Alpha-Hexylcinnamaldehyde & & $\mathrm{X}$ & $\mathrm{X}$ \\
\hline 1-Octadecene & $\mathrm{X}$ & $\mathrm{X}$ & \\
\hline Isopropyl Myristate & $\mathrm{X}$ & & $\mathrm{X}$ \\
\hline Galaxolide & & & $\mathrm{X}$ \\
\hline 1-Hexadecanol & $\mathrm{X}$ & $\mathrm{X}$ & $\mathrm{X}$ \\
\hline Homomenthyl Salicylate & & $\mathrm{X}$ \\
\hline Methyl palmitate & & \\
\hline Isopropyl Palmitate & & \\
\hline
\end{tabular}

A wide series of PCA score plots and loading plots were constructed to evaluate the differentiation of the three Caucasian female age groups. Initially, a score plot built by taking into consideration the original 28 compounds indicated that $18-30$ year old and 55+ year old females cannot be differentiated (Figure 37). This was concluded as a result of having observed an overlap between the female subjects in these two age groups while being evaluated under the influence of all 28 VOCs. However, when reducing the list of 
variables to only the 15 compounds included in the secondary list for these two groups, female subjects from each group were seen to agglomerate according to their corresponding age category (Figure 38 - left). This agglomeration noticeably enhanced the separation between the groups, yet it did not favor a complete separation. A slight overlap between some females of the 55+ year group with a minority of females from the 18-30 year group was still observed. After comparing the percentage of variation in PC1 and PC2, for both the initial score plot and the secondary list score plot obtained for this group set, an increase in variation was observed for both principal components. This increase in variation resulted from having adjusted the discrimination variables to only those compounds identified as noticeably different between these groups. The percentage in PC1 increased from $22.5 \%$ to $25.2 \%$; while in PC2 the increase was from $18.9 \%$ to 20.6\%. According to the secondary list loading plot (Figure 38 - right), the compounds found to be most influential in PC1's increased variation were Methyl palmitate, Methyl tridecanoate, Methyl laurate, 2,4-Diisopropylphenol and Galaxolide (in decreasing order of influence). In the same token, the compounds found to be more influential in achieving the $20.6 \%$ of variation in PC2 were Gamma-Hexylcinnamaldehyde, Isoamyl salicylate, nHexyl salicylate, Trans-Beta-Ionone and Galaxolide (also in decreasing order).

In the case of the 18-30 and 35-50 year age groups, the PCA score plot obtained for all 28 VOC variables did not show separation between the groups (Figure 39). This initial score plot portrayed most subjects being spread out and mixed towards the center of the plot. On the contrary, the secondary list score plot displayed subjects from both age groups having a tendency to cluster around those of their corresponding age category (Figure 40 - left). This response to the adjusted discrimination criteria favored a fairly 
clear distinction of both groups. Moreover, it was also observed that as the spread of the 35-50 year group was reduced with the secondary list VOCs, the spread of the 18-30 year group seemed to slightly increase. Hence, although an enhancement in the separation of the groups was noted, changes in the spread of each group were not enough to completely separate and distinguish all individuals from these two age groups. According to the secondary list loading plot (Figure 40 - right), both PC1 and PC2 had an increase in variation percent. When evaluating group differentiation using all 28 VOCs, PC1 showed a $24.3 \%$ and PC2 showed 18\%. After having adjusted compound list, the variation percentages in PC1 and PC2 increased to $35.6 \%$ and $19.5 \%$, respectively. All observations gathered for this age group set suggest that, although variation increased in both principal components, there might still be a need to improve the discrimination criteria for female differentiation between these two age groups. Nevertheless, among the most influential compounds in driving variation in PC1 were identified Heptadecane, Methyl palmitate, Isopropyl Palmitate, Pentadecane, and Galaxolide (in decreasing order of influence). On the other hand those VOCs of higher impact in PC2 were n-Hexyl salicylate, 1-Hexadecanol, Alpha-Hexylcinnamaldehyde, 1-Octadecene, and Methyl laurate (also in decreasing order of influence).

When evaluating the 35-50 and the 55+ year age groups, the first score plot showed most subjects from both groups mixed towards the center of the plot (Figure 41). This did not favor a clear distinction between the two age groups. On the contrary, when using the compounds from the secondary list as discrimination criteria, an improvement was observed in cluster formation for both groups (Figure 42 - left). While the score plot including all variables showed no clear separation between the clusters, this last score 
plot showed subjects from both groups appearing closer together and with minimal overlapping between groups. The slight overlapping between the groups did not allow a complete separation, but it was still possible to distinguish both groups with ease because of the significant enhancement in the separation between their subjects. In this comparison, the variation percentages showed by the initial score plot were $23.6 \%$ in PC1 and $14.7 \%$ in PC2. Once the discrimination criteria were adjusted, variation in PC1 increased to $28.6 \%$ and in PC2 to $18.8 \%$. This suggests that, once again, the variation in both principal components was improved by reducing the number of compounds and using only those that were noticeably different as discrimination criteria. In the case of these two age groups, the loading plot (Figure 42 - right) showed that the VOCs with heavier impact in PC1's variation were 1-Octadecene, 1-Hexadecanol, Trans-BetaIonone, Methyl tridecanoate, and Isoamyl salicylate (in decreasing order). In the same token, those with most impact in PC2 were found to be Dioctyl ether, Pentadecane, Homomenthyl Salicylate, Methyl palmitate, and Isopropyl Myristate (also in decreasing order).

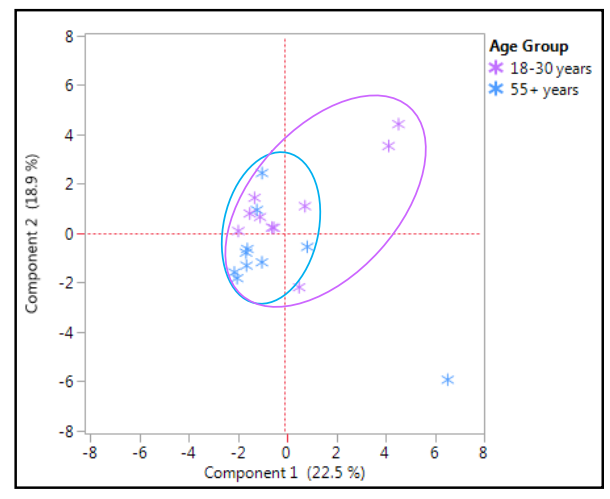

Figure 37: PCA score plot of 18-30 vs. 55+ year old Caucasian females including all VOCs 

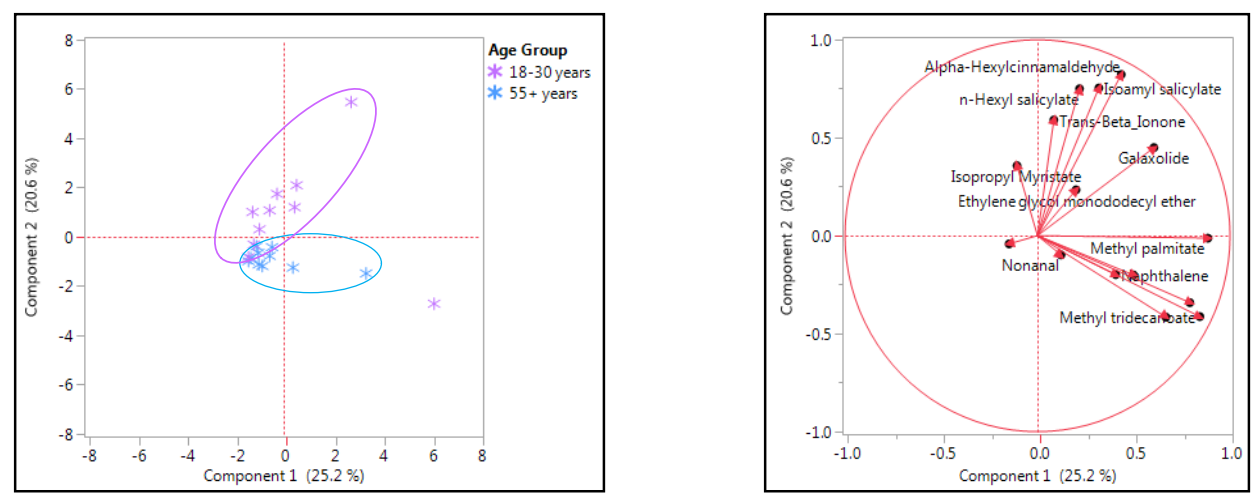

Figure 38: PCA score plot (left) and loading plot (right) of 18-30 vs. 55+ year old Caucasian females including VOCs from secondary list

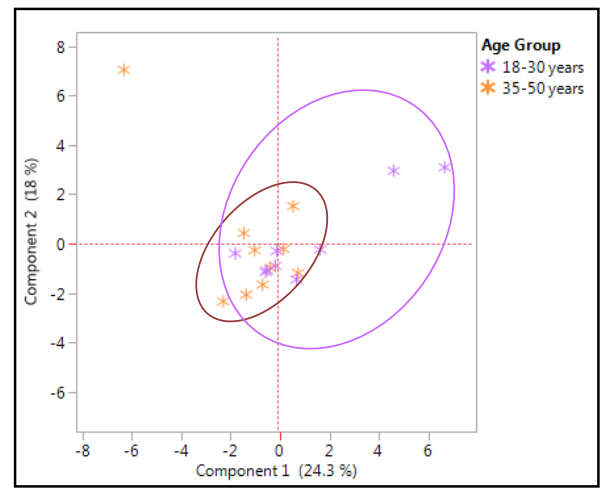

Figure 39: PCA score plot of 18-30 vs. 35-50 year old Caucasian females including all VOCs
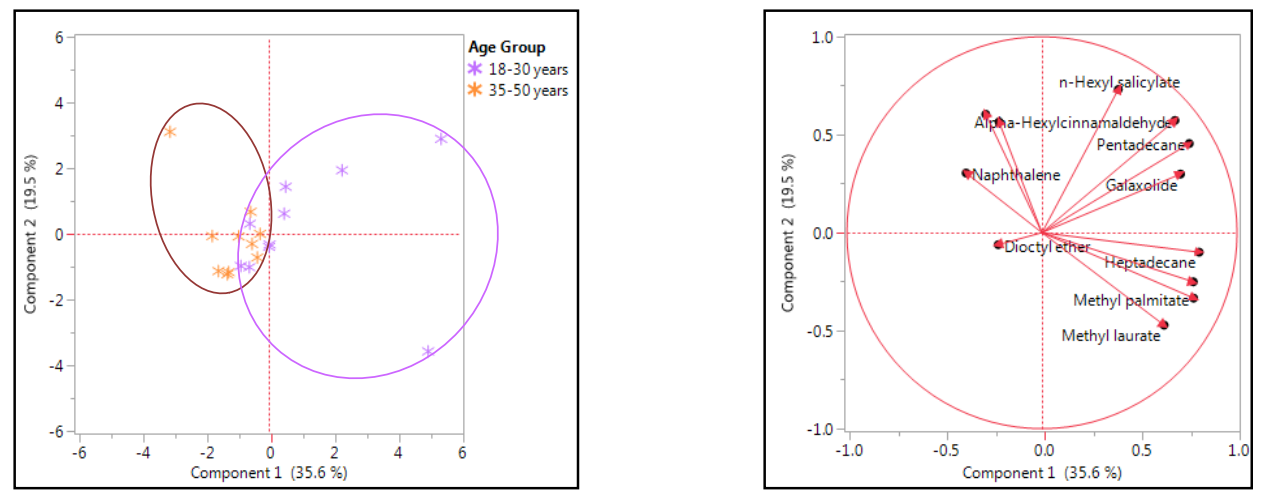

Figure 40: PCA score plot (left) and loading plot (right) of 18-30 vs. 35-50 year old Caucasian females including VOCs from secondary list 


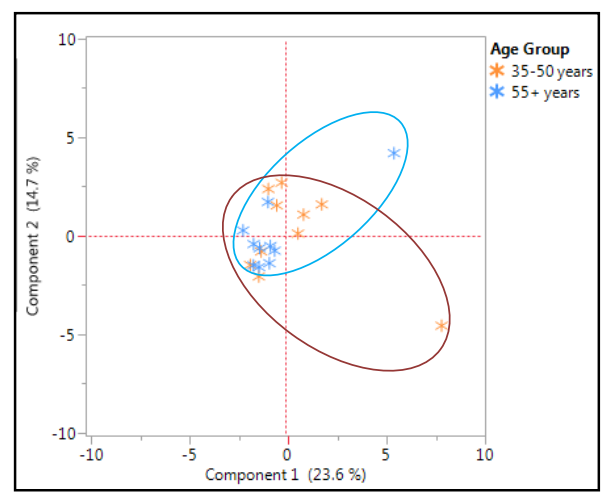

Figure 41: PCA score plot of 35-50 vs. 55+ year old Caucasian females including all VOCs
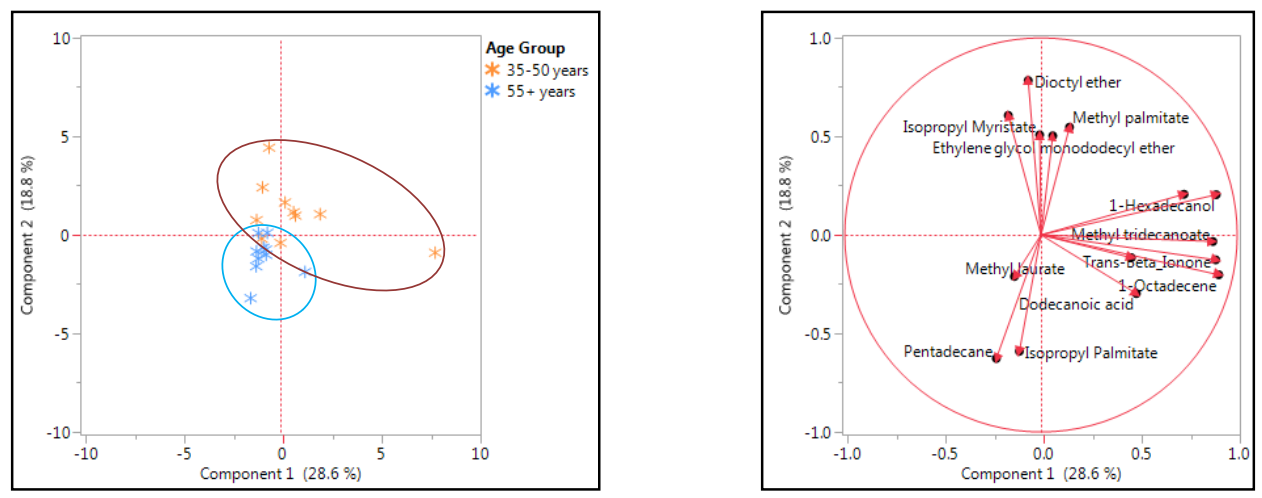

Figure 42: PCA score plot (left) and loading plot (right) of 35-50 vs. 55+ year old Caucasian females including VOCs from secondary list

Comparison of Males of Different Age Groups

In the same way it was done for Caucasian females, an evaluation of the average VOC amount extracted from male odor samples was performed. Out of the initial 28 compounds, males in the 18-30 year age group showed the highest average compound amount for seven VOCs. Males in the 35-50 year group showed the highest average amount in eight compounds, and males in the 55+ year category were the group with the highest average amount in the majority of the VOCs. It was observed that Hexyl salicylate and Alpha-Hexylcinnamaldehyde were two compounds for which Caucasian 
males showed to have an increase, in both occurrence and amount, with age. This observation contradicts the findings obtained in the analysis of Caucasian females, as well as previous reports in which these two compounds have been stated as being most abundant in younger subjects. ${ }^{21}$ According to Gallagher et al., high amounts of these exogenous compounds could be associated to a high cosmetic product usage. ${ }^{21}$ This in itself represents a reason for variation. Moreover, it may be an indication that males in the Caucasian population may be using less cosmetic products that contain these VOCs than females in the same population. On the other hand in the case of the average amount of Nonanal, compound established by Gallagher et al. as an age biomarker for older subjects, Caucasian males showed an increase in amount with age. ${ }^{21}$ The highest occurrence and highest average amount for Nonanal was observed in the group of 55+ year old males. In the same token, as it was noted for females, males showed a higher difference between the amounts of Nonanal in the youngest and oldest subjects, than in between those of the 35-50 and 55+ year age groups.

A selection of 14 compounds was made after having performed a visual evaluation and considering their amounts to be different enough, between the groups, to be evaluated as possible age markers in Caucasian males (Figure 43). Out of those 14 compounds, nine were found to be noticeably different between individuals in the 18-30 and the 55+ year categories, and included in the secondary list for these two groups. The 18-30 year vs. 35-50 year and the 35-50 year vs. 55+ year groups showed seven and ten noticeable differences, or secondary list VOCs, respectively (Table 16). For the most part, variation was noted in the secondary list VOCs identified for each of the different 
age group sets. Nevertheless, two compounds appeared to be consistently included in the secondary lists of all three age group sets: Dodecanoic acid and Naphthalene. 


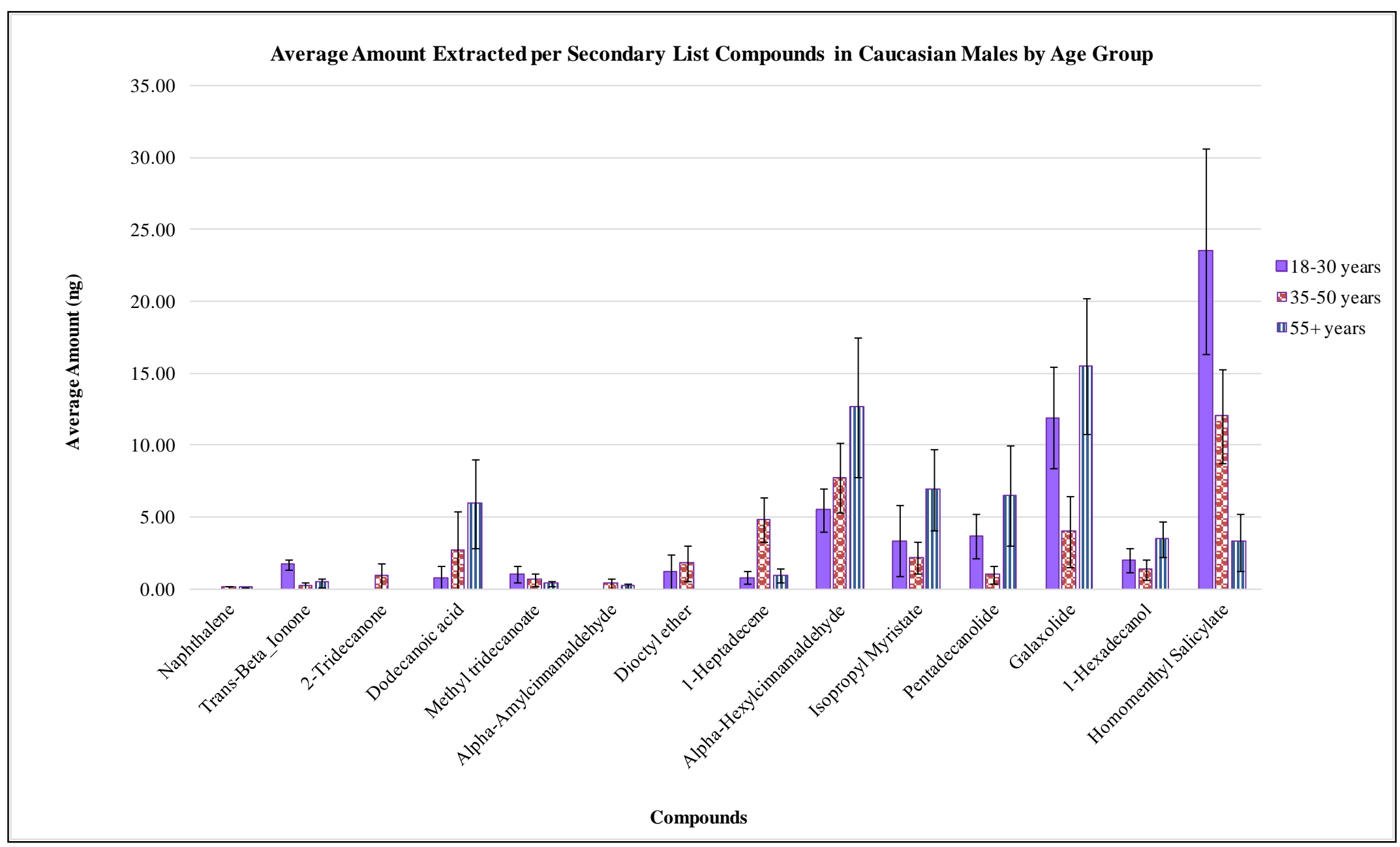

Figure 43: Comparison of average extracted amounts obtained for the VOCs considered in the secondary lists of Caucasian males from different age groups 
Table 16: Compounds considered in secondary lists made for comparing Caucasian males of different age groups

\begin{tabular}{|c|c|c|c|}
\hline $\begin{array}{c}\text { Compounds included in Secondary } \\
\text { Lists for Males }\end{array}$ & $\begin{array}{c}\mathbf{1 8 - 3 0} \text { vs. 35-50 } \\
\text { years }\end{array}$ & $\begin{array}{c}\text { 18-30 vs. 55+ } \\
\text { years }\end{array}$ & $\begin{array}{c}\text { 35-50 vs. 55+ } \\
\text { years }\end{array}$ \\
\hline Naphthalene & $\mathrm{X}$ & $\mathrm{X}$ & $\mathrm{X}$ \\
\hline Trans-Beta-Ionone & $\mathrm{X}$ & $\mathrm{X}$ & $\mathrm{X}$ \\
\hline 2-Tridecanone & $\mathrm{X}$ & $\mathrm{X}$ & $\mathrm{X}$ \\
\hline Dodecanoic acid & & $\mathrm{X}$ & \\
\hline Methyl tridecanoate & $\mathrm{X}$ & $\mathrm{X}$ & \\
\hline Alpha-Amylcinnamaldehyde & & $\mathrm{X}$ & $\mathrm{X}$ \\
\hline Dioctyl ether & $\mathrm{X}$ & & $\mathrm{X}$ \\
\hline 1-Heptadecene & & $\mathrm{X}$ & $\mathrm{X}$ \\
\hline Alpha-Hexylcinnamaldehyde & & & $\mathrm{X}$ \\
\hline Isopropyl Myristate & $\mathrm{X}$ & & $\mathrm{X}$ \\
\hline Pentadecanolide & $\mathrm{X}$ & $\mathrm{X}$ & $\mathrm{X}$ \\
\hline Galaxolide & & & \\
\hline 1-Hexadecanol & & & \\
\hline Homomenthyl Salicylate & & & \\
\hline
\end{tabular}

After performing PCA with the initial 28 compounds, males in the 18-30 year group and in the 55+ year groups were seen to equally spread out and overlap across all four quadrants of the score plot (Figure 44). Individuals in these two groups did not show a clear tendency to separate. However, when these two groups were evaluated under the influence of only the secondary list VOCs, a significant improvement was observed in group discrimination (Figure 45 - left). With the exception of one male from the 18-30 year age group, all other males grouped together with their corresponding age group. This allowed almost a complete separation and discrimination between males of these two age groups. In this comparison, the first score plot showed a variation percent of $20.7 \%$ in PC1 and 14\% in PC2; while the secondary list score plot showed an increase in variation to $23.4 \%$ and a $17.5 \%$, respectively. The increase in variation in the case of this comparison resulted in an efficient differentiation between the youngest and oldest age groups. These results suggest that the compounds selected to be part of the secondary list 
did serve as good discrimination criteria between the two groups. According to the loading plot obtained for this comparison (Figure 45 - right) the most influential compounds in the variation percent shown by PC1 were (in decreasing order): TransBeta-Ionone, Methyl tridecanoate, Dodecanoic acid, Alpha-Hexylcinnamaldehyde, and Dioctyl ether. On the other hand, those of most influence in PC2's variation were AlphaHexylcinnamaldehyde, Isopropyl Myristate, Homomenthyl Salicylate, Dioctyl ether, and Alpha-Amylcinnamaldehyde.

In the comparison of the 18-30 and the 35-50 year groups, the PCA score plot that was done including all variables did not show any separation between males of different age categories (Figure 46). All males appeared being spread out and overlapping in the score plot. Nevertheless, when only considering the secondary list VOCs selected for this age group set, it was noticed that the spread from the 18-30 year males was significantly reduced. This caused the formation of a tighter cluster for that age group. Males in the 35-50 year group continued to appear fairly spread out throughout the score plot, maintaining a partial overlap between the groups (Figure 47 - left). This partial overlap did not favor a total separation between these two age groups. However, if the majority of the males from each group were to be considered, it would probably still be possible to associate most subjects with their corresponding age group. The variation percentages obtained for PC1 and PC2, in the secondary list score plot, were higher than the percentages obtained for the initial score plot. The variation increased from $19.8 \%$ to 23.9\% in PC1 and $15.2 \%$ to $19.4 \%$ in PC2. Nevertheless, the fact that a total separation of the age groups was not achieved suggests that there is still room for improvement in the discrimination criteria being employed to classify males in these age groups. 
According to the loading plot (Figure 47 - right), the compounds of most influence to PC1's variation were 1-Heptadecene, Alpha-Amylcinnamaldehyde, Galaxolide, Pentadecanolide, and 2-Tridecanone (in decreasing order). On the other hand, those most influential to PC2 were Pentadecanolide, Naphthalene, Galaxolide, 2-Tridecanone, and Dodecanoic acid (also in decreasing order).

When comparing the 35-50 and 55+ year age groups, the results obtained were similar to those of the previous comparison (18-30 years vs. 35-50 years). The score plot including all variables showed no clear separation between the groups, but instead showed males from both groups spreading out without any tendency to cluster (Figure 48). On the other hand, when considering only the secondary list VOCs, a significant reduction was observed in the spread of all males in each group (Figure 49 - left). This resulted in an almost complete separation between the groups, although there were still some subjects from opposite groups that continued to overlap. Nevertheless, it remained fairly clear that both age groups had a tendency to be separated under the influence of the selected discrimination criteria. The variation percentages observed for PC1 and PC2 in the initial score plot were seen to increase in the secondary list score plot. In addition, it was noticed that, in the case of this comparison, PC2 had a larger increase in variation. This suggests that the impact of the variables in this component were a significant factor behind the evident changes in appearance of both clusters. According to the secondary list loading plot (Figure 49 - right), the most influential compounds in PC1 were 1Hexadecanol, Pentadecanolide, 1-Heptadecene, 2-Tridecanone, and Isopropyl Myristate. On the other hand, those of most influence in PC2's variation were Dioctyl ether, 2- 
Tridecanone, 1-Heptadecene, Galaxolide, and Pentadecanolide (both in decreasing order of influence).

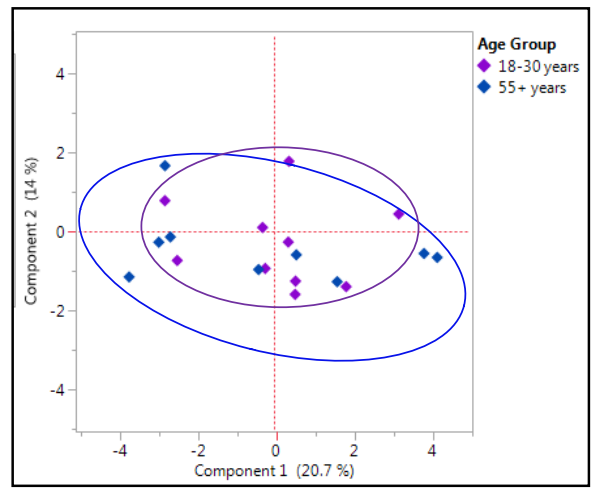

Figure 44: PCA score plot of 18-30 vs. 55+ year old Caucasian males including all VOCs
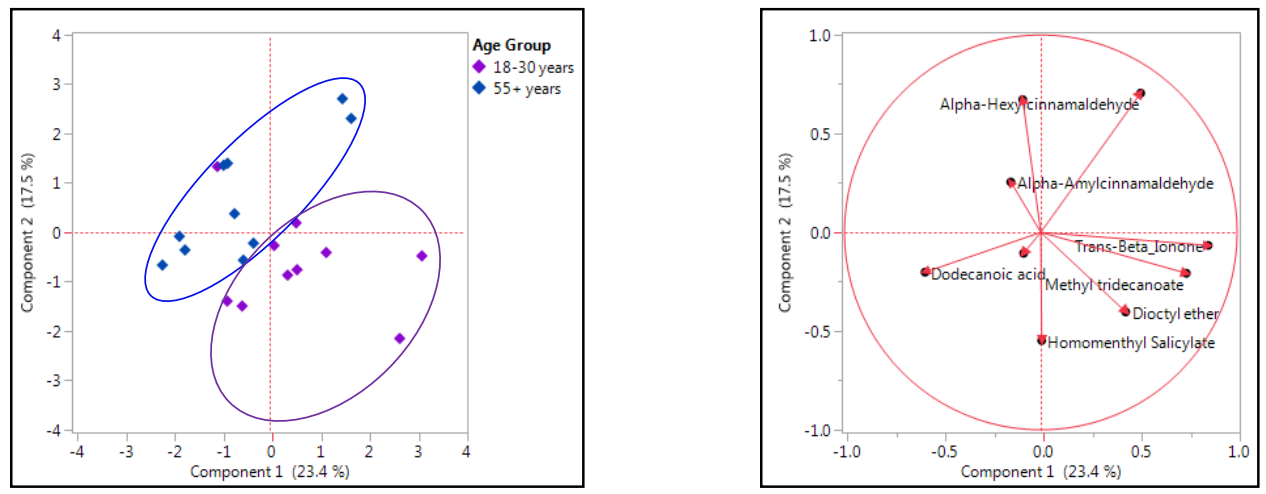

Figure 45: PCA score plot (left) and loading plot (right) of 18-30 vs. 55+ year old Caucasian males including VOCs from secondary list 


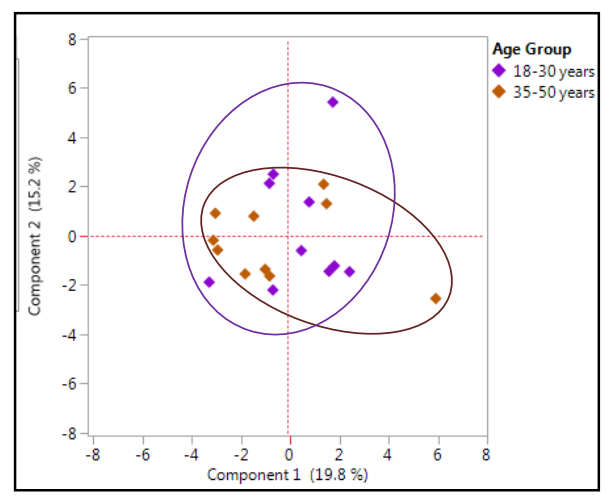

Figure 46: PCA score plot of 18-30 vs. 35-50 year old Caucasian males including all VOCs
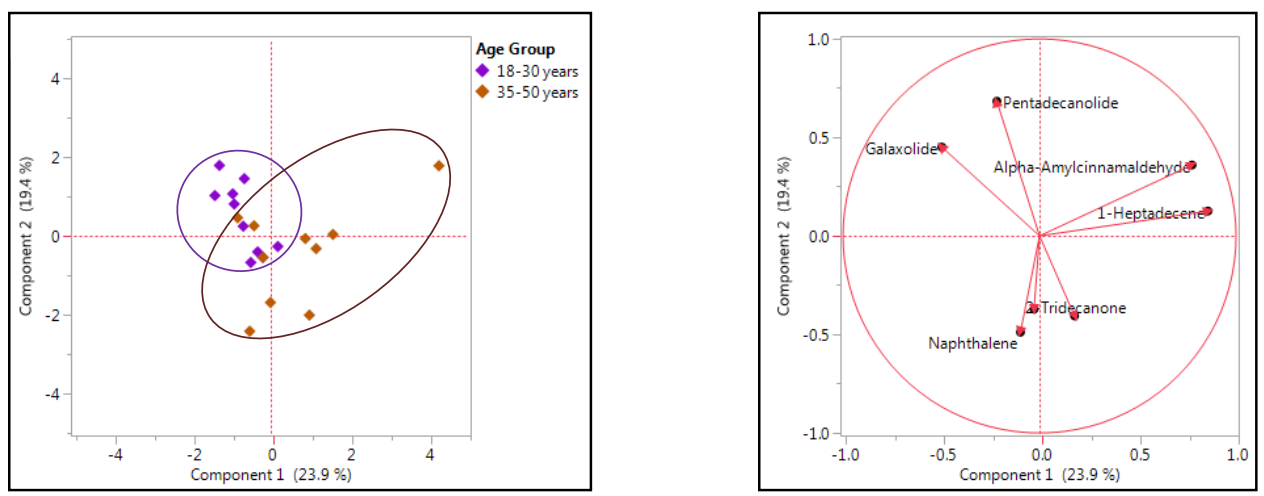

Figure 47: PCA score plot (left) and loading plot (right) of 18-30 vs. 35-50 year old Caucasian males including VOCs from secondary list

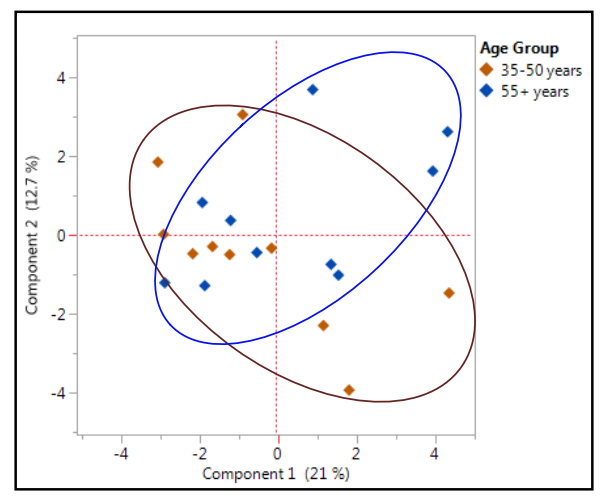

Figure 48: PCA score plot of 35-50 vs. 55+ year old Caucasian males including all VOCs 

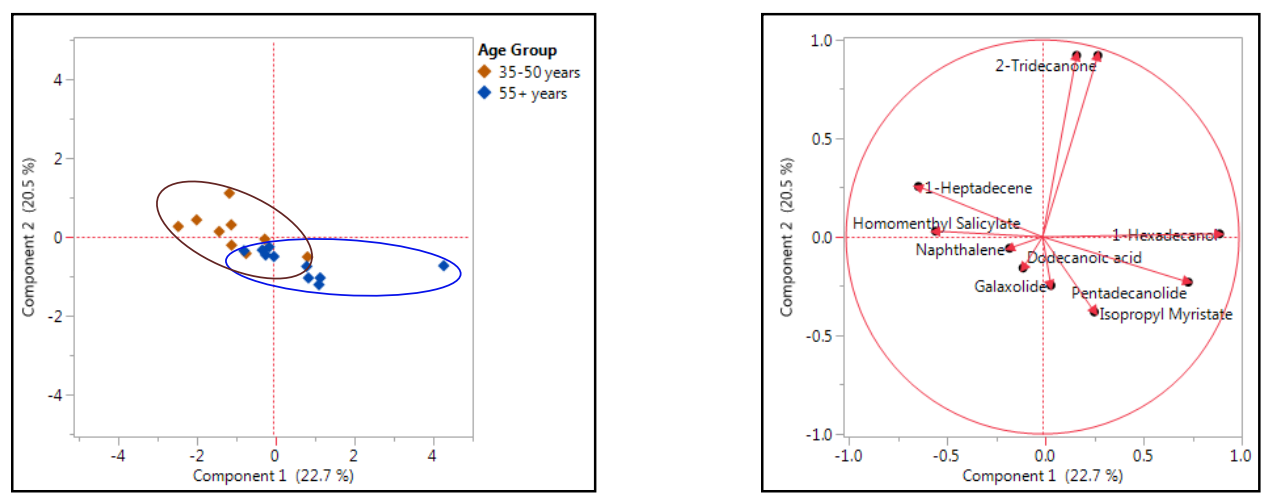

Figure 49: PCA score plot (left) and loading plot (right) of 35-50 vs. 55+ year old Caucasian males including VOCs from secondary list

\subsubsection{Results from Hispanic population}

\subsection{Qualitative Analysis}

The original VOC list employed as part of the data analysis for the Hispanic population consisted of a total of 30 VOCs. These compounds were considered for both the qualitative and quantitative analysis of the different gender and age groups (Table 17). The occurrence of all 30 VOCs was evaluated in all individuals, from each age group, by surveying the presence and absence of each compound in the samples collected from their right underarm. Odor profiles were constructed for each individual on the basis of the VOC occurrence reflected in their scent samples (Figure 50 - Figure 55). 


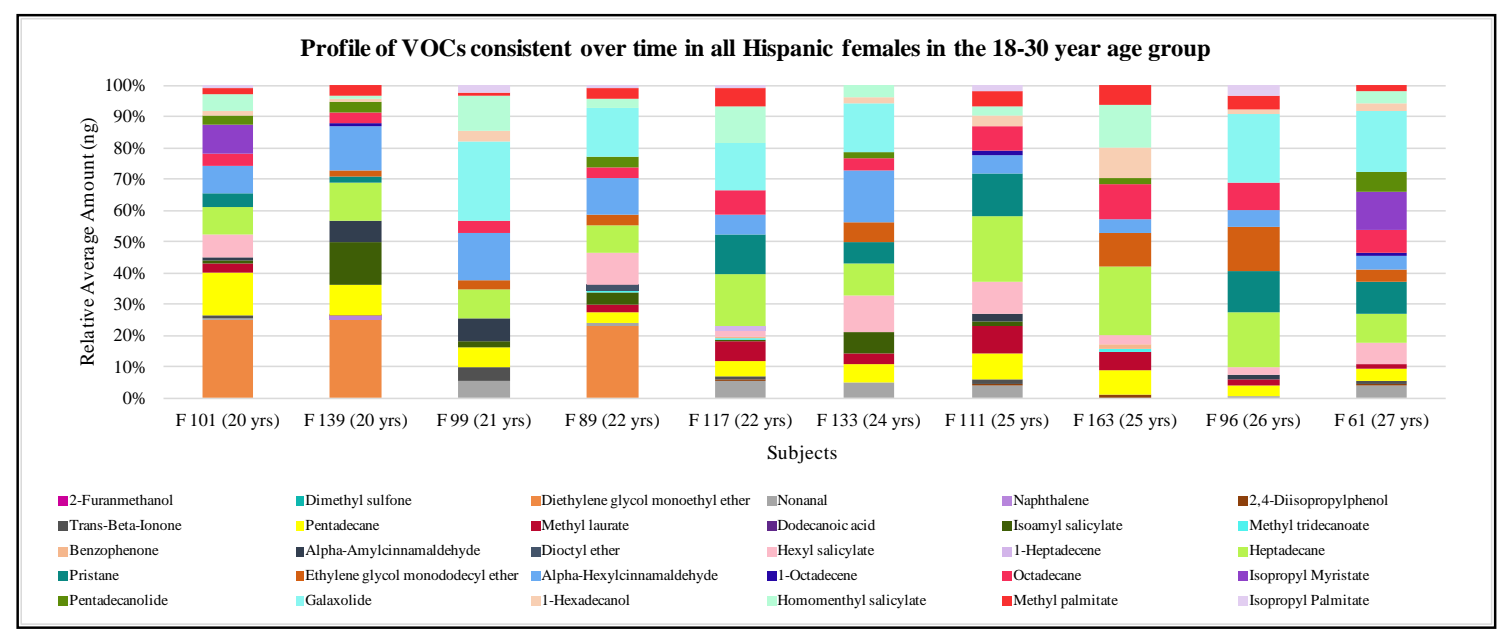

Figure 50: Scent profiles obtained for all 18-30 year old Hispanic females

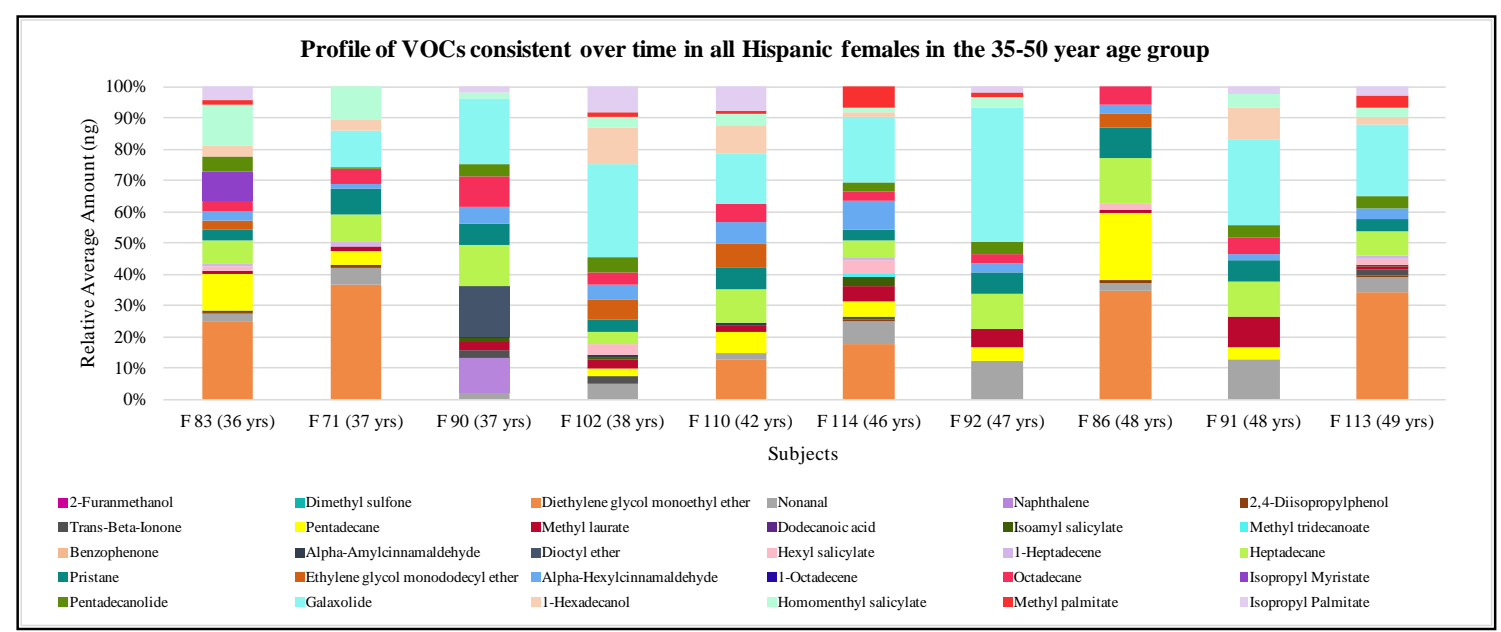

Figure 51: Scent profiles obtained for all 35-50 year old Hispanic females 


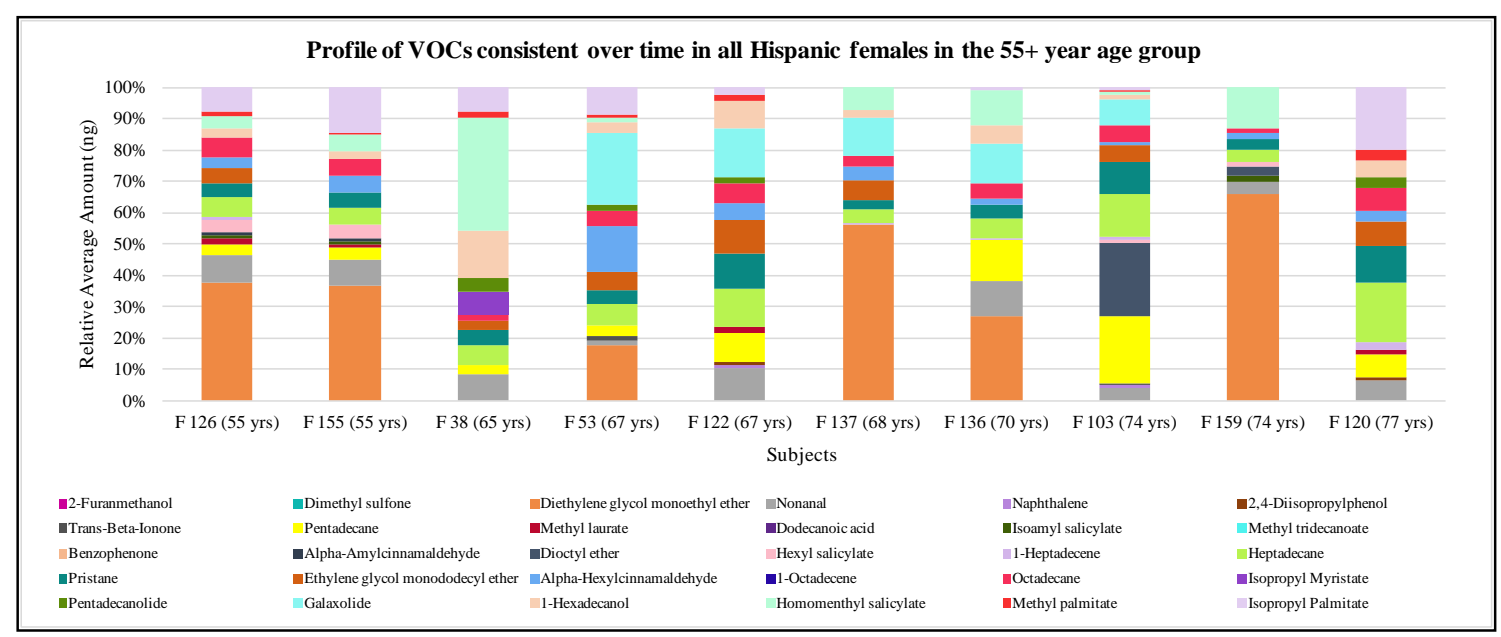

Figure 52: Scent profiles obtained for all 55+ year old Hispanic females

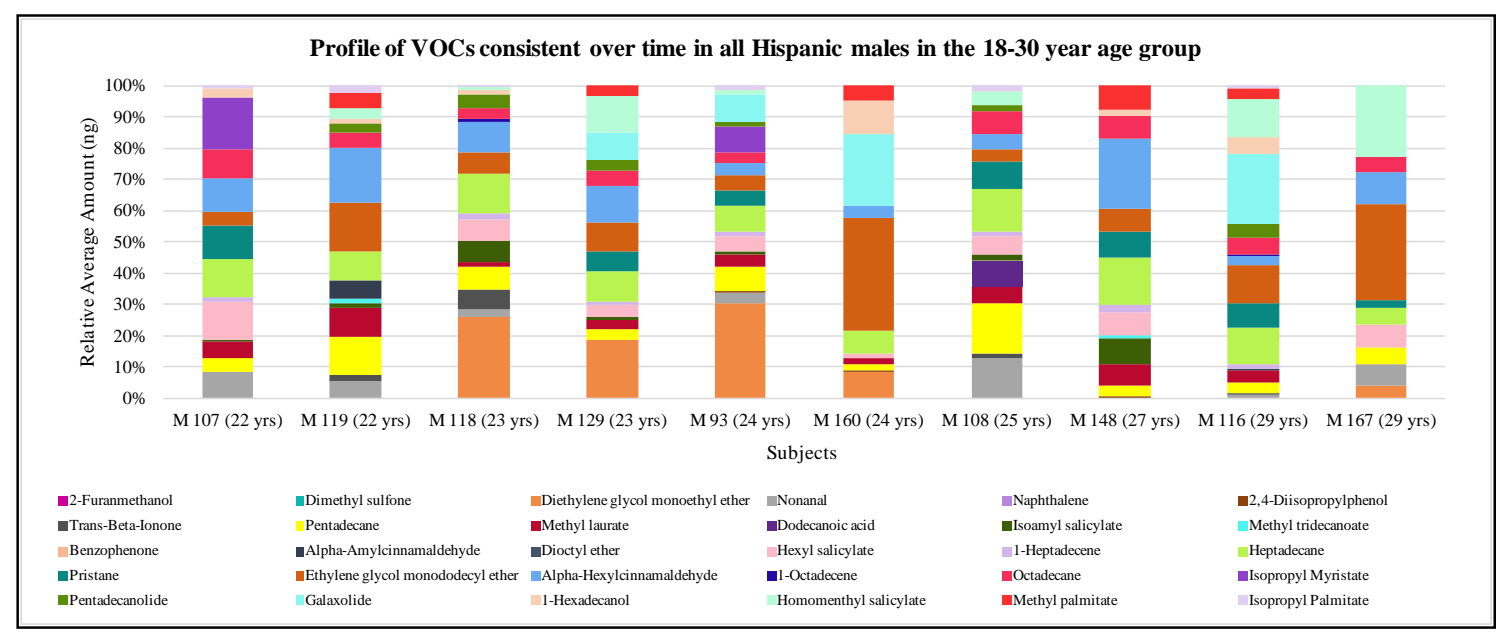

Figure 53: Scent profiles obtained for all 18-30 year old Hispanic males 


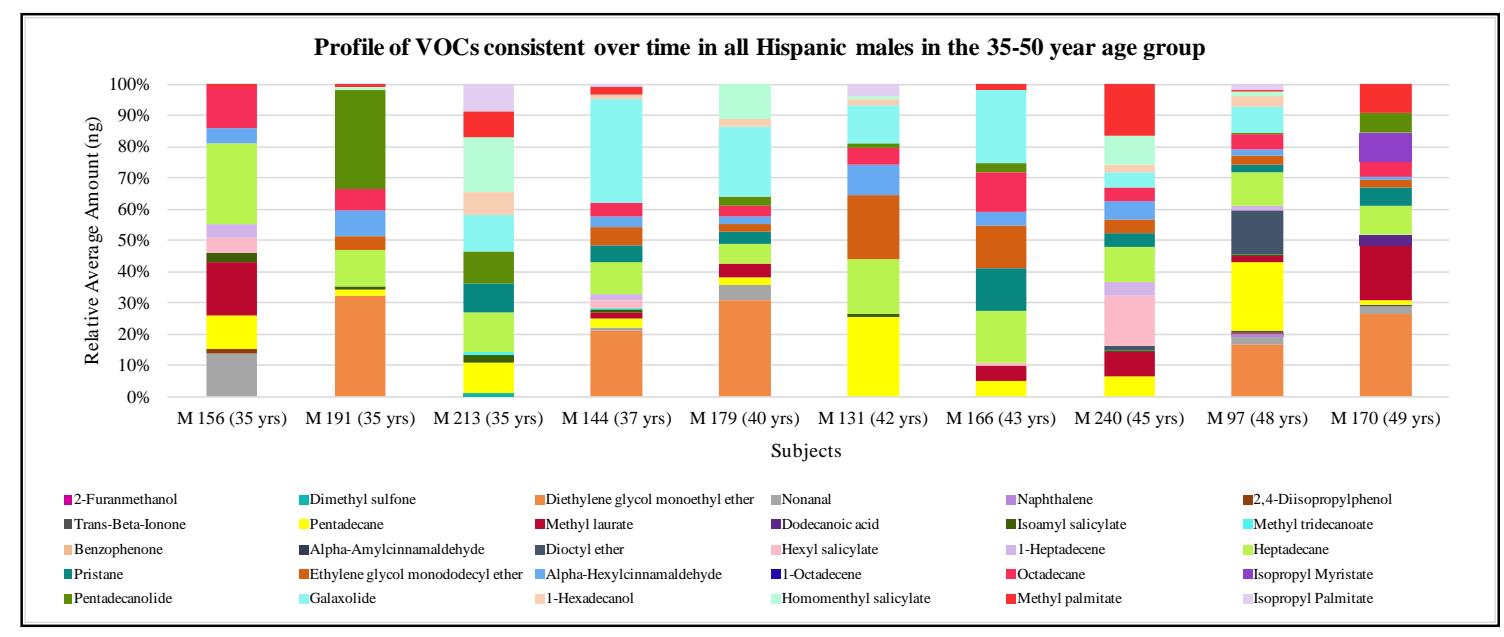

Figure 54: Scent profiles obtained for all 35-50 year old Hispanic males

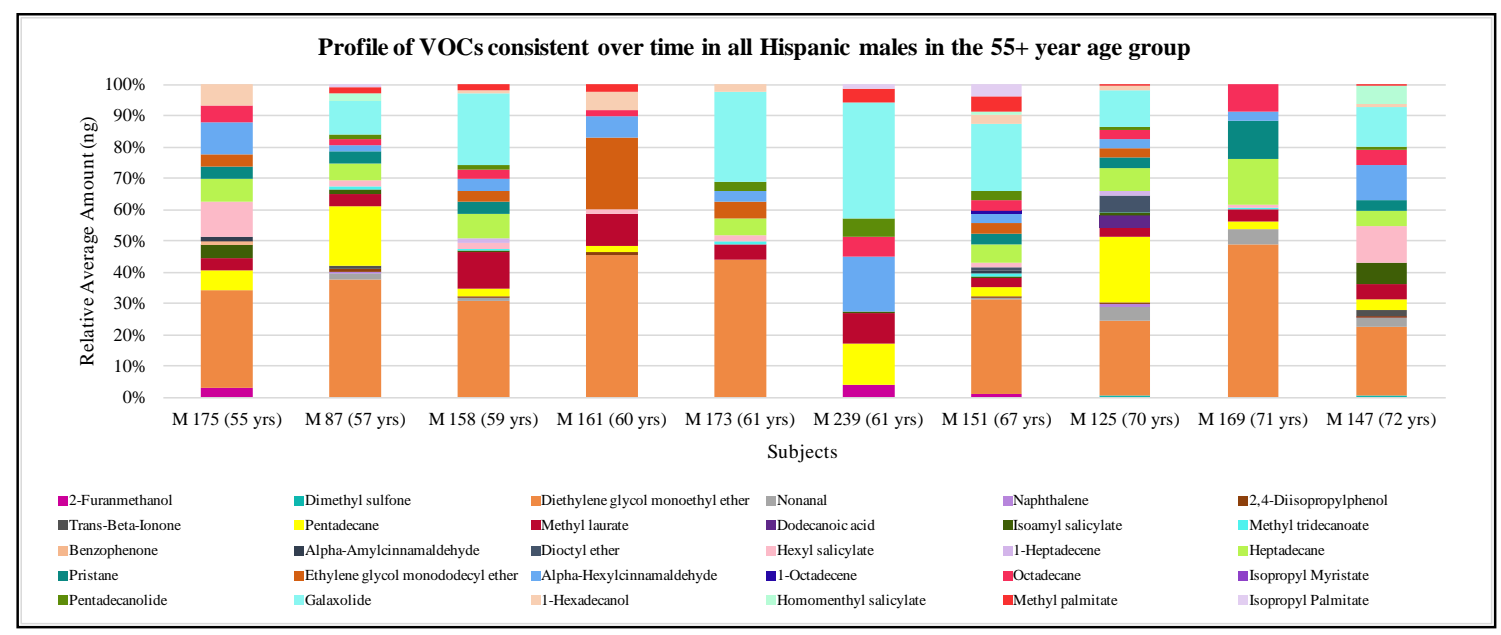

Figure 55: Scent profiles obtained for all 55+ year old Hispanic males

When evaluating the profiles of those individuals in the 18-30 year age group, it was noted that Alpha-Amylcinnamaldehyde occurred in 50\% of females, while it only occurred in $20 \%$ of the males (Table 17). On the other hand, 1-Heptadecene showed much higher occurrence in males than in females, showing a $70 \%$ versus a $10 \%$ of occurrence in females. It was observed that Naphthalene, Benzophenone, and Dioctyl ether were only found in female odor profiles for this age group, while Dodecanoic acid 
was only found in males. Moreover, 2-Furanmethanol and Dimethyl sulfone were not found in any subjects in the 18-30 year age group. Reports from Gallagher et al. had previously described Dimethyl sulfone as a biomarker for increased age. ${ }^{21}$ The study considered "increased age" to be 41-79 years old, which could be a reason for this compound not being observed in subjects of any gender within the 18-30 year category. Isopropyl palmitate had been previously reported by Penn et al. as a gender marker, after an evaluation of its relative abundance and detection frequency. ${ }^{37}$ In my study, Isopropyl palmitate displayed the same percent of occurrence for individuals of both genders. Nevertheless, females displayed, on average, a higher amount of this compound than males $($ females $=2.73 \mathrm{ng}$; males $=1.44 \mathrm{ng}$ ). This observation agrees with the results reported by Penn et al. in regards to Isopropyl palmitate’s abundance. ${ }^{37}$

The qualitative analysis of odor profiles from individuals in the 35-50 year age group, revealed that the rate of occurrence for Nonanal, 2,4-Diisopropylphenol, and Trans-Beta-Ionone in females was at least $100 \%$ or more that of males. On the contrary, the occurrence of Ethylene glycol monododecyl ether in males was observed to double that of females (Table 17). Some compounds, such as 2-Furanmethanol, Benzophenone and 1-Octadecene were not found in either gender for this age group. However, other compounds were seen exclusively in the odor profiles of females or males in this age group. Dimethyl sulfone and Dodecanoic acid were only found to occur in the odor profiles of males, while Alpha-Amylcinnamaldehyde was only observed in females. In the case of Isopropyl palmitate, a higher compound occurrence was noted in females (70\%) than in males (40\%). In the same token, the average amount extracted for this compound in females was $6.96 \mathrm{ng}$, while for males it was $1.88 \mathrm{ng}$. The fact that $35-50$ 
year old females showed a higher average amount of this compound, in addition to its increased occurrence in comparison to males, continues to support Penn et al.'s statement of this compound being a gender marker. ${ }^{37}$

In the 55+ year age group, 2-Furanmethanol, Dimethyl sulfone, Dodecanoic acid, Benzophenone, Methyl tridecanoate, and 1-Octadecene, were only found present in male odor profiles (Table 17). On the contrary, Isopropyl myristate was only found present in $10 \%$ of female odor profiles for this age group. Methyl laurate, 2,4-Diisopropylphenol, Isoamyl salicylate and Hexyl salicylate had a percent of occurrence in males of $100 \%$ or more as that obtained for females. Conversely, the percent of occurrence for Isopropyl palmitate in females was found to be more than twice as high as that of males. The fact that this VOC occurred more in female odor profiles than in males', and that its average amount extracted was higher (10.64 ng) in females than in males (1.28 ng), supports Pen et al.’s report on this compound as a marker for gender. ${ }^{37}$

Relevant information was also obtained after performing a qualitative analysis per gender group. In the case of females, it was observed that the occurrence of Isoamyl Salicylate, Methyl tridecanoate, Alpha-Amylcinnamaldehyde, and Hexyl salicylate tended to decrease with age (Table 17). However, the occurrence of Isopropyl palmitate and 1-Heptadecene appeared to consistently increase with age. Also, it was noted that Methyl tridecanoate was present in the odor profiles of females of all age groups, except those of the 55+ year old females. Similarly, Benzophenone and 1-Octadecene were only seen to occur in the profiles of 18-30 year old females. On the other hand, in the case of males, 1-Heptadecene, Ethylene glycol monododecyl ether, Homomenthyl salicylate, and Isopropyl Palmitate showed a decrease in their occurrence with age. In the case of this 
last compound, Hispanic males displayed the opposite of Hispanic females, who showed an increase in Isopropyl palmitate's occurrence with age. Nevertheless, it was observed that Diethylene glycol monoethyl ether consistently increased its occurrence with age in Hispanic males, and that 2-Furanmethanol and Benzophenone only occurred in the odor profiles of 55+ year old males.

Gallagher et al. had reported Dimethyl sulfone as a biomarker for increased age on the basis of its abundance in individuals older than 40 years. ${ }^{21}$ In my study, this compound did not occur in at least $50 \%$ of all males. However, the $10 \%$ of total occurrence that was noted was because of this compound's detection in the odor profiles of one individual of 35 years, another of 70, and another of 72 years. The fact that one of the individuals with Dimethyl sulfone in the odor profile was 35 years old, disagrees with Gallagher et al.’s report. My finding suggests that there could be variations in terms of the minimum age for which this particular compound could be used as a marker for increased age. Moreover, it is important to note that the study by Gallagher et al. did not consider any Hispanic males among its subjects, and evaluated scent samples from different body regions (e.g., back and forearms) to those considered in my study. These differences in experimental design may explain the observed discrepancy. Nevertheless, it is important to highlight that, in my study, Dimethyl sulfone was not observed in males from the youngest age group, and that this confirms the compound's relevance in odor profiles of individuals older than 18-30 years old. 
Table 17: VOCs emanating from Hispanic subjects' underarm odor samples detected and quantified by solid-phase microextraction GC/MS

\begin{tabular}{|c|c|c|c|c|c|c|c|c|}
\hline \multirow{4}{*}{ CAS no. } & \multirow{4}{*}{$\mathrm{RT}(\min )$} & \multirow{4}{*}{ Compound Name } & \multicolumn{6}{|c|}{ Frequency of Occurrence in Subjects } \\
\hline & & & \multicolumn{3}{|c|}{ Females $(n=30)$} & \multicolumn{3}{|c|}{ Males $(n=30)$} \\
\hline & & & $18-30$ & $35-50$ & $55+$ & $18-30$ & $35-50$ & $55+$ \\
\hline & & & years & years & years & years & years & years \\
\hline $98-00-0$ & 7.842 & 2-Furanmethanol ${ }^{d}$ & $0 \%$ & $0 \%$ & $0 \%$ & $0 \%$ & $0 \%$ & $30 \%$ \\
\hline $67-71-0$ & 9.471 & Dimethyl sulfone $^{d}$ & $0 \%$ & $0 \%$ & $0 \%$ & $0 \%$ & $10 \%$ & $20 \%$ \\
\hline $111-90-0$ & 11.168 & Diethylene glycol monoethyl ether $^{\mathrm{c}}$ & $30 \%$ & $60 \%$ & $60 \%$ & $40 \%$ & $50 \%$ & $90 \%$ \\
\hline $124-19-6$ & 13.066 & Nonanal c, d & $80 \%$ & $100 \%$ & $90 \%$ & $70 \%$ & $50 \%$ & $60 \%$ \\
\hline $91-20-3$ & 14.883 & Naphthalene $^{\text {c, d, e }}$ & $10 \%$ & $10 \%$ & $20 \%$ & $0 \%$ & $10 \%$ & $30 \%$ \\
\hline 2934-05-6 & 18.800 & 2,4-Diisopropylphenol & $50 \%$ & $60 \%$ & $20 \%$ & $30 \%$ & $20 \%$ & $60 \%$ \\
\hline $79-77-6$ & 20.638 & Trans-Beta-Ionone $^{\text {c, e }}$ & $50 \%$ & $50 \%$ & $20 \%$ & $40 \%$ & $20 \%$ & $20 \%$ \\
\hline $629-62-9$ & 20.796 & Pentadecane $^{\text {c, e }}$ & $100 \%$ & $80 \%$ & $80 \%$ & $100 \%$ & $100 \%$ & $90 \%$ \\
\hline $111-82-0$ & 21.296 & Methyl laurate ${ }^{\mathrm{c}, \mathrm{d}}$ & $80 \%$ & $100 \%$ & $40 \%$ & $90 \%$ & $70 \%$ & $100 \%$ \\
\hline $143-07-7$ & 22.156 & Dodecanoic acid c, d, e & $0 \%$ & $0 \%$ & $0 \%$ & $10 \%$ & $10 \%$ & $10 \%$ \\
\hline $87-20-7$ & 22.331 & Isoamyl salicylate $^{\mathrm{c}, \mathrm{e}}$ & $70 \%$ & $40 \%$ & $30 \%$ & $70 \%$ & $70 \%$ & $70 \%$ \\
\hline $1731-88-0$ & 23.263 & Methyl tridecanoate $^{\mathrm{d}}$ & $30 \%$ & $10 \%$ & $0 \%$ & $20 \%$ & $20 \%$ & $40 \%$ \\
\hline $119-61-9$ & 23.503 & Benzophenone ${ }^{\text {c, d, e }}$ & $20 \%$ & $0 \%$ & $0 \%$ & $0 \%$ & $0 \%$ & $10 \%$ \\
\hline $122-40-7$ & 23.819 & Alpha-Amylcinnamaldehyde c, e & $50 \%$ & $30 \%$ & $20 \%$ & $20 \%$ & $0 \%$ & $20 \%$ \\
\hline $629-82-3$ & 24.037 & Dioctyl ether & $10 \%$ & $10 \%$ & $20 \%$ & $0 \%$ & $20 \%$ & $20 \%$ \\
\hline $6259-76-3$ & 24.383 & Hexyl salicylate $^{\mathrm{c}, \mathrm{d}}$ & $80 \%$ & $50 \%$ & $40 \%$ & $80 \%$ & $40 \%$ & $80 \%$ \\
\hline $6765-39-5$ & 24.537 & 1-Heptadecene ${ }^{\mathrm{d}}$ & $10 \%$ & $40 \%$ & $50 \%$ & $70 \%$ & $40 \%$ & $20 \%$ \\
\hline $629-78-7$ & 24.657 & Heptadecane $^{\text {c, e }}$ & $100 \%$ & $100 \%$ & $100 \%$ & $100 \%$ & $100 \%$ & $80 \%$ \\
\hline $1921-70-6$ & 24.764 & Pristane & $70 \%$ & $100 \%$ & $100 \%$ & $70 \%$ & $70 \%$ & $70 \%$ \\
\hline $4536-30-5$ & 24.965 & Ethylene glycol monododecyl ether & $70 \%$ & $40 \%$ & $70 \%$ & $90 \%$ & $80 \%$ & $60 \%$ \\
\hline $101-86-0$ & 25.521 & Alpha-Hexylcinnamaldehyde c, d, e & $100 \%$ & $100 \%$ & $90 \%$ & $100 \%$ & $90 \%$ & $100 \%$ \\
\hline $112-88-9$ & 26.102 & 1-Octadecene & $30 \%$ & $0 \%$ & $0 \%$ & $20 \%$ & $0 \%$ & $10 \%$ \\
\hline $593-45-3$ & 26.209 & Octadecane $^{\text {c }}$ & $100 \%$ & $90 \%$ & $100 \%$ & $90 \%$ & $90 \%$ & $90 \%$ \\
\hline
\end{tabular}




\begin{tabular}{|c|c|c|c|c|c|c|c|c|}
\hline \multirow{3}{*}{ CAS no. } & \multirow{3}{*}{$\mathrm{RT}(\min )$} & \multirow{3}{*}{ Compound Name } & \multicolumn{6}{|c|}{ Frequency of Occurrence in Subjects } \\
\hline & & & \multicolumn{3}{|c|}{ Females $(n=30)$} & \multicolumn{3}{|c|}{ Males $(n=30)$} \\
\hline & & & $\begin{array}{l}18-30 \\
\text { years }\end{array}$ & $\begin{array}{l}35-50 \\
\text { years }\end{array}$ & $\begin{array}{c}55^{+} \\
\text {years }\end{array}$ & $\begin{array}{l}18-30 \\
\text { years }\end{array}$ & $\begin{array}{l}35-50 \\
\text { years }\end{array}$ & $\begin{array}{c}55^{+} \\
\text {years }\end{array}$ \\
\hline $110-27-0$ & 26.564 & Isopropyl Myristate $^{\mathrm{c}}$ & $20 \%$ & $10 \%$ & $10 \%$ & $20 \%$ & $10 \%$ & $0 \%$ \\
\hline $106-02-5$ & 26.808 & Pentadecanolide $^{\mathrm{C}}$ & $60 \%$ & $80 \%$ & $40 \%$ & $60 \%$ & $70 \%$ & $70 \%$ \\
\hline $1222-05-5$ & 27.068 & Galaxolide $^{\text {c, e }}$ & $50 \%$ & $80 \%$ & $50 \%$ & $40 \%$ & $70 \%$ & $70 \%$ \\
\hline $36653-82-4$ & 27.274 & 1-Hexadecanol c, d & $\mathbf{8 0 \%}$ & $70 \%$ & $90 \%$ & $60 \%$ & $60 \%$ & $70 \%$ \\
\hline 52253-93-7 & 27.475 & Homomenthyl salicylate & $90 \%$ & $90 \%$ & $80 \%$ & $70 \%$ & $60 \%$ & $30 \%$ \\
\hline $112-39-0$ & 27.838 & Methyl palmitate ${ }^{\mathrm{c}, \mathrm{d}}$ & $90 \%$ & $60 \%$ & $70 \%$ & $50 \%$ & $80 \%$ & $70 \%$ \\
\hline $142-91-6$ & 28.821 & Isopropyl Palmitate ${ }^{\mathrm{c}, \mathrm{d}}$ & $60 \%$ & $70 \%$ & $80 \%$ & $60 \%$ & $40 \%$ & $30 \%$ \\
\hline
\end{tabular}

a. $\mathrm{RT}=$ Retention Time

b. Compounds with a frequency of occurrence equal or higher to 50\% of subjects are denoted in bold

c. Compounds of possible exogenous origin - reported as being typically used as fragrance ingredients in consumer goods ${ }^{122}$

d. Reported by previous studies as present in human skin emanations, human VOC profiles, characteristic axillary odors or as individual axillary marker compounds ${ }^{21,33,36-38,123}$

e. Compounds were also quantified in unscented deodorant or soap blanks 


\subsection{Gender comparison: a quantitative analysis of VOC profiles by age group}

A gender comparison was performed for the Hispanic population, in the same way it was assessed for the Caucasian population. A quantitative analysis by age group was pursued to differentiate Hispanic females and males, within each group, using all 30 compounds as discrimination criteria (Figure 56 - Figure58). A visual evaluation of the average amount extracted, per VOC, for each gender was performed for all age categories. Subsequently, tailored versions of the initial compound list (secondary lists) were created for gender discrimination in each of the age categories. Both VOC lists were compared in regards to their potential to discriminate individuals of different gender within each age group. Ultimately, the main objective of this approach was to obtain a generalized profile for each gender in the Hispanic population. The generalized profile was meant to provide information on potential VOC biomarkers that could be useful in the classification of Hispanic individuals on the basis of gender. 


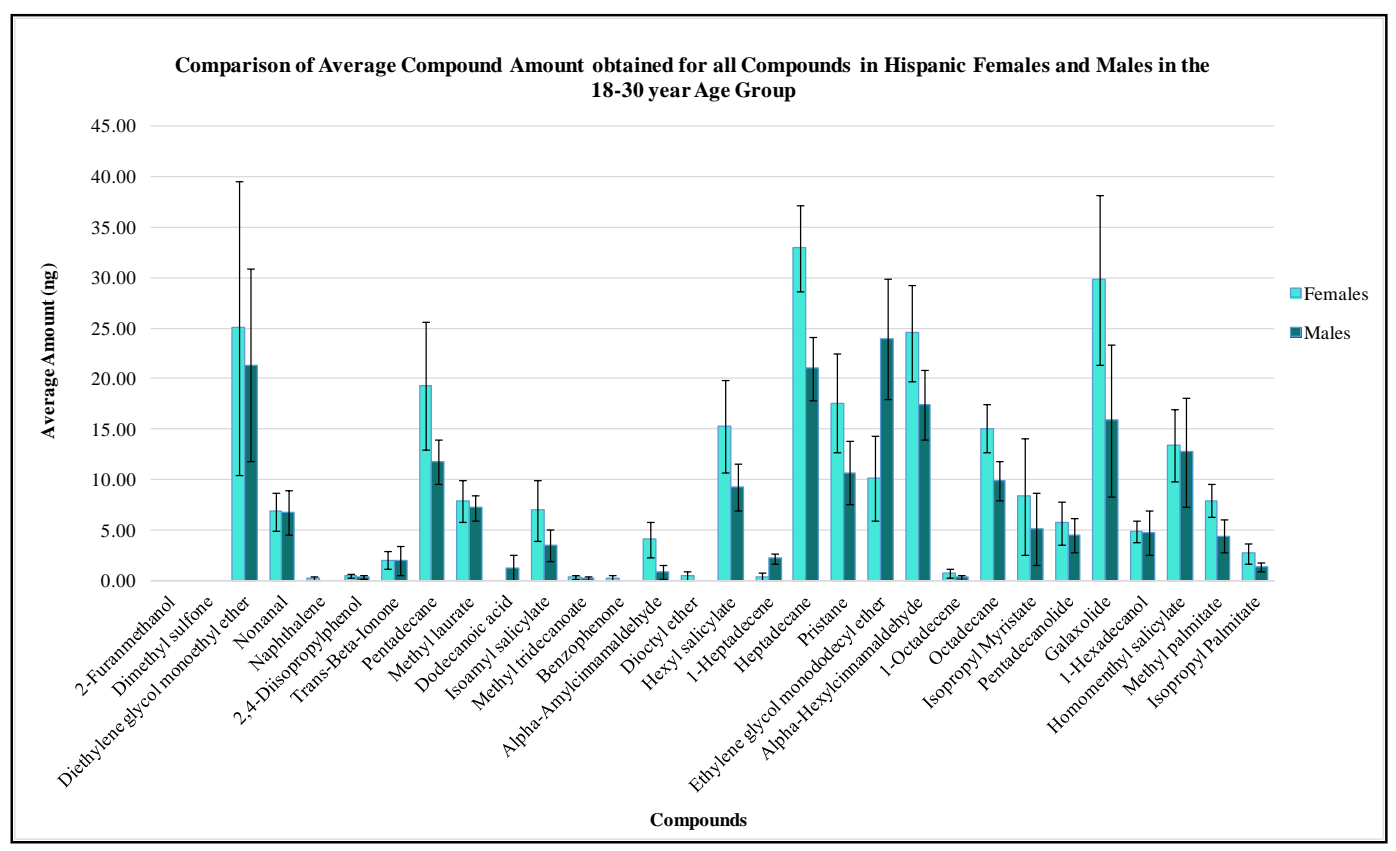

Figure 56: Comparison of the average extracted amounts obtained for all 30 VOCs in 18-30 year old Hispanic females and males

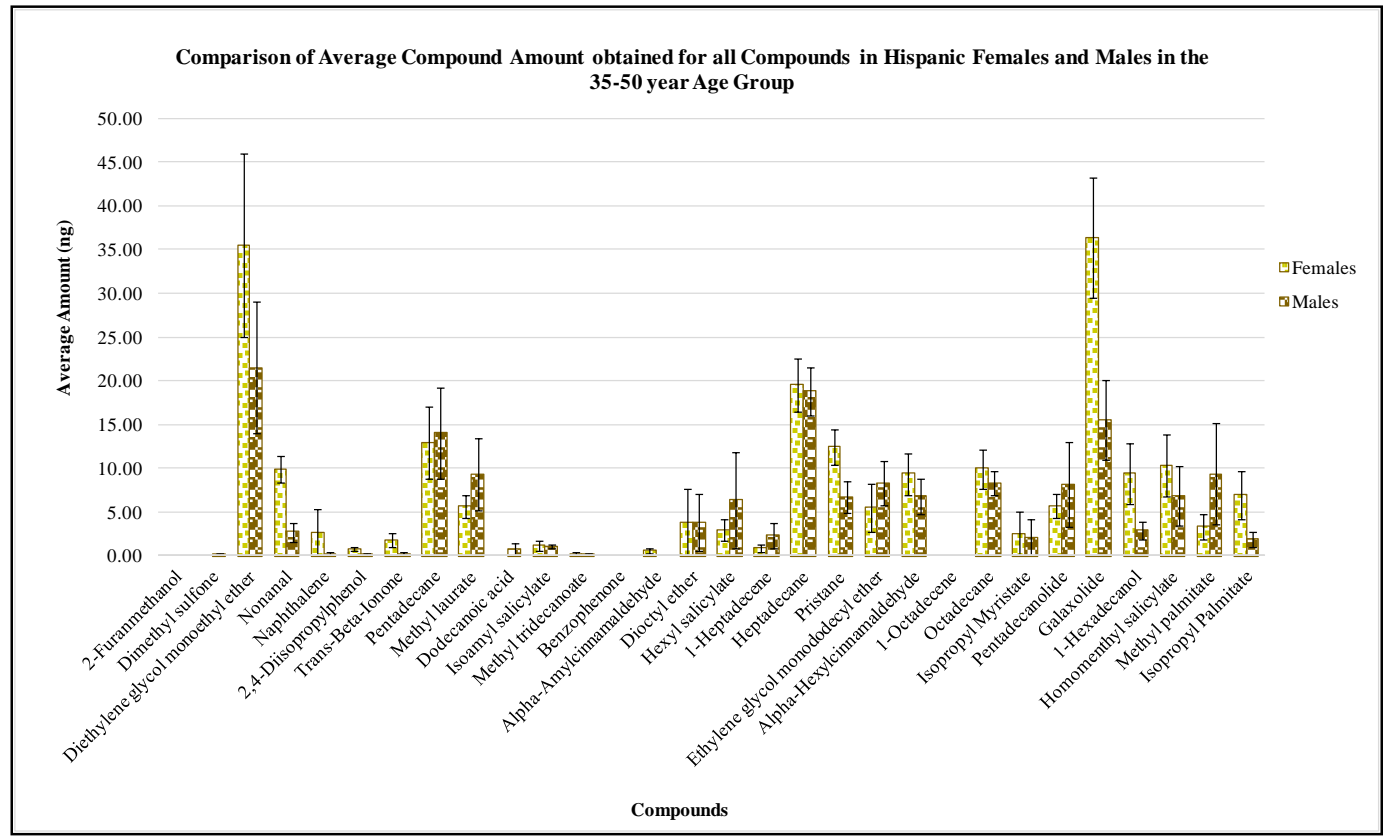

Figure 57: Comparison of the average extracted amounts obtained for all 30 VOCs in 35-50 year old Hispanic females and males 


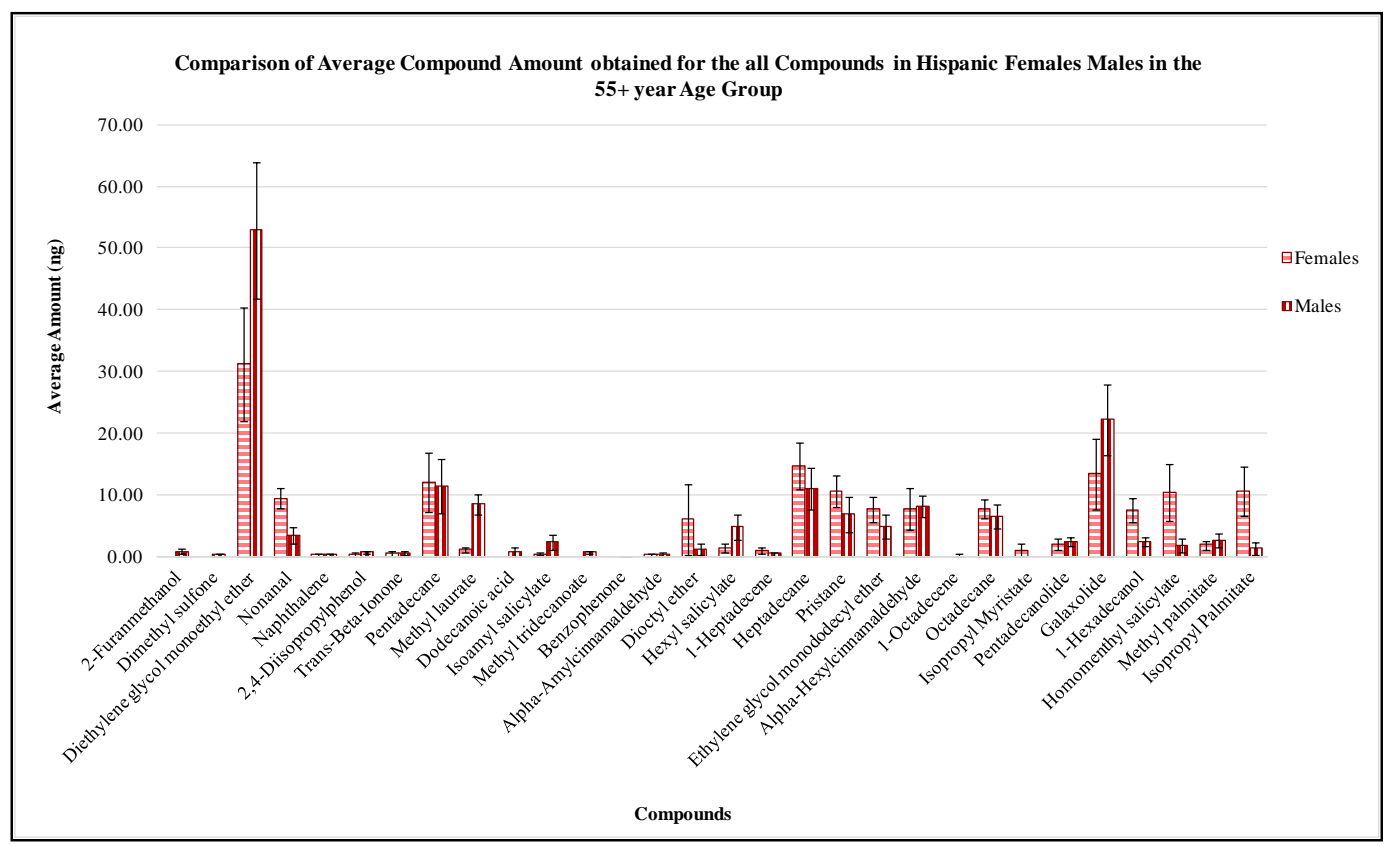

Figure 58: Comparison of the average extracted amounts obtained for all 30 VOCs in 55+ year old Hispanic females and males

\section{8-30 year Age Group}

Out of the initial list of 30 compounds, it was observed that only 10 compounds showed differences in occurrence, or in average amounts (100\% or more), between females and males of this category (Table 18). From this selection of compounds, seven showed higher average amounts in females and only three in males. In this case, as well as when considering all 30 VOCs, my study revealed that the majority of compounds presented higher amounts in female odor profiles than in males.

Principal component analysis was performed to study the VOCs’ power as criteria to distinguish individuals of different gender in the 18-30 year age group. The PCA score plot created for the evaluation of all 30 VOCs did not show any separation between individuals of different gender in this age group (Figure 59). Most samples from individuals of both genders were equally clustered towards the center of the score plot. 
On the other hand, when a score plot was made by only taking into consideration the secondary list compounds, a small improvement was achieved for gender discrimination (Figure 60 - left). In this case the first and second principal components (PC1 and PC2) accounted for $24.9 \%$ and $16.2 \%$ of the data's variation, respectively; while in the case of the initial score plot these only accounted for $18 \%$ and $13.2 \%$. Although clear clusters of individuals from each gender were not observed, differentiation between the groups was still slightly possible. The spread shown before by male subjects was reduced, causing a clearer separation between the groups. However, the fact that most female subjects still continued to be spread out, and some showed overlap with the male's cluster, did not allow a complete separation of the groups.

A loading plot was also created to determine which VOCs served as leading variables in the group differentiation of this last PCA score plot (Figure 60 - right). It was found that some of the most influential compounds in PC1's variation were Naphthalene, Isoamyl salicylate, Alpha-Amylcinnamaldehyde, 1-Octadecene and Ethylene glycol monododecyl ether (in decreasing order of influence). Moreover, the decreasing order of compounds with most influence in PC2 was as follows: 1-Heptadecene, Isopropyl Palmitate, Benzophenone, Alpha-Amylcinnamaldehyde and Ethylene glycol monododecyl ether.

\section{5-50 year Age Group}

From the initial list of 30 compounds, 15 compounds were selected as being different between males and females in the 35-50 year age group (Table 19). Out of those 15 VOCs, females showed a higher average amount than males in ten compounds. This 
shows that, for most VOCs in this age group, there was a tendency for females to have predominating abundances over males. This trend was also observed in the evaluation of gender groups for the 18-30 year category.

When performing PCA with all initial 30 compounds as differentiation variables, it was observed that the majority of females and males clustered together towards the center of the plot (Figure 61). The score plot obtained for this specific scenario showed a variation of $18.3 \%$ and $13.7 \%$ in PC1 and PC2, respectively. However, once only the compounds from the secondary list were taken into account, a significant enhancement was observed in the discrimination of both genders within this age group (Figure 62 left). In this case, the PCA score plot showed an increase in the variation of both principal components. PC1 showed a variation of $25.2 \%$, while PC2 showed a $19.2 \%$. Although a slight overlap was still observed towards the center of the score plot for some subjects of different gender, the changes observed in the spread and distribution of the subjects from each group, in response to the influence of the variables being considered, served for a clear and efficient differentiation between the groups.

The loading plot (Figure 62 - right) obtained for the second PCA score plot indicated that the compounds with heavier influence on PC1 were (in decreasing order) Alpha-Amylcinnamaldehyde, Isopropyl Palmitate, 1-Hexadecanol, Galaxolide and TransBeta-Ionone. On the other hand, it also revealed that the compounds responsible for the highest level of variation in PC2 were Hexyl salicylate, Methyl palmitate, 1-Heptadecene, Pristane and, as it was found in PC1, 1-Hexadecanol (also in decreasing order). 
55+ year Age Group

After having evaluated the data obtained for females and males in the $55+$ year age category, it was noted that, out of the initial 30 compounds, males showed a higher average amount for 17 compounds and females did for 13. A series of 16 compounds from this list were identified to be included in the secondary list, from which males showed to have the highest amount in nine of the compounds (Table 20). On the other hand, females only showed a higher average amount in seven of the compounds. This continued to demonstrate male predominance, in this age group, for VOC abundance in odor profiles. The tendency to higher VOC abundances in the odor profiles of 55+ year old males, instead of in 55+ year old females, was opposite to the observations made for the other two age groups. In the other two cases, females seemed to display higher amounts for most of the VOCs under consideration.

A PCA score plot was also created to assess the differentiation of 55+ year old females and males using all 30 compounds as variables. As part of this score plot it was possible to see that samples from both genders within this age group could not be clearly discriminated with such set of variables (Figure 63). The PCA score plot showed that only a $17.6 \%$ and $14.9 \%$ of variation was observed for PC1 and PC2, respectively. However, when employing the selection of 16 secondary list VOCs as the variable set, a clear separation between the genders was observed (Figure 64 - left). In this case PC1 showed a $23.2 \%$ of variation and PC2 a $15.9 \%$. This enhancement supports the fact that the compounds selected were able to serve as efficient discrimination criteria for the genders in this age group. Results obtained from the analysis of the loading plot (Figure 64 - right), generated along with the secondary list score plot, showed that the leading 
compounds in the variation observed in PC1 were 1-Hexadecanol, Isopropyl Myristate, Nonanal, Hexyl salicylate and Methyl laurate (in decreasing order). In the case of PC2, the decreasing order for most influential compounds was Dimethyl sulfone, Isoamyl salicylate, Hexyl salicylate, 2-Furanmethanol, and 1-Octadecene.

Table 18: Average VOC amount for secondary list compounds in 18-30 year old Hispanic females and males

\begin{tabular}{|c|c|c|}
\hline $\begin{array}{c}\text { Secondary List of Compounds for Males } \\
\text { and Females of 18-30 years }\end{array}$ & $\begin{array}{c}\text { Female Average } \\
\text { Compound } \\
\text { Amount (ng) }\end{array}$ & $\begin{array}{c}\text { Male Average } \\
\text { Compound } \\
\text { Amount (ng) }\end{array}$ \\
\hline Naphthalene & 0.25 & 0 \\
\hline Dodecanoic acid & 0 & 1.29 \\
\hline Isoamyl salicylate & 7.01 & 3.54 \\
\hline Benzophenone & 0.32 & 0 \\
\hline Alpha-Amylcinnamaldehyde & 4.15 & 0.91 \\
\hline Dioctyl ether & 0.50 & 0 \\
\hline 1-Heptadecene & 0.42 & 2.22 \\
\hline Ethylene glycol monododecyl ether & 10.19 & 23.94 \\
\hline 1-Octadecene & 0.75 & 0.38 \\
\hline Isopropyl Palmitate* & 2.73 & 1.44 \\
\hline
\end{tabular}

Table 19: Average VOC amount for secondary list compounds in 35-50 year old Hispanic females and males

\begin{tabular}{|c|c|c|}
\hline $\begin{array}{c}\text { Secondary List of Compounds for Males } \\
\text { and Females of 35-50 years }\end{array}$ & $\begin{array}{c}\text { Female Average } \\
\text { Compound } \\
\text { Amount (ng) }\end{array}$ & $\begin{array}{c}\text { Male Average } \\
\text { Compound } \\
\text { Amount (ng) }\end{array}$ \\
\hline Dimethyl sulfone & 0 & 0.12 \\
\hline Nonanal & 9.86 & 2.73 \\
\hline Naphthalene & 2.69 & 0.23 \\
\hline 2,4-Diisopropylphenol & 0.77 & 0.18 \\
\hline Trans-Beta-Ionone & 1.82 & 0.24 \\
\hline Dodecanoic acid & 0 & 0.74 \\
\hline Methyl tridecanoate* & 0.20 & 0.15 \\
\hline Alpha-Amylcinnamaldehyde & 0.57 & 0 \\
\hline Hexyl salicylate & 2.94 & 6.35 \\
\hline 1-Heptadecene & 0.87 & 2.37 \\
\hline Pristane* & 12.44 & 6.76 \\
\hline Galaxolide & 36.34 & 15.57 \\
\hline 1-Hexadecanol & 9.40 & 2.88 \\
\hline Methyl palmitate & 3.31 & 9.36 \\
\hline Isopropyl Palmitate & 6.96 & 1.88 \\
\hline
\end{tabular}


Table 20: Average VOC amount for secondary list compounds in 55+ year old Hispanic females and males

\begin{tabular}{|c|c|c|}
\hline $\begin{array}{c}\text { Secondary List of Compounds for Males } \\
\text { and Females of 55+ years }\end{array}$ & $\begin{array}{c}\text { Female Average } \\
\text { Compound } \\
\text { Amount (ng) }\end{array}$ & $\begin{array}{c}\text { Male Average } \\
\text { Compound } \\
\text { Amount (ng) }\end{array}$ \\
\hline 2-Furanmethanol & 0 & 0.79 \\
\hline Dimethyl sulfone & 0 & 0.23 \\
\hline Nonanal & 9.42 & 3.35 \\
\hline Methyl laurate & 1.07 & 8.43 \\
\hline Dodecanoic acid & 0 & 0.71 \\
\hline Isoamyl salicylate & 0.39 & 2.38 \\
\hline Methyl tridecanoate & 0 & 0.66 \\
\hline Benzophenone & 0 & 0.04 \\
\hline Dioctyl ether & 6.04 & 1.20 \\
\hline Hexyl salicylate & 1.39 & 4.75 \\
\hline 1-Heptadecene & 0.96 & 0.43 \\
\hline 1-Octadecene & 0 & 0.19 \\
\hline Isopropyl Myristate & 1.01 & 0 \\
\hline 1-Hexadecanol & 7.51 & 2.43 \\
\hline Homomenthyl salicylate & 10.43 & 1.83 \\
\hline Isopropyl Palmitate & 10.64 & 1.28 \\
\hline & & \\
\hline & & \\
\hline
\end{tabular}

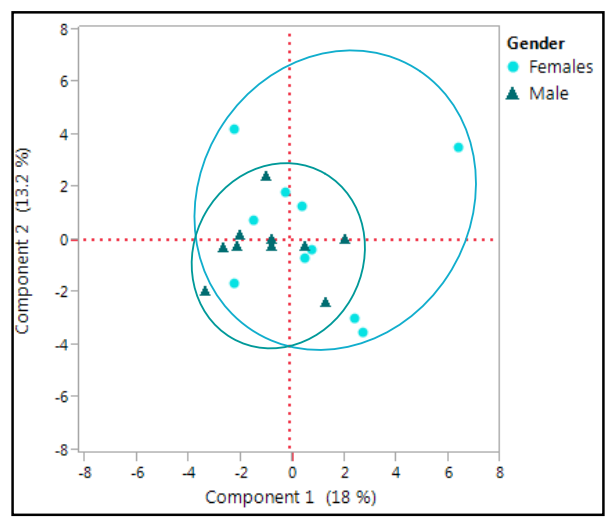

Figure 59: PCA score plot of 18-30 year old Hispanic females and males including all VOCs 

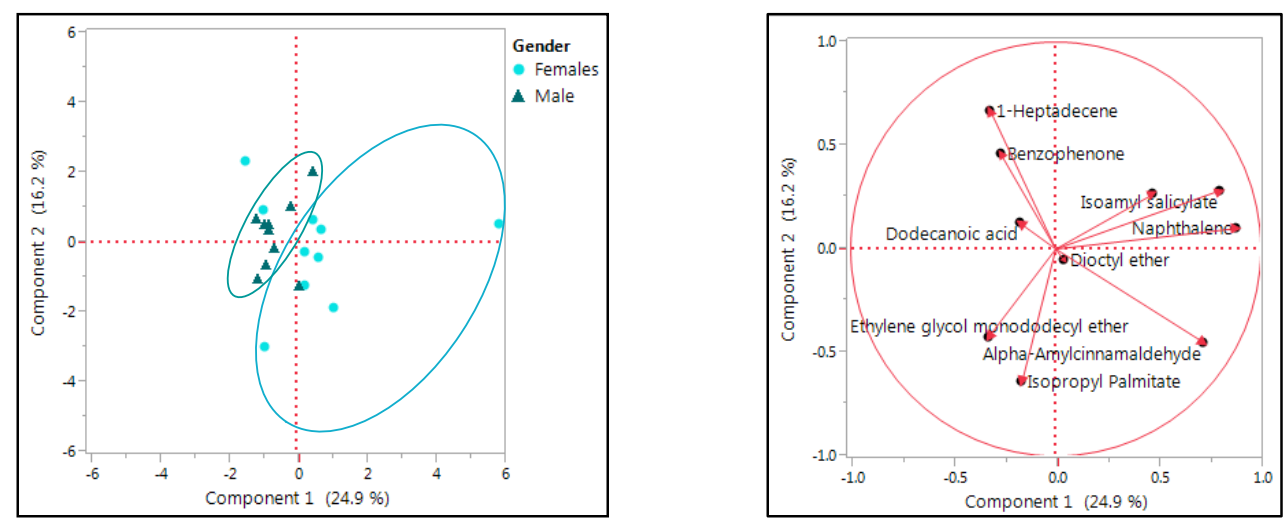

Figure 60: PCA score plot (left) and loading plot (right) of 18-30 year old Hispanic females and males including VOCs from secondary list

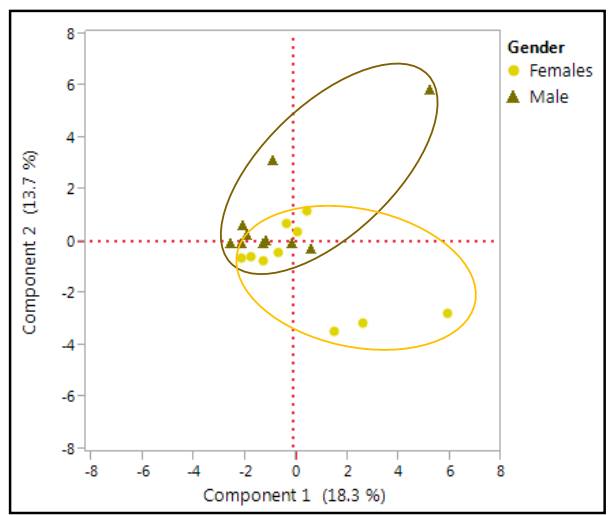

Figure 61: PCA score plot of 35-50 year old Hispanic females and males including all VOCs
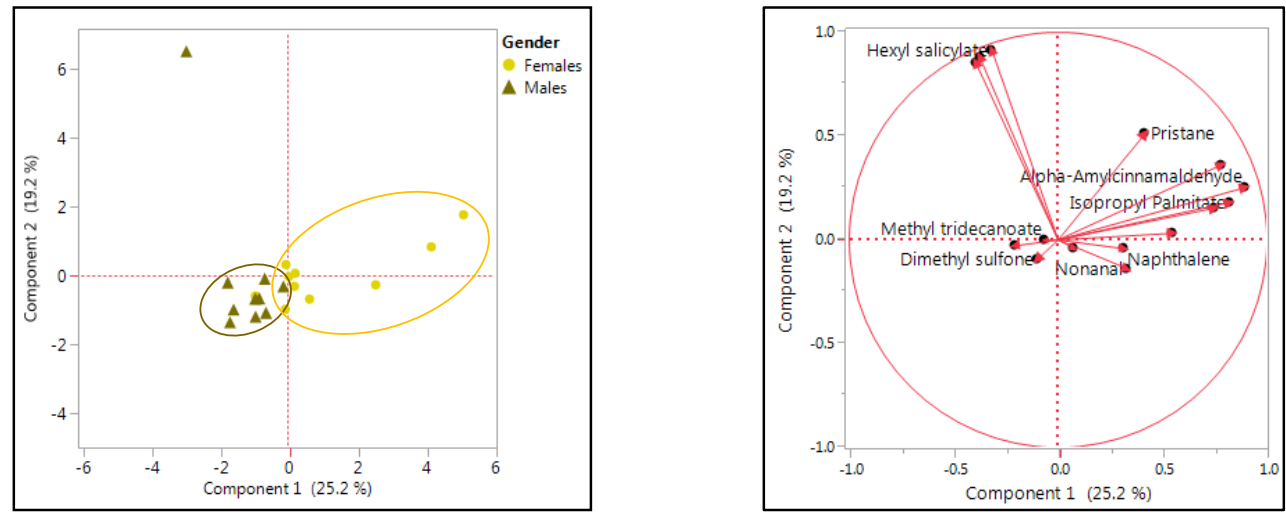

Figure 62: PCA score plot (left) and loading plot (right) of 35-50 year old Hispanic females and males including VOCs from secondary list 


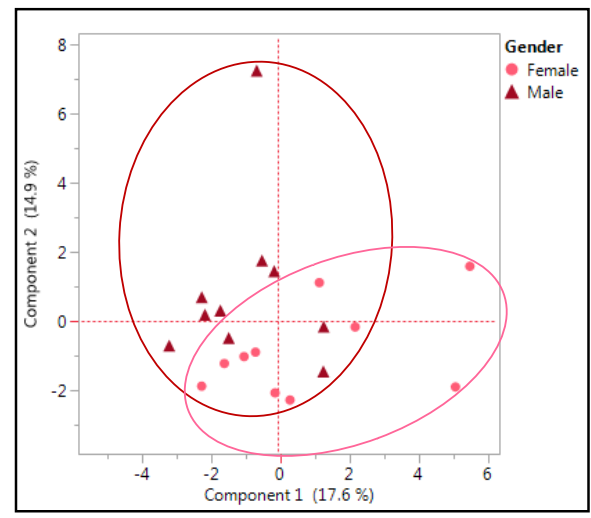

Figure 63: PCA score plot of 55+ year old Hispanic females and males including all VOCs
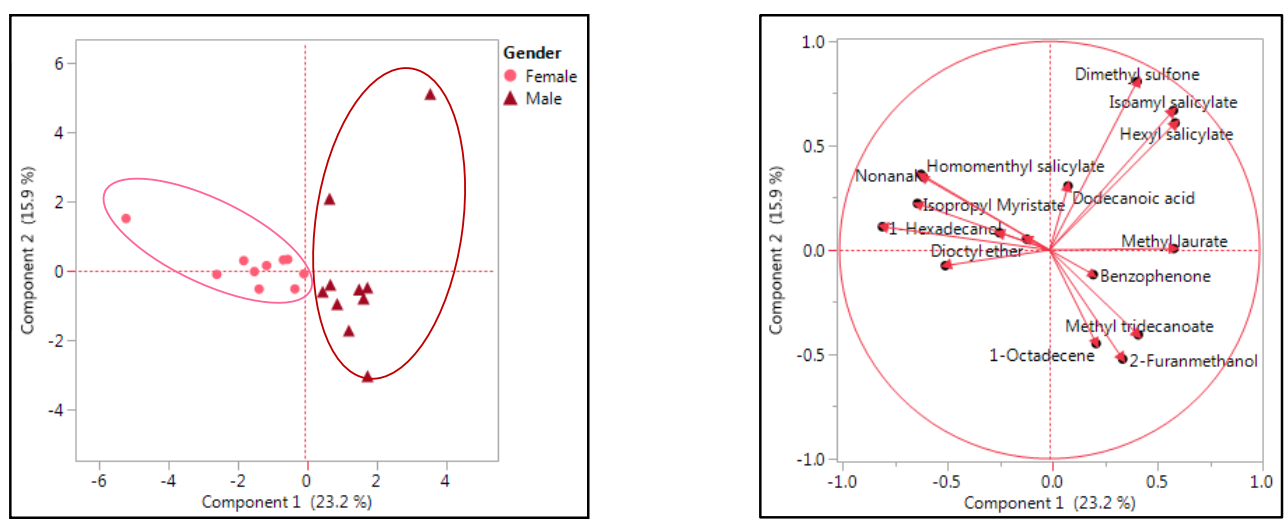

Figure 64: PCA score plot (left) and loading plot (right) of 55+ year old Hispanic females and males including VOCs from secondary list

\subsection{Age group comparison: a quantitative analysis of VOC profiles by gender}

An age group comparison was also performed for the Hispanic population. This was assessed by evaluating the average VOC amount extracted for each compound in each of the different age groups (Figure 65 - Figure 66) within each gender. A visual evaluation, of sets of two age groups at a time, was performed and secondary lists were created for age group discrimination in each gender. This comparison, allowed the identification of each VOCs impact in a subject's odor profile as a result of their age. 
Therefore, the approach facilitated the identification of those compounds that could serve as potential age biomarkers to classify Hispanic individuals of a same gender.

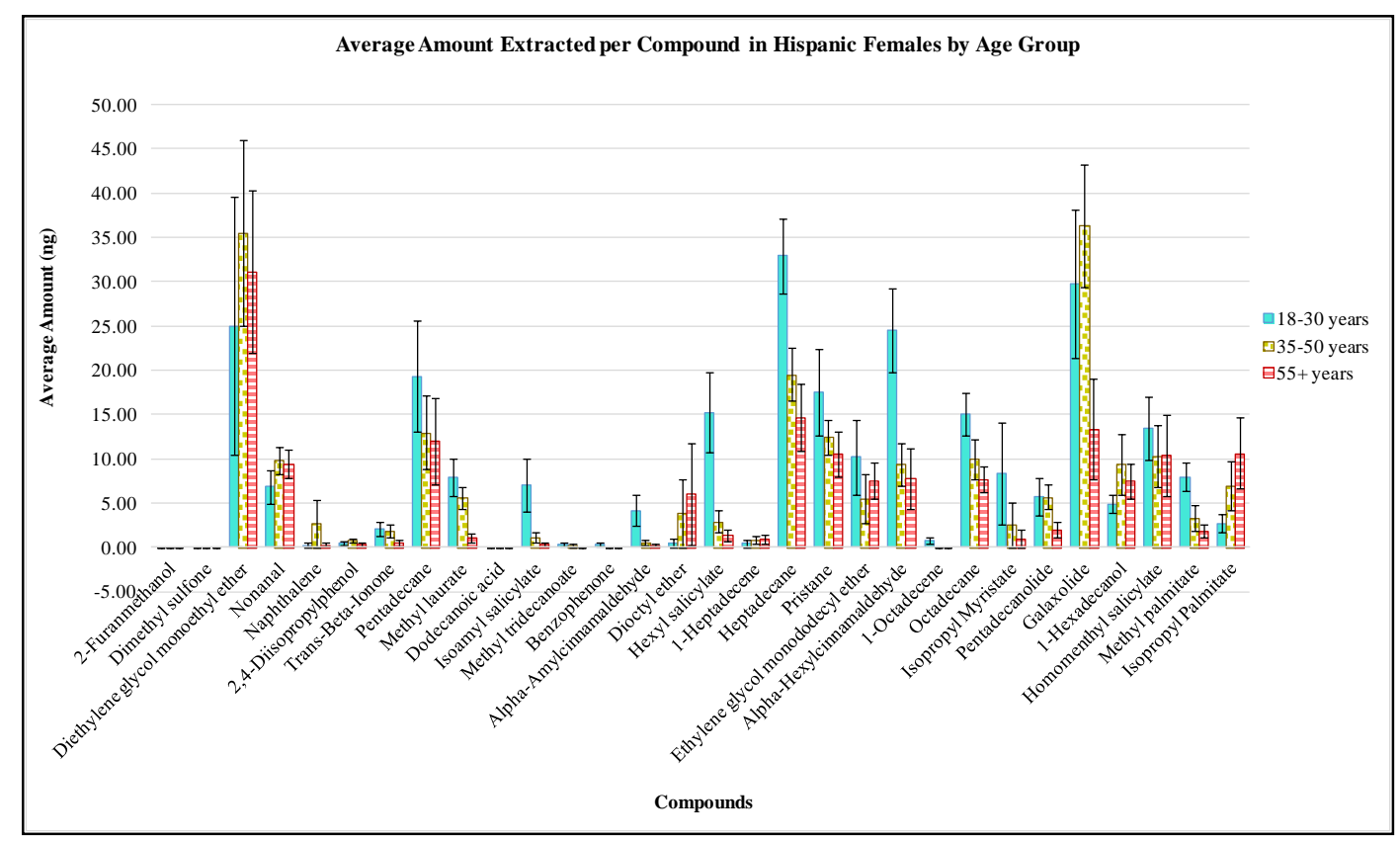

Figure 65: Comparison of the average extracted amounts obtained for all 30 VOCs in Hispanic females of all age groups 


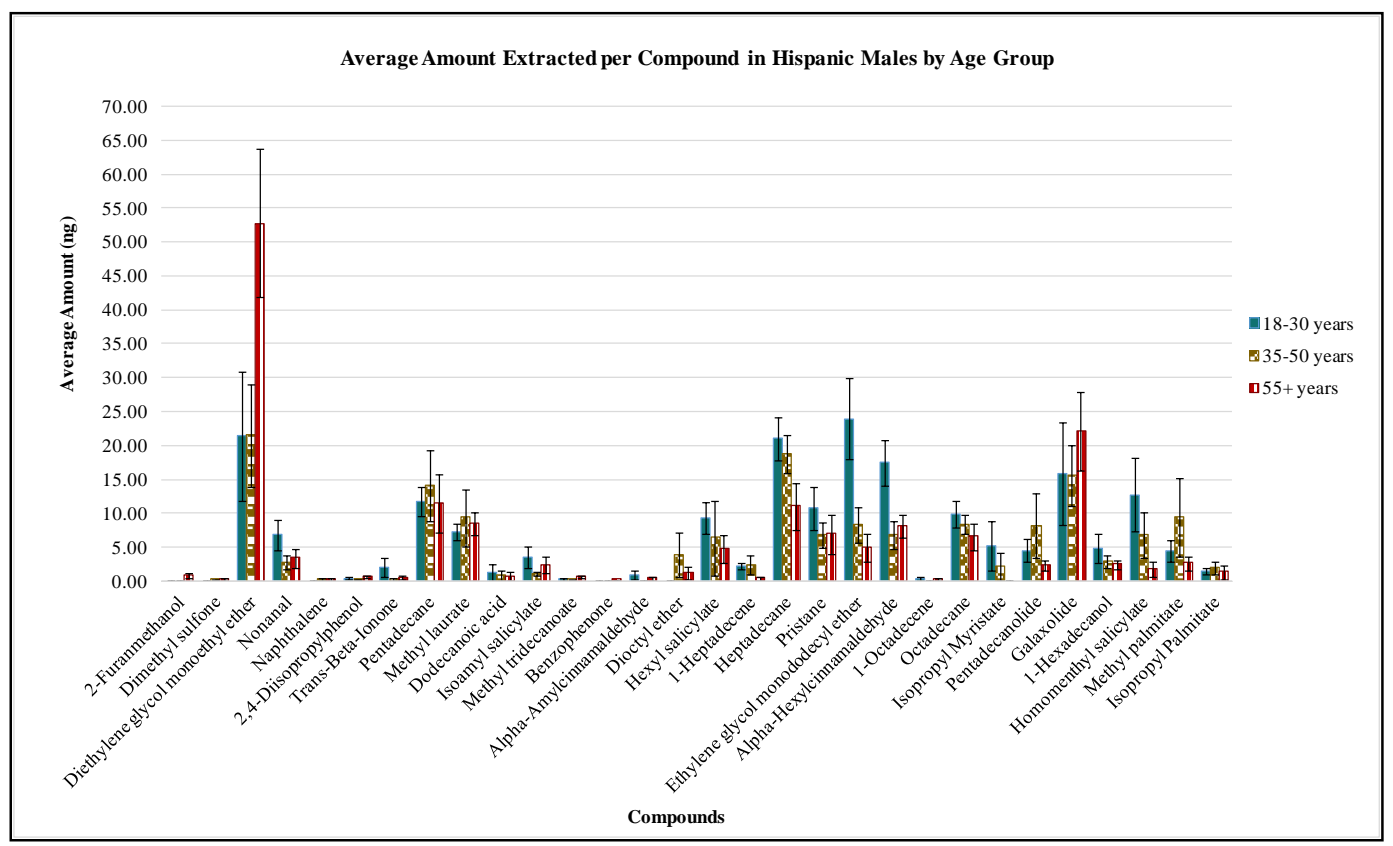

Figure 66: Comparison of the average extracted amounts obtained for all 30 VOCs in Hispanic males of all age groups

\section{Comparison of Females of Different Age Groups}

After comparing the average compound amounts obtained by females in each age group, it was noted that out of 30 compounds being considered, 18-30 year old females displayed the highest amount, out of the rest of the groups, for most compounds. The 3550 year old females had the highest average amount in the case of six compounds and 55+ year old females in only three compounds: Dioctyl ether, 1-Heptadecene and Isopropyl palmitate. According to Gallagher et al., who evaluated skin VOCs from the back and forearm of different individuals, Hexyl salicylate and AlphaHexylcinnamaldehyde are significantly more abundant in younger subjects (19-40 years). ${ }^{21}$ In the case of my study, these two compounds showed the greatest average amount in females that belonged to the 18-30 year age group. For both VOCs in this case, the average amounts were higher than twice of those obtained by females in the other two 
age groups. Also, it was noted that the average amount for these two compounds reduced with age. Therefore, all of these observations are consistent with Gallagher et al.'s previous findings. Dimethyl sulfone was not seen to occur in Hispanic female odor profiles. On the contrary, Nonanal, showed the lowest average amount in 18-30 year old females, while the 35-50 year and 55+ year olds showed almost the same amount (9.86 ng and 9.42 ng were reported respectively). Both of these last compounds had been previously reported as biomarkers for age on the basis of their increased abundance in older subjects (41-79 year olds). ${ }^{21}$ Therefore, in the case of Nonanal, these findings suggest that there is a possibility that the age limits for this compound to be considered a biomarker can be revised.

Following the visual evaluation, a total of 21 compounds were selected for the secondary lists to compare each female age group set (Figure 67). Compounds incorporated in the secondary list of each group set were evaluated for their potential as biomarkers for the age groups under study. Out of 21 compounds, 17 compounds were included in the secondary list for comparing the 18-30 year category and the 55+ year category. The secondary lists used to compare 18-30 year olds vs. 35-50 year olds, and 35-50 year olds vs. 55+ year olds, included ten compounds each (Table 21). Variation was noted in the compounds included in the secondary lists of all age group comparisons. Only four compounds out of 21 were consistently considered throughout the comparison of all age group sets: Isoamyl salicylate, Alpha-Amylcinnamaldehyde, Hexyl salicylate, and Isopropyl Myristate. This continues to suggest that, despite of the different group sets having VOCs in common as noticeable differences, the discrimination of these groups might be relying on a combination of compounds, instead of on a series of VOCs that 
portray particular differences in specific group sets. For 14 out of 21 compounds, females in the 18-30 year age group showed to have the highest average amount out of all groups. On the other hand, females in the 35-50 year age category showed the highest average amounts for Naphthalene, 2,4-Diisopropylphenol, Galaxolide and 1-Hexadecanol. Females in the 55+ year age group showed the highest values for Dioctyl ether, 1Heptadecene and Isopropyl Palmitate. 


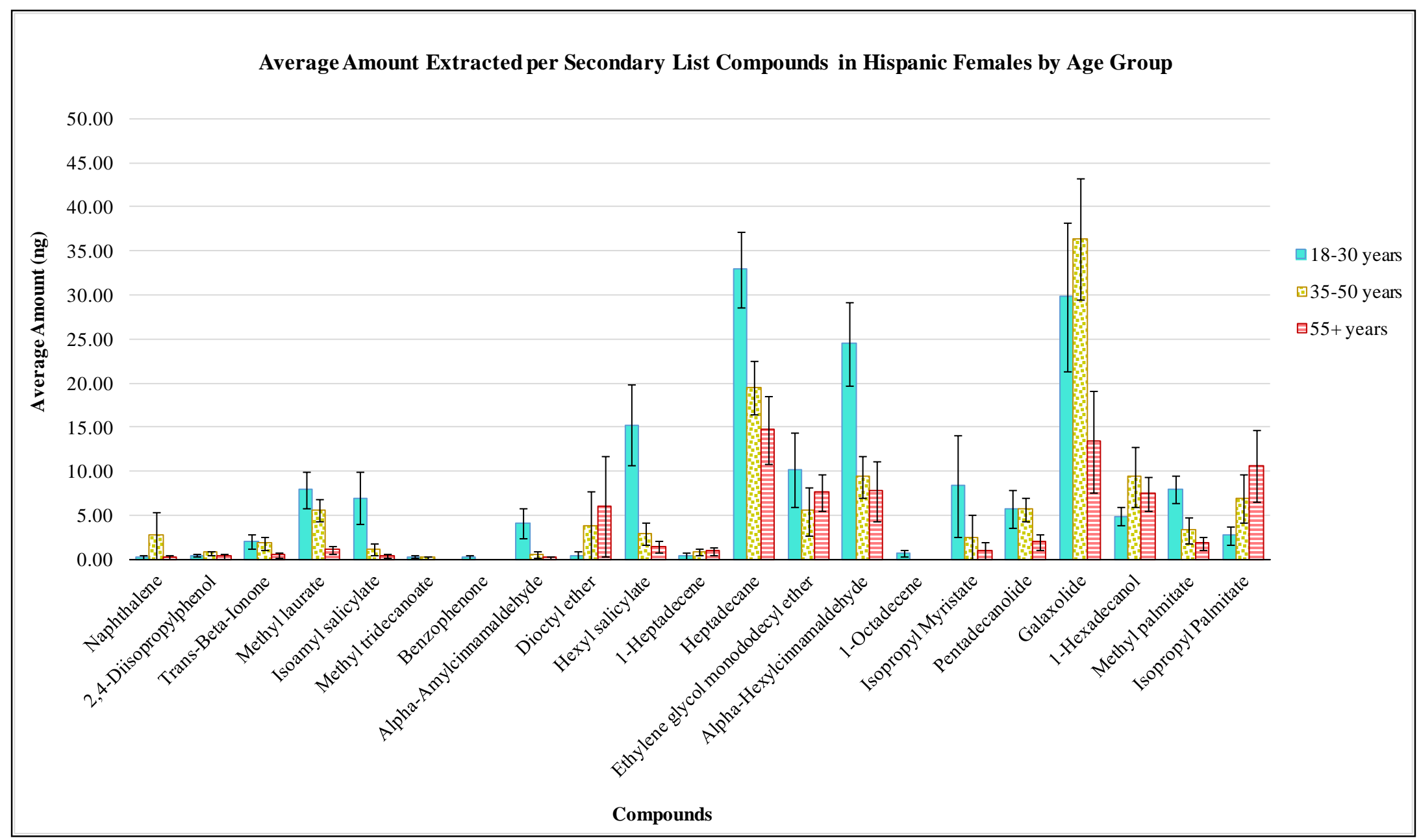

Figure 67: Comparison of average extracted amounts obtained for the VOCs considered in the secondary lists of Hispanic females from different age groups 
Table 21: Compounds considered in secondary lists made for comparing Hispanic females of different age groups

\begin{tabular}{|l|c|c|c|}
\hline $\begin{array}{c}\text { Compounds included in } \\
\text { Secondary Lists for Females }\end{array}$ & $\begin{array}{c}\mathbf{1 8 - 3 0} \text { vs. 35-50 } \\
\text { years }\end{array}$ & $\begin{array}{c}\mathbf{1 8 - 3 0} \text { vs. 55+ } \\
\text { years }\end{array}$ & $\begin{array}{c}\text { 35-50 vs. 55+ } \\
\text { years }\end{array}$ \\
\hline Naphthalene & $\mathrm{X}$ & & $\mathrm{X}$ \\
\hline 2,4-Diisopropylphenol & & $\mathrm{X}$ & $\mathrm{X}$ \\
\hline Trans-Beta-Ionone & & $\mathrm{X}$ & $\mathrm{X}$ \\
\hline Methyl laurate & $\mathrm{X}$ & $\mathrm{X}$ & $\mathrm{X}$ \\
\hline Isoamyl salicylate & & $\mathrm{X}$ & $\mathrm{X}$ \\
\hline Methyl tridecanoate & $\mathrm{X}$ & $\mathrm{X}$ & $\mathrm{X}$ \\
\hline Benzophenone & $\mathrm{X}$ & $\mathrm{X}$ & \\
\hline Alpha-Amylcinnamaldehyde & $\mathrm{X}$ & $\mathrm{X}$ & $\mathrm{X}$ \\
\hline Dioctyl ether & $\mathrm{X}$ & $\mathrm{X}$ & \\
\hline Hexyl salicylate & & $\mathrm{X}$ & \\
\hline 1-Heptadecene & $\mathrm{X}$ & $\mathrm{X}$ & \\
\hline Heptadecane & & $\mathrm{X}$ & \\
\hline Alpha-Hexylcinnamaldehyde & $\mathrm{X}$ & $\mathrm{X}$ & $\mathrm{X}$ \\
\hline 1-Octadecene & & $\mathrm{X}$ & $\mathrm{X}$ \\
\hline Isopropyl Myristate & & $\mathrm{X}$ & $\mathrm{X}$ \\
\hline Pentadecanolide & $\mathrm{X}$ & $\mathrm{X}$ & \\
\hline Galaxolide & $\mathrm{X}$ & $\mathrm{X}$ & \\
\hline Methyl palmitate & & & \\
\hline Isopropyl Palmitate & & & \\
\hline
\end{tabular}

Principal component analysis score plots and loading plots were prepared for the evaluation of female age group differentiation. In this case, it was observed that when comparing females in the 18-30 year age group versus those in the 55+ year age group, the score plot containing all 30 VOC variables already almost displayed a complete differentiation between the groups (Figure 68). This initial score plot showed a variation of 21.8\% in PC1 and 15\% in PC2. Nevertheless, it was noted that the secondary list score plot showed an enhancement in the separation between the groups (Figure 69 - left). In the case of this second score plot, variation in PC1 was slightly increased to $26 \%$ and in PC2 to $17.4 \%$. Such increases in variation resulted enough to cause a complete separation 
between these two female groups being compared. After evaluating the loading plot obtained for this second score plot (Figure 69 - right), it was found that the compounds leading the variation in PC1 were Hexyl salicylate, Methyl laurate, AlphaHexylcinnamaldehyde, Heptadecane, and Methyl palmitate, in decreasing order of influence. On the other hand, the most influential VOC for PC2 were Benzophenone, 1Heptadecene, Methyl palmitate, Methyl tridecanoate, and Heptadecane.

When comparing females in the 18-30 year age group with those in the 35-50 year category, the initial PCA score plot did not show separation between the groups. In this case, the plot presented all female subjects overlapping towards the center of the score plot, regardless of their age (Figure 70). The first principal component showed a variation of $15.9 \%$, while PC2 only showed $11.9 \%$. On the other hand, the score plot done with the secondary list VOCs, revealed that females from both groups had a tendency to cluster around those of their same age group (Figure 71 - left). A fairly clear distinction of both groups was possible by looking at this particular score plot, with PC1 and PC2 showing $23.5 \%$ and $19.3 \%$ of variation, respectively. However, it was observed that two females, one from each age group, still seemed to overlap with the clusters formed by females of their opposite age group. Hence, these two subjects precluded the complete separation of these two groups. However, there is a possibility that evaluating a larger number of individuals could have helped establish these two subjects as outliers, or as being incorporated into their respective groups. The loading plot obtained for this comparison (Figure 71 - right) showed that the most influential compounds in PC1 were AlphaHexylcinnamaldehyde, Hexyl salicylate, Isoamyl salicylate, Alpha-Amylcinnamaldehyde and Isopropyl Myristate, in decreasing order. On the other hand, variation in PC2 was 
mostly led by Naphthalene, Dioctyl ether, 1-Heptadecene, Benzophenone, and Methyl Palmitate (also in decreasing order of influence).

In the comparison of females of 35-50 years versus females of 55+ years, it was observed, once again, that the score plot that took into consideration all 30 VOCs did not show a clear separation between the groups (Figure 72). Most subjects from both groups seemed to spread out and overlap fairly equitably around the score plot, showing a variation of $20.8 \%$ in PC1 and 18\% in PC2, respectively. On the other hand, when using only the ten compounds from the corresponding secondary list for this comparison, almost a complete discrimination was achieved between females of these age categories (Figure 73 - left). Variation in PC1 and PC2 was increased to $35.4 \%$ and $18.2 \%$ respectively, which caused a noticeable enhancement in the ability to discriminate between subjects of the different groups. After evaluating the loading plot (Figure 73 right), it was noted that the compounds of most influence in the variation of PC1 were (in decreasing order) Isoamyl salicylate, Methyl tridecanoate, Hexyl salicylate, Methyl laurate and Trans-Beta-Ionone. On the other hand, those seen to be most influential in PC2 were Galaxolide, Alpha-Amylcinnamaldehyde, Isopropyl Myristate, 2,4Diisopropylphenol and Methyl tridecanoate. 


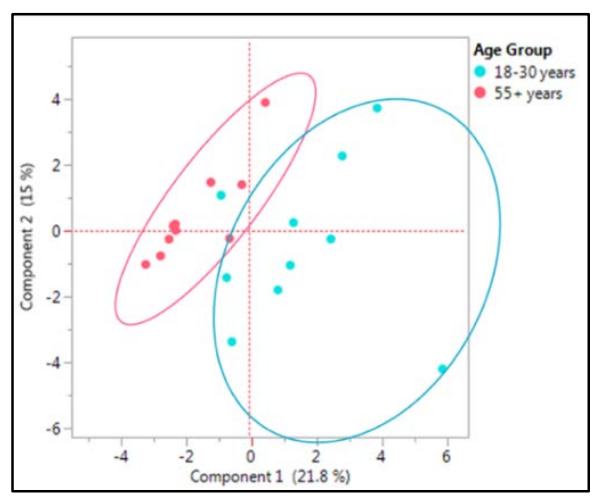

Figure 68: PCA score plot of 18-30 vs. 55+ year old Hispanic females including all VOCs
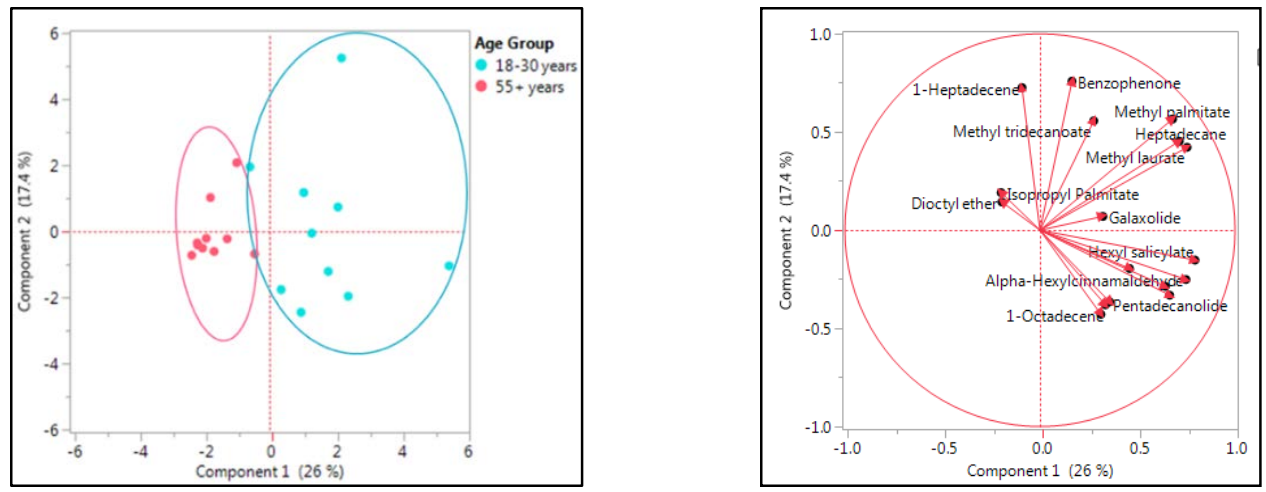

Figure 69: PCA score plot (left) and loading plot (right) of 18-30 vs. 55+ year old Hispanic females including VOCs from secondary list

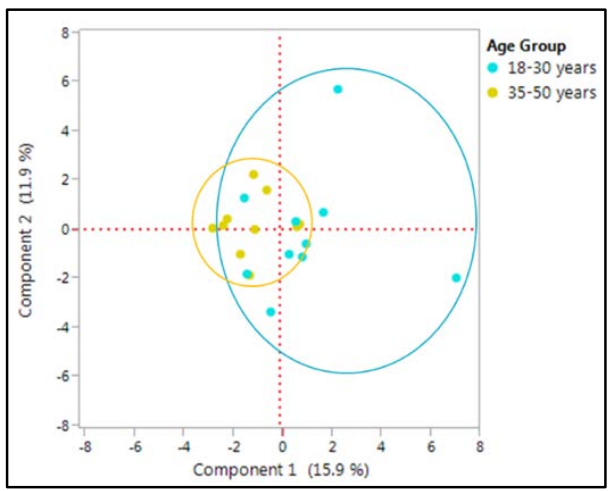

Figure 70: PCA score plot of 18-30 vs. 35-50 year old Hispanic females including all VOCs 

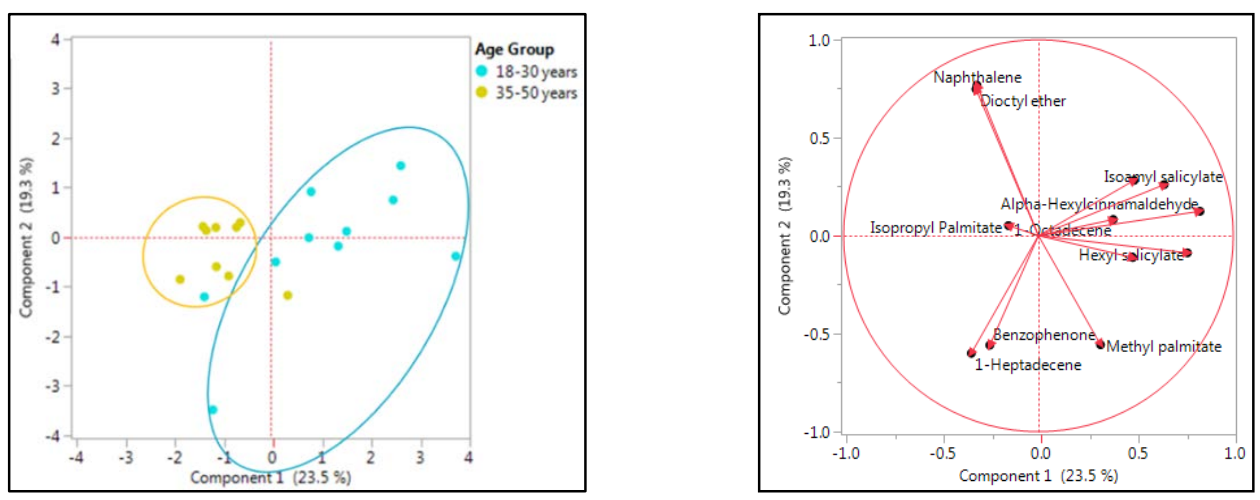

Figure 71: PCA score plot (left) and loading plot (right) of 18-30 vs. 35-50 year old Hispanic females including VOCs from secondary list

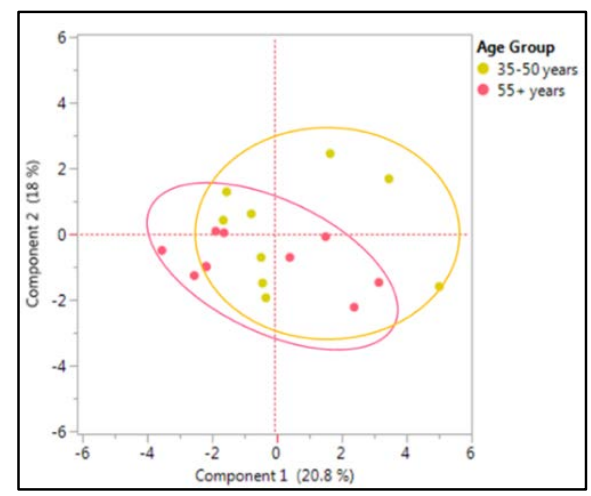

Figure 72: PCA score plot of 35-50 vs. 55+ year old Hispanic females including all VOCs
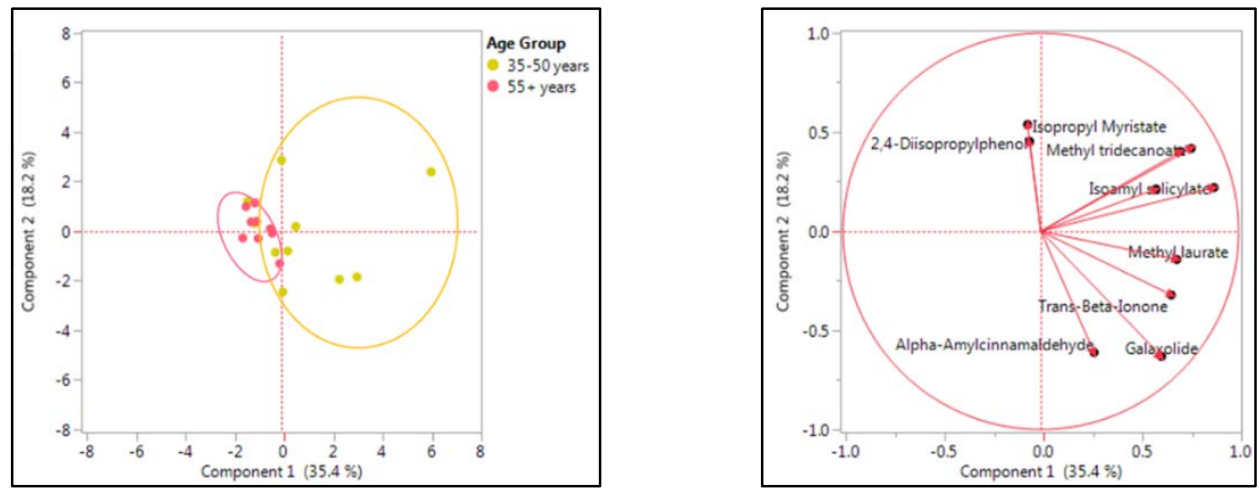

Figure 73: PCA score plot (left) and loading plot (right) of 35-50 vs. 55+ year old Hispanic females including VOCs from secondary list 


\section{Comparison of Males of Different Age Groups}

A comparison of the VOC average amounts was also performed for Hispanic males in each of the age groups under study. Out of 30 compounds, males in the 18-30 age groups showed a higher average amount for 15 compounds, while 35-50 year olds did for seven compounds, and 55+ year olds did for 8 . As it was previously reported for females, Hexyl salicylate and Alpha-Hexylcinnamaldehyde were seen to display a higher average amount in males from the 18-30 year old age group. These findings support Gallagher et al.'s report from $2008 .{ }^{21}$ On the other hand, in my study, $60 \%$ of all males showed to have Nonanal as part of their odor profile, but it was 18-30 year old males that showed the highest amount of this VOC. Males in the 35-50 year old category showed the least amount. Therefore, in the case of these results, no evidence was found to support previous reports that state this compound as being a biomarker for increased age.

Secondary lists were also created to assess male odor profiles. This time, a total of 22 compounds were selected for consideration in the secondary lists of the different male age group sets (Figure 74). These compounds were evaluated for their potential as possible biomarkers for age in Hispanic males. Table 22 lists the compounds included in the secondary lists of each male age group set. In this table, an asterisk has been used to mark certain compounds that, despite not having complied with the selection criteria, were also incorporated in the secondary lists. These exceptions were made after noticing an increase in group discrimination as a result of those compounds' inclusion as discrimination criteria.

From the total of 22 compounds, 20 were included in the secondary list to compare the $18-30$ year category and the $55^{+}$year category. The secondary list used to 
compare subjects of 18-30 years versus those of 35-50 years included 17 compounds, and the list used for comparison of 35-50 year old and 55+ year old males consisted of 16 VOCs. As for females, variation was noticed in the case of males for those compounds included in the secondary lists of all age group sets. As a result, it could be understood that the discrimination between male age groups might also be relying on the nature of the different VOC combinations of each group set. Nonetheless, a total of 16 VOCs were still consistently considered in the comparison of all male age group sets. Out of the list of 22 compounds being considered for secondary lists, males in the 18-30 year age group portrayed the highest average amount for 15 VOCs. On the other hand, males in the 3550 year age group showed the highest average amount for Pentadecane, Methyl laurate, Dioctyl ether, 1-Heptadecene, Pentadecanolide, Methyl palmitate and Isopropyl palmitate. Moreover, males in the 55+ year category showed the highest average amount for 2-Furanmethanol, Dimethyl sulfone, Diethylene glycol monoethyl ether, Naphthalene, 2,4-Diisopropylphenol, Methyl tridecanoate, Benzophenone and Galaxolide. 


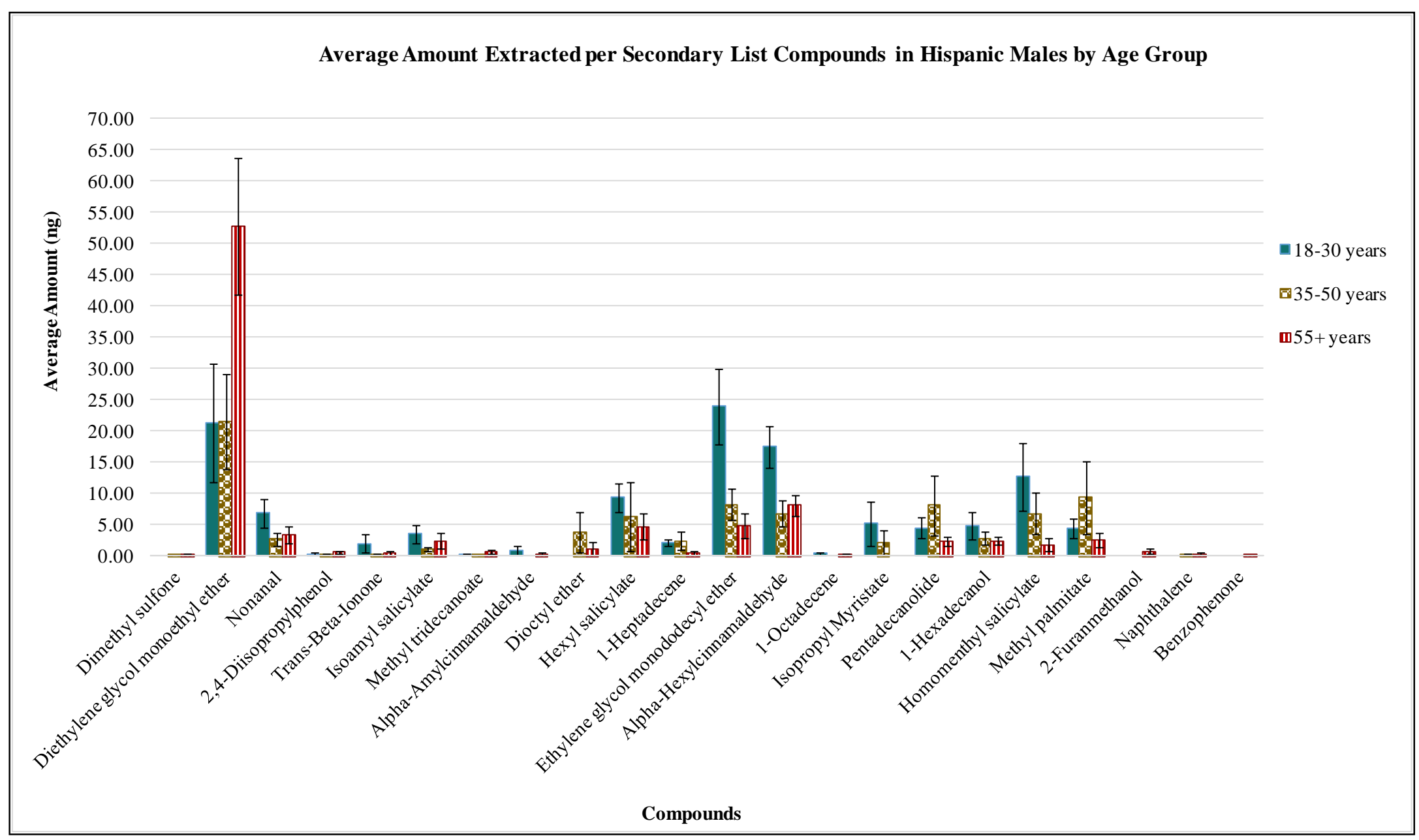

Figure 74: Comparison of average extracted amounts obtained for the VOCs considered in the secondary lists of Hispanic males from different age groups 
Table 22: Compounds considered in secondary lists made for comparing Hispanic males of different age groups

\begin{tabular}{|l|c|c|c|}
\hline $\begin{array}{c}\text { Compounds included in } \\
\text { Secondary Lists for Males }\end{array}$ & $\begin{array}{c}\mathbf{1 8 - 3 0} \text { vs. 35-50 } \\
\text { years }\end{array}$ & $\begin{array}{c}\mathbf{1 8 - 3 0} \text { vs. 55+ } \\
\text { years }\end{array}$ & $\begin{array}{c}\text { 35-50 vs. 55+ } \\
\text { years }\end{array}$ \\
\hline Dimethyl sulfone & $\mathrm{X}$ & & $\mathrm{X}^{*}$ \\
\hline Diethylene glycol monoethyl ether & $\mathrm{X}$ & $\mathrm{X}$ & $\mathrm{X}$ \\
\hline Nonanal & $\mathrm{X}^{*}$ & $\mathrm{X}^{*}$ & $\mathrm{X}$ \\
\hline 2,4-Diisopropylphenol & $\mathrm{X}$ & $\mathrm{X}$ & $\mathrm{X}$ \\
\hline Trans-Beta-Ionone & $\mathrm{X}$ & & $\mathrm{X}$ \\
\hline Isoamyl salicylate & $\mathrm{X}^{*}$ & $\mathrm{X}$ & $\mathrm{X}$ \\
\hline Methyl tridecanoate & $\mathrm{X}$ & $\mathrm{X}$ & $\mathrm{X}$ \\
\hline Alpha-Amylcinnamaldehyde & $\mathrm{X}$ & $\mathrm{X}$ & $\mathrm{X}$ \\
\hline Dioctyl ether & & $\mathrm{X}$ & \\
\hline Hexyl salicylate & & $\mathrm{X}$ & \\
\hline 1-Heptadecene & $\mathrm{X}$ & $\mathrm{X}$ & $\mathrm{X}^{*}$ \\
\hline Ethylene glycol monododecyl ether & $\mathrm{X}$ & $\mathrm{X}$ & \\
\hline Alpha-Hexylcinnamaldehyde & $\mathrm{X}$ & $\mathrm{X}^{*}$ & $\mathrm{X}$ \\
\hline 1-Octadecene & $\mathrm{X}$ & $\mathrm{X}$ & $\mathrm{X}$ \\
\hline Isopropyl Myristate & $\mathrm{X}^{*}$ & $\mathrm{X}^{*}$ & $\mathrm{X}$ \\
\hline Pentadecanolide & $\mathrm{X}^{*}$ & $\mathrm{X}^{*}$ & \\
\hline 1-Hexadecanol & $\mathrm{X}^{*}$ & $\mathrm{X}$ & $\mathrm{X}$ \\
\hline Homomenthyl salicylate & $\mathrm{X}$ & $\mathrm{X}^{*}$ & $\mathrm{X}$ \\
\hline Methyl palmitate & & $\mathrm{X}$ & $\mathrm{X}$ \\
\hline 2-Furanmethanol & $\mathrm{X}$ & $\mathrm{X}$ & \\
\hline Naphthalene & & $\mathrm{X}$ & $\mathrm{X}$ \\
\hline Benzophenone & & & \\
\hline
\end{tabular}

Principal component analysis was performed to evaluate male age group differentiation. In the comparison established between the 18-30 year age group and the 55+ year category, the PCA score plot including all 30 variables showed all subjects from the two age groups spreading equally throughout all quadrants of the plot (Figure 75). No clear clusters were formed by the individuals in any of the age groups and PC1 and PC2 showed a variation percent of $17.4 \%$ and $15.2 \%$, respectively. On the contrary, as soon as only the compounds from the secondary list of that age group set were used, a significant 
enhancement was observed for the differentiation of both groups (Figure 76 - left). Variation in PC1 and PC2 was increased to $18.6 \%$ and $16.9 \%$, respectively, and individuals of each age group formed defined clusters. According to the loading plot (Figure 76 - right) obtained for this comparison, the top compounds leading variation in PC1 were Pentadecanolide, Homomenthyl salicylate, Ethylene glycol monododecyl ether, Alpha-Hexylcinnamaldehyde and 1-Octadecene (in decreasing order of influence). Moreover, those causing most variation in PC2 were Hexyl salicylate, Nonanal, Isopropyl Myristate Methyl palmitate and 1-Heptadecene (also in decreasing order).

In the first PCA score plot used to compare males in the 18-30 year and $35-50$ year category, it was observed that all 30 VOCs did not serve as efficient criteria for the discrimination of the two groups (Figure 77). The variation in the first two principal components (17.7\% in PC1 and 11\% in PC2) was not enough to group individuals by their corresponding age group, but instead made all individuals from one age group to appear mixed with those of the opposite group. On the other hand, when performing PCA with the compounds from the secondary list for this age group set, a slight enhancement was observed in terms of the formation of individual clusters in the score plot (Figure 78 - left). Although there was still some overlap observed between the clusters of both age groups, the majority of individuals for both cases grouped with those of their same age category. This second score plot showed a variation of $16.8 \%$ in PC1 and 14\% in PC2. Nevertheless, despite the decrease in variation in the first component, the increase in variation percent in the second component resulted enough to cause a visible improvement in the score plot's appearance. The loading plot obtained using the VOCs from the secondary list (Figure 78 - right) revealed that 1-Octadecene, Trans-Beta- 
Ionone, Isoamyl salicylate, Alpha-Hexylcinnamaldehyde, and Naphthalene, were the most influential VOCs in PC1's variation (in decreasing order). In addition, it showed that Trans-Beta-Ionone, Methyl palmitate, Isoamyl salicylate, Ethylene glycol monododecyl ether, and Nonanal were, in decreasing order, the VOCs of highest influence in PC2.

In the case of the comparison between the 35-50 year age group and the 55+ year age group, the initial PCA score plot with all 30 VOCs served as another example for which all VOCs did not provide a successful discrimination between the age groups (Figure 79). The initial score plot showed a variation of 18\% and 13.9\% in PC1 and PC2, respectively. Also, it showed individuals from the two different age groups being mixed among those of the opposite group, and no clear signs for the formation of any age group cluster. However, once only the compounds from the secondary list were considered as discrimination variables, the variation in PC1 was increased to $19.7 \%$ and in PC2 to 17.7\%. This increase in variation caused a significant decrease in the spread of all individuals in the 35-50 year age group. As a result, a clear distinction between males that belonged to each of the different age groups was accomplished, and it was possible to visibly differentiate the formation of two clusters (Figure 80 - left). The loading plot (Figure 80 - right) obtained for this last score plot, revealed that the compounds of highest influence in PC1 were Alpha-Amylcinnamaldehyde, 1-Octadecene, Methyl tridecanoate, 2-Furanmethanol, and 2,4-Diisopropylphenol (in decreasing order). On the other hand, variation in the second component was found to be mainly driven by TransBeta-Ionone, Isoamyl salicylate, Dimethyl sulfone, 2,4-Diisopropylphenol, and Ethylene glycol monododecyl ether (also in decreasing order). 


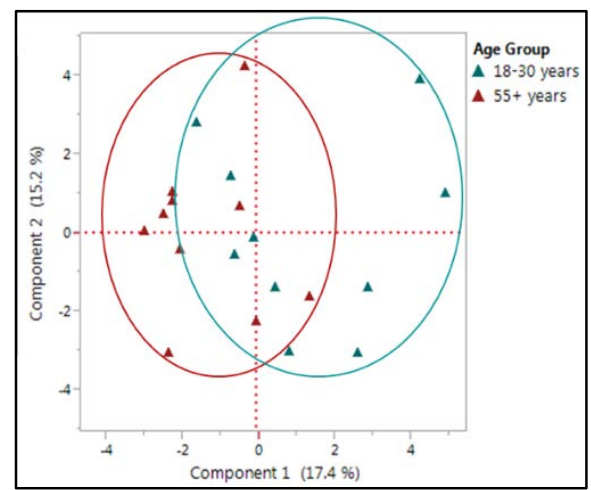

Figure 75: PCA score plot of 18-30 vs. 55+ year old Hispanic males including all VOCs
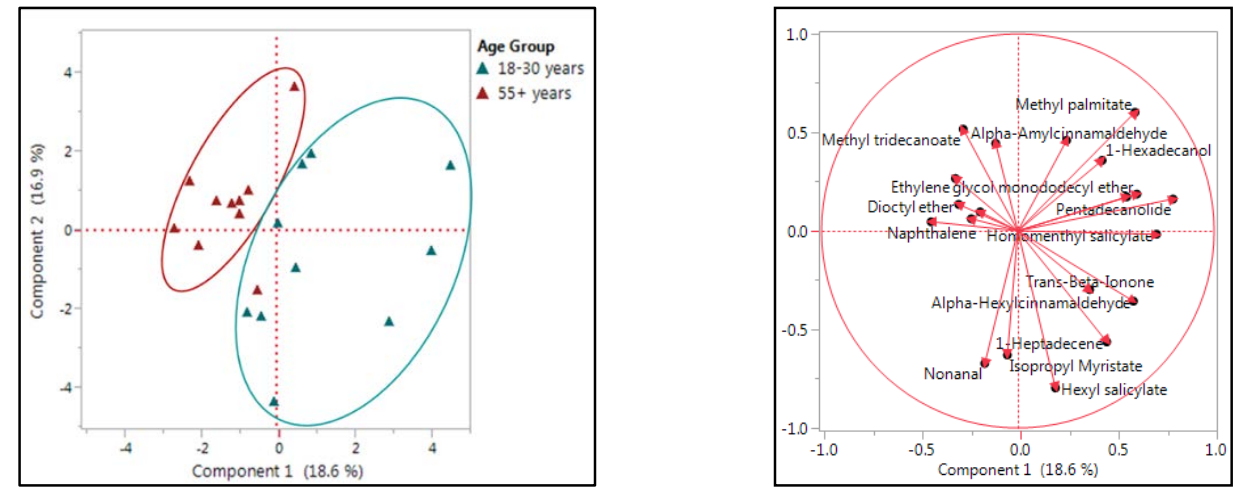

Figure 76: PCA score plot (left) and loading plot (right) of 18-30 vs. 55+ year old Hispanic males including VOCs from secondary list

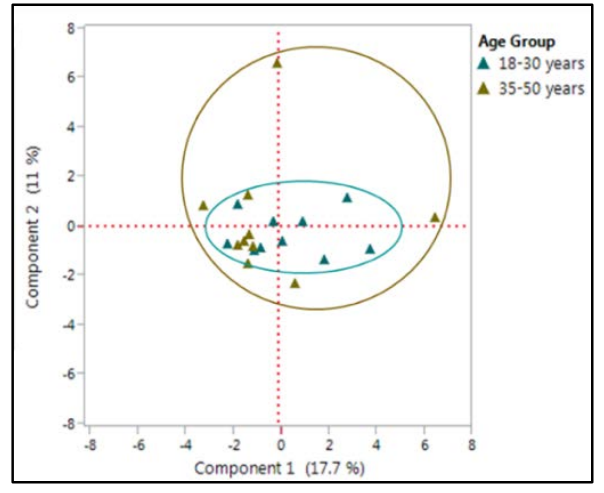

Figure 77: PCA score plot of 18-30 vs. 35-50 year old Hispanic males including all VOCs 

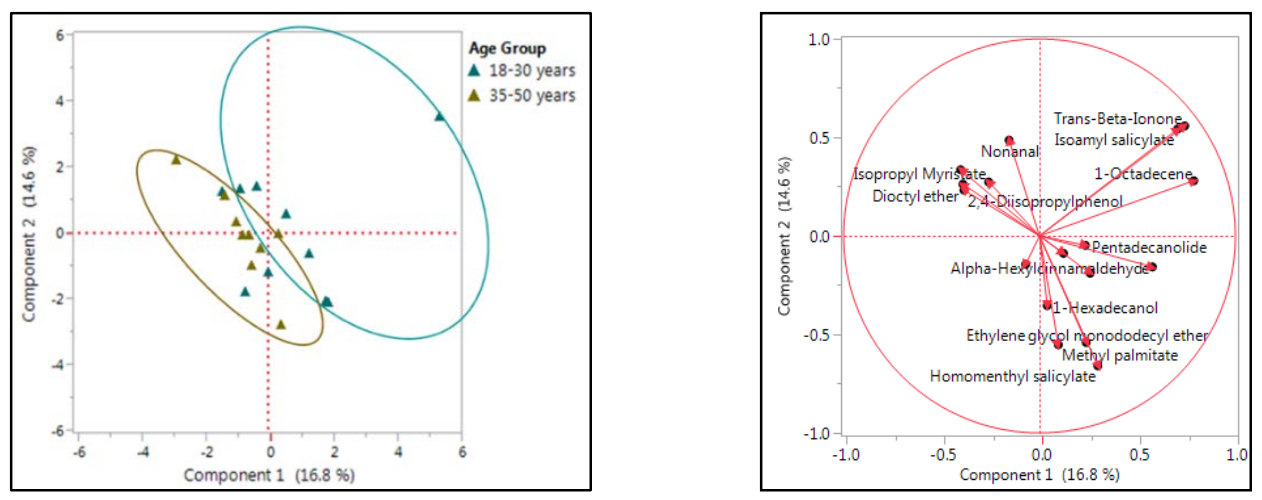

Figure 78: PCA score plot (left) and loading plot (right) of 18-30 vs. 35-50 year old Hispanic males including VOCs from secondary list

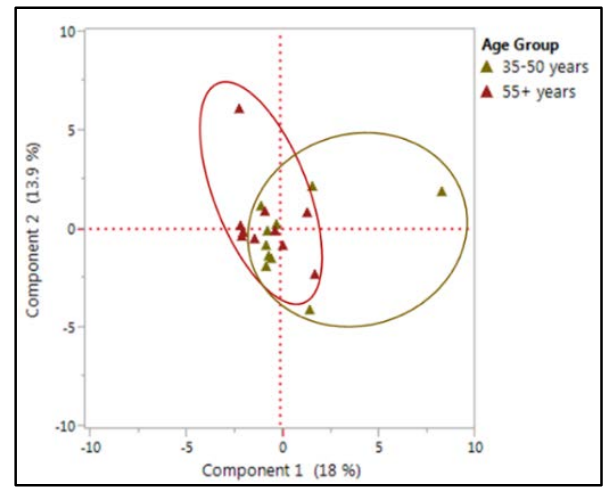

Figure 79: PCA score plot of 35-50 vs. 55+ year old Hispanic males including all VOCs
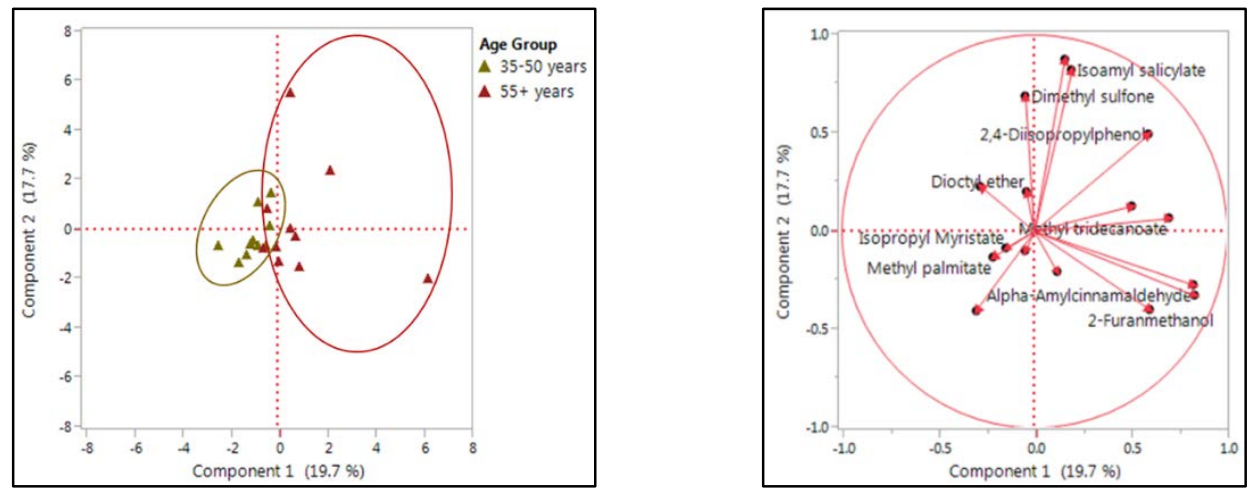

Figure 80: PCA score plot (left) and loading plot (right) of 35-50 vs. 55+ year old Hispanic males including VOCs from secondary list 


\subsubsection{Results from East Asian population}

\subsection{Qualitative Analysis}

A total of 27 VOCs were initially considered in the data analysis for the East Asian population. The 27 compounds were employed in a qualitative and quantitative assessment of the different gender and age groups under study (Table 23). A survey was made of the occurrence of each compound in each of the samples collected from the participants' right underarms. Consequently, odor profiles were constructed for each East Asian individual on the basis of each person's characteristic pattern of VOC occurrence in their scent samples (Figure 81 - Figure 86).

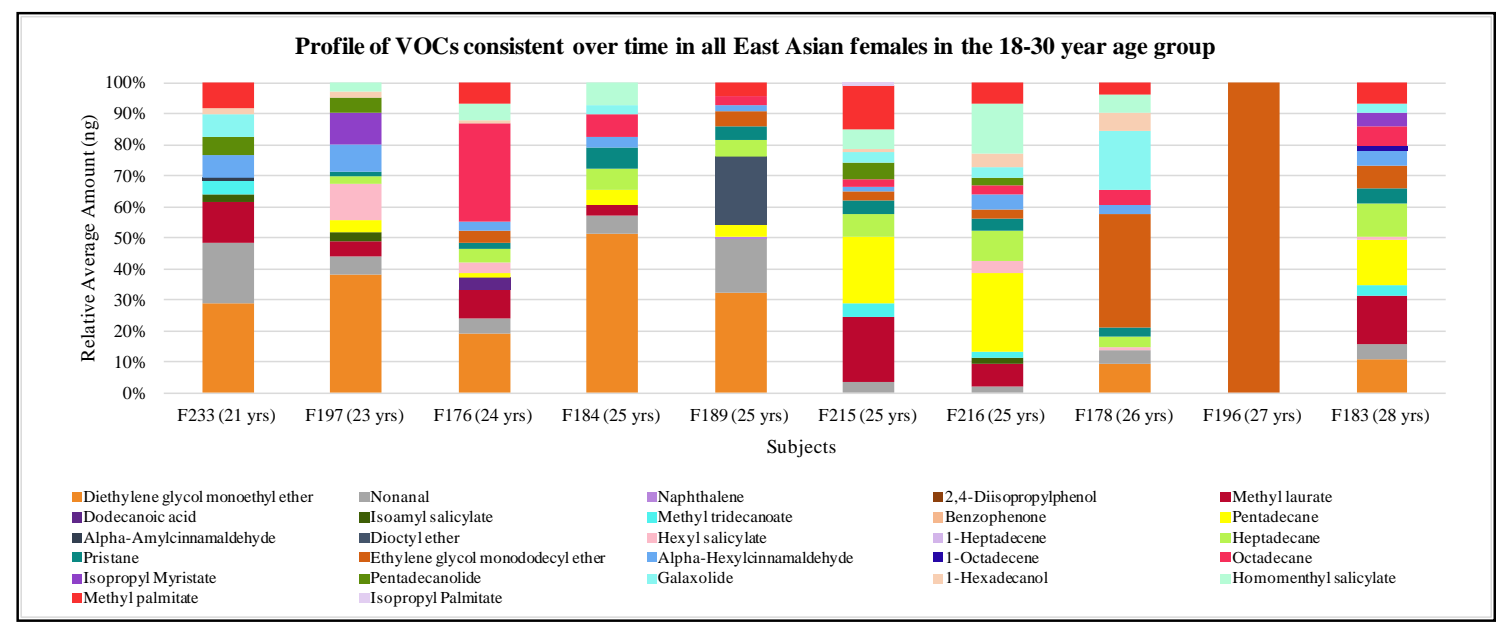

Figure 81: Scent profiles obtained for all 18-30 year old East Asian females 


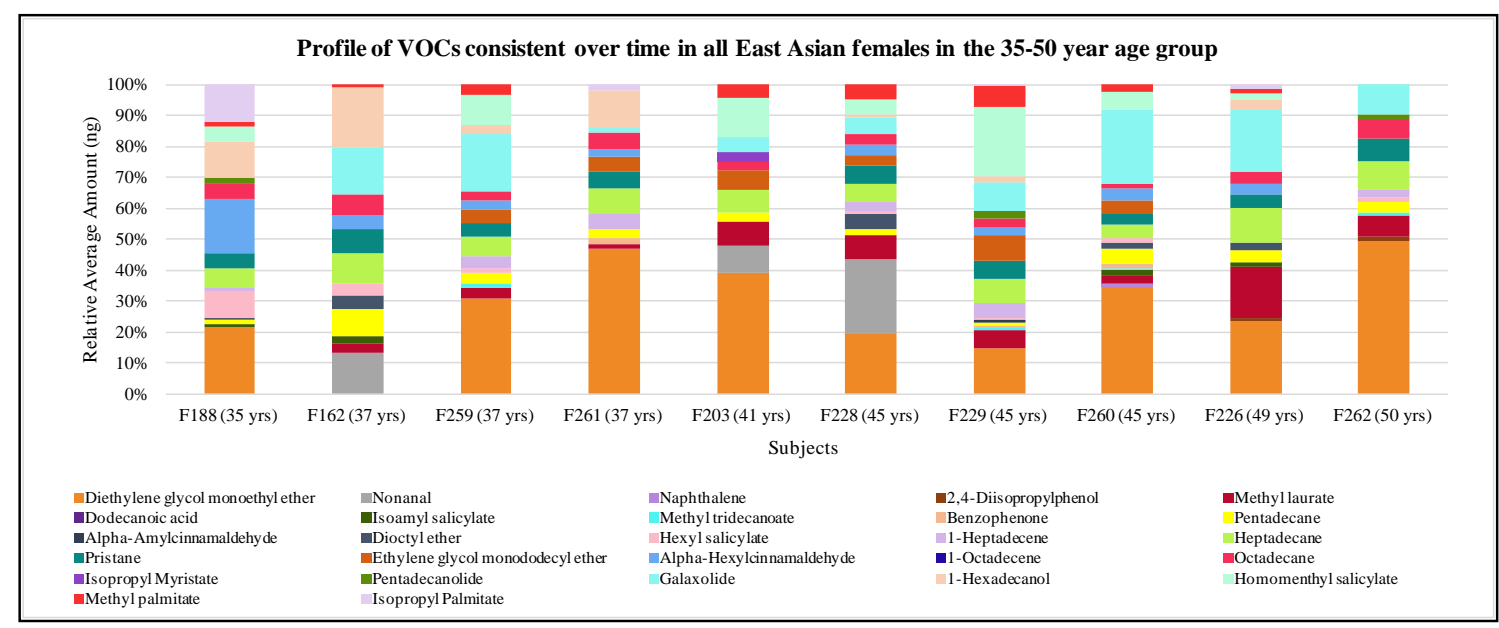

Figure 82: Scent profiles obtained for all 35-50 year old East Asian females

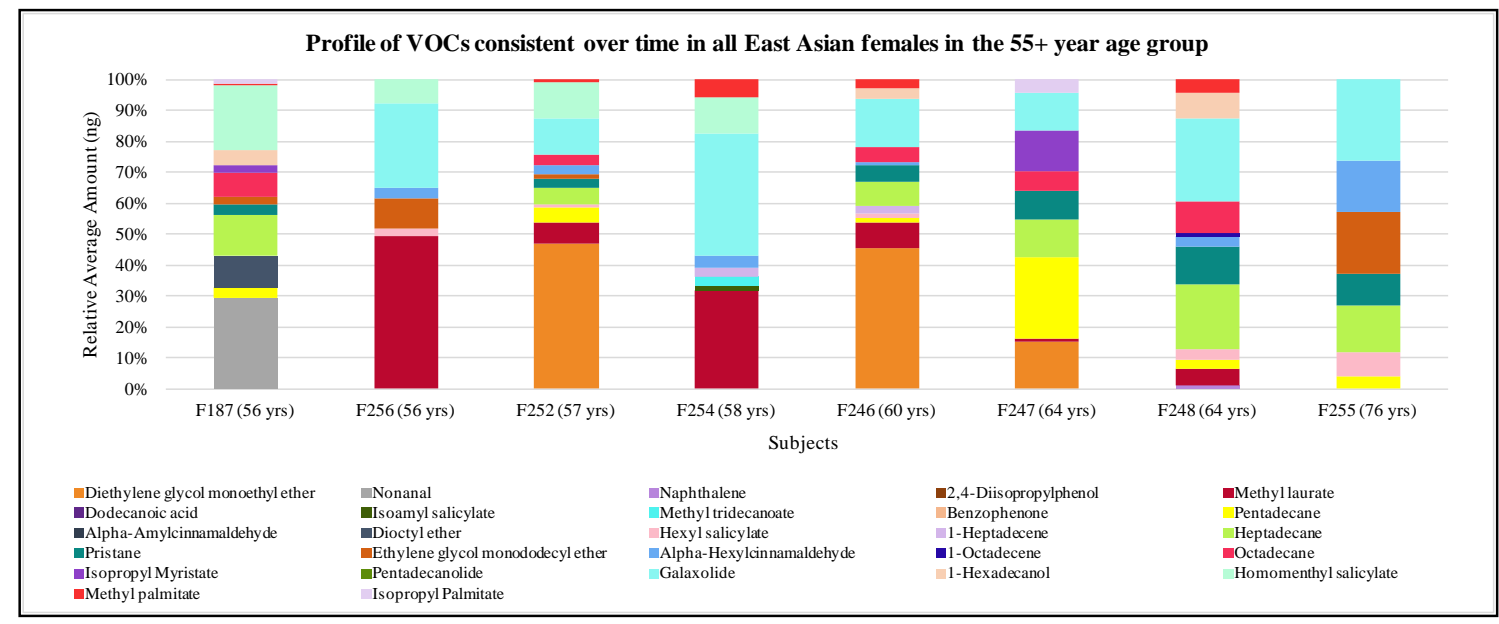

Figure 83: Scent profiles obtained for all 55+ year old East Asian females 


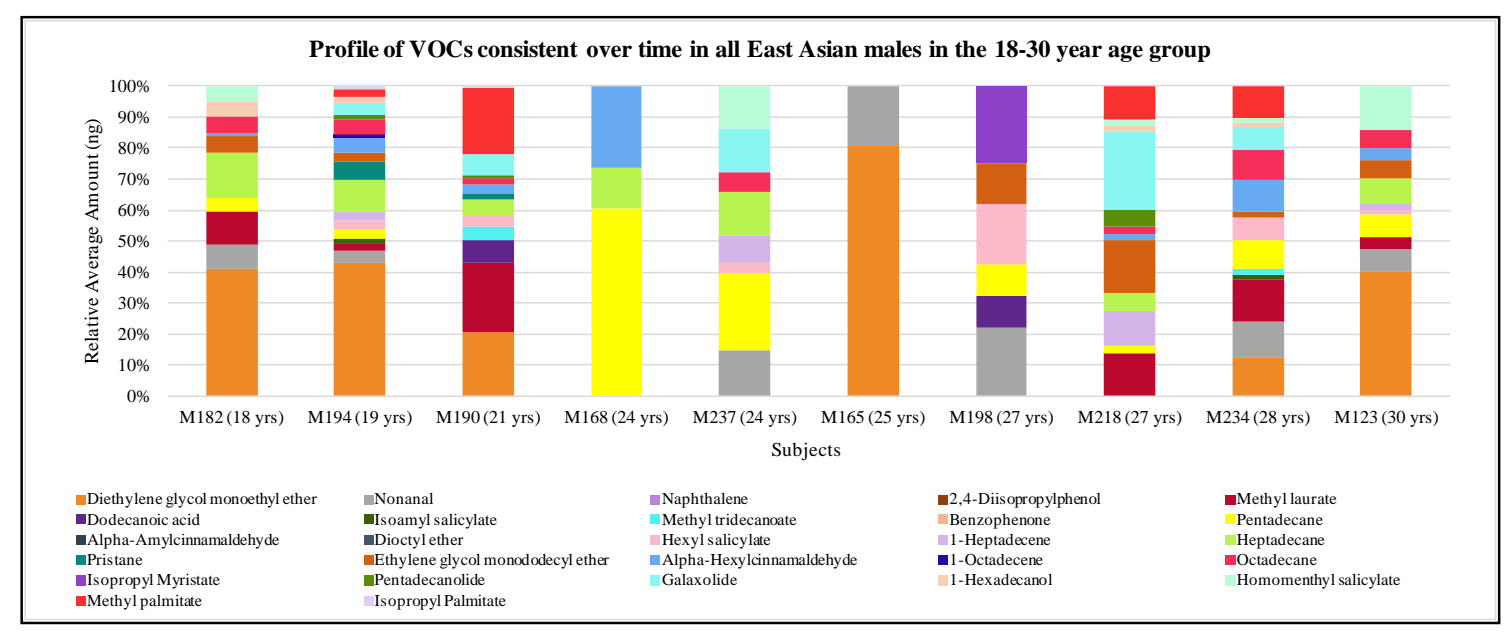

Figure 84: Scent profiles obtained for all 18-30 year old East Asian males

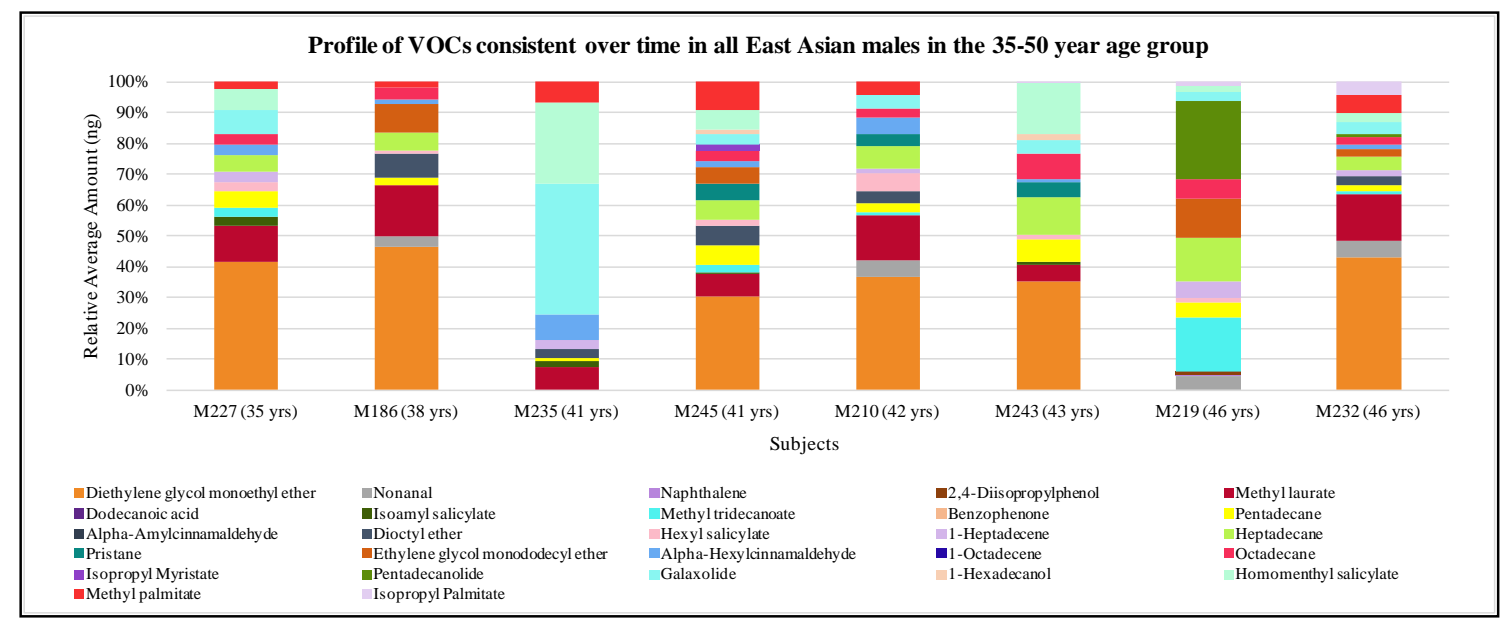

Figure 85: Scent profiles obtained for all 35-50 year old East Asian males 


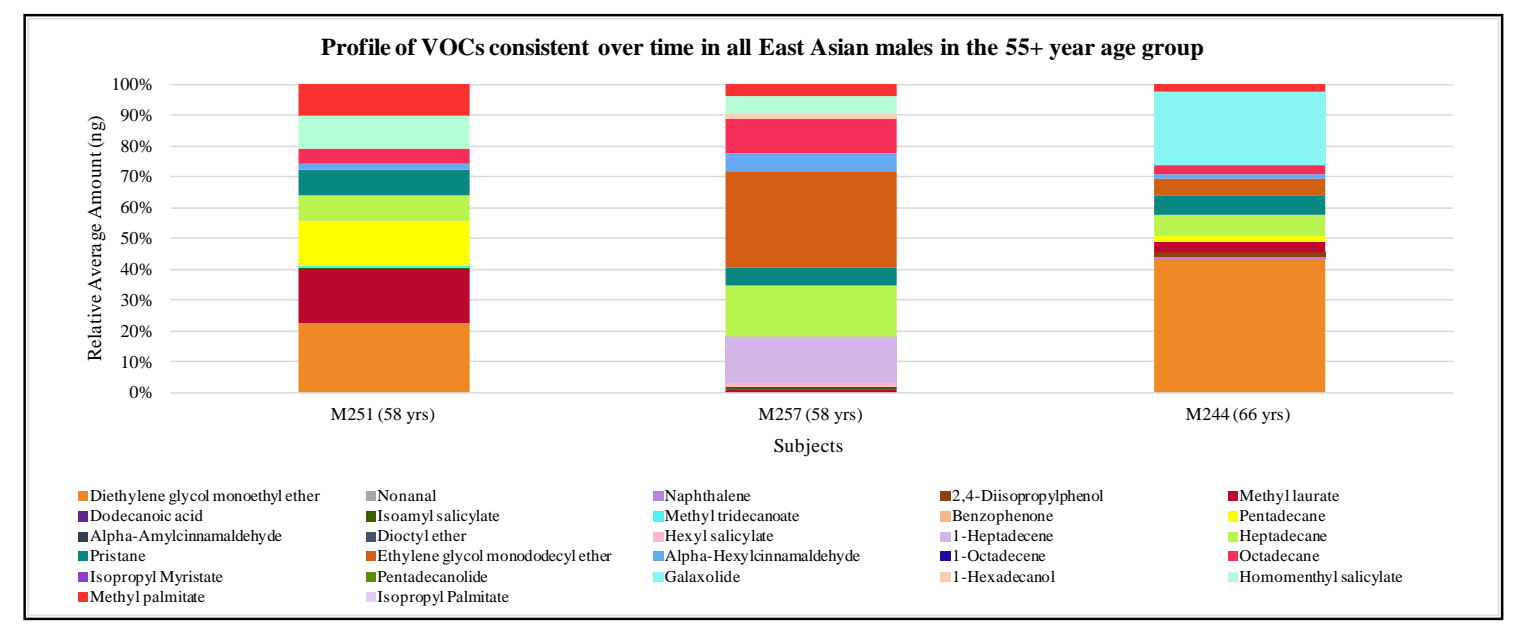

Figure 86: Scent profiles obtained for all 55+ year old East Asian males

A thorough evaluation of the odor profiles of all 18-30 year old East Asian individuals, allowed noting that the occurrence of Methyl tridecanoate and Isopropyl myristate in females doubled that of males (Table 23). In addition, it revealed that Pristane is much more occurent in females than in males, by showing an $80 \%$ and a $20 \%$ for each of the genders, respectively. On the other hand, it was also observed that the occurrence of Dodecanoic acid and Isopropyl Palmitate, in East Asian male odor profiles, doubled that of East Asian females. In my study Isopropyl palmitate, previously reported by Penn et al. as a gender marker, also displayed this same trend for 18-30 year old Caucasian individuals. ${ }^{37}$ In addition, this compound portrayed very similar average amounts in both genders of the East Asian population, yet 18-30 year old males portrayed a slightly higher abundance of this compound in their profiles $(0.30 \mathrm{ng})$ than females of the same age group (0.21 ng). This particular observation was not in accordance with findings from Penn et al. and represents the first case in the 18-30 year age category for which the trend in occurrence or abundance of this compound shows a discrepancy from 
previous findings. ${ }^{37}$ However, the fact that the average amounts of this compound in East Asian females and males are so close, suggests that further investigation is needed prior to establishing this group as an exception. A deeper investigation, considering a larger number of individuals, may help ensure the results are not a mere manifestation of chance and confirm that insignificant differences are not favoring misleading conclusions. Among other things that were noted from the qualitative evaluation of this age group were that 1-Heptadecene was only found in male odor profiles, while 2,4Diisopropylphenol and Benzophenone were not found in the odor profiles of any gender group for the 18-30 year old East Asians. Also, Naphthalene, Dioctyl ether and AlphaAmylcinnamaldehyde were all compounds only found in female odor profiles for this age group.

The qualitative analysis of the odor profiles of 35-50 year old East Asian individuals, showed the majority of the compounds as having similar percentages of occurrence in both genders (Table 23). The occurrences of Pristane and 1-Hexadecanol in females were seen to surpass those of males by more than $100 \%$. Nevertheless, no cases were observed in which the occurrence of a compound for males was higher than that of females by $100 \%$ or more. On the other hand, Alpha-Amylcinnamaldehyde and Benzophenone were each only found in female odor profiles, with an occurrence of $20 \%$ and 30\%, respectively. Dodecanoic acid and 1-Octadecene were not seen to occur in any of the odor profiles obtained for this age group and, contrary to the observations made for 18-30 year old East Asians, Isopropyl palmitate was found to have very similar occurrence in both genders in this age group (females $40 \%$ and males $38 \%$ ). The average amount of Isopropyl palmitate for females, in the 35-50 yrs age group, was more than the 
amount extracted for males (3.89 ng and $1.23 \mathrm{ng}$ respectively). Once again, this shows agreement with previous findings that present Isopropyl palmitate as a gender marker. ${ }^{37}$

The qualitative evaluation of the odor profiles of East Asian females in the 55+ year category revealed the occurrence of Galaxolide in females was more than twice the that of East Asian males in the same age group (Table 23). The opposite was observed in the case of Naphthalene, Isoamyl salicylate, Methyl tridecanoate and Octadecane, where males showed a percentage of occurrence that was twice or more of that shown by the group of females. Alpha-Amylcinnamaldehyde, Benzophenone, Dodecanoic acid and Pentadecanolide were not found in any odor profiles for this age group, while other compounds like Nonanal, Dioctyl ether, 1-Octadecene, and Isopropyl Myristate were only found in female odor profiles from the 55+ year age group. Moreover, it was observed that 2,4-Diisopropylphenol was only present in male odor profiles and was seen to increase from $13 \%$ to $33 \%$ of occurrence from the $35-50$ to the $55+$ age group. On the other hand, Isopropyl palmitate was only observed in the odor profiles of 55+ year old females, and was seen to decrease its occurrence in comparison to the East Asian females of $35-50$ years of age (from $40 \%$ to $25 \%$ ). The fact that this compound was not observed in 55+ year old male odor profiles could be counted as supporting evidence for this compound's previous classification as a gender marker for females. ${ }^{37}$

After performing a qualitative analysis per individual age groups for East Asian females it was noted that Nonanal, Dodecanoic acid, Methyl palmitate, Ethylene glycol monododecyl ether, Alpha-Hexylcinnamaldehyde, and Pentadecanolide tended to decrease their occurrence with age (Table 23). Naphthalene appeared to be the only compound to show a consistent increase in its occurrence as age increased, and 
Dodecanoic acid was only noted as present in the youngest of all female age groups. Benzophenone and 2,4-Diisopropylphenol were only observed in the odor profiles of 3550 year old females, and 1-Octadecene was observed in all female age groups except the 35-50 year group. Contrary to females, in the case of Methyl palmitate and AlphaHexylcinnamaldehyde, East Asian males showed an increasing trend of occurrence with age. A consistent increase in occurrence with age was also observed for Methyl laurate, Isoamyl salicylate, Heptadecane, Pristane, and Octadecane. On the other hand, Male odor profiles also showed a decreasing pattern in occurrence for Nonanal and Pentadecanolide as age increased, as it was observed in East Asian females. Nonanal has been previously reported as marker compound of increased age on the basis of its increased abundance. ${ }^{21}$ However, despite Nonanal being present in the odor profiles of $52 \%$ of the males under study, previous findings on this compound were not seen to be fully supported. Moreover, Dodecanoic Acid and 1-Octadecene were only seen to occur in the youngest of all male age groups, and Alpha-Amylcinnamaldehyde was not seen to occur in any East Asian male odor profiles. Dioctyl ether was only noted in the odor profiles of 35-50 year old males, and no compounds were seen to only occur in the 55+ year age group for males. 
Table 23: VOCs emanating from East Asian subjects’ underarm odor samples detected and quantified by solid-phase microextraction GC/MS

\begin{tabular}{|c|c|c|c|c|c|c|c|c|}
\hline \multirow{3}{*}{ CAS no. } & \multirow{3}{*}{$\mathrm{RT}$ (min) } & \multirow{3}{*}{ Compound Name } & \multicolumn{6}{|c|}{ Frequency of Occurrence in Subjects } \\
\hline & & & \multicolumn{3}{|c|}{ Females $(n=28)$} & \multicolumn{3}{|c|}{ Males $(n=21)$} \\
\hline & & & $\begin{array}{l}18-30 \\
\text { years }\end{array}$ & $\begin{array}{l}\text { 35-50 } \\
\text { years }\end{array}$ & $\begin{array}{c}55+ \\
\text { years }\end{array}$ & $\begin{array}{l}18-30 \\
\text { years }\end{array}$ & $\begin{array}{l}\text { 35-50 } \\
\text { years }\end{array}$ & $\begin{array}{c}55+ \\
\text { years }\end{array}$ \\
\hline $111-90-0$ & 11.168 & Diethylene glycol monoethyl ether $^{\mathrm{c}}$ & $70 \%$ & $90 \%$ & $38 \%$ & $60 \%$ & $75 \%$ & $67 \%$ \\
\hline $124-19-6$ & 13.066 & Nonanal ${ }^{\mathrm{c}, \mathrm{d}}$ & $90 \%$ & $30 \%$ & $13 \%$ & $70 \%$ & $50 \%$ & $0 \%$ \\
\hline $91-20-3$ & 14.883 & Naphthalene ${ }^{\text {c, d, e }}$ & $10 \%$ & $10 \%$ & $13 \%$ & $0 \%$ & $13 \%$ & $33 \%$ \\
\hline 2934-05-6 & 18.800 & 2,4-Diisopropylphenol & $0 \%$ & $20 \%$ & $0 \%$ & $0 \%$ & $13 \%$ & $33 \%$ \\
\hline $629-62-9$ & 20.796 & Pentadecane $^{\mathrm{c}, \mathrm{e}}$ & $70 \%$ & $90 \%$ & $75 \%$ & $60 \%$ & $88 \%$ & $100 \%$ \\
\hline $111-82-0$ & 21.296 & Methyl laurate ${ }^{\text {c, d }}$ & $10 \%$ & $0 \%$ & $0 \%$ & $20 \%$ & $0 \%$ & $0 \%$ \\
\hline $143-07-7$ & 22.156 & Dodecanoic acid $^{\text {c, d, e }}$ & $30 \%$ & $40 \%$ & $13 \%$ & $20 \%$ & $50 \%$ & $67 \%$ \\
\hline $87-20-7$ & 22.331 & Isoamyl salicylate $^{\mathrm{c}, \mathrm{e}}$ & $40 \%$ & $40 \%$ & $13 \%$ & $20 \%$ & $63 \%$ & $33 \%$ \\
\hline $1731-88-0$ & 23.263 & Methyl tridecanoate $^{d}$ & $0 \%$ & $30 \%$ & $0 \%$ & $0 \%$ & $0 \%$ & $0 \%$ \\
\hline $119-61-9$ & 23.503 & Benzophenone ${ }^{\mathrm{c}, \mathrm{d}, \mathrm{e}}$ & $70 \%$ & $100 \%$ & $75 \%$ & $80 \%$ & $100 \%$ & $67 \%$ \\
\hline $122-40-7$ & 23.819 & Alpha-Amylcinnamaldehyde ${ }^{\mathrm{c}, \mathrm{e}}$ & $10 \%$ & $20 \%$ & $0 \%$ & $0 \%$ & $0 \%$ & $0 \%$ \\
\hline $629-82-3$ & 24.037 & Dioctyl ether & $10 \%$ & $40 \%$ & $13 \%$ & $0 \%$ & $63 \%$ & $0 \%$ \\
\hline $6259-76-3$ & 24.383 & Hexyl salicylate ${ }^{\mathrm{c}, \mathrm{d}}$ & $50 \%$ & $70 \%$ & $63 \%$ & $60 \%$ & $75 \%$ & $33 \%$ \\
\hline $6765-39-5$ & 24.537 & 1-Heptadecene ${ }^{\mathrm{d}}$ & $0 \%$ & $60 \%$ & $25 \%$ & $40 \%$ & $63 \%$ & $33 \%$ \\
\hline $629-78-7$ & 24.657 & Heptadecane ${ }^{\text {c, e }}$ & $80 \%$ & $100 \%$ & $75 \%$ & $70 \%$ & $\mathbf{8 8 \%}$ & $100 \%$ \\
\hline $1921-70-6$ & 24.764 & Pristane & $80 \%$ & $90 \%$ & $75 \%$ & $20 \%$ & $38 \%$ & $100 \%$ \\
\hline $4536-30-5$ & 24.965 & Ethylene glycol monododecyl ether & $70 \%$ & $60 \%$ & $50 \%$ & $60 \%$ & $50 \%$ & $67 \%$ \\
\hline $101-86-0$ & 25.521 & Alpha-Hexylcinnamaldehyde c, d, e & $90 \%$ & $80 \%$ & $75 \%$ & $70 \%$ & $88 \%$ & $100 \%$ \\
\hline $112-88-9$ & 26.102 & 1-Octadecene & $10 \%$ & $0 \%$ & $13 \%$ & $10 \%$ & $0 \%$ & $0 \%$ \\
\hline $593-45-3$ & 26.209 & Octadecane $^{\mathrm{c}}$ & $70 \%$ & $100 \%$ & $50 \%$ & $70 \%$ & $88 \%$ & $100 \%$ \\
\hline $110-27-0$ & 26.564 & Isopropyl Myristate $^{\mathrm{c}}$ & $20 \%$ & $10 \%$ & $25 \%$ & $10 \%$ & $13 \%$ & $0 \%$ \\
\hline $106-02-5$ & 26.808 & Pentadecanolide $^{\mathrm{c}}$ & $40 \%$ & $30 \%$ & $0 \%$ & $30 \%$ & $25 \%$ & $0 \%$ \\
\hline $1222-05-5$ & 27.068 & Galaxolide $^{\mathrm{c}, \mathrm{e}}$ & $60 \%$ & $90 \%$ & $88 \%$ & $50 \%$ & $88 \%$ & $33 \%$ \\
\hline
\end{tabular}




\begin{tabular}{|c|c|c|c|c|c|c|c|c|}
\hline \multirow{3}{*}{ CAS no. } & \multirow{3}{*}{$\mathrm{RT}$ (min) } & \multirow{3}{*}{ Compound Name } & \multicolumn{6}{|c|}{ Frequency of Occurrence in Subjects } \\
\hline & & & \multicolumn{3}{|c|}{ Females $(n=30)$} & \multicolumn{3}{|c|}{ Males $(n=30)$} \\
\hline & & & $\begin{array}{l}18-30 \\
\text { years }\end{array}$ & $\begin{array}{l}\text { 35-50 } \\
\text { years }\end{array}$ & $\begin{array}{c}55+ \\
\text { years }\end{array}$ & $\begin{array}{l}18-30 \\
\text { years }\end{array}$ & $\begin{array}{l}35-50 \\
\text { years }\end{array}$ & $\begin{array}{c}55+ \\
\text { years }\end{array}$ \\
\hline $36653-82-4$ & 27.274 & 1-Hexadecanol c, d & $60 \%$ & $70 \%$ & $38 \%$ & $40 \%$ & $25 \%$ & $33 \%$ \\
\hline 52253-93-7 & 27.475 & Homomenthyl salicylate & $60 \%$ & $70 \%$ & $50 \%$ & $50 \%$ & $75 \%$ & $67 \%$ \\
\hline $112-39-0$ & 27.838 & Methyl palmitate ${ }^{c, d}$ & $70 \%$ & $80 \%$ & $63 \%$ & $40 \%$ & $75 \%$ & $100 \%$ \\
\hline $142-91-6$ & 28.821 & Isopropyl Palmitate ${ }^{\mathrm{c}, \mathrm{d}}$ & $10 \%$ & $40 \%$ & $25 \%$ & $20 \%$ & $38 \%$ & $0 \%$ \\
\hline
\end{tabular}

a. $\mathrm{RT}=$ Retention Time

b. Compounds with a frequency of occurrence equal or higher to $50 \%$ of subjects are denoted in bold

c. Compounds of possible exogenous origin - reported as being typically used as fragrance ingredients in consumer goods ${ }^{122}$

d. Reported by previous studies as present in human skin emanations, human VOC profiles, characteristic axillary odors or as individual axillary marker compounds $\mathrm{s}^{21,33,36-38,123}$

e. Compounds were also quantified in unscented deodorant or soap blanks 


\subsection{Gender comparison: a quantitative analysis of VOC profiles by age group}

A comparison of the odor profiles from East Asian females and males was established in the same way that it was done for the Hispanic and Caucasian individuals. A quantitative analysis by age group was pursued to differentiate the genders in each group by using all 27 compounds as discrimination criteria (Figure 87 - Figure 89). The average amounts extracted per VOC, for females and males from a same age group, were subjected to a visual evaluation in all age categories. Secondary lists were also created in this population to be used for gender discrimination in each of the age categories. Subsequently, the potential of both VOC lists to discriminate individuals of different

gender, within each age group, was compared. The comparison intended to evaluate a generalized profile for each gender in the East Asian population. This evaluation would facilitate the identification of potential VOC biomarkers for the classification of East Asian individuals by gender. 


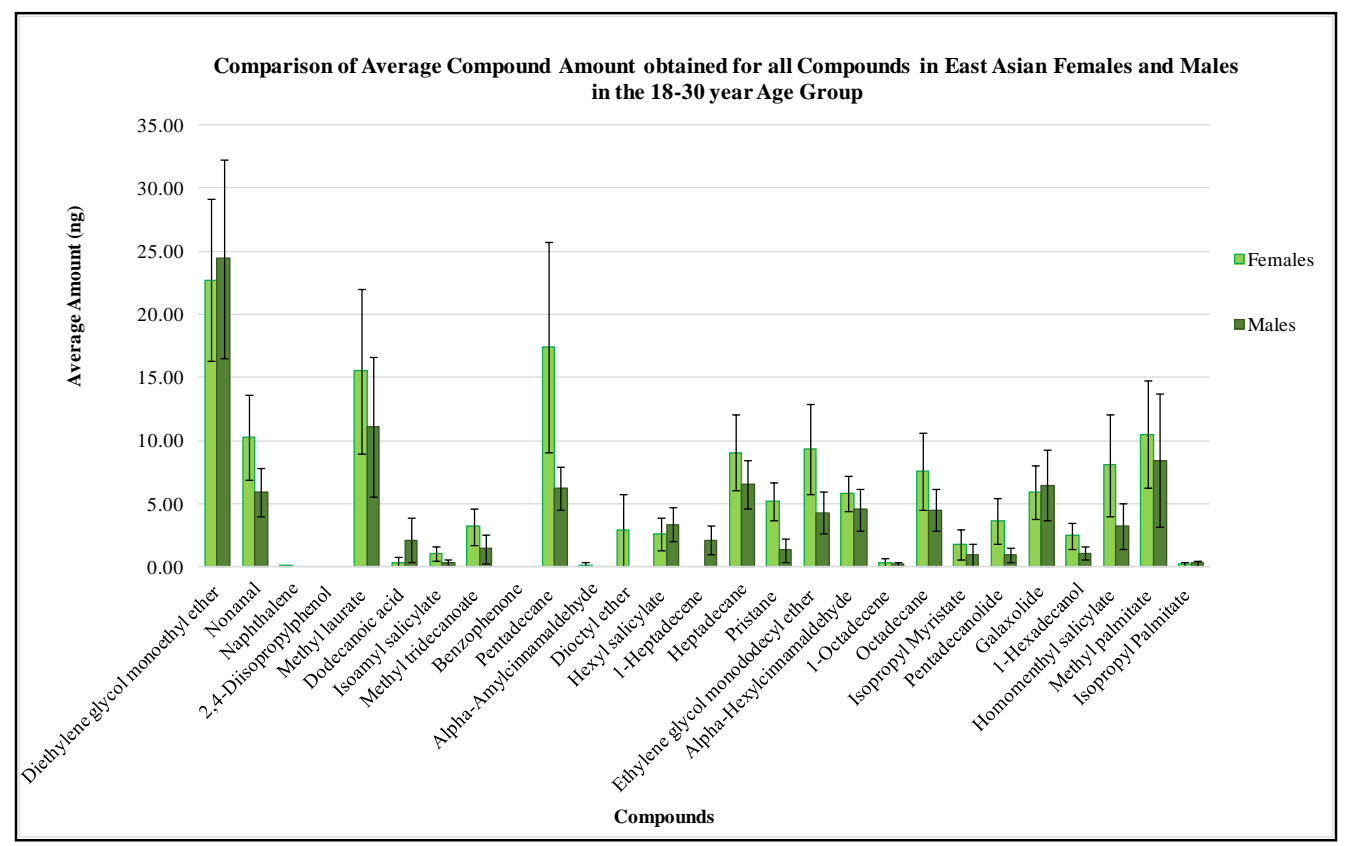

Figure 87: Comparison of the average extracted amounts obtained for all 27 VOCs in 18-30 year old East Asian females and males

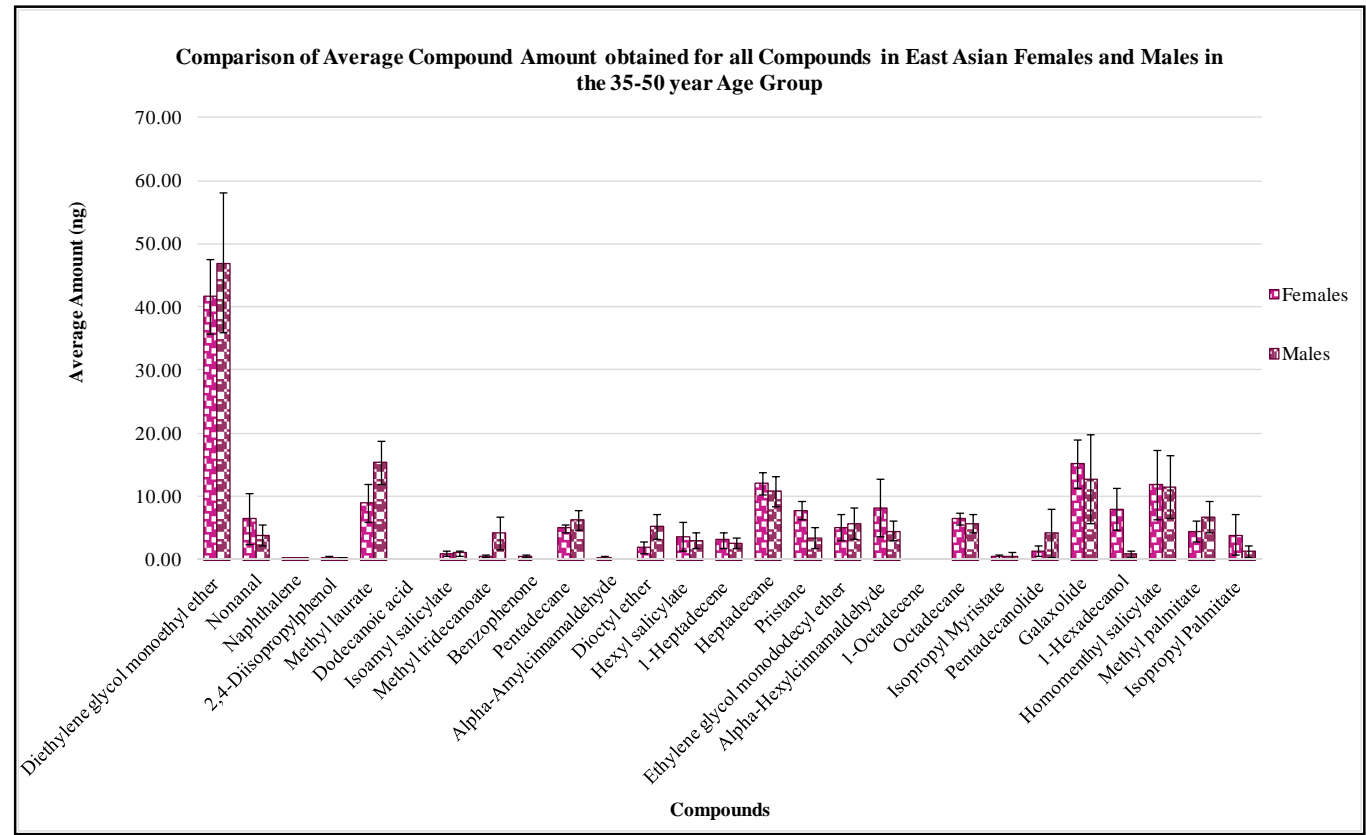

Figure 88: Comparison of the average extracted amounts obtained for all 27 VOCs in 35-50 year old East Asian females and males 


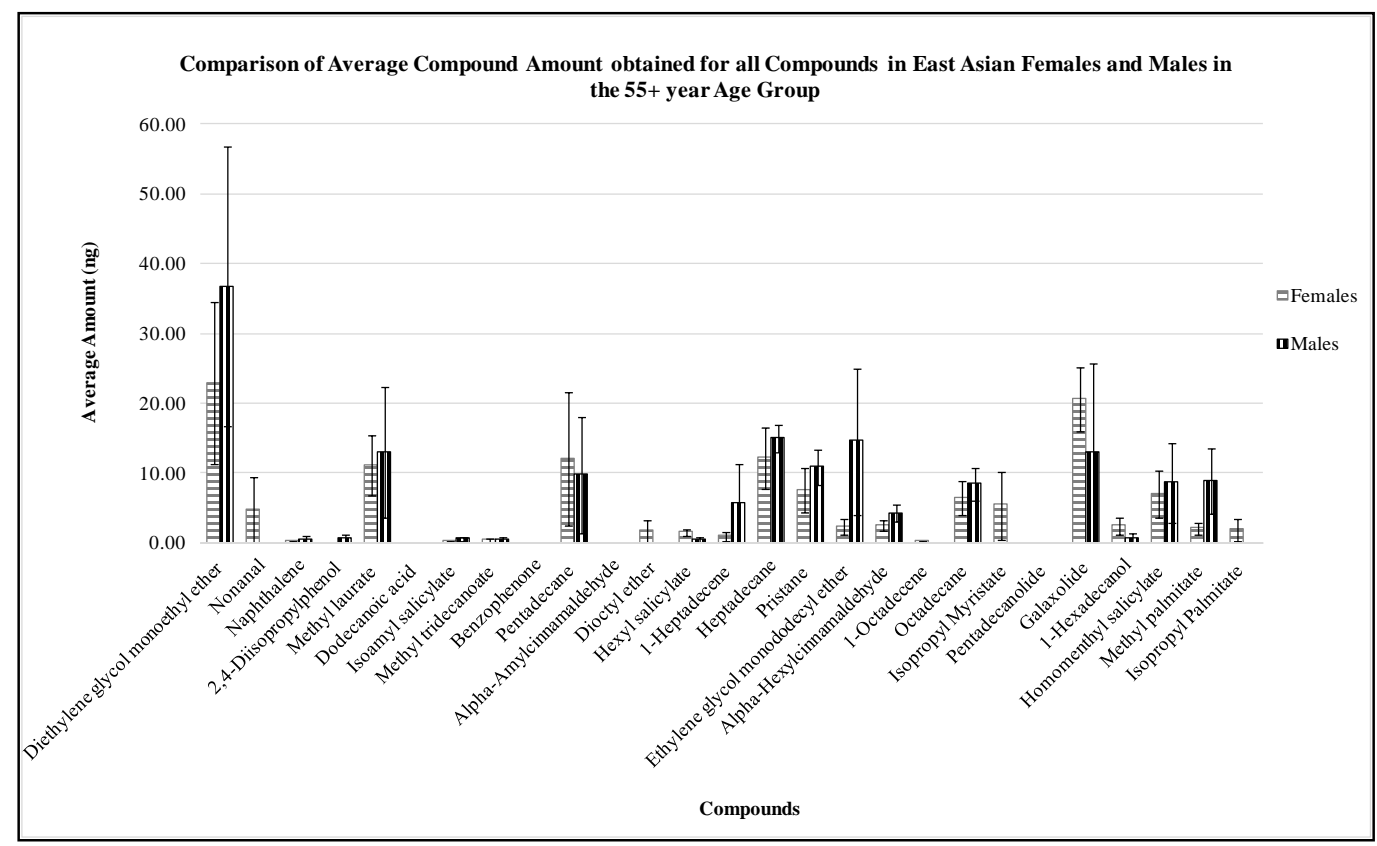

Figure 89: Comparison of the average extracted amounts obtained for all 27 VOCs in 55+ year old East Asian females and males

\section{8-30 year Age Group}

From the initial list of 27 compounds, a total of 13 compounds were included in the secondary list for gender differentiation in the 18-30 year age group (Table 24). Out of the 13 compounds selected, only two VOCs showed higher average amounts in males, while the rest did in females. This shows that, as it had been observed in other groups, females in this age group also had a tendency to show higher average amounts than males for the great majority of compounds.

The PCA performed to evaluate the VOC lists as discrimination criteria for 18-30 year old females and males, provided limited insight on the possibility of distinguishing East Asian individuals by gender. The score plot created for the evaluation of the initial compound list (27 VOCs) portrayed individuals of both genders being concentrated and mixed in the center of the score plot (Figure 90). No clear clusters were identified as 
being formed by any of the groups. Similarly, when the PCA score plot was made using only the secondary list VOCs, a total separation between the groups was still not achieved (Figure 91 - left). A decrease in the spread of male individuals led to the formation of a clear cluster for this gender. However, this change did not cause enough separation between females and males to be able to differentiate individuals from both groups. The first and second principal components (PC1 and PC2) for this last score plot represented a $30.4 \%$ and $16.2 \%$ of the data's variation, respectively. These percentages showed an increase in variation from that exhibited in the initial score plot (PC1: 26.3\% and PC2: 12.4\%). However, the appearance of each of the score plots demonstrates that the increase in variation was not enough to induce an effective differentiation of the genders. The results obtained suggest that further investigation is necessary in order to possibly improve the discrimination criteria (compound lists). This would enable continuing to explore if there are really differences, between females and males of this age group, that are significant enough to cause gender differentiation.

A loading plot was created on the basis of the secondary list to determine which VOCs showed the most influence in differentiating the groups under consideration (Figure 91 - right). According to the plot, the compounds leading the variation observed in PC1 were Pentadecane, Pentadecanolide, Homomenthyl salicylate, 1-Hexadecanol and Methyl tridecanoate (in decreasing order of influence). In the case of the variation observed in PC2, Naphthalene, Dioctyl ether, Pristane, Alpha-Amylcinnamaldehyde, and Pentadecane were found to be the VOCs with most impact. 


\section{5-50 year Age Group}

Out of the initial list of 27 compounds, 35-50 year old females portrayed a higher average amount for 15 compounds, while males in the same age category did for ten. Also, out of the 27 compounds, only nine VOCs were selected to form part of the secondary list that would be used to evaluate the differentiation of females and males in this age group (Table 25). From the VOCs in the secondary list, females showed a higher average amount than males in six compounds. This shows that there is also a tendency for females in this age group to predominate over males in terms of the abundance of most of the compounds present in odor profiles.

The initial PCA for this age group, performed including all 27 VOCs as variables, showed the bulk of female and male subjects forming overlapping clusters towards the center of the score plot (Figure 92). As a result, no separation between the two genders was made evident. On the other hand, after only considering the compounds from the secondary list as criteria for group differentiation, an increase in the spread of both clusters was observed (Figure 93 - left). In specific, the female cluster seemed to become less defined under the influence of the nine secondary list VOCs. This response seemed to increase the separation between females and males, but at the expense of also increasing the scatter amongst female subjects. Therefore, in the case of 35-50 year old East Asians, the continuous overlap between the female and male clusters, and the spread between subjects of a same group, did not allow a definite separation between the genders. The initial PCA score plot showed a variation in PC1 of $20.3 \%$ and $15.4 \%$ in PC2. On the other hand, the PCA score plot obtained when using compounds from the secondary list showed a $29.6 \%$ and $25.1 \%$ of variation, for PC1 and PC2 respectively. 
The increase in variation in both principal components explains the slight separation achieved between females and males. However, the variation achieved under the influence of the secondary list compounds alone, seemed to remain insufficient to achieve a total separation of the groups. The loading plot obtained for the secondary list analysis (Figure 93 - right) reported that 1-Hexadecanol, Pristane, Isopropyl Palmitate, Alpha-Amylcinnamaldehyde, and Methyl tridecanoate provided the highest level of variation between the groups (in decreasing order) for PC1. Moreover, it revealed that Pentadecanolide, Methyl tridecanoate, Dioctyl ether, 2,4-Diisopropylphenol, and Isopropyl Palmitate (also in decreasing order) were the most influential compounds in the variation of PC2.

55+ year Age Group

The quantitative analysis of East Asian females and males in the 55+ year category showed that, out of an initial list of 27 compounds, females in this group showed a higher average amount for nine compounds, and males did for 13. A total of 13 compounds were observed to have a $100 \%$ or higher difference in average amount, between males and females, which led to the selection of such compounds for further use as discrimination criteria between the genders in this age group (Table 26). From the list of selected compounds, females showed a higher average amount than males in the case of seven VOCs. This observation confirms that, out of both genders in the East Asian age groups, all females tend to display higher VOC abundances in their odor profiles than males. It is important to note that, contrary to this finding, in both the case of 55+ year 
old Caucasians and Hispanics, males showed a tendency to have predominating abundances for most VOCs in comparison to females.

The PCA score plot performed taking into consideration all initial 27 compounds, displayed the majority of female and male subjects overlapping and forming one main cluster towards the center of the quadrants (Figure 94). As a result, no separation was observed between the two genders. However, once the secondary compound list was considered, an increase in the spread of individuals from both groups was seen in the new score plot (Figure 95- left). Even though a completely clear separation was not possible, there was an improvement in the ability to differentiate individuals of different gender. The female cluster did not seem defined; however, most female individuals appeared towards the right side of the score plot, while male individuals remained towards the left side. An important fact concerning the nature of the score plots in this age group is that, contrary to the plots obtained for other groups, these consider a smaller number of individuals. Therefore, in this case, the reduced sample size limits the certitude of the analysis. For this reason, these findings need to be confirmed under the scope of a larger sample size. Despite the sample size limitations, PCA was able to display an enhancement in the separation of the groups. The initial score plot presented a variation in PC1 of $26.8 \%$ and $16.3 \%$ in PC2, while the PCA score plot obtained with the secondary list VOCs showed a $22.8 \%$ and $20.8 \%$ of variation, in PC1 and PC2 respectively. The variation percentages from the secondary list score plot demonstrate that the improvements observed for gender differentiation were mostly a result of the impact of certain compounds in the second principal component. However, employing a greater sample size to confirm the efficiency of the secondary list VOCs, as 
discrimination criteria for 55+ year old East Asian females and males, continues to be suggested. According to the loading plot (Figure 95 - right), Isoamyl salicylate, 1Heptadecene, Ethylene glycol monododecyl ether, Dioctyl ether, and Nonanal provided the highest level of variation between the groups (in decreasing order) in PC1. On the other hand Isopropyl Palmitate, Isopropyl Myristate, 1-Octadecene, Hexyl salicylate, and Naphthalene were found to be the most influential compounds in the variation of PC2 (also in decreasing order).

Table 24: Average VOC amount for secondary list compounds in 18-30 year old East Asian females and males

\begin{tabular}{|c|c|c|}
\hline $\begin{array}{c}\text { Secondary List of Compounds for } \\
\text { Males and Females of 18-30 years }\end{array}$ & $\begin{array}{c}\text { Female Average } \\
\text { Compound } \\
\text { Amount (ng) }\end{array}$ & $\begin{array}{c}\text { Male Average } \\
\text { Compound } \\
\text { Amount (ng) }\end{array}$ \\
\hline Naphthalene & 0.06 & 0 \\
\hline Dodecanoic acid & 0.39 & 2.14 \\
\hline Isoamyl salicylate & 1.09 & 0.39 \\
\hline Methyl tridecanoate & 3.20 & 1.43 \\
\hline Pentadecane & 17.40 & 6.24 \\
\hline Alpha-Amylcinnamaldehyde & 0.19 & 0 \\
\hline Dioctyl ether & 2.88 & 0 \\
\hline 1-Heptadecene & 0 & 2.14 \\
\hline Pristane & 5.22 & 1.35 \\
\hline Ethylene glycol monododecyl ether & 9.34 & 4.28 \\
\hline Pentadecanolide & 3.69 & 0.94 \\
\hline 1-Hexadecanol & 2.50 & 1.11 \\
\hline Homomenthyl salicylate & 8.06 & 3.19 \\
\hline
\end{tabular}


Table 25: Average VOC amount for secondary list compounds in 35-50 year old East Asian females and males

\begin{tabular}{|c|c|c|}
\hline $\begin{array}{c}\text { Secondary List of Compounds for } \\
\text { Males and Females of 35-50 years }\end{array}$ & $\begin{array}{c}\text { Female Average } \\
\text { Compound } \\
\text { Amount (ng) }\end{array}$ & $\begin{array}{c}\text { Male Average } \\
\text { Compound } \\
\text { Amount (ng) }\end{array}$ \\
\hline 2,4-Diisopropylphenol & 0.33 & 0.15 \\
\hline Methyl tridecanoate & 0.55 & 4.19 \\
\hline Benzophenone & 0.53 & 0 \\
\hline Alpha-Amylcinnamaldehyde & 0.26 & 0 \\
\hline Dioctyl ether & 1.88 & 5.19 \\
\hline Pristane & 7.81 & 3.39 \\
\hline Pentadecanolide & 1.38 & 4.13 \\
\hline 1-Hexadecanol & 8.02 & 0.87 \\
\hline Isopropyl Palmitate & 3.89 & 1.23 \\
\hline
\end{tabular}

Table 26: Average VOC amount for secondary list compounds in 55+ year old East Asian females and males

\begin{tabular}{|c|c|c|}
\hline $\begin{array}{c}\text { Secondary List of Compounds for } \\
\text { Males and Females of 55+ years }\end{array}$ & $\begin{array}{c}\text { Female Average } \\
\text { Compound } \\
\text { Amount (ng) }\end{array}$ & $\begin{array}{c}\text { Male Average } \\
\text { Compound } \\
\text { Amount (ng) }\end{array}$ \\
\hline Nonanal & 4.66 & 0 \\
\hline Naphthalene & 0.15 & 0.45 \\
\hline 2,4-Diisopropylphenol & 0 & 0.58 \\
\hline Isoamyl salicylate & 0.14 & 0.57 \\
\hline Dioctyl ether & 1.63 & 0 \\
\hline Hexyl salicylate & 1.41 & 0.39 \\
\hline 1-Heptadecene & 0.88 & 5.63 \\
\hline Ethylene glycol monododecyl ether & 2.31 & 14.52 \\
\hline 1-Octadecene & 0.10 & 0 \\
\hline Isopropyl Myristate & 5.36 & 0 \\
\hline 1-Hexadecanol & 2.36 & 0.64 \\
\hline Methyl palmitate & 2.11 & 8.85 \\
\hline Isopropyl Palmitate & 1.86 & 0 \\
\hline
\end{tabular}




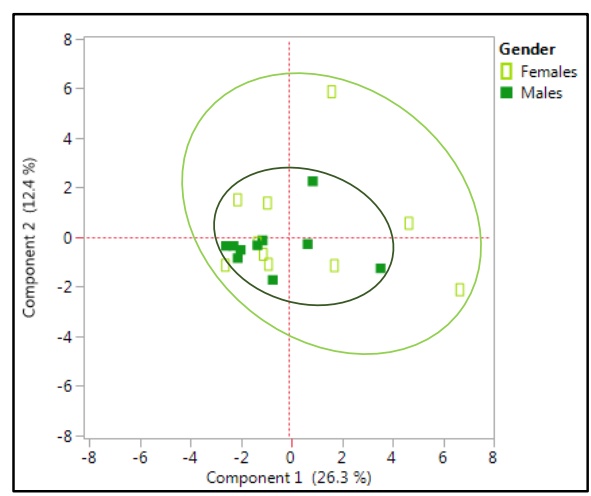

Figure 90: PCA score plot of 18-30 year old East Asian females and males including all VOCs
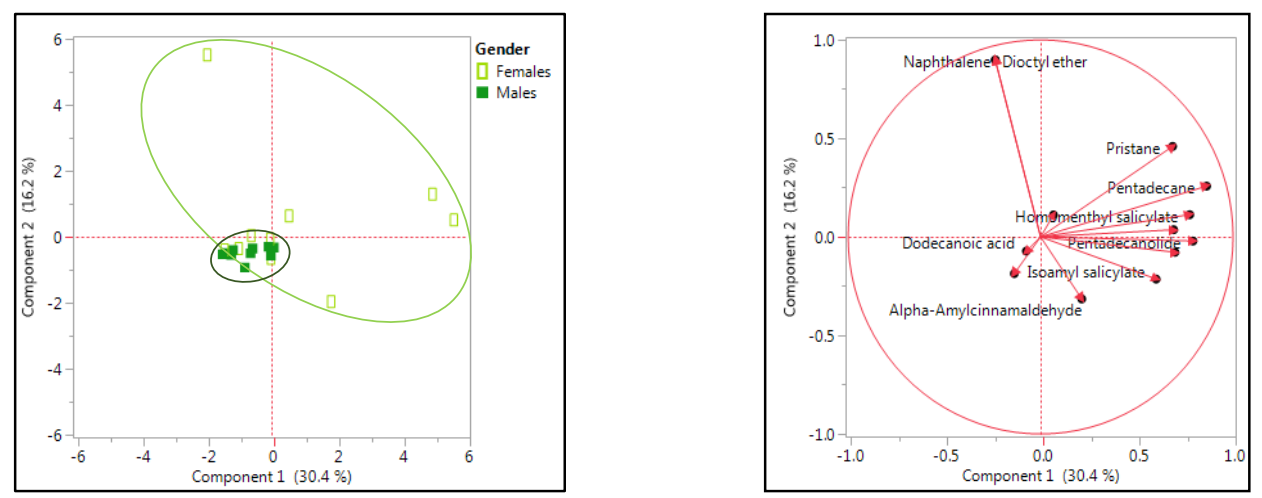

Figure 91: PCA score plot (left) and loading plot (right) of 18-30 year old East Asian females and males including VOCs from secondary list

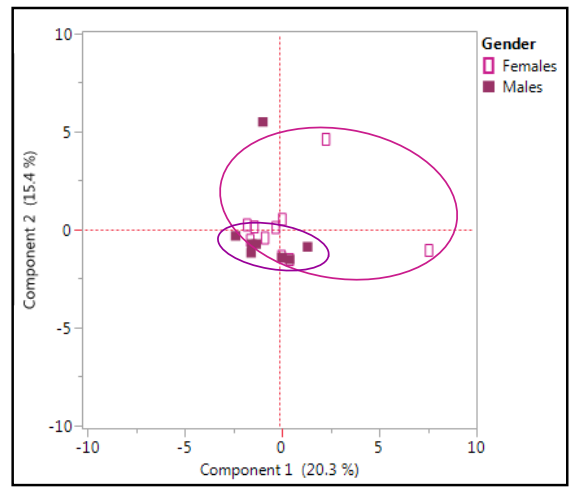

Figure 92: PCA score plot of 35-50 year old East Asian females and males including all VOCs 

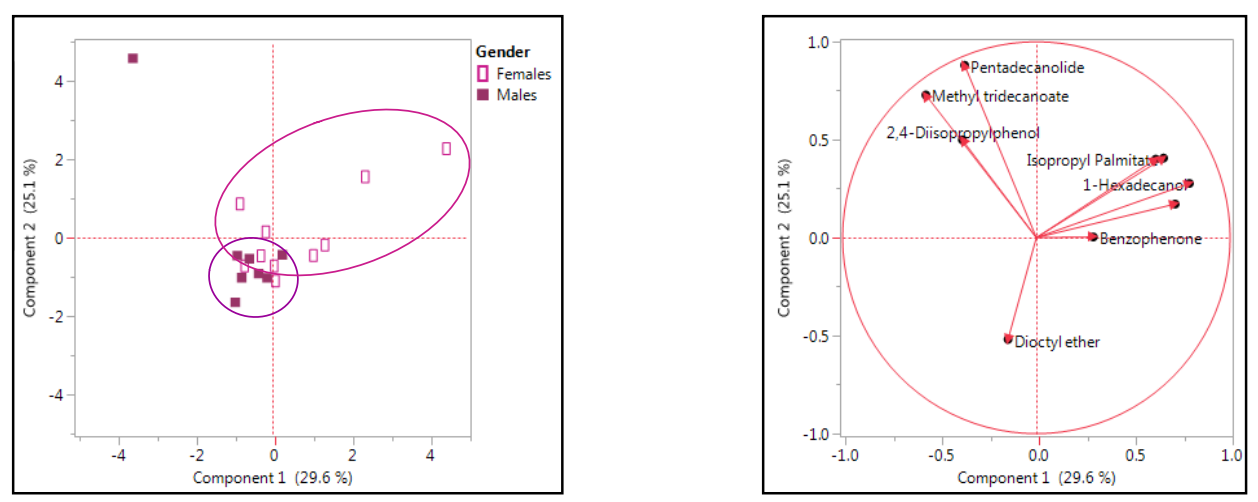

Figure 93: PCA score plot (left) and loading plot (right) of 35-50 year old East Asian females and males including VOCs from secondary list

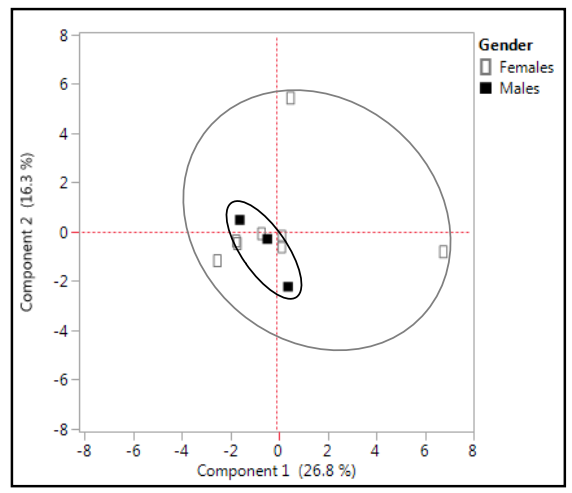

Figure 94: PCA score plot of 55+ year old East Asian females and males including all VOCs
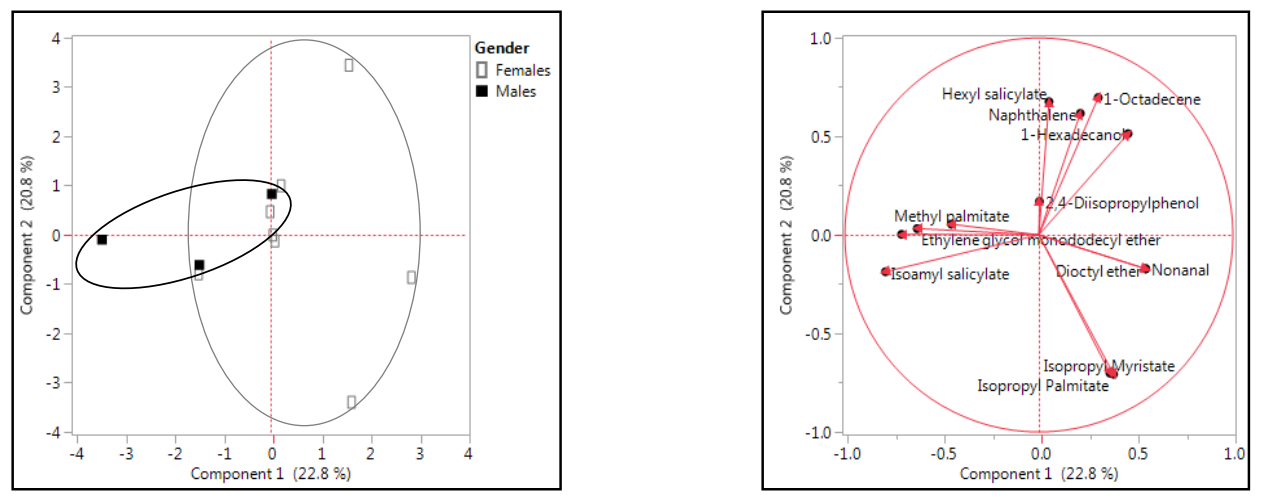

Figure 95: PCA score plot (left) and loading plot (right) of 55+ year old East Asian females and males including VOCs from secondary list 


\subsection{Age group comparison: a quantitative analysis of VOC profiles by gender}

The variation amongst scent profiles from different age groups was assessed for each gender in the East Asian population. The average amount extracted for each compound, in each of the different age groups, (Figure 96 - Figure 97) was evaluated for each gender. A comparison was established for sets of two age groups at a time, and tailored secondary lists were created for age group discrimination amongst females and males. As a result of this comparison, it was possible to identify the impact of each VOC in the scent profile of an East Asian individual in accordance to their age group. These results facilitated the evaluation of VOCs as potential age biomarkers to classify East Asian individuals.

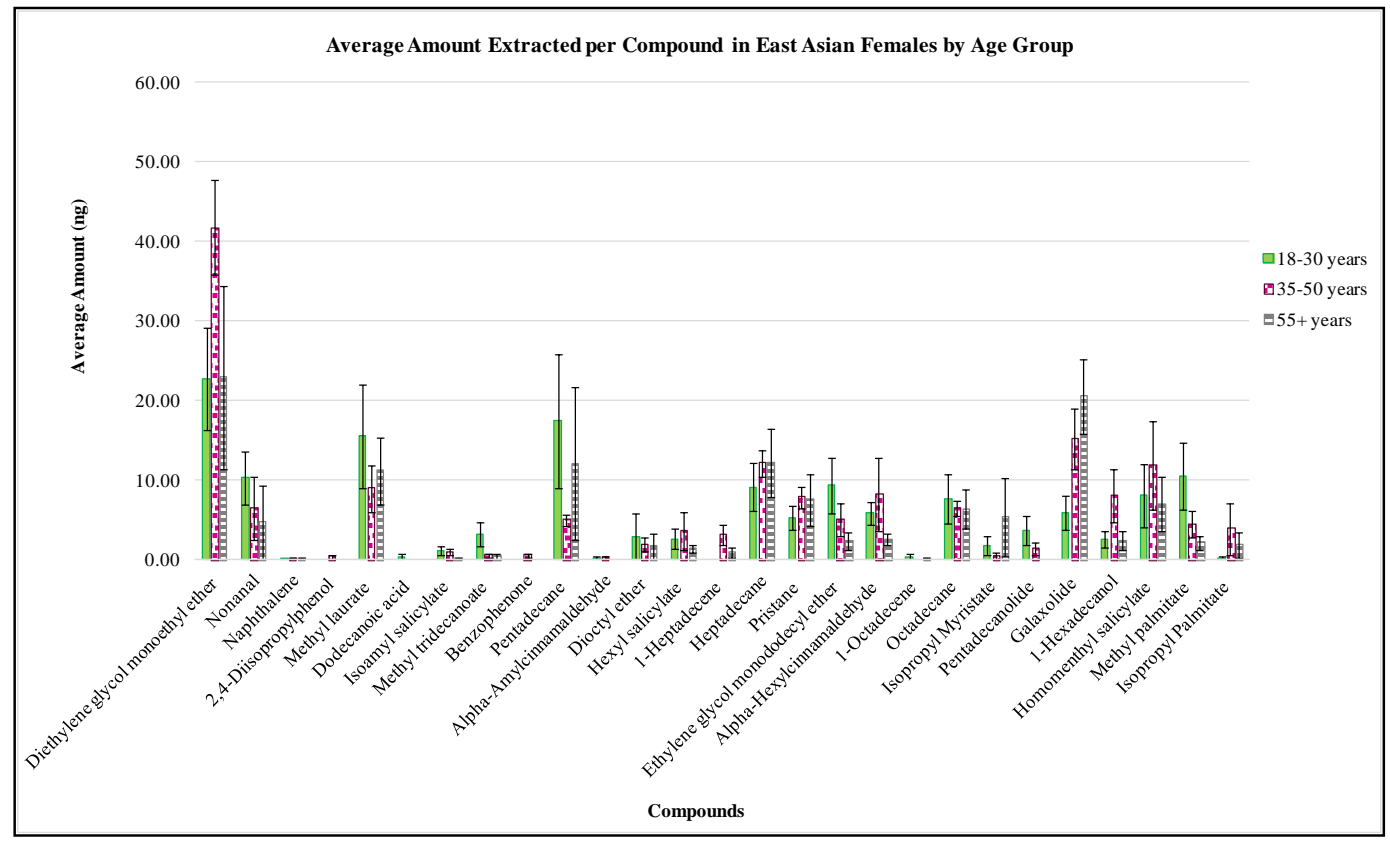

Figure 96: Comparison of the average extracted amounts obtained for all 27 VOCs in East Asian females of all age groups 


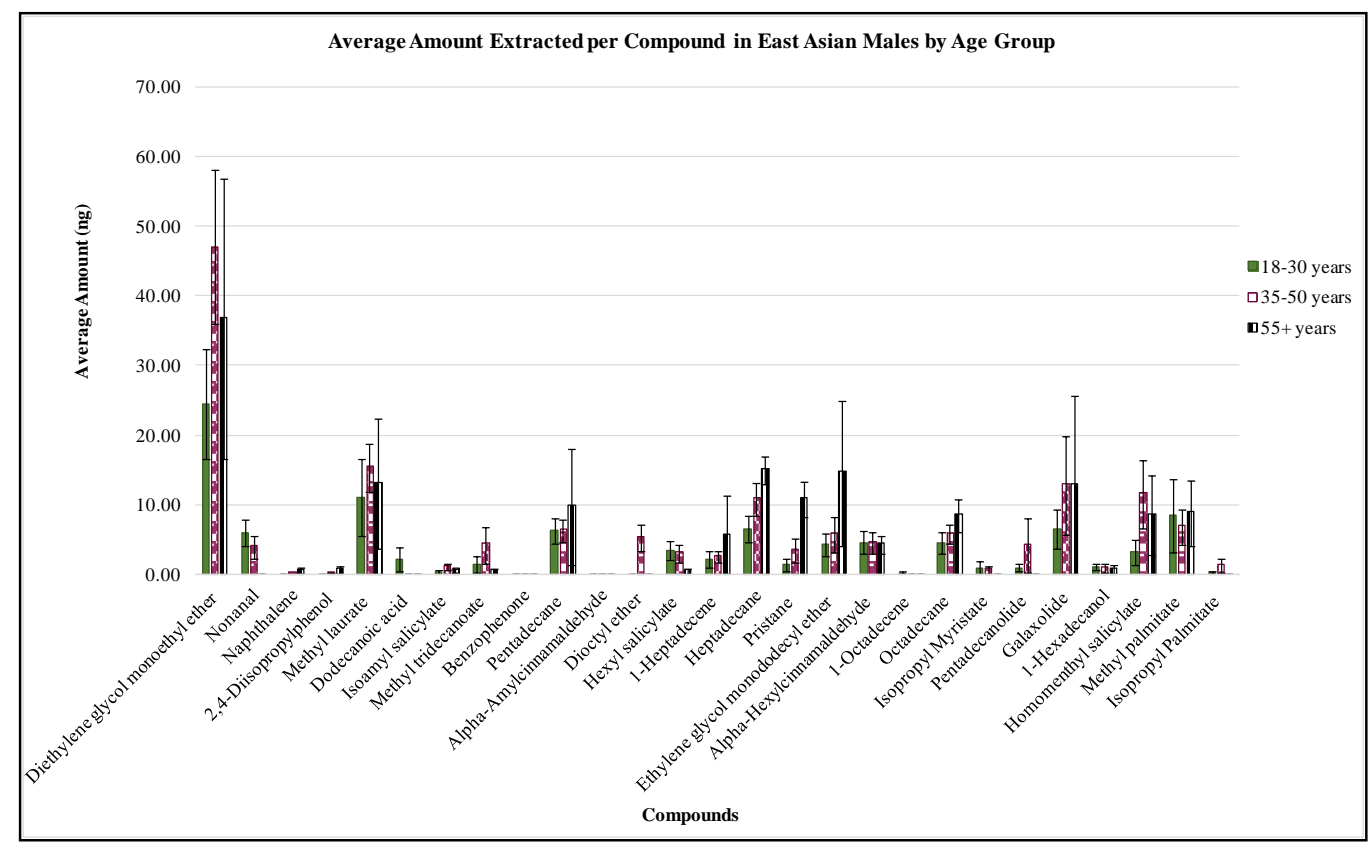

Figure 97: Comparison of the average extracted amounts obtained for all 27 VOCs in East Asian males of all age groups

\section{Comparison of Females of Different Age Groups}

Through the assessment of female scent profiles by age group, the18-30 year group was observed to have the highest average amount for the majority of compounds in the initial VOC list. The 35-50 year olds followed, by showing the highest average amount in 11 compounds, while the 55+ year old group showed the highest amount for four compounds: Naphthalene, Heptadecane, and Isopropyl Myristate and Galaxolide. A total of 19 compounds (Figure 98) were selected to take part in the secondary lists that would be used to compare female age group sets. From these 19 VOCs, the 18-30 year age group showed the highest average amount in nine compounds, while the 35-50 year olds and 55+ year olds showed the highest amounts for eight and two compounds, respectively. Hence, in both instances, younger females displayed predominating average 
amounts over the other groups under study. Previous research showed that Hexyl salicylate and Alpha-Hexylcinnamaldehyde, are significantly more abundant in younger subjects (19-40 years). ${ }^{21}$ Nevertheless, according to my study, these two compounds display greater amounts in 35-50 year old females; rather than in the 18-30 year old group. In the East Asian population these two compounds seemed to have peak abundance in the odor profiles of females in the middle age group and continued to decrease as age was above 50 years. Moreover, the same type of observation was made for Nonanal, previously stated as a marker compound for individuals of increased age. ${ }^{21}$ In this study, out of all groups, Nonanal was seen to be both less occurent and abundant in 55+ year old East Asian females.

The secondary list created for the comparison of 18-30 year and 55+ year old females consisted specifically of 14 VOCs, while the lists to compare the 18-30 year and 35-50 year groups, and the 35-50 year and 55+ year groups, consisted of 13 and 15 VOCs respectively (Table 27). Variation was noted in the composition of each age group set's secondary list. Only five compounds were selected as being simultaneously different across the three age groups: 1-Heptadecene, Isopropyl Myristate, Pentadecanolide, Methyl palmitate, and Isopropyl Palmitate. Once again, this type of observation continued to suggest that despite those differences that are common to all groups, the basis of group differentiation is more dependent on a combination of VOCs. 


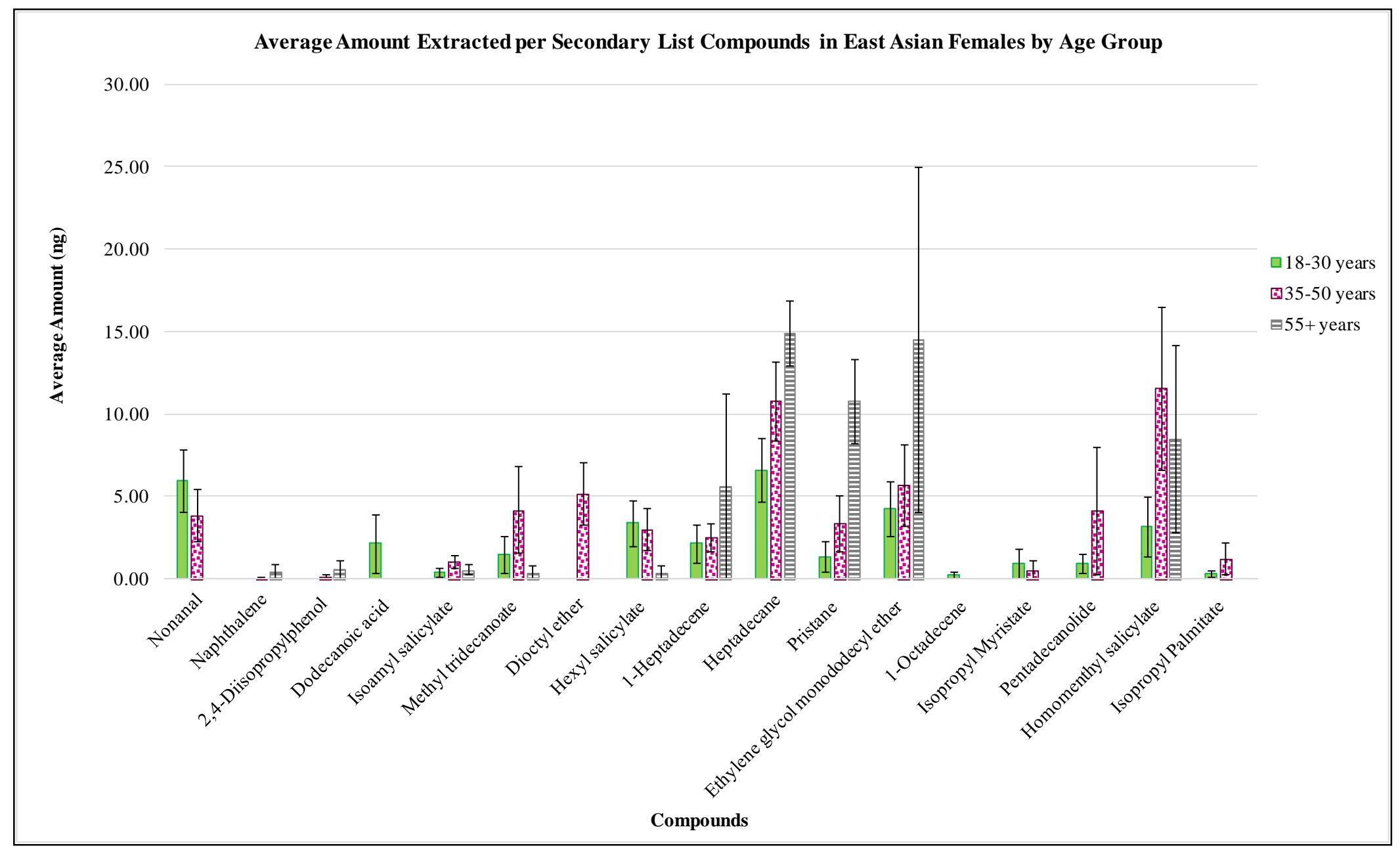

Figure 98: Comparison of average extracted amounts obtained for the compounds in the VOC secondary lists of East Asian females from different age groups 
Table 27: Compounds considered in secondary lists made for comparing East Asian females of different age groups

\begin{tabular}{|c|c|c|c|}
\hline $\begin{array}{c}\text { Compounds included in } \\
\text { Secondary Lists for Females }\end{array}$ & $\begin{array}{c}18-30 \text { vs. } \\
35-50 \text { years }\end{array}$ & $\begin{array}{l}18-30 \text { vs. } \\
55+\text { years }\end{array}$ & $\begin{array}{l}35-50 \text { vs. } \\
55+\text { years }\end{array}$ \\
\hline Nonanal & & $\mathrm{X}$ & \\
\hline 2,4-Diisopropylphenol & $\mathrm{X}$ & & $\mathrm{X}$ \\
\hline Dodecanoic acid & $\mathrm{X}$ & $\mathrm{X}$ & \\
\hline Isoamyl salicylate & & $\mathrm{X}$ & $\mathrm{X}$ \\
\hline Methyl tridecanoate & $\mathrm{X}$ & $\mathrm{X}$ & \\
\hline Benzophenone & $\mathrm{X}$ & & $\mathrm{X}$ \\
\hline Pentadecane & $\mathrm{X}$ & & $\mathrm{X}$ \\
\hline Alpha-Amylcinnamaldehyde & & $\mathrm{X}$ & $\mathrm{X}$ \\
\hline Hexyl salicylate & & & $\mathrm{X}$ \\
\hline 1-Heptadecene & $\mathrm{X}$ & $\mathrm{X}$ & $\mathrm{X}$ \\
\hline Ethylene glycol monododecyl ether & & $\mathrm{X}$ & $\mathrm{X}$ \\
\hline Alpha-Hexylcinnamaldehyde & & $\mathrm{X}$ & $\mathrm{X}$ \\
\hline 1-Octadecene & $\mathrm{X}$ & $\mathrm{X}$ & $\mathrm{X}$ \\
\hline Isopropyl Myristate & $\mathrm{X}$ & $\mathrm{X}$ & $\mathrm{X}$ \\
\hline Pentadecanolide & $\mathrm{X}$ & $\mathrm{X}$ & $\mathrm{X}$ \\
\hline Galaxolide & $\mathrm{X}$ & $\mathrm{X}$ & \\
\hline 1-Hexadecanol & $\mathrm{X}$ & & $\mathrm{X}$ \\
\hline Methyl palmitate & $\mathrm{X}$ & $\mathrm{X}$ & $\mathrm{X}$ \\
\hline Isopropyl palmitate & $\mathrm{X}$ & $\mathrm{X}$ & $\mathrm{X}$ \\
\hline
\end{tabular}

Principal component analysis score plots and loading plots were made to evaluate the potential discrimination of East Asian females on the basis of their age group. The first score plot was made for the 18-30 year and the 55+ year groups using all 27 variables (Figure 99). Despite showing a slight separation between some of the females sampled in both groups, the score plot still showed an overlap between females of opposite groups. It was evident that the slight separation being observed did not result enough to consider the complete set of variables the most efficient discrimination alternative for these age groups. On the other hand, the score plot made considering the secondary list VOCs as differentiation variables, showed female subjects from each 
group agglomerating according to their age group (Figure 100 - left). A slight overlap between the clusters was still observed in the center of the score plot, yet it was still possible to group East Asian females in accordance to their age. Clear clusters of female subjects were formed and this caused the separation between the age groups to be enhanced.

The percentage of variation in PC1 and PC2, for both the initial score plot and the secondary list score plot, also reflected changes. The first principal component (PC1) increased its variation from $21.1 \%$ to $29.3 \%$, while the percentage in PC2 was seen to decrease from $18.8 \%$ to $16.4 \%$. These changes in percentage suggest that the variation observed in PC1 had a more significant impact in the discrimination enhancement between the groups. The compounds stated by the loading plot as most influential in PC1's increased variation (in decreasing order of influence) were Pentadecanolide, Methyl tridecanoate, Alpha-Hexylcinnamaldehyde, Methyl palmitate and Isoamyl salicylate (Figure 100 - right). In the same token, the compounds found to be more influential on PC2 were Isopropyl Palmitate, Isopropyl Myristate, Galaxolide, Ethylene glycol monododecyl ether, and Dodecanoic acid (also in decreasing order).

On the other hand, in the case of the 18-30 year and 35-50 year age groups, the PCA that included all variables showed the majority of the females from both groups clustering altogether in the middle of the score plot (Figure 101). A separation between the groups was not observed. Nevertheless, after reevaluating the score plot with only the compounds from the secondary list, an enhancement was noted in group discrimination (Figure 102 - left). East Asian females from the 18-30 and 35-50 year groups showed a tendency to cluster around those of their corresponding age group, which allowed a fairly 
clear distinction between the groups in the score plot. It was observed that the spread of the 35-50 year group was reduced, and that served as the main cause for cluster separation. Although there was one female from the 35-50 year group that remained overlapping with the opposite cluster, it is possible that with a larger sample size this type of observation can get neglected. The variation percentages obtained for the initial score plot were $21.2 \%$ for PC1 and $15.6 \%$ for PC2, while an increase was observed in the variation percentages obtained for the second score plot. As only the compounds from the secondary list were considered as discrimination variables, PC1 and PC2 showed 26.8\% and $16.9 \%$ variation. The increase in variation for both principal components favored the differentiation of females from the two age groups. Moreover, the loading plot obtained along with this last score plot (Figure 102 - right) presented the most influential compounds in the variation of PC1 as being Methyl tridecanoate, Methyl palmitate, Pentadecane, Pentadecanolide, and 1-Octadecene (in decreasing order). On the other hand, those of highest influence in PC2 were observed to be Isopropyl Myristate, 1Hexadecanol, 1-Octadecene, Isopropyl Palmitate, and 1-Heptadecene (also in decreasing order).

The comparison of the 35-50 and 55+ year age groups taking into account all initial compounds showed that, although the majority of the females in both age groups appeared mixed towards the center of the plot, there was still a slight tendency for them to cluster around those of their same group (Figure 103). Females in the 55+ year age group seemed to incline more towards the left of the score plot, while 35-50 year old females appeared more towards the right of 55+ year old females. However, contrary to the results from other age group comparisons, the score plot obtained from the evaluation 
of these two groups when considering the secondary list VOCs did not show any discrimination enhancement (Figure 104 - left). The cluster formed by 55+ year old females appeared to reduce its spread, which created a more concise cluster for that group. However, this also made the cluster overlap more with 35-50 year old females. Therefore, in the case of East Asian females in the 35-50 year and 55+ year age groups, it was not possible to achieve a discrimination enhancement between the groups by adjusting the discrimination variables. The variation percentages obtained in the initial score plot were $22.1 \%$ in PC1 and $19.6 \%$ in PC2. The second score plot, which only included VOCs from the secondary list showed 31.0\% and 24.3\% for PC1 and PC2. Once again, it was noted that the variation percentages of both principal components underwent an increase when reducing the number of compounds as discrimination criteria. However, it appears that in the case of these score plots, the changes in variation from the principal components mainly affected the formation of a tighter cluster for $55+$ year old females. These changes were therefore not enough to allow an efficient group differentiation. Despite of this observation, it was noted that the compounds with heavier impact in PC1's variation were Pentadecanolide, Alpha-Amylcinnamaldehyde, AlphaHexylcinnamaldehyde, Methyl palmitate, and 1-Hexadecanol in decreasing order (Figure 104 - right). In the same token, the loading plot showed that those of more impact in PC2 were Ethylene glycol monododecyl ether, Isopropyl Palmitate, 1-Heptadecene, Hexyl salicylate, and Methyl palmitate. Therefore, further evaluation of these compounds, in addition to other possible discrimination variables, could lead to an improvement in the differentiation capabilities for East Asian females in the 35-50 and 55+ year age groups. 


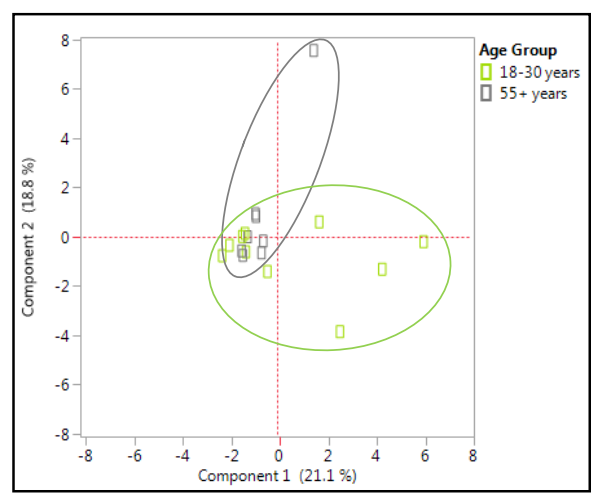

Figure 99: PCA score plot of 18-30 vs. 55+ year old East Asian females including all VOCs
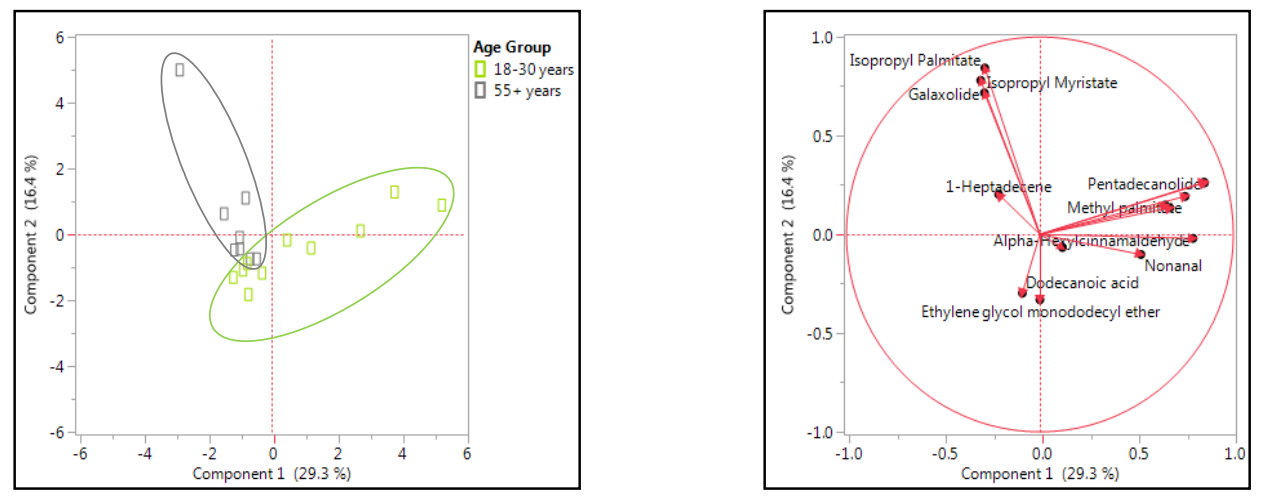

Figure 100: PCA score plot (left) and loading plot (right) of 18-30 vs. 55+ year old East Asian females including VOCs from secondary list

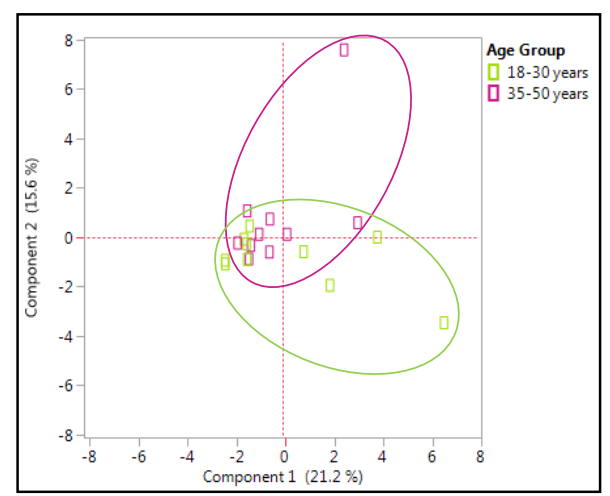

Figure 101: PCA score plot of 18-30 vs. 35-50 year old East Asian females including all VOCs 

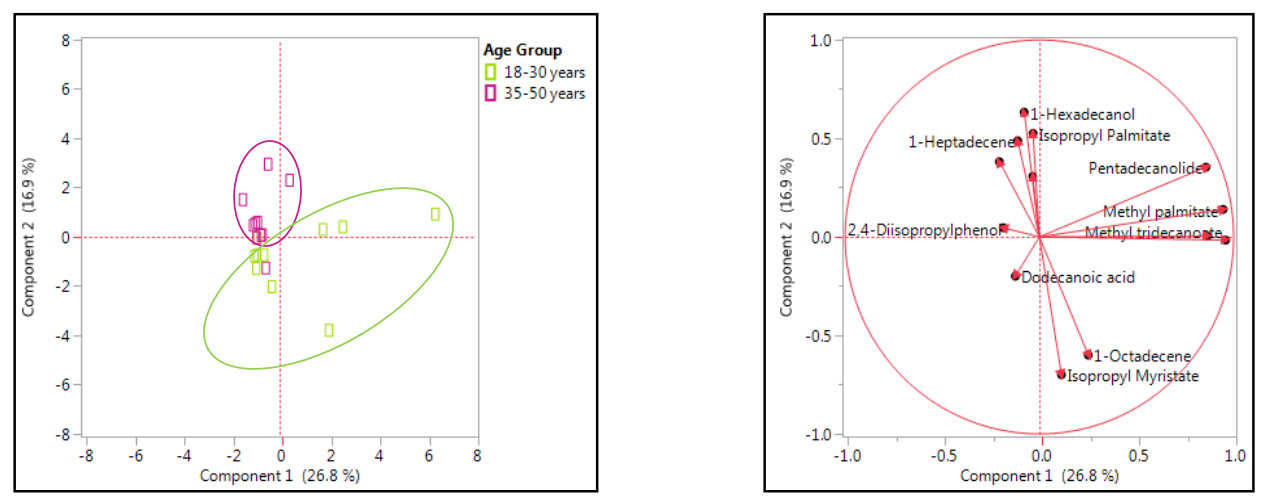

Figure 102: PCA score plot (left) and loading plot (right) of 18-30 vs. 35-50 year old East Asian females including VOCs from secondary list

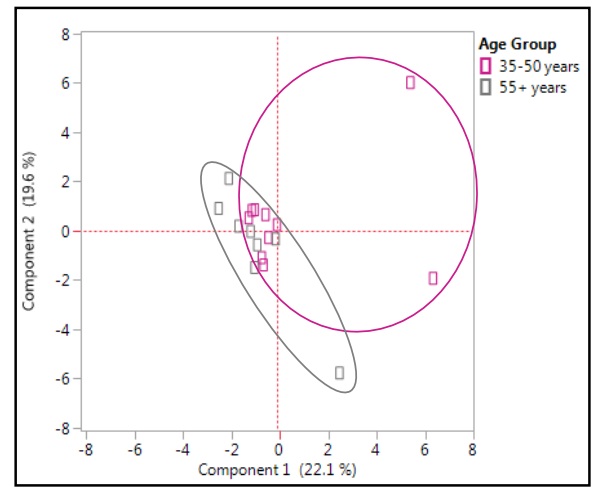

Figure 103: PCA score plot of 35-50 vs. 55+ year old East Asian females including all VOCs
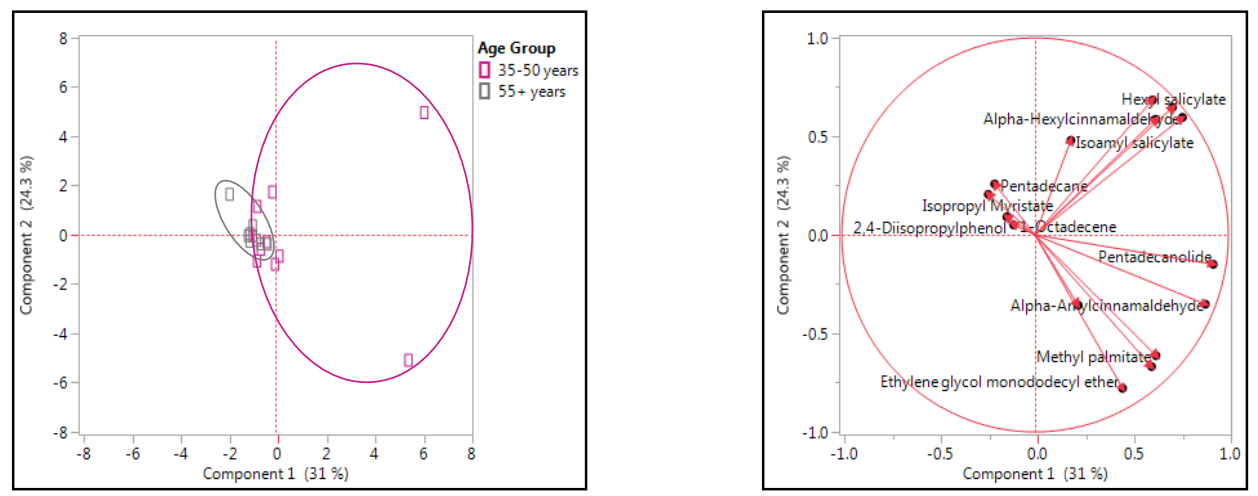

Figure 104: PCA score plot (left) and loading plot (right) of 35-50 vs. 55+ year old East Asian females including VOCs from secondary list 


\section{Comparison of Males of Different Age Groups}

The comparison of the average compound amounts for East Asian males showed that males in the 18-30 year age group had the highest amount for seven out of the initial 27 compounds under consideration. The 35-50 year, and 55+ year, age groups displayed the highest amount for eight and ten compounds, respectively. The abundance of Hexyl salicylate and Gamma-Hexylcinnamaldehyde decreased with age for East Asian males, providing supporting evidence to findings from previous studies. ${ }^{21}$ On the other hand, Nonanal showed that its average amount and occurrence is reduced as East Asian males age. This does not support the previous claims of this compound being considered an age marker. It is important to note that the same trends, in abundance and occurrence, were also observed for Nonanal in East Asian females. Therefore, the fact that this compound should be considered a marker that is indicative of old age for all populations must be reevaluated; as East Asians seem to represent an exception.

The initial list of 27 VOCs was evaluated for the creation of secondary compound lists for the East Asian male age group sets. A total of 17 compounds were selected for consideration in these lists (Figure 105). These compounds' potential to become biomarkers for age in East Asian males was assessed. Out of 17 compounds, 15 were included in the secondary list to compare the 18 -30 year category and the 55+ year category, while both the secondary list of the 18-30 year versus 35-50 year, and the list of the 35-50 year versus the 55+ year age groups, consisted of 11 VOCs (Table 28). Variation was observed in the compounds present in the secondary lists of the different age group sets. For this reason, it could also be understood that the discrimination between East Asian male age groups might be relying on the nature of different VOC 
combinations for each group set. Nonetheless, a total of five VOCs were still observed to be equally included in all VOC lists used to compare East Asian male age groups. 


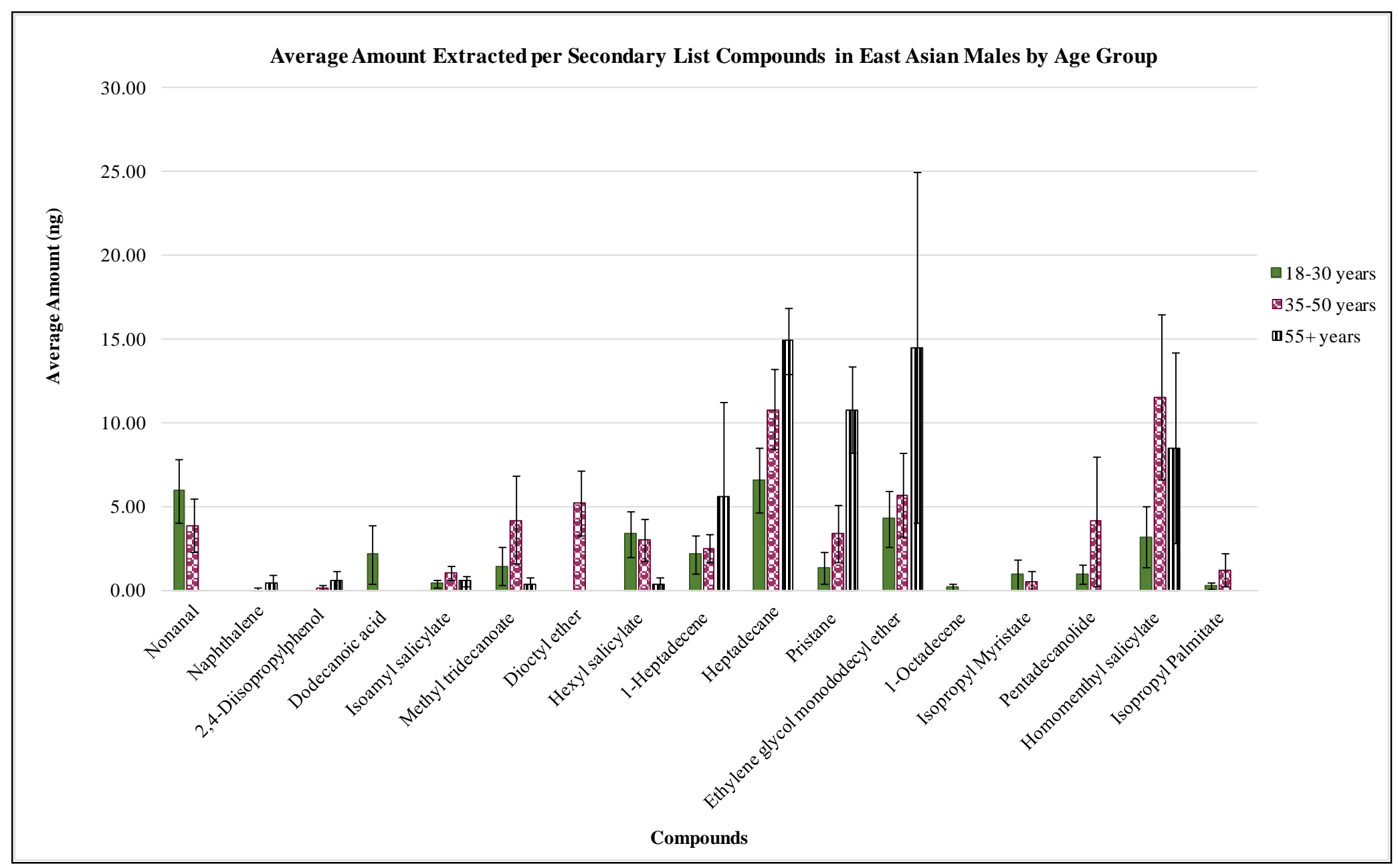

Figure 105: Comparison of average extracted amounts obtained for the compounds in the VOC secondary lists of East Asian males from different age groups 
Table 28: Compounds considered in secondary lists made for comparing East Asian males of different age groups

\begin{tabular}{|c|c|c|c|}
\hline $\begin{array}{c}\text { Compounds included in } \\
\text { Secondary Lists for Males }\end{array}$ & $\begin{array}{c}\text { 18-30 vs. 35-50 } \\
\text { years }\end{array}$ & $\begin{array}{c}\text { 18-30 vs. 55+ } \\
\text { years }\end{array}$ & $\begin{array}{c}\text { 35-50 vs. 55+ } \\
\text { years }\end{array}$ \\
\hline Nonanal & $\mathrm{X}$ & $\mathrm{X}$ & $\mathrm{X}$ \\
\hline Naphthalene & $\mathrm{X}$ & $\mathrm{X}$ & $\mathrm{X}$ \\
\hline 2,4-Diisopropylphenol & $\mathrm{X}$ & $\mathrm{X}$ & \\
\hline Dodecanoic acid & $\mathrm{X}$ & $\mathrm{X}$ & $\mathrm{X}$ \\
\hline Isoamyl salicylate & $\mathrm{X}$ & $\mathrm{X}$ & $\mathrm{X}$ \\
\hline Methyl tridecanoate & $\mathrm{X}$ & $\mathrm{X}$ & $\mathrm{X}$ \\
\hline Dioctyl ether & & $\mathrm{X}$ & $\mathrm{X}$ \\
\hline Hexyl salicylate & & $\mathrm{X}$ & $\mathrm{X}$ \\
\hline 1-Heptadecene & $\mathrm{X}$ & $\mathrm{X}$ & \\
\hline Heptadecane & $\mathrm{X}$ & $\mathrm{X}$ & $\mathrm{X}$ \\
\hline Pristane & $\mathrm{X}$ & $\mathrm{X}$ & $\mathrm{X}$ \\
\hline Ethylene glycol monododecyl ether & $\mathrm{X}$ & $\mathrm{X}$ & \\
\hline 1-Octadecene & & & \\
\hline Isopropyl Myristate & & $\mathrm{X}$ & $\mathrm{X}$ \\
\hline Pentadecanolide & & $\mathrm{X}$ & $\mathrm{X}$ \\
\hline Homomenthyl salicylate & & $\mathrm{X}$ & $\mathrm{X}$ \\
\hline Isopropyl Palmitate & & & $\mathrm{X}$ \\
\hline
\end{tabular}

The principal component analysis performed to evaluate the differentiation of males in the 18-30 and the 55+ year categories, revealed that using all 27 VOCs is not effective as discrimination criteria. The score plot obtained showed no clear separation between males in these two age groups. All subjects appeared equally spread out across the four quadrants, and without a noticeable tendency to separate in the plot (Figure 106). On the contrary, when the list of compounds being taken into consideration to discriminate the groups was reduced to those in the secondary list, a significant improvement in the discrimination of the groups was observed (Figure 107 - left). All male subjects grouped in accordance to their corresponding age group allowing a full discrimination between males in the $18-30$ and $55+$ year categories. On the basis of this 
observation, it can be stated that the 15 compounds selected as discrimination criteria seem to be efficient in the differentiation of East Asian males in these groups. However, due to the sample size limitations, this finding should be further confirmed to ensure accuracy and applicability of the results. The initial score plot showed a variation percent of $22.4 \%$ in PC1 and $16.9 \%$ in PC2, while the final score plot showed a $23.0 \%$ and a 20.7\%, respectively. As made evident, the increase in variation from both principal components in this case resulted in an efficient discrimination between the groups. On the other hand, the loading plot (Figure 107 - right) obtained after only considering the secondary list VOCs, revealed that the most influential compounds in PC1 were (in decreasing order): Ethylene glycol monododecyl ether, Methyl tridecanoate, Nonanal, Heptadecane, and Dodecanoic acid. The compounds of most influence in PC2 were found to be Isopropyl Palmitate, Dodecanoic acid, Heptadecane, Methyl tridecanoate, and Pristane (also in decreasing order).

The PCA score plot created, using all variables, to evaluate the 18-30 and 35-50 year olds presented all males forming one single cluster (Figure 108). Therefore, a separation of the groups was not achieved. On the other hand, the consideration of only the secondary compound list VOCs led to a reduction in the spread of males from both age groups (Figure 109 - left). Although this reduction in the spread of each group caused the initial cluster to have a more condensed aspect, it was still not enough to favor group separation. Therefore, in the case of East Asian males, an effective differentiation between males in the 18-30 and 35-50 year categories could not be established using the evaluated criteria. In the case of this comparison, it was observed that the variation percentage of PC 1 and PC2 increased from the initial score plot to the one made with the 
secondary compound list. The percentages went from $19.7 \%$ to $36.0 \%$. for PC1 and 16.1\% to $16.2 \%$ in PC2. Nevertheless, it appears that the increase in variation percentage was not enough to enhance the separation between the groups, but only to have an impact in the spread amongst male subjects. These findings prove that there is still room for improvement in the discrimination criteria being employed to classify males in these age groups and that further investigation is required to improve the accuracy of the these results. The loading plot (Figure 109 - right) obtained after the evaluation that employed the secondary list VOCs, stated that the compounds of higher influence to PC1's variation were Pentadecanolide, 2,4-Diisopropylphenol, Naphthalene, Methyl tridecanoate, and Homomenthyl salicylate (in decreasing order of influence). In addition, it revealed that the most influential compounds to PC2 were Isoamyl salicylate, Pristane, Homomenthyl salicylate, 1-Octadecene, and Dioctyl ether (also in decreasing order).

Similarly to the pattern described before, the evaluation of the 35-50 and 55+ year age groups, using all 27 VOCs as differentiation criteria, produced a score plot with no clear separation between the groups (Figure 110). Once the compounds from the secondary list were used as discrimination criteria, two noticeable clusters were formed: on per each age group (Figure 111 - left). A complete separation between the groups was achieved for East Asian males in these two age groups. Nonetheless, further confirmation might be necessary to ensure the results are applicable when considering larger populations, especially due to the sample size employed for the 55+ year age group. The variation percentages observed for PC1 and PC2 in the initial score plot were seen to be lower than those obtained for the score plot performed with the secondary list VOCs (increased from $22.1 \%$ to $28.3 \%$ in PC1 and $18.0 \%$ to $21.5 \%$ in PC2). These increments 
in variation provided an explanation to the enhancement in group differentiation. In this evaluation, the loading plot (Figure 111 - right) revealed that the most influential compounds in PC1 were Pentadecanolide, 2,4-Diisopropylphenol, Methyl tridecanoate, Naphthalene, and Dioctyl ether; while in PC2 the VOCs were Nonanal, Pristane, Isopropyl Palmitate, Naphthalene, and Dioctyl ether (in both cases in decreasing order of influence).

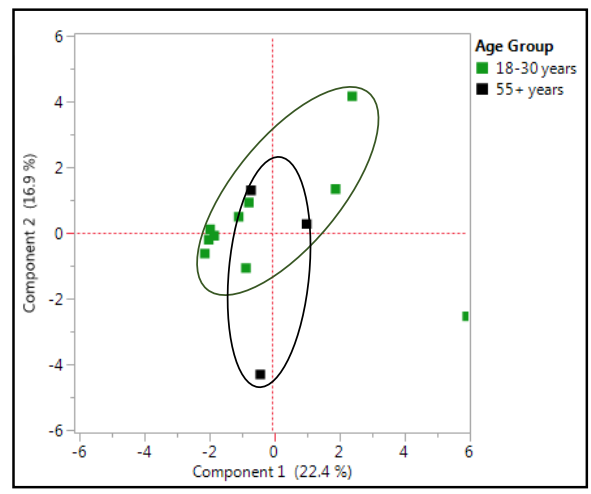

Figure 106: PCA score plot of 18-30 vs. 55+ year old East Asian males including all VOCs
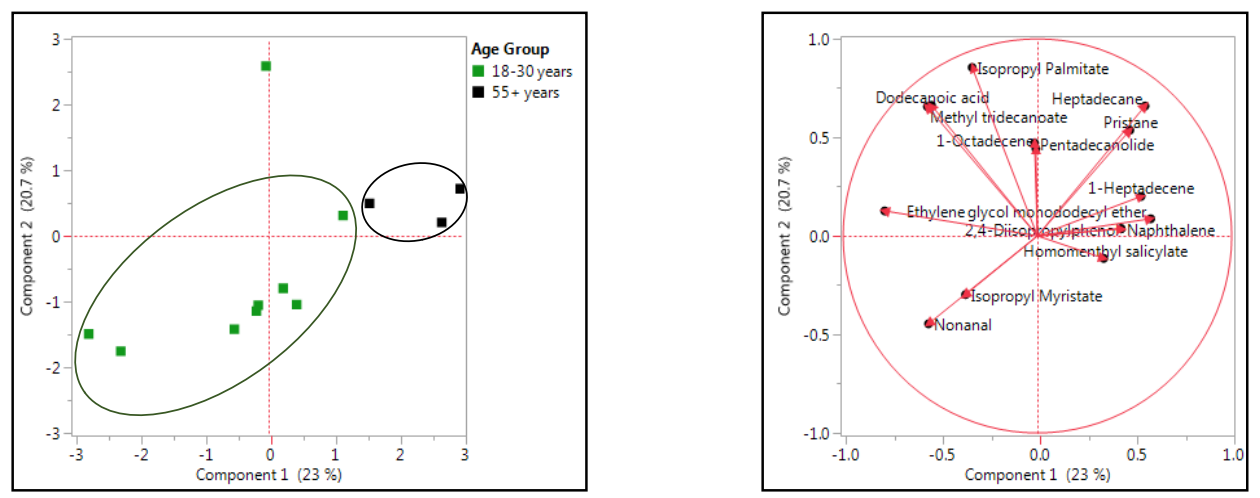

Figure 107: PCA score plot (left) and loading plot (right) of 18-30 vs. 55+ year old East Asian males including VOCs from secondary list 


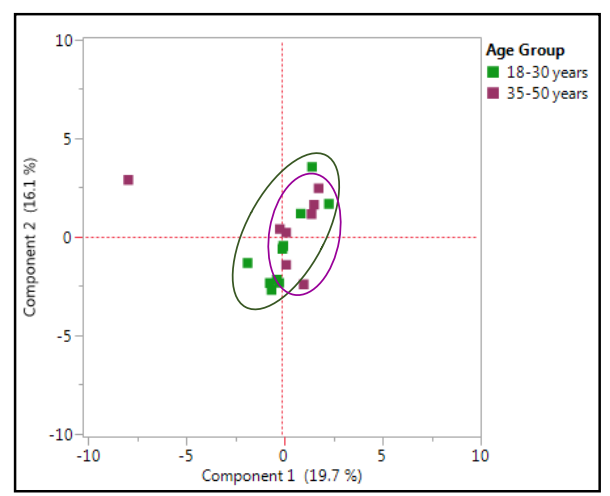

Figure 108: PCA score plot of 18-30 vs. 35-50 year old East Asian males including all VOCs
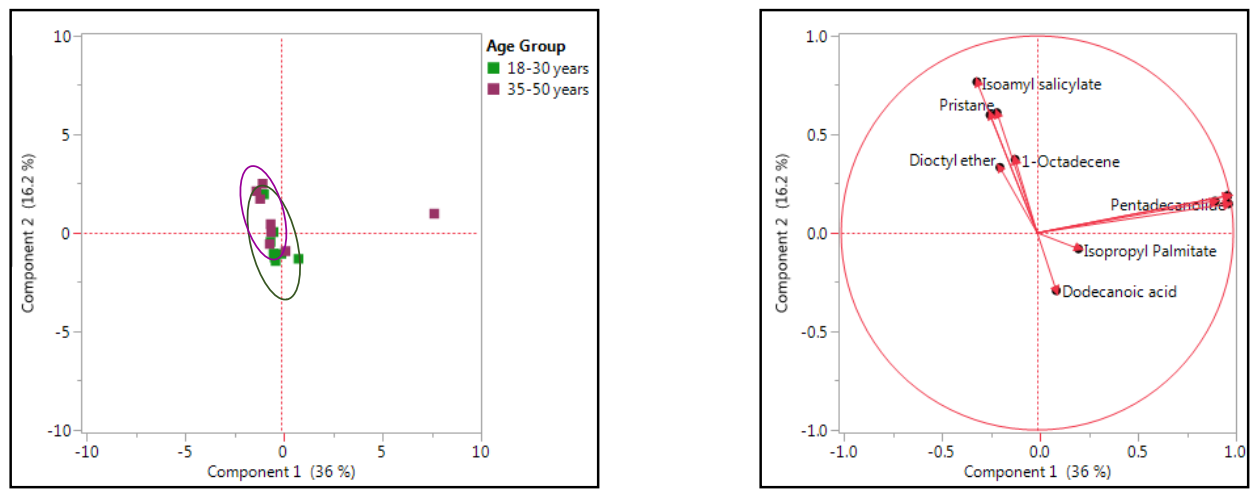

Figure 109: PCA score plot (left) and loading plot (right) of 18-30 vs. 35-50 year old East Asian males including VOCs from secondary list

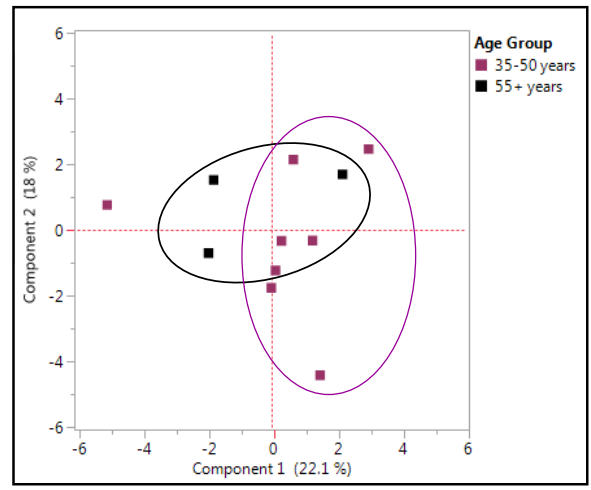

Figure 110: PCA score plot of $35-50$ vs. 55+ year old East Asian males including all VOCs 

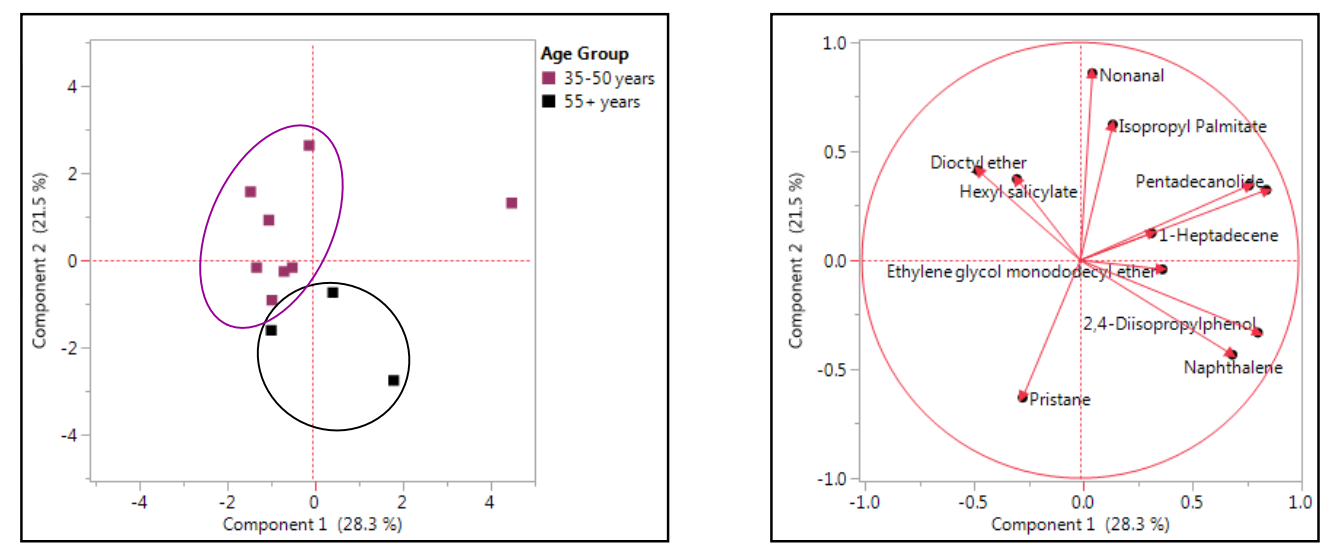

Figure 111: PCA score plot (left) and loading plot (right) of 35-50 vs. 55+ year old East Asian males including VOCs from secondary list

\subsubsection{Overall comparison across all populations under study}

The evaluation of odor profiles from different individuals within a same racial or ethnic population, allowed reaching conclusions that were specific to each population. Also, it provided insight on how individual characteristics can correlate with VOC expression within a particular group. These comparisons surveyed odor profile peculiarities that could arise amongst individuals of a same race/ethnicity, under the influence of their expression of other traits, such as gender and age. Nevertheless, the following comparison seeks to study the overall VOC characteristics that could serve as classification criteria for an individual when considering a diverse population. The identification of VOC features that are specific to a race, ethnicity, gender or age group could uncover biomarkers for individual classification on the basis of any of these traits.

An initial list of 26 compounds was put together to perform an overall comparison of all the individuals under study. The list was a selection of all VOCs that could be compared across all the different groups under study. This approach was intended to focus the analysis on a set of parameters that would facilitate an objective evaluation of 
all groups; while allowing the identification of potential VOC biomarkers. Three conditions, or traits, served as primary elements of comparison between groups: Race/ Ethnicity, Age and Gender. A scent profile, constituted by each of the 26 compounds from the initial list, was evaluated for each individual under study. Subsequently, the potential for classification of each individual in accordance to their corresponding trait, and the characteristics expressed in their VOC profile, was evaluated using Linear Discriminant Analysis (LDA). In the attempt to obtain optimal results for group differentiation by trait, a series of three different approaches were tested in each comparison. In all cases, a comparison was first established using all 26 compounds from the initial list as discrimination criteria. Then, the discrimination criteria were reduced to a selection of compounds that constituted a secondary list. As in previous comparisons, a secondary list was created by including all compounds that showed a difference in amount of $100 \%$ or more in the profiles of the different groups. On the other hand, a third set of discrimination criteria was also created and evaluated. In this case, a stepwise regression method was employed to select specific VOC variables according to their impact in group differentiation. This last method provided an additional alternative for the determination and evaluation of differentiation criteria between the groups. After having proceeded with all three approaches, their individual classification efficiencies were compared. This served to establish conclusions in regards to the best suited discrimination technique and potential scent biomarkers for each trait. The statistical significance of the differences in average VOC amounts, between each of the groups under study, was also assessed. Nevertheless, this information did not serve as foundation for any of the classification approaches attempted. The main reason for this was the fact 
that the information lacked power when being employed by itself as a resource for individual classification. Therefore, significant differences between the average VOC amounts of different groups have been reported in this comparison for mere reference purposes. Details on the results of all statistical significance tests performed to evaluate average VOC amounts can be found in Appendix 7 and Appendix 8.

\subsection{Determination of VOC markers for individual classification by age}

To determine whether individuals can be classified according to their age group, all individuals in this study were considered part of a single pool of samples; regardless of their race/ethnicity and gender. Average VOC amounts of all 26 compounds were obtained for each one of the three age groups under study (Figure 112), and used to evaluate the possibility of individual classification based on any VOC characteristics that could be linked to age as a trait. 


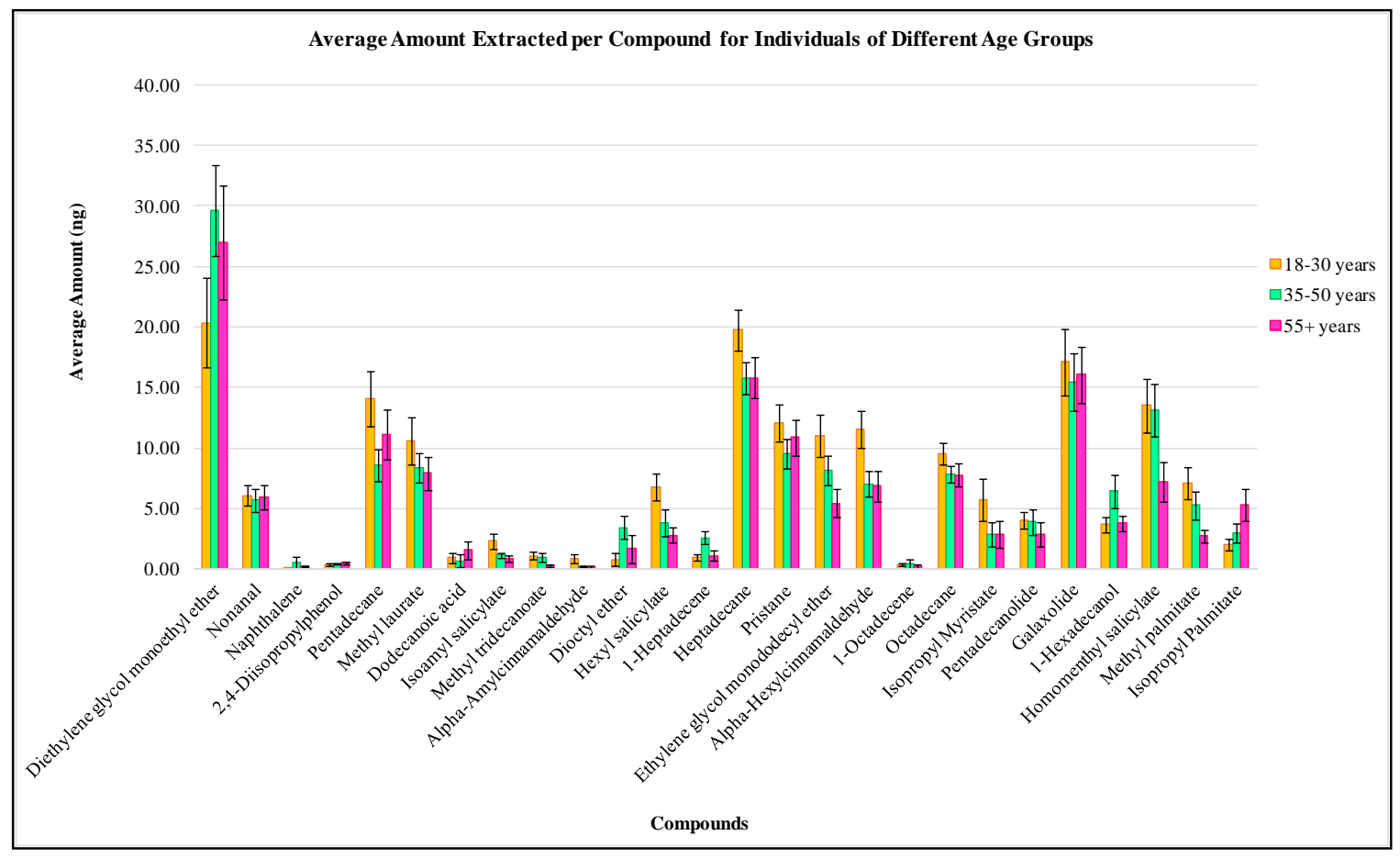

Figure 112: Average VOC amounts per age group for all 26 VOCs in the initial compound list

Initially, a Linear Discriminant Analysis was performed using the complete list of 26 VOCs as variables for individual classification (Table 29). The LDA analysis, classified 66 individuals, out of a pool of 169, in the incorrect age groups. This led to achieving only $61 \%$ of overall accuracy in the classification of individuals through this method. Out of a total of 60 individuals in the 18-30 year old group, a total of nine were misclassified as 35-50 year olds and 15 as 55+ year olds. On the other hand, out of 58 individuals in the 35-50 year group, eight were misclassified as 18-30 year olds and 16 as 55+ year olds. Moreover, from a total of 51 individuals in the 55+ year category, eight were classified incorrectly under the 18-30 year category and ten under the 35-50 year old category. The classification predictions obtained through this method suggest that there is difficulty in achieving a clear differentiation between individuals in the middle 
(35-50) and oldest (55+) of age groups. This also shows that $36 \%$ of all misclassifications were caused by lack of accuracy in the classification of 18-30 year olds. Although the canonical plot revealed a fairly clear distinction between the different age groups (Figure 113), the overall accuracy percentage achieved through this approach suggests there is still room for improvement in regards to the VOC variables being considered as classification criteria.

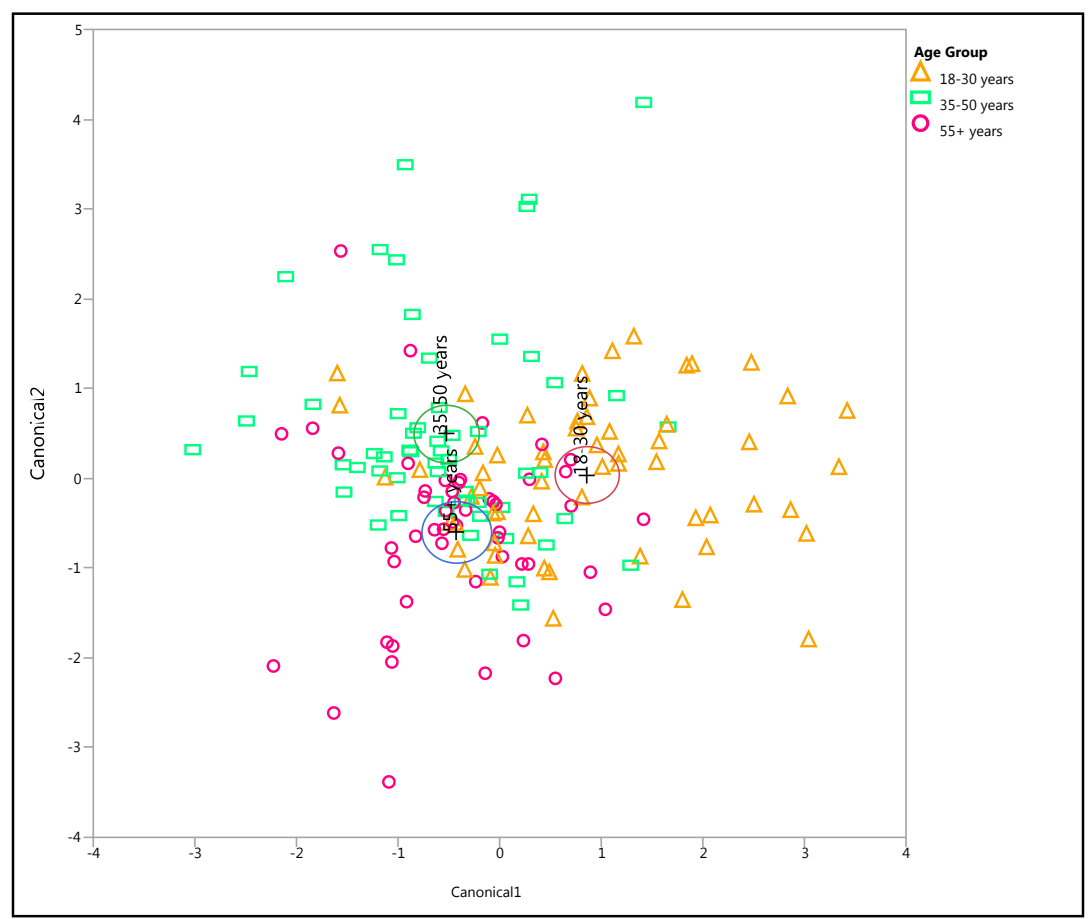

Figure 113: LDA canonical plot using all 26 VOCs as criteria for subject differentiation by age group

As a second approach in the assessment of optimal age group differentiation, LDA was performed by only using the compounds selected for the secondary VOC list in this analysis. In this case, the canonical plot revealed a reduction in the separation between the age groups, yet continued to show that it was still possible to differentiate 
them (Figure 114). Compared to the 26 VOCs employed in the first approach, a total of 12 VOCs were considered in this method (Table 29). Therefore, the loss of accuracy observed in this case seems to be linked to the exclusion of certain compounds that seemed to contribute to the enhancement of group differentiation in the previous case. The fact that the overall classification accuracy was reduced from $61 \%$ in the previous approach, to $56 \%$ in this one, supports this statement. Out of 60 individuals in the 18-30 year category, 43\% were misclassified: seven as 35-50 year olds and 19 as 55+ year olds. In the same token, out of 58 subjects in the 35-50 year old category, 53\% were classified incorrectly (14 as 18-30 year olds and 17 as 55+ year olds). The 55+ year category showed the least number of misclassifications with only a $33 \%$ of all individuals being classified incorrectly. From this 33\%, nine subjects were misclassified as 18-30 year olds and eight as 35-50 year olds.

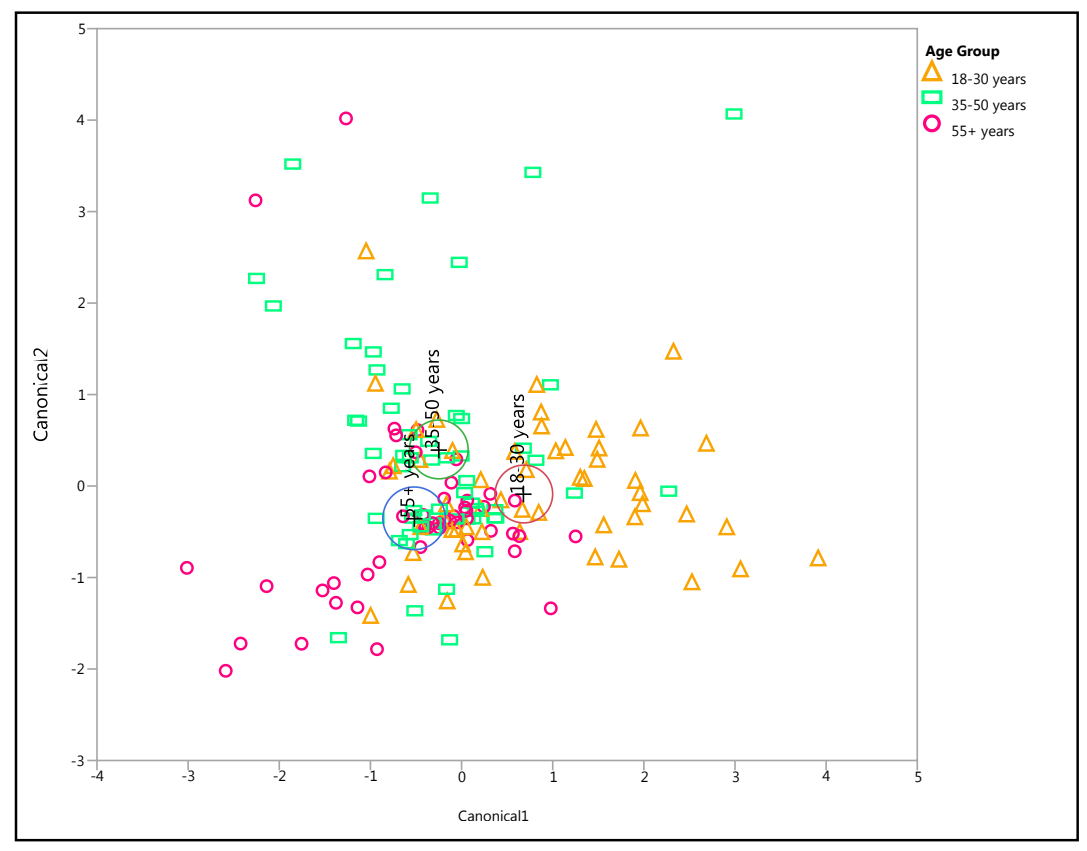

Figure 114: LDA canonical plot using compounds from secondary list as criteria for subject differentiation by age group 
The third approach used to attempt to find the best conditions for age group differentiation, involved the use of a stepwise regression method for the selection of the classification criteria. A total of 12 compounds were considered for the creation of the canonical plot, on the basis of their level of influence in group differentiation. As a result, the plot revealed that it was possible to differentiate the three different age groups with an overall accuracy of 63\% (Figure 115). None of the confidence level (CL) ellipses of any of the groups were seen to overlap or intercept, which means there is enough variation, caused by the VOCs being considered, to differentiate all groups. From a total of 60 subjects in the 18-30 year old category, 12 were misclassified as 55+year olds and nine as 35-50 year olds. Also, from a total of 58 subjects in the 35-50 year old category, 17 were misclassified as 55+ year olds and seven as $18-30$ year olds. Moreover, in the case of 55+ year old individuals, 12 were misclassified as 35-50 year olds and only five as 18-30 year olds. These numbers show that individuals in the 55+ year age group provided the highest classification accuracy of all groups (67\%).

Table 29 displays the 12 compounds that were considered for the creation of the canonical plot in this method. Since the stepwise regression approach was found to be the most efficient classification method, the loading scores for these compounds have been listed for reference. The absolute value of the loading scores' magnitude represents the level of influence each one of these VOCs have in age group differentiation. The higher the magnitude of the loading score, the greater is the impact of the VOC on the level of differentiation presented by the canon. On the other hand, the sign presented by each value corresponds to the direction towards which the influence was exerted by the compound in the canon. Therefore, the loading scores obtained for each of these 
compounds disclose their strength to discriminate individuals from different age groups, regardless of any other traits they might possess. Table 29 shows the list of 12 VOCs arranged in decreasing order of their influence on age group differentiation.

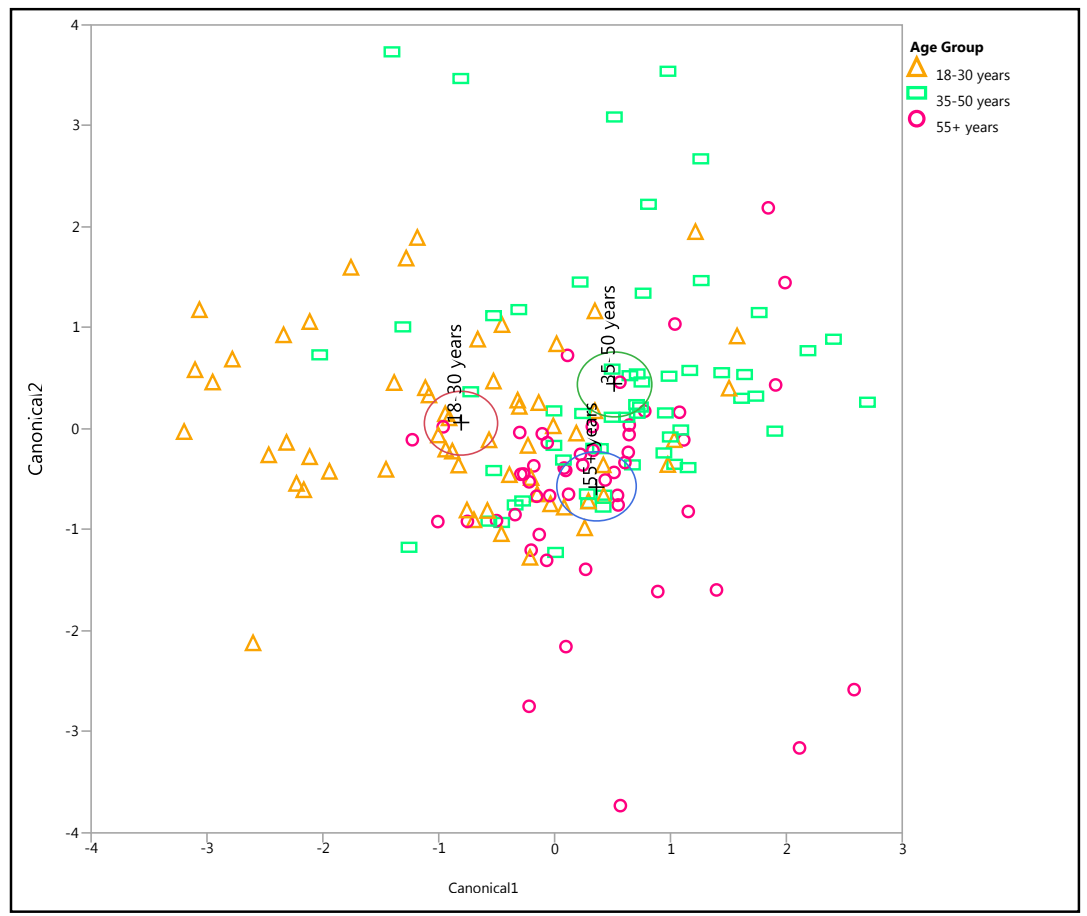

Figure 115: LDA canonical plot using compounds from stepwise regression method as criteria for subject differentiation by age group

The compounds identified as the most efficient differentiation criteria for the age groups under study, were also seen to play a role in all cases of age differentiation comparisons performed in the separate population studies. Nine out of the 12 VOCs identified as having the most impact on overall age group differentiation (Table 29) were also present among the compounds considered in the secondary lists of Caucasian females for age differentiation. On the other hand, in the case of Caucasian males, six out of the 12 compounds were considered in the secondary lists used for age group 
differentiation. Among the compounds found to be consistent between the secondary lists and the stepwise regression VOC list, were included the five compounds of highest influence for overall age group differentiation: 1-Heptadecene, 1-Hexadecanol, Hexyl salicylate, Ethylene glycol monododecyl ether and 1-Octadecene. As a matter of fact, there were cases in which these five compounds were also considered to be among the most influential compounds for age differentiation within the Caucasian population. Therefore, the constancy observed in both studies demonstrates how the results from the Caucasian study correlate with those obtained in the overall comparison study.

In the case of Hispanic females and males, nine and 11 of the best suited VOCs for overall age differentiation were also included in the secondary lists used for age differentiation in the Hispanic population. Among these compounds in the secondary lists of the Hispanic population, the five VOCs of highest influence for overall age group differentiation were noted (e.g., 1-Heptadecene, 1-Hexadecanol, Hexyl salicylate, Ethylene glycol monododecyl ether, and 1-Octadecene). Therefore, observations obtained from this study also demonstrate a correlation between the results from the Hispanic study and those gathered from the overall comparison.

On the other hand, East Asian female secondary lists included nine out of 12 of the VOCs established as the most influential for overall age differentiation, while male secondary lists included eight VOCs. Among the compounds in common between the VOC lists, the five VOCs found to be most influential for overall age differentiation were also included. In specific, 1-Heptadecene and 1-Octadecene were among the compounds of higher occurrence in East Asian secondary lists. Nonetheless, 1-Hexadecanol, Hexyl salicylate, and Ethylene glycol monododecyl ether were also found to display frequent 
occurrence. Moreover, 1-Octadecene was identified as a VOC of high influence in age differentiation for both the independent population study and the overall comparison. Therefore, in the same way it was seen in the other two race/ethnic populations, the East Asian study also revealed findings that were correlated to the overall comparison.

The fact that the results from all the independent population studies and the overall comparison displayed constancy, confirms the importance of the reported VOC combination for the differentiation of individuals by their age. Also, it provides a point of origin for the conclusions made in regards to these compounds and eases the understanding of the overall results. 
Table 29: VOC lists employed as criteria for subject differentiation by age group in Linear Discriminant Analysis

\begin{tabular}{|c|c|c|c|c|}
\hline \multirow{2}{*}{ Initial VOC List } & \multirow{2}{*}{ Secondary VOC List } & \multicolumn{3}{|c|}{ Stepwise Regression Method } \\
\hline & & VOC List & Canon 1 & Canon 2 \\
\hline Diethylene glycol monoethyl ether & Naphthalene & *1-Heptadecene & 0.584 & 0.409 \\
\hline Nonanal & Dodecanoic acid & 1-Hexadecanol & 0.486 & 0.552 \\
\hline Naphthalene & *Isoamyl salicylate & *Hexyl salicylate & -0.483 & 0.161 \\
\hline 2,4-Diisopropylphenol & Methyl tridecanoate & *Ethylene glycol monododecyl ether & -0.436 & 0.208 \\
\hline Pentadecane & Alpha-Amylcinnamaldehyde & 1-Octadecene & -0.394 & -0.248 \\
\hline Methyl laurate & Dioctyl ether & Alpha-Amylcinnamaldehyde & -0.375 & 0.123 \\
\hline Dodecanoic acid & *Hexyl salicylate & Diethylene glycol monoethyl ether & 0.350 & 0.202 \\
\hline *Isoamyl salicylate & *1-Heptadecene & *Isopropyl Palmitate & 0.315 & -0.514 \\
\hline Methyl tridecanoate & *Ethylene glycol monododecyl ether & Dioctyl ether & 0.315 & 0.271 \\
\hline Alpha-Amylcinnamaldehyde & 1-Octadecene & Pentadecane & -0.263 & -0.171 \\
\hline Dioctyl ether & *Methyl palmitate & Homomenthyl salicylate & -0.211 & 0.408 \\
\hline *Hexyl salicylate & *Isopropyl Palmitate & Methyl tridecanoate & -0.140 & 0.378 \\
\hline \multicolumn{5}{|l|}{ *1-Heptadecene } \\
\hline \multicolumn{5}{|l|}{ Heptadecane } \\
\hline \multicolumn{5}{|l|}{ Pristane } \\
\hline \multicolumn{5}{|l|}{ *Ethylene glycol monododecyl ether } \\
\hline \multicolumn{5}{|l|}{ *Alpha-Hexylcinnamaldehyde } \\
\hline \multicolumn{5}{|l|}{ 1-Octadecene } \\
\hline \multicolumn{5}{|l|}{ Octadecane } \\
\hline \multicolumn{5}{|l|}{ Isopropyl Myristate } \\
\hline \multicolumn{5}{|l|}{ Pentadecanolide } \\
\hline \multicolumn{5}{|l|}{ Galaxolide } \\
\hline \multicolumn{5}{|l|}{ 1-Hexadecanol } \\
\hline \multicolumn{5}{|l|}{ Homomenthyl salicylate } \\
\hline *Methyl palmitate & & & & \\
\hline *Isopropyl Palmitate & & & & \\
\hline
\end{tabular}

* Compounds for which the average VOC amounts were seen to be significantly different between the age groups 


\subsection{Determination of VOC markers for individual classification by race/ethnicity}

In order to evaluate the capacity of using human scent as a tool for human classification based on race (e.g., Caucasian and East Asian) or ethnicity (e.g., Hispanic), all individuals under study were considered part of a single pool of samples, regardless of their age group and gender. The average VOC amounts were calculated for all 26 compounds per race/ethnic group (Figure 116) and used to establish the possibility of differentiating individuals based on VOC characteristics that could be associated to their specific race or ethnicity.

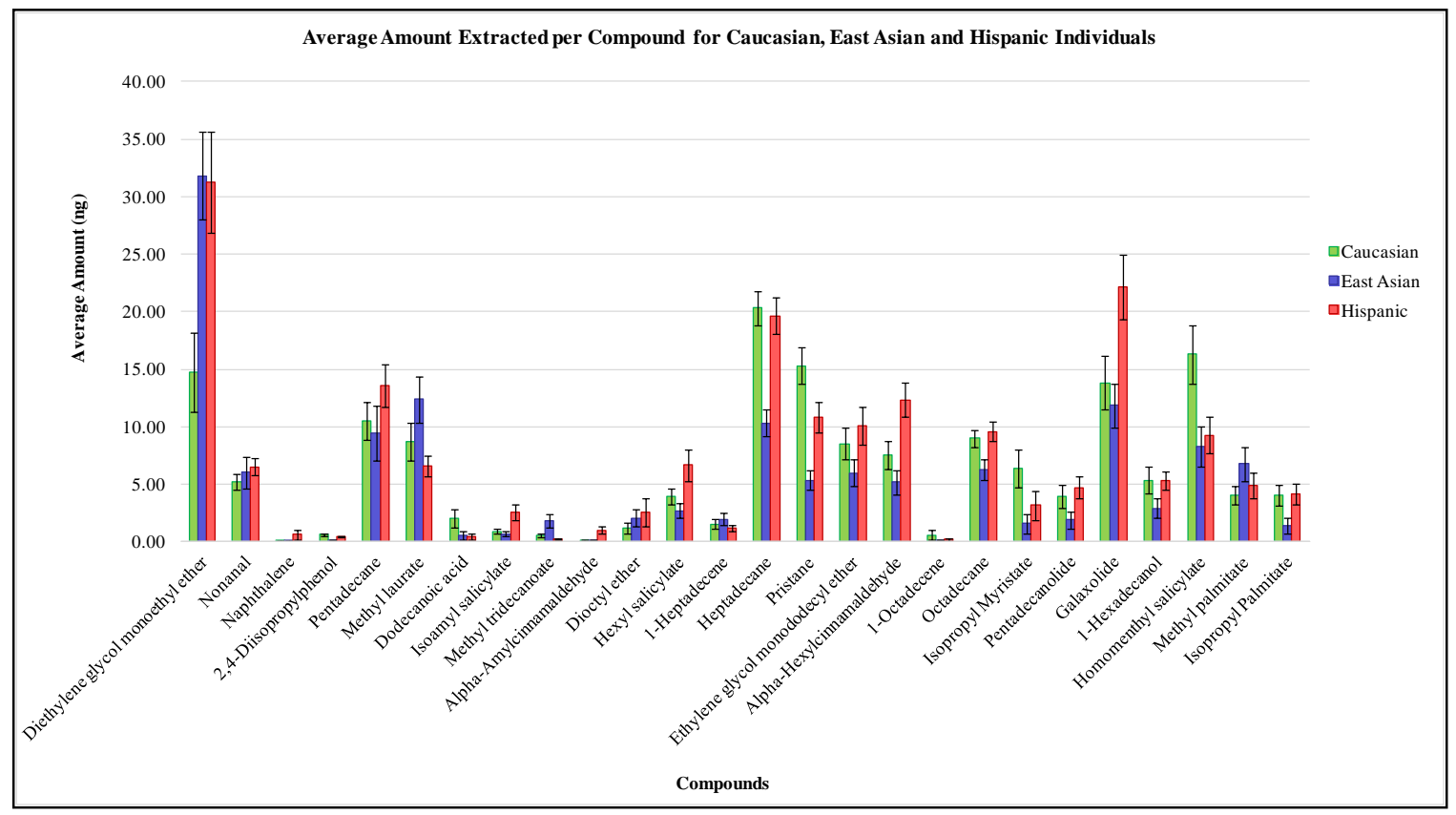

Figure 116: Average VOC amounts per racial/ethnic group for all 26 VOCs in the initial compound list

Linear Discriminant Analysis was initiated using all 26 VOCs as variables for individual classification per racial/ethnic group (Table 30). The canonical plot obtained showed that it was possible to classify individuals according to their race/ethnicity with 
an accuracy of 69\% (Figure 117). Out of a total of 169 subjects, 60 Caucasian, 60 Hispanic and 49 East Asian, there were a total of 53 misclassifications. This qualifies for a $31 \%$ of all subjects being misclassified. From a total of 60 Caucasians, six were misclassified as East Asian and 14 were misclassified as Hispanic. From a total of 49 East Asians, three were misclassified as Caucasian and five as Hispanic, while in the case of Hispanics, 12 subjects were misclassified as Caucasian and 13 as East Asian. Therefore, East Asians showed the highest percentage of correct classification, 84\%, followed by Caucasians with $67 \%$ and Hispanics with $58 \%$.

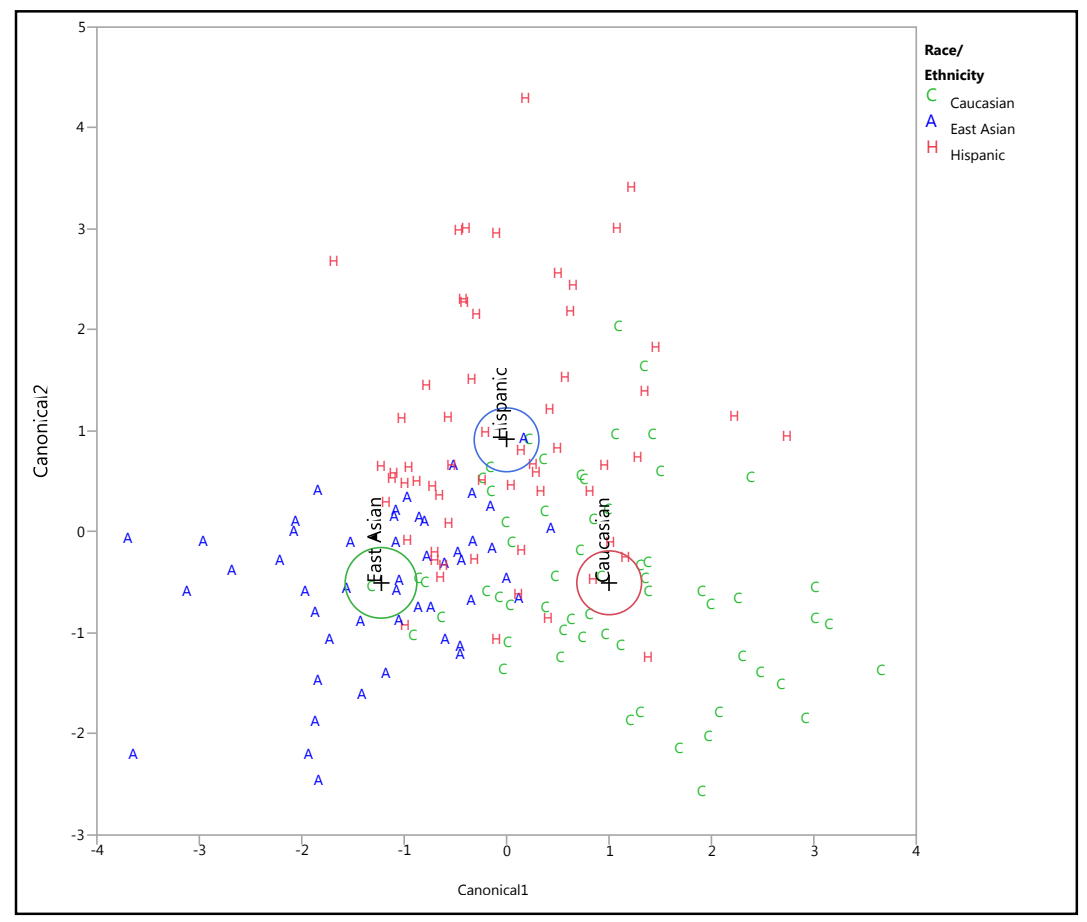

Figure 117: LDA canonical plot using all 26 VOCs as criteria for subject differentiation by racial/ethnic group

As the second approach for LDA, only those compounds noted to have a difference in amount of $100 \%$ or more, between any of the racial/ethnic groups, were 
considered as variables for discrimination. A total of 15 compounds (Table 30) were considered in the creation of the canonical plot. A reduction in the accuracy percentage was observed, as only a 67\% was achieved (Figure 118). This reduction was made evident in the position of all groups in the plot and the distance between the 95\% CL ellipses surrounding each group's mean. Although they were still not seen to intersect, the distance between the ellipses in this plot was reduced, which indicates there is a lower level of differentiation between the groups. In this case, 55 misclassifications were observed (33\%), and the majority of them were in the Hispanic group. Only $48 \%$ of all Hispanic individuals were classified correctly, while $88 \%$ and $70 \%$ were classified correctly for East Asians and Caucasians, respectively. Although, overall, this approach was not a better method for group classification than the method including all 26 VOCs, it still showed that East Asians seem to be the group with most classification accuracy, and Hispanics those with the highest tendency for misclassification. 


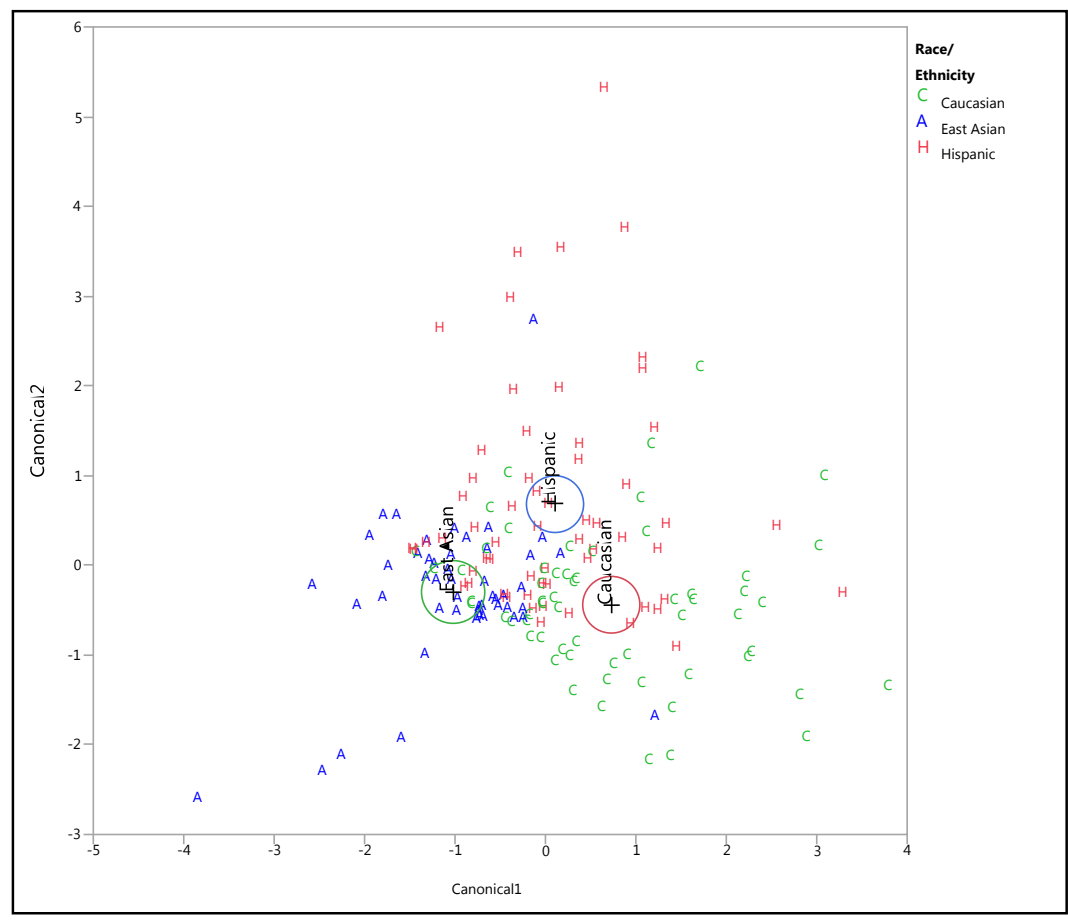

Figure 118: LDA canonical plot using compounds from secondary list as criteria for subject differentiation by racial/ethnic group

As the final LDA approach for the racial/ethnic differentiation of individuals, a stepwise regression method was performed. Through this method, a total of 21 compounds (Table 30) were identified as being able to provide a $70 \%$ of classification accuracy; the highest percentage attained for this comparison so far. The canonical plot obtained (Figure 119) showed, once again, a clear differentiation between the groups. As observed in the other comparisons, the CL ellipses did not appear intercepted between any of the groups, and showed a greater distance between them than those presented in the plot created in the previous approach. This is explained by the fact that, using this selection of compounds, only a $30 \%$ of all subjects under study were misclassified. The fact that only $32 \%$ of all Caucasians, $16 \%$ of all East Asians and 38\% of all Hispanics 
were misclassified in this approach proves an efficient differentiation between the groups based on race/ethnicity when using this selection of VOCs.

Table 30 presents, in decreasing order, the compounds selected through this method and their influence in differentiating race/ethnic groups. This influence is represented by the loading of each compound on each canon. The five compounds found to portray a higher influence on overall race/ethnic differentiation were Octadecane, Pristane, Heptadecane, Dodecanoic acid, and 2,4-Diisopropylphenol.

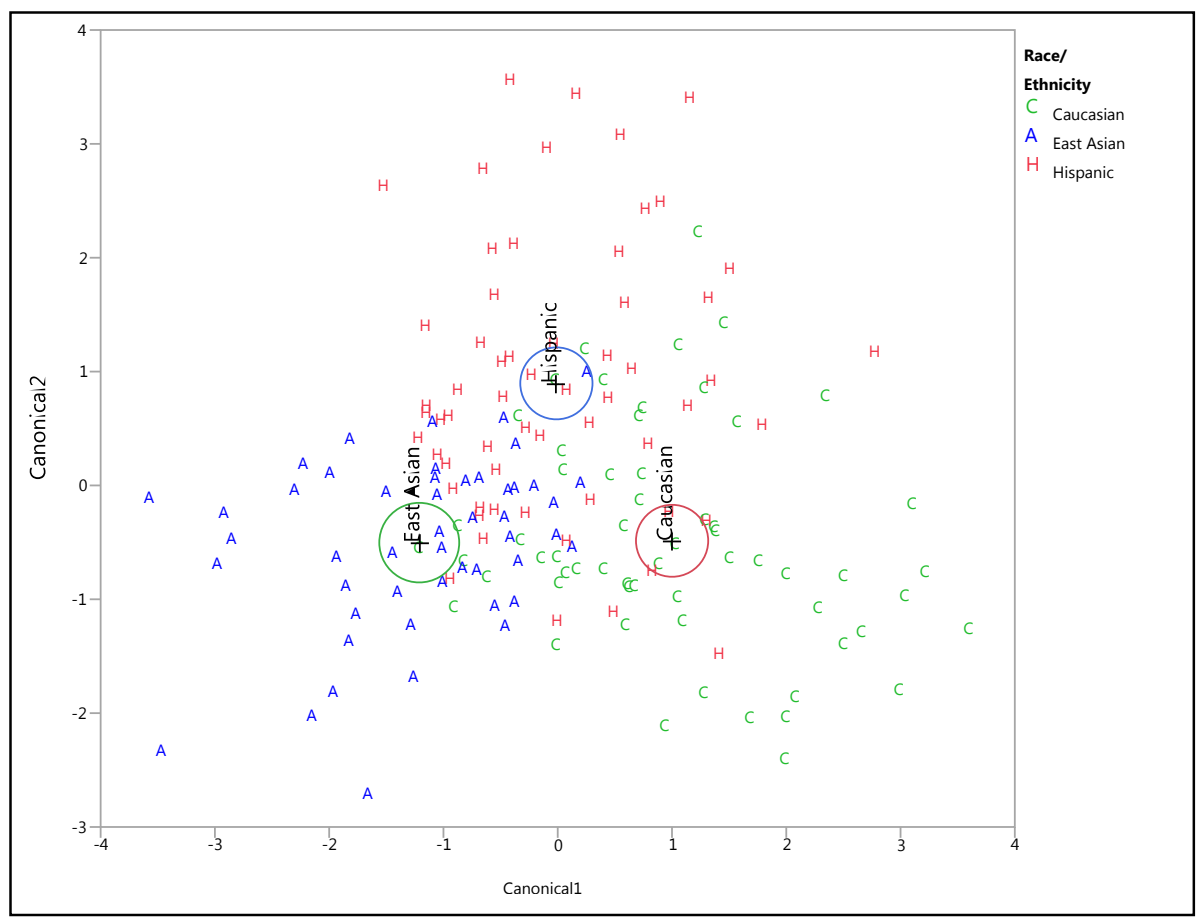

Figure 119: LDA canonical plot using compounds from stepwise regression method as criteria for subject differentiation by racial/ethnic group 
Table 30: VOC lists employed as criteria for subject differentiation by racial/ethnic group in Linear Discriminant Analysis

\begin{tabular}{|c|c|c|c|c|}
\hline \multirow{2}{*}{ Initial VOC List } & \multirow{2}{*}{ Secondary VOC List } & \multicolumn{3}{|c|}{ Stepwise Regression Method } \\
\hline & & VOC List & Canon 1 & Canon 2 \\
\hline *Diethylene glycol monoethyl ether & *Diethylene glycol monoethyl ether & *Octadecane & -0.538 & 0.004 \\
\hline Nonanal & Naphthalene & *Pristane & 0.521 & -0.358 \\
\hline Naphthalene & *2,4-Diisopropylphenol & *Heptadecane & 0.476 & 0.395 \\
\hline *2,4-Diisopropylphenol & Dodecanoic acid & Dodecanoic acid & 0.462 & -0.075 \\
\hline Pentadecane & *Isoamyl salicylate & *2,4-Diisopropylphenol & 0.439 & 0.094 \\
\hline Methyl laurate & Methyl tridecanoate & Methyl laurate & -0.390 & -0.251 \\
\hline Dodecanoic acid & Alpha-Amylcinnamaldehyde & Homomenthyl salicylate & 0.365 & -0.272 \\
\hline *Isoamyl salicylate & Dioctyl ether & 1-Heptadecene & -0.349 & -0.172 \\
\hline Methyl tridecanoate & Hexyl salicylate & *Diethylene glycol monoethyl ether & -0.330 & 0.313 \\
\hline Alpha-Amylcinnamaldehyde & *Pristane & 1-Octadecene & 0.284 & -0.015 \\
\hline Dioctyl ether & *Alpha-Hexylcinnamaldehyde & Methyl tridecanoate & -0.281 & -0.369 \\
\hline Hexyl salicylate & 1-Octadecene & *Isopropyl Myristate & 0.245 & -0.403 \\
\hline 1-Heptadecene & *Isopropyl Myristate & Pentadecane & -0.234 & 0.204 \\
\hline *Heptadecane & Pentadecanolide & *Alpha-Hexylcinnamaldehyde & 0.210 & -0.342 \\
\hline *Pristane & Isopropyl Palmitate & Pentadecanolide & 0.192 & 0.251 \\
\hline Ethylene glycol monododecyl ether & & Nonanal & -0.123 & 0.275 \\
\hline *Alpha-Hexylcinnamaldehyde & & *Galaxolide & -0.098 & 0.421 \\
\hline 1-Octadecene & & Alpha-Amylcinnamaldehyde & -0.078 & 0.429 \\
\hline *Octadecane & & Hexyl salicylate & -0.070 & 0.614 \\
\hline *Isopropyl Myristate & & Ethylene glycol monododecyl ether & 0.050 & 0.318 \\
\hline Pentadecanolide & & Naphthalene & 0.002 & 0.174 \\
\hline \multicolumn{5}{|l|}{ *Galaxolide } \\
\hline \multicolumn{5}{|l|}{ 1-Hexadecanol } \\
\hline \multicolumn{5}{|l|}{ Homomenthyl salicylate } \\
\hline \multicolumn{5}{|l|}{ Methyl palmitate } \\
\hline Isopropyl Palmitate & & & & \\
\hline
\end{tabular}

* Compounds for which the average VOC amounts were seen to be significantly different between the age groups 


\subsection{Determination of VOC markers for individual classification by gender}

The evaluation of scent as viable information for the classification of human individuals by their gender was also assessed. Once again, all individuals under study were considered part of a single pool of samples, this time regardless of their age group and race/ ethnicity. The average VOC amounts of all 26 initial compounds were calculated for both females and males (Figure 120). Subsequently, these values were used to identify VOC profile characteristics that could be associated to each gender and serve as gender biomarkers.

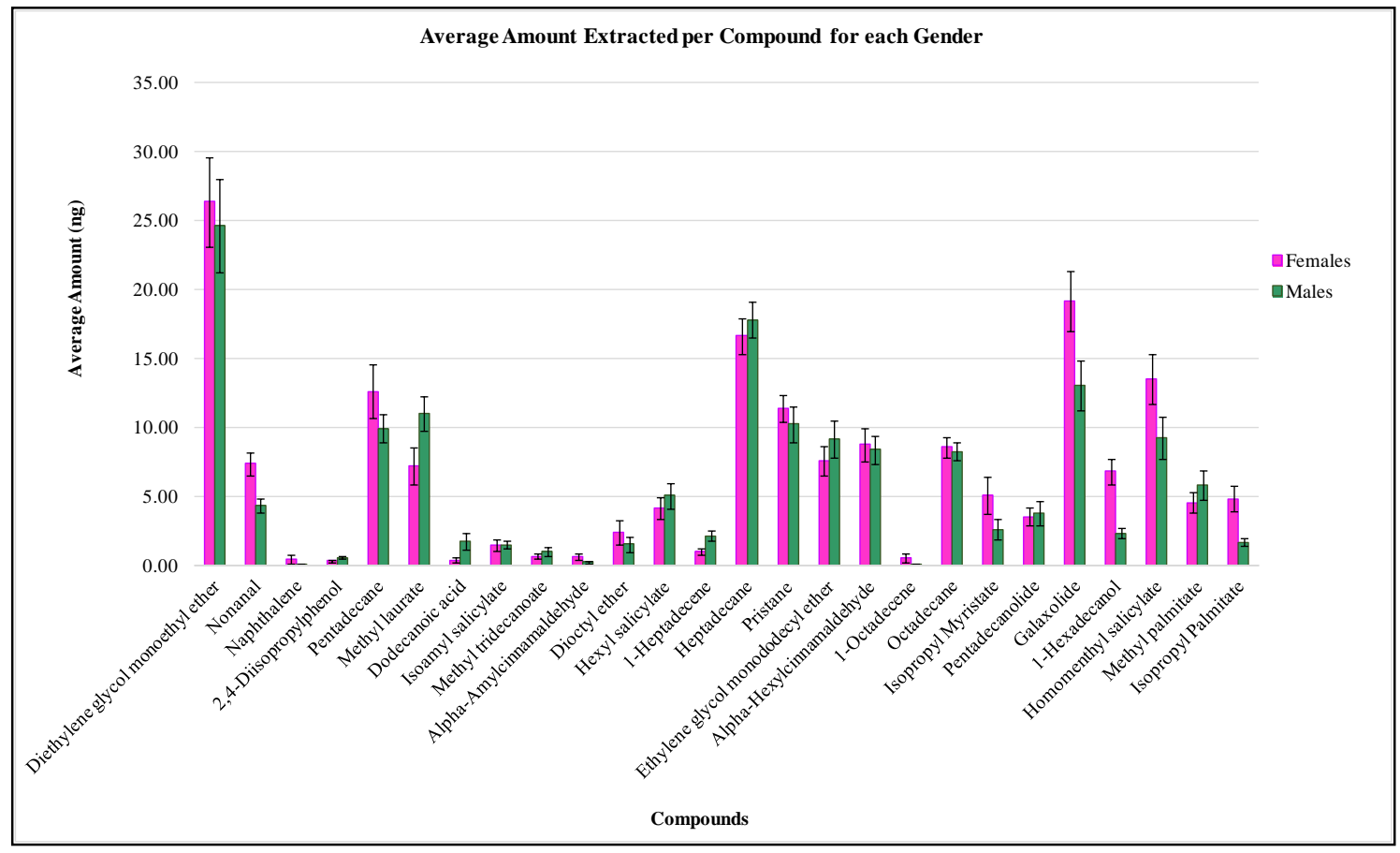

Figure 120: Average VOC amounts per gender group for all 26 VOCs in the initial compound list

The LDA performed using all original 26 VOCs as classification criteria (Table 31) provided a canonical plot that showed a clear separation between genders (Figure 
121). The CL ellipses around each of the groups' mean were not seen to intercept, which indicates an efficient differentiation of the individuals when considering gender as a trait. It was possible to classify individuals by gender with $79 \%$ accuracy, since only 35 out of 169 individuals were misclassified. From the individuals that were classified incorrectly, 21 out of 88 were females and 14 out of 81 were males. This suggests that males could be classified with a higher level of accuracy than females (76\% and $83 \%$ accuracy for females and males, respectively).

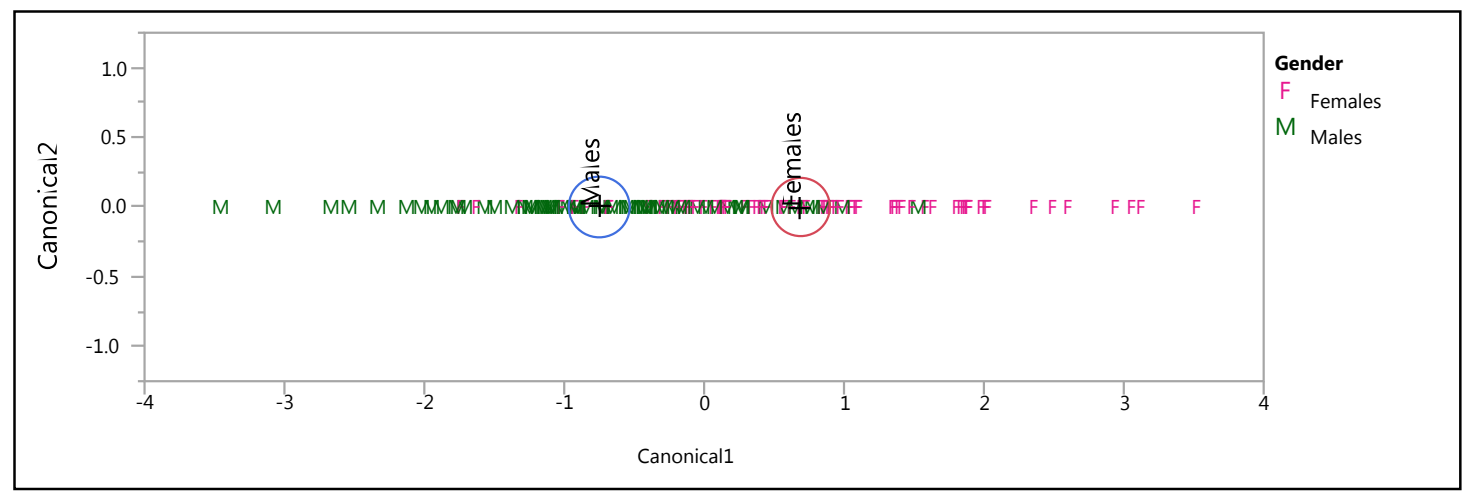

Figure 121: LDA canonical plot using all 26 VOCs as criteria for subject differentiation by gender

In an attempt to examine the possibility of improving the classification accuracy for individuals of different gender, the number of VOCs being used as differentiation criteria was reduced. A total of seven compounds selected for the secondary VOC list were taken into consideration and LDA was performed once again (Table 31). As a result of this approach it was observed that, despite of showing a slight reduction in the separation between the groups, the canonical plot still continued to portray a clear separation between individuals of different gender (Figure 122). However, with the slight reduction in distance between the groups came also a reduction in the percentage of 
classification accuracy. Compared to the previous approach, the set of variables employed in this method for individual classification only allowed a $67 \%$ accuracy of classification. The number of misclassified individuals in this case increased from 35 to 55 , and the increase was the result of a misclassification of $38 \%$ of the females and $27 \%$ of the males being considered.

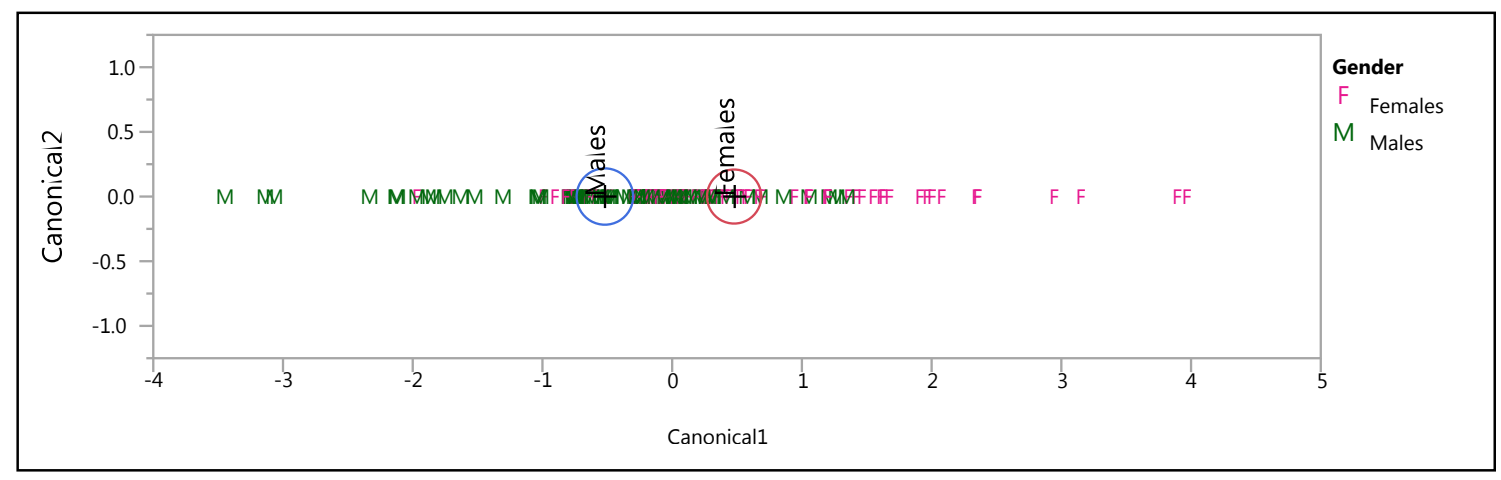

Figure 122: LDA canonical plot using compounds from secondary list as criteria for subject differentiation by gender

On the other hand, the stepwise regression approach was seen to enhance the ability to differentiate genders. As part of this method, 17 compounds were considered as variables for individual classification, leading to the achievement of an $80 \%$ of classification accuracy. The canonical plot showed, once again, a clear distinction between individuals of different genders and no overlap between the CL ellipses of both groups (Figure 123). There was only 20\% misclassification obtained through this method, which indicates that, in comparison to the two previous approaches, the stepwise method is more effective for the classification of individuals by their gender. A $77 \%$ of all females and an $84 \%$ of all males being considered were accurately classified in their corresponding groups. Therefore, these results suggest that the set of variables employed 
in this method, for individual classification by gender, represents the best criteria for such purposes.

Table 31 details the compounds selected through the stepwise regression method, along with the loading scores shown by each one for the first canon of the plot. In this table, each VOC has also been arranged in decreasing order of their influence on age group differentiation. The five compounds found to portray a higher influence on overall gender differentiation are the following: 1-Hexadecanol, 2,4-Diisopropylphenol, Pristane, Nonanal and Heptadecane.

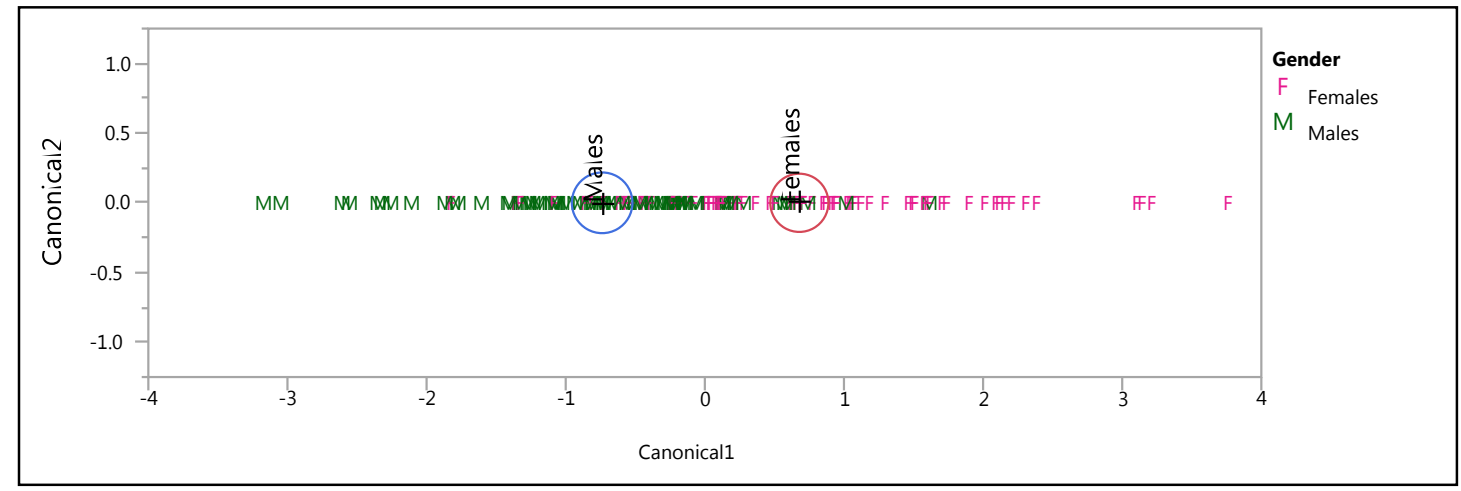

Figure 123: LDA canonical plot using compounds from stepwise regression method as criteria for subject differentiation by gender

The compounds identified as the most efficient criteria for overall gender differentiation, were also observed to exert impact in all cases of gender differentiation attempted in the independent population studies. In the Caucasian population, the secondary list used for the differentiation of females and males in the 18-30 old category included five of the 17 VOCs identified as most influential in overall gender differentiation (Table 31). On the other hand, both the secondary lists obtained to differentiate females and males in the 35-50 and 55+ year categories contained eight of 
these 17 VOCs. From the compounds found to coincide between the different secondary lists and the most influential VOCs for overall gender differentiation, 2,4Diisopropylphenol and 1-Hexadecanol were seen to frequently concur. These two compounds, considered the two VOCs with highest influence on overall gender differentiation, were also found to be among the compounds with most impact for some of the independent gender differentiations performed in the Caucasian study. An example of this was the differentiation of 18-30 year old Caucasian females and males, for which these two compounds were seen to also be highly influential factors.

In the case of the Hispanic population, the secondary lists used for gender differentiation in the 18-30, 35-50 and 55+ year age groups included seven, ten and eight VOCs, respectively, of the 17 most influential compounds for overall gender differentiation. Amongst those common compounds, the top four most influential compounds on overall gender differentiation (1-Hexadecanol, 2,4-Diisopropylphenol, Pristane and Nonanal) were found. Moreover, 1-Hexadecanol, Pristane and Nonanal were also seen to play an important and influential role on gender differentiation within the Hispanic study. This was particularly observed for gender differentiation in the 35-50 and $55+$ year categories.

Gender differentiation in the East Asian population was explored with secondary lists that contained ten, five and eight of the 17 VOCs stated as most influential for overall gender differentiation, respectively. Included in those coinciding VOCs were 1Hexadecanol and Pristane, compounds considered highly influential in the overall gender differentiation. In addition, these two compounds were also seen among the most influential compounds for gender differentiation in the East Asian study, which proves a 
correlation between the findings from both the overall and the independent population assessments.

The correlation observed between the differentiation criteria of both the overall study and the studies performed for each of the independent race/ethnic populations, supports the importance of using these VOCs for gender differentiation. The fact that the results from the different assessments are related provides a foundation for the findings being presented on this dissertation, and the potential use of human scent for gender differentiation. 
Table 31: VOC lists employed as criteria for subject differentiation by gender group in Linear Discriminant Analysis

\begin{tabular}{|c|c|c|c|}
\hline \multirow[b]{2}{*}{ Initial VOC List } & \multirow[b]{2}{*}{ Secondary VOC List } & \multicolumn{2}{|c|}{ Stepwise Regression Method } \\
\hline & & VOC List & $\begin{array}{c}\text { Canon } \\
1\end{array}$ \\
\hline $\begin{array}{l}\text { Diethylene glycol } \\
\text { monoethyl ether }\end{array}$ & Naphthalene & *1-Hexadecanol & 0.508 \\
\hline *Nonanal & Dodecanoic acid & *2,4-Diisopropylphenol & -0.456 \\
\hline Naphthalene & $\begin{array}{c}\text { Alpha- } \\
\text { Amylcinnamaldehyde }\end{array}$ & Pristane & 0.448 \\
\hline *2,4-Diisopropylphenol & *1-Heptadecene & *Nonanal & 0.398 \\
\hline Pentadecane & 1-Octadecene & Heptadecane & -0.355 \\
\hline *Methyl laurate & *1-Hexadecanol & *1-Heptadecene & -0.351 \\
\hline *Dodecanoic acid & Isopropyl Palmitate & $\begin{array}{c}\text { Alpha- } \\
\text { Hexylcinnamaldehyde }\end{array}$ & -0.348 \\
\hline Isoamyl salicylate & & Isopropyl Palmitate & 0.343 \\
\hline Methyl tridecanoate & & *Dodecanoic acid & -0.277 \\
\hline $\begin{array}{c}\text { Alpha- } \\
\text { Amylcinnamaldehyde }\end{array}$ & & *Galaxolide & 0.265 \\
\hline Dioctyl ether & & Homomenthyl salicylate & 0.222 \\
\hline Hexyl salicylate & & $\begin{array}{c}\text { Alpha- } \\
\text { Amylcinnamaldehyde }\end{array}$ & 0.170 \\
\hline *1-Heptadecene & & Isoamyl salicylate & 0.154 \\
\hline Heptadecane & & Pentadecane & 0.152 \\
\hline Pristane & & $\begin{array}{c}\text { Ethylene glycol } \\
\text { monododecyl ether }\end{array}$ & -0.150 \\
\hline $\begin{array}{c}\text { Ethylene glycol } \\
\text { monododecyl ether }\end{array}$ & & Naphthalene & 0.135 \\
\hline $\begin{array}{c}\text { Alpha- } \\
\text { Hexylcinnamaldehyde }\end{array}$ & & *Methyl laurate & -0.125 \\
\hline 1-Octadecene & & & \\
\hline Octadecane & & & \\
\hline Isopropyl Myristate & & & \\
\hline Pentadecanolide & & & \\
\hline *Galaxolide & & & \\
\hline *1-Hexadecanol & & & \\
\hline Homomenthyl salicylate & & & \\
\hline Methyl palmitate & & & \\
\hline Isopropyl Palmitate & & & \\
\hline
\end{tabular}

* Compounds for which the average VOC amounts were seen to be significantly different between the age groups 


\subsection{Determination of VOC markers for individual classification by race/ethnicity}

and gender

All tests presented so far have focused on evaluating the classification of the individuals under study in accordance with each one of the traits of interest separately. However, an attempt was also made to classify individuals by simultaneously considering two of these traits: race/ethnicity, and gender. According to all previous studies, the classification approach involving the VOCs from the secondary lists provided the lowest classification accuracy out of all methods evaluated. Therefore, for this test, only the approaches including the initial VOC list and the VOCs selected through the stepwise regression method were performed. The idea behind this test was to determine whether it was possible to still classify and differentiate individuals when considering two different traits at once. This would provide a better idea of the level of singularity expressed in a scent profile by influence of an individual's gender and race/ethnicity (regardless of age). For this purpose, the average VOC amounts of all 26 initial compounds were calculated for both females and males in each race/ethnicity (Figure 124). Subsequently, these values were used to identify VOC characteristics, in the different odor profiles, that could be associated to each gender in each race. 


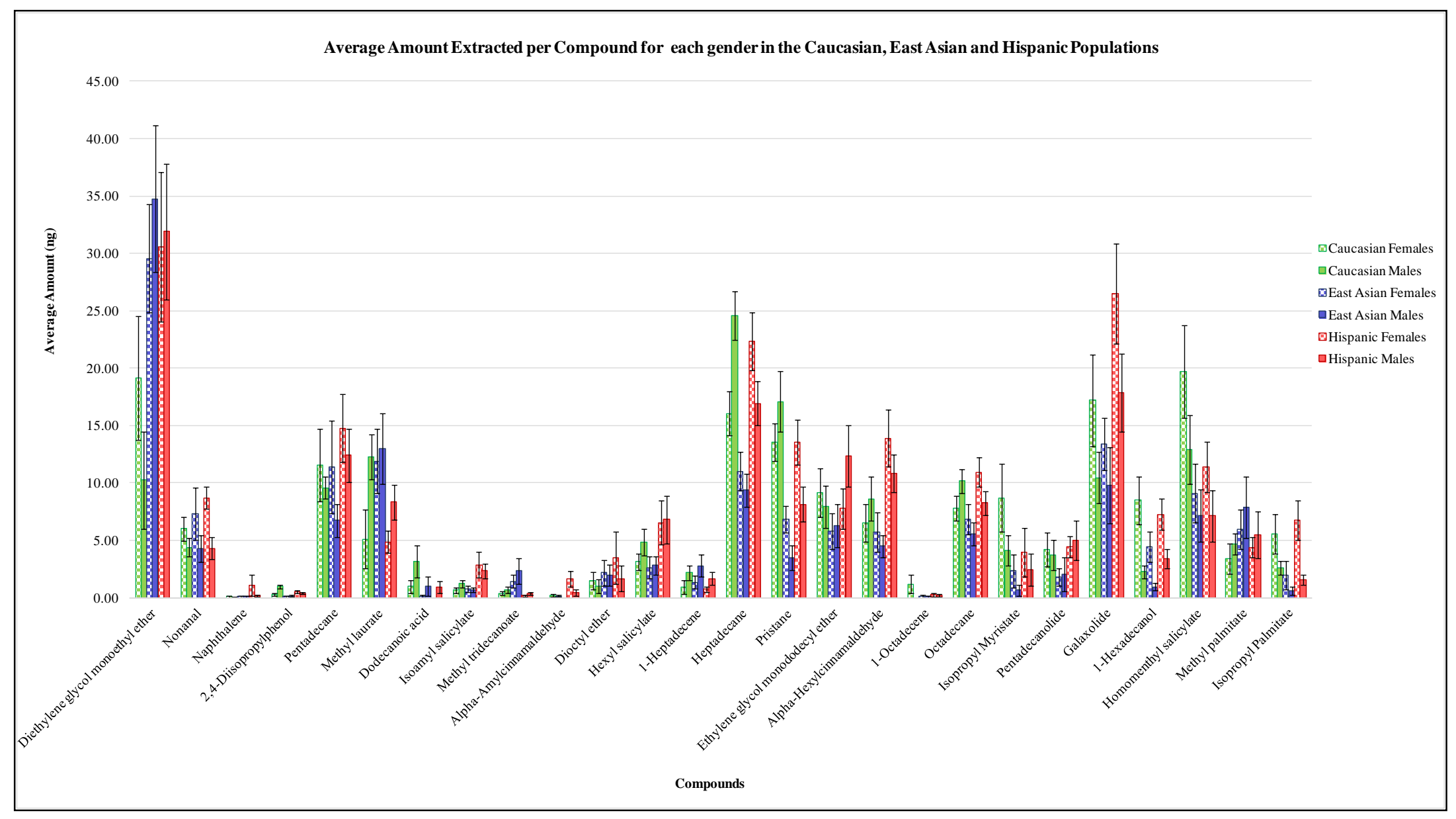

Figure 124: Average VOC amounts per race/ethnicity and gender for all 26 VOCs in the initial compound list 
As a result of this test, it was found that both the initial VOC list method and the stepwise regression method provide the highest accuracy when all 26 compounds are considered as variables. In both cases, 61\% accuracy of classification was achieved with 66 of 169 subjects being classified incorrectly. The LDA canonical plot showed, unlike the rest of the LDA plots previously presented, that the CL ellipses of certain groups intercepted (Figure 125). This means that the use of these 26 compounds as classification criteria was not able to provide an efficient differentiation for such groups. The canonical plot showed an overlap between the ellipses of East Asian Males and Females, East Asian Females and Hispanic Males, Caucasian Females and Hispanic Females, and Caucasian Females and Hispanic Males. Therefore, the odor profiles for each of these groups seemed to share similarities that did not facilitate a clear differentiation and correct classification of individuals in these groups.

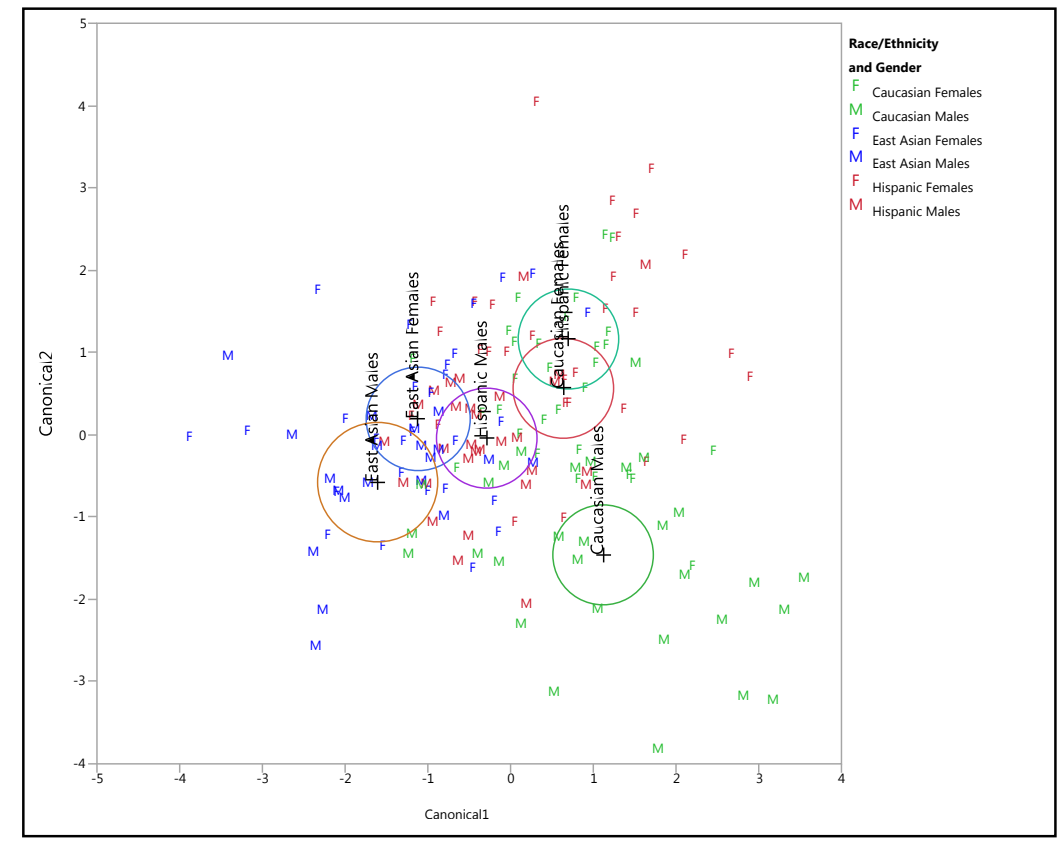

Figure 125: LDA canonical plot obtained through both the initial list and the stepwise regression methods for subject differentiation by race/ethnicity and gender 
In an attempt to reduce any possible "noise" being induced in the canonical plot as a result of so many groups being considered at one time, the same analysis was performed by breaking down the sample pool into females and males of different races/ethnicities. In the case of both females and males, considering the initial VOC list as differentiation variables provided an overall classification accuracy of $83 \%$. Out of a total of 88 females and 81 males, only 15 and 14 were misclassified, respectively. From a total of 30 Caucasian females, one was misclassified as East Asian and three as Hispanic. Also, of 28 East Asian females, two were incorrectly classified as Hispanic and one as Caucasian while, from 30 Hispanic females, six were classified as East Asian and only two as Caucasian. The misclassification distribution resulted a little different for males. Out of 30 Caucasian and 30 Hispanic males, four males were misclassified as East Asian and two as Hispanic in both cases. On the other hand, from a total of 21 East Asian males, only two were misclassified as Hispanic. No East Asian male was found to be misclassified in the Caucasian category. In both the canonical plot obtained for females and males independently (Figure 126 and Figure 127), a clear separation of the racial/ethnic groups was observed. There were no cases in which the CL ellipses were seen to overlap for any groups. Therefore, it was noted that the 26 compounds served as good differentiation criteria and facilitated an efficient classification of individuals from both genders. In addition, it was found that the compounds of highest influence in achieving this differentiation were Heptadecane, Octadecane, Pristane, 2,4Diisopropylphenol and Dodecanoic acid (in decreasing order). 


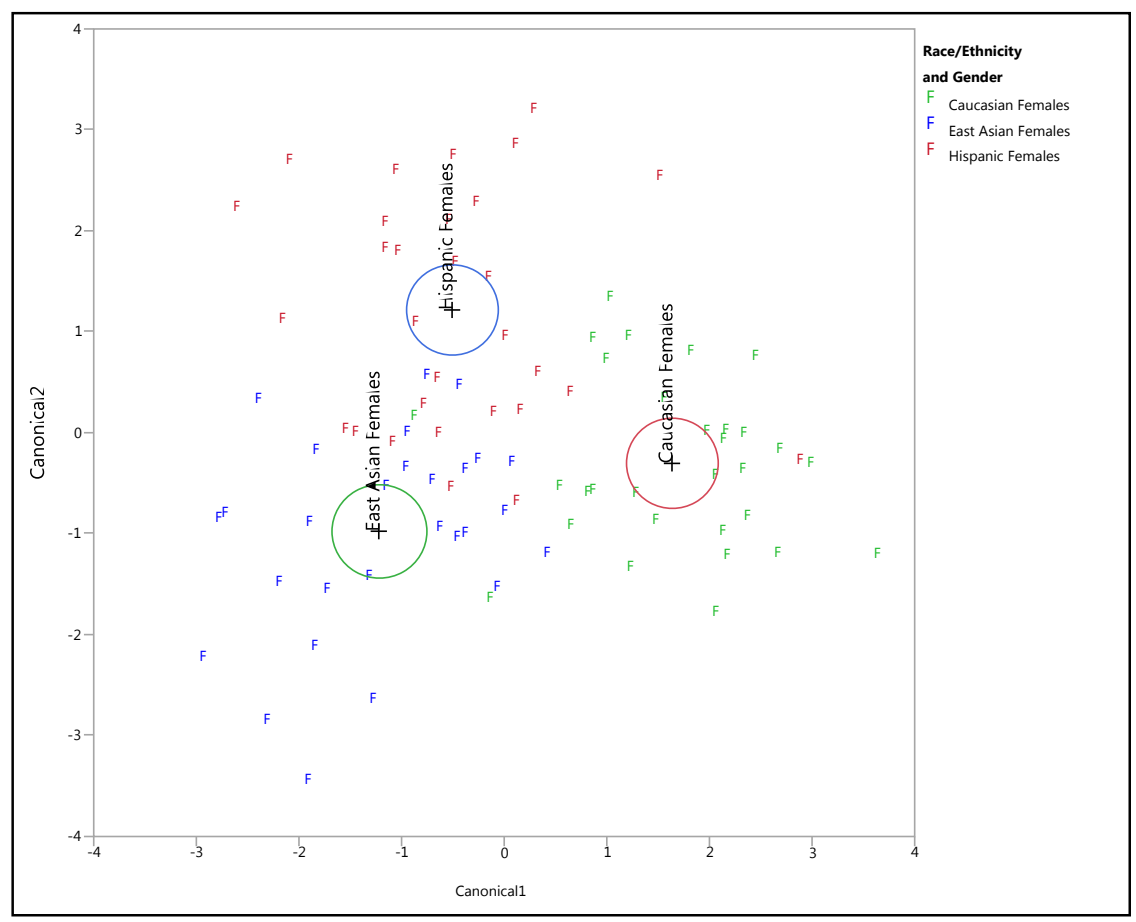

Figure 126: LDA canonical plot obtained using the initial VOC list for female subject differentiation by race/ethnicity

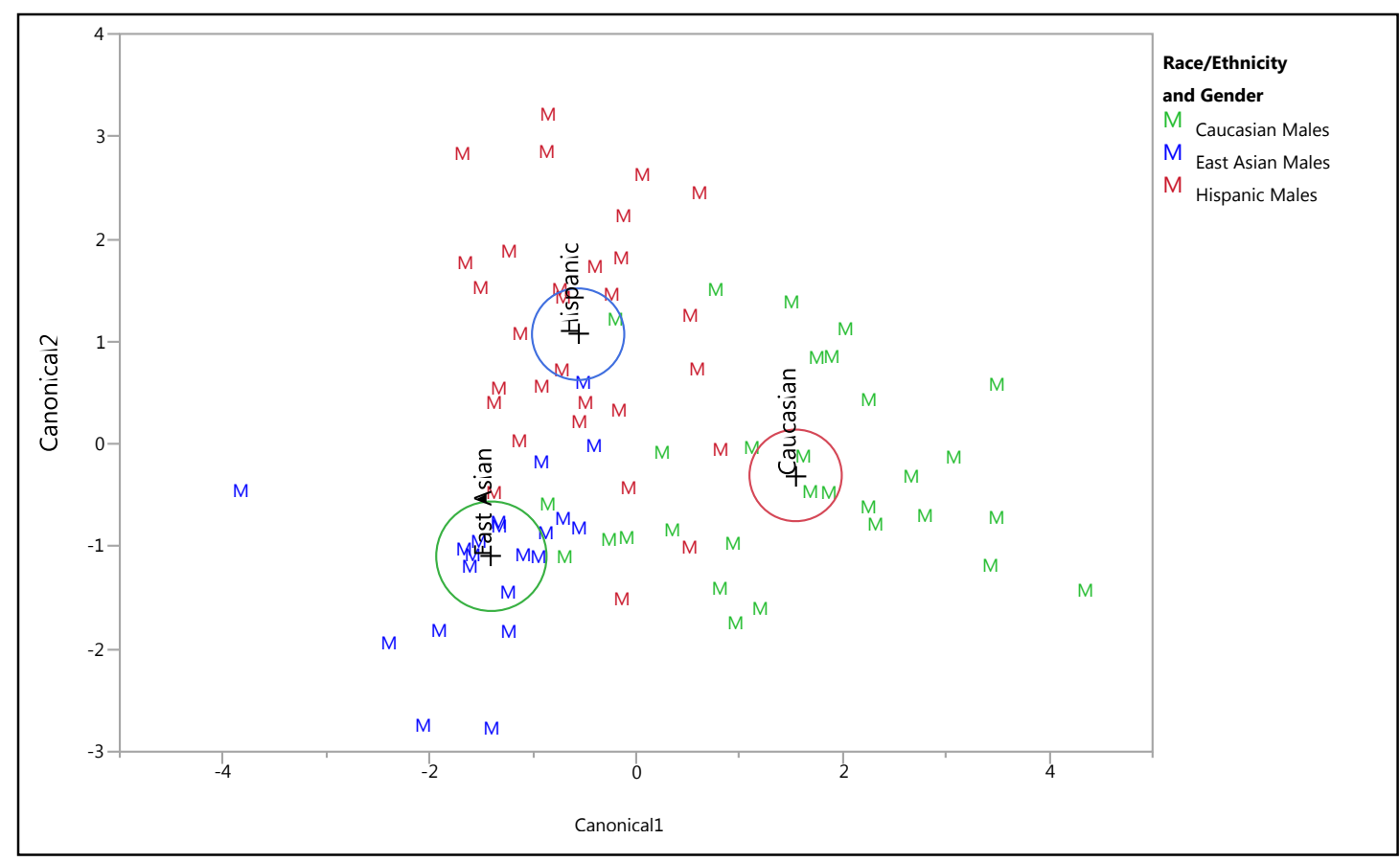

Figure 127: LDA canonical plot obtained using the initial VOC list for male subject differentiation by race/ethnicity 
After having observed that in previous tests the stepwise regression method provided the most efficient approach for individual classification, the same approach was also implemented in the scope of this analysis. In the case of females, the number of discrimination criteria was reduced to 19 VOCs (Table 32), from which Pristane, Dodecanoic acid, Octadecane, 1-Heptadecene, and Homomenthyl salicylate were seen to be the most influential on differentiation. This modification in the selection of VOCs caused the overall classification accuracy percent to increase from $83 \%$ to $85 \%$. Therefore, the new VOC selection enhanced the differentiation power for females of different racial/ethnic groups. The slight improvement in classification accuracy was caused by a reduction in the number of misclassified females from the Caucasian and Hispanic groups. Unlike before, only two Caucasian females were misclassified as Hispanic, while only five Hispanic females were misclassified as East Asian. No changes in the number of misclassified subjects were seen for East Asian females. This suggests that the changes in the VOC selection only served to increase the level of variation between Hispanic females and those from the other two groups. The canonical plot obtained for this approach (Figure 128) depicts the positioning of all female groups as a result of the influence of all VOCs considered. 


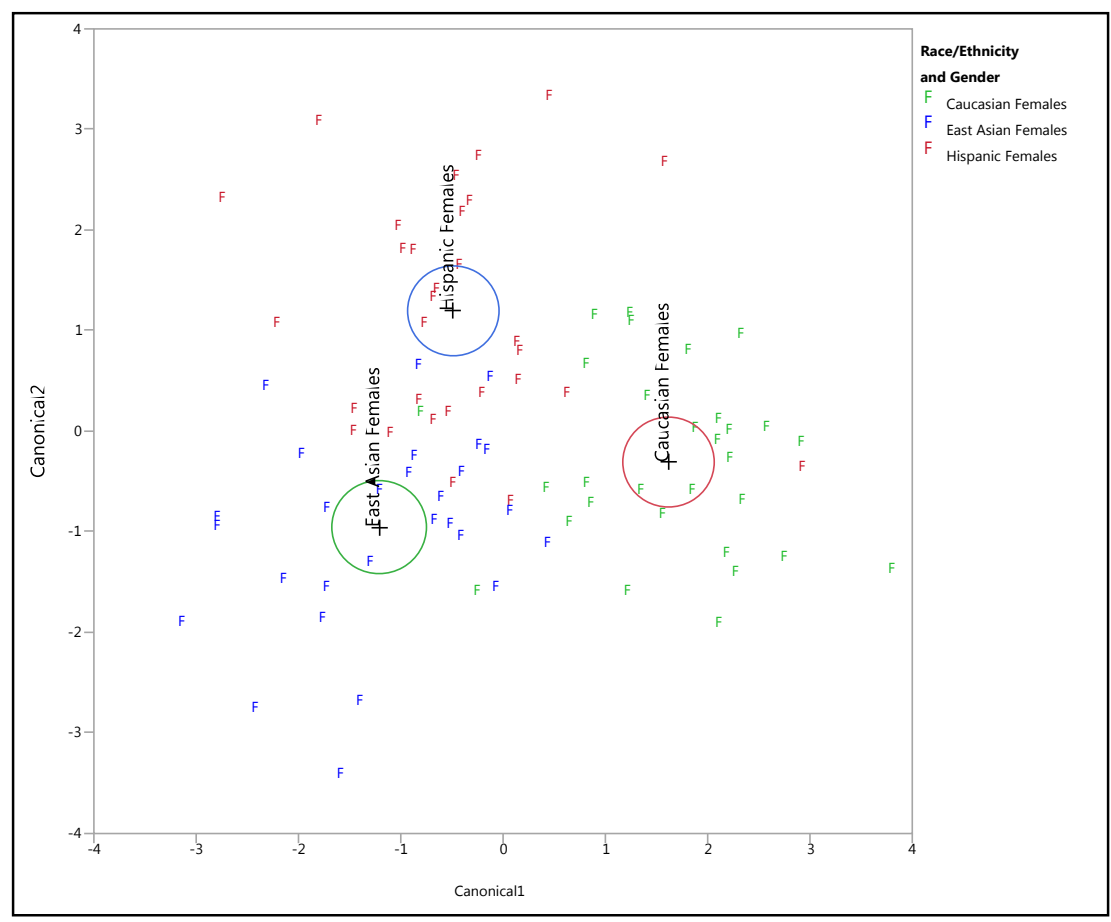

Figure 128: LDA canonical plot using compounds from stepwise regression method as criteria for female subject differentiation by race/ethnicity 
Table 32: VOC lists employed as criteria for female differentiation by racial/ethnic group in Linear Discriminant Analysis

\begin{tabular}{|c|c|c|c|}
\hline \multirow[b]{2}{*}{ Initial VOC List } & \multicolumn{3}{|c|}{ Stepwise Regression Method } \\
\hline & VOC List & $\begin{array}{c}\text { Canon } \\
1\end{array}$ & $\begin{array}{c}\text { Canon } \\
2\end{array}$ \\
\hline Diethylene glycol monoethyl ether & Pristane & 1.357 & -0.239 \\
\hline Nonanal & Dodecanoic acid & 1.084 & -0.355 \\
\hline Naphthalene & Octadecane & -1.020 & 0.055 \\
\hline 2,4-Diisopropylphenol & 1-Heptadecene & -0.860 & -0.359 \\
\hline Pentadecane & Homomenthyl salicylate & 0.660 & 0.087 \\
\hline Methyl laurate & Hexyl salicylate & -0.568 & 0.528 \\
\hline Dodecanoic acid & Methyl laurate & -0.510 & -0.679 \\
\hline Isoamyl salicylate & 1-Octadecene & 0.446 & 0.399 \\
\hline Methyl tridecanoate & Pentadecanolide & 0.434 & 0.249 \\
\hline *Alpha-Amylcinnamaldehyde & Pentadecane & -0.417 & -0.118 \\
\hline Dioctyl ether & *Alpha-Hexylcinnamaldehyde & 0.383 & -0.509 \\
\hline Hexyl salicylate & Galaxolide & -0.324 & 0.547 \\
\hline 1-Heptadecene & Isopropyl Myristate & 0.309 & -0.445 \\
\hline *Heptadecane & Nonanal & -0.245 & 0.267 \\
\hline Pristane & Diethylene glycol monoethyl ether & -0.232 & 0.262 \\
\hline Ethylene glycol monododecyl ether & *Alpha-Amylcinnamaldehyde & -0.202 & 0.458 \\
\hline *Alpha-Hexylcinnamaldehyde & 2,4-Diisopropylphenol & -0.167 & 0.396 \\
\hline 1-Octadecene & Heptadecane & 0.145 & 0.668 \\
\hline Octadecane & Isopropyl Palmitate & 0.045 & 0.297 \\
\hline \multicolumn{4}{|l|}{ Isopropyl Myristate } \\
\hline \multicolumn{4}{|l|}{ Pentadecanolide } \\
\hline \multicolumn{4}{|l|}{ Galaxolide } \\
\hline \multicolumn{4}{|l|}{ 1-Hexadecanol } \\
\hline \multicolumn{4}{|l|}{ Homomenthyl salicylate } \\
\hline \multicolumn{4}{|l|}{ Methyl palmitate } \\
\hline Isopropyl Palmitate & & & \\
\hline
\end{tabular}

* Compounds for which the average VOC amounts were seen to be significantly different between the age groups 
On the other hand, in the case of males, the stepwise regression method involved the use of 17 VOCs (Table 33). From these 17 compounds, the five VOCs to portray most influence on differentiation were Heptadecane, 2,4-Diisopropylphenol, Octadecane, Diethylene glycol monoethyl ether, and Methyl tridecanoate. On the other hand, the reduction in the number of classification variables did not represent a change in the percentage of classification accuracy for this case. In both cases, 83\% accuracy was achieved, yet different numbers of differentiation criteria were needed to achieve that specific level of accuracy. Hence, this presents the possibility of being able to assess male classification for different racial/ethnic groups with high accuracy, without having to take into consideration a set of criteria as vast as the initial VOC list. As it was previously presented for females, the canonical plot obtained for this approach (Figure 129) shows the positioning of all male groups in response to the influence exerted by the VOCs considered on the different canons.

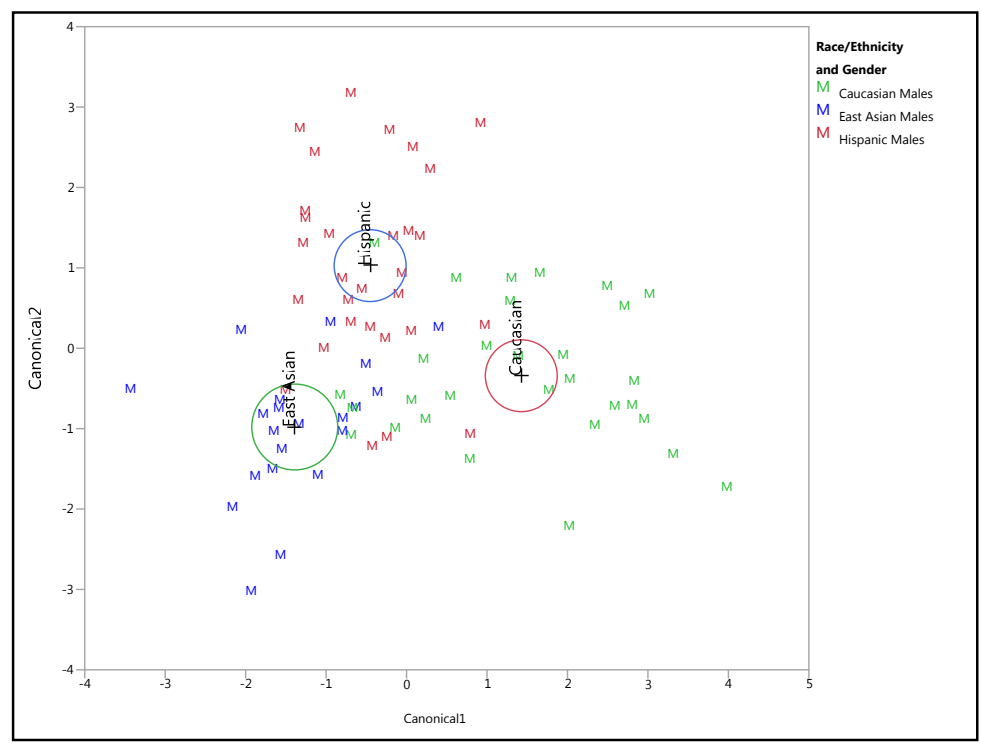

Figure 129: LDA canonical plot using compounds from stepwise regression method as criteria for male subject differentiation by race/ethnicity 
Table 33: VOC lists employed as criteria for male differentiation by racial/ethnic group in Linear Discriminant Analysis

\begin{tabular}{|c|c|c|c|}
\hline \multirow[b]{2}{*}{ Initial VOC List } & \multicolumn{3}{|c|}{ Stepwise Regression Method } \\
\hline & VOC List & $\begin{array}{c}\text { Canon } \\
1\end{array}$ & $\begin{array}{c}\text { Canon } \\
2\end{array}$ \\
\hline $\begin{array}{l}\text { *Diethylene glycol monoethyl } \\
\text { ether }\end{array}$ & *Heptadecane & 1.011 & 0.441 \\
\hline Nonanal & *2,4-Diisopropylphenol & 0.694 & -0.404 \\
\hline Naphthalene & *Octadecane & -0.455 & 0.061 \\
\hline *2,4-Diisopropylphenol & $\begin{array}{l}\text { *Diethylene glycol monoethyl } \\
\text { ether }\end{array}$ & -0.417 & 0.124 \\
\hline Pentadecane & Methyl tridecanoate & -0.377 & -0.264 \\
\hline Methyl laurate & Pentadecane & -0.332 & 0.398 \\
\hline Dodecanoic acid & Dodecanoic acid & 0.294 & 0.100 \\
\hline Isoamyl salicylate & 1-Heptadecene & -0.287 & -0.531 \\
\hline Methyl tridecanoate & Galaxolide & -0.237 & 0.623 \\
\hline Alpha-Amylcinnamaldehyde & *Alpha-Hexylcinnamaldehyde & 0.152 & -0.525 \\
\hline Dioctyl ether & 1-Octadecene & -0.144 & -0.346 \\
\hline Hexyl salicylate & Methyl laurate & -0.110 & -0.458 \\
\hline 1-Heptadecene & Alpha-Amylcinnamaldehyde & 0.104 & 0.410 \\
\hline *Heptadecane & Homomenthyl salicylate & 0.097 & -0.396 \\
\hline *Pristane & Isoamyl salicylate & -0.091 & 0.707 \\
\hline Ethylene glycol monododecyl ether & Ethylene glycol monododecyl ether & -0.042 & 0.475 \\
\hline *Alpha-Hexylcinnamaldehyde & Hexyl salicylate & -0.019 & 0.530 \\
\hline \multicolumn{4}{|l|}{ 1-Octadecene } \\
\hline \multicolumn{4}{|l|}{ *Octadecane } \\
\hline \multicolumn{4}{|l|}{ Isopropyl Myristate } \\
\hline \multicolumn{4}{|l|}{ Pentadecanolide } \\
\hline \multicolumn{4}{|l|}{ Galaxolide } \\
\hline \multicolumn{4}{|l|}{ 1-Hexadecanol } \\
\hline \multicolumn{4}{|l|}{ Homomenthyl salicylate } \\
\hline \multicolumn{4}{|l|}{ Methyl palmitate } \\
\hline *Isopropyl Palmitate & & & \\
\hline
\end{tabular}

* Compounds for which the average VOC amounts were seen to be significantly different between the age groups 


\subsection{Conclusion on the determination of scent markers in underarm odor samples}

The assessment of underarm odor profiles within specific race/ethnic populations facilitated the identification of characteristic VOC tendencies that can be associated to traits (e.g., gender and age) in individuals from different populations. In the case of most populations and compounds under study, females were characterized by having higher average VOC amounts than males in their scent profiles. Isopropyl palmitate was one of the compounds for which this particular trend was observed. This compound had been previously reported as a gender marker for females after displaying a higher abundance in odor profiles of such gender in comparison to those of males. ${ }^{21,37}$ Therefore, the fact that the current study identified this trend in the majority of female odor profiles supports previous reports. There were only two cases in which male averaged VOC profiles displayed higher abundance of Isopropyl palmitate. Caucasian males in the 35-50 year group, and East Asian males in the 18-30 year group, displayed higher average amounts of this VOC than females in their corresponding age groups. However, the differences in abundance between genders were very close in magnitude. This observation agrees with findings from previous research work that suggests there should not be a significant difference between the amounts of this VOC in both genders. ${ }^{21}$ Therefore, this could explain why these exceptions happened to be observed in the underarm odor assessment. On the other hand, there were other trends observed in this study that suggested deviations from previous published peer reviewed reports. According to Gallagher et al., whose study considered nine Caucasian, and three African American 41-79 year olds, Nonanal is a biomarker for increased age as a consequence of its higher abundance in older individuals. ${ }^{21}$ However, the results reported in this dissertation indicate that $55+$ 
year old Hispanic males, East Asian females and East Asian males possess the lowest VOC abundance for this compound in their underarm odor profiles. These results suggest that Nonanal may not necessarily be an age biomarker for all racial/ethnic populations. The possibility of this statement is also supported by the fact that the results obtained for the Caucasian population surveyed in this dissertation did support Gallagher et al.'s findings, contrary to the Asian and Hispanic groups. Consequently, these results create awareness of the fact that generalizing this type of conclusion on scent biomarkers could lead to incorrect reports. Moreover, these results suggest that further confirmation should be attained by surveying a greater diversity of racial/ethnic populations prior to reporting this type of conclusions.

On the other hand, among the most important observations that were made during the assessment of underarm odor profiles within each individual racial/ethnic population was that the characteristic properties of each group rely on sets of combined VOCs, rather than on the influence of specific VOCs. This finding served to expand on the work of Penn et al., in which it was stated that body odor differences between females and males are characterized by a multivariate distribution of marker compounds. ${ }^{37}$ The data analyzed and discussed in this dissertation supports this statement, and shows that this is not only applicable when considering gender differences, but also for age and race/ethnicity differences. Moreover, another interesting finding from the analysis of the results in this dissertation was that secondary lists share a type of VOC "backbone”. This "backbone” consists of compounds that are present in the characteristic VOC assortment of all groups taking part in a specific comparison. For instance, Dodecanoic acid, 1Heptadecene and Isopropyl palmitate were equally included in the secondary lists created 
for Hispanic gender comparison in all the different age groups. Hence, these compounds served as "backbone" to the characteristic VOC assortments associated to each of these age groups for gender differentiation. The compounds in these "backbones" are thought to serve as consistent sources of variation between groups. The reason for this is that, despite of these "backbone" VOCs being identified more consistently than other compounds, they still don't preclude the singularity of different secondary lists. Therefore, the occurrence of these VOC "backbones" in the VOC assortments identified for each group results in a significant enhancement for group differentiation. Moreover, this assessment also revealed that underarm odor VOC assortments included compounds for which the average amount displayed, by the groups being differentiated, showed statistical significant difference. Despite the fact that such compounds were not found to be enough to achieve optimum group differentiation, the statistically significant differences do explain the presence of these compounds in each group's characteristic VOC assortment.

Besides investigating human scent peculiarities in the underarm odor profiles of individuals of different age and gender, within specific racial/ethnic populations, this study also pursued another relevant objective. The identification of human scent biomarkers that could be associated to traits shared amongst individuals, in specific age, gender, and race or ethnicity, stood as the main goal of this project. Investigating the scent profiles of individuals that belonged to a same age, gender or race/ethnic group allowed the association of specific VOC characteristics for each trait. Therefore, this investigation facilitated the identification of those VOCs useful for organizing and grouping individuals in accordance to their age, gender and race/ethnicity. These three 
traits have been, without a doubt, seen to play a role on scent expression. Variations in the qualitative and quantitative nature of odor profiles, amongst groups with differing traits, have acted as primary sources of evidence for such influence. Nevertheless, the assessment of underarm odor profiles has also revealed that the characteristic compound combinations identified for each trait are constituted by VOCs that could be both of endogenous or exogenous nature to the human body. ${ }^{21,48,122,124}$ This represents an important observation because it suggests that, despite the great correlation between trait expression and characteristic human scent features, there are also external factors that help shape the expression of these traits' influence in human odor. The presence of potential exogenous compounds in the characteristic VOC assortment of each group was not seen to preclude, but instead enhance group differentiation in this study. For this reason, it is necessary to establish that VOCs in these assortments should be considered human scent "markers" instead of "biomarkers". The fact that external factors have been seen to consistently influence the level of differentiation between the groups rejects the idea that group differentiation is only dependent on biomarker differences. Hence, from this point forward, characteristic VOCs will be referred to in this dissertation as "human scent markers”. Nevertheless, the development of further studies that seek to associate the origin and exudation of endogenous human scent compounds, with the already identified VOC characteristics of each group, will most likely advance the ability to differentiate groups by traits. Ensuring all VOCs associated to a group's characteristic human scent are strictly exuded by the human body, requires work outside of the scope of this dissertation. Findings from this study provide the foundation for other studies to expand knowledge on the nature of VOC expression and its direct association with specific traits. 
The results obtained from this assessment led to the identification of "VOC marker combinations" associated to age, gender and race/ethnicity as common or shared traits. This achievement represents the fulfillment of one of this dissertation's most important objectives.

The power of these VOC marker combinations has been shown by the level of accuracy achieved in the classification of all individuals under study into their corresponding groups. According to the data, the classification accuracy obtained when using underarm odor profiles for group differentiation was $61 \%$ or more. This means that in all cases the reported VOC marker combinations allowed the correct classification of more than half of the individuals under study. The case in which $61 \%$ accuracy was achieved considered the classification of all individuals under study by both race/ethnicity and gender. Hence, the fact that the number of VOC variables being used for group differentiation resulted too limited to asses these two types of simultaneous classifications, may explain why this differentiation attempt achieved the lowest percent of classification accuracy. On the other hand, when the classification attempt was made for females and males on an independent basis, it was seen that females were accurately classified by race/ethnic group with $85 \%$ accuracy, while males showed and accuracy of 83\%. From the other classification attempts pursued, it was found that classifying all individuals under study by age group, regardless of their gender and race/ethnicity, provided the next lowest accuracy level, 63\%. Considering age as a trait for individual classification was expected to display a higher level of inaccuracy; especially between those age groups that were closest to one another and for which age's impact on odor profiles may have appeared less defined. The canonical plots obtained for this 
classification attempt demonstrated that, in fact, the highest level of discrimination was achieved between those age groups that were further apart (18-30 and 55+ years). This observation supports that the lack of abrupt differences between the odor profiles of individuals in closer age groups, may have affected the classification accuracy achieved. On the other hand, the classification of all individuals under study by race/ethnicity was achieved with $70 \%$ accuracy, and $80 \%$ accuracy was achieved for the attempt to classify individuals by gender. These last percentages represent the best examples of how the VOC marker combinations reported in this study could become efficient tools for individual classification by specific traits. Moreover, it was also noticed that some of the VOCs that had been previously identified as important for subject differentiation in the independent population studies, also coincided with VOCs from the marker combinations established during the overall comparison study. This type of correlation between the results of both underarm odor assessments highlights the relevance and potential of using the VOCs reported for scent profile differentiation.

The assessment of underarm odor profiles served as starting point to inquire on the way age, gender, and race/ethnicity can influence the way an individual portrays human scent. The characteristics and trends identified in the VOC profiles, from each of the groups under study, made possible to evaluate the feasibility of subject differentiation on the basis of characteristic features. Therefore, discovering that underarm odor profiles display VOC marker combinations as a result of these traits represents a significant step for human scent investigation. 


\subsubsection{Determination of scent marker consistency in hand odor samples}

Hand odor profiles (Figure 130 - Figure 135) were created for a total of 105 individuals (Table 34) to assess the potential identification of VOC characteristics in hand odor composition. In the same manner it was done in the evaluation of underarm odor samples, the identification of these characteristics aimed to determine their association with age, gender, and race/ethnicity, as traits in a diverse population of individuals. This type of association would allow evaluating the use of a combination of hand odor VOCs as markers for the classification of individuals on the basis of these traits. In addition, this approach was also intended to facilitate a comparison between VOC marker combinations identified for each trait in both hand and underarm odor samples. The comparison of marker combinations from both body regions would allow determining any consistencies between the two types of scent profiles under study. This assessment would not only provide additional support to the already identified VOC marker combinations found in underarm scent profiles, but would also highlight the relevance and feasibility of applying this type of finding in the Forensic Science field. Hand odor is often found in crime scenes because of its ease of transfer as perpetrators and victims touch the scene. Therefore, the availability of hand odor as human scent evidence makes the assessment of marker combinations in hands be significant to forensic investigations. 


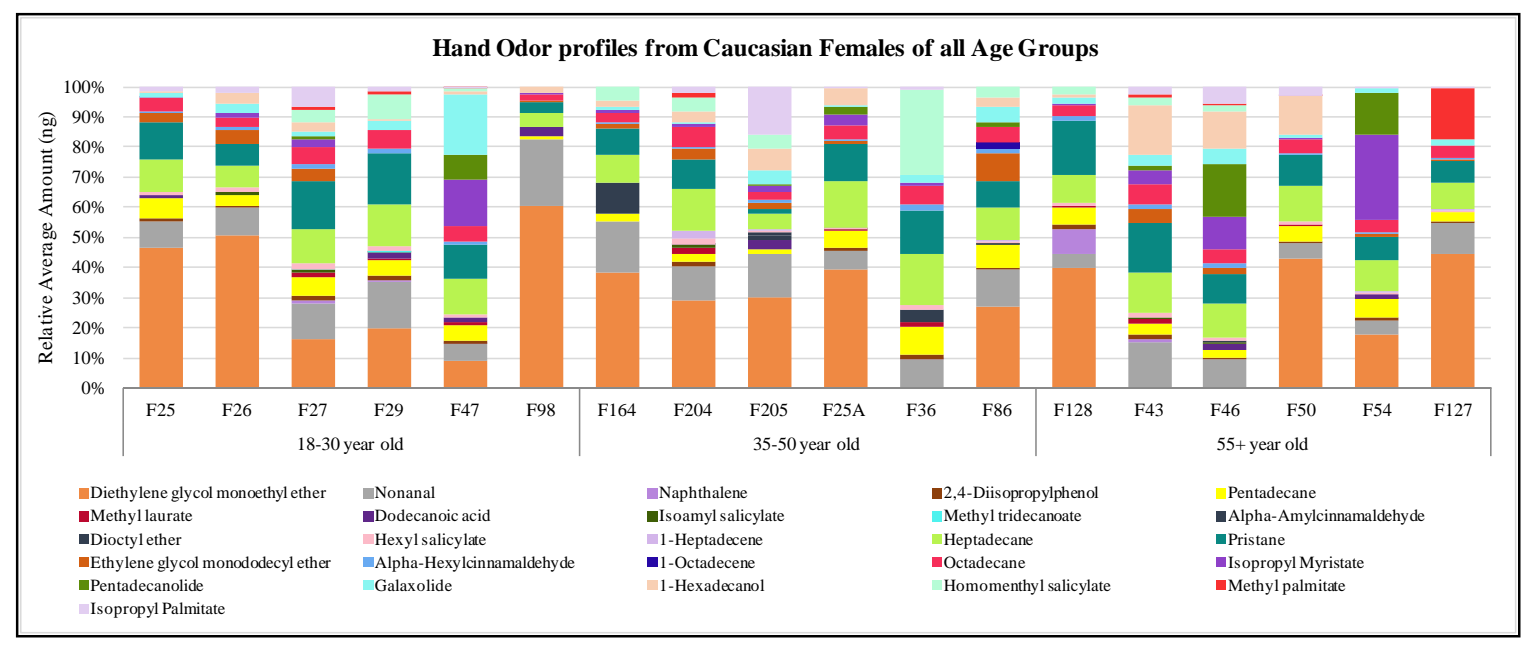

Figure 130: Hand odor profiles from Caucasian females of all age groups

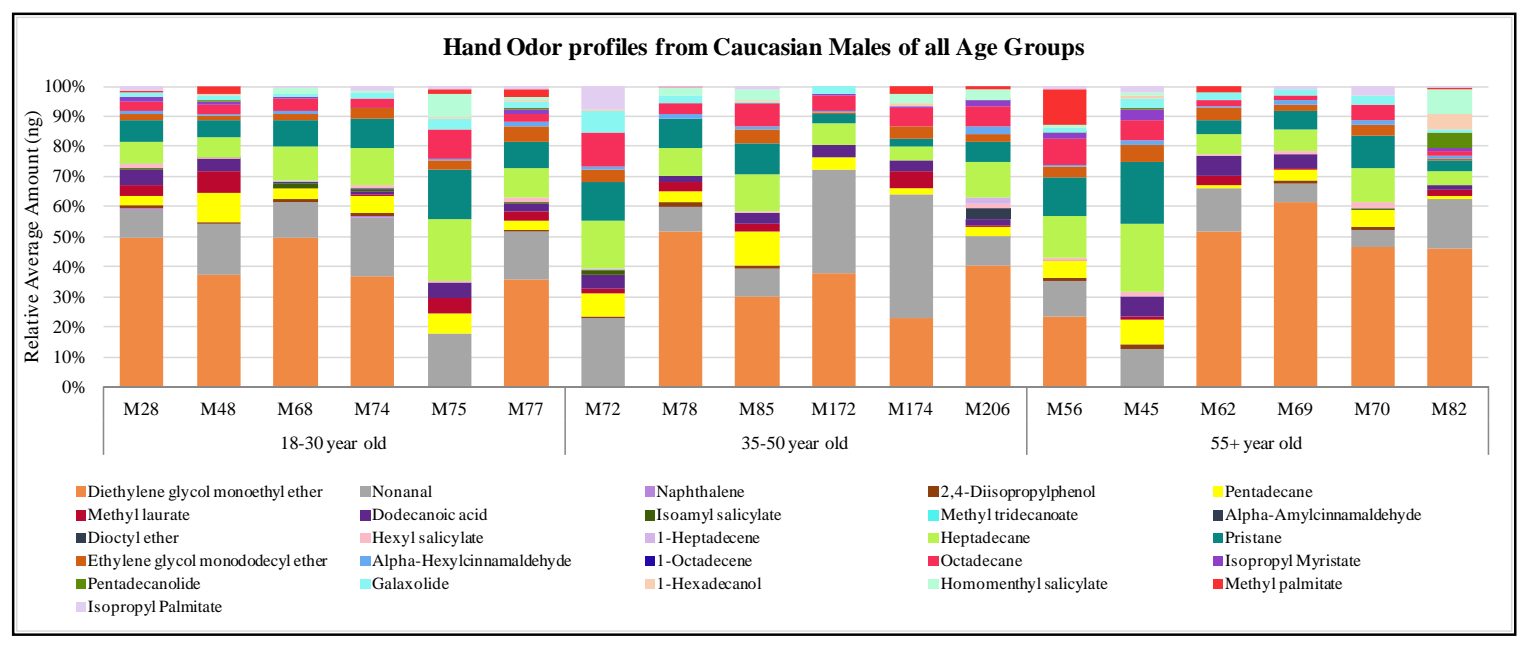

Figure 131: Hand odor profiles from Caucasian males of all age groups 


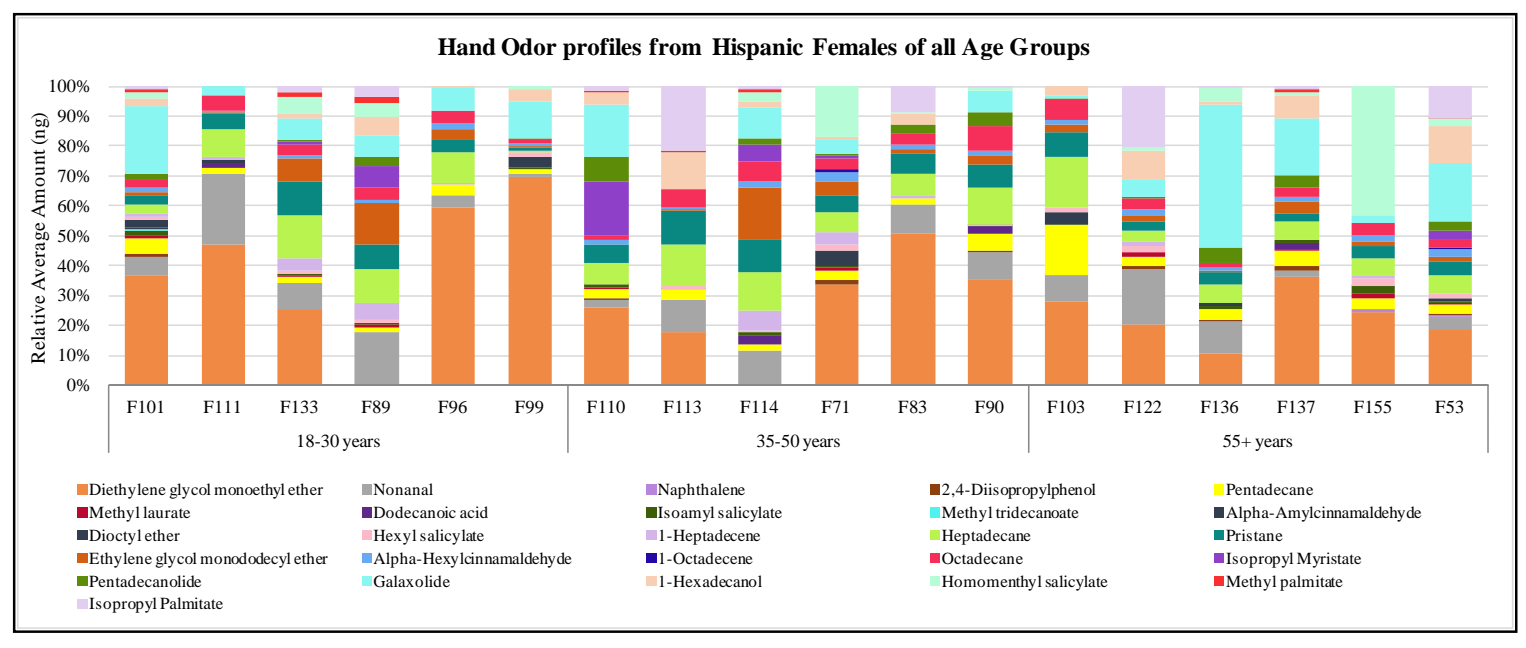

Figure 132: Hand odor profiles from Hispanic females of all age groups

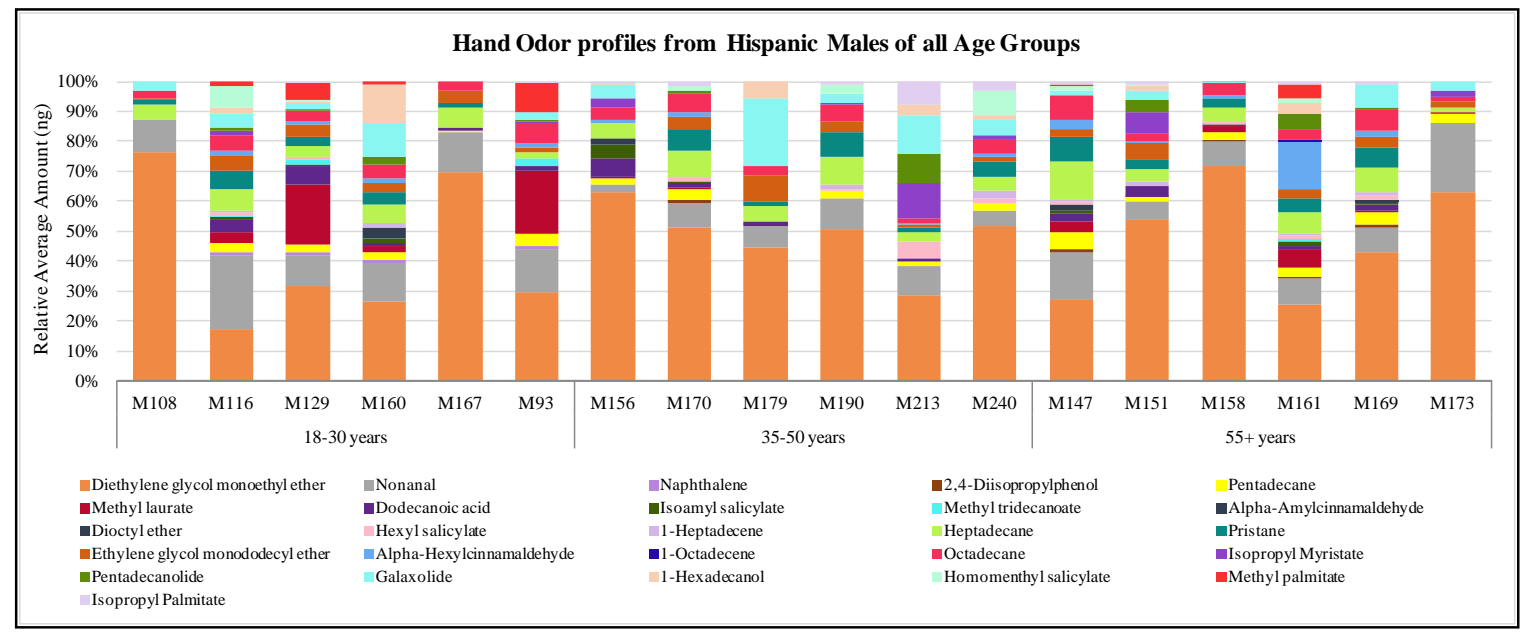

Figure 133: Hand odor profiles from Hispanic males of all age groups 


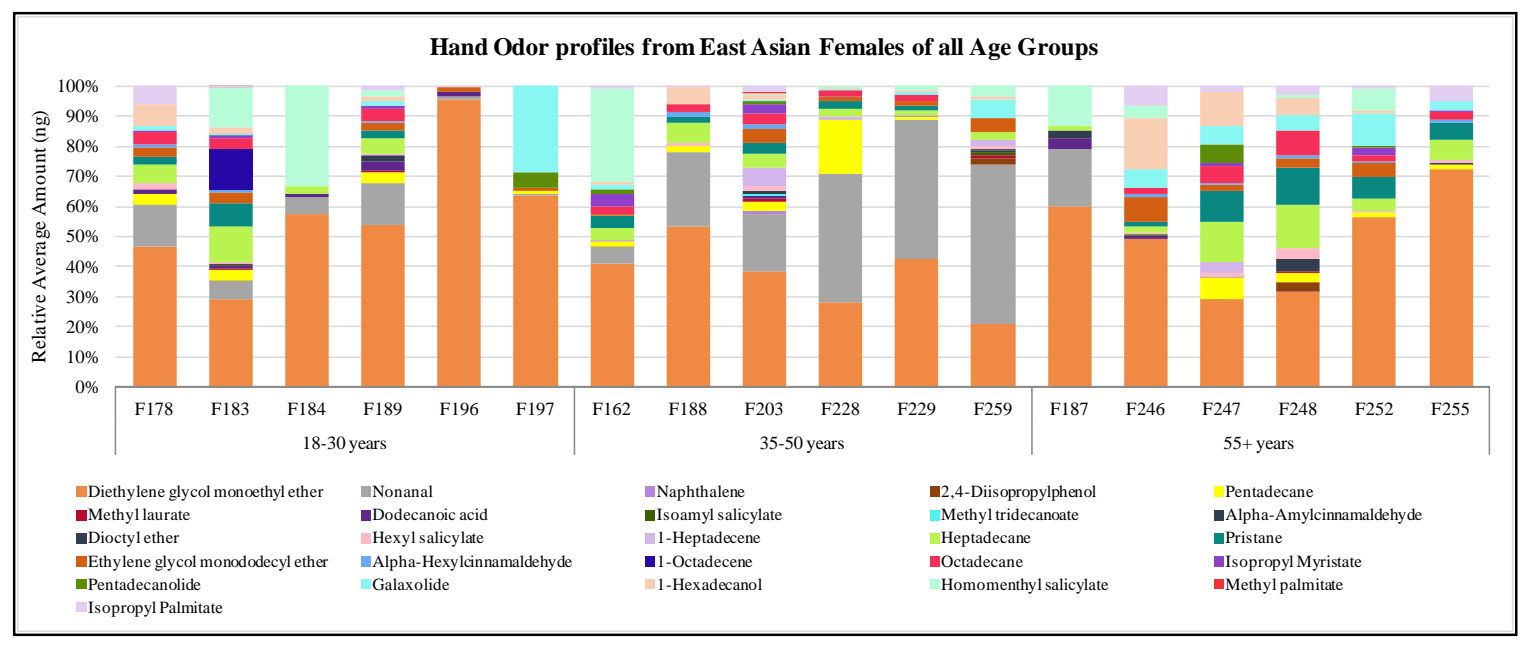

Figure 134: Hand odor profiles from East Asian females of all age groups

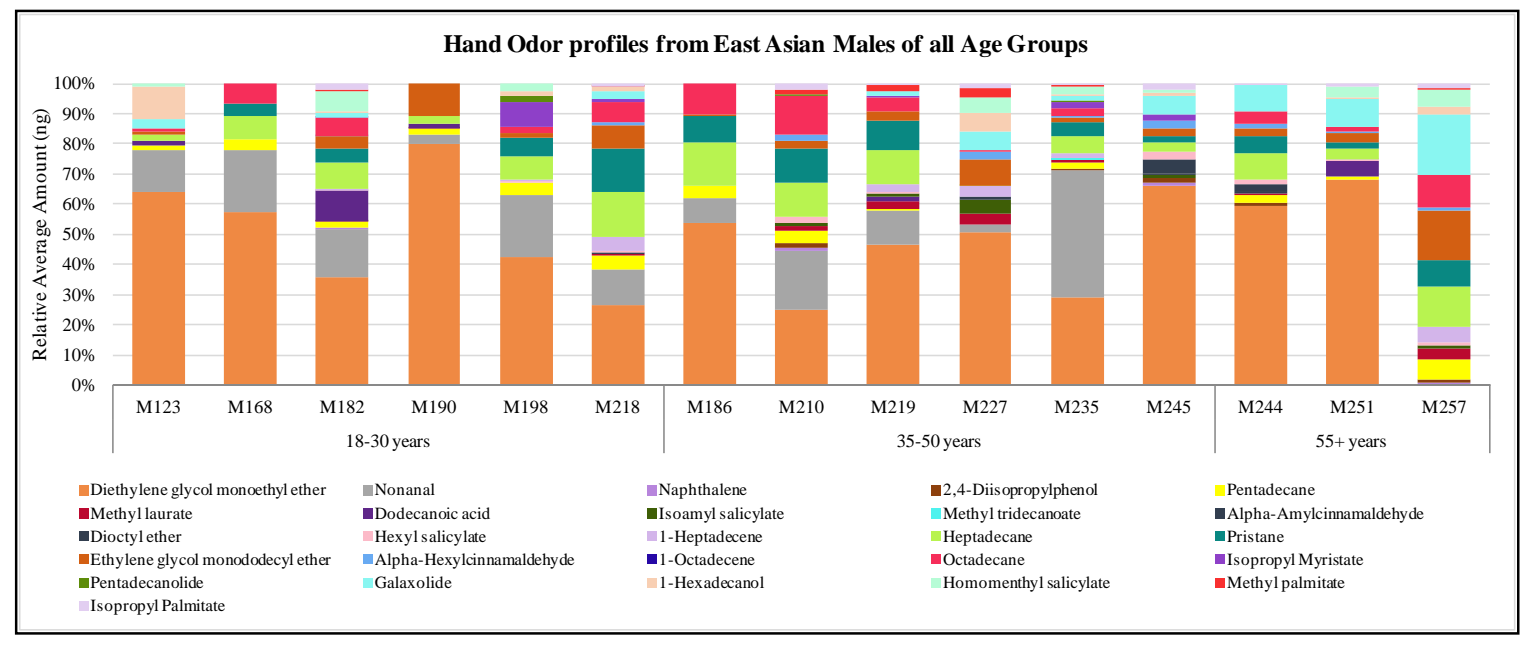

Figure 135: Hand odor profiles from East Asian males of all age groups

Table 34: Demographic information of the population surveyed for hand odor VOCs

\begin{tabular}{|c|c|c|c|c|c|c|}
\hline \multirow{2}{*}{ Age Group } & \multicolumn{2}{|c|}{ Caucasians } & \multicolumn{2}{c|}{ Hispanics } & \multicolumn{2}{c|}{ East Asians } \\
\cline { 2 - 7 } & Females & Males & Females & Males & Females & Males \\
\hline 18-30 years & 6 & 6 & 6 & 6 & 6 & 6 \\
\hline 35-50 years & 6 & 6 & 6 & 6 & 6 & 6 \\
\hline 55+ years & 6 & 6 & 6 & 6 & 6 & 3 \\
\hline Total Subjects & 18 & 18 & 18 & 18 & 18 & 15 \\
\hline
\end{tabular}


A list of 26 VOCs was initially considered to perform an overall comparison of the population under study. Compounds were selected for this list on the basis of their potential for comparison across the different demographic groups. Scent profiles were created for each individual using the 26 VOCs under consideration and, once again, the impact exerted by race/ethnicity, age, and gender on these profiles became the main focus of this study. The potential classification of each individual in accordance to their corresponding trait and their expressed VOC markers was evaluated using Linear Discriminant Analysis (LDA). In the case of the hand odor assessment, individual classification was attempted in all comparisons using two different sets of criteria: the initial list of 26 VOCs and a list of VOCs created using a stepwise regression method. The first approach was employed as a way to obtain a comprehensive depiction of the role of VOC profiles in potential individual classification and differentiation by traits. On the other hand, the stepwise regression method VOCs were selected as the second set of criteria, after having observed the efficiency of this approach for individual classification in the assessment of underarm odor profiles. In the case of the study of hand odor profiles, the stepwise regression method also facilitated the identification of those VOC variables capable of exerting a higher impact in group differentiation. Therefore, this approach allowed the optimum fulfillment of the study's interests: the determination of VOC marker combinations in hand odor.

After completing the evaluation of the previously described sets of differentiation criteria, for each of the comparisons being assessed in the hand odor study, the individual classification efficiency of both approaches was determined. The efficiency of both classification methods was compared and conclusions were established in regards to the 
best VOC variables for group discrimination. The identities of potential VOC marker combinations for age, gender, and race/ethnicity, in hand odor, were evaluated. In addition, the consistency between hand and underarm odor marker combinations was also assessed. The evaluation of these elements served to complement and support the information obtained thus far on how age, gender and race/ethnicity can influence human scent. Moreover, the statistical significance of the differences in average VOC amounts, between each of the groups under study, was also determined for reference purposes. The determination of statistical significance between VOC amounts was intended to facilitate the identification of any trends that may show correlation with the efficient differentiation of the groups under study. However, findings from these tests were not employed in this study as basal elements for group differentiation. Results on the statistical significance tests, performed to evaluate differences on average VOC amounts, can be found in Appendix 9 and Appendix 10.

\subsubsection{Determination of VOC markers for individual classification by age}

The hand odor profiles obtained for all 105 subjects were used to test the feasibility of using hand odor VOCs to classify individuals on the basis of their age. In this assessment, all individuals were considered as part of a single sample pool, regardless of their race/ethnicity and gender. Individual classification by age was pursued by associating individuals with any of the three age group categories being considered in this dissertation: 18-30 years, 35-50 years and 55+ years. In addition, average VOC amounts were obtained for each one of the three age groups under study (Figure 136). 
These VOC amounts were used to display VOC distribution in the averaged odor profile of each age group and to associate any potential trends with age group differentiation.

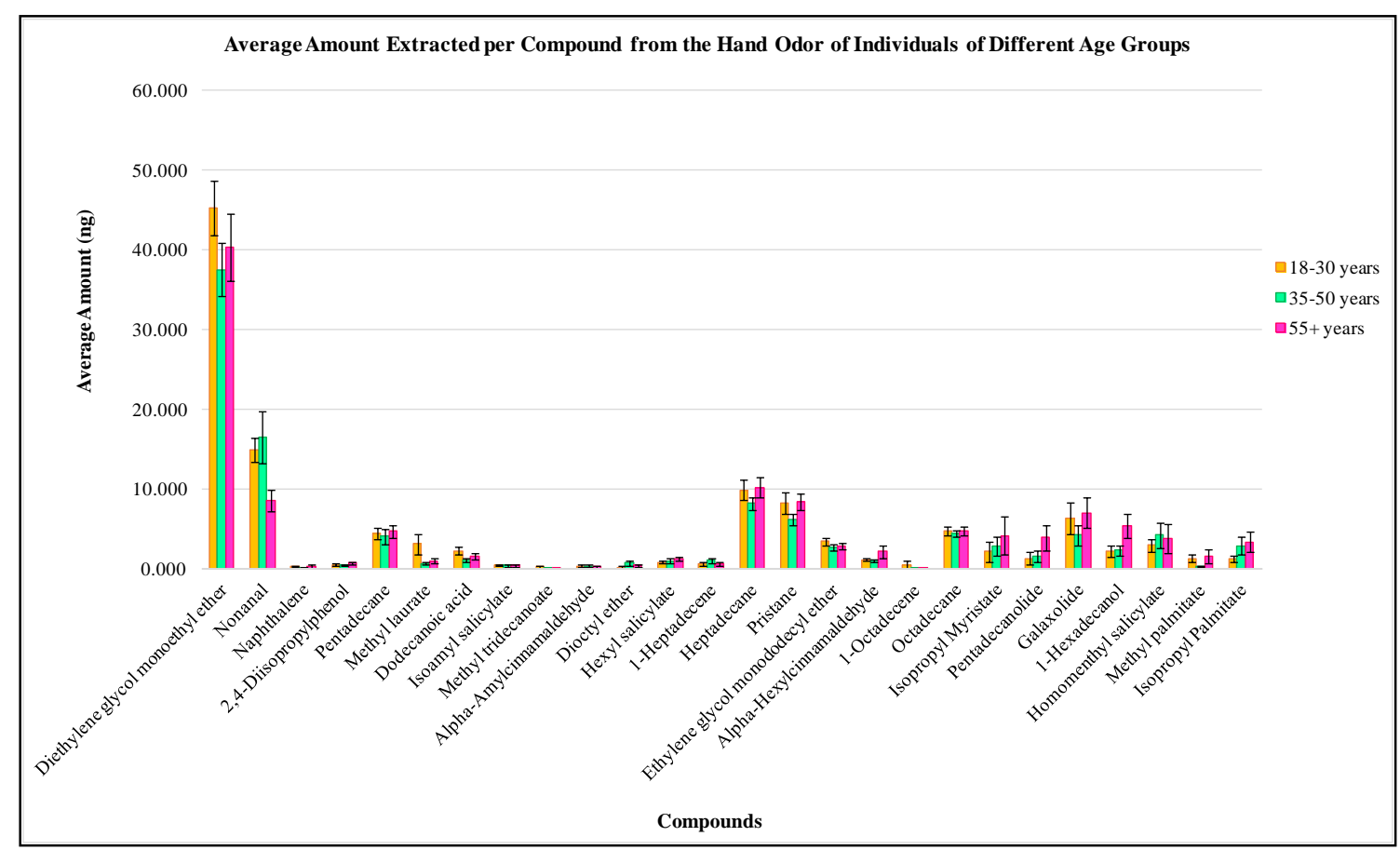

Figure 136: Average amounts of hand VOCs per age group

Linear Discriminant Analysis was performed using the 26 VOCs as variables for individual classification (Table 35). As a result, it was noted that 37 out of 105 subjects were classified in an incorrect age group. The occurrence of such misclassifications limited the overall classification accuracy of this approach to $65 \%$ of all subjects. From a total of 36 individuals in the 18-30 years category, seven were misclassified as 35-50 year olds, and five as 55+ year olds. In the case of the misclassifications observed for 36 individuals in the 35-50 year group, two were seen to occur under the 18-30 year age group, and seven were in the 55+ year group. On the other hand, nine out of 33 subjects from the 55+ year category were misclassified as 35-50 year olds, while six were 
misclassified as 18-30 year olds. The information discovered in this assessment revealed that, as it was also observed in underarm odor, there is a higher resemblance between the VOC profiles of individuals in the 35-50 and 55+ year age groups. Hence a higher level of difficulty was, once again, observed when trying to differentiate these two groups. In addition, it was noted that $41 \%$ of all misclassifications were caused by lack of accuracy in the classification of 55+ year olds. This specific observation was found to be different from findings from the underarm odor assessment. In the underarm odor assessment it was noted that classifying 18-30 year olds was more inaccurate than the classification of individuals into any of the other groups being compared.

The canonical plot obtained to evaluate individual classification by age revealed that, despite of the noted misclassifications, age differentiation was still possible (Figure 137). None of the confidence level (CL) ellipses were seen to overlap, which suggests the 26 VOCs used as differentiation criteria were capable of differentiating members from different age groups. The stepwise regression approach was subsequently pursued in an attempt to improve the total classification accuracy achieved in the comparison of the different age groups. 


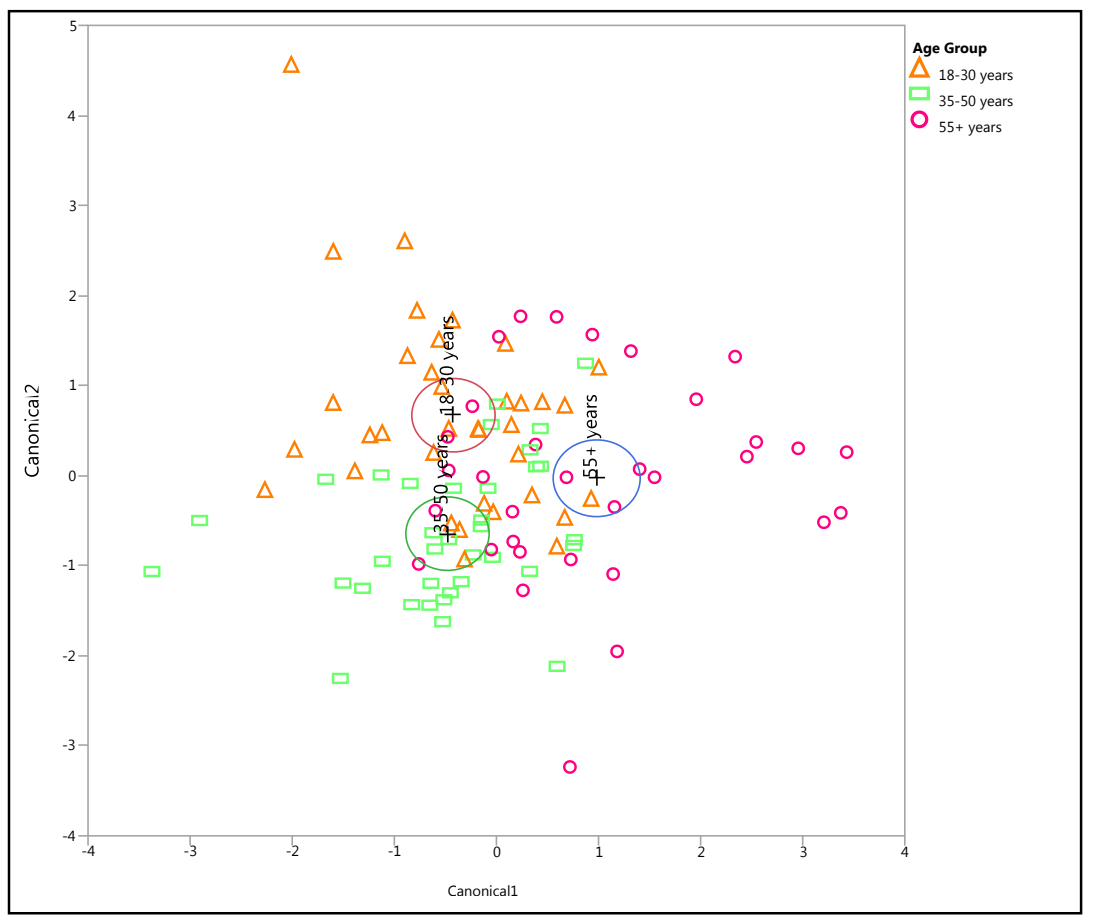

Figure 137: LDA canonical plot using all 26 VOCs as criteria for subject differentiation by age group using hand odor

The stepwise regression method revealed that the maximum accuracy that could be obtained for the classification of individuals on the basis of their age was $65 \%$, as it was demonstrated with the initial approach. The canonical plot obtained and the distribution of misclassified subjects was found to be identical to that obtained when using all 26 VOCs as differentiation criteria. However, the stepwise regression method did reveal that it was possible to attain 65\% accuracy using fewer VOCs as differentiation criteria. From the initial 26 VOCs, only 23 (Table 35) were necessary to achieve optimum age group differentiation. These 23 compounds displayed enough influence in group differentiation to ensure the best individual classification accuracy possible. As a result, these compounds were established as the VOC marker combination for subject differentiation by age. The loading scores obtained for these compounds have been listed 
in Table 35, in decreasing order, for reference of their impact level on age group differentiation.

Table 35: VOC lists employed as criteria for subject differentiation by age group in Linear Discriminant Analysis

\begin{tabular}{|c|c|c|c|}
\hline \multirow{2}{*}{ Initial VOC List } & \multicolumn{3}{|c|}{ Stepwise Regression Method } \\
\cline { 2 - 4 } & VOC List & Canon 1 & Canon 2 \\
\hline Diethylene glycol monoethyl ether & 1-Hexadecanol & 0.901 & 0.230 \\
\hline *Nonanal & Methyl laurate & -0.742 & -0.280 \\
\hline Naphthalene & Pristane & -0.713 & 0.789 \\
\hline 2,4-Diisopropylphenol & *Nonanal & -0.625 & -0.267 \\
\hline Pentadecane & 2,4-Diisopropylphenol & 0.557 & -0.124 \\
\hline Methyl laurate & Methyl palmitate & 0.554 & 0.020 \\
\hline Dodecanoic acid & Pentadecane & 0.404 & 0.045 \\
\hline Isoamyl salicylate & Dodecanoic acid & 0.398 & 0.352 \\
\hline Methyl tridecanoate & Alpha-Hexylcinnamaldehyde & 0.365 & -0.034 \\
\hline Alpha-Amylcinnamaldehyde & Isoamyl salicylate & -0.350 & -0.113 \\
\hline Dioctyl ether & Alpha-Amylcinnamaldehyde & -0.335 & 0.063 \\
\hline Hexyl salicylate & Isopropyl Palmitate & -0.275 & -0.411 \\
\hline 1-Heptadecene & Naphthalene & 0.266 & 0.045 \\
\hline Heptadecane & Homomenthyl salicylate & 0.215 & -0.147 \\
\hline Pristane & Galaxolide & -0.197 & 0.298 \\
\hline Ethylene glycol monododecyl ether & 1-Octadecene & -0.192 & 0.179 \\
\hline Alpha-Hexylcinnamaldehyde & Pentadecanolide & 0.156 & -0.350 \\
\hline 1-Octadecene & Methyl tridecanoate & 0.103 & 0.740 \\
\hline Octadecane & Octadecane & -0.081 & -0.455 \\
\hline Isopropyl Myristate & 1-Heptadecene & -0.077 & -0.299 \\
\hline Pentadecanolide & Dioctyl ether & -0.074 & -0.340 \\
\hline Galaxolide & Diethylene glycol monoethyl ether & -0.039 & 0.378 \\
\hline 1-Hexadecanol & Ethylene glycol monododecyl ether & -0.006 & 0.305 \\
\hline Homomenthyl salicylate & & & \\
\hline Methyl palmitate & & & \\
\hline Isopropyl Palmitate & & & \\
\hline
\end{tabular}

* Compounds for which the average VOC amounts were seen to be significantly different between the age groups

\subsubsection{Determination of VOC markers for individual classification by race/ethnicity}

The evaluation of hand odor, as a tool for human classification on the basis of race or ethnicity, considered all individuals as being part of a single pool of samples, regardless of their age group and gender. Average VOC amounts were calculated for the 
three race/ethnic groups (e.g., Caucasian, East Asian and Hispanic) under study (Figure 138). These amounts were used to display VOC distribution in the averaged odor profile of each group, and to associate any potential trends with the ability to classify individuals on the basis of their race/ethnicity.

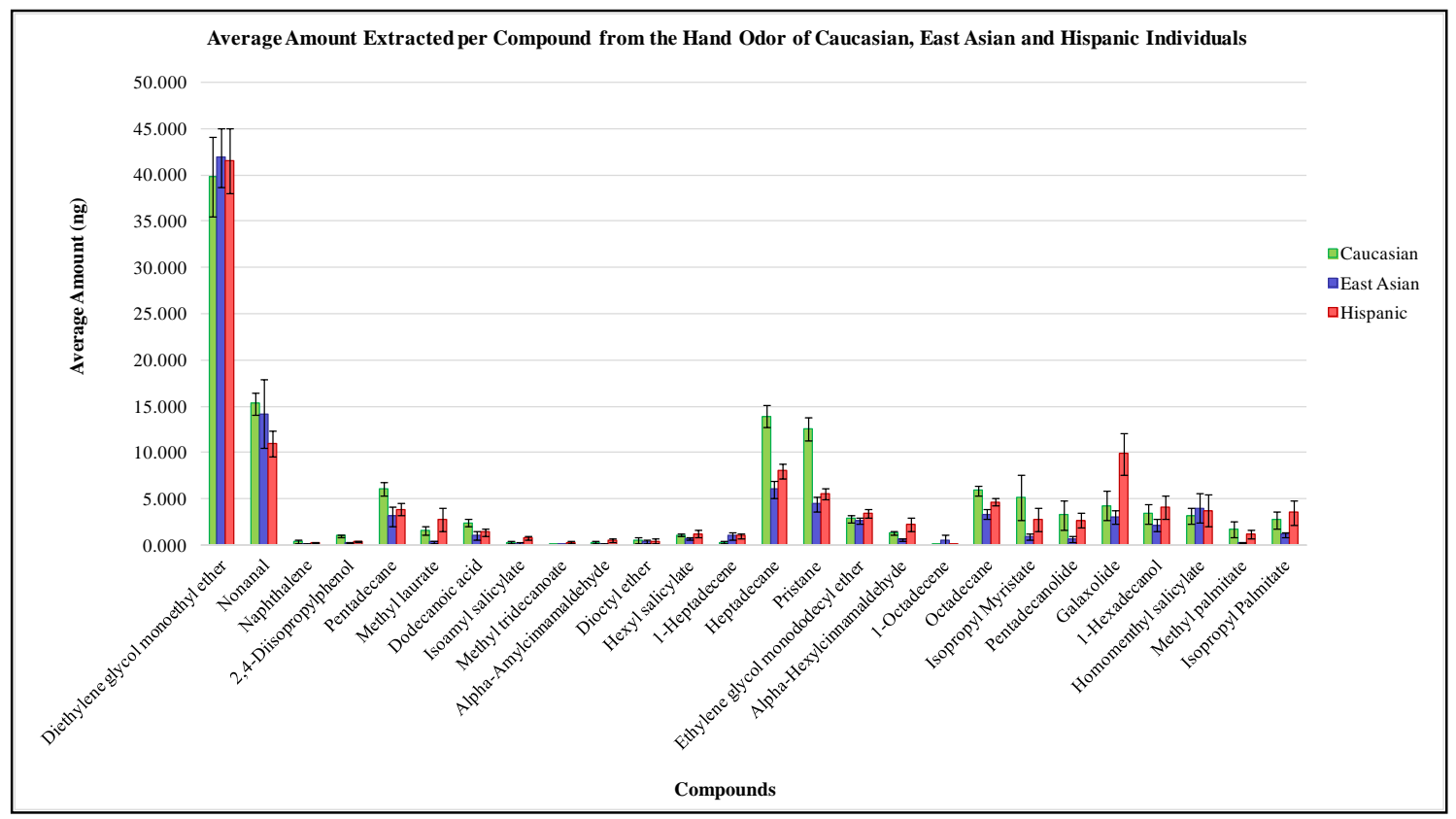

Figure 138: Average amounts of hand VOCs per race/ethnic group

Linear Discriminant Analysis was performed using all 26 VOCs as variables for individual classification per racial/ethnic group (Table 36). Results from this analysis revealed that individuals could be classified in accordance to their race/ethnicity with a total accuracy of $71 \%$ and that all three groups could be successfully differentiated (Figure 139). From a total of 105 subjects, there were only a total of 30 misclassifications observed (only 29\% of all individuals). Out of 36 Caucasians, six were misclassified as East Asian, and five as Hispanic. In the case of East Asians, two of 33 subjects were misclassified as Caucasian, and seven as Hispanic. On the other hand, out of 36 
Hispanics, ten subjects were misclassified as East Asian and none as Caucasians. After comparing the classification accuracy of the three groups, it was observed that East Asians showed the highest percentage of correct classification (73\%). This observation agrees with the results obtained in the evaluation of underarm odor for individual classification by race/ethnicity. The results obtained suggest that the odor profiles from East Asian individuals tend to portray more defined factors that aid in race/ethnic differentiation. On the other hand, classification of Hispanic and Caucasian individuals was achieved with $72 \%$ and $69 \%$ accuracy, respectively. Despite the fact that the accuracy percentages achieved for these two groups did not follow the magnitude order observed in the underarm odor assessment, it was noted that the accuracy percentage achieved for Caucasians remained very similar $(67 \%=$ underarms vs. $69 \%=$ hands $)$. Nevertheless, a more noticeable increase was observed in the accuracy achieved for the classification of Hispanics using hand odor. An accuracy of 58\% was achieved using underarm odor, while $72 \%$ was achieved when using hand odor. This increase in classification accuracy supports the fact that hand odor profiles can be a well suited resource for the classification of Hispanic individuals on the basis of their ethnicity. 


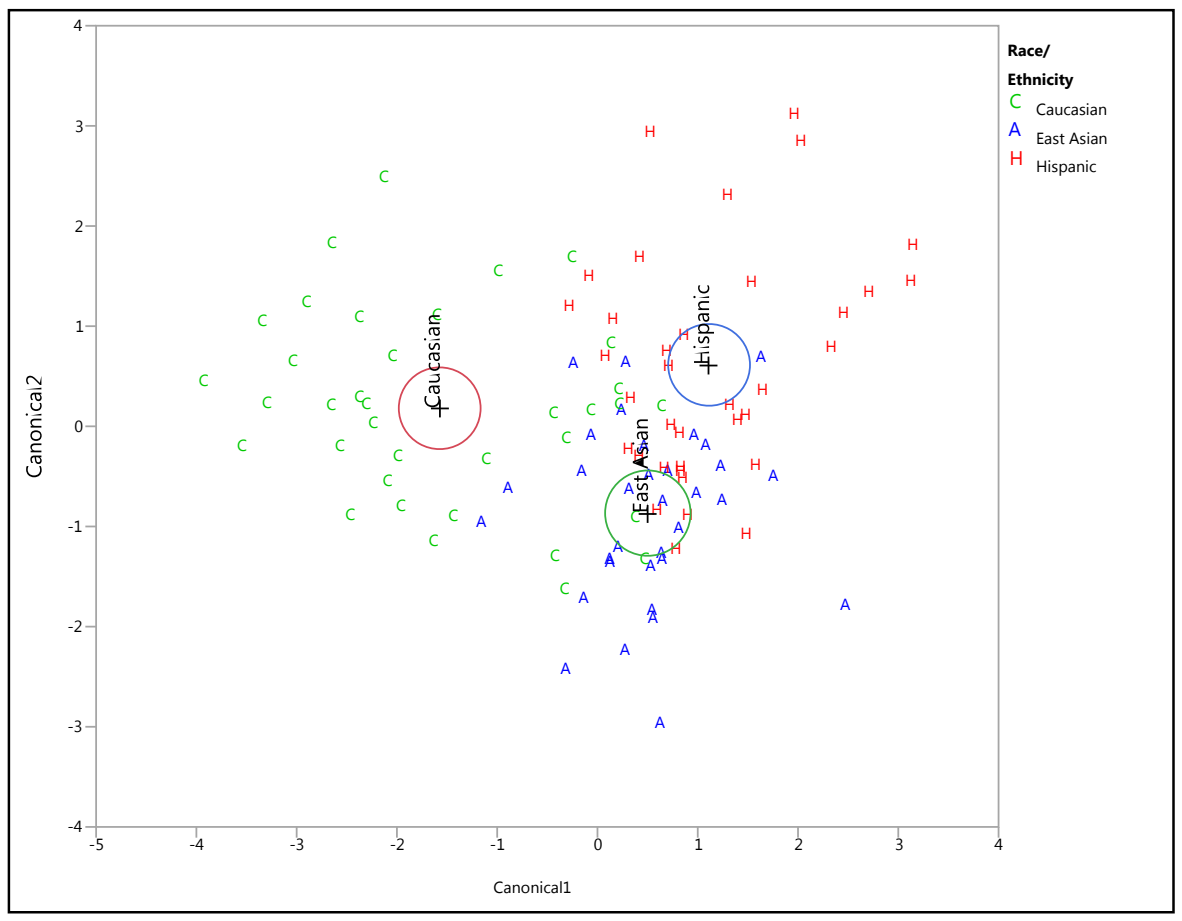

Figure 139: LDA canonical plot using all 26 VOCs as criteria for subject differentiation by race/ethnic group using hand odor

The stepwise regression method applied in this assessment revealed that a total of 15 VOC variables provided optimum differentiation for the race/ethnic groups. Therefore, these 15 compounds were established as constituents of the VOC marker combination for subject differentiation by race/ethnicity. Table 36 lists, in decreasing order, the VOCs in this marker combination and their level of influence in group differentiation (loading values).

A total classification accuracy of $72 \%$ was achieved when using the VOC variables from the stepwise regression method. This represents a slight, but definitive, improvement in the differentiation level achieved when using the complete list of VOCs as discrimination variables. From a total of 36 Caucasians, three subjects were misclassified as Hispanic, and seven as East Asian. In the case of Hispanics, 12 
misclassifications were observed with the East Asian group, and none were seen with the Caucasian population. Moreover, out of 33 East Asians, three subjects were misclassified in each of the other two populations.

The canonical plot obtained (Figure 140) revealed a clear differentiation of the groups by displaying individuals of equal races/ethnicities clustering together, and with a tendency to appear separated from those of other groups. Once again, the CL ellipses of each group were not seen to intercept, and it was observed that the Caucasian group showed a bigger distance from the other two groups than any other group. This last observation explains why only a few of East Asian individuals were misclassified as Caucasian, and no misclassifications of this type were seen in the case of Hispanics. The distance between the Caucasian population and the rest of the groups led to the achievement of $72 \%$ of total classification accuracy for Caucasian individuals. On the other hand, the Hispanic and the East Asian groups were seen to display a higher individual overlap in the canonical plot. This overlap suggests that, out of the three groups, the odor profiles from these two specific populations may contain factors that are of more similar impact to race/ethnic differentiation. Nevertheless, the achievement of $82 \%$ and $67 \%$ classification accuracy for East Asians and Hispanics, respectively, demonstrated that the VOC marker combination was successful in the differentiation of individuals by their race/ethnicity. 


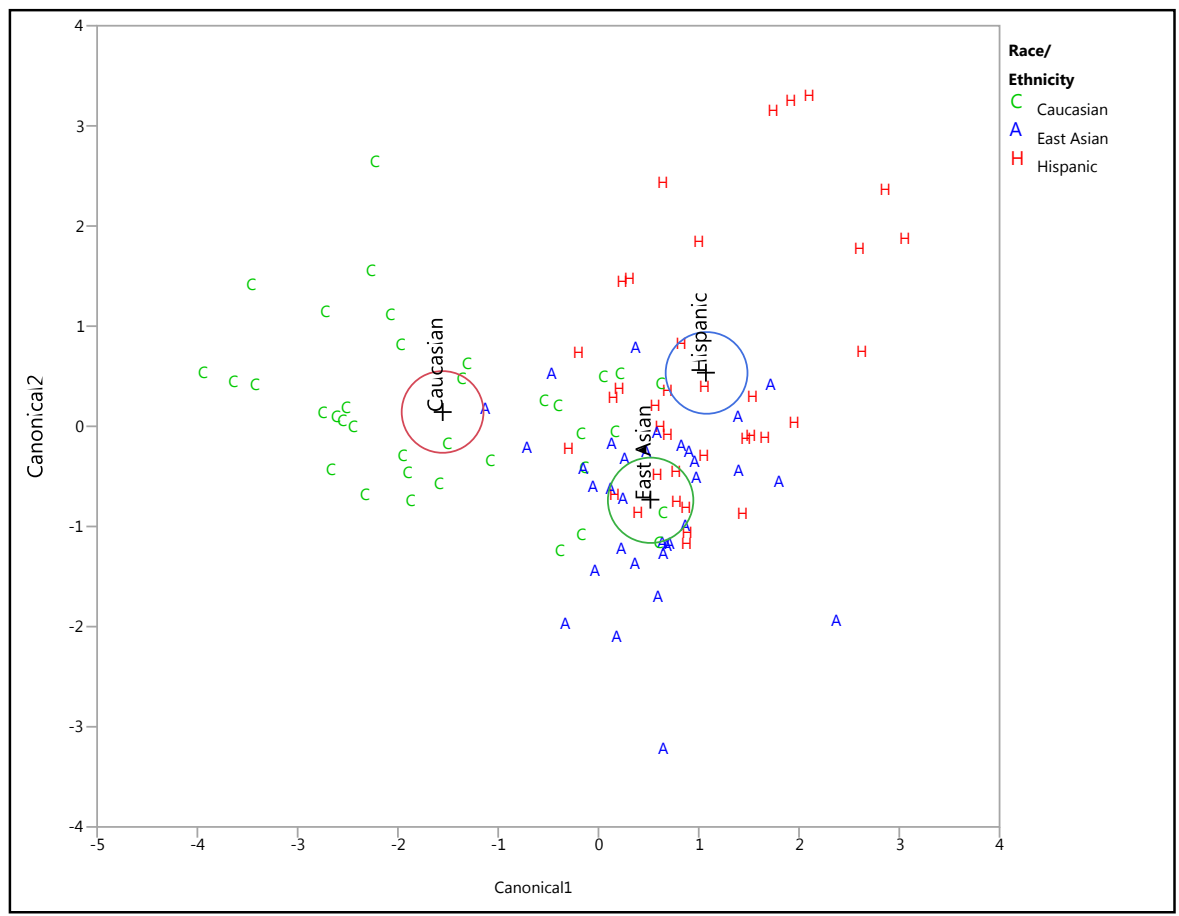

Figure 140: LDA canonical plot of stepwise regression VOCs as criteria for subject differentiation by race/ethnic group using hand odor 
Table 36: VOC lists employed as criteria for subject differentiation by race/ethnic group in Linear Discriminant Analysis

\begin{tabular}{|c|c|c|c|}
\hline \multirow{2}{*}{ Initial VOC List } & \multicolumn{3}{|c|}{ Stepwise Regression Method } \\
\cline { 2 - 4 } & VOC List & Canon 1 & Canon 2 \\
\hline Diethylene glycol monoethyl ether & *Pristane & -1.176 & 0.073 \\
\hline Nonanal & *Galaxolide & 0.602 & 0.323 \\
\hline Naphthalene & *Octadecane & 0.596 & 0.355 \\
\hline *2,4-Diisopropylphenol & Dodecanoic acid & -0.426 & -0.003 \\
\hline *Pentadecane & Methyl palmitate & -0.369 & 0.137 \\
\hline Methyl laurate & $*$ 2,4-Diisopropylphenol & -0.345 & -0.058 \\
\hline Dodecanoic acid & 1-Heptadecene & 0.284 & -0.390 \\
\hline *Isoamyl salicylate & Alpha-Amylcinnamaldehyde & -0.280 & 0.123 \\
\hline Methyl tridecanoate & Methyl tridecanoate & 0.265 & 0.169 \\
\hline Alpha-Amylcinnamaldehyde & $*$ Alpha-Hexylcinnamaldehyde & 0.237 & 0.192 \\
\hline Dioctyl ether & Dioctyl ether & -0.203 & 0.034 \\
\hline Hexyl salicylate & $*$ Isoamyl salicylate & 0.155 & 0.369 \\
\hline 1-Heptadecene & Ethylene glycol monododecyl ether & 0.147 & 0.354 \\
\hline *Heptadecane & Nonanal & -0.134 & -0.264 \\
\hline *Pristane & 1-Octadecene & 0.132 & -0.353 \\
\hline Ethylene glycol monododecyl ether & & & \\
\hline *Alpha-Hexylcinnamaldehyde & & & \\
\hline 1-Octadecene & & & \\
\hline *Octadecane & & & \\
\hline Isopropyl Myristate & & & \\
\hline Pentadecanolide & & & \\
\hline *Galaxolide & & & \\
\hline 1-Hexadecanol & & & \\
\hline Homomenthyl salicylate & & & \\
\hline Methyl palmitate & & & \\
\hline Isopropyl Palmitate & & & \\
\hline & & & \\
\hline
\end{tabular}

* Compounds for which the average VOC amounts were seen to be significantly different between the age groups 


\subsubsection{Determination of VOC markers for individual classification by gender}

The use of hand odor to classify individuals by gender was also studied by considering all individuals part of a single pool of samples, regardless of their age group and race/ ethnicity. Average VOC amounts were calculated for all 26 compounds in both genders (Figure 141). These values were used to identify any VOC characteristics in hand odor that might be directly related to gender as a trait.

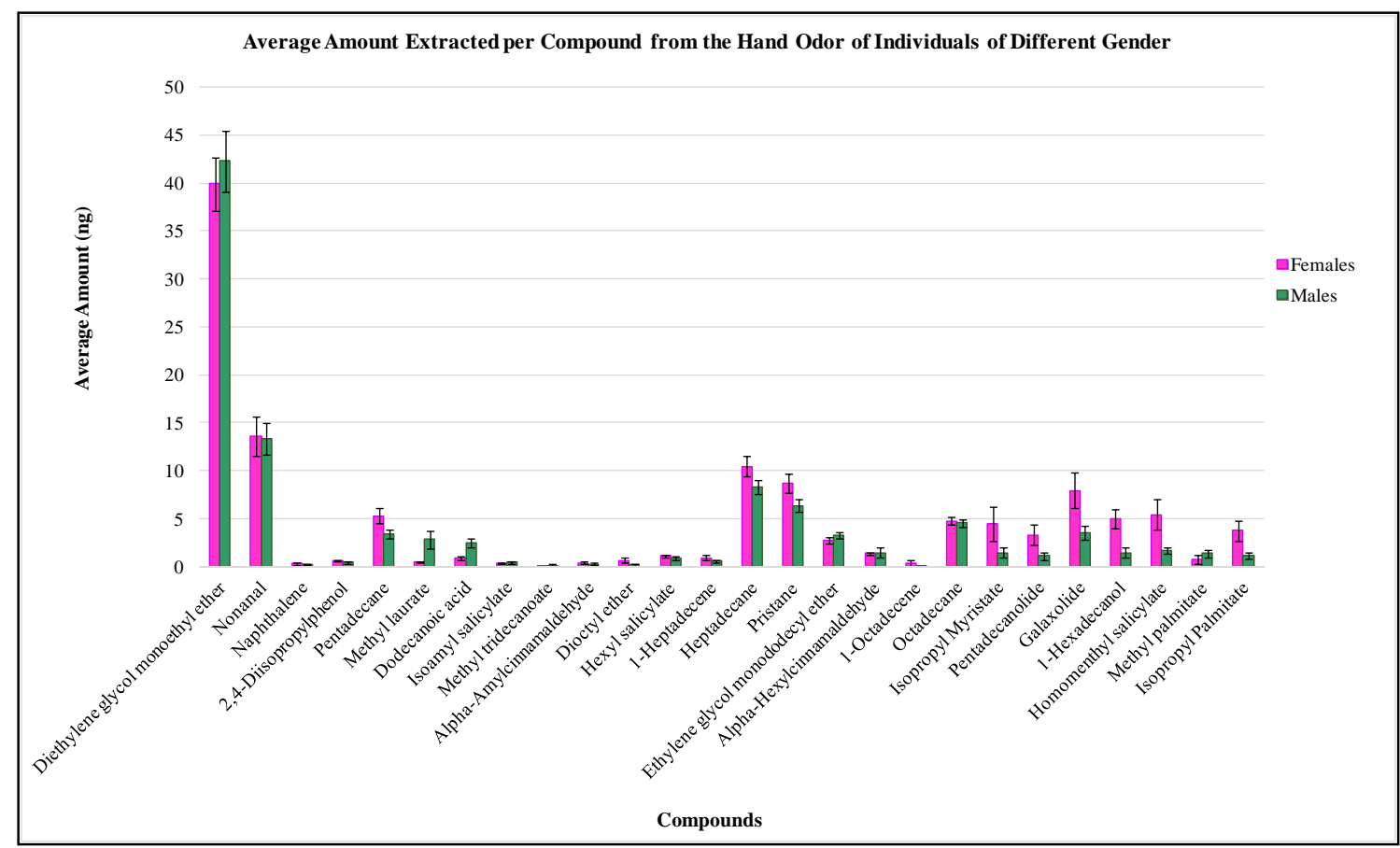

\section{Figure 141: Average amounts of hand VOCs per gender}

The use of all 26 VOCs as differentiation criteria between the genders in the LDA, allowed the achievement of a total classification accuracy of 79\%. The canonical plot obtained revealed no overlap between the CL ellipses of the groups, which points to an efficient gender differentiation (Figure 142). Out of 105 subjects, 15 females were misclassified as males and only seven males were misclassified as females. These results 
revealed that there was a higher likelihood for females to be misclassified than males. The classification accuracy achieved for females and males, specifically, was $86 \%$ and $72 \%$, respectively.

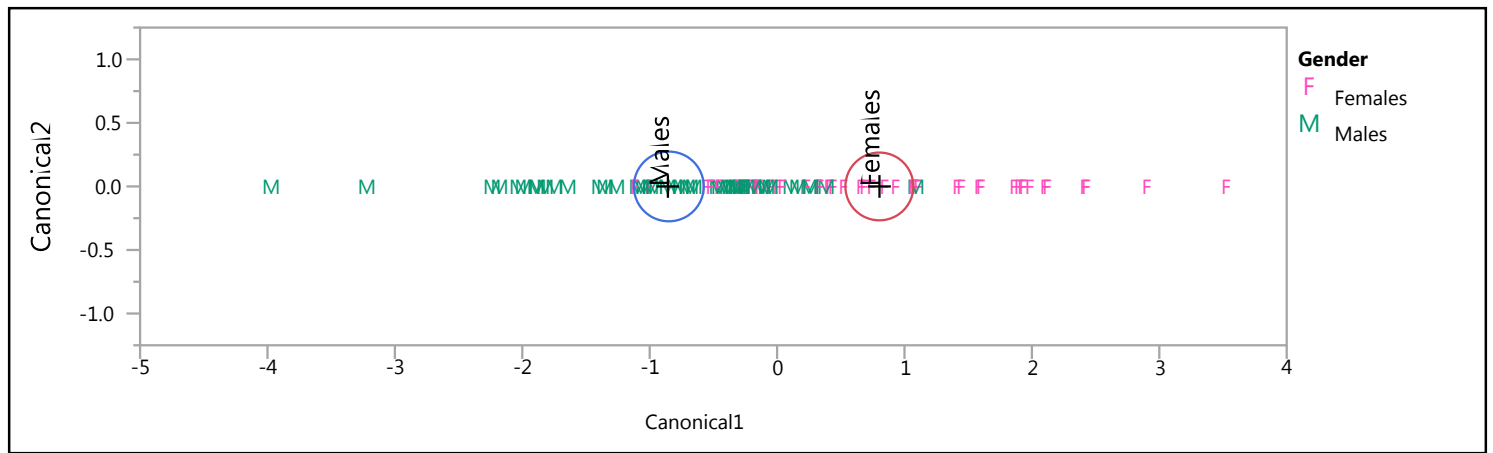

\section{Figure 142: LDA canonical plot using all 26 VOCs as criteria for subject differentiation by gender}

On the other hand, differences were observed in gender differentiation when using the stepwise regression method. In this case, only 13 compounds were found to be necessary to provide optimum gender differentiation (Table 37). These compounds were established as the VOC marker combination for subject differentiation by gender. Despite the fact that the canonical plot (Figure 143) obtained for this approach did not reveal any drastic changes from the plot obtained in the previous approach, an increase in the accuracy for female classification was observed. Only 13 females were misclassified using the VOC marker combination, which led this approach to achieve $76 \%$ of classification accuracy for this gender. On the contrary, it was noted that the accuracy achieved for male classification was reduced. The reduction was caused by a slight increase in male misclassification. A total of eight males were misclassified in the stepwise regression approach. Nevertheless, all of these differences resulted in an 
increase in accuracy for the overall assessment. The VOC marker combination used allowed achieving an accuracy of $80 \%$ for the classification of all subjects on the basis of their gender.

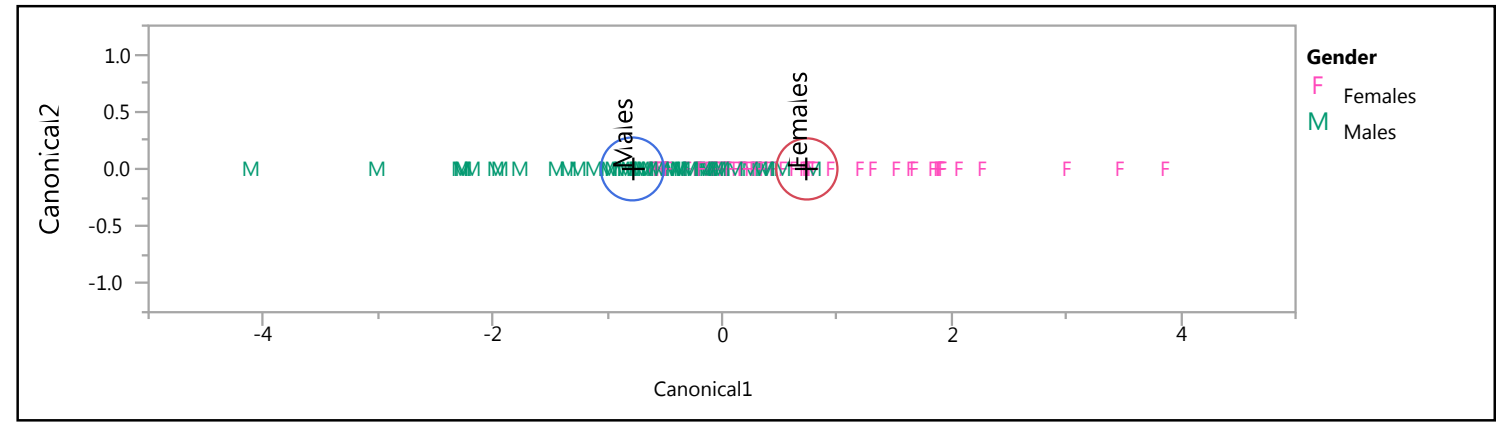

Figure 143: LDA canonical plot using compounds from stepwise regression method as criteria for subject differentiation by gender 
Table 37: VOC lists employed as criteria for subject differentiation by gender group in Linear Discriminant Analysis

\begin{tabular}{|c|c|c|}
\hline \multirow{2}{*}{ Initial VOC List } & \multicolumn{2}{|c|}{ Stepwise Regression Method } \\
\cline { 2 - 3 } & VOC List & Canon 1 \\
\hline Diethylene glycol monoethyl ether & *Methyl laurate & -0.745 \\
\hline Nonanal & Methyl tridecanoate & 0.599 \\
\hline Naphthalene & Homomenthyl salicylate & 0.548 \\
\hline 2,4-Diisopropylphenol & *Pentadecane & 0.478 \\
\hline *Pentadecane & *1-Hexadecanol & 0.464 \\
\hline *Methyl laurate & *Dodecanoic acid & -0.419 \\
\hline *Dodecanoic acid & Isoamyl salicylate & -0.343 \\
\hline Isoamyl salicylate & Hexyl salicylate & -0.331 \\
\hline Methyl tridecanoate & Isopropyl Myristate & 0.266 \\
\hline Alpha-Amylcinnamaldehyde & *Isopropyl Palmitate & 0.241 \\
\hline Dioctyl ether & Octadecane & -0.231 \\
\hline Hexyl salicylate & Dioctyl ether & 0.199 \\
\hline 1-Heptadecene & Galaxolide & 0.180 \\
\hline Heptadecane & & \\
\hline Pristane & & \\
\hline Ethylene glycol monododecyl ether & & \\
\hline Alpha-Hexylcinnamaldehyde & & \\
\hline 1-Octadecene & & \\
\hline Octadecane & & \\
\hline Isopropyl Myristate & & \\
\hline Pentadecanolide & & \\
\hline Galaxolide & & \\
\hline *1-Hexadecanol & & \\
\hline Homomenthyl salicylate & & \\
\hline Methyl palmitate & & \\
\hline *Isopropyl Palmitate & & \\
\hline
\end{tabular}

* Compounds for which the average VOC amounts were seen to be significantly different between the age groups

5.4.4.4. Determination of VOC markers for individual classification by race/ethnicity and gender

Attempting to classify individuals by means of their race/ethnicity and gender, as simultaneous traits, was also part of the hand odor assessment. The purpose of this specific evaluation was to test whether it was possible to still classify and differentiate individuals, on the basis of their hand odor profiles, when considering two different traits at once. Average amounts were calculated for both genders, in each race/ethnic group, for 
the initial 26 VOCs (Figure 144). Averaged values were used to identify any potential VOC characteristics in hand odor that could be associated to members of each gender in each race/ethnicity. 


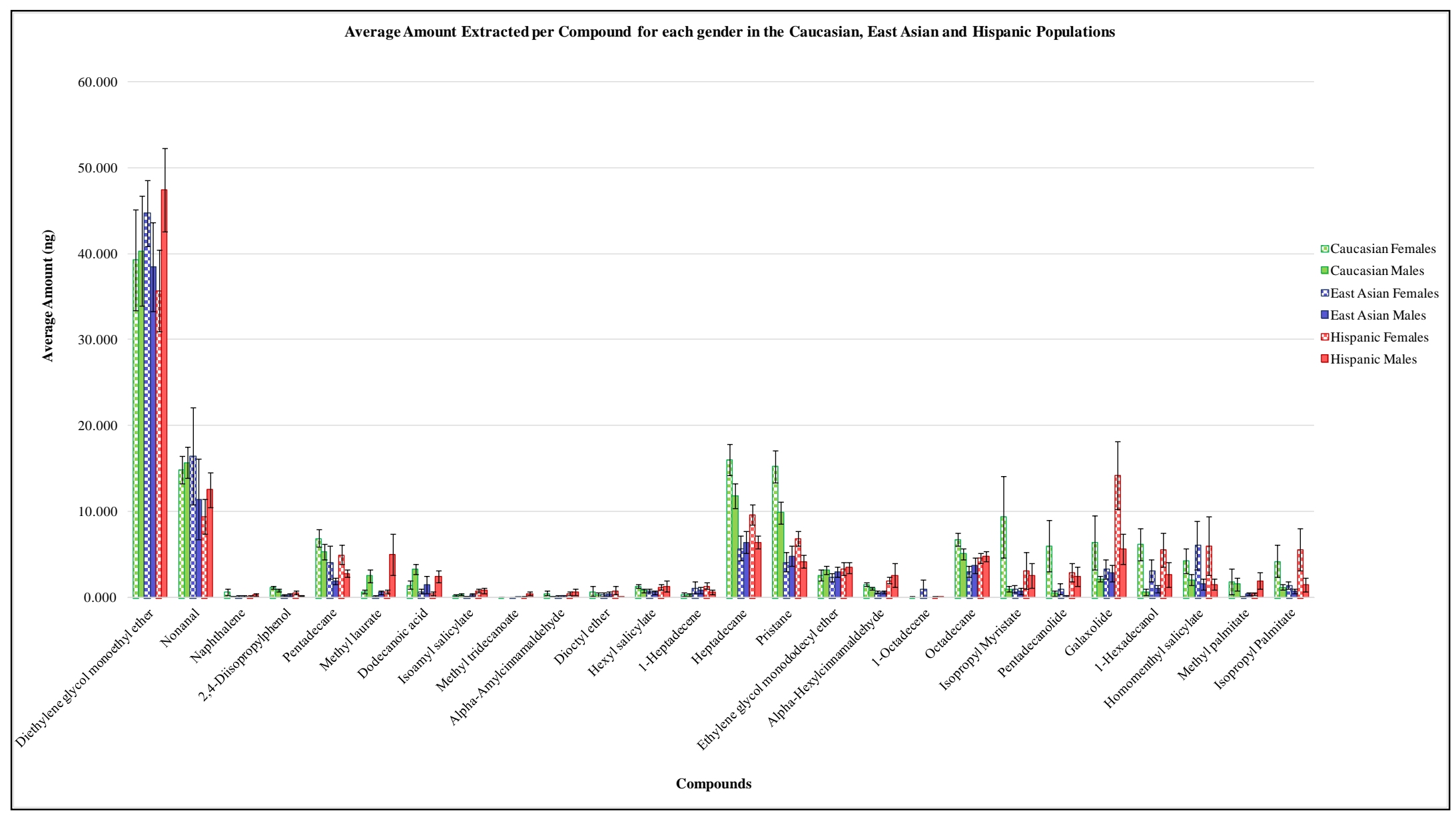

Figure 144: Average VOC amounts per race/ethnicity and gender 
The LDA performed using all 26 compounds as discrimination criteria revealed that the majority of the groups being compared had a high tendency to overlap with each other. The canonical plot obtained (Figure 145) displayed the CL ellipses of five out of six groups being intercepted by the CL ellipse of at least one other group. This observation suggests that the 26 VOC variables, employed as differentiation criteria, were not able to provide a total and clear differentiation of the groups being considered. Out of all groups, Caucasian females were the only one that did not overlap with any other group. The lack of overlap with other groups allowed this group to achieve $72 \%$ of classification accuracy. Nevertheless, despite of the high accuracy achieved, some Caucasian females were still classified incorrectly into other groups; with the highest misclassification incidence being two Caucasian females that were classified as East Asian males. On the other hand, Hispanic females also achieved $72 \%$ of classification accuracy. Despite having seen the CL ellipse of Hispanic females only overlapping with the CL ellipse from the East Asian female group, one Hispanic female was misclassified as East Asian female, another as a Hispanic male, and three other as East Asian males. Both Caucasian and Hispanic females achieved the highest percentage of classification accuracy (72\%) in this approach; while East Asian females displayed the lowest classification accuracy of all groups (44\%). Overall, out of a total of 105 subjects, 41 ended up being misclassified by using this approach. As a result, a total classification accuracy of $61 \%$ was achieved. 


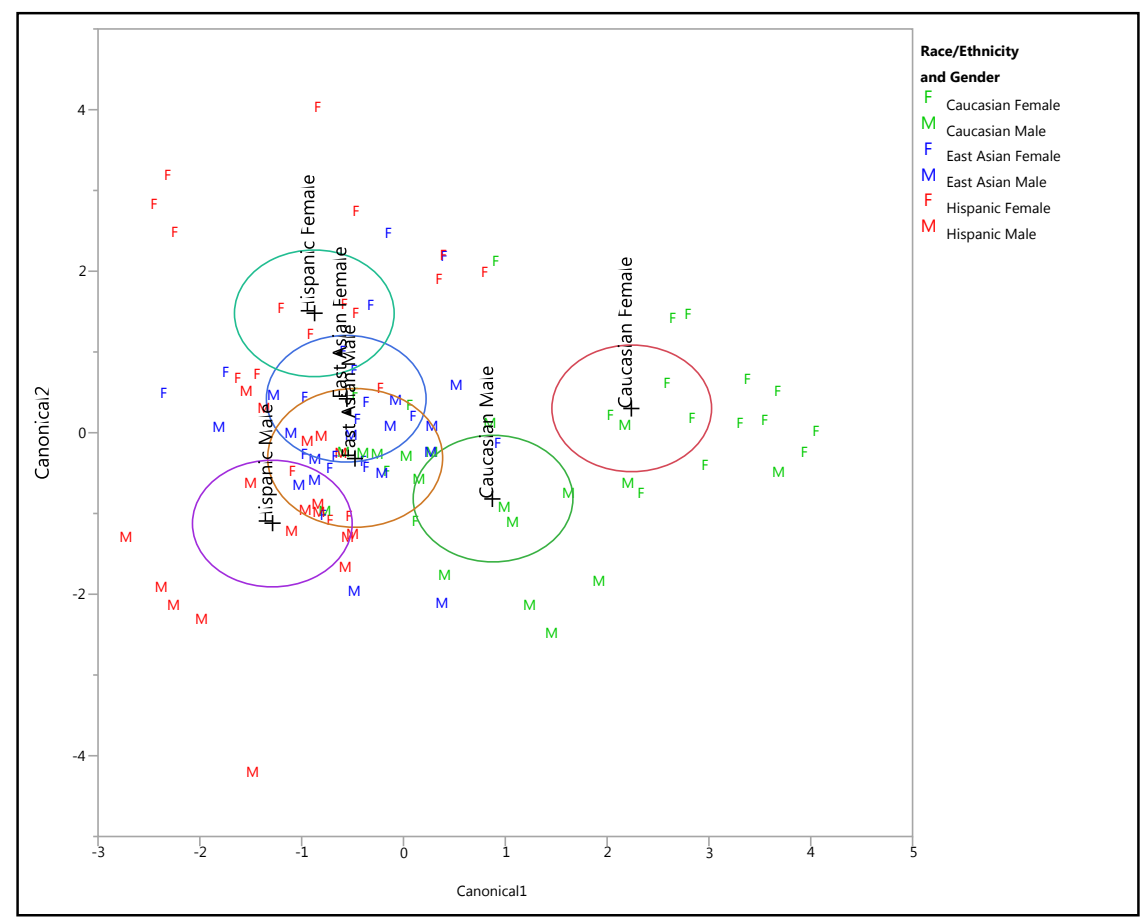

Figure 145: LDA canonical plot obtained using all 26 VOCs for subject differentiation by race/ethnicity and gender

The stepwise regression method was used to evaluate if any improvement could be accomplished by changing the set of differentiation variables being considered. In this case, 21 VOCs (Table 38) were considered as discrimination criteria between the groups after seeing they provided the best degree of differentiation possible. These VOCs were considered the VOC marker combination for this specific differentiation attempt.

In terms of appearance, the canonical plot obtained with this approach did not reveal any significant differences (Figure 146). However, a reduction on the number of misclassifications was accomplished. From 41 misclassified subjects obtained in the initial approach, only 39 subjects were misclassified in this attempt. This decrease in the number of misclassifications increased the total classification accuracy achieved from $61 \%$ to $63 \%$. In this approach, Caucasian females were, once again, seen to achieve the 
highest percentage of classification accuracy (72\%). On the other hand, Hispanic females were seen to increase their misclassification rate in the Hispanic male category. Consequently, the accuracy achieved for the classification of Hispanic females was reduced to $67 \%$. East Asian females and Hispanic males were both seen to achieve 56\%, becoming the two groups with lowest classification accuracy.

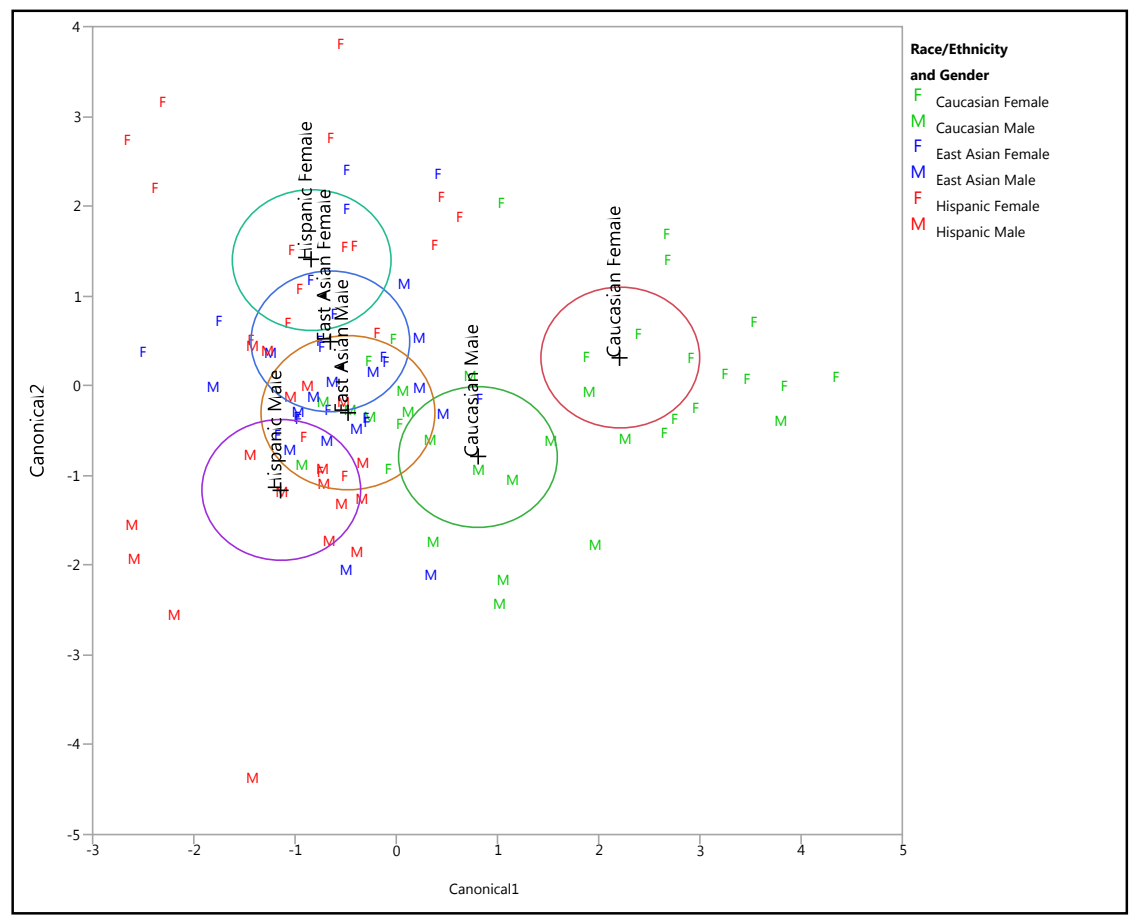

Figure 146: LDA canonical plot obtained using the stepwise regression method for subject differentiation by race/ethnicity and gender 
Table 38: VOC lists employed as criteria for racial/ethnic group differentiation in Linear Discriminant Analysis

\begin{tabular}{|c|c|c|c|}
\hline \multirow{2}{*}{ Initial VOC List } & \multicolumn{3}{|c|}{ Stepwise Regression Method } \\
\hline & VOC List & Canon 1 & Canon 2 \\
\hline Diethylene glycol monoethyl ether & *Pristane & 1.431 & -0.296 \\
\hline Nonanal & *Galaxolide & -0.573 & 0.492 \\
\hline Naphthalene & *Octadecane & -0.524 & -0.553 \\
\hline *2,4-Diisopropylphenol & Methyl palmitate & 0.391 & 0.026 \\
\hline *Pentadecane & *Heptadecane & -0.381 & 0.599 \\
\hline *Methyl laurate & *Methyl laurate & -0.365 & -0.670 \\
\hline *Dodecanoic acid & 1-Heptadecene & -0.348 & 0.376 \\
\hline Isoamyl salicylate & Alpha-Amylcinnamaldehyde & 0.337 & -0.315 \\
\hline Methyl tridecanoate & Isoamyl salicylate & -0.301 & -0.091 \\
\hline Alpha-Amylcinnamaldehyde & Dioctyl ether & 0.298 & 0.206 \\
\hline Dioctyl ether & *2,4-Diisopropylphenol & 0.286 & 0.101 \\
\hline Hexyl salicylate & *Dodecanoic acid & 0.280 & -0.374 \\
\hline 1-Heptadecene & 1-Hexadecanol & 0.220 & 0.155 \\
\hline *Heptadecane & Isopropyl Myristate & 0.170 & -0.038 \\
\hline *Pristane & 1-Octadecene & -0.153 & 0.037 \\
\hline Ethylene glycol monododecyl ether & Hexyl salicylate & -0.139 & -0.530 \\
\hline Alpha-Hexylcinnamaldehyde & Methyl tridecanoate & 0.083 & 0.519 \\
\hline 1-Octadecene & Homomenthyl salicylate & 0.082 & 0.489 \\
\hline *Octadecane & Diethylene glycol monoethyl ether & -0.061 & -0.174 \\
\hline Isopropyl Myristate & *Pentadecane & 0.055 & 0.356 \\
\hline Pentadecanolide & Isopropyl Palmitate & 0.018 & 0.519 \\
\hline \multicolumn{4}{|l|}{ *Galaxolide } \\
\hline \multicolumn{4}{|l|}{ 1-Hexadecanol } \\
\hline \multicolumn{4}{|l|}{ Homomenthyl salicylate } \\
\hline \multicolumn{4}{|l|}{ Methyl palmitate } \\
\hline Isopropyl Palmitate & & & \\
\hline
\end{tabular}

* Compounds for which the average VOC amounts were seen to be significantly different between the age groups 
The changes obtained using the VOC criteria from the stepwise regression method did not represent a significant improvement on the differentiation of individuals on the basis of their race/ethnicity and gender. Therefore, as it had been previously done in the underarm odor assessment, the analysis was continued by breaking down the sample pool into females and males of different races/ethnicities. The purpose of focusing in the evaluation of smaller populations was to minimize any potential "noise" being generated in the initial canonical plot, while maximizing the expression of the differentiation power provided by hand odor profiles.

When only evaluating Hispanic, Caucasian and East Asian females, both the stepwise regression method and the initial VOC list approach indicated that all 26 compounds provided the best conditions for group differentiation (Table 39). For this reason, all 26 compounds were considered to constitute the VOC marker combination in this case. The canonical plot obtained displayed a complete differentiation of the three groups (Figure 147). No overlap was seen between any of the CL ellipses and only three subjects were misclassified in the assessment. All of these factors allowed the achievement of $94 \%$ classification accuracy in the differentiation of females from the three different race/ethnic groups. Out of the three groups, East Asian females displayed no misclassifications, while Hispanic females only showed one misclassification in the East Asian group. On the other hand, Caucasian females were the most misclassified by having classified one subject incorrectly in each one of the other two groups. The fact that the lowest percentage of classification accuracy achieved in this evaluation was $89 \%$ suggests that all 26 VOCs served as an efficient set of differentiation criteria for the groups. 


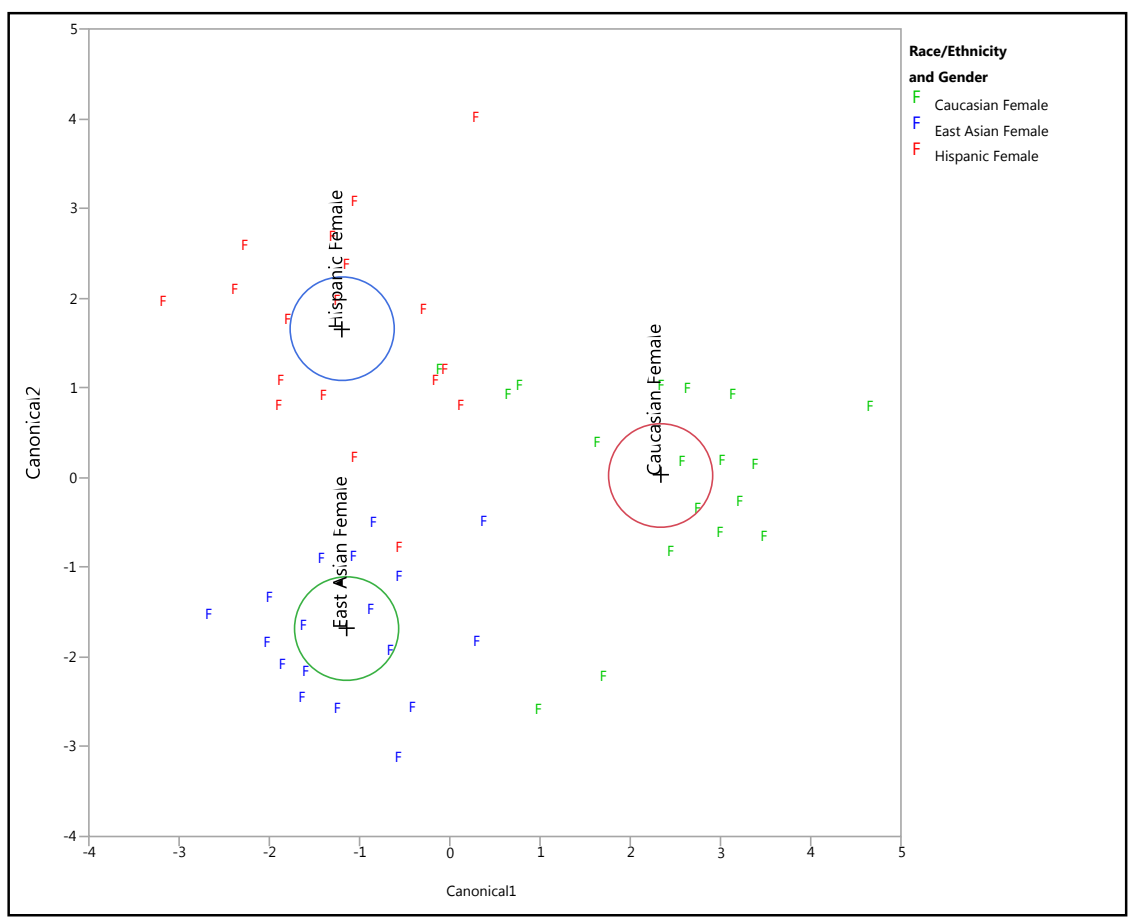

Figure 147: LDA canonical plot obtained through both the initial list and the stepwise regression methods for female differentiation by race/ethnicity 
Table 39: VOC list employed as criteria for female differentiation by racial/ethnic group in Linear Discriminant Analysis

\begin{tabular}{|c|c|c|}
\hline VOC List & Canon 1 & Canon 2 \\
\hline *Pristane & 1.198 & -0.380 \\
\hline *Octadecane & -0.882 & 0.930 \\
\hline *Alpha-Hexylcinnamaldehyde & -0.658 & 1.058 \\
\hline Galaxolide & -0.644 & 0.054 \\
\hline *2,4-Diisopropylphenol & 0.627 & 0.238 \\
\hline Dodecanoic acid & 0.611 & -0.389 \\
\hline Alpha-Amylcinnamaldehyde & 0.604 & 0.152 \\
\hline 1-Heptadecene & -0.596 & 0.103 \\
\hline *Heptadecane & 0.531 & 0.521 \\
\hline Ethylene glycol monododecyl ether & 0.513 & 0.167 \\
\hline Methyl palmitate & 0.425 & -0.068 \\
\hline Diethylene glycol monoethyl ether & 0.414 & -0.213 \\
\hline 1-Octadecene & -0.410 & -0.390 \\
\hline Isopropyl Myristate & -0.402 & -0.191 \\
\hline Dioctyl ether & 0.345 & 0.135 \\
\hline Methyl laurate & 0.338 & 0.036 \\
\hline Methyl tridecanoate & -0.305 & -0.350 \\
\hline Naphthalene & 0.297 & 0.089 \\
\hline *Isoamyl salicylate & 0.281 & 1.221 \\
\hline 1-Hexadecanol & 0.222 & -0.932 \\
\hline Isopropyl Palmitate & -0.174 & 0.950 \\
\hline Nonanal & 0.167 & -0.392 \\
\hline Pentadecanolide & 0.151 & -0.383 \\
\hline Pentadecane & -0.134 & 0.103 \\
\hline Hexyl salicylate & -0.046 & -1.797 \\
\hline Homomenthyl salicylate & 0.031 & -0.348 \\
\hline
\end{tabular}

* Compounds for which the average VOC amounts were seen to be significantly different between the age groups

On the other hand, the use of all 26 VOCs as criteria for the differentiation of Hispanic, Caucasian and East Asian males resulted in a total of nine subject misclassifications. From those nine, two Caucasian males were misclassified as East Asian, two East Asian males were misclassified as Hispanic and five Hispanic males 
were misclassified as East Asian. Although the canonical plot obtained for this analysis displayed no overlap between the CL ellipses of any of the groups, it did show that Hispanic and Caucasian males were most likely to be confused with East Asian males (Figure 148). The reason for this is that the cluster formed by East Asian males appeared located in between the other two groups formed. The position of this cluster in the canonical plot suggests that, in comparison to the Hispanic and Caucasian profiles, there are a lower number of factors in East Asian hand odor profiles to influence a drastic differentiation between that population and males from the other two groups. Nevertheless, a total classification accuracy of $82 \%$ was achieved by using the initial 26 VOCs. This accuracy demonstrates an efficient differentiation between the groups when using such VOC variables.

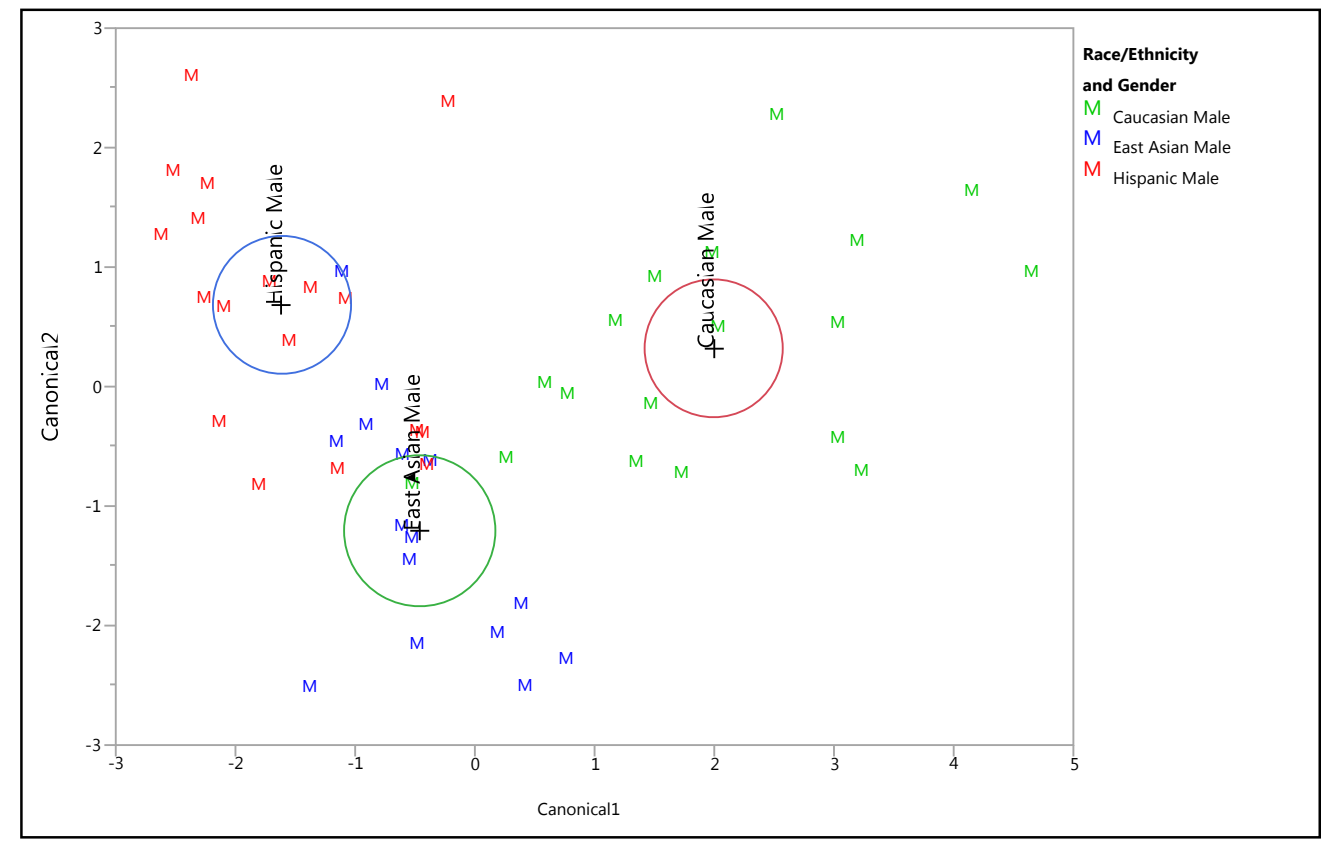

Figure 148: LDA canonical plot obtained using the initial VOC list for male subject differentiation by race/ethnicity 
Moreover, the modification of the VOC criteria, using the stepwise regression method, favored an improvement in the differentiation of males from different race/ethnic groups. A total of 24 VOCs were employed in this case as variables for group differentiation (Table 40). These 24 compounds were established as the VOC marker combination for the differentiation of males by race/ethnicity after being seen to have a positive impact on the results. The exclusion of Naphthalene and Heptadecane resulted in having the classification accuracy increase from $82 \%$ to $84 \%$. The LDA results indicated that excluding these two compounds reduced the misclassification of Hispanic males in the East Asian category from five to four subjects. Therefore, this caused a slight improvement in the overall accuracy of this analysis. Although the canonical plot obtained did not reveal any major differences from the one obtained using all 26 VOCs, it still continued to display separate clusters for each group (Figure 149). Caucasian males showed a classification accuracy of $89 \%$, East Asian males of $87 \%$ and Hispanic of $78 \%$. These percentages prove that the 24 compounds evaluated provide good enough information for a highly efficient differentiation of the groups. 


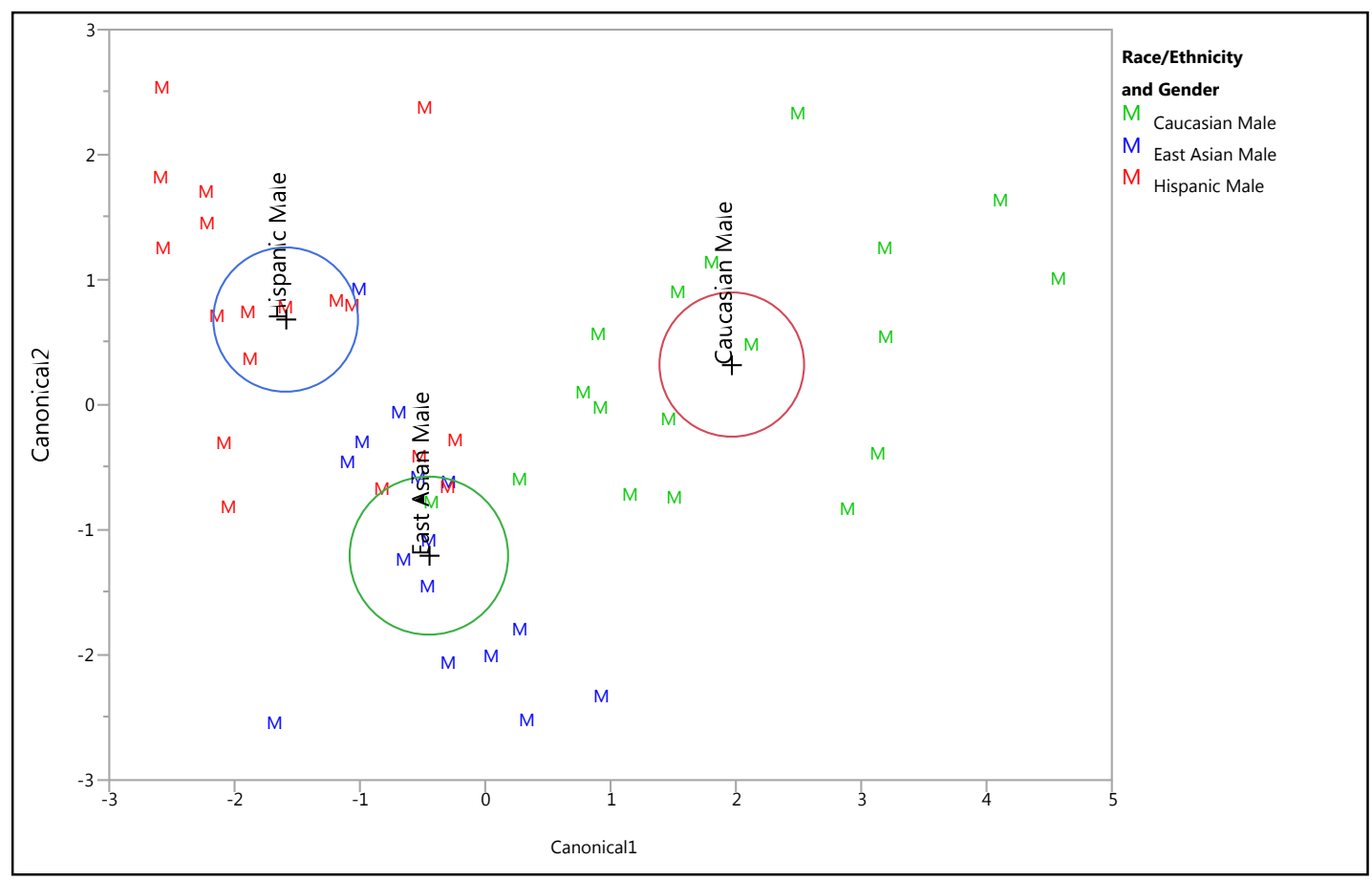

Figure 149: LDA canonical plot obtained using VOCs from the stepwise regression method for male subject differentiation by race/ethnicity 
Table 40: VOC lists employed as criteria for male differentiation by racial/ethnic group in Linear Discriminant Analysis

\begin{tabular}{|c|c|c|c|}
\hline \multirow{2}{*}{ Initial VOC List } & \multicolumn{3}{|c|}{ Stepwise Regression Method } \\
\hline & VOC List & Canon 1 & Canon 2 \\
\hline Diethylene glycol monoethyl ether & 1-Octadecene & -2.281 & -6.675 \\
\hline Nonanal & *Pristane & 1.517 & 0.454 \\
\hline Naphthalene & Isopropyl Myristate & -1.512 & 0.713 \\
\hline *2,4-Diisopropylphenol & Pentadecanolide & 1.499 & -0.515 \\
\hline *Pentadecane & Alpha-Hexylcinnamaldehyde & 1.413 & 7.785 \\
\hline Methyl laurate & Octadecane & -1.395 & -0.316 \\
\hline Dodecanoic acid & Hexyl salicylate & -1.276 & -1.159 \\
\hline Isoamyl salicylate & Isopropyl Palmitate & 1.180 & 0.830 \\
\hline Methyl tridecanoate & Methyl palmitate & 0.898 & 0.322 \\
\hline Alpha-Amylcinnamaldehyde & Dioctyl ether & 0.820 & -0.557 \\
\hline Dioctyl ether & Alpha-Amylcinnamaldehyde & -0.536 & 0.859 \\
\hline Hexyl salicylate & Methyl tridecanoate & -0.535 & -1.377 \\
\hline 1-Heptadecene & Dodecanoic acid & 0.495 & -0.567 \\
\hline *Heptadecane & *Pentadecane & 0.475 & 0.388 \\
\hline *Pristane & Galaxolide & -0.464 & 0.419 \\
\hline Ethylene glycol monododecyl ether & Ethylene glycol monododecyl ether & -0.462 & -0.017 \\
\hline Alpha-Hexylcinnamaldehyde & Homomenthyl salicylate & -0.461 & -0.203 \\
\hline 1-Octadecene & Methyl laurate & -0.427 & 0.788 \\
\hline Octadecane & Nonanal & 0.400 & 0.364 \\
\hline Isopropyl Myristate & 2,4-Diisopropylphenol & -0.315 & -1.401 \\
\hline Pentadecanolide & 1-Heptadecene & -0.306 & -1.230 \\
\hline Galaxolide & Diethylene glycol monoethyl ether & -0.178 & 0.444 \\
\hline 1-Hexadecanol & Isoamyl salicylate & 0.168 & -0.561 \\
\hline Homomenthyl salicylate & 1-Hexadecanol & 0.127 & -0.883 \\
\hline \multicolumn{4}{|l|}{ Methyl palmitate } \\
\hline Isopropyl Palmitate & & & \\
\hline
\end{tabular}

* Compounds for which the average VOC amounts were seen to be significantly different between the age groups

5.4.4.5. Confirmation of the forensic value of previously determined VOC marker combinations

Human scent is known to be often found in crime scenes as forensic evidence. Its ease of transfer and persistence makes of this type of evidence a valuable source of 
information for forensic investigators to associate criminals and/or victims to specific scenes. Employing VOC profiles as aids to identify, differentiate, and classify individuals by means of their age, gender and race/ethnicity has the potential to greatly assist in improving the process of criminal investigations.

Despite the fact that human scent could be transferred onto a crime scene from any region of an individual's body, the likelihood of collecting samples of hand odor in this type of scenario surpasses that of any other type of human scent. This is explained by the fact that both victims and perpetrators normally come in contact with their surroundings using primarily their hands. For this reason, the VOC marker combinations identified, in this dissertation, for hand odor were cross validated. Cross validation was performed by LDA, using the VOC marker combinations established for hand odor as criteria to predict the age, race/ethnicity and gender of new individuals.

Two types of cross validation methods were employed in LDA to test the classification accuracy of each VOC marker combination: the 5-fold cross validation method and an external validation method. The 5-fold cross validation consisted in dividing the data set (individual hand odor profiles), being evaluated under a specific VOC marker combination, into five different subsets or "folds". In this case, four folds were considered part of a training set for the LDA, while the fifth fold was considered a validation set. The classification accuracy was calculated, for all of the folds established, by alternating which folds were included in the LDA training set. Once all folds had been evaluated, a percentage for averaged accuracy was obtained for the classification of subjects on the basis of each VOC marker combination tested. 
In the external validation method, the training set consisted of all the hand odor profiles evaluated in this dissertation. In addition, the validation set consisted of ten hand odor profiles that were not part of the original data set. These ten odor profiles were obtained from individuals that were not considered in the original hand odor assessment, and that were sampled with the intention to consider their profiles as an independent data set for validation. In this case, these profiles represented the type of unknown samples that would be evaluated if these VOC marker combinations were to be used as a forensic resource for subject classification.

Both cross validation methods aimed to test the efficiency, of using the previously established VOC marker combinations, to predict the traits of a new or "unknown" sample. These tests were meant to confirm the feasibility of incorporating this dissertation's findings as tools for forensic applications. Therefore, they were evaluated in unison to obtain a broader perspective on the potential of VOC marker combinations as a subject classification tool.

The results from the 5-fold cross validation (Table 41) revealed that the average classification accuracy obtained for the different traits under study ranged between 33\% and $71 \%$. Subject classification by age was found to display the highest misclassification rate, managing to only provide $33 \%$ of averaged classification accuracy. On the other hand, subject classification by simultaneously considering race/ethnicity and gender, provided 38\% classification accuracy. These two classification attempts were also seen to provide the lowest classification accuracy percentages during the initial hand odor assessment, and were therefore expected to display the lowest accuracy during validation. Nevertheless, the fact that the validation percentages obtained for these two cases 
resulted close to half of the percentages achieved in the initial assessment, does not reflect an efficient application of the marker combinations evaluated. The independent evaluation of female and male classification, by the simultaneous consideration of race/ethnicity and gender, also provided accuracy percentages that were close to half of those achieved in the initial assessment. This also suggests limitations in the application of the VOC marker combinations used for these types of subject classifications. Moreover, the VOC marker combinations used for subject classification by race/ethnicity, and by gender, proved to facilitate an efficient classification for more than half of the individuals under evaluation. An averaged classification accuracy of $57 \%$ was achieved when attempting to classify individuals by their race/ethnicity; while $71 \%$ was achieved when pursuing their classification by gender. According to the initial assessment, $80 \%$ and $72 \%$ of accuracy were achieved for the classification of subjects by gender and race/ethnicity, respectively. This proves that using the corresponding VOC marker combinations for the classification of individuals by gender and race/ethnicity results in the most efficient methods for subject differentiation.

Table 41: Results obtained for the 5-fold cross validation of hand odor VOC marker combinations

\begin{tabular}{|c|c|c|c|c|c|c|}
\hline \multirow{2}{*}{ Fold Number } & \multicolumn{6}{|c|}{ Traits for Validated VOC Marker Combinations } \\
\cline { 2 - 7 } & Age & Gender & $\begin{array}{c}\text { Race/ } \\
\text { Ethnicity }\end{array}$ & $\begin{array}{c}\text { Race and Gender } \\
\text { (Both Genders) }\end{array}$ & $\begin{array}{c}\text { Race and Gender } \\
\text { (Only Females) }\end{array}$ & $\begin{array}{c}\text { Race and Gender } \\
\text { (Only Males) }\end{array}$ \\
\hline 1 & $8 / 21$ & $14 / 21$ & $12 / 21$ & $7 / 21$ & $4 / 11$ & $4 / 10$ \\
\hline 2 & $9 / 21$ & $16 / 21$ & $10 / 21$ & $9 / 21$ & $6 / 11$ & $2 / 10$ \\
\hline 3 & $8 / 21$ & $14 / 21$ & $13 / 21$ & $9 / 21$ & $9 / 11$ & $2 / 10$ \\
\hline 4 & $5 / 21$ & $16 / 21$ & $12 / 21$ & $6 / 21$ & $4 / 11$ & $6 / 11$ \\
\hline 5 & $9 / 21$ & $17 / 21$ & $11 / 21$ & $9 / 21$ & $5 / 11$ & $4 / 10$ \\
\hline $\begin{array}{c}\text { Averaged } \\
\text { Classification } \\
\text { Accuracy }\end{array}$ & $7 / 21$ & $15 / 21$ & $12 / 21$ & $8 / 21$ & $45 \%$ & $40 \%$ \\
\hline $\begin{array}{c}\text { Percentage for } \\
\text { averaged Classification } \\
\text { Accuracy }\end{array}$ & $33 \%$ & $71 \%$ & $57 \%$ & $38 \%$ & & 4 \\
\hline
\end{tabular}


On the other hand, the external validation provided additional insight on the efficiency of VOC marker combinations as resources for subject classification and differentiation. Figure 150 displays the hand odor profiles of all individuals considered in the validation data set for this method, while Table 42 provides details on each individual's demographic information.

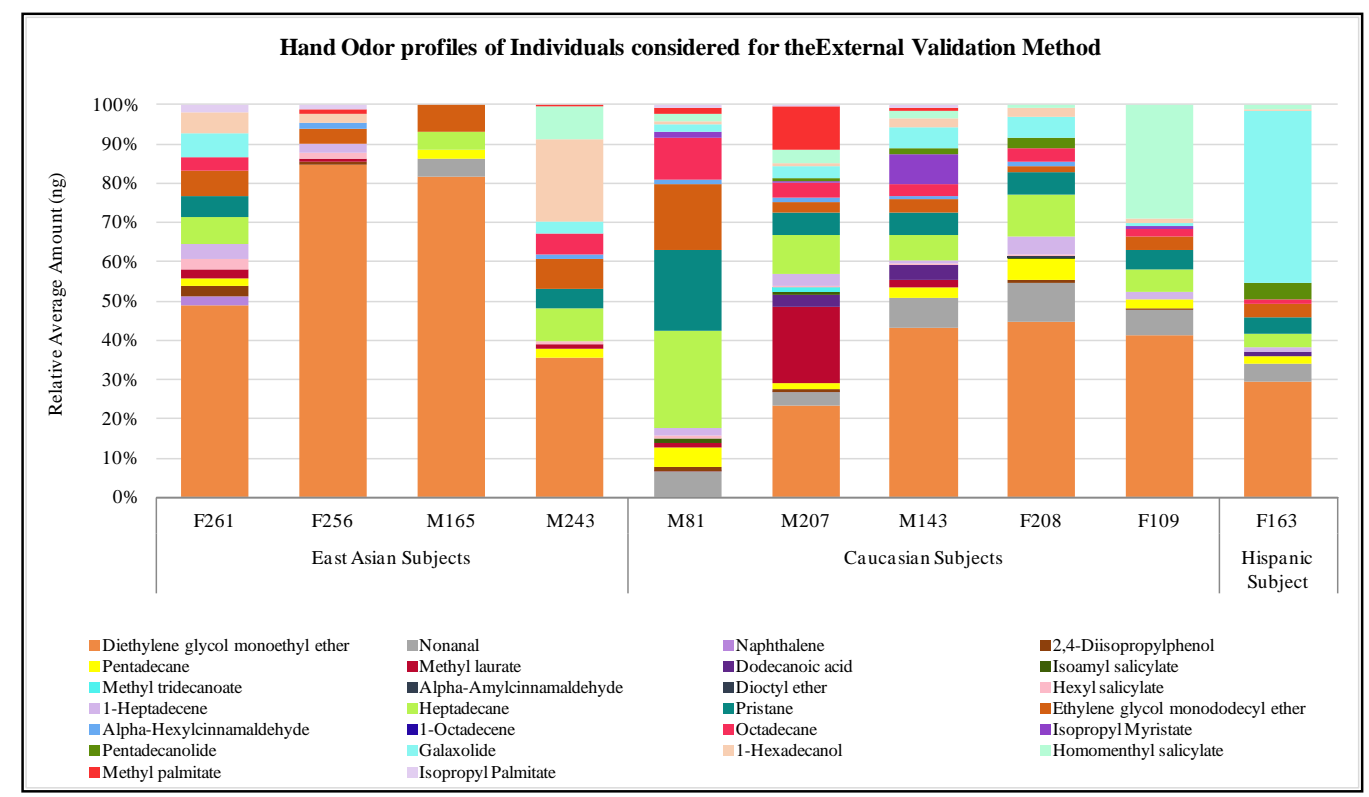

Figure 150: Hand odor profiles of individuals considered for the external validation method

Table 42: Demographic information of individuals included in external validation

\begin{tabular}{|c|c|c|c|}
\hline Subject Number & Race/Ethnicity & Gender & Age Group \\
\hline F261 & East Asian & Female & $35-50$ years \\
\hline F256 & East Asian & Female & $55+$ years \\
\hline M165 & East Asian & Male & $18-30$ years \\
\hline M243 & East Asian & Male & $35-50$ years \\
\hline M81 & Caucasian & Male & $18-30$ years \\
\hline M207 & Caucasian & Male & $35-50$ years \\
\hline M143 & Caucasian & Male & $55+$ years \\
\hline F208 & Caucasian & Female & $35-50$ years \\
\hline F109 & Caucasian & Female & $18-30$ years \\
\hline F163 & Hispanic & Female & $18-30$ years \\
\hline
\end{tabular}


The classification of each of these individuals was attempted using the VOC marker combinations for age, gender and race/ethnicity. As a result, it was discovered that $50 \%$ of the subjects were classified correctly, on the basis of their age, when using the external validation method. In addition, it was found that $60 \%$ of all subjects were classified correctly by their gender, as well as by their race/ethnicity, when evaluating the two traits separately. On the other hand, when attempting to differentiate all subjects by both race/ethnicity and gender, simultaneously, a classification accuracy of $50 \%$ was achieved. Once members of each gender were separated into different groups to assess this type of differentiation, $40 \%$ and $60 \%$ of classification accuracy was attained for females and males, respectively.

Overall, the external validation provided better accuracy percentages than the 5fold validation. This was shown by the fact that, with the exception of the classification of females by race/ethnicity, $50 \%$ or more of all subjects were correctly associated with their traits when using this method. In the 5-fold validation, such level of accuracy was only achieved for subject classification by gender, and by race/ethnicity. The differences observed between the classification accuracies achieved with these two methods can be explained by their innate qualities and their assessment process. The 5-fold method considers a smaller training data set than the external validation. This limits the criteria used by such method to establish accurate associations between the subjects and their traits. Moreover, contrary to the external validation, in the 5-fold method the data from both the training and validation sets are interchangeably selected from a same pool of samples. This difference provides another reason why a better level of accuracy can be achieved with the external validation. The external validation utilizes data that is 
independent from the training data set to validate the LDA results. Therefore, despite their differences, the results obtained from both validation methods should be considered complementary sources of information on the true value of VOC marker combinations as tools for subject classification.

The two types of cross validation, provided percentages that were mostly below those achieved in the initial hand odor assessment. This decrease in accuracy was expected as a result of having a limited sample size for both the training and validation data sets considered in each of the cross validation methods. Nevertheless, cross validation results still suggest that there is potential for the use of VOC marker combinations as subject classification criteria, and show the feasibility of using age, gender, and race/ethnicity as traits to associate or differentiate individuals. The cross validations performed demonstrated that the VOC markers discussed in this dissertation can be an efficient and accurate resource for subject classification.

Table 43: Classification accuracy results obtained from external validation

\begin{tabular}{|c|c|c|}
\hline $\begin{array}{c}\text { Traits for Validated VOC Marker } \\
\text { Combinations }\end{array}$ & $\begin{array}{c}\text { Classification } \\
\text { Accuracy }\end{array}$ & $\begin{array}{c}\text { Percentage of } \\
\text { Classification Accuracy }\end{array}$ \\
\hline Age & $5 / 10$ & $50 \%$ \\
\hline Gender & $6 / 10$ & $60 \%$ \\
\hline Race/Ethnicity & $6 / 10$ & $60 \%$ \\
\hline Race and Gender (Both Genders) & $5 / 10$ & $50 \%$ \\
\hline Race and Gender (Only Females) & $2 / 5$ & $40 \%$ \\
\hline Race and Gender (Only Males) & $3 / 5$ & $60 \%$ \\
\hline
\end{tabular}

5.4.4.6. Conclusion on the determination of scent marker consistency in hand odor samples

The evaluation of hand odor profiles as a resource to classify individuals on the basis of age, gender, and race/ethnicity provided insightful information on the feasibility 
of this type of practice. The study allowed the identification of those VOC combinations in hand odor acting as differentiating markers between groups of different traits. This achievement was confirmed after cross validating the results obtained for subject differentiation by trait. Cross validation demonstrated the feasibility of subject classification by means of these VOC marker combinations, providing essential support to this dissertation's findings. Moreover, this study revealed details on the presence and relationship of these markers, in the different types of scent samples evaluated in this dissertation.

As part of this assessment, it was found that all hand odor marker combinations included compounds for which the average amount displayed statistical significant differences among the groups being differentiated. This was also observed in the majority of the marker combinations identified in underarm odor. However, in none of these cases, was subject differentiation seen to solely rely on the compounds that portrayed significant differences in amounts. Compounds for which no statistical significant differences were identified were seen to be as important for the composition of hand and underarm odor marker combinations. Nevertheless, it is important to note that the statistical significant differences identified for certain compounds explain the presence of such compounds in VOC marker combinations. On the other hand, as it was also noted in underarm odor profiles, the marker combinations identified in hand odor profiles, for each of the traits of interest, were found to be different. Despite of these differences found between the marker combinations identified for each trait, there were still some compounds that were found to be in common across all combinations. Dodecanoic Acid, Isoamyl salicylate, Methyl tridecanoate, Dioctyl ether, Octadecane, Galaxolide and Methyl palmitate were 
seen to be present in all marker combinations identified for hand odor. For this reason, these compounds were considered to act analogously to Pentadecane, AlphaAmylcinnamaldehyde, 1-Heptadecene and Homomenthyl salicylate in underarm odor marker combinations. In both hand and underarm odor profiles, each specific list of compounds served as characteristic backbone for the type of scent sample being studied. The fact that no compounds were found in common between the VOC backbones identified for underarm and hand odor samples could be caused by the fundamental differences in nature of each of these types of scent samples (e.g., region of origin in human body, level of exposure to environment, etc.). Nevertheless, the consistency of each of these sets of compounds, across each of the marker combinations associated with each sample type, reveals useful information for the future analysis and discrimination of human scent. Moreover, the fact that hand odor profiles were found to provide equal or higher total classification accuracies than underarm odor profiles for human classification by trait, evidences the high potential of this type of sample as a forensic tool (Table 44).

Table 44: Subject classification accuracy percentages obtained in hand and underarm odor assessments

\begin{tabular}{|c|c|c|}
\hline \multirow{2}{*}{ Traits considered for Subject Differentiation } & \multicolumn{2}{|c|}{$\begin{array}{c}\text { Subject Classification } \\
\text { Accuracy }\end{array}$} \\
\cline { 2 - 3 } & $\begin{array}{c}\text { Hand Odor } \\
\text { Profiles }\end{array}$ & $\begin{array}{c}\text { Underarm } \\
\text { Odor Profiles }\end{array}$ \\
\hline Race/Ethnicity vs. Race/Ethnicity & $72 \%$ & $70 \%$ \\
\hline Age vs. Age & $65 \%$ & $63 \%$ \\
\hline Gender vs. Gender & $80 \%$ & $80 \%$ \\
\hline $\begin{array}{c}\text { Race/Ethnicity and Gender vs. Race/Ethnicity and Gender } \\
\text { (Both Genders Together) }\end{array}$ & $63 \%$ & $61 \%$ \\
\hline $\begin{array}{c}\text { Race/Ethnicity and Gender vs. Race/Ethnicity and Gender } \\
\text { (Only Females) }\end{array}$ & $94 \%$ & $85 \%$ \\
\hline $\begin{array}{c}\text { Race/Ethnicity and Gender vs. Race/Ethnicity and Gender } \\
\text { (Only Males) }\end{array}$ & $84 \%$ & $83 \%$ \\
\hline
\end{tabular}


The VOC marker combinations identified in hand and underarm odor profiles for the differentiation of individuals on the basis of age, race/ ethnicity and gender, were compared. Marker combinations of different samples types were not seen to be identical in any of the cases. For instance, a total of 23 compounds were found to be the optimum criteria in hand odor for the differentiation of individuals on the basis of age, while in the case of underarm odor a total of 12 were required. Notwithstanding, a total of 11 compounds were found to be consistently considered part of both VOC marker combinations (Table 45). In the case of subject differentiation by race/ethnicity, hand odor displayed a total of 15 marker VOCs, out of which 12 were also included in the VOC marker combination identified from underarm odor profiles. Moreover, the hand odor marker combination determined for optimum subject differentiation by gender, consisted of 13 compounds. Out of those 13 compounds, eight were also among the 17 VOCs constituting the underarm odor marker combination for the same type of subject differentiation.

When differentiating individuals by their race/ethnicity and gender, simultaneously, the hand odor marker combination was seen to provide the lowest percentage of classification accuracy out of all other percentages achieved. This was also seen to occur in the underarm odor assessment. According to the hand odor marker combination, 21 VOCs provided the best possible differentiation of race/ethnic and gender groups when being considered all together. On the other hand, 26 compounds were found to be necessary in underarm odor to achieve the best differentiation possible in this case. The comparison of both VOC marker combinations revealed that all 21 compounds identified as hand odor markers for this type of subject differentiation were 
also included in the marker combination obtained for underarm odor. This could have been caused by the possibility that more VOC variables were required for an effective differentiation of so many groups at a time. However, it also served to point out relevant scent constituents for human classification and differentiation. Once individuals from both genders were considered independently, the marker combinations for female hand and underarm odor profiles were seen to involve 26 and 19 compounds, respectively. In this case, all compounds included in the underarm odor marker combination were also seen to be considered part of the hand odor marker combination. Furthermore, the marker combination for male hand odor profiles included a total of 24 compounds. Out of those 24, 16 VOCs were found to be consistent in the marker combination obtained for male underarm odor profiles. 
Table 45: VOCs found to be consistent in marker combinations identified for hand and underarm odor

\begin{tabular}{|c|c|c|c|c|c|c|}
\hline Compounds & 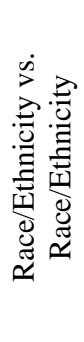 & 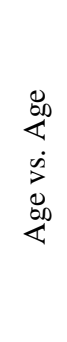 & 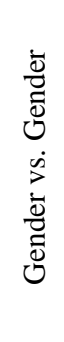 & 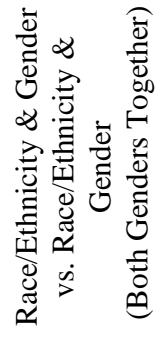 & 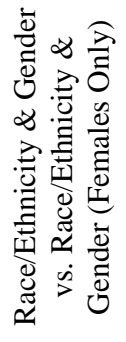 & 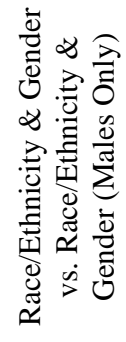 \\
\hline Nonanal & $\mathrm{X}$ & & & & $\mathrm{X}$ & \\
\hline 2,4-Diisopropylphenol & $\mathrm{X}$ & & & $\mathrm{X}$ & $\mathrm{X}$ & $\mathrm{X}$ \\
\hline Dodecanoic acid & $\mathrm{X}$ & & $\mathrm{X}$ & $\mathrm{X}$ & $\mathrm{X}$ & $\mathrm{X}$ \\
\hline Methyl tridecanoate & $\mathrm{X}$ & $\mathrm{X}$ & & $\mathrm{X}$ & & $\mathrm{X}$ \\
\hline Alpha-Amylcinnamaldehyde & $\mathrm{X}$ & $\mathrm{X}$ & & $\mathrm{X}$ & $\mathrm{X}$ & $\mathrm{X}$ \\
\hline 1-Heptadecene & $\mathrm{X}$ & $\mathrm{X}$ & & $\mathrm{X}$ & $\mathrm{X}$ & $\mathrm{X}$ \\
\hline Pristane & $\mathrm{X}$ & & & $\mathrm{X}$ & $\mathrm{X}$ & \\
\hline Ethylene glycol monododecyl ether & $\mathrm{X}$ & $\mathrm{X}$ & & & & $\mathrm{X}$ \\
\hline Alpha-Hexylcinnamaldehyde & $\mathrm{X}$ & & & & $\mathrm{X}$ & $\mathrm{X}$ \\
\hline 1-Octadecene & $\mathrm{X}$ & $\mathrm{X}$ & & $\mathrm{X}$ & $\mathrm{X}$ & $\mathrm{X}$ \\
\hline Octadecane & $\mathrm{X}$ & & & $\mathrm{X}$ & $\mathrm{X}$ & $\mathrm{X}$ \\
\hline Galaxolide & $\mathrm{X}$ & & $\mathrm{X}$ & $\mathrm{X}$ & $\mathrm{X}$ & $\mathrm{X}$ \\
\hline Diethylene glycol monoethyl ether & & $\mathrm{X}$ & & $\mathrm{X}$ & $\mathrm{X}$ & $\mathrm{X}$ \\
\hline Pentadecane & & $\mathrm{X}$ & $\mathrm{X}$ & $\mathrm{X}$ & $\mathrm{X}$ & $\mathrm{X}$ \\
\hline Dioctyl ether & & $\mathrm{X}$ & & $\mathrm{X}$ & & \\
\hline 1-Hexadecanol & & $\mathrm{X}$ & $\mathrm{X}$ & $\mathrm{X}$ & & \\
\hline Homomenthyl salicylate & & $\mathrm{X}$ & $\mathrm{X}$ & $\mathrm{X}$ & $\mathrm{X}$ & $\mathrm{X}$ \\
\hline Isopropyl Palmitate & & $\mathrm{X}$ & & & & \\
\hline Methyl laurate & & & $\mathrm{X}$ & $\mathrm{X}$ & $\mathrm{X}$ & $\mathrm{X}$ \\
\hline Isoamyl salicylate & & & $\mathrm{X}$ & $\mathrm{X}$ & & $\mathrm{X}$ \\
\hline Isopropyl Palmitate & & & $\mathrm{X}$ & $\mathrm{X}$ & $\mathrm{X}$ & \\
\hline Hexyl salicylate & & & & $\mathrm{X}$ & $\mathrm{X}$ & $\mathrm{X}$ \\
\hline Heptadecane & & & & $\mathrm{X}$ & $\mathrm{X}$ & \\
\hline Isopropyl Myristate & & & & $\mathrm{X}$ & $\mathrm{X}$ & \\
\hline Methyl palmitate & & & & $\mathrm{X}$ & & \\
\hline Pentadecanolide & & & & & $\mathrm{X}$ & \\
\hline
\end{tabular}

Overall, it was noted that more than half of the VOCs included in the shortest marker combination list (underarms' or hands') were also consistent in the marker 
combination list determined for the alternate odor profile type. This finding suggests that, despite the fact these marker combinations might have some characteristics determined by factors associated with the scent type (hand or underarm), they also include factors that are determined by an individual's traits. Age, gender and race/ethnicity, were seen to impact marker combinations in the two types of scent samples under study. The consistent presence of some of these compounds in both types of odor profiles, despite of the innate differences these profiles involve, highlight the influence of these traits in human scent expression. Moreover, this consistency also highlights the active role some of these compounds may exert as resources for human classification and differentiation on the basis of age race/ethnicity and gender.

In addition to all the previously described findings, another interesting observation was made from comparing the results from the underarm and hand odor assessments. In the majority of attempts made to differentiate subjects by trait, some of the most influential VOC markers in hand odor were also seen to coincide with some of the most influential VOC markers in underarm odor. Table 46 lists, in decreasing order, the five VOCs found to be of most influence for subject differentiation in each of the comparisons assessed using hand odor. From the compounds identified as most influential for subject differentiation by age and by gender, using hand odor, only 1 Hexadecanol was also amongst the most influential VOCs in underarm odor for the same purposes. On the other hand, Pristane, Octadecane, and Dodecanoic acid were all seen to be considered of high impact to subject differentiation by race/ethnicity when using hand and underarm odor profiles. In the case of the subject differentiation on the basis of both race/ethnicity and gender, simultaneously, Pristane, Octadecane and Heptadecane were 
seen to be among the top five VOCs in both hand and underarm odor profiles. However, when evaluating females apart from males, only Pristane and Octadecane were found to coincide in both types of odor profiles for females. On the contrary, for males, no compounds were found to be in common between the top five compounds in hand and underarm odor profiles. The consistency of highly influential VOCs for subject differentiation, across the two types of scent profiles under study, continues to support the feasibility of using human scent to classify individuals on the basis of shared traits. 
Table 46: VOCs of most influence in subject differentiation by trait comparison using hand odor

\begin{tabular}{|c|c|}
\hline Traits considered for Subject Differentiation & $\begin{array}{c}\begin{array}{c}\text { Most Influential Compounds } \\
\text { per Comparison }\end{array} \\
\end{array}$ \\
\hline \multirow{5}{*}{ Race/Ethnicity vs. Race/Ethnicity } & Pristane \\
\hline & Galaxolide \\
\hline & Octadecane \\
\hline & Dodecanoic acid \\
\hline & Methyl palmitate \\
\hline \multirow{5}{*}{ Age vs. Age } & 1-Hexadecanol \\
\hline & Methyl laurate \\
\hline & Pristane \\
\hline & Nonanal \\
\hline & 2,4-Diisopropylphenol \\
\hline \multirow{5}{*}{ Gender vs. Gender } & Methyl laurate \\
\hline & Methyl tridecanoate \\
\hline & Homomenthyl salicylate \\
\hline & Pentadecane \\
\hline & 1-Hexadecanol \\
\hline \multirow{5}{*}{$\begin{array}{l}\text { Race/Ethnicity \& Gender vs. Race/Ethnicity \& Gender } \\
\text { (Both Genders Together) }\end{array}$} & Pristane \\
\hline & Galaxolide \\
\hline & Octadecane \\
\hline & Methyl palmitate \\
\hline & Heptadecane \\
\hline \multirow{5}{*}{$\begin{array}{l}\text { Race/Ethnicity \& Gender vs. Race/Ethnicity \& Gender } \\
\text { (Only Females) }\end{array}$} & Pristane \\
\hline & Octadecane \\
\hline & Alpha-Hexylcinnamaldehyde \\
\hline & Galaxolide \\
\hline & 2,4-Diisopropylphenol \\
\hline \multirow{5}{*}{$\begin{array}{l}\text { Race/Ethnicity \& Gender vs. Race/Ethnicity \& Gender } \\
\text { (Only Males) }\end{array}$} & 1-Octadecene \\
\hline & Pristane \\
\hline & Isopropyl Myristate \\
\hline & Pentadecanolide \\
\hline & Alpha-Hexylcinnamaldehyde \\
\hline
\end{tabular}

The fact that accurate human classification by age, gender and race/ethnicity was made possible using hand odor profiles represents advancement to the forensic analysis of human scent as we currently know it. This knowledge not only broadens the spectrum of possibilities for the analysis of human scent evidence in forensic investigations, but also provides new elements to support the basis and credibility of human scent as a 
forensic resource. In addition, the constancy observed in the findings from the subject differentiation using underarm and hand odor profiles strengthens the foundation of human scent's application for subject differentiation by age, race/ethnicity, and gender. The steadiness observed in the results enhances, and confirms, the level of dependability and trust that can be achieved when considering the type of human scent information that has been contemplated in this dissertation. Therefore, the findings from this dissertation present a new and hopeful alternative to the forensic investigation of human scent evidence.

6. EVALUATION OF HS-SPME AND SOLVENT EXTRACTION METHODS AS COMPLEMENTARY TECHNIQUES FOR THE EXTRACTION OF HUMAN SCENT SAMPLES

\subsection{TASK OVERVIEW}

The evaluation of a solvent extraction method was included among the tasks to be assessed in this dissertation. This particular task was intended to explore another extraction technique as an alternative to perform VOC extraction of human scent samples. In addition the potential of using a solvent extraction method to complement the HS-SPME technique by enhancing the VOC information obtained from scent samples was evaluated. 


\subsection{OPTIMIZATION OF SOLVENT EXTRACTION TECHNIQUE}

\subsubsection{Methodology}

Prior to this task’s assessment, three different organic solvents (Fisher Scientific, Pittsburgh, PA) were tested to determine the most favorable extraction agent: HPLC grade Methanol (polar), Methylene Chloride (moderately polar), and Hexane (non polar). This particular selection was made to test the extraction efficiency of solvents characterized by different levels of polarity. In addition, two different extract volumes, $1 \mathrm{~mL}$ and $2 \mathrm{~mL}$, were tested and compared to determine the optimum amount necessary for an efficient analysis of the extracts.

Solvent optimization was assessed by initially placing three clean cotton gauze pads inside $10 \mathrm{~mL}$ glass vials, and spiking them with $100 \mu \mathrm{L}$ of a $100 \mathrm{ppm}$ standard mix solution. Following a 24 hour period, $10 \mathrm{~mL}$ of each solvent were added to each solvent's corresponding gauze pad and VOC extraction was performed in all cases for another 24 hours. After finalizing the extraction, fractions of $1 \mathrm{~mL}$ and $2 \mathrm{~mL}$ from each solvent's extracts were transferred to $4 \mathrm{~mL}$ glass vials containing a screw top with PTFE/Silicone septa (SUPELCO, Bellefonte, PA). Nitrogen gas was used to evaporate the extracts, and the VOCs extracted were pre-concentrated by adding $300 \mathrm{uL}$ of each of the corresponding solvents into the vials once again. Once the samples had been preconcentrated, the extracts were transferred into $2 \mathrm{~mL}$ glass vials with PTFE/Silicone lined caps, and analyzed sequentially in the GC-MS.

Blank extracts were also analyzed alongside the extract samples. The consideration of the extracts from blank samples allowed the correction of any overestimation of the VOCs extracted. The determination of the optimum solvent, along 
with the extract volume, was made on the basis of the VOC amount extracted for each of the VOCs being considered. The total VOC amount extracted, by each of the solvent methods tested, was also considered for this determination. The individual and total VOC amounts extracted in all solvent cases were compared upon completion of the test. The selection of the final solvent conditions, to be used in the evaluation of the solvent extraction method with respect to HS-SPME, was made as a result of such comparison.

\subsubsection{Results on the determination of best suited solvent extraction method}

Solvent extraction of human scent VOC reference standards (Appendix 6) was performed, using Methanol, Methylene Chloride and Hexane. In addition, two different volumes of extract, $1 \mathrm{~mL}$ and $2 \mathrm{~mL}$ were evaluated. After GC-MS analysis of the different extract fractions, the VOC amount extracted from each volume, with each solvent, was calculated. In the case of Methylene Chloride and Methanol, the highest VOC amount for the majority of the compounds was extracted using a $2 \mathrm{~mL}$ extract volume (Figure 151 and Figure 152). Also, out of these two solvents, it was observed that the amounts extracted by Methylene Chloride surpassed those extracted by Methanol. In the case of Hexane, the VOC amounts extracted for the majority of the compounds seemed to be very similar regardless of the extract volume used (Figure 153). However, different to the other two methods, a slightly better extraction seemed to be accomplished for most VOCs using an extract volume of $1 \mathrm{~mL}$. The fact that both of Hexane's extract volumes provided almost the same results, suggested that its capabilities as a solvent were not the optimum for the solvent extraction method. This was further confirmed by comparing the overall extraction efficiency accomplished by each one of the extract 
volumes tested for each solvent (Figure 154). In this comparison the total VOC amounts extracted with each method were compared, and it was found that, in fact, Hexane was not the optimum solvent for extraction. Although, all methods were seen to extract VOC amounts that were higher than those extracted from the blank samples (Average amount: $55.23 \mathrm{ng}$ ), using $2 \mathrm{~mL}$ of Methylene Chloride resulted the best conditions for solvent extraction. Using such extract volume conditions and solvent allowed extracting the highest total VOC amount achieved by any method. Therefore, these conditions were selected to ensure maximum solvent extraction efficiency in the succeeding assessment of this technique's evaluation with respect to HS-SPME.

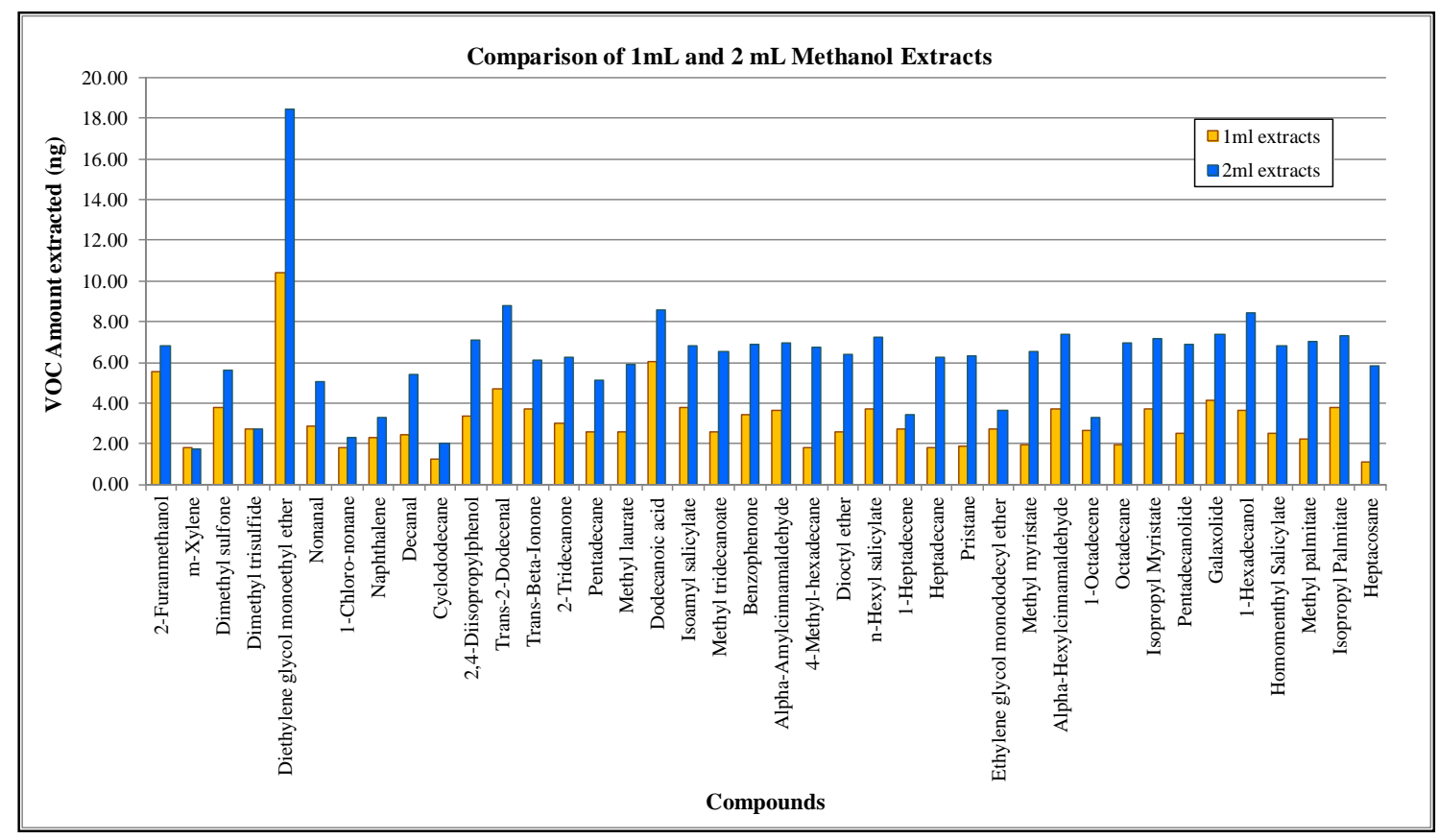

Figure 151: VOC Amount extracted per compound for $1 \mathrm{~mL}$ and $2 \mathrm{~mL}$ Methanol extracts 


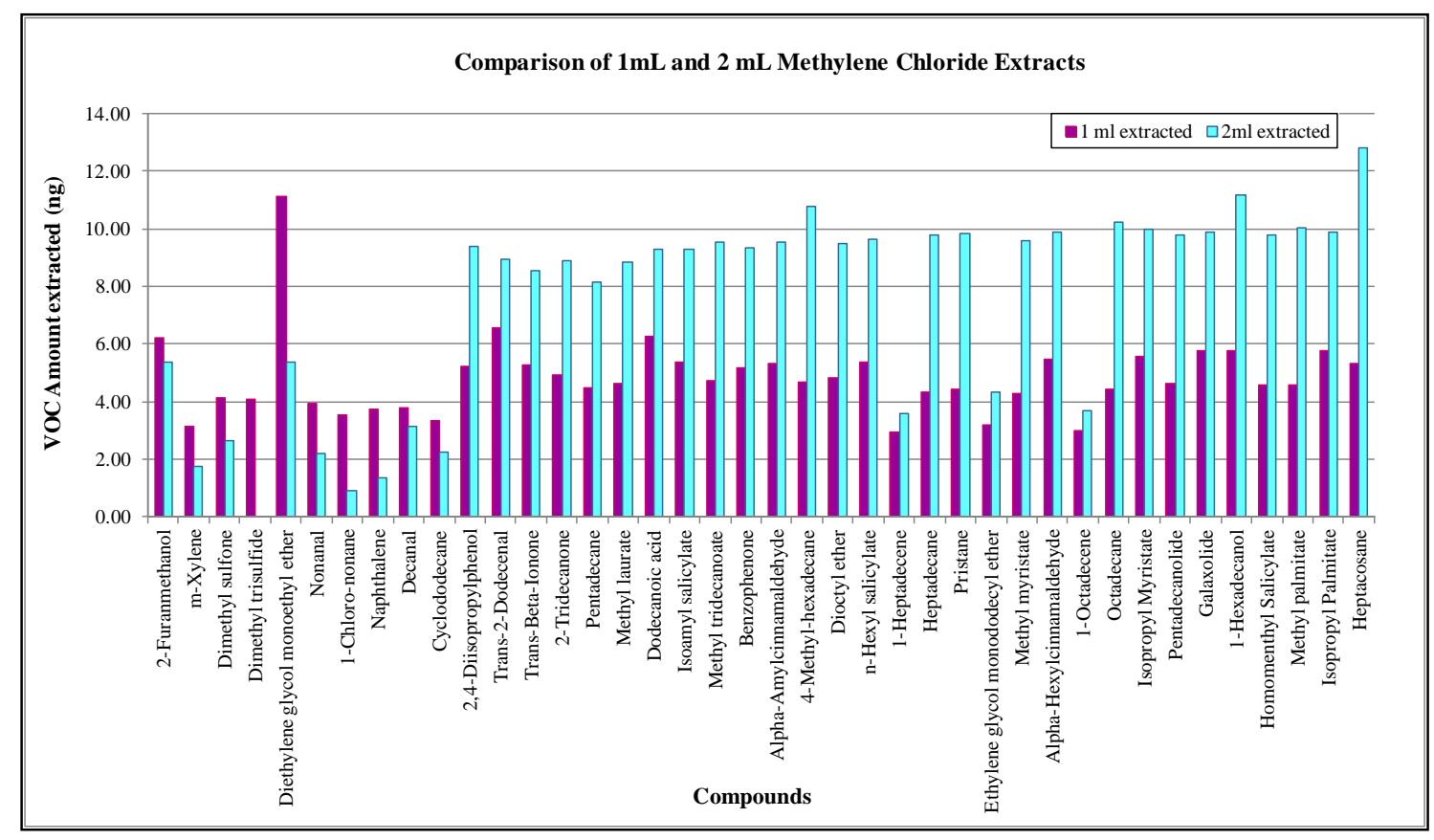

Figure 152: VOC Amount extracted per compound for $1 \mathrm{~mL}$ and $2 \mathrm{~mL}$ Methylene Chloride extracts

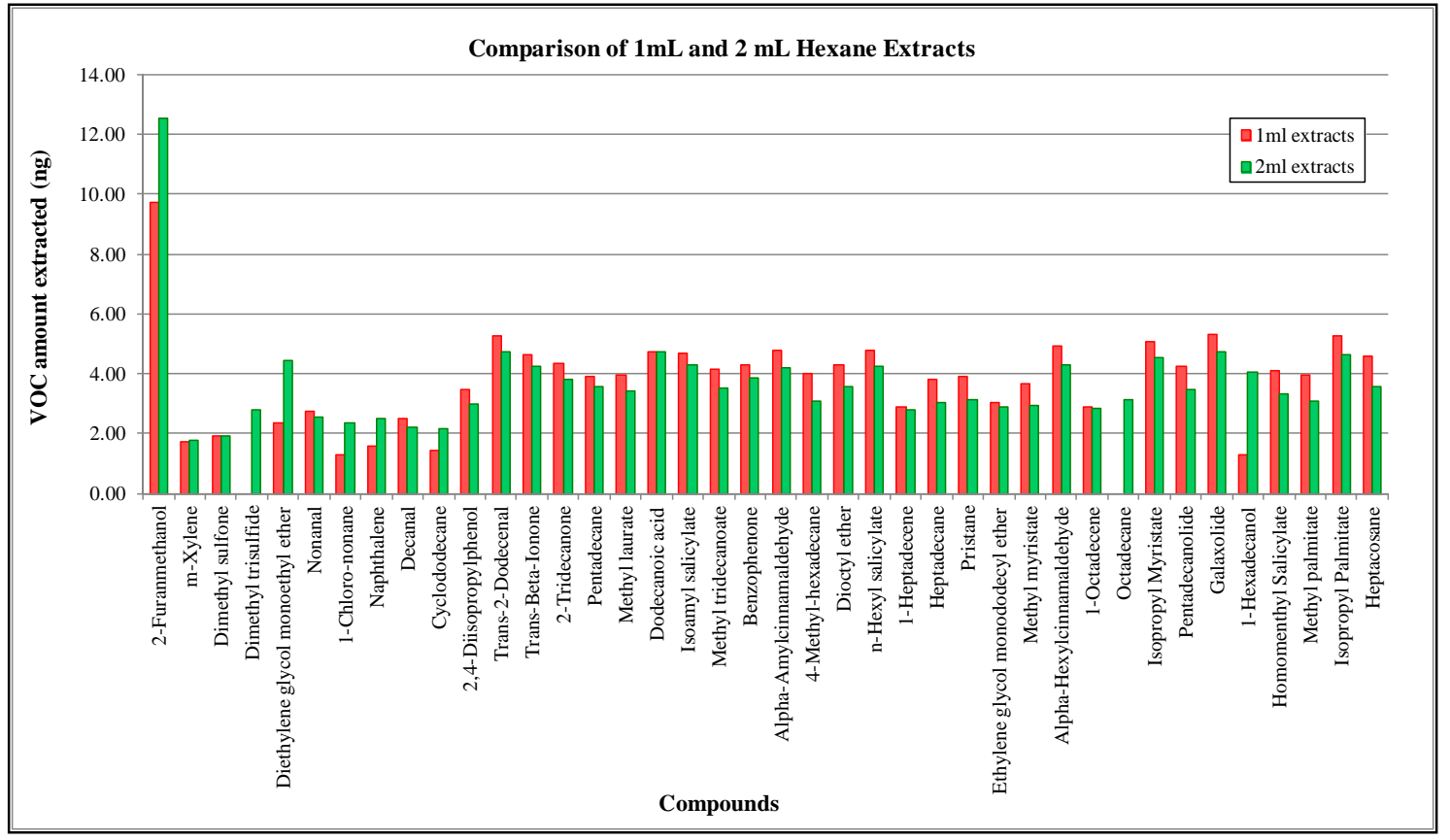

Figure 153: VOC Amount extracted per compound for $1 \mathrm{~mL}$ and $2 \mathrm{~mL}$ Hexane extracts 


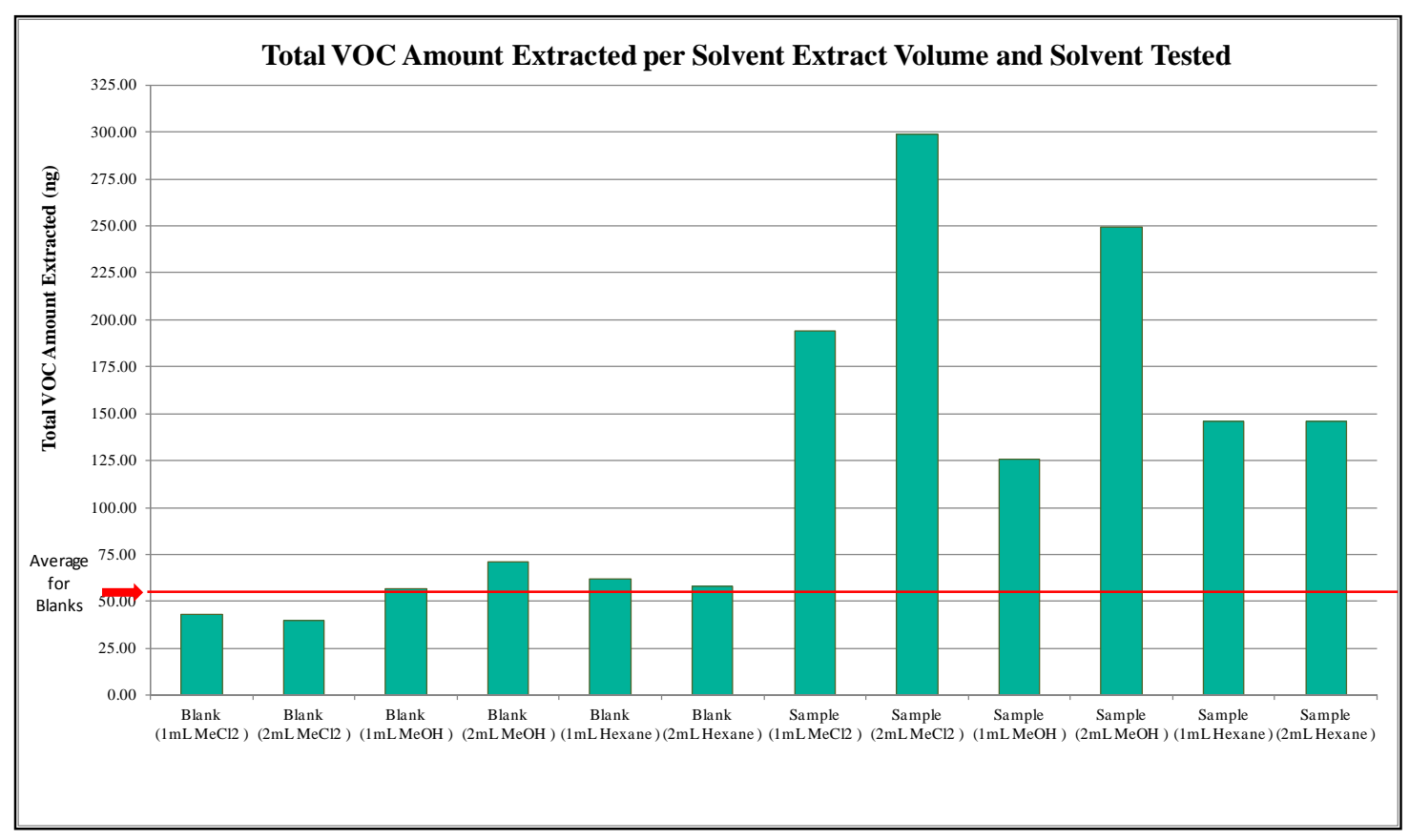

Figure 154: Comparison of total VOC amounts obtained by different extract volumes and solvents tested

\subsection{COMPARISON OF SOLVENT EXTRACTION AND HS-SPME TECHNIQUES \\ IN THE EXTRACTION OF SCENT SAMPLES}

\subsubsection{Methodology}

Scent samples from 12 Caucasian subjects (six of each gender) were extracted for this proof of concept. In addition, two different age groups were considered for each gender: three subjects corresponded to the 18-30 year age group and three to the 55+ year age group. The procedure was initiated by performing the usual HS-SPME on the 12 samples, and completing the analysis by GC-MS (specific methodologies have been previously described in sections 5.3.3. and 5.3.5.). Following headspace extraction, samples were stored in a freezer, at an average temperature of $-20{ }^{\circ} \mathrm{C}$, until solvent extraction was performed. The solvent extraction procedure consisted in adding $10 \mathrm{~mL}$ of 
methylene chloride to each scent sample, to extract the remaining VOCs for a period of 24 hours. After finalizing the extraction, $2 \mathrm{~mL}$ of the extract were transferred to $4 \mathrm{~mL}$ glass vials, and nitrogen gas was used to evaporate the extract fraction. Once evaporated, the extracts were pre-concentrated by re adding $300 \mathrm{uL}$ of methylene chloride into the vial. Following the pre-concentration step, extracts were transferred into $2 \mathrm{~mL}$ glass vials and analyzed sequentially in the GC-MS.

The extraction of the scent samples using the HS-SPME and solvent extraction techniques facilitated a comparison of the methods under study. The VOCs extracted with each method were identified and compiled to reach conclusions on the complementarity of both extraction techniques (Appendix 6 and Appendix 19). Principal Component Analysis (previously described in section 5.3.5.4.) was also used to create graphical representations of the results and depict the impact level of both techniques on the data of scent samples.

\subsubsection{Results on the evaluation of a solvent extraction method as a complementary} technique for the HS-SPME of scent samples As previously established, human scent samples collected from all individuals were extracted and analyzed using HS-SPME and GC-MS, respectively. Nevertheless, in order to evaluate solvent extraction as a complementary extraction method to HS-SPME, samples from 12 different Caucasian subjects (three females 18-30 year old, three males 18-30 year old, three females 55+ year old and three males 55+ year old) were selected at random. After having been extracted using HS-SPME, these samples were also extracted using the solvent extraction technique. Scent profiles were obtained for each subject from 
the analysis of the VOCs extracted by each method. The profiles were evaluated to compare the compounds extracted through each method and determine whether the information provided by the solvent extraction method served to complement that from the HS-SPME technique.

The GC-MS analysis of the solvent extracts revealed that, the solvent extraction method results efficient for the extraction of semi volatile and non-volatile compounds, characterized by high molecular weight and high boiling points. This is explained by the fact that highly volatile compounds are prone to evaporation into the sample's headspace, which eases their extraction by HS-SPME. Therefore, the chromatograms obtained from the analysis of the solvent extracts mainly displayed late eluting compounds that were, in their majority, undetected by HS-SPME. Quantitation of the VOCs extracted by solvent extraction was performed by means of comparison to an external calibration curve. Chemical standards were used to prepare reference solutions of 1 ppm, 5 ppm, 20 ppm, 40 ppm, 60 ppm, 80 ppm and 100 ppm, which included the compounds of interest. In total, there were seven compounds quantified using the solvent extraction method. These VOCs have been listed in Appendix 19.

On the other hand, when comparing the solvent extraction chromatograms with those of HS-SPME, it was noted that the HS-SPME method is capable of mainly detecting low molecular weight and low boiling point compounds (Figure 155). The chromatograms also showed that, in comparison to solvent extraction, this method has more limitations in the extraction of high molecular weight and boiling point compounds. 


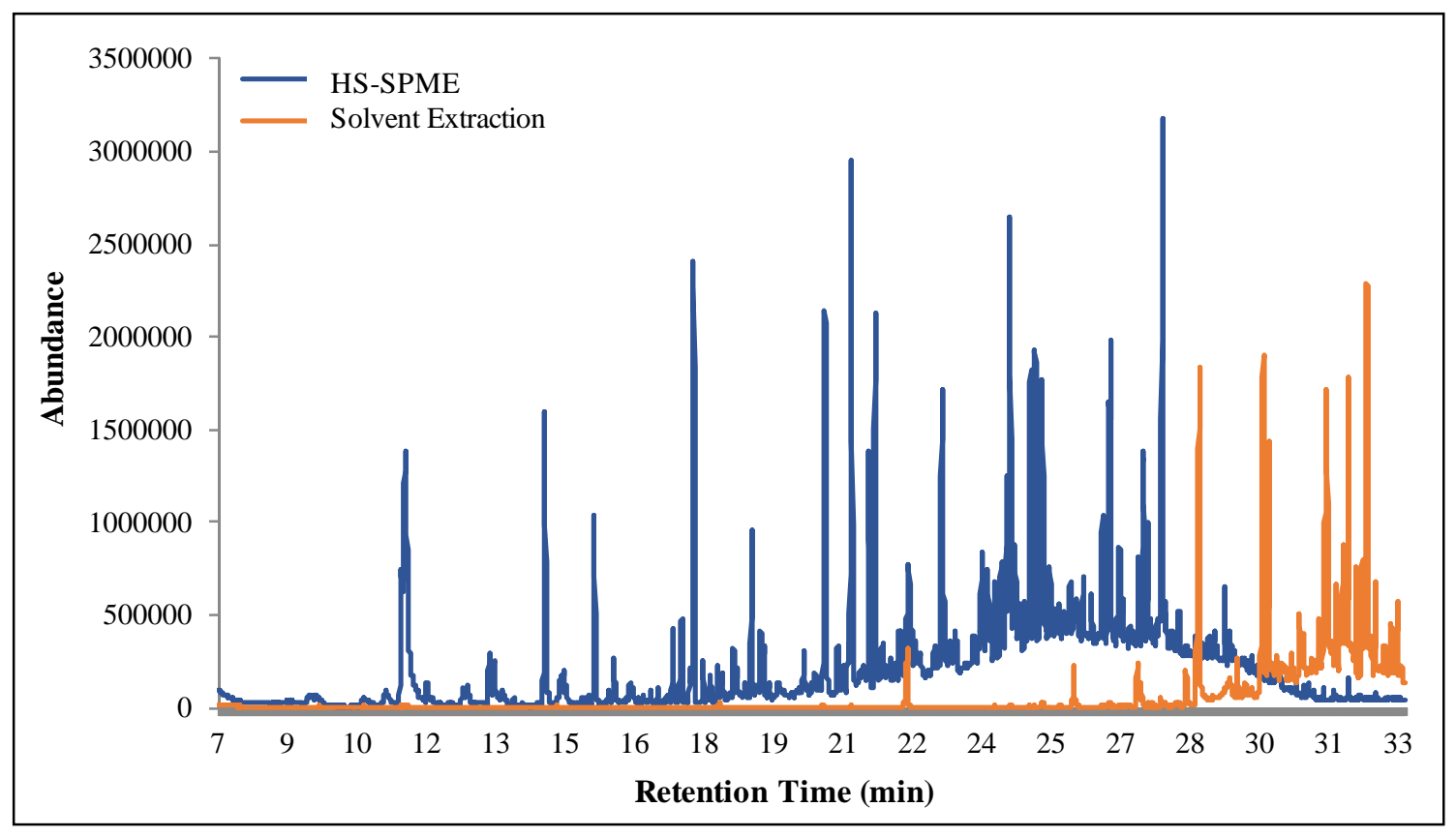

Figure 155: Overlaid chromatograms depicting VOCs extracted by solvent extraction and HS-SPME from a human scent sample

The individual scent profiles obtained through HS-SPME and solvent extraction were also evaluated to test the potential complementarity of solvent extraction as an alternate technique. Profiles were used to assess group differentiation, and determine if the VOC information obtained through solvent extraction was capable of enhancing the differentiation achieved by using HS-SPME alone. Principal component analysis was performed and score plots were used for the graphic representation of the differentiation achieved between the groups.

Differentiation of 18-30 and 55+ year old females was initially assessed by using the HS-SPME data. In this case, it was noted that the data presented in the PCA score plot already allowed a clear differentiation between the groups (Figure 156). However, after compiling the HS-SPME and solvent extraction data in the PCA analysis, the score plot showed a reduction in the spread of the cluster formed by 55+ year old females 
(Figure 157). Although there were no noticeable changes in the cluster of 18-30 year old females, the fact that the spread of the other cluster was reduced helped define group clusters. Nevertheless, a reduction in the variation percentage of both principal components was observed from one score plot to the other. This means that including the compounds extracted by solvent extraction, in the PCA differentiation criteria, did not aid in enhancing the differentiation of $18-30$ and 55+ year old females.

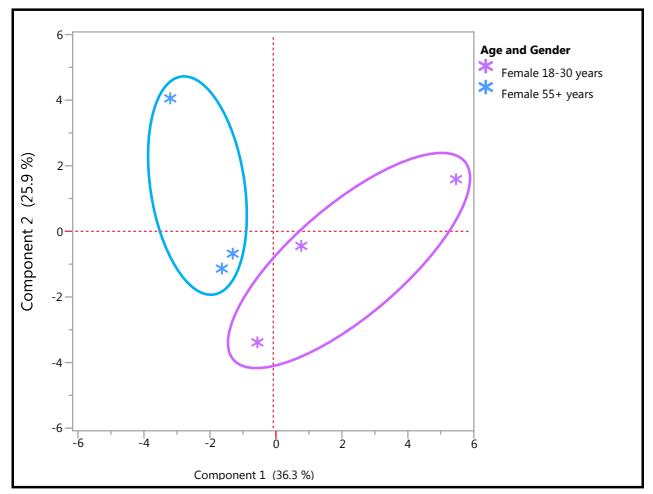

Figure 156: Differentiation of Caucasian females by age group using HS-SPME data

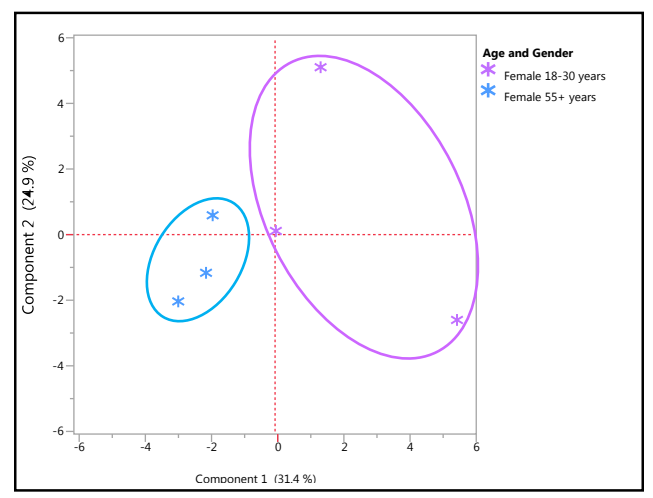

Figure 157: Differentiation of Caucasian females by age group using compiled HSSPME and solvent extraction data

The principal component analysis performed for the differentiation of 18-30 and $55+$ year old males, using the HS-SPME data, revealed that one of the 55+ year old males 
appeared very close to the 18-30 year old males' cluster (Figure 158). This observation suggested a good differentiation between the groups was not in place. However, including the data from the solvent extraction, among the PCA differentiation variables, caused a complete separation of the clusters (Figure 159). An increase in the variation percentages of both principal components was also noted from one score plot to the other; which indicates that the differentiation of males by age groups was enhanced.

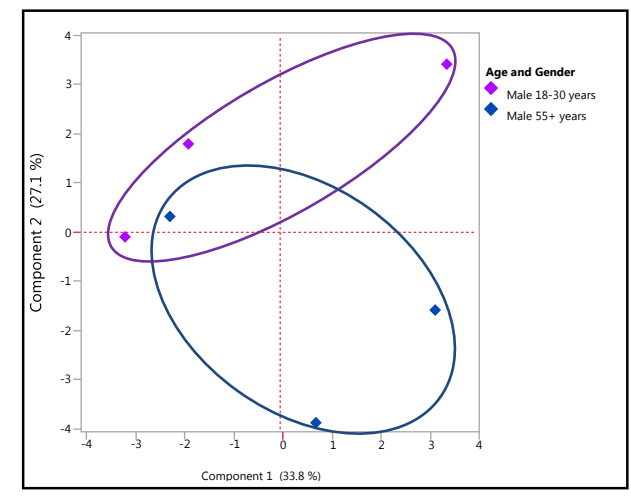

Figure 158: Differentiation of males by age group using HS-SPME data

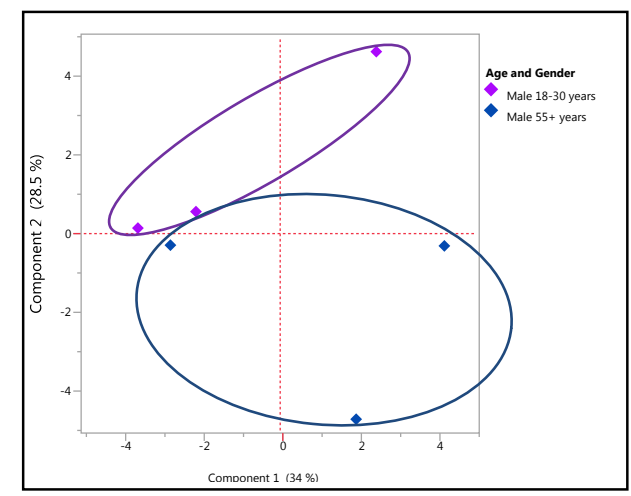

Figure 159: Differentiation of males by age group using compiled HS-SPME and solvent extraction data

On the other hand, PCA was also performed for the differentiation of males and females of a same age group. The PCA score plot created using the HS-SPME data, from the scent profiles of females and males in the 18-30 year category, displayed the two 
clusters being very close to each other (Figure 160). Therefore, no clear differentiation between the groups could be established in this case. On the contrary, the inclusion of the solvent extraction data, as variables being considered in the score plot, caused a slight improvement in the separation of the groups (Figure 161). In this case, the variation percentage in PC1 was seen to decrease from $39.6 \%$ to $35.7 \%$, while those of PC2 increased from $28.6 \%$ to $30.2 \%$. This means that although there was no variation enhancement reflected in the first principal component, an enhancement did take place in PC2.

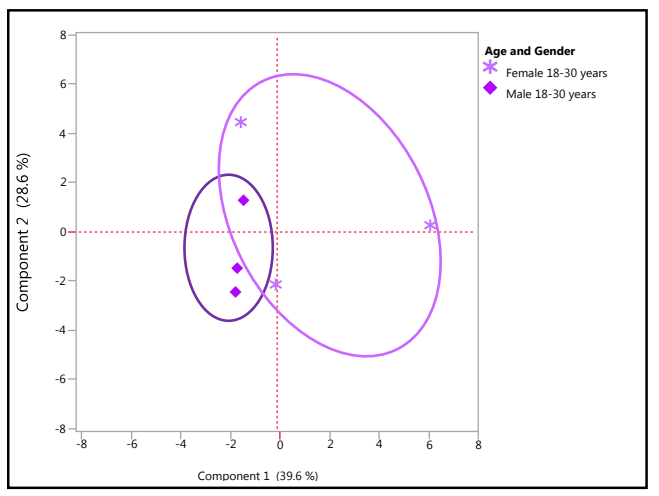

Figure 160: Differentiation of 18-30 year old females and males using HS-SPME data

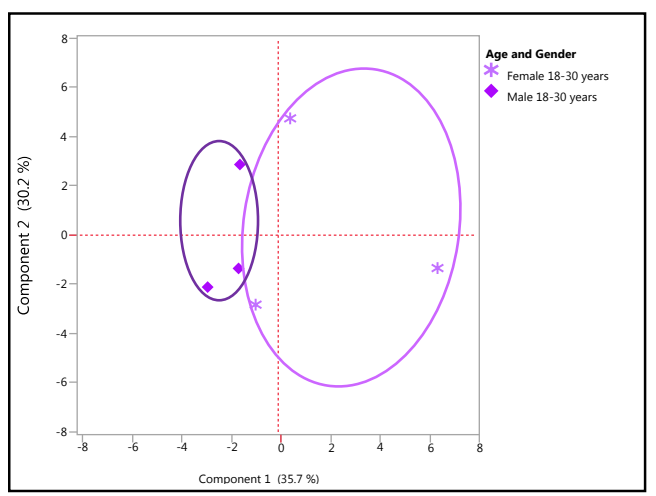

Figure 161: Differentiation of 18-30 year old females and males using compiled HSSPME and solvent extraction data 
Moreover, when using PCA to evaluate the differentiation of females and males in the 55+ year category, one of the females appeared very close to the male cluster (Figure 162). The inclusion of the solvent extraction data as score plot variables altered the position of this individual, causing two discernible clusters to be displayed (Figure 163). Once again, in this case, a decrease in PC1's variation percentage was observed (44.2\% to $37.9 \%)$. However, as it was observed when differentiating $18-30$ year old females and males, the variation percentage in PC2 was increased (24.7\% to 25.8\%). Therefore, although no variation enhancement was seen to occur in PC1, the second principal component reflected the complementarity of the solvent extraction method.

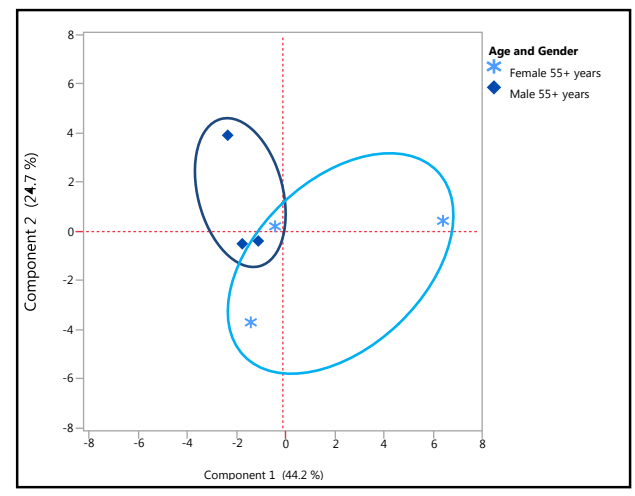

Figure 162: Differentiation of 55+ year old females and males using HS-SPME data 


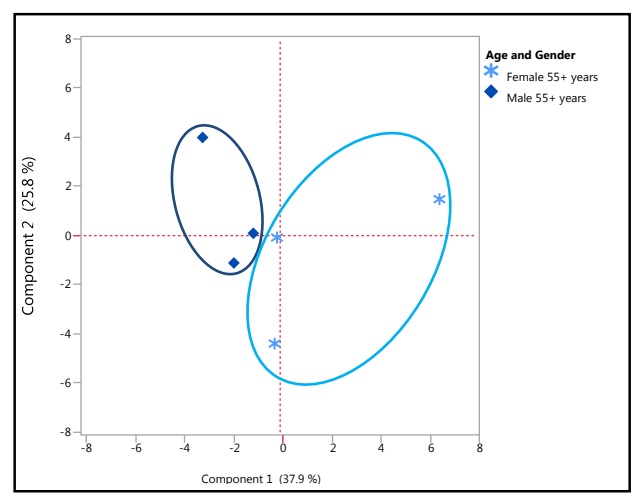

Figure 163: Differentiation of 55+ year old females and males using compiled HSSPME and solvent extraction data

\subsection{CONCLUSION ON THE COMPLEMENTARITY OF SOLVENT EXTRACTION}

\section{AND HS-SPME METHODS}

Regardless of whether it improved the definition of group clusters or enhanced the differentiation of the groups, solvent extraction data was observed to complement HSSPME data in all cases of PCA evaluated. The PCA score plots created using the HSSPME and solvent extraction compiled data allowed in all cases the visual identification of two discernible groups. Moreover, the chromatographic evaluation of the extracts and physical properties of the compounds extracted through each of these two methods also provided substantial evidence of their complementarity. For this reason, it is possible to propose the use of solvent extraction as a feasible alternative for VOC extraction, to complement the information obtained through the use of HS-SPME.

\section{OVERALL CONCLUSION}

Over the years, human scent has been used as a useful resource to obtain information on individuals that are of interest for forensic investigations. The assessment 
of scent profiles has facilitated the association of subjects to committed crimes, this way helping expedite the resolution of forensic investigations. Nevertheless, the consistent challenges faced by this type of forensic evidence throughout the years, has urged the search for new ways to strengthen the power and credibility of human scent as a forensic tool. As a result, this dissertation focused on investigating a new angle of human scent. So far, variations in scent profile composition across different individuals have been the core to human scent's forensic relevance. However, the research discussed in this dissertation presents a new approach for the evaluation of scent profiles as a forensic resource.

The current dissertation presents new information on the nature and chemical composition of scent profiles, and is the first of its kind to do so on such a large scale, sampling 190 individuals. The human scent of individuals from different age groups, gender, and races/ethnicities was evaluated to determine the influence of common traits on human scent expression. Therefore, this dissertation discusses the first attempt to use human scent as a means to obtain general details on an individual's identity. The determination of this type of details, along with the individualizing information human scent is known to often provide, can significantly enhance the scope of forensic investigations. As a result, law enforcement processes are bound to become more efficient, which may lead to a faster resolution of criminal cases.

The research in this dissertation revolved around the study of human scent profiles, and the determination of their efficiency as tools for individual differentiation by common traits. The fulfillment of all research objectives was founded upon the successful optimization of all the instrumental methods and analytical procedures involved in such 
assessment. A pilot study was also pursued to refine important research details that eased the further achievement of scientific findings. On the other hand, the scent profiles of Caucasian, Hispanic and East Asian individuals, from different age groups and genders, were examined. This was done with the objective of evaluating the influence of age, gender, and race/ethnicity in human scent expression, and determining how such influence is portrayed in the chemical composition of scent profiles. Underarm odor profiles were assessed, within each of the race/ethnic groups under study, to facilitate the identification of characteristic scent features in individuals from each group. Subsequently, such features were used to determine VOC marker combinations for age, gender, and race/ethnicity, as generic traits. Marker combinations were found to portray the influence of these traits on individual scent profiles, across different populations. Hence, they were also found to be useful criteria for the differentiation of individuals of different age, gender and race/ethnic groups, on the basis of underarm odor.

The fact that this type of association could be established between a scent profile and its human source, highlighted the incredible impact such finding could have on forensic applications. Therefore, VOC marker combinations were also determined for hand odor; the most common type of human scent evidence found in forensic settings. The potential of hand odor profiles, to reveal information on the age, gender and race/ethnicity of individuals from different populations, was evaluated by comparing hand and underarm odor VOC marker combinations. The comparison of both types of scent profiles revealed consistency between the information provided, by each body region, for the different traits under consideration. As a result, the potential of using VOC marker combinations as criteria for individual differentiation in forensic applications was 
strengthened. Also, the fact that LDA results revealed that VOC marker combinations favored a successful classification of individuals, on the basis of the three traits of interest, further supported the use of these VOCs as differentiation criteria.

The results discussed in this dissertation have demonstrated that it is possible to enhance the power of human scent evidence in forensic investigations. The fact that scent profiles can disclose information on the common traits expressed by an individual, offers a wider range of knowledge for forensic investigators to address criminal cases. In addition, other findings from this research have also facilitated alternatives to overcome current limitations in the assessment of human scent. In this dissertation, the capability of enhancing human scent information was also tested by using two different analytical approaches to study scent profiles. Results from the solvent extraction and HS-SPME of human scent samples were compared; and each technique’s capability to provide details on the chemical composition of scent profiles was evaluated. The evaluation of each technique's performance revealed that the use of both methods provides complementary information on the chemical composition of human scent profiles. Hence, the implementation of both techniques has been suggested as a feasible alternative for cases in which a comprehensive assessment of human scent profiles results imperative. This option represents an additional method to continue to improve human scent investigations on the basis of evidence collected at a crime scene.

The current research allowed the fulfillment of all pre-established objectives. Nevertheless, there are certain factors involved in this project's execution that must be taken into consideration for further studies. One of these factors is the fact that the data used in the underarm odor assessments only considered those compounds that showed 
consistent occurrence, on both days of sampling, for each individual. This conservative approach was crucial to reducing the number of external influences in odor profiles and being able to pursue the identification of true human scent markers. On the other hand, another necessary remark is that, despite of the large number of individuals sampled in this study, the results obtained for some of the subject differentiation attempts might have been limited by the number of individuals considered. The power of the different statistical analyses performed in this study depended on the number of individuals and differentiation variables under consideration. Therefore, larger sample sizes in the different groups being evaluated might have favored the identification of clearer trends and higher accuracy levels in the results. The level of complexity and time dependence involved in the sampling procedures and instrumental analysis performed for this research, limited the sample sizes to those considered. Nevertheless, despite the possible impact of these experimental limitations, the study was able to be completed and all its objectives were able to be efficiently fulfilled.

The discoveries presented in this dissertation demonstrate that human scent, is not only useful to obtain information that is exclusive to an individual, but that it also allows establishing general categorizations on the basis of an individual's shared or common traits. This capability encourages the development of new approaches that can be implemented to improve the assessment of human scent as forensic evidence. The use of new alternatives to evaluate scent profiles would promote an improvement in the methods being currently used to investigate human scent evidence. At the same time, this may have a direct and positive impact on the level of reliability associated with this type of forensic evidence in the court of law. Increasing the amount of information attainable 
from human scent profiles can reduce the uncertainty that often surrounds the use of scent samples to associate individuals with a crime. Human scent marker combinations could be used to develop canine training aids, which could assist in ensuring optimum canine performance in forensic investigations that involve subject identification. On the other hand, analytical instrumentation could be employed to corroborate canine alerts and provide concrete meaning to human scent evidence. The opportunity to rely in both of these approaches could add on great value to the admissibility of human scent evidence in court.

The completion of this research has exposed findings that set the foundation for future and more specialized investigations on the use of human scent for subject classification. The development of in depth studies, for each of the populations that have been assessed in this research, represents an example of a very feasible approach for further research. In depth studies could not only confirm the current findings, but also facilitate ways to expand on all the information that has been discovered so far. These studies would have a solid starting point, and would allow modifying different aspects of the current experimental design, to continue to reach the investigation's maximum potential. The simplification of sampling procedures, an increase in the number of samples being evaluated, the evaluation of genetic profiles as a complement to scent analysis, and the evaluation of individuals from new populations (e.g., Sub-Saharan African descent), could be key factors in the development of these new investigations. Therefore, this study has marked the direction towards which new research should be headed in order to achieve more significant discoveries in the field of human scent. On the other hand, the fact that there is such a wide range of possibilities for new 
advancements to be made in the human scent field, continues to open room for new ways to apply human scent in forensic investigations. There is no doubt that there are many more ways in which human scent evidence could be used to bring major advancements to both the forensic and law enforcement communities. Hence, curiosity and research engagement must continue to be encouraged as the driving forces behind the pursuit of justice. 


\section{REFERENCES}

1. People v. Wade. Akd.111. (Akd. 2007).

2. U.S.Attorney's Office . Joshua Wade Sentenced to Life in Prison for the Murders of Mindy Schloss and Della Brown. 2-7-2010. The Federal Bureau of Investigation - Anchorage Division.

3. Brott v. State, 70 Neb. 395 (Neb. 1903).

4. Prada, P.; Furton, K. Human Scent Detection: A Review of its Developments and Forensic Applications. Revista de Ciencias Forenses, 2008, pp 81-87.

5. Ensminger, J.; Jezierski, T.; McCullock, M. Scent Identification in Criminal Investigations and Prosecutions: New Protocol Designs Improve Forensic Reliability; Social Science Research Network: 10.

6. People v. Willis, 115 Cal. App. 4th 379 (2004).

7. Scent. Merriam-Webster Dictionary . 2015. Merriam-Webster Incorporated, An Encyclopedia Britannica Company. 2-8-2015.

8. Curran, A. M.; Rabin, S. I.; Furton, K. G. Analysis of the Uniqueness and Persistence of Human Scent. Forensic Science Communications 2005, 7 (2).

9. Albano, R. E.; Forslind, B.; Lindberg, M. M. D.; NetLibrary, I. Skin, hair, and nails structure and function; M. Dekker: New York, 2004.

10. Burns, T. F.; Rook, A. Rook's textbook of dermatology; 8th ed. / edited by Tony Burns ... [et al.]. ed.; Wiley-Blackwell: Chichester, West Sussex, UK ; Hoboken, NJ, 2010.

11. Ebling, F. J. G. Human Skin. Encyclopaedia Britannica . 2015. Encyclopaedia Britannica Inc. 3-14-2015.

12. Zouboulis, C. C.; Baron, J. M.; Böhm, M.; Kippenberger, S.; Kurzen, H.; Reichrath, J.; Thielitz, A. Frontiers in sebaceous gland biology and pathology. Experimental Dermatology 2008, 17 (6), 542-551.

13. Picardo, M.; Ottaviani, M.; Camera, E.; Mastrofrancesco, A. Sebaceous gland lipids. Dermatoendocrinol 2009, 1 (2), 68-71.

14. Greene, R. S.; Downing, D. T.; Pochi, P. E.; Strauss, J. S. Anatomical Variation in the Amount and Composition of Human Skin Surface Lipid. J Investig Dermatol 1970, 54 (3), 240-247. 
15. Saga, K. Histochemical and Immunohistochemical Markers for Human Eccrine and Apocrine Sweat Glands: An Aid for Histopathologic Differentiation of Sweat Gland Tumors. J Investig Dermatol Symp Proc 2001, 6 (1), 49-53.

16. Romanowski, P.; Schueller, R. Axillary Odor: Its Physiology, Microbiology and Chemistry. In Beginning cosmetic chemistry: practical knowledge for the cosmetic industry, Allured Books: Carol Stream, IL, 2009; pp 87-91.

17. Wilke, K.; Martin, A.; Terstegen, L.; Biel, S. S. A short history of sweat gland biology. International Journal of Cosmetic Science 2007, 29 (3), 169-179.

18. Sato, K.; Leidal, R.; Sato, F. Morphology and Development of An Apoeccrine Sweat Gland in Human Axillae. American Journal of Physiology 1987, 252 (1), R166-R180.

19. Sato, K.; Sato, F. Sweat secretion by human axillary apoeccrine sweat gland in vitro. American Journal of Physiology - Regulatory, Integrative and Comparative Physiology 1987, 252 (1), R181-R187.

20. Wysocki, C. J.; Preti, G. Facts, fallacies, fears, and frustrations with human pheromones. Anatomical Record Part A-Discoveries in Molecular Cellular and Evolutionary Biology 2004, 281A (1), 1201-1211.

21. Gallagher, M.; Wysocki, C. J.; Leyden, J. J.; Spielman, A. I.; Sun, X.; Preti, G. Analyses of volatile organic compounds from human skin. British Journal of Dermatology 2008, 159 (4), 780-791.

22. Egelrud, T. Desquamation in the stratum corneum. Acta Dermato VenereologicaSupplement 2000, 208, 44-45.

23. Doyle, C. Secret cloud that surrounds us. Family Health , 32-35. 1970.

24. Syrotuck, W. Scent and the Scenting Dog; Barkleigh Productions: Mechanicsburg, 2000.

25. Leyden, J. J.; Mcginley, K. J.; Holzle, E.; Labows, J. N.; Kligman, A. M. The Microbiology of the Human Axilla and Its Relationship to Axillary Odor. Journal of Investigative Dermatology 1981, 77 (5), 413-416.

26. Grice, E. A.; Segre, J. A. The skin microbiome. Nat Rev Microbiol 2011, 9 (4), 244-253.

27. Chiller, K.; Selkin, B. A.; Murakawa, G. J. Skin microflora and bacterial infections of the skin. Journal of Investigative Dermatology Symposium Proceedings 2001, 6 (3), 170-174. 
28. Taylor, D.; Daulby, A.; Grimshaw, S.; James, G.; Mercer, J.; Vaziri, S. Characterization of the microflora of the human axilla. International Journal of Cosmetic Science 2003, 25 (3), 137-145.

29. Fredrich, E.; Barzantny, H.; Brune, I.; Tauch, A. Daily battle against body odor: towards the activity of the axillary microbiota. Trends in Microbiology 2013, 21 (6), 305-312.

30. Zeng, X. N.; Leyden, J. J.; Lawley, H. J.; Sawano, K.; Nohara, I.; Preti, G. Analysis of Characteristic Odors from Human Male Axillae. Journal of Chemical Ecology 1991, 17 (7), 1469-1492.

31. Troccaz, M.; Borchard, G.; Vuilleumier, C.; Raviot-Derrien, S.; Niclass, Y.; Beccucci, S.; Starkenmann, C. Gender-Specific Differences between the Concentrations of Nonvolatile (R)/(S)-3-Methyl-3-Sulfanylhexan-1-Ol and (R)/(S)-3-Hydroxy-3-Methyl-Hexanoic Acid Odor Precursors in Axillary Secretions. Chemical Senses 2009, 34 (3), 203-210.

32. Natsch, A.; Derrer, S.; Flachsmann, F.; Schmid, J. A Broad Diversity of Volatile Carboxylic Acids, Released by a Bacterial Aminoacylase from Axilla Secretions, as Candidate Molecules for the Determination of Human-Body Odor Type. Chemistry \& Biodiversity 2006, 3 (1), 1-20.

33. Curran, A. M.; Ramirez, C. F.; Schoon, A. A.; Furton, K. G. The frequency of occurrence and discriminatory power of compounds found in human scent across a population determined by SPME-GC/MS. Journal of Chromatography BAnalytical Technologies in the Biomedical and Life Sciences 2007, 846 (1-2), 8697.

34. Brown, J. S.; Prada, P. A.; Curran, A. M.; Furton, K. G. Applicability of emanating volatile organic compounds from various forensic specimens for individual differentiation. Forensic Science International 2013, 226 (1-3), 173182.

35. Wilson, M. A. Microbial inhabitants of humans : their ecology and role in health and disease; Cambridge University Press: Cambridge, UK ; New York, 2005.

36. Zeng, X. N.; Leyden, J. J.; Spielman, A. I.; Preti, G. Analysis of characteristic human female axillary odors: Qualitative comparison to males. Journal of Chemical Ecology 1996, 22 (2), 237-257.

37. Penn, D. J.; Oberzaucher, E.; Grammer, K.; Fischer, G.; Soini, H. A.; Wiesler, D.; Novotny, M. V.; Dixon, S. J.; Xu, Y.; Brereton, R. G. Individual and gender fingerprints in human body odour. Journal of the Royal Society Interface 2007, 4 (13), 331-340. 
38. Curran, A.; Rabin, S.; Prada, P.; Furton, K. Comparison of the Volatile Organic Compounds Present in Human Odor Using Spme-GC/MS. Journal of Chemical Ecology 2005, 31 (7), 1607-1619.

39. Mebazaa, R.; Mahmoudi, A.; Rega, B.; Ben Cheikh, R.; Camel, V. Analysis of human male armpit sweat after fenugreek ingestion: Instrumental and sensory optimisation of the extraction method. Food Chemistry 2010, 120 (3), 771-782.

40. Curran, A. M.; Prada, P. A.; Furton, K. G. The Differentiation of the Volatile Organic Signatures of Individuals Through SPME-GC/MS of Characteristic Human Scent Compounds. Journal of Forensic Sciences 2010, 55 (1), 50-57.

41. Dormont, L.; Bessière, J. M.; McKey, D.; Cohuet, A. New methods for field collection of human skin volatiles and perspectives for their application in the chemical ecology of human-pathogen-vector interactions. Journal of Experimental Biology 2013, 216 (15), 2783-2788.

42. Logan, J.; Birkett, M.; Clark, S.; Powers, S.; Seal, N.; Wadhams, L.; Mordue, L.; Pickett, J. Identification of Human-Derived Volatile Chemicals that Interfere with Attraction of Aedes aegypti Mosquitoes. J Chem Ecol 2008, 34 (3), 308-322.

43. Natsch, A.; Gfeller, H.; Gygax, P.; Schmid, J.; Acuna, G. A specific bacterial aminoacylase cleaves odorant precursors secreted in the human axilla. Journal of Biological Chemistry 2003, 278 (8), 5718-5727.

44. James, A. G.; Casey, J.; Hyliands, D.; Mycock, G. Fatty acid metabolism by cutaneous bacteria and its role in axillary malodour. World Journal of Microbiology and Biotechnology 2004, 20 (8), 787-793.

45. Austin, C.; Ellis, J. Microbial pathways leading to steroidal malodour in the axilla. The Journal of Steroid Biochemistry and Molecular Biology 2003, 87 (1), 105-110.

46. Wedekind, C.; Penn, D. MHC genes, body odours, and odour preferences. Nephrology Dialysis Transplantation 2000, 15 (9), 1269-1271.

47. Jain, A. K.; Li, S. Z.; Li, S. Z.; SpringerLink (Online service) Encyclopedia of biometrics; Springer: New York, 2009.

48. Penn, D.; Potts, W. How Do Major Histocompatibility Complex Genes Influence Odor and Mating Preferences?*. In Advances in Immunology, Volume 69 ed.; Frank, J. D., Ed.; Academic Press: 1998; pp 411-436.

49. Wedekind, C.; Seebeck, T.; Bettens, F.; Paepke, A. J. MHC-Dependent Mate Preferences in Humans. Proceedings of the Royal Society of London B: 
Biological Sciences 1995, 260 (1359), 245-249.

50. Wedekind, C.; Füri, S. Body odour preferences in men and women: do they aim for specific MHC combinations or simply heterozygosity? Proc Biol Sci 1997, 264 (1387), 1471-1479.

51. Herz, R.; Cahill, E. Differential use of sensory information in sexual behavior as a function of gender. Hum Nat 1997, 8 (3), 275-286.

52. Strimbu, K.; Tavel, J. A. What are Biomarkers? Curr Opin HIV AIDS 2010, 5 (6), 463-466.

53. Manolis, A. The diagnostic potential of breath analysis. Clinical Chemistry 1983, 29 (1), 5-15.

54. Willis, C. M.; Church, S. M.; Guest, C. M.; Cook, W. A.; McCarthy, N.; Bransbury, A. J.; Church, M. R. T.; Church, J. C. T. Olfactory detection of human bladder cancer by dogs: proof of principle study. BMJ 2004, 329 (7468), 712.

55. Syhre, M.; Manning, L.; Phuanukoonnon, S.; Harino, P.; Chambers, S. T. The scent of Mycobacterium tuberculosis - Part II breath. Tuberculosis 2009, 89 (4), 263-266.

56. Buljubasic, F.; Buchbauer, G. The scent of human diseases: a review on specific volatile organic compounds as diagnostic biomarkers. Flavour Fragr. J. 2015, 30 (1), 5-25.

57. Biomarkers Definitions Working Group Biomarkers and surrogate endpoints: Preferred definitions and conceptual framework. Clinical Pharmacology \& Therapeutics 2001, 69 (3), 89-95.

58. Havlicek, J.; Lenochova, P. The Effect of Meat Consumption on Body Odor Attractiveness. Chemical Senses 2006, 31 (8), 747-752.

59. Wallace, P. Individual discrimination of humans by odor. Physiology \& Behavior 1977, 19 (4), 577-579.

60. Hepper, P. G. The Discrimination of Human Odor by the Dog. Perception 1988, 17 (4), 549-554.

61. Hudson, D. T. Variables Affecting the Collection and Preservation of Human Scent Components through Instrumental and Biological Evaluations. Florida International University, 2009.

62. Pinc, L.; Bartos, L.; Reslova, A.; Kotrba, R. Dogs Discriminate Identical Twins. 
Plos One 2011, 6 (6).

63. Hurst, J.; Beynon, R. J.; Roberts, S. C.; Wyatt, T. Chemical Signals in Vertebrates 11; Springer: 2007.

64. Roberts, S. C.; Gosling, L. M.; Spector, T. D.; Miller, P.; Penn, D. J.; Petrie, M. Body Odor Similarity in Noncohabiting Twins. Chemical Senses 2005, 30 (8), 651-656.

65. Yoshiura, K. e. al. A SNP in the ABCC11 gene is the determinant of human earwax type. Nature Genetics 2006, 38 (3).

66. Matsunaga, E. The dimorphism in human normal cerumen. Annals of Human Genetics 1962, 25 (4), 273-286.

67. Prokop-Prigge, K. A.; Thaler, E.; Wysocki, C. J.; Preti, G. Identification of volatile organic compounds in human cerumen. Journal of Chromatography B 2014, 953-954 (0), 48-52.

68. Hart, R. Human Body Odor. Nexus 1980, 1 (1).

69. Hurley, H. J. a. S. W. B. The Human Apocrine Sweat Gland in Health and Disease; Charles C Thomas: Springfield, 1960.

70. Bates, L. A.; Sayialel, K. N.; Njiraini, N. W.; Moss, C. J.; Poole, J. H.; Byrne, R. W. Elephants classify human ethnic groups by odor and garment color. Current Biology 2007, 17 (22), 1938-1942.

71. Mitro, S.; Gordon, A. R.; Olsson, M. J.; Lundstrom, J. N. The Smell of Age: Perception and Discrimination of Body Odors of Different Ages. Plos One 2012, 7 (5).

72. Haze, S.; Gozu, Y.; Nakamura, S.; Kohno, Y.; Sawano, K.; Ohta, H.; Yamazaki, K. 2-Nonenal newly found in human body odor tends to increase with aging. Journal of Investigative Dermatology 2001, 116 (4), 520-524.

73. Asano, K. G.; Bayne, C. K.; Horsman, K. M.; Buchanan, M. V. Chemical composition of fingerprints for gender determination. Journal of Forensic Sciences 2002, 47 (4), 805-807.

74. Stockham, R.; Slavin, D.; Kift, W. Specialized Use of Human Scent in Criminal Investigations. Forensic Science Communications, 2004.

75. Brown, J. S. Determination of Signature Volatile Odor Chemicals Emanating from Novel Biological Specimens by Non-invasive Analytical Techniques for the 
Potential Use in Forensic Identifications . Florida International University, 2012.

76. Pandey, S. K.; Kim, K. H. Human body-odor components and their determination. TrAC Trends in Analytical Chemistry 2011, 30 (5), 784-796.

77. Ras, M. R.; Borrull, F.; Marcé, R. M. Sampling and preconcentration techniques for determination of volatile organic compounds in air samples. TrAC Trends in Analytical Chemistry 2009, 28 (3), 347-361.

78. Scheppers Wercinski, S. A. Solid Phase Microextraction : A Practical Guide; CRC Press: New York, 1999.

79. Demeestere, K.; Dewulf, J.; De Witte, B.; Van Langenhove, H. Sample preparation for the analysis of volatile organic compounds in air and water matrices. Journal of Chromatography A 2007, 1153 (1-2), 130-144.

80. Wade, L. G. Organic Chemistry; 6 ed.; Pearson Prentice Hall: NJ, 2006.

81. Sample Preparation; Nova Science Publishers, Inc.: NY, 2007.

82. Olivas, R. M. o.; Cámara, C.; Bouyssiere, B.; Szpunar, J.; Potin-Gautier, M.; Lobinski, R.; Hlavay, J.; Polyák, K. Sample Preparation. In Handbook of Elemental Speciation: Techniques and Methodology, John Wiley \& Sons, Ltd: 2003; pp 73-146.

83. Ramirez, N.; Cuadras, A.; Rovira, E.; Borrull, F.; Marcé, R. M. Comparative study of solvent extraction and thermal desorption methods for determining a wide range of volatile organic compounds in ambient air. Talanta 2010, 82 (2), 719-727.

84. Dormont, L.; Bessière, J. M.; Cohuet, A. Human Skin Volatiles: A Review. J Chem Ecol 2013, 39 (5), 569-578.

85. Ara, K.; Hama, M.; Akiba, S.; Koike, K.; Okisaka, K.; Hagura, T.; Kamiya, T.; Tomita, F. Foot odor due to microbial metabolism and its control. Canadian Journal of Microbiology 2006, 52 (4), 357-364.

86. Vas, G.; Vekey, K. Solid-phase microextraction: a powerful sample preparation tool prior to mass spectrometric analysis. Journal of Mass Spectrometry 2004, 39 (3), 233-254.

87. Mindrup, R.; Shirey, R. Improved Performance of SPME Fibers and Applications. 2001. Bellefonte, PA, Sigma-Aldrich Co.

88. Chai, M.; Pawliszyn, J. Analysis of Environmental Air Samples by Solid-Phase 
Microextraction and Gas Chromatography/Ion Trap Mass Spectrometry. Environ. Sci. Technol. 1995, 29 (3), 693-701.

89. Zhang, Z.; Pawliszyn, J. Analysis of organic compounds in environmental samples by headspace solid phase microextraction. J. High Resol. Chromatogr. 1993, 16 (12), 689-692.

90. Yang, X.; Peppard, T. Solid-Phase Microextraction for Flavor Analysis. J. Agric. Food Chem. 1994, 42 (9), 1925-1930.

91. Wang, Y.; Yang, C.; Li, S.; Yang, L.; Wang, Y.; Zhao, J.; Jiang, Q. Volatile characteristics of 50 peaches and nectarines evaluated by HP-SPME with GC-MS. Food Chemistry 2009, 116 (1), 356-364.

92. Fucci, N.; De Giovanni, N.; Chiarotti, M. Simultaneous detection of some drugs of abuse in saliva samples by SPME technique. Forensic Science International 2003, 134 (1), 40-45.

93. Junting, L.; Peng, C.; Suzuki, O. Solid-phase microextraction (SPME) of drugs and poisons from biological samples. Forensic Science International 1998, 97 (23), 93-100.

94. Mebazaa, R.; Rega, B.; Camel, V. r. Analysis of human male armpit sweat after fenugreek ingestion: Characterisation of odour active compounds by gas chromatography coupled to mass spectrometry and olfactometry. Food Chemistry 2011, 128 (1), 227-235.

95. Sparkman, O. D.; Penton, Z.; Kitson, F. G. Gas Chromatography and Mass Spectrometry : A Practical Guide (2nd Edition); Academic Press: Saint Louis, MO, USA, 2011.

96. Skoog, D. A.; Crouch, S. R.; Holler, F. J. Principles of instrumental analysis; 6th ed. / Douglas A. Skoog, F. James Holler, Stanley R. Crouch. ed.; Thomson Brooks/Cole: Belmont, CA, 2007.

97. GC Columns Stationary Phase Applications Guide. 2015. Agilent Technologies.

98. Leone, A. M.; Gustafsson, L. E.; Francis, P. L.; Persson, M. G.; Wiklund, N. P.; Moncada, S. Nitric Oxide Is Present in Exhaled Breath in Humans: Direct GC-MS Confirmation. Biochemical and Biophysical Research Communications 1994, 201 (2), 883-887.

99. Pennsylvania v. Michaux, 360 Pa.Super 452, 456, 520 A.2d 1177, 1178 (Pa.Supr.Ct. 1987). 
100. State v. Iverson, 187 N.W.2d 1 (N.D. 1971).

101. Cranford v. State, 130 Ark. 101 (Ark. 1917).

102. State v. Wallace, 181 Conn. 237 (Conn. 1980).

103. Prada, P. a.; Curran, A. M.; Furton, K. G. Human scent evidence; CRC Press/Taylor \& Francis Group: Boca Raton, 2015.

104. Hodge v. State, 98 Ala. 10 (Ala. 1893).

105. State v. Hall, 4 Ohio Dec. 147, 148 (Ohio Misc. 1896).

106. Pedigo v. Commonwealth, 103 Ky. 41 (Ky. 1898).

107. California v. Salcido, GA052057 (Los Angeles Superior Court, March 11, 2005). 2005. Los Angeles Superior Court.

108. ABC Good Morning America . DNA May Change Killer profile. 2012. 10-10012 .

109. Fullwiley, D. Can DNA "Witness" Race? Council for Responsible Genetics . 2012. 10-1-0012.

110. Hasegawa, Y.; Yabuki, M.; Matsukane, M. Identification of new odoriferous compounds in human axillary sweat. Chemistry \& Biodiversity 2004, 1 (12), 2042-2050.

111. Zeng, X. N.; Leyden, J.; Brand, J.; Spielman, A.; McGinley, K.; Preti, G. An investigation of human apocrine gland secretion for axillary odor precursors. J Chem Ecol 1992, 18 (7), 1039-1055.

112. Akutsu, T.; Sekiguchi, K.; Ohmori, T.; Sakurada, K. Individual comparisons of the levels of (E)-3-methyl-2-hexenoic acid, an axillary odor-related compound, in Japanese. Chemical Senses 2006, 31 (6), 557-563.

113. Caraballo, N. I. Identification of Characteristic Volatile Organic Compounds Released during the Decomposition Process of Human Remains and Analogues. Florida International University, 2014.

114. DeGreeff, L. E. Development of a Dynamic Headspace Concentration Technique for the Non-Contact Sampling of Human Odor Samples and the Creation of Canine Training Aids. Florida International University, 2010.

115. Prada, P. A.; Curran, A. M.; Furton, K. G. Comparison of extraction methods for 
the removal of volatile organic compounds (VOCs) present in sorbents used for human scent evidence collection. Analytical Methods 2010, 2 (5), 470-478.

116. Prada, P. A.; Curran, A. M.; Furton, K. G. The Evaluation of Human Hand Odor Volatiles on Various Textiles: A Comparison Between Contact and Noncontact Sampling Methods. Journal of Forensic Sciences 2011, 56 (4), 866-881.

117. Miller J.N.; Miller J.C. Statistics and Chemometrics for Analytical Chemistry; 5th Edition ed.; Pearson Education Limited: 2005.

118. Lowry, R. Concepts and Applications of Inferential Statistics; 1998.

119. Compare Means: All Pairs Tukey HSD. 2015. SAS Institute Inc.

120. Lehman, A. JMP for Basic Univariate and Multivariate Statistics : A Step-by-step Guide; SAS Press: Cary, NC, 2005.

121. The Discriminant Analysis Report. 2015. SAS Institute Inc.

122. International Fragrance Association . Ingredients. 2015. International Fragrance Association.

123. Bernier, U. R.; Kline, D. L.; Barnard, D. R.; Schreck, C. E.; Yost, R. A. Analysis of Human Skin Emanations by Gas Chromatography/Mass Spectrometry. 2. Identification of Volatile Compounds That Are Candidate Attractants for the Yellow Fever Mosquito (Aedes aegypti). Anal. Chem. 2000, 72 (4), 747-756.

124. Labows, J.; Preti, G.; Hoelzle, E.; Leyden, J.; Kligman, A. Analysis of Human Axillary Volatiles - Compounds of Exogenous Origin. Journal of Chromatography 1979, 163 (3), 294-299.

125. Chemical Book . Amylcinnamaldehyde (122-40-7). 2008. 11-21-2015.

126. Chemical Book . 2,4-Diisopropylphenol (2934-05-6). 2008. 11-21-2015.

127. Chemical Book . Galaxolide (1222-05-5). 2008. 11-21-2015.

128. Hexyl salicylate. 2008. 11-21-2015.

129. Chemical Book . Trans-2-Dodecenal. 2008. 11-21-2015.

130. National Institute of Standards and Technology . 4-Methyl Hexadecane. 2011. 11-21-0015.

131. The Good Scents Company . 4-Methyl-Hexadecane. 2015. 11-21-2015. 
132. National Institute of Standards and Technology . Galaxolide. 2011. 11-21-2015.

133. National Institute of Standards and Technology . n-Hexyl salicylate. 2011. 11-212015.

134. ALS Environmental . Homomenthyl salicylate. 2014. 11-21-2015.

135. PubChem Compound Database . Homosalate. 2015. National Center for Biotechnology Information. 11-21-2015.

136. Chemical Rubber Company CRC handbook of chemistry and physics; Electronic ed. ed.; CRC Press : Taylor \& Francis: Boca Raton, Fla, 1978. 
8.

Appendix 1: Flyer used in the recruitment of 35-50 year old Caucasian individuals

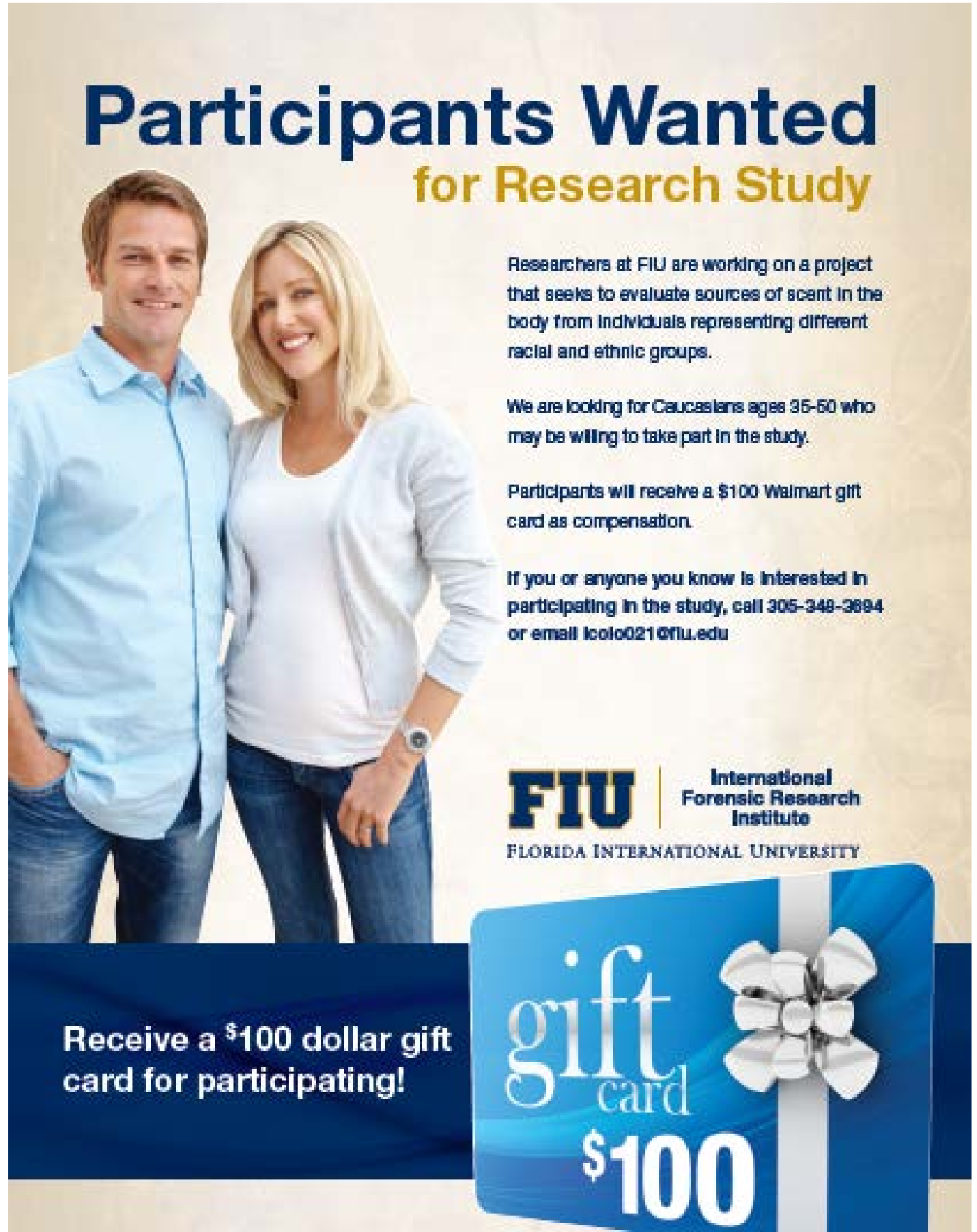


Appendix 2: Flyer used in the recruitment of 55+ year old Caucasian individuals

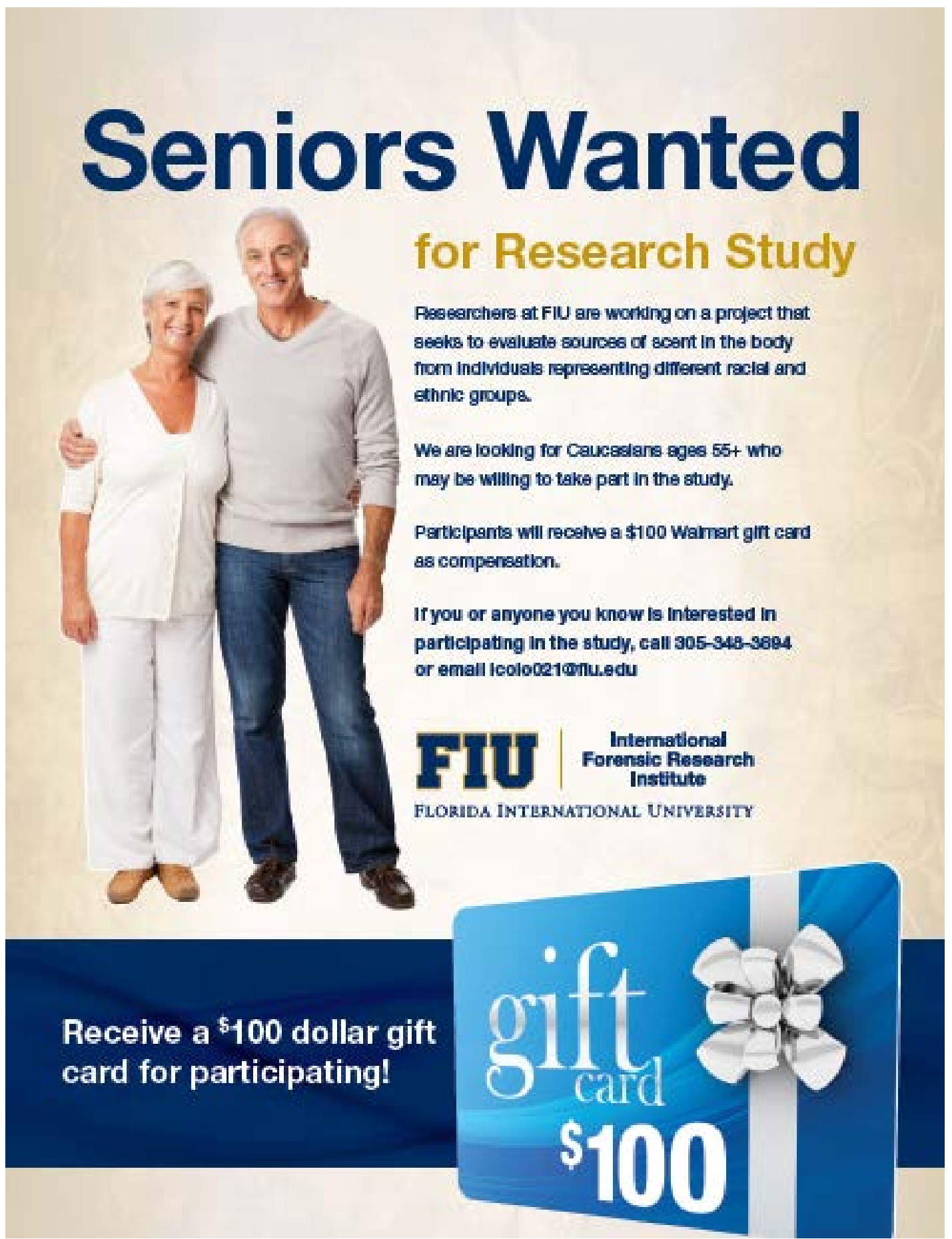




\section{Appendix 3: Flyer used in the recruitment of Hispanic individuals}

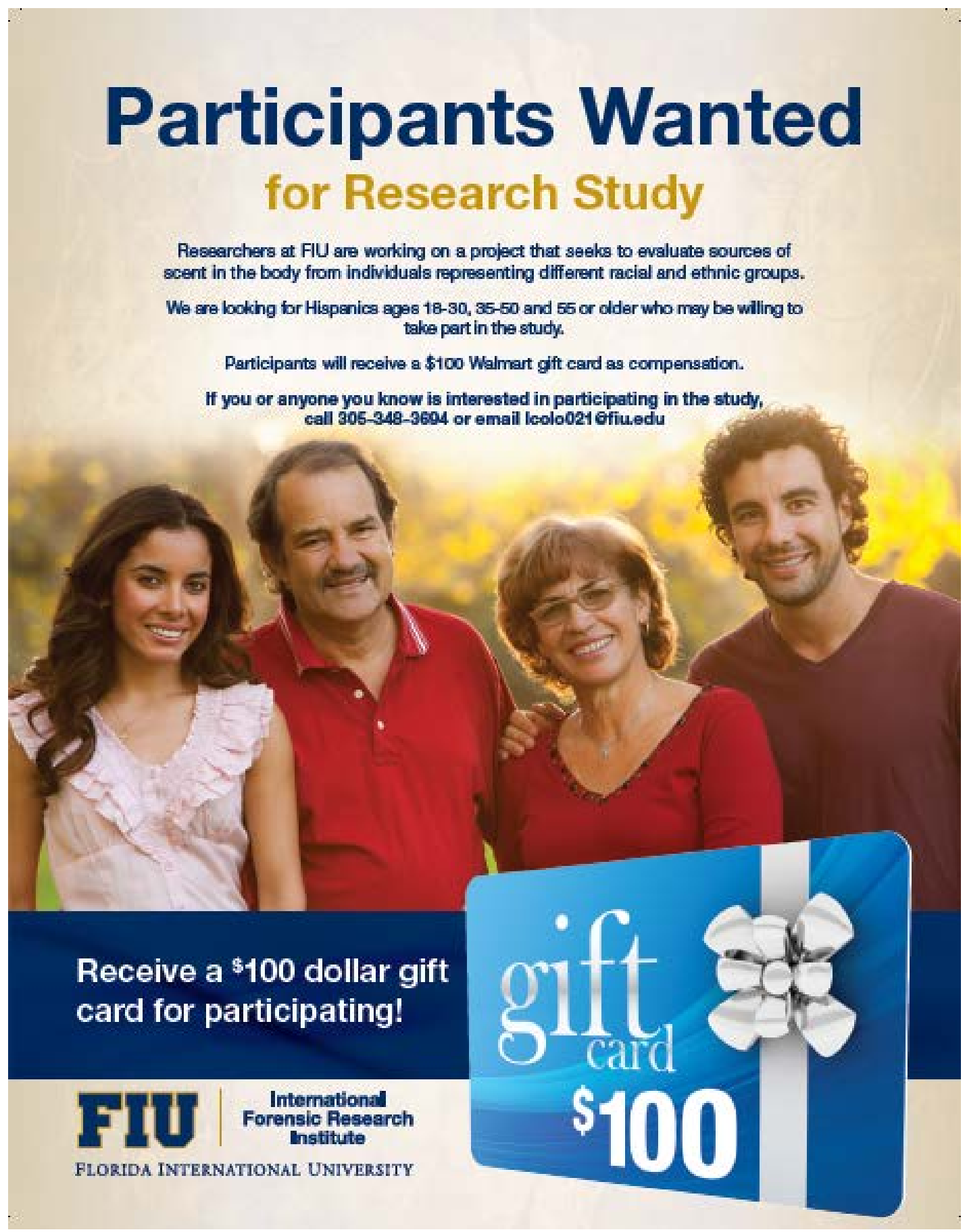


Appendix 4: Flyer used in the recruitment of East Asian individuals

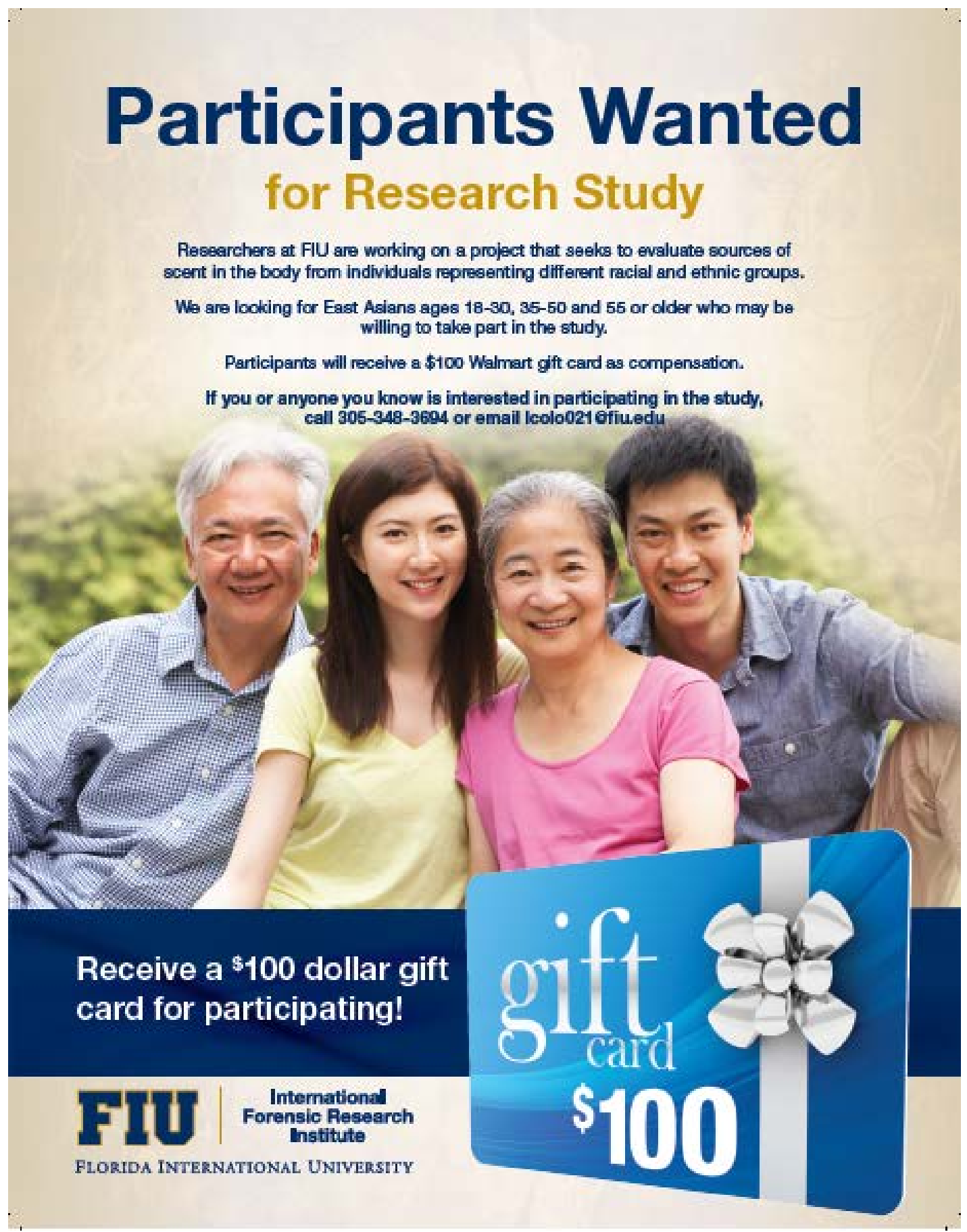




\section{Appendix 5: List of VOCs considered in Optimization Studies}

\begin{tabular}{|c|c|c|c|c|c|c|c|c|c|c|}
\hline \multicolumn{6}{|c|}{ VOC properties and information } & \multicolumn{5}{|c|}{ VOC List per optimization study } \\
\hline $\begin{array}{l}\text { Functional } \\
\text { Group }\end{array}$ & Compound Name & $\begin{array}{c}\text { CAS } \\
\text { Number }\end{array}$ & $\begin{array}{c}\text { Molecular } \\
\text { weight } \\
\text { (g/mol) }\end{array}$ & $\begin{array}{c}\text { Boiling Point } \\
\left({ }^{\circ} \mathrm{C}\right)\end{array}$ & $\begin{array}{c}\text { Literature } \\
\text { Review } \\
\text { Reference }\end{array}$ & $\begin{array}{c}\text { GC-MS } \\
\text { Column } \\
\text { and } \\
\text { Method }\end{array}$ & $\begin{array}{l}\text { HS-SPME } \\
\text { Fiber }\end{array}$ & $\begin{array}{c}\text { HS-SPME } \\
\text { Extraction } \\
\text { Time }\end{array}$ & $\begin{array}{c}\text { HS-SPME } \\
\text { Extraction } \\
\text { Temperature }\end{array}$ & $\begin{array}{c}\text { Underarm } \\
\text { Sampling } \\
\text { Method }\end{array}$ \\
\hline Haloalkane & 1-Chloro-nonane & $2473-01-0$ & 162.7 & $204(3)$ & 38 & $\mathrm{X}$ & $\mathrm{X}$ & $\mathrm{X}$ & $\mathrm{X}$ & \\
\hline Alcohol & 1-Hexadecanol & $36653-82-4$ & 242.44 & $325(2)$ & 36 & $\mathrm{X}$ & $\mathrm{X}$ & & $\mathrm{X}$ & $\mathrm{X}$ \\
\hline $\begin{array}{c}\text { Alcohol, Cyclic } \\
\text { ether }\end{array}$ & 2-Furanmethanol & $98-00-0$ & 98.101 & $168(2)$ & 38 & $\mathrm{X}$ & $\mathrm{X}$ & $\mathrm{X}$ & $\mathrm{X}$ & $\mathrm{X}$ \\
\hline Ketone & Benzophenone & 119-61-9 & 182.217 & $305.9(0.2)$ & 94 & $\mathrm{X}$ & $\mathrm{X}$ & $\mathrm{X}$ & $\mathrm{X}$ & $\mathrm{X}$ \\
\hline Aldehyde & Decanal & $112-31-2$ & 156.265 & $212(3)$ & 8 & $\mathrm{X}$ & $\mathrm{X}$ & $\mathrm{X}$ & $\mathrm{X}$ & $\mathrm{X}$ \\
\hline Sulfide & Dimethyl trisulfide & $3658-80-8$ & 126.264 & $41^{6}$ & 113 & $\mathrm{X}$ & $\mathrm{X}$ & $\mathrm{X}$ & & \\
\hline Alkane & Heptadecane & $629-78-7$ & 240.468 & $303(2)$ & 8 & $\mathrm{X}$ & $\mathrm{X}$ & $\mathrm{X}$ & $\mathrm{X}$ & $\mathrm{X}$ \\
\hline Ester & Methyl palmitate & $112-39-0$ & 270.451 & 417 & 8,36 & $\mathrm{X}$ & $\mathrm{X}$ & & $\mathrm{X}$ & $\mathrm{X}$ \\
\hline Ester & Methyl tridecanoate & 1731-88-0 & 228.371 & $92^{1}$ & 8 & $\mathrm{X}$ & $\mathrm{X}$ & $\mathrm{X}$ & & $\mathrm{X}$ \\
\hline Aromatic & m-Xylene & $108-38-3$ & 106.165 & $139.1(0.4)$ & 94 & $\mathrm{X}$ & $\mathrm{X}$ & $\mathrm{X}$ & $\mathrm{X}$ & \\
\hline Aromatic & Naphthalene & $91-20-3$ & 128.171 & $218.0(0.1)$ & 8 & $\mathrm{X}$ & $\mathrm{X}$ & $\mathrm{X}$ & $\mathrm{X}$ & \\
\hline Aromatic & Toluene & $108-88-3$ & 92.139 & $110.60(0.07)$ & 8 & $\mathrm{X}$ & $\mathrm{X}$ & $\mathrm{X}$ & $\mathrm{X}$ & \\
\hline Carboxylic acid & Lactic acid & $10326-41-7$ & 90.078 & $103^{2}$ & 94 & $\mathrm{X}$ & & & & \\
\hline Carboxylic acid & $\begin{array}{l}\text { Cyclohexanecarboxylic } \\
\text { acid }\end{array}$ & $98-89-5$ & 128.169 & $233(6)$ & 36 & $\mathrm{X}$ & $\mathrm{X}$ & $\mathrm{X}$ & & \\
\hline Cyclic ester & Y-Nonalactone & $104-61-0$ & 156.222 & $134^{12}$ & 36 & $\mathrm{X}$ & & & & \\
\hline Ester & Methyl caprylate & $111-11-5$ & 158.238 & $194.1(0.9)$ & 8 & $\mathrm{X}$ & $\mathrm{X}$ & $\mathrm{X}$ & & \\
\hline Alkenes & Squalene & $111-02-4$ & 410.718 & 421.3 & 94 & $\mathrm{X}$ & & & $\mathrm{X}$ & \\
\hline Amine & Pyridine & $110-86-1$ & 79.101 & $115.2(0.1)$ & 8 & $\mathrm{X}$ & $\mathrm{X}$ & $\mathrm{X}$ & & \\
\hline Ketone & 2-pentanone & $107-87-9$ & 86.132 & $102.2(0.1)$ & 94 & $\mathrm{X}$ & & & & \\
\hline Sulfide & Dimethyl sulfide & $75-18-3$ & 62.134 & $37.32(0.05)$ & 113 & $\mathrm{X}$ & & $\mathrm{X}$ & & \\
\hline Carboxylic acid & Undecanoic acid & $112-37-8$ & 186.292 & 280 & $36,110,111$ & $\mathrm{X}$ & $\mathrm{X}$ & & & \\
\hline Cyclic ester & Y-Decalactone & 706-14-9 & 170.249 & $301(8)$ & 110 & $\mathrm{X}$ & & & & \\
\hline Alcohol & 1-Undecanol & $112-42-5$ & 172.308 & $246(2)$ & 110 & $\mathrm{X}$ & $\mathrm{X}$ & $\mathrm{X}$ & $\mathrm{X}$ & \\
\hline Aldehyde & 3-Methylbutanal & $590-86-3$ & 86.132 & $92.5(0.3)$ & 94 & $\mathrm{X}$ & & & & \\
\hline Aldehyde & Citral & $141-27-5$ & 152.233 & 229 & 94 & $\mathrm{X}$ & $\mathrm{X}$ & $\mathrm{X}$ & $\mathrm{X}$ & $\mathrm{X}$ \\
\hline Aldehyde & Hexanal & 66-25-1 & 100.158 & $129.6(0.4)$ & 8 & $\mathrm{X}$ & $\mathrm{X}$ & $\mathrm{X}$ & & \\
\hline Sulfonyl & Dimethyl sulfone & 67-71-0 & 94.133 & $238(5)$ & 36 & $\mathrm{X}$ & $\mathrm{X}$ & & $\mathrm{X}$ & \\
\hline
\end{tabular}

* Superscripts indicate the pressure (in $\mathrm{mmHg}$ ) for boiling points not available at normal conditions

** Parentheses indicate combined expanded uncertainties (level of confidence, approximately $95 \%$ ) as listed in the CRC Handbook of Chemistry and Physics ${ }^{76}$ 


\section{Appendix 6: Tailored VOC list created in Pilot Study ${ }^{125-136}$}

\begin{tabular}{|c|c|c|c|c|c|}
\hline \multicolumn{6}{|c|}{ Tailored VOC list created in Pilot Study } \\
\hline Functional Group & Compound Name & CAS Number & $\begin{array}{c}\text { Molecular weight } \\
\text { (g/mol) }\end{array}$ & $\begin{array}{c}\text { Boiling Point } \\
\left({ }^{\circ} \mathrm{C}\right)\end{array}$ & LOD (ng) \\
\hline Haloalkane & 1-Chloro-nonane & $2473-01-0$ & 162.7 & $204(3)$ & 2.35 \\
\hline Alkene & 1-Heptadecene & $6765-39-5$ & 238.452 & $301(3)$ & 2.25 \\
\hline Alcohol & 1-Hexadecanol & $36653-82-4$ & 242.44 & $325(2)$ & 2.81 \\
\hline Alkene & 1-Octadecene & $112-88-9$ & 252.479 & $179^{15}$ & 1.96 \\
\hline Alcohol & 2,4-Diisopropylphenol & 2934-05-6 & 178.27 & $79-80$ & 2.51 \\
\hline Alcohol, Cyclic ether & 2-Furanmethanol & $98-00-0$ & 98.101 & $168(2)$ & 7.03 \\
\hline Ketone & 2-Tridecanone & $593-08-8$ & 198.344 & $268(1)$ & 4.41 \\
\hline Alkane & 4-Methyl-hexadecane & $25117-26-4$ & 240.468 & 296.3 & 2.48 \\
\hline Aldehyde & Alpha-Amylcinnamaldehyde & $122-40-7$ & 202.29 & $287-290$ & 2.60 \\
\hline Aldehyde & Alpha-Hexylcinnamaldehyde & $101-86-0$ & 216.319 & 252 & 2.98 \\
\hline Ketone & Benzophenone & $119-61-9$ & 182.217 & $305.9(0.2)$ & 3.10 \\
\hline Cyclic alkane & Cyclododecane & $294-62-2$ & 168.319 & $244.0(0.5)$ & 2.58 \\
\hline Aldehyde & Decanal & $112-31-2$ & 156.265 & $212(3)$ & 4.50 \\
\hline Ether & Diethylene glycol monoethyl ether & $111-90-0$ & 134.173 & $202(3)$ & 0.87 \\
\hline Sulfonyl & Dimethyl sulfone & $67-71-0$ & 94.133 & $238(5)$ & 2.26 \\
\hline Sulfonyl & Dimethyl trisulfide & $3658-80-8$ & 126.264 & $41^{6}$ & 3.18 \\
\hline Ether & Dioctyl ether & $629-82-3$ & 242.440 & $289(3)$ & 1.98 \\
\hline Carboxylic Acid & Dodecanoic acid & $143-07-7$ & 200.318 & $225^{100}$ & 8.92 \\
\hline Ether & Ethylene glycol monododecyl ether & $4536-30-5$ & 230.387 & $143^{0.8}$ & 3.40 \\
\hline Cyclic Ether & Galaxolide & $1222-05-5$ & 258.398 & 304 & 6.94 \\
\hline Alkane & Heptacosane & $593-49-7$ & 380.734 & 442 & 4.23 \\
\hline Alkane & Heptadecane & $629-78-7$ & 240.468 & $303(2)$ & 2.03 \\
\hline Ester & Hexyl salicylate & $6259-76-3$ & 222.280 & 290 & 3.10 \\
\hline Ester & Homomenthyl Salicylate & 52253-93-7 & 262.344 & $322-329$ & 2.88 \\
\hline
\end{tabular}




\begin{tabular}{|c|c|c|c|c|c|}
\hline Functional Group & Compound Name & CAS Number & $\begin{array}{c}\text { Molecular weight } \\
(\mathbf{g} / \mathbf{m o l})\end{array}$ & $\begin{array}{c}\text { Boiling Point } \\
\left({ }^{\circ} \mathbf{C}\right)\end{array}$ & LOD (ng) \\
\hline Ester & Isoamyl salicylate & $87-20-7$ & 208.253 & 278 & 2.72 \\
\hline Ester & Isopropyl Myristate & $110-27-0$ & 270.451 & $193^{20}$ & 2.33 \\
\hline Ester & Isopropyl palmitate & $142-91-6$ & 298.504 & $160^{2}$ & 2.69 \\
\hline Ester & Methyl laurate & $111-82-0$ & 214.344 & $268(2)$ & 2.35 \\
\hline Ester & Methyl myristate & $124-10-7$ & 242.398 & $295(10)$ & 2.21 \\
\hline Ester & Methyl palmitate & $112-39-0$ & 270.451 & 417 & 2.45 \\
\hline Ester & Methyl tridecanoate & $1731-88-0$ & 228.371 & $92^{1}$ & 2.53 \\
\hline Aromatic & m-Xylene & $108-38-3$ & 106.165 & $139.1(0.4)$ & 9.89 \\
\hline Aromatic & Naphthalene & $91-20-3$ & 128.171 & $218.0(0.1)$ & 2.14 \\
\hline Aldehyde & Nonanal & $124-19-6$ & 142.238 & $195(3)$ & 5.02 \\
\hline Alkane & Octadecane & $593-45-3$ & 254.495 & $316(2)$ & 2.04 \\
\hline Alkane & Pentadecane & $629-62-9$ & 212.415 & $270.6(0.4)$ & 5.47 \\
\hline Cyclic Ester & Pentadecanolide & $106-02-5$ & 240.382 & $176^{15}$ & 2.54 \\
\hline Alkane & Pristane & $1921-70-6$ & 268.521 & $306(6)$ & 2.15 \\
\hline Aldehyde & Trans-2-Dodecenal & $20407-84-5$ & 182.30 & $93^{0.5}$ & 4.88 \\
\hline Ketone & Trans-Beta-Ionone & $79-77-6$ & 192.297 & 124 & 3.85 \\
\hline
\end{tabular}

*Note: Despite the listed LODs, compounds were often detected down to $0.50 \mathrm{ng}$ amounts. 
Appendix 7: Results obtained from ANOVA and Tukey Tests performed on the average underarm VOC amounts across all populations under study

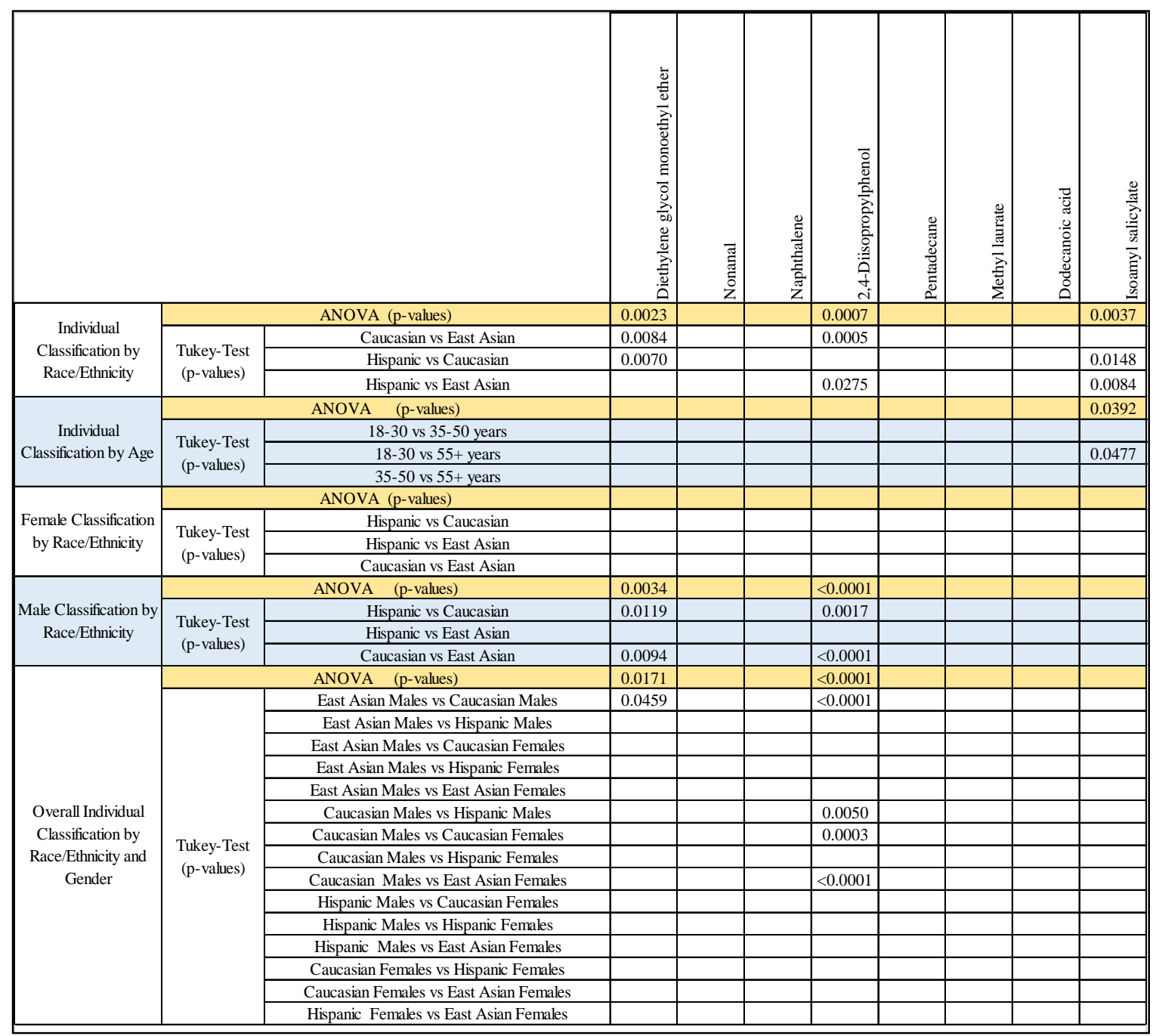




\begin{tabular}{|c|c|c|c|c|c|c|c|c|c|c|}
\hline & & & 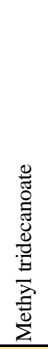 & 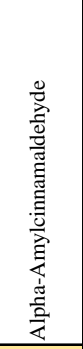 & 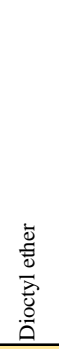 & 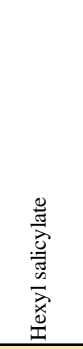 & 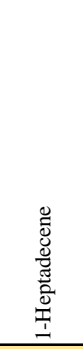 & 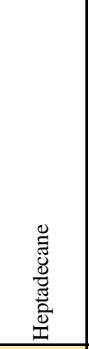 & 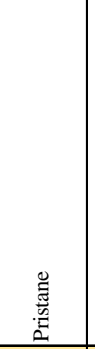 & 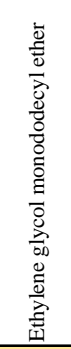 \\
\hline \multirow{4}{*}{$\begin{array}{c}\text { Individual } \\
\text { Classification by } \\
\text { Race/Ethnicity }\end{array}$} & \multirow{4}{*}{$\begin{array}{c}\text { Tukey-Test } \\
\text { (p-values) }\end{array}$} & ANOVA (p-values) & & & & & & $<0.0001$ & $<0.0001$ & \\
\hline & & Caucasian vs East Asian & & & & & & $<0.0001$ & $<0.0001$ & \\
\hline & & Hispanic vs Caucasian & & & & & & & \begin{tabular}{l|l}
0.0342 \\
\end{tabular} & \\
\hline & & Hispanic vs East Asian & & & & & & $<0.0001$ & 0.0121 & \\
\hline \multirow{4}{*}{$\begin{array}{c}\text { Individual } \\
\text { Classification by Age }\end{array}$} & \multirow{4}{*}{$\begin{array}{l}\text { Tukey-Test } \\
\text { (p-values) }\end{array}$} & ANOVA $\quad$ (p-values) & & & & 0.0156 & 0.0091 & & & 0.0252 \\
\hline & & $18-30$ vs $35-50$ years & & & & & 0.0131 & & & \\
\hline & & $18-30$ vs $55+$ years & & & & 0.0169 & & & & 0.0187 \\
\hline & & $35-50$ vs $55+$ years & & & & & 0.0394 & & & \\
\hline \multirow{4}{*}{$\begin{array}{c}\text { Female Classification } \\
\text { by Race/Ethnicity }\end{array}$} & \multirow{4}{*}{$\begin{array}{l}\text { Tukey-Test } \\
\text { (p-values) }\end{array}$} & ANOVA (p-values) & & 0.0016 & & & & $<0.0001$ & & \\
\hline & & Hispanic vs Caucasian & & 0.0028 & & & & & & \\
\hline & & Hispanic vs East Asian & & 0.0119 & & & & 0.0013 & & \\
\hline & & Caucasian vs East Asian & & & & & & & & \\
\hline \multirow{4}{*}{$\begin{array}{l}\text { Male Classification by } \\
\text { Race/Ethnicity }\end{array}$} & \multirow{4}{*}{$\begin{array}{l}\text { Tukey-Test } \\
\text { (p-values) }\end{array}$} & ANOVA (p-values) & & & & & & $<0.0001$ & $<0.0001$ & \\
\hline & & Hispanic vs Caucasian & & & & & & 0.0124 & \begin{tabular}{l|l}
0.0038 \\
\end{tabular} & \\
\hline & & Hispanic vs East Asian & & & & & & 0.0275 & & \\
\hline & & Caucasian vs East Asian & & & & & & $<0.0001$ & $<0.0001$ & \\
\hline \multirow{16}{*}{$\begin{array}{l}\text { Overall Individual } \\
\text { Classification by } \\
\text { Race/Ethnicity and } \\
\text { Gender }\end{array}$} & \multirow{16}{*}{$\begin{array}{l}\text { Tukey-Test } \\
\text { (p-values) }\end{array}$} & ANOVA $\quad$ (p-values) & & 0.0016 & & & & $<0.0001$ & $<0.0001$ & \\
\hline & & East Asian Males vs Caucasian Males & & & & & & $<0.0001$ & $<0.0001$ & \\
\hline & & East Asian Males vs Hispanic Males & & & & & & & & \\
\hline & & East Asian Males vs Caucasian Females & & & & & & & 0.0043 & \\
\hline & & East Asian Males vs Hispanic Females & & 0.0092 & & & & 0.0005 & 0.0045 & \\
\hline & & East Asian Males vs East Asian Females & & & & & & & & \\
\hline & & Caucasian Males vs Hispanic Males & & & & & & & \begin{tabular}{l|l}
0.0055 \\
\end{tabular} & \\
\hline & & Caucasian Males vs Caucasian Females & & & & & & 0.0308 & & \\
\hline & & Caucasian Males vs Hispanic Females & & 0.0121 & & & & & & \\
\hline & & Caucasian Males vs East Asian Females & & & & & & $<0.0001$ & \begin{tabular}{l|l}
0.0011 \\
\end{tabular} & \\
\hline & & Hispanic Males vs Caucasian Females & & & & & & & & \\
\hline & & Hispanic Males vs Hispanic Females & & & & & & & & \\
\hline & & Hispanic Males vs East Asian Females & & & & & & & & \\
\hline & & Caucasian Females vs Hispanic Females & & 0.0028 & & & & & & \\
\hline & & Caucasian Females vs East Asian Females & & & & & & & & \\
\hline & & Hispanic Females vs East Asian Females & & 0.0119 & & & & 0.0013 & & \\
\hline
\end{tabular}




\begin{tabular}{|c|c|c|c|c|c|c|c|c|c|c|c|c|}
\hline & & & 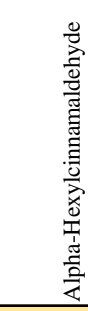 & 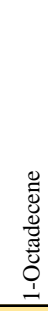 & 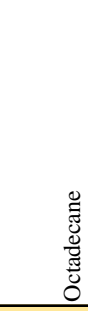 & 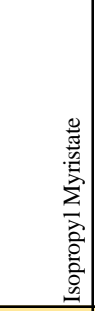 & 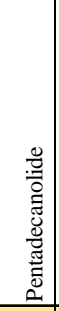 & 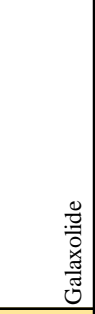 & 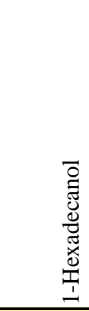 & 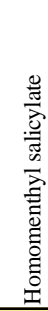 & 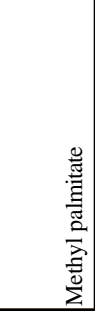 & 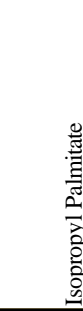 \\
\hline \multirow{4}{*}{$\begin{array}{c}\text { Individual } \\
\text { Classification by } \\
\text { Race/Ethnicity } \\
\end{array}$} & \multicolumn{2}{|r|}{ ANOVA (p-values) } & 0.0006 & & 0.0148 & 0.0397 & & 0.0068 & & & & \\
\hline & \multirow{3}{*}{$\begin{array}{l}\text { Tukey-Test } \\
\text { (p-values) }\end{array}$} & Caucasian vs East Asian & & & & 0.0374 & & & & & & \\
\hline & & Hispanic vs Caucasian & 0.0217 & & & & & 0.0345 & & & & \\
\hline & & Hispanic vs East Asian & 0.0006 & & 0.0156 & & & 0.0102 & & & & \\
\hline \multirow{4}{*}{$\begin{array}{c}\text { Individual } \\
\text { Classification by Age }\end{array}$} & \multirow{4}{*}{$\begin{array}{l}\text { Tukey-Test } \\
\text { (p-values) }\end{array}$} & ANOVA (p-values) & 0.0180 & & & & & & & & 0.0222 & 0.0348 \\
\hline & & $18-30$ vs $35-50$ years & 0.0397 & & & & & & & & & \\
\hline & & $18-30$ vs $55+$ years & 0.0383 & & & & & & & & 0.0162 & 0.0298 \\
\hline & & $35-50$ vs $55+$ years & & & & & & & & & & \\
\hline \multirow{4}{*}{$\begin{array}{c}\text { Female Classification } \\
\text { by Race/Ethnicity }\end{array}$} & \multirow{4}{*}{$\begin{array}{c}\text { Tukey-Test } \\
\text { (p-values) }\end{array}$} & ANOVA (p-values) & 0.0045 & & & & & & & & & \\
\hline & & Hispanic vs Caucasian & 0.0447 & & & & & & & & & \\
\hline & & Hispanic vs East Asian & 0.0220 & & & & & & & & & \\
\hline & & Caucasian vs East Asian & & & & & & & & & & \\
\hline \multirow{4}{*}{$\begin{array}{c}\text { Male Classification by } \\
\text { Race/Ethnicity }\end{array}$} & \multirow{4}{*}{$\begin{array}{l}\text { Tukey-Test } \\
\text { (p-values) }\end{array}$} & ANOVA (p-values) & 0.0410 & & 0.0139 & & & & & & & 0.0322 \\
\hline & & Hispanic vs Caucasian & & & & & & & & & & \\
\hline & & Hispanic vs East Asian & 0.0319 & & & & & & & & & \\
\hline & & Caucasian vs East Asian & & & 0.0099 & & & & & & & 0.0261 \\
\hline \multirow{16}{*}{$\begin{array}{c}\text { Overall Individual } \\
\text { Classification by } \\
\text { Race/Ethnicity and } \\
\text { Gender }\end{array}$} & \multirow{16}{*}{$\begin{array}{l}\text { Tukey-Test } \\
\text { (p-values) }\end{array}$} & ANOVA (p-values) & 0.0045 & & 0.0155 & & & 0.0074 & 0.0003 & & & 0.0023 \\
\hline & & East Asian Males vs Caucasian Males & & & & & & & & & & \\
\hline & & East Asian Males vs Hispanic Males & & & & & & & & & & \\
\hline & & East Asian Males vs Caucasian Females & & & & & & & 0.0023 & & & \\
\hline & & East Asian Males vs Hispanic Females & 0.0119 & & 0.0262 & & & 0.0171 & 0.0181 & & & 0.0130 \\
\hline & & East Asian Males vs East Asian Females & & & & & & & & & & \\
\hline & & Caucasian Males vs Hispanic Males & & & & & & & & & & \\
\hline & & Caucasian Males vs Caucasian Females & & & & & & & 0.0076 & & & \\
\hline & & Caucasian Males vs Hispanic Females & & & & & & 0.0096 & & & & \\
\hline & & Caucasian Males vs East Asian Females & & & & & & & & & & \\
\hline & & Hispanic Males vs Caucasian Females & & & & & & & & & & \\
\hline & & Hispanic Males vs Hispanic Females & & & & & & & & & & 0.0249 \\
\hline & & Hispanic Males vs East Asian Females & & & & & & & & & & \\
\hline & & Caucasian Females vs Hispanic Females & 0.0447 & & & & & & & & & \\
\hline & & Caucasian Females vs East Asian Females & & & & & & & & & & \\
\hline & & Hispanic Females vs East Asian Females & 0.0220 & & & & & & & & & \\
\hline
\end{tabular}


Appendix 8: Results obtained from T-test performed on the average underarm VOC amounts of all females and males (regardless of any other classification trait)

\begin{tabular}{|c|c|}
\hline \multicolumn{2}{|c|}{ Individual Classification by Gender: Females vs Males } \\
\hline Compounds & T-Test (p-values) \\
\hline Diethylene glycol monoethyl ether & 0.0036 \\
\hline Nonanal & 0.0283 \\
\hline Naphthalene & \\
\hline 2,4-Diisopropylphenol & 0.0355 \\
\hline Pentadecane & 0.0314 \\
\hline Methyl laurate & \\
\hline Dodecanoic acid & \\
\hline Isoamyl salicylate & \\
\hline Methyl tridecanoate & \\
\hline Alpha-Amylcinnamaldehyde & 0.0174 \\
\hline Dioctyl ether & \\
\hline Hexyl salicylate & \\
\hline 1-Heptadecene & \\
\hline Heptadecane & \\
\hline Pristane & \\
\hline Ethylene glycol monododecyl ether & \\
\hline Alpha-Hexylcinnamaldehyde & \\
\hline 1-Octadecene & \\
\hline Octadecane & \\
\hline Isopropyl Myristate & \\
\hline Pentadecanolide & \\
\hline Galaxolide & \\
\hline 1-Hexadecanol & \\
\hline Homomenthyl salicylate & \\
\hline Methyl palmitate & \\
\hline Isopropyl Palmitate & \\
\hline & \\
\hline & \\
\hline
\end{tabular}


Appendix 9: Results obtained from ANOVA and Tukey Tests performed on the average hand VOC amounts across all populations under study

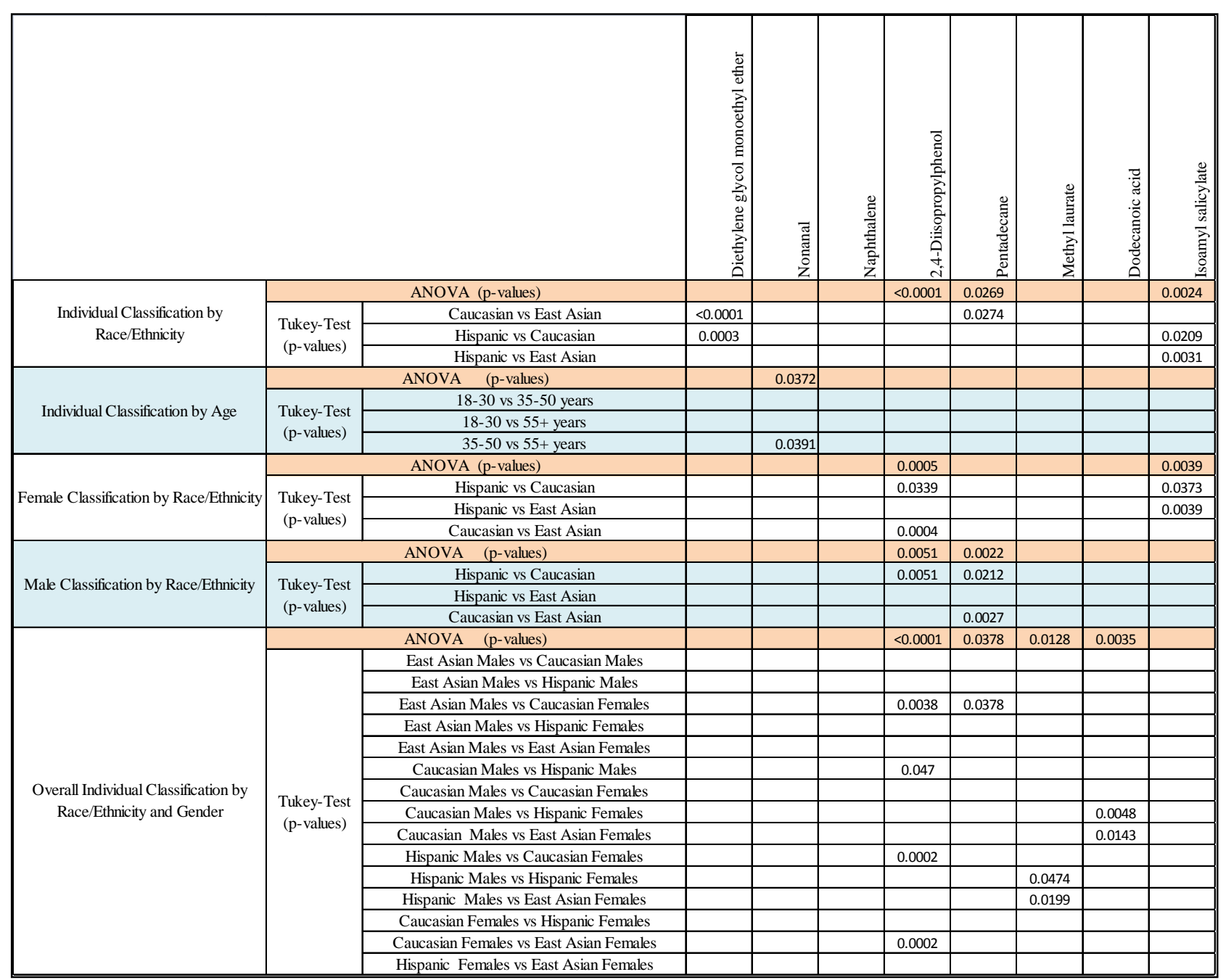




\begin{tabular}{|c|c|c|c|c|c|c|c|c|c|c|c|}
\hline & & & 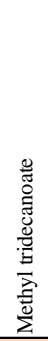 & 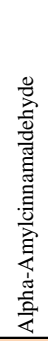 & 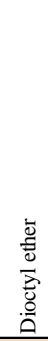 & 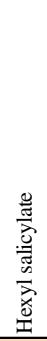 & 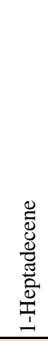 & 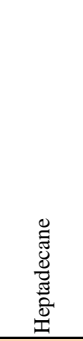 & 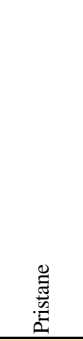 & 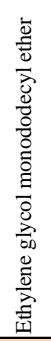 & 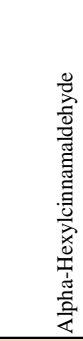 \\
\hline \multirow{4}{*}{$\begin{array}{l}\text { Individual Classification by } \\
\text { Race/Ethnicity }\end{array}$} & \multicolumn{2}{|r|}{ ANOVA (p-values) } & & & & & & $<0.0001$ & $<0.0001$ & & 0.0329 \\
\hline & \multirow{3}{*}{$\begin{array}{l}\text { Tukey-Test } \\
\text { (p-values) }\end{array}$} & Caucasian vs East Asian & & & & & & $<0.0001$ & $<0.0001$ & & \\
\hline & & Hispanic vs Caucasian & & & & & & 0.0001 & $<0.0001$ & & \\
\hline & & Hispanic vs East Asian & & & & & & & & & 0.0262 \\
\hline \multirow{4}{*}{ Individual Classification by Age } & \multirow{4}{*}{$\begin{array}{l}\text { Tukey-Test } \\
\text { (p-values) }\end{array}$} & ANOVA (p-values) & & & & & & & & & \\
\hline & & $18-30$ vs $35-50$ years & & & & & & & & & \\
\hline & & $18-30$ vs $55+$ years & & & & & & & & & \\
\hline & & $35-50$ vs $55+$ years & & & & & & & & & \\
\hline \multirow{4}{*}{ Female Classification by Race/Ethnicity } & \multirow{4}{*}{$\begin{array}{l}\text { Tukey-Test } \\
\text { (p-values) }\end{array}$} & ANOVA (p-values) & & & & & & $<0.0001$ & $<0.0001$ & & 0.0026 \\
\hline & & Hispanic vs Caucasian & & & & & & 0.012 & 0.0002 & & \\
\hline & & Hispanic vs East Asian & & & & & & & & & 0.0021 \\
\hline & & Caucasian vs East Asian & & & & & & $<0.0001$ & $<0.0001$ & & 0.0496 \\
\hline \multirow{4}{*}{ Male Classification by Race/Ethnicity } & \multirow{4}{*}{$\begin{array}{l}\text { Tukey-Test } \\
\text { (p-values) }\end{array}$} & ANOVA (p-values) & & & & & & 0.0019 & 0.0005 & & \\
\hline & & Hispanic vs Caucasian & & & & & & 0.0046 & 0.0009 & & \\
\hline & & Hispanic vs East Asian & & & & & & & & & \\
\hline & & Caucasian vs East Asian & & & & & & 0.0073 & 0.0054 & & \\
\hline \multirow{16}{*}{$\begin{array}{l}\text { Overall Individual Classification by } \\
\text { Race/Ethnicity and Gender }\end{array}$} & \multirow{16}{*}{$\begin{array}{l}\text { Tukey-Test } \\
\text { (p-values) }\end{array}$} & ANOVA (p-values) & & & & & & $<0.0001$ & $<0.0001$ & & \\
\hline & & East Asian Males vs Caucasian Males & & & & & & & & & \\
\hline & & East Asian Males vs Hispanic Males & & & & & & & & & \\
\hline & & East Asian Males vs Caucasian Females & & & & & & $<0.0001$ & $<0.0001$ & & \\
\hline & & East Asian Males vs Hispanic Females & & & & & & & & & \\
\hline & & East Asian Males vs East Asian Females & & & & & & & & & \\
\hline & & Caucasian Males vs Hispanic Males & & & & & & & 0.0150 & & \\
\hline & & Caucasian Males vs Caucasian Females & & & & & & & 0.0267 & & \\
\hline & & Caucasian Males vs Hispanic Females & & & & & & & & & \\
\hline & & Caucasian Males vs East Asian Females & & & & & & 0.0224 & 0.0132 & & \\
\hline & & Hispanic Males vs Caucasian Females & & & & & & $<0.0001$ & $<0.0001$ & & \\
\hline & & Hispanic Males vs Hispanic Females & & & & & & & & & \\
\hline & & Hispanic Males vs East Asian Females & & & & & & & & & \\
\hline & & Caucasian Females vs Hispanic Females & & & & & & 0.0141 & $<0.0001$ & & \\
\hline & & Caucasian Females vs East Asian Females & & & & & & $<0.0001$ & $<0.0001$ & & \\
\hline & & $\begin{array}{l}\text { Hispanic Females vs East Asian Females } \\
\end{array}$ & & & & & & & & & \\
\hline
\end{tabular}




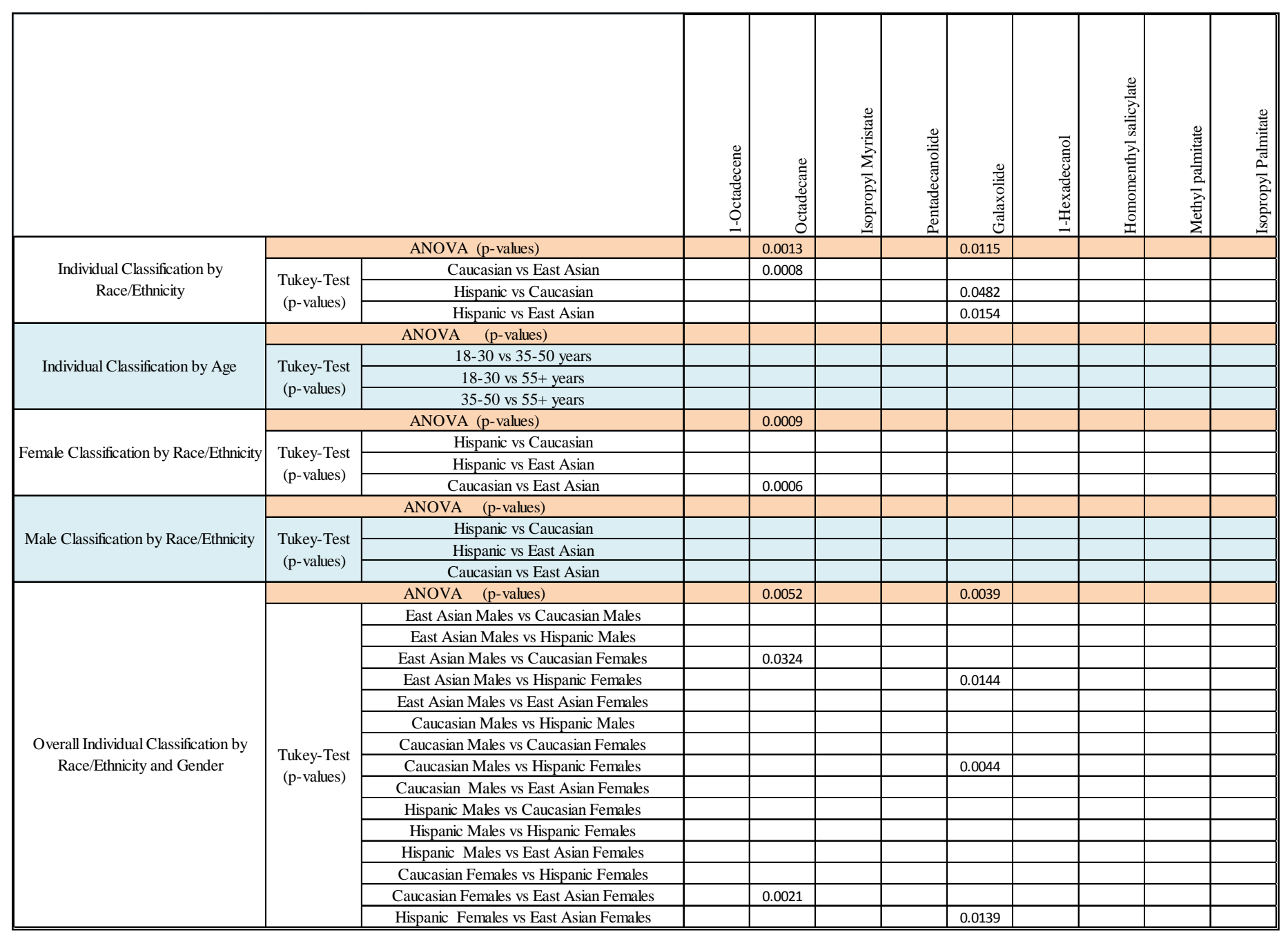


Appendix 10: Results obtained from T-test performed on the average hand VOC amounts of all females and males (regardless of any other classification trait)

\begin{tabular}{|c|c|}
\hline \multicolumn{2}{|c|}{ Individual Classification by Gender: Females vs Males } \\
\hline Compounds & T-Test (p-values) \\
\hline Diethylene glycol monoethyl ether & \\
\hline Nonanal & \\
\hline Naphthalene & 0.0457 \\
\hline 2,4-Diisopropylphenol & 0.0106 \\
\hline Pentadecane & 0.0009 \\
\hline Methyl laurate & \\
\hline Dodecanoic acid & \\
\hline Isoamyl salicylate & \\
\hline Methyl tridecanoate & \\
\hline Alpha-Amylcinnamaldehyde & \\
\hline Dioctyl ether & \\
\hline Hexyl salicylate & \\
\hline 1-Heptadecene & \\
\hline Heptadecane & \\
\hline Pristane & \\
\hline Ethylene glycol monododecyl ether & \\
\hline Alpha-Hexylcinnamaldehyde & \\
\hline 1-Octadecene & \\
\hline Octadecane & \\
\hline Isopropyl Myristate & \\
\hline Pentadecanolide & \\
\hline Galaxolide & \\
\hline 1-Hexadecanol & \\
\hline Homomenthyl salicylate & \\
\hline Methyl palmitate & \\
\hline Isopropyl Palmitate & \\
\hline & \\
\hline & \\
\hline & \\
\hline & \\
\hline
\end{tabular}




\section{Appendix 11: Average VOC amounts evaluated for the determination of age markers in underarm odor}

\begin{tabular}{|c|c|c|c|}
\hline \multirow{2}{*}{ Compound List } & \multicolumn{3}{|c|}{$\begin{array}{l}\text { Average Extracted Amounts per Age } \\
\text { Group }\end{array}$} \\
\hline & 18-30 years & $35-50$ years & $55+$ years \\
\hline Diethylene glycol monoethyl ether & 20.37 & 29.60 & 26.94 \\
\hline Nonanal & 6.08 & 5.68 & 5.94 \\
\hline Naphthalene & 0.05 & 0.57 & 0.20 \\
\hline 2,4-Diisopropylphenol & 0.36 & 0.42 & 0.50 \\
\hline Pentadecane & 14.10 & 8.56 & 11.13 \\
\hline Methyl laurate & 10.59 & 8.33 & 7.94 \\
\hline Dodecanoic acid & 0.94 & 0.69 & 1.57 \\
\hline Isoamyl salicylate & 2.32 & 1.12 & 0.83 \\
\hline Methyl tridecanoate & 1.11 & 0.96 & 0.30 \\
\hline Alpha-Amylcinnamaldehyde & 0.87 & 0.21 & 0.16 \\
\hline Dioctyl ether & 0.77 & 3.44 & 1.68 \\
\hline Hexyl salicylate & 6.79 & 3.83 & 2.78 \\
\hline 1-Heptadecene & 0.95 & 2.55 & 1.11 \\
\hline Heptadecane & 19.75 & 15.73 & 15.81 \\
\hline Pristane & 12.07 & 9.54 & 10.86 \\
\hline Ethylene glycol monododecyl ether & 11.00 & 8.17 & 5.43 \\
\hline Alpha-Hexylcinnamaldehyde & 11.54 & 7.02 & 6.84 \\
\hline 1-Octadecene & 0.36 & 0.40 & 0.19 \\
\hline Octadecane & 9.50 & 7.85 & 7.78 \\
\hline Isopropyl Myristate & 5.69 & 2.90 & 2.85 \\
\hline Pentadecanolide & 4.03 & 3.90 & 2.85 \\
\hline Galaxolide & 17.09 & 15.48 & 16.03 \\
\hline 1-Hexadecanol & 3.68 & 6.43 & 3.77 \\
\hline Homomenthyl salicylate & 13.52 & 13.14 & 7.17 \\
\hline Methyl palmitate & 7.12 & 5.26 & 2.70 \\
\hline Isopropyl Palmitate & 2.02 & 2.96 & 5.28 \\
\hline
\end{tabular}

*Each compound's highest average value is denoted in bold. 
Appendix 12: Average VOC amounts evaluated for the determination of race/ethnicity markers in underarm odor

\begin{tabular}{|c|c|c|c|}
\hline \multirow{2}{*}{ Compound List } & \multicolumn{3}{|c|}{$\begin{array}{l}\text { Average Extracted Amounts per } \\
\text { Race/Ethnic Group }\end{array}$} \\
\hline & Caucasians & East Asians & Hispanics \\
\hline Diethylene glycol monoethyl ether & 14.70 & 31.78 & 31.22 \\
\hline Nonanal & 5.20 & 6.02 & 6.51 \\
\hline Naphthalene & 0.07 & 0.09 & 0.63 \\
\hline 2,4-Diisopropylphenol & 0.63 & 0.13 & 0.46 \\
\hline Pentadecane & 10.56 & 9.41 & 13.60 \\
\hline Methyl laurate & 8.68 & 12.37 & 6.61 \\
\hline Dodecanoic acid & 2.06 & 0.51 & 0.46 \\
\hline Isoamyl salicylate & 0.93 & 0.72 & 2.59 \\
\hline Methyl tridecanoate & 0.54 & 1.82 & 0.27 \\
\hline Alpha-Amylcinnamaldehyde & 0.09 & 0.09 & 1.04 \\
\hline Dioctyl ether & 1.24 & 2.08 & 2.57 \\
\hline Hexyl salicylate & 3.98 & 2.70 & 6.67 \\
\hline 1-Heptadecene & 1.54 & 1.96 & 1.21 \\
\hline Heptadecane & 20.31 & 10.32 & 19.65 \\
\hline Pristane & 15.31 & 5.38 & 10.82 \\
\hline Ethylene glycol monododecyl ether & 8.54 & 6.01 & 10.07 \\
\hline Alpha-Hexylcinnamaldehyde & 7.55 & 5.19 & 12.35 \\
\hline 1-Octadecene & 0.58 & 0.12 & 0.22 \\
\hline Octadecane & 8.98 & 6.28 & 9.59 \\
\hline Isopropyl Myristate & 6.41 & 1.61 & 3.19 \\
\hline Pentadecanolide & 3.95 & 1.90 & 4.73 \\
\hline Galaxolide & 13.83 & 11.84 & 22.19 \\
\hline 1-Hexadecanol & 5.37 & 2.94 & 5.33 \\
\hline Homomenthyl salicylate & 16.31 & 8.26 & 9.26 \\
\hline Methyl palmitate & 4.05 & 6.77 & 4.93 \\
\hline Isopropyl Palmitate & 4.06 & 1.40 & 4.16 \\
\hline
\end{tabular}

*Each compound's highest average value is denoted in bold. 
Appendix 13: Average VOC amounts evaluated for the determination of gender markers in underarm odor

\begin{tabular}{|c|c|c|}
\hline \multirow{2}{*}{ Compound List } & \multicolumn{2}{|c|}{$\begin{array}{c}\text { Average Extracted Amounts per } \\
\text { Gender Group }\end{array}$} \\
\hline & Females & Males \\
\hline Diethylene glycol monoethyl ether & 26.35 & 24.62 \\
\hline Nonanal & 7.35 & 4.33 \\
\hline Naphthalene & 0.43 & 0.11 \\
\hline 2,4-Diisopropylphenol & 0.31 & 0.55 \\
\hline Pentadecane & 12.61 & 9.89 \\
\hline Methyl laurate & 7.19 & 10.99 \\
\hline Dodecanoic acid & 0.38 & 1.77 \\
\hline Isoamyl salicylate & 1.44 & 1.48 \\
\hline Methyl tridecanoate & 0.66 & 0.99 \\
\hline Alpha-Amylcinnamaldehyde & 0.61 & 0.23 \\
\hline Dioctyl ether & 2.38 & 1.50 \\
\hline Hexyl salicylate & 4.13 & 5.03 \\
\hline 1-Heptadecene & 1.00 & 2.14 \\
\hline Heptadecane & 16.61 & 17.79 \\
\hline Pristane & 11.39 & 10.24 \\
\hline Ethylene glycol monododecyl ether & 7.61 & 9.15 \\
\hline Alpha-Hexylcinnamaldehyde & 8.76 & 8.36 \\
\hline 1-Octadecene & 0.53 & 0.09 \\
\hline Octadecane & 8.56 & 8.26 \\
\hline Isopropyl Myristate & 5.05 & 2.60 \\
\hline Pentadecanolide & 3.52 & 3.76 \\
\hline Galaxolide & 19.16 & 13.03 \\
\hline 1-Hexadecanol & 6.78 & 2.33 \\
\hline Homomenthyl salicylate & 13.50 & 9.28 \\
\hline Methyl palmitate & 4.54 & 5.81 \\
\hline Isopropyl Palmitate & 4.83 & 1.69 \\
\hline
\end{tabular}

*Each compound's highest average value is denoted in bold. 
Appendix 14: Average VOC amounts evaluated for the determination of underarm odor markers for gender and race/ethnicity

\begin{tabular}{|c|c|c|c|c|c|c|}
\hline \multirow{2}{*}{ Compound List } & \multicolumn{2}{|c|}{ Caucasian } & \multicolumn{2}{|c|}{ East Asian } & \multicolumn{2}{|c|}{ Hispanic } \\
\hline & Females & Males & Females & Males & Females & Males \\
\hline Diethylene glycol monoethyl ether & 19.16 & 10.25 & 29.55 & 34.76 & 30.55 & 31.89 \\
\hline Nonanal & 6.01 & 4.38 & 7.31 & 4.29 & 8.71 & 4.30 \\
\hline Naphthalene & 0.09 & 0.05 & 0.10 & 0.09 & 1.08 & 0.18 \\
\hline 2,4-Diisopropylphenol & 0.27 & 0.99 & 0.12 & 0.14 & 0.54 & 0.39 \\
\hline Pentadecane & 11.55 & 9.57 & 11.42 & 6.74 & 14.79 & 12.41 \\
\hline Methyl laurate & 5.12 & 12.25 & 11.91 & 12.98 & 4.87 & 8.34 \\
\hline Dodecanoic acid & 0.98 & 3.14 & 0.14 & 1.02 & 0 & 0.91 \\
\hline Isoamyl salicylate & 0.66 & 1.20 & 0.76 & 0.66 & 2.85 & 2.33 \\
\hline Methyl tridecanoate & 0.40 & 0.68 & 1.44 & 2.33 & 0.18 & 0.36 \\
\hline Alpha-Amylcinnamaldehyde & 0.00 & 0.19 & 0.16 & 0 & 1.65 & 0.43 \\
\hline Dioctyl ether & 1.49 & 0.99 & 2.17 & 1.98 & 3.47 & 1.68 \\
\hline Hexyl salicylate & 3.14 & 4.82 & 2.62 & 2.81 & 6.53 & 6.81 \\
\hline 1-Heptadecene & 0.91 & 2.17 & 1.35 & 2.78 & 0.75 & 1.67 \\
\hline Heptadecane & 16.07 & 24.55 & 11.02 & 9.38 & 22.36 & 16.93 \\
\hline Pristane & 13.54 & 17.09 & 6.81 & 3.47 & 13.52 & 8.12 \\
\hline Ethylene glycol monododecyl ether & 9.13 & 7.94 & 5.80 & 6.28 & 7.76 & 12.37 \\
\hline Alpha-Hexylcinnamaldehyde & 6.48 & 8.63 & 5.71 & 4.48 & 13.89 & 10.82 \\
\hline 1-Octadecene & 1.17 & 0.00 & 0.15 & 0.09 & 0.25 & 0.19 \\
\hline Octadecane & 7.79 & 10.17 & 6.83 & 5.53 & 10.93 & 8.25 \\
\hline Isopropyl Myristate & 8.69 & 4.14 & 2.32 & 0.65 & 3.97 & 2.42 \\
\hline Pentadecanolide & 4.17 & 3.72 & 1.81 & 2.02 & 4.46 & 5.00 \\
\hline Galaxolide & 17.19 & 10.46 & 13.39 & 9.77 & 26.50 & 17.87 \\
\hline 1-Hexadecanol & 8.48 & 2.26 & 4.43 & 0.95 & 7.28 & 3.38 \\
\hline Homomenthyl salicylate & 19.70 & 12.92 & 9.10 & 7.14 & 11.39 & 7.13 \\
\hline Methyl palmitate & 3.39 & 4.70 & 5.95 & 7.86 & 4.38 & 5.47 \\
\hline Isopropyl Palmitate & 5.54 & 2.59 & 1.99 & 0.61 & 6.78 & 1.53 \\
\hline
\end{tabular}




\section{Appendix 15: Average VOC amounts evaluated for the determination of age markers in hand odor}

\begin{tabular}{|c|c|c|c|}
\hline \multirow{2}{*}{ Compound List } & \multicolumn{3}{|c|}{$\begin{array}{l}\text { Average Extracted Amounts per Age } \\
\text { Group }\end{array}$} \\
\hline & 18-30 years & $35-50$ years & $55+$ years \\
\hline Diethylene glycol monoethyl ether & 45.26 & 37.52 & 40.29 \\
\hline Nonanal & 14.93 & 16.51 & 8.57 \\
\hline Naphthalene & 0.34 & 0.13 & 0.33 \\
\hline 2,4-Diisopropylphenol & 0.52 & 0.44 & 0.76 \\
\hline Pentadecane & 4.49 & 4.06 & 4.72 \\
\hline Methyl laurate & 3.14 & 0.72 & 1.00 \\
\hline Dodecanoic acid & 2.30 & 1.09 & 1.58 \\
\hline Isoamyl salicylate & 0.50 & 0.44 & 0.42 \\
\hline Methyl tridecanoate & 0.27 & 0.03 & 0.04 \\
\hline Alpha-Amylcinnamaldehyde & 0.38 & 0.40 & 0.25 \\
\hline Dioctyl ether & 0.21 & 0.74 & 0.41 \\
\hline Hexyl salicylate & 0.85 & 0.99 & 1.25 \\
\hline 1-Heptadecene & 0.68 & 1.05 & 0.60 \\
\hline Heptadecane & 9.89 & 8.22 & 10.24 \\
\hline Pristane & 8.24 & 6.25 & 8.41 \\
\hline Ethylene glycol monododecyl ether & 3.48 & 2.68 & 2.85 \\
\hline Alpha-Hexylcinnamaldehyde & 1.15 & 1.06 & 2.17 \\
\hline 1-Octadecene & 0.53 & 0.08 & 0.03 \\
\hline Octadecane & 4.77 & 4.47 & 4.83 \\
\hline Isopropyl Myristate & 2.19 & 2.89 & 4.15 \\
\hline Pentadecanolide & 1.35 & 1.63 & 3.90 \\
\hline Galaxolide & 6.32 & 4.23 & 7.06 \\
\hline 1-Hexadecanol & 2.26 & 2.32 & 5.39 \\
\hline Homomenthyl salicylate & 2.94 & 4.22 & 3.79 \\
\hline Methyl palmitate & 1.30 & 0.35 & 1.63 \\
\hline Isopropyl Palmitate & 1.28 & 2.90 & 3.42 \\
\hline
\end{tabular}

*Each compound's highest average value is denoted in bold. 
Appendix 16: Average VOC amounts evaluated for the determination of race/ethnicity markers in hand odor

\begin{tabular}{|c|c|c|c|}
\hline \multirow{2}{*}{ Compound List } & \multicolumn{3}{|c|}{$\begin{array}{l}\text { Average Extracted Amounts per } \\
\text { Race/Ethnic Group }\end{array}$} \\
\hline & Caucasians & East Asians & Hispanics \\
\hline Diethylene glycol monoethyl ether & 39.77 & 41.88 & 41.55 \\
\hline Nonanal & 15.29 & 14.19 & 10.99 \\
\hline Naphthalene & 0.39 & 0.14 & 0.25 \\
\hline 2,4-Diisopropylphenol & 1.00 & 0.28 & 0.39 \\
\hline Pentadecane & 6.11 & 3.13 & 3.90 \\
\hline Methyl laurate & 1.60 & 0.37 & 2.84 \\
\hline Dodecanoic acid & 2.41 & 1.08 & 1.44 \\
\hline Isoamyl salicylate & 0.33 & 0.20 & 0.82 \\
\hline Methyl tridecanoate & 0.02 & 0.03 & 0.28 \\
\hline Alpha-Amylcinnamaldehyde & 0.30 & 0.17 & 0.55 \\
\hline Dioctyl ether & 0.51 & 0.41 & 0.44 \\
\hline Hexyl salicylate & 1.08 & 0.70 & 1.26 \\
\hline 1-Heptadecene & 0.33 & 1.02 & 1.00 \\
\hline Heptadecane & 13.92 & 6.05 & 8.03 \\
\hline Pristane & 12.57 & 4.45 & 5.55 \\
\hline Ethylene glycol monododecyl ether & 2.91 & 2.65 & 3.44 \\
\hline Alpha-Hexylcinnamaldehyde & 1.31 & 0.64 & 2.30 \\
\hline 1-Octadecene & 0.05 & 0.57 & 0.06 \\
\hline Octadecane & 5.92 & 3.32 & 4.69 \\
\hline Isopropyl Myristate & 5.21 & 0.93 & 2.83 \\
\hline Pentadecanolide & 3.27 & 0.65 & 2.69 \\
\hline Galaxolide & 4.27 & 3.10 & 9.92 \\
\hline 1-Hexadecanol & 3.40 & 2.18 & 4.11 \\
\hline Homomenthyl salicylate & 3.16 & 4.03 & 3.77 \\
\hline Methyl palmitate & 1.73 & 0.22 & 1.20 \\
\hline Isopropyl Palmitate & 2.73 & 1.15 & 3.53 \\
\hline
\end{tabular}

*Each compound's highest average value is denoted in bold. 
Appendix 17: Average VOC amounts evaluated for the determination of gender markers in hand odor

\begin{tabular}{|c|c|c|}
\hline \multirow{2}{*}{ Compound List } & \multicolumn{2}{|c|}{$\begin{array}{c}\text { Average Extracted Amounts per } \\
\text { Gender Group }\end{array}$} \\
\hline & Females & Males \\
\hline Diethylene glycol monoethyl ether & 39.88 & 42.28 \\
\hline Nonanal & 13.60 & 13.34 \\
\hline Naphthalene & 0.32 & 0.21 \\
\hline 2,4-Diisopropylphenol & 0.66 & 0.46 \\
\hline Pentadecane & 5.33 & 3.45 \\
\hline Methyl laurate & 0.51 & 2.84 \\
\hline Dodecanoic acid & 0.89 & 2.47 \\
\hline Isoamyl salicylate & 0.42 & 0.50 \\
\hline Methyl tridecanoate & 0.05 & 0.18 \\
\hline Alpha-Amylcinnamaldehyde & 0.39 & 0.29 \\
\hline Dioctyl ether & 0.66 & 0.23 \\
\hline Hexyl salicylate & 1.11 & 0.92 \\
\hline 1-Heptadecene & 0.96 & 0.59 \\
\hline Heptadecane & 10.46 & 8.33 \\
\hline Pristane & 8.76 & 6.40 \\
\hline Ethylene glycol monododecyl ether & 2.78 & 3.25 \\
\hline Alpha-Hexylcinnamaldehyde & 1.39 & 1.49 \\
\hline 1-Octadecene & 0.42 & 0.01 \\
\hline Octadecane & 4.79 & 4.57 \\
\hline Isopropyl Myristate & 4.53 & 1.47 \\
\hline Pentadecanolide & 3.33 & 1.11 \\
\hline Galaxolide & 7.98 & 3.58 \\
\hline 1-Hexadecanol & 4.96 & 1.46 \\
\hline Homomenthyl salicylate & 5.46 & 1.72 \\
\hline Methyl palmitate & 0.80 & 1.37 \\
\hline Isopropyl Palmitate & 3.77 & 1.17 \\
\hline
\end{tabular}

*Each compound's highest average value is denoted in bold. 
Appendix 18: Average VOC amounts evaluated for the determination of hand odor markers for gender and race/ethnicity

\begin{tabular}{|c|c|c|c|c|c|c|}
\hline \multirow{2}{*}{ Compound List } & \multicolumn{2}{|c|}{ Caucasian } & \multicolumn{2}{c|}{ East Asian } & \multicolumn{2}{c|}{ Hispanic } \\
\cline { 2 - 6 } & Females & Males & Females & Males & Females & Males \\
\hline Diethylene glycol monoethyl ether & 39.24 & 40.31 & 44.74 & 38.44 & 35.65 & 47.45 \\
\hline Nonanal & 14.86 & 15.72 & 16.48 & 11.44 & 9.44 & 12.53 \\
\hline Naphthalene & 0.67 & 0.11 & 0.13 & 0.15 & 0.16 & 0.35 \\
\hline 2,4-Diisopropylphenol & 1.18 & 0.82 & 0.22 & 0.35 & 0.58 & 0.20 \\
\hline Pentadecane & 6.89 & 5.33 & 4.11 & 1.96 & 5.00 & 2.80 \\
\hline Methyl laurate & 0.68 & 2.52 & 0.19 & 0.59 & 0.65 & 5.04 \\
\hline Dodecanoic acid & 1.48 & 3.34 & 0.73 & 1.50 & 0.46 & 2.42 \\
\hline Isoamyl salicylate & 0.30 & 0.36 & 0.12 & 0.30 & 0.83 & 0.80 \\
\hline Methyl tridecanoate & 0.05 & 0.00 & 0.02 & 0.04 & 0.09 & 0.47 \\
\hline Alpha-Amylcinnamaldehyde & 0.53 & 0.07 & 0.17 & 0.17 & 0.49 & 0.61 \\
\hline Dioctyl ether & 0.75 & 0.27 & 0.41 & 0.42 & 0.83 & 0.04 \\
\hline Hexyl salicylate & 1.34 & 0.82 & 0.77 & 0.61 & 1.23 & 1.29 \\
\hline 1-Heptadecene & 0.38 & 0.29 & 1.14 & 0.89 & 1.35 & 0.65 \\
\hline Heptadecane & 16.02 & 11.82 & 5.73 & 6.44 & 9.64 & 6.42 \\
\hline Pristane & 15.25 & 9.89 & 4.12 & 4.85 & 6.91 & 4.20 \\
\hline Ethylene glycol monododecyl ether & 2.61 & 3.20 & 2.35 & 3.01 & 3.38 & 3.50 \\
\hline Alpha-Hexylcinnamaldehyde & 1.55 & 1.08 & 0.63 & 0.65 & 2.00 & 2.60 \\
\hline 1-Octadecene & 0.10 & 0.00 & 1.05 & 0.00 & 0.10 & 0.02 \\
\hline Octadecane & 6.75 & 5.10 & 3.01 & 3.70 & 4.61 & 4.78 \\
\hline Isopropyl Myristate & 9.41 & 1.00 & 1.05 & 0.77 & 3.12 & 2.53 \\
\hline Pentadecanolide & 6.03 & 0.51 & 1.03 & 0.20 & 2.92 & 2.46 \\
\hline Galaxolide & 6.40 & 2.15 & 3.32 & 2.83 & 14.21 & 5.62 \\
\hline 1-Hexadecanol & 6.19 & 0.61 & 3.14 & 1.03 & 5.56 & 2.66 \\
\hline Homomenthyl salicylate & 4.27 & 2.06 & 6.09 & 1.56 & 6.03 & 1.52 \\
\hline Isopropyl Palmitate & 1.89 & 1.58 & 0.06 & 0.42 & 0.44 & 1.96 \\
\hline
\end{tabular}


Appendix 19: Compounds quantified in extracts by Solvent Extraction ${ }^{136}$

\begin{tabular}{|c|c|c|c|}
\hline \multicolumn{4}{|c|}{ Compounds quantified in extracts obtained by Solvent Extraction } \\
\hline $\begin{array}{c}\text { CAS } \\
\text { Number }\end{array}$ & Compound & $\begin{array}{c}\text { Molecular weight } \\
\text { (g/mol) }\end{array}$ & $\begin{array}{c}\text { Boiling Point } \\
\left({ }^{\circ} \mathbf{C}\right)\end{array}$ \\
\hline $111-90-0$ & $\begin{array}{c}\text { Diethylene glycol monoethyl } \\
\text { ether }\end{array}$ & 134.173 & $202(3)$ \\
\hline $544-63-8$ & Tetradecanoic acid & 228.371 & $250^{100}$ \\
\hline $1002-84-2$ & Pentadecanoic acid & 242.398 & $257^{100}$ \\
\hline $57-10-3$ & n-Hexadecanoic acid & 256.424 & $351(6)$ \\
\hline $630-01-3$ & Hexacosane & 366.707 & $415(11)$ \\
\hline $117-81-7$ & Bis(2-ethylhexyl) phthalate & 390.557 & 384 \\
\hline $630-02-4$ & Octacosane & 394.761 & $432(6)$ \\
\hline
\end{tabular}

${ }^{*}$ Superscripts indicate specific pressure for boiling point measurements (mmHg). The remaining values reported are Normal boiling points (760 mmHg).

${ }^{* *}$ Parentheses indicate the combined expanded uncertainty of the boiling point values 


\section{LAUREN JANICE COLÓN CRESPO}

1988

$2006-2011$

$2008-2010$

$2009-2010$

$2010-2011$

$2011-2012$

$2012-2016$
Born, San Juan, Puerto Rico

B. Sc., Chemistry

University of Puerto Rico

Río Piedras, Puerto Rico

Undergraduate Research Student

Laboratory Intern

Food and Drug Administration

San Juan, Puerto Rico

Undergraduate Research Assistant

University of Puerto Rico

Río Piedras, Puerto Rico

Teaching Assistant

Florida International University

Miami, Florida

Research Assistant

Florida International University

Miami, Florida

\section{PUBLICATIONS AND PRESENTATIONS}

Colón-Crespo, L.J.; Herrera-Hernández, D.; Holness, H.; Kabir, A.; Furton, K.G.; Fan, A.; Du-Thumm, L., Use of Human Scent as a Classification Tool for Individuals, Florida Annual Meeting and Exposition (FAME), Tampa, Florida, May 8 - May 10, 2014.

Herrera-Hernández, D.; Colón-Crespo, L.J.; Holness, H.; Furton, K.G.; Fan, A.; DuThumm, L., Enhancement of Human Scent Detection through the use of Exhaustive Liquid Extraction Methods, Florida Annual Meeting and Exposition (FAME), Tampa, Florida, May 8 - May 10, 2014.

Colón-Crespo, L.J.; Herrera-Hernández, D.; Holness, H.; Kabir, A.; Furton, K.G.; Fan, A.; Du-Thumm, L., Evaluation of Human Scent As Possible Classification Evidence, 3rd Annual International Forensic Research Institute (IFRI) Symposium, Florida International University, Miami, Florida, April 30 - May 1, 2014. 
Herrera-Hernández, D.; Colón-Crespo, L.J.; Holness, H.; Furton, K.G.; Fan, A.; DuThumm, L., Enhancement of Human Scent Detection through the use of Exhaustive Liquid Extraction Methods, 3rd Annual International Forensic Research Institute (IFRI) Symposium, Florida International University, Miami, Florida, April 30 - May 1, 2014.

Colón-Crespo, L.J.; Herrera-Hernández, D.; Holness, H.; Kabir, A.; Furton, K.G.; Fan, A.; Du-Thumm, L., Evaluation of Human Scent As Possible Classification Evidence, Graduate Student Appreciation Week’s (GSAW) Scholarly Forum, Florida International University, Miami, Florida, March 31 - April 1, 2014.

Colón-Crespo, L.J.; Herrera-Hernández, D.; Holness, H.; Kabir, A.; Furton, K.G.; Fan, A.; Du-Thumm, L., Evaluation of Human Scent As Possible Classification Evidence, 66th Annual Scientific Meeting of the Academy of Forensic Sciences (AAFS), Seattle,WA, February 17-22, 2014.

Herrera-Hernández, D.; Colón-Crespo, L.J.; Holness, H.; Furton, K.G.; Fan, A.; DuThumm, L., Enhancement of Human Scent Detection through the use of Exhaustive Liquid Extraction Methods, 66th Annual Scientific Meeting of the Academy of Forensic Sciences (AAFS), Seattle,WA, February 17-22, 2014.

Colón-Crespo, L.J.; Brown, J.S.; Caraballo, N.I.; Furton, K.G., Advances in the Forensic Use of Human Scent, 2nd Annual International Forensic Research Institute (IFRI) Symposium, Florida International University, Miami, Florida, March 13-14, 2013.

Colón-Crespo, L.J.; Brown, J.S.; Caraballo, N.I.; Furton, K.G., Advances in the Forensic Use of Human Scent, 65th Annual Scientific Meeting of the Academy of Forensic Sciences (AAFS), Washington, D.C., February 18-23, 2013.

Colón, Lauren; Rivera, Brenda; Rosario, Osvaldo, Thin Layer Chromatography for the Analysis of Food Colorings, The Puerto Rico Interdisciplinary Scientific Meeting (PRISM), Mayagüez, Puerto Rico, March 13, 2010.

Colón, Lauren; Mayol-Bracero, Olga; Formenti, Paola; Mazzei, Federico, Atmospheric Aerosols in the Guánica Dry Forest, American Geophysical Union (AGU) Joint Assembly, Toronto, Canada, May 24-27, 2009.

Colón, Lauren; Mayol-Bracero, Olga; Formenti, Paola; Mazzei, Federico, Atmospheric Aerosols in the Guánica Dry Forest: African Dust, Marine, and Anthropogenic Particles, The Puerto Rico Interdisciplinary Scientific Meeting (PRISM), Río Piedras, Puerto Rico, March 14, 2009. 